\title{
Woods Hole FORTH
}

\author{
by
}

John J. Akens

Woods Hole Oceanographic Institution

Woods Hole, Massachusetts 02543

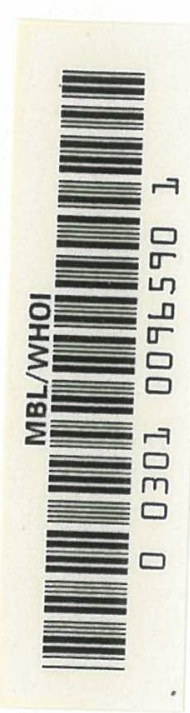

. December 1984

Technical Report

Funding was provided by the National Science Foundation under Grant number OCE 83-20508.

Reproduction in whole or in part is permitted for any purpose of the United States Government.

This report should be cited as: Woods Hole Oceanog. Inst. Tech. Rept., WHOI-84-46.

Approved for public release; distribution unlimited.

Approved for Distribution:

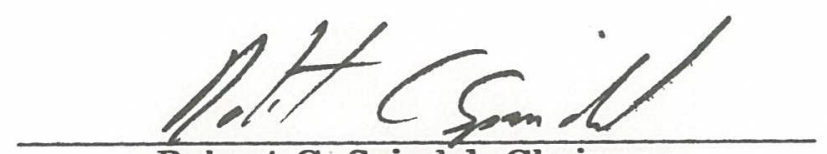

Robert C. Spindel, Chairman

Department of Ocean Engineering 



\section{Abstract}

Woods Hole FORTH, called WFORTH, is a real time language for the RCA 1806 microprocessor. It follows the fig-FORTH standard, and is used by Alvin for data acquisition and display tasks, plus playback and processing tasks at sea and ashore. WFORTH is optimized for simple networking with RS-232 uarts. Programs can be installed in Eprom for use in unattended systems and can be configured to run a specific task when power is applied. The error handling can be customized to permit continued operation when errors are encountered. Fxtensive utilities and development tools are included such as full screen editor; the fig line editor; a single pass assembler; floating point support; disk file access; communication utilities for uploading or downloading files or blocks of memory; and documentation utilities. The complete sources and a glossary for WFORTH are included in this publication. Versions are supplied which include compiled development, floating point, and assembler utilities. 


\section{Acknowledgements}

I would like to thank the following individuals and groups who assisted in the preparation of this document.

The National Science Foundation, which provided the financial support for this project, under NSF grant OCE 83-20508 .

Barrie Walden, Woods Hole Oceanographic Institution, who proofread several versions of this document, and offered many suggestions.

Dick Berger, Micro Amp Designs, who provided the original source used in writing Woods Hole FORTH.

Jim McDaniels, Geometrics Incorporated, who wrote the original version of the assembler.

George Stetten, Woods Hole Oceanographic Institution, who did much of the work involved in linking the floating point words.

George Meier, Woods Hole Oceanographic Institution, who built all of the hardware prototypes.

Terri Nielsen, Woods Hole Oceanographic Institution, who typed the original version of the glossary.

The FORTH Interest Group, for their many fine publications and the figFORTH standard.

Charles Moore, Forth Incorporated, the inventor of the language FORTH.

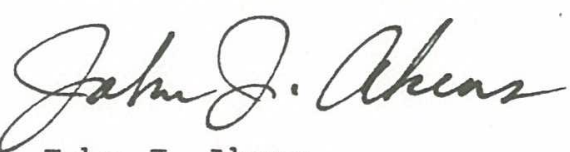

John J. Akens

Research Associate,

Submersible Engineering and Operations

Woods Hole Oceanographic Institution 
Woods Hole FORTH

Table of Contents

Abstract i

Acknowledgements ii

$1 \quad$ Introduction and notation summary 1

$2 \quad$ System Configuration and Special Hardware 5

3 FORTH Editor and Glossary 10

$4 \quad$ FORTH Disk Operating System 16

$5 \quad$ FORTH Assembler and Glossary 18

6 Communication Utilities 20

7 Memory Map and Parameter Table 22

$8 \quad$ Developing Eprom Application Programs 28

$9 \quad$ FORTH Floating Point 31

$10 \quad$ Documentation Utilities 36

11 Disk Directories and Cluster Allocation Tables 37

$12 \quad$ Screen Index 39

13 Vocabulary list (in order of creation) 43

$14 \quad$ Vocabulary List (alphabetically) 46

15 USER Variables 49

16 CONSTANTS, VARIABLES, and VOCABULARIES 51

$17 \quad$ Screen Listings 53

$18 \quad$ Vocabulary List with Full Attributes 97

19 Memory Dump 106

$20 \quad$ Examples 129

$21 \quad$ Source Listing 143

$22 \quad$ Cross Reference Listing for the Source 211

23 Known Deviations from fig-FORTH 222

$24 \quad$ References 226

$\begin{array}{lll}25 & \text { Glossary } & 229\end{array}$

$26 \quad$ Numbered Errors and Text Messages 286 



\section{Introduction}

Woods Hole FORTH is a version of fig-FORTH, written for the RCA CDP 1806A microprocessor, used by the ALVIN Group and the Deep Submergence Laboratory at the Woods Hole Oceanographic Institution (WHOI) to perform numerous realtime data acquisition, display, and control functions. Woods Hole FORTH, hereafter called WFORTH, is an extension of the language FORTH optimized for serial communications (RS-232), and program development for systems that will ultimately be placed in Prom or Eprom. WFORTH is fully compatible with the RCA disk operating system, MDOS 1.0. An extremely powerful decompiler is included to help in debugging new words, or merely understand existing words. Floating point support is included as a series of screens and a file called FLOAT.CM. A version of WFORTH which includes floating point support has been provided as a file named FFORTH.CM . In addition, a version containing a single pass Assembler is included for writing FORTH words in machine code. This is the file AFORTH.CM. WFORTH conforms closely to the fig-FORTH model. With the exception of code words and I/O words, WFORTH words are fully transportable to other fig-FORTH systems.

A complete Woods Hole FORTH system occupies three disks. The first, called the ALVIN OPERATING SYSTEM, normally occupies drive zero. It contains many copyrighted programs from RCA MICRODOS 1.0. The user should copy those files not found in the disk directory for the ALVIN Operating system in Chapter 11, from MDOS 1.0. This disk also contains backup copies of FORTH and WFORTH, several SUBMIT files, and many files and programs used in acquiring and playing back data on the submersible Alvin.

The second disk, WOODS HOLE FORTH SCREFN FILE, contains all of the source screens which have been used to create WFORTH.CM, FFORTH.CM, and AFORTH.CM from the file FORTH.CM plus numerous utility screens and 6 files: FORTH.CM, WFORTH.CM, FFORTH.CM, AFORTH.CM, ASM4TH.CM, and FLOAT.CM. FORTH.CM, based upon the fig-FORTH model, has been generated from the source file WFORTH.SRC. FLOAT.CM and ASM4TH.CM are files which are loaded by FFORTH.CM and AFORTH.CM respectively, and are not intended to be called by the user directly. This disk should also include the files COPY.CM and SYSGEN.CM, which the user must copy from the RCA MDOS disk. Chapter 11 provides a directory for this disk and also shows the cluster allocation table (CAT), which has been marked to reserve the sectors which correspond to FORTH screens 65 through 249. These are the screens normally used in WFORTH and at 
present, there are still about 60 (d) sectors available on the disk. I sector equals 512 (d) bytes. This is enough space to store 2 or more additional application versions of FORTH.

The following explanation demonstrates the relationships between the major files.

WFORTH.CM has been created from FORTH.CM by the sequence:

>FORTH:I ( $\mathrm{Cr}$ ) (load and run fig-FORTH generated by the assembler)

OK

66 LOAD 65 LOAD SAVE WFORTH.CM:I (cr) (compile new words and save as the file WFORTH.CM:I)

FFORTH.CM has been created from WFORTH.CM by the sequence:

$>$ WFORTH:I (cr) (load and run WFORTH.CM)

OK

FLOATING NEN-FENCE SAVE FFORTH.CM:I (cr) (compile floating point words and save as the file FFORTH.CM:1)

AFORTH.CM has been created from WFORTH.CM by the sequence:

>WFORTH:I ( $\mathrm{Cr}$ ) (load and run WFORTH.CM)

OK

77 LOAD (cr) (load assembler master load screen)

$>$ MEM ( $\mathrm{Cr}$ )

MEMORY SAVE PROGRAM

FIRST ADDR? $\mathrm{C600}$ (cr)

IAST ADDR? CFFF (cr)

WRITE? ASM4TH.MEM:I (cr)

>CDSBIN ASM4TH.MEM:I ASM4TH.CM:1;9005 (cr)

The third disk, WOODS HOLE FORTH SOURCE AND LIST, contains the source file WFORTH.SRC; the output of the Assembler which is a list file called WFORTH.IST; and the file FORTH.CM. FORTH.CM is the binary loadable version of WFORTH.LST. Many SUBMIT files are also included to facilitate recursive development of the source, and ultimately, FORTH.CM. 
FORTH.CM has been created from WFORTH.SRC by the sequence:

>SUBMIT ASMB:I (cr) (calls all programs required to convert the source file to a binary loadable .CM file.)

These three disks are all that are required to completely maintain FORTH. If it becomes desirable to change FORTH, begin by editing the file WFORTH.SRC, then assemble to create the files WFORTH.LST and FORTH.CM. Finally, switch to the high level environment of FORTH, compile screens and save the resulting program, either with MEM.CM and CDSBIN.CM in the operating system, or SAVE from within WFORTH.

\section{NOTATION}

The following is a summary of the notation used in this document.

Examples

Meaning

FORTH

Any word which is capitalized represents a FORTH word. BEEP

MEM.CM Capitalized words which end with the string . CM are programs created or used by the operating system MDOS 1.0 .

Filespec Filespec is used to indicate any file or program running under MDOS 1.0, using RCA's notation. For instance: AFORTH.CM:I, identifies the version of FORTH which has the assembler precompiled, which is located on drive 1.

ANY.WORD These indicate that any FORTH word can be used.

ANY.NAME

There are eight general notations used for numbers, with some obvious variations. For instance, STRING.ADDRESS indicates a number, interpreted as an address, which points to a string. BLOCK. NUMBER indicates a number which specifies a block. 
NUMBER

Nl

$\mathrm{N}$

$\mathrm{U}$

DOUBLE

D2

D

UD

F

CHARACTER

CHAR

ADDRESS

$A D D R$

FLAG

$\mathrm{f}$

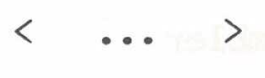

(cr)
These represent signed 16 bit numbers.

$\mathrm{U}$ represents an unsigned 16 bit number.

These represent signed 32 bit numbers (double precision).
UD represents an unsigned 32 bit double number.

F represents a 32 bit signed floating point number.

These represent a 16 bit number which is interpreted as as an 8 bit character. The least significant 8 bits are used.

These represent 16 bit numbers which are interpreted as memory addresses.

These represent 16 bit numbers which are interpreted as logical flags. The flag is true if the number is non-zero. The flag is false if the number is equal to zero.

This notation is used to represent the relative contents of the computation stack before and after a word is executed. The notation <N1 N2 N3 ... N4 N5 > is explained as follows. Before the word is executed, the stack has three numbers of interest, N1, N2, and N3, with $\mathrm{N} 3$ being the top number. After the word is executed, there is one less number on the stack. N5 is the top number, and N4 is below N5.

(cr) indicates that a carriage return is required to terminate the command line and cause execution of that line. 
Getting started; system configuration and special hardware

First, working copies should be made of the three disks supplied. Format three disks by installing the SYSTEM disk in drive 0, and a blank diskette in drive 1, and typing: FORMAT (cr). After formatting three disks, type >SYSGEN ¡E (cr) to copy the system disk. Without leaving SYSGEN, replace the SYSTEM disk in drive 0 with the SCREEN FIIE disk. Replace the copy of the SYSTEM in drive 1 with another formatted disk. Type C (cr) to copy the second disk. Replace the SCREFN FILE disk in drive 0 with the SOURCE disk and the SCREEN FILE copy in drive 1 with the final formatted disk and type C (cr). After this is done, type $\mathrm{Q}(\mathrm{cr})$ to return to the operating system. Then copy all MDOS files onto the three disks. Use the directory in chapter 11 as a guide. Replace the SOURCE in drive 0 with the SYSTEM disk copy. Type WFORTH:I to start FORTH. You are now running WFORTH. Type:

65 LIST (cr)

to see the master load screen.

The minimum required hardware is one RCA MS 2000 development system with a CDP 1806A installed, and a terminal. It is preferrable, at least for editing, to use a terminal at 9600 or 19.2K BAUD which does not require handshaking, such as a Televideo 910t. The communication utilities require either a CDP 18S641A uart card or a CDP 185653 modem card. Both are supported simultaneously. The Woods Hole uart switcher card is required if it is desirable to communicate with more than 1 other system (up to 8 ) with a single uart. The schematic of this card is included. The Woods Hole video card is required for memory mapped video and the schematic is included. Graphics requires the Woods Hole graphics ram, schematic included. A parallel printer requires the RCA CDP 18 S646 card. A serial printer requires the CDP 18S641 uart or CDP $18 \mathrm{~S} 653$ modem. The CDP $18 \mathrm{~S} 402$ or $18 \mathrm{~S} 602$ card is required to burn EPROMS, using the operating system program PROM25. No special hardware is required for floating point support.

The RCA 2 level group number I/O convention is utilized and fully supported. This convention is a simple method of extending the number of I/O ports available for the 1806 processor. Port 1 is reserved for the group number. All I/O hardware must latch the value sent to port 1 , and respond to I/O requests only if the value in port 1 ( the group number) corresponds to the designated port number for that particular I/O device. There are some 
restrictions on actually assigning group numbers. For a complete discussion, refer to RCA MPM 201C; the documentation for the 1800 series microprocessors.

The group numbers in use are shown in the following list.

Group Number

l(h)

$4(\mathrm{~h})$

$8(\mathrm{~h})$

$10(\mathrm{~h})$

$\mathrm{EO}(\mathrm{h})$

$\mathrm{FO}(\mathrm{h})$
Card Name

CDP 18S605 Terminal uart and

CDP 18 S646 parallel printer

CDP 18S640 Prom programmer

CDP 18S651 Floppy disk controller

WHOI Video ram and Graphics ram cards

CDP 18S641A Communication uart and

WHOI uart switcher

CDP 185653 MODEM
FORTH pointer

TERM.GRP

COMM.GRP

MODEM.GRP 


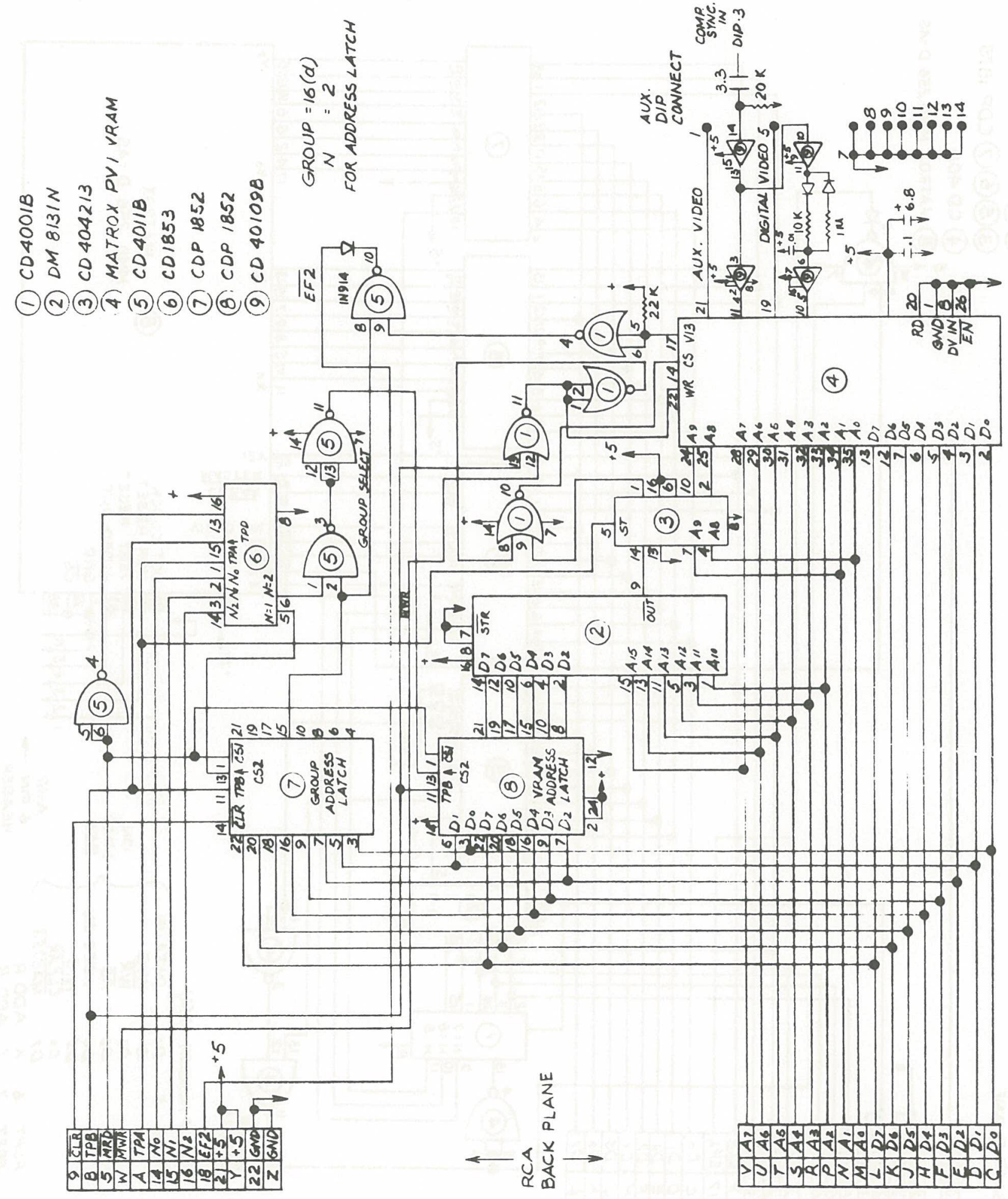

WH.O.I. VIDEO RAM 


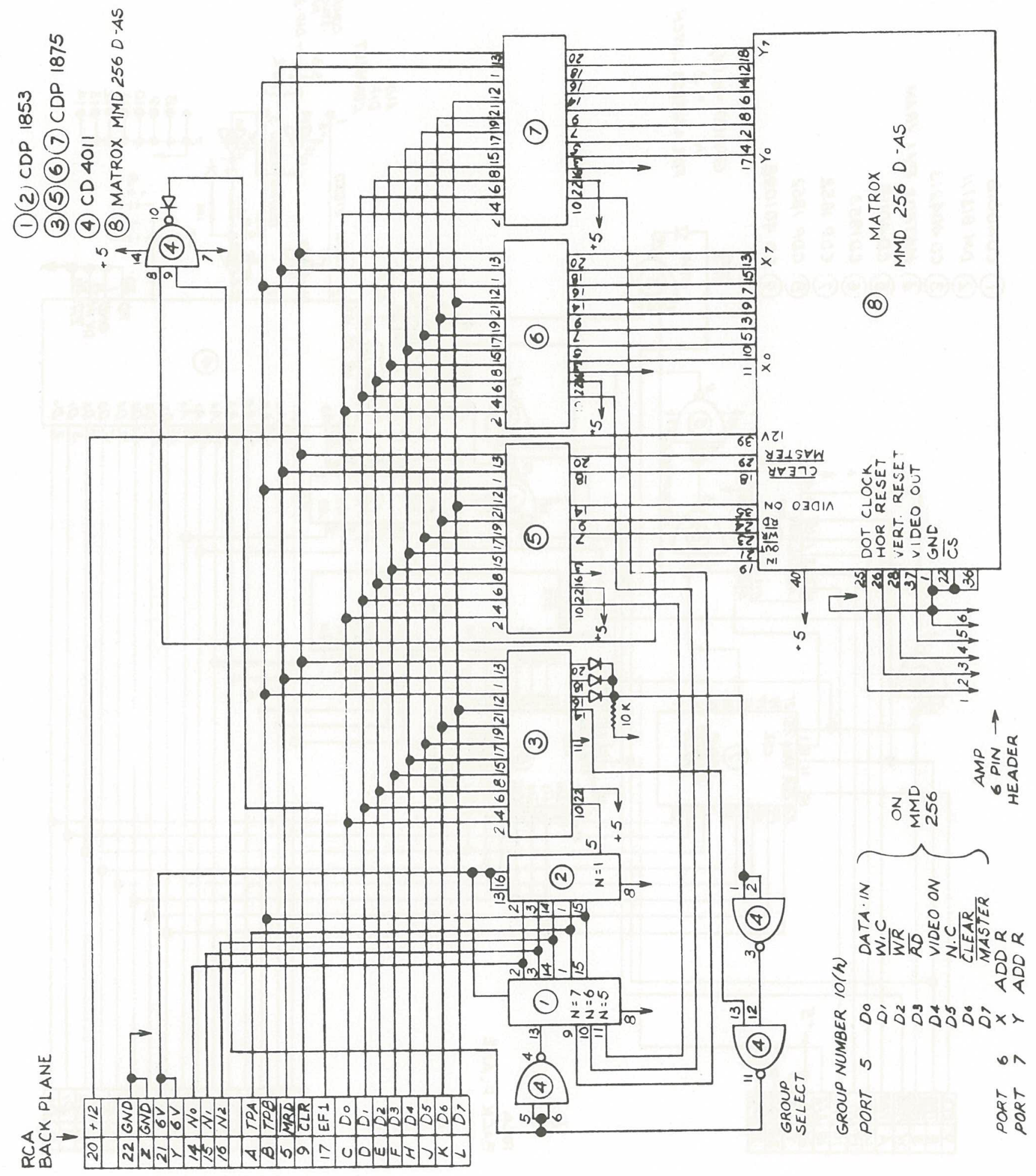

W.H.OI. GRAPHICS RAM

Page 8 


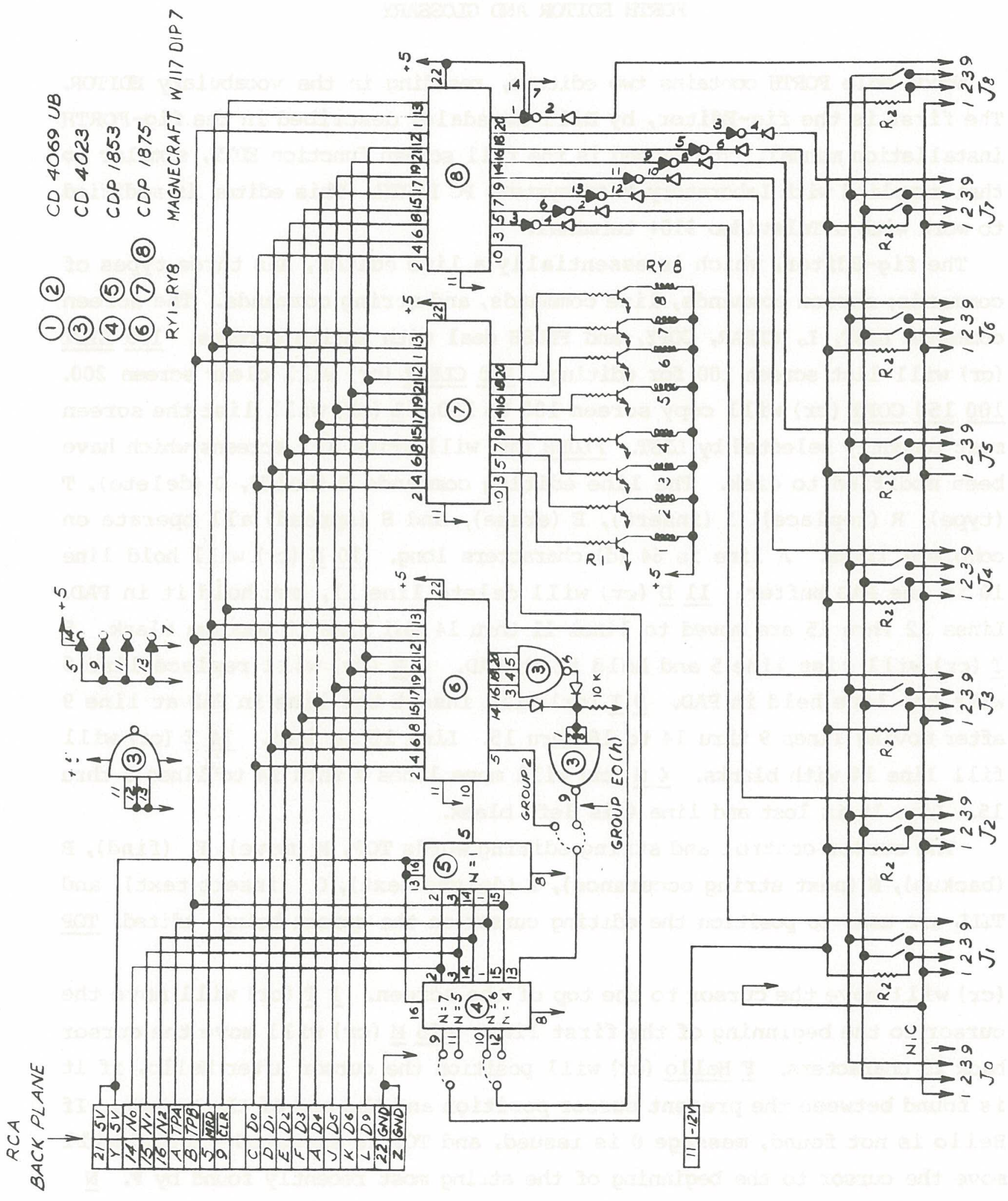

W.H.O.I. UART SWITCHER 


\section{FORTH EDITOR AND GLOSSARY}

Woods Hole FORTH contains two editors, residing in the vocabulary EDITOR. The first is the fig-Editor, by Bill Ragsdale, described in the fig-FORTH installation manual. The second is the full screen function EDIT, similar to that supplied with Laboratory Microsystems PC FORTH. This editor is modified to work with a Televideo $910+$ terminal.

The fig-Editor, which is essentially a line editor, has three types of commands; screen commands, line commands, and string commands. The screen commands LIST, L, CLFAR, COPY, and FLUSH deal with entire screens. $100 \underline{\text { LIST }}$ (cr) will list screen 100 for editing. 200 CLFAR (cr) will clear screen 200. 100150 COPY (cr) will copy screen 100 to 150. I (cr) will list the screen most recently selected by LIST. FLUSH (cr) will write all screens which have been modified to disk. The line editing commands $\mathrm{H}$ (hold), D (delete), T (type), R (replace), I (insert), E (erase), and S (spread) all operate on complete lines. A line is 64 (d) characters long. $10 \underline{\mathrm{H}}$ (cr) will hold line 10 in the PAD buffer. 11 D (Cr) will delete line 11, but hold it in PAD. Lines 12 thru 15 are moved to lines 11 thru 14 and line 15 becomes blank. $\underline{5}$ $\underline{T}$ (cr) will list line 5 and hold it in PAD. $\underline{6} \underline{\mathrm{R}}(\mathrm{cr})$ will replace line 6 with the line held in PAD. $\underline{9} I(\mathrm{cr})$ will insert the line in PAD at line 9 after moving lines 9 thru 14 to 10 thru 15. Line 15 is lost. 14 E (cr) will fill line 14 with blanks. $\underline{4} \underline{\mathrm{S}}$ (cr) will move lines 4 thru 14 to lines 5 thru 15. Line 15 is lost and line 4 is left blank.

The cursor control and string editing words TOP, M (move), F (find), B (backup), N (next string occurance), X (delete text), C (insert text), and TIL are used to position the editing cursor on the screen being edited. TOP

(cr) will move the cursor to the top of the screen. $1 \underline{T}$ (cr) will move the cursor to the beginning of the first line. $-25 \mathrm{M}$ (cr) will move the cursor back 25 characters. F $\underline{\text { Hello }}$ ( $\mathrm{cr}$ ) will position the cursor after Hello, if it is found between the present cursor position and the end of the screen. If Hello is not found, message 0 is issued, and TOP is executed. B (cr) will move the cursor to the beginning of the string most recently found by $\mathrm{F}$. $\underline{N}$ (cr) will find the next occurrance of the text found by F. $\mathrm{X}$ BAD.WORD (cr) will find and delete the string BAD.WORD. $\underline{\mathrm{C} E W . T E X T}$ (cr) will insert NEW.TEXT at the present cursor position. If characters are shifted past the 
end of the current line, they are lost. TILL TEXT (cr) will delete from the present cursor position to the end of the string TEXT. $\underline{C}(\mathrm{cr})$ will cause a fig-Editor bug. A NULL character is inserted at the present cursor position. The NULU will prematurely terminate loading. To remove the NUL, type:

$\underline{T O P} \underline{X}(\mathrm{cr})$.

There are many other words in the Editor vocabulary; they are generally used to define the above words, rather than being executed by the user.

EDIT, the full screen editor is a variation of "EDIT" supplied with Laboratory Microsystems PC FORTH. It has been modified to work with a Televideo 910+ terminal, and may require changes for other terminals. The source screens for EDIT are screens 121 and 122. In particular, the three words GOTOXY, SETEDIT, and NOEDIT may require change. After changing these words, execute the following sequence:

65 LOAD SAVE WFORTH.CM:I (cr).

This will rewrite the file WFORTH.CM to preserve the new version of EDIT. If you are using the assembler or floating point versions of WFORTH, it will also be necessary to regenerate AFORTH.CM and FFORTH.CM, as shown in chapter 1.

To use EDIT, type: SCREEN.NUMBER EDIT (cr) or: EDITL (cr). EDITL will select the screen most recently accessed by LIST. When in EDIT, eight keys have special significance. (ESC) terminates full screen editing. The cursor movement keys control cursor position. Control-I (^I) causes a horizontal tab. Control-E ( $\mathrm{A})$ clears the entire screen and ( $\mathrm{Cr}$ ) moves the cursor to the beginning of the next line. The delete key (del) only functions as a non-destructive backspace. 
\#LAG is a primative used by the EDITOR.

\#LFAD

<... LINE.ADDRESS OFFSET.TO.CURSOR >

Number Lead

\#IFAD is a primative used by the EDITOR.

\#LOCATE

$\langle\quad \ldots \mathrm{N} 1 \mathrm{~N} 2\rangle$

Number Locate

From the cursor position, determine the line number, Nl and the offset into the line, N2.

-MOVE

$\langle$ ADDRESS.LINE.NO ... > >

Dash Move

Move a line of text from ADDRESS to the line no. of the current screen.

IIINE

$$
<\ldots \text { FLAG > }
$$

1 Line

Scan the cursor line for a match to PAD text. Return flag and update the sursor $R \#$ to the end of matching text, or to the start of the next line if no match is found.

B

$$
\langle\ldots\rangle
$$

Backup

Backup cursor by the text in PAD.

C

$$
\langle\ldots\rangle
$$

Insert

Characters

Spread at the cursor and copy the following text into the cursor line.

CIFAR

$$
\langle\mathrm{N} \ldots\rangle>\quad \text { Clear }
$$

Clear screen N. CLFAR can be used to select screen $\mathrm{N}$ for editing. 
COPY

$\langle\mathrm{N} 1 \mathrm{~N} 2 \ldots\rangle$

Copy

Copy screen N1 to screen N2.

D

$\langle\mathrm{N} \ldots\rangle$

Delete

Delete line $\mathrm{N}$, but hold it in PAD. Line 15 is blank.

DEIETE

$\langle\mathrm{N} \ldots\rangle$

Delete

Delete $\mathrm{N}$ characters prior to the cursor.

$\mathrm{E}$

$\langle\mathrm{N} \ldots\rangle$

Erase

Erase line $\mathrm{N}$ with blanks.

F

FIND

FLUSH

$\langle\ldots\rangle$

Flush

Write all updated buffers to disk.

$\mathrm{H}$

$\langle\mathrm{N} \ldots\rangle$

Hold

Hold line $\mathrm{N}$ in $\mathrm{PAD}$.

I

$\langle\mathrm{N} \ldots\rangle$

Insert Line

Spread at line $\mathrm{N}$ and insert the text from PAD.

L

$\langle\ldots\rangle$

List

List the current screen. 
Leave ADDRESS of line $\mathrm{N}$ of current screen. The address will be in the disk buffer area.

Move cursor by the signed amount $\mathrm{N}$, and print the cursor line.

Match the string at STR.ADDR with all strings on the cursor line forward from the cursor. The arguements left allow the cursor R\# to be updated either to the end of the matching text or to the start of the line text.

$\mathrm{N}$

$\langle\ldots\rangle$

Next

Find the next occurrence of the text in PAD.

$\langle\mathrm{N} \ldots\rangle\rangle$

Put

Put the following text on line $\mathrm{N}$.

$\mathrm{R}$

$\langle\mathrm{N} \ldots\rangle$

Replace

Replace line $\mathrm{N}$ with the line in PAD.

R\#

$\langle\ldots$ ADDRESS $>$

R Number

$R \#$ is a USER variable which contains the offset of the editing cursor from the start of the screen.

Spread lines starting at line N. Lines $\mathrm{N}$ through 14 move down 1 line. Line $\mathrm{N}$ becomes blank, and line 15 is lost.

$\mathrm{T}$

$$
\langle\mathrm{N} \ldots\rangle>\text { Type }
$$

Type line $\mathrm{N}$ and save it in $\mathrm{PAD}$. 
Accept the following text to pad. CHARACTER is the text delimeter.

TILU

$\langle\ldots\rangle$ Till

Delete on the cursor line, from the cursor to the end of the following text.

TOP

$\langle\ldots\rangle$

Top

Position the editing cursor at the top of the screen.

WHERE

$$
\langle\mathrm{Nl} \mathrm{N} 2 \ldots\rangle \text { Where }
$$

$\mathrm{N} 2$ is the block number, $\mathrm{N} 1$ is the offset into the block. If an error is found in the source when loading from disk, the word ERROR leaves these values on the stack to help the user locate the error. WHERE uses these to print the screen and line numbers where the error occurred.

$\mathrm{X}$

$$
\langle\ldots\rangle \text { Delete Text }
$$

Delete the next occurrence of the text following $\mathrm{x}$. 
FORTH DISK OPERATING SYSTEM

Woods Hole FORTH includes a group of words used to access files created by the operating system MDOS 1.0. This group of words is collectively referred to as FORTH DOS. WFORTH accesses the disk via the routines Seek, Dread, and Dwrite in UT71, the RCA ROM utility program. WFORTH is fully compatible with all functions of RCA's MDOS, but some guidelines must be followed. WFORTH protects screens 65 thru 249 from the operating system by the word CATUPDATE, located on screen 226. This word marks the cluster allocation table of the disk in the selected drive (DRO or DRI) to show that clusters 130 thru 499 are utilized. Once this has been done, MDOS will not write any files over those sectors. The screens are not named as a file, and MDOS is unable to find the screens; to MDOS they look like occupied sectors. The operating system sequence:

\section{$\underline{\text { SYSGEN }} \underline{\text { iE }}$ (cr)}

will correctly copy a disk with screens. If you are not required to store files on a disk, the full range of screens 0 thru 314 is available on each disk. It is always possible for FORTH to write over files, because FORTH DOS does not examine the cluster allocation table. To prevent this, use only screens 65 through 249. The word .CAT, also on screen 226, will show the cluster allocation table for the selected disk. Refer to RCA MPM $241 \mathrm{Pl}$, Appendix E, the document for MDOS, for a complete discussion of the cluster allocation table.

WFORTH has several user words which manipulate MDOS files. The word DIR will print the disk directory, and starting sector number for each file for the selected disk. The word OPEN, followed by a file name will make a file available for reading or modifying. After OPEN is executed, the word GETSEC will read the next sequential sector of the file into buffer memory, and return the address of the start of that sector, or it will return 0 if the end of file is reached. A sector is $200(\mathrm{~h}$ ) bytes long. The word NEWLSN (new logical sector number) allows random access to the open file. NEWLSN works like GETSEC and returns the buffer address of the new sector. Once NEWLSN is executed (preceded by the new sector number), calls to GETSEC will return the address of the next sector. The word FIIEID will print the name of the opened file. The word DISKID will print the disk ID field specified when the disk was created by SYSGEN.CM (an operating system program, not a FORTH 
word). The word GETSEC stores the sector in FORTH's screen buffer area. Therefore, if there is any chance that the buffers may be disturbed by words such as FLUSH, or by reading many more new sectors or screens, then the sector should be copied from the disk buffer space to an unused area of ram (C000h - FFFFh is a good place).

The word DOWNLOAD is used to send a file on disk to a target computer which is connected to the uart at COMM.GRP. It requires that the target system be running a utility program that supports the I (insert) command (UT62, UT70, or UT71). See the glossary entry for DOWNLOAD for more details.

The word FIIFIOAD is used to load a file directly into memory, using the address contained in the file. This word does not sense the actual end of file but simply loads the number of complete sectors contained in the file. In most cases, this is not a problem, but problems can arise when loading files within a block of memory that is used for other purposes. The word ASSEMBLER is an example of a word which uses FILELOAD.

The word SAVE is used to save the present version of the program FORTH as a file. The file must already exist on the disk; it is merely updated to reflect the present environment. The sequence:

65 IOAD SAVE WFORTH.CM:1 (cr)

will load screen 65, the master load screen, and save the compiled result as the file WFORTH.CM:I. SAVE is the easiest way to preserve a special application version of FORTH, and is much faster than the operating system sequence:

$\underline{M E M} \underline{0}=\underline{3 F F F} \underline{\text { WFORTH.MEM }}(\mathrm{cr})$

CDSBIN WFORTH.MEM. (cr)

FORTH DOS is intended only to be a FORTH accessible subset of the RCA MDOS 1.0 operating system. It is still necessary to open files, format disks, copy disks, get directories with full attributes, and so on using MDOs. FORTH DOS is not a substitute for the operating system. Rather, it is intended to provide some functions, such as disk directory display and file updating without leaving FORTH. This extends the usefulness of both systems. 


\section{THE FORTH ASSEMBIER}

Woods Hole FORTH includes a single pass assembler based upon the assembler by Jim McDaniels, supplied with RCA's Microboard FORTH. Labels LABI: thru LAB4: are supported. The label must be defined first by a sequence such as: IAB4: 5 SEP, and then utilized by: LBR IAB4 or BR LAB4, as examples. If it is necessary to refer to a label before it is defined, the address must be computed by trial and error. The sequence HEX HERE $44+$ IBR will assemble to a forward long branch $44(\mathrm{~h})$ bytes higher. The assembler is useful when it is necessary for FORTH words to be written in machine code due to timing considerations.

In order to write code words, it is necessary to understand the register utilization of the 1806 microprocessor by WFORTH. Register 3 is used as the program counter for code words. Register 4 is the $\mathrm{X}$ register and the computation stack pointer. In Woods Hole FORTH, the stack pointer always points to the high byte of the top item on the stack. Successive bytes are retrieved by increasing the stack pointer. When a code word is finished, it should return to the FORTH word NEXT, by changing the program counter to register 5. Registers A, B, C, D, and F are available for general use. In addition, the other registers may be useful for interaction with FORTH. See the source listing for a complete description of register utilization.

The assembler is merely a high level interface to the FORTH word CREATE, and a lookup table for mneumonics. With this in mind, it is useful to first examine the structure of a no operation word that has been defined by CREATE. CREATE NO-OP HERE DUP 2- $\underline{\text { D5 }}$ C, SMUDGE (cr)

The above sequence will create a word in the FORTH vocabulary which does nothing. When it is defined, it is named NO-OP. The sequence HERE DUP 2- ! makes it a CODE definition by storing the parameter field address of the word NO-OP in the code field of the word NO-OP. The sequence D5 C, stores the 1806 opcode D5 in the parameter field. When the word NO-OP is executed, this single machine instruction will be executed resulting in a return to the FORTH word NEXT by setting the program counter to register 5. The word SMUDGE makes the word NO-OP visible to dictionary searches. Although this is a trivial example, it shows the essential structure of CODE words, and the mechanism for building them. 
As a second example, examine the definition of SETQ (set the Q line true). HEX CREATE SETQ HERE DUP 2-! 7BD5 $上$ SMUDGE (cr)

This definition is on screen 91. Refer also to the assembler example load screen, screen 241. Using the assembler, the identical run time code can be created by the sequence:

CODE SETQ SEQ $\underline{5}$ SEP ENDCODE (cr)

These simple examples show the basic utilization of the assembler. Screen 220 contains a non-trivial example of assembler utilization.

There are a few additional assembler directive words. $C_{\text {; }}$ and ENDCODE are pseudonyms for END-CODE. A.O takes a 16 bit number from the stack and stores the low byte in the dictionary. A.1 takes a 16 bit number from the stack and stores the high byte in the dictionary. PAGE moves the dictionry pointer to the next page boundary (XXOOh). PAGEADJUST takes a number $\mathrm{N}$ from the stack and ensures that the following $\mathrm{N}$ bytes are on the same page. Additional assembler directives are located on screen 242. All RCA 1806 mneumonics are supported except four: B1, B2, B3, and B4. They are not supported because if they were, it would be impossible to define the hex numbers BI thru B4. Instead, these branch on flag instructions have the modified mneumonics SBI, SB2, SB3, and SB4. Five FORTH words are redefined in the assembler: RO, OUT, $\mathrm{OR}, \mathrm{AND}$, and XOR. These are all 1806 mneumonics and in order to use the corresponding FORTH words it is necessary to execute FORTH first; for example: FORTH 6 OUT ASSEMBLER will place 2 numbers on the stack, the address of the USER variable OUT, and the number 6 , and then return to the ASSEMBIER vocabulary (rather than assemble the op code for OUT 6 ). 


\section{COMMUNICATION UTILITIES}

The FORTH communication utilities are intended to facilitate terminal emulation including full telephone dialing capabilities, handle downloading of MDOS files and uploading or downloading of blocks of memory in other RCA systems, facilitate the transfer of screens with another FORTH system, handle simple RS-232 tree networking at 1200 baud without handshaking or software protocol, and send MDOS files as formatted strings of ASCII-hex characters to any other computer.

The simplest communication word, UART initiates 2 way conversation between a device connected to the uart at TERM.GRP and a device connected to the uart at COMM.GRP. Usually this means that an operators' terminal is connected to a uart at Group 1 and a uart communicating with another computer is connected at group E0h.

With all communication words, the escape key (esc) is used to terminate the operation. If it is desirable to send an escape character to the target system, the following sequence will work:

(esc) ESC' U (cr) for a uart; and

(esc) ESC' RE-ENTER (cr) for a modem.

The sequence DIAL (PHONE。NUMBER) ORIGINATE (cr) will dial the phone number and set the modem for originate mode. The word DIAL will only dial dual-tone numbers, but the modem is capable of dialing pulse numbers. Refer to the documentation for the $18 \$ 653$ modem and screens 103 and 104 for more details.

The word ANSWER is included, although the behavior of this word is presently somewhat primative. ANSWER will set the modem to answer mode, answer after two rings, and then give full control of the FORTH system to the calling system. This is a convenient way to run the system remotely. The successful implementation of ANSWER depends upon the following programming convention. Any word which uses group select I/O must use the word TERM to restore the terminal group after I/O, instead of the sequence 11 OUTPUT. This is because Woods Hole FORTH does not assume that the terminal is assigned to Group 1 ; the actual terminal group number is contained in the USER variable TERM.GRP. If this convention is followed, then full remote operation via the modem is possible.

The words SEND-SCREEN and GET-SCREEN al low transfer of screens between FORTH systems. The sequence: 
Nl GET-SCREEN (cr)

typed at the receiving system will set up the system to receive screen $\mathrm{Nl}$. Then:

N2 SEND-SCREEN (cr)

typed at the transmitting system will send screen N2. These words include no handshaking or error checking. Note that screen N2 is sent and stored as screen $\mathrm{Nl}$ in the receiving system, overwriting the existing screen $\mathrm{Nl}$. Both systems must have uarts assigned to the COMM.GRP, which are connected together, to transfer the data.

XMIT and $V$ are two words used to pass blocks of data resident in memory between RCA systems only. The target system must be running an RCA utility program which supports the D (dump) and I (insert) commands (UT62, UT63, UT70, and UT71). The sequence:

SOURCE.ADDR LENGTH DESTINATION.ADDR XMIT (cr)

will send a block of data from SOURCE.ADDR in the local computer to DESTINATION.ADDR in the target computer for LENGTH bytes. The sequence: SOURCE.ADDR LENGTH DESTINATION.ADDR $\underline{\mathrm{V}}$ (cr)

will receive L.FNGTH bytes from SOURCE.ADDR in the target computer and store it at DESTINATION.ADDR in the local computer. In the case of XMIT and V, the local computer connects via the uart at COMM.GRP to the terminal input of the target computer.

The word DOWNLOAD will move a file from the disk to a target computer that is running an RCA utility program. The usage is:

TARGET.ADDR DOWNLOAD FILESPEC (cr)

This will move the file named in FILESPEC, to TARGET.ADDR in the target computer. The length of the transfer is determined by the file itself. The initial values of TFRM.GRP, COMM.GRP, and MODEM.GRP are set by items 36, 38, and 40 in the parameter table and be changed by changing these items. See chapter 7, page 26 for a description of this procedure. 
MEMORY MAP AND PARAMETER TABLE

\begin{tabular}{|c|c|c|}
\hline ADDRESS & POINIER & SIGNIFICANCE \\
\hline 0000 & COID & $\begin{array}{l}\text { This is the entry point for FORTH. Location } 0000 \\
\text { contains a long branch to location } 0005 \text {. This lon } \\
\text { branch may be patched to vector to another locatiol } \\
\text { after a system reset. This is useful in Eprom } \\
\text { systems if something other than FORTH is desired } \\
\text { after a system reset. FORTH may be entered } \\
\text { externally by branching to location } 0005 \text {. COID } \\
\text { initializes the USER variables } 10(h) \text { through } 1 \mathrm{E}(\mathrm{h}) \\
\text { ( } 8 \text { numbers) from the initial values in the } \\
\text { parameter table and then executes WARM. }\end{array}$ \\
\hline 0003 & WARM & $\begin{array}{l}\text { This is the warm re-entry point for FORTH. Warm } \\
\text { initializes registers } 2,4,5,6,7, \text { and } 8 \text {. It } \\
\text { also initializes USER variables } 0 \text { through OE ( } 8 \\
\text { numbers). All initial values are taken from the } \\
\text { parameter table. }\end{array}$ \\
\hline
\end{tabular}

184l(h) PARAMETER HORIGIN The parameter table holds all the values that are TABLE used to initialize registers, USER variables (considered system variables) and CONSTANTS when WARM and COID are executed. By manipulating values contained in the parameter table, it is possible to customize the behavior of the FORTH system, even to the extent of defining the initial task that FORTH will execute. Refer to the follow section of this chapter for complete details of the parameter table.

varies Fence FENCE The fence is a marker, below which the system will not FORGET words. The value of FENCE Is initialized by COID and WARM from a value in the parameter table. The word NEW-FENCE will set the 
initial value of FENCE in the parameter table to include (protect) all words currently defined. This is done by making the value of FENCE equal to the value of the dictionary pointer (DP).

$\begin{array}{ll}\text { varies } & \text { Dictionary DP } \\ & \text { Pointer }\end{array}$

$\mathrm{DP}+48(\mathrm{~h}) \quad \mathrm{PAD}$

55FE(h) Computation Stack
PAD PAD is the USER variable which points to the dynamically assigned scratch pad buffer located $48(h)$ bytes past the dictionary pointer. PAD is used in two ways. The area below PAD is used to construct output number strings by the words $<\#$ \# and \# >. This area must, therefore, be in Ram if an Eprom application program is to print numbers. The area above PAD is used by the FDITOR as a temporary string buffer. PAD's location is always 48 bytes above DP, so it is merely necessary to move DP to point to Ram for PAD to work properly in Eprom applications.

S0 The computation stack, pointed to by S0, is the primary stack in FORTH. It grows downward in memory to meet PAD. Message 2 is issued by CRFATE if there is any danger of the stack and the scratch pad colliding.

$5600(\mathrm{~h})$ Terminal TIB The terminal input buffer is $80(\mathrm{~h})$ bytes long. It Input is used to store the input command line from the terminal. It grows upward in memory towards the return stack. 


\begin{tabular}{|c|c|c|}
\hline $56 \mathrm{FE}(\mathrm{h})$ & $\begin{array}{l}\text { Return } \\
\text { Stack }\end{array}$ & $\begin{array}{l}\text { The return stack is used by the FORTH interpreter } \\
\text { to store the addresses of calling words. The } \\
\text { return stack can also be used to store numbers in } \\
\text { a fashion similar to the computation stack, with } \\
\text { the words }>\mathrm{R} \text { and } \mathrm{R}>\text {, but only within the definition } \\
\text { of a word. If the use of }>\mathrm{R} \text { and } \mathrm{R}>\text { is not balanced } \\
\text { within a definition, the system will crash. The } \\
\text { return stack grows downward toward the terminal } \\
\text { input buffer. }\end{array}$ \\
\hline $5700(h)$ & $\begin{array}{l}\text { User } \\
\text { Variable } \\
\text { Area } \\
\text { (register 7) }\end{array}$ & $\begin{array}{l}\text { The USER variable area is the area where the } \\
\text { actual values of USER variables are stored. It is } \\
\text { D4 ( } h \text { ) bytes long so D4 ( } h \text { )/2 (l06d) USER variables } \\
\text { are available. The USER variable area must start } \\
\text { on a page boundary. The allocation of USER } \\
\text { variables is most easily handled by NEXTU ( Refer } \\
\text { to the glossary). The use of USER variables is } \\
\text { mandatory in Eprom applications because the values } \\
\text { are stored in the RAM USER variable area, not in the } \\
\text { PROM dictionary, as VARIABLES are. See the glossary } \\
\text { entry for USER for an explanation. }\end{array}$ \\
\hline
\end{tabular}

57D9(h) Start of FIRST The screen buffer is used to store screens while Screen being accessed by FORTH. In addition, the screen Buffers buffers are used by FORTH's Dos when reading files. Each screen buffer is $404(\mathrm{~h})$ bytes long. The first two bytes are the block number. The most significant bit of the first byte is the UPDATE bit. If it is 1 , then the screen will be updated (written to disk) by FLUSH, or when the buffer is needed for other screens. The next 400 bytes are the screen itself. The last 2 bytes are unused. When reassigning the screen buffer area by changing the constants FIRST and LIMIT, make sure that the area is an integral multiple of $404(\mathrm{~h})$ bytes long. 


\begin{tabular}{|c|c|c|c|}
\hline $7 F F F(h)$ & $\begin{array}{l}\text { End of } \\
\text { Screen } \\
\text { Buffers }\end{array}$ & LIMIT & $\begin{array}{l}\text { IIMIT Is a constant which contains a pointer to the } \\
\text { last byte of the screen buffer area. When } \\
\text { floating point support is compiled, IIMIT is } \\
\text { changed to } 6 \mathrm{FEF}(\mathrm{h}) \text {, and the floating point code } \\
\text { occupies the area from } 7000 \text { to } 7 \mathrm{FFF}(\mathrm{h}) \text {. }\end{array}$ \\
\hline $\begin{array}{l}8000(h)- \\
87 F F(h)\end{array}$ & $\begin{array}{l}\text { Utility } \\
\text { Program }\end{array}$ & & $\begin{array}{l}\text { The RCA utility program UT7l is required if disks } \\
\text { are used by FORTH. UT70 will work if the SUBMIT } \\
\text { feature of the operating system is not required. }\end{array}$ \\
\hline $87 \mathrm{FO}(\mathrm{h})$ & & MON & The FORTH word MON enterstheutilityprogram here. \\
\hline $\begin{array}{l}8000(h)- \\
8 F F F(h)\end{array}$ & & & The utility programs use this area. It must be Ram. \\
\hline $\begin{array}{l}9000(h)- \\
B F F F(h)\end{array}$ & MDOS & & $\begin{array}{l}\text { The RCA operating system MDOS } 1.0 \text { resides here, } \\
\text { although FORTH does not use the operating system } \\
\text { directly. When developing programs, it is } \\
\text { convenient to have MDOS resident. }\end{array}$ \\
\hline $9005(\mathrm{~h})$ & & DOS & The FORTH word DOS enters MDOS here. \\
\hline $\begin{array}{l}\mathrm{C} 700(\mathrm{H})- \\
\mathrm{CFFF}(\mathrm{h})\end{array}$ & Assembler & & The assembler is compiled as a transient here. \\
\hline $\begin{array}{l}\mathrm{D} 000(h)- \\
F F F F(h)\end{array}$ & unused & & This area of memory is unused by FORTH. \\
\hline
\end{tabular}


THE PARAMETER TABIE:

The parameter table is used by FORTH to initialize the FORTH system at COID or WARM startup. By manipulating values in the parameter table, FORTH can be configured for special applications. It is possible to specify the first word to be executed when FORTH starts up. As an example, the sequence: N1 는 +ORIGIN ! ( $\mathrm{cr}$ ) where $\mathrm{N} 1$ is the Value, and $\mathrm{N} 2$ is the Item\#, will update Item\# with the new Value in the parameter table. The new Values are installed by executing the words COID or WARM. The following 6 Values are used to initialize registers at COID or WARM start:

\begin{tabular}{llll} 
ADDR Value & Item\# & \multicolumn{1}{l}{ Register } & \multicolumn{1}{c}{ Name } \\
1841 56FE: & 0 & 2 & Return stack position \\
1843 55FE: & 2 & 4 & Computation stack \\
1845 0098: & 4 & 5 Inner interpreter \\
1847 0016: & 6 & 6 Cold start vector \\
1849 5700: & 8 & 7 User area \\
$184 \mathrm{~B}$ 11EB: & 10 & 8 Initial task
\end{tabular}

The next 8 values are placed in USER variables 0 thru $\mathrm{OE}$ at COID or WARM start:

$\underline{\text { ADDR Value }}$

Item\# (d)

$\underline{\mathrm{USER}} \# \underline{(\mathrm{h})}$

Name

VARIABLE

184D 18FE:

12

184F 007F:

1851 5700:

1853 55FE:

1855 56FE:

18575600 :

1859 001F:

185B 0000:
14

16

18

20

22

24

26
0 Last name field addr

2 Backspace character

4 User area

6 Computation stack

8 Return stack

A Terminal input buffer

C Name field width

E Warning 
The following 8 values are inserted in USER variables 10 thru $1 \mathrm{E}$ at COID start only:

\begin{tabular}{|c|c|c|c|c|}
\hline$\underline{\mathrm{ADDR}}$ & Value & Item\# $(d)$ & 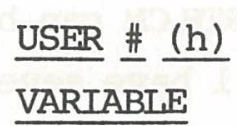 & Name \\
\hline 185D & 192A: & 28 & 10 & Fence \\
\hline $185 \mathrm{~F}$ & 192A: & 30 & 12 & Dictionary pointer \\
\hline 1861 & 183F: & 32 & 14 & VOC-LINK in FORTH \\
\hline 1863 & 013B: & 34 & 16 & Screens per disk \\
\hline 1865 & OOF0: & 36 & 18 & Modem group \\
\hline 1867 & 0001: & 38 & $I \mathrm{~A}$ & Terminal group \\
\hline 1869 & OOE0: & 40 & IC & Comm uart group \\
\hline $186 \mathrm{~B}$ & 0050: & 42 & $1 \mathrm{E}$ & Next user variable \\
\hline
\end{tabular}

The sequence:

DECIMAL '- ANY.WORD 10 +ORIGIN ! (cr)

will force FORTH to execute ANY.WORD initially. The word ANY.WORD must end with the word QUIT, however, to enter the FORTH interpretive loop when ANY.WORD has completed execution. The word NEW-FENCE will update items 12, $28,30,32$, and 42 in the parameter table to insure that all words and USER variables presently defined are protected in the dictionary. This is required when saving FORTH as a disk file. See the chapter describing Eprom applications programming for more details on changing the parameter table. 


\section{DEVELOPING EPROM APPLICATION PROGRAMS}

Woods Hole FORTH lends itself to Eprom, Prom, or Rom applications. The files FORTH.CM or WFORTH.CM can be placed into Eprom with no changes, but the resulting version will have several problems because many FORTH pointers are stored within the dictionary and will be frozen in Eprom. The terminal input buffer is located just beyond the end of the dictionary; therefore, unless this is in Ram, it will contain garbage. Also, the word FORTH contains a pointer to the most recent word of the FORTH vocabulary, and no new words can be defined unless this pointer can be changed. In order to solve these problems, the word ROMIT is supplied. ROMIT assumes that a combination of 2716 Eproms and 6116 Rams will be used for memory. ROMIT moves all pointers associated with the end of the dictionary to the next $800(h)$ byte boundary beyond the dictionary pointer, and prints the memory limits for the dictionary.

The memory map for the Eprom system is:

0 to HERE-1

Eproms, Proms, or Roms

HERE to HERE+800(h) (terminal input buffer and PAD) 16116 Ram

Computation stack to end of USER variables

End of USER variables to 7FFF(h) (FIRST to LIMIT)

(only if screen buffers are required)

$7000(h)$ to $7 \mathrm{FFF}(\mathrm{h})$

(only if floating point is required)
16116 Ram

6116 Rams

Eproms, Proms, or Roms

In practical terms, this means that a $32 \mathrm{~K}$ memory board (18S626 or 18S628) may conveniently contain an entire application system. The beginning is comprised of 2716 Eproms, and the remainder is composed of 6116 Rams. The word ROMIT must be executed after all application words are defined, to realign the pointers for an Eprom system.

If memory resources are severely limited and the memory map must be changed, the following list is useful in understanding the affected areas. 
1. FIRST and LIMIT point to the start and end of the screen buffer area. If screens will be used, for instance to debug in-situ application systems, then the constants FIRST and LIMIT may be changed. The number of bytes between FIRST and LIMIT must be an integral number of $404(\mathrm{~h})$ bytes. If screens are not to be used, then FIRST and IIMIT can be ignored. Note, however, that EMPTY-BUFFERS, called in ABORT, fills memory between FIRST and LIMIT with zeroes. This would destroy any user data between FIRST and LIMIT.

2. Computation stack. The location of the computation stack can be changed by changing items 2 and $18(d)$ in the parameter tble. Remember that the stack grows downward.

3. Return stack. The location of the return stack can be changed by changing items 0 and $20(d)$ in the parameter table.

4. USER variable area. The location of the USER variable area can be changed by changing items 8 and $16(d)$ in the parameter table. The USER area must be $100(\mathrm{~h})$ bytes long and start on a page boundary.

5. Terminal input buffer. The location of the terminal input buffer can be changed by changing item 22 (d) in the parameter table. The terminal input buffer must be $80(\mathrm{~h})$ bytes long. This buffer is only required if input from a terminal will be used.

If it is desirable to have FORTH immediately begin execution of a word, then the following sequence should be executed:

DECIMAL '- ANY.WORD 10 +ORIGIN ! (cr)

The definition of ANY.WORD must include QUIT as the last item of the definition if you want to enter the normal interpretive loop after ANY.WORD is executed.

In an Eprom version of fig-FORTH, it is not possible to define new words into existing vocabularies because the pointer to the last word is frozen in the vocabulary defining word(ie FORTH, EDITOR, ASSEMBLER, etc.) located within the dictionary and therefore within Prom. However, it is possible to start a new vocabulary and define words into it with the following sequence: 
VOCABULARY ANY.VOCAB IMMEDIATE HERE 4 = DUP CURRENT ! CONTEXT ! (cr) At this point it is possible to define new words into the vocabulary ANY.VOCAB, which is linked to the FORTH vocabulary. Sufficient ram must exist above the dictionary pointer to contain the new entries. This technique is very valuable for debugging Prom systems. 


\section{FLOATING POINT}

Woods Hole FORTH has optional floating point functions. They are based on a software package from RCA (see RCA documentaiton MPM-207) which was adapted to be callable from FORTH. The file is called FLOAT.CM (Floating Point Arithmetic Subroutines) and is loaded separately in high memory by the word FLOATING. The file FFORTH.CM has floating point already compiled.

The internal representaton of the floating point numbers is 32 bits; 1 byte exponent and 3 byte mantissa stored in 2's complement notation. The exponent and the mantissa must have a leading decimal point. Since floating point numbers are 32 bits long, the same as FORTH double precision numbers, words such as 2DUP and 2ROT may be used to manipulate them.

A functional description of the Floating Point words is given below. Wherever possible, words correspond to Laboratory Microsystems PC FORTH for the IBM PC, and secondly to Laboratory Microsystems 280 FORTH for CP/M systems.

The following symbols are used when describing stack behavior:

$$
\begin{array}{ll}
F=\text { signed floating point number } & \text { (32 bits) } \\
D=\text { signed double precision fixed point number } & \text { (32 bits) } \\
N=\text { signed single precision fixed point number } & \text { (16 bits) } \\
\mathrm{f}=\mathrm{flag} ; \text { false }=0, \text { true }=\text { non-zero } & \text { (16 bits) }
\end{array}
$$

FIOAT $\langle\ldots F\rangle$ Float Usage: FLOAT -. $x x x x x x-y y$. FLOAT converts a six digit mantissa and two digit exponent (base 10). All digits and decimal points must be present. Negative signs are optional. The floating point number, $F$, is left on the stack.
$\mathrm{D}->\mathrm{F}$
$\langle D \ldots F\rangle$
Convert Double Precision to Floating Point D->F converts a signed double precision fixed point number to a floating point number. D must be between $7 F F F F F$ and $-7 F F F F F(h)$ and is interpreted as an integer without decimal point.
F. $\langle\mathrm{F} \ldots\rangle$ Floating Point 
Print

F. prints the floating point number in the format -. $\mathrm{xxxxxx}-\mathrm{yy}$. All digits are printed.

FQUAD

$\langle$ F1 F2 F3 ...F F4 F5 $\rangle$

Floating Point Quadratic

solution

FQUAD solves the equations $F 1(x * * 2)+F 2(x)+F 3=0$ and returns the roots $\mathrm{F} 4$ and $\mathrm{F} 5$.

F@

$\langle\mathrm{ADDR} \ldots \mathrm{F}\rangle$

Floating Point Fetch

Fetch the floating point number $F$ from $A D D R$.

F!

$\langle\mathrm{FADDR} \ldots\rangle$

Floating Point Store

Store floating point number $F$ at ADDR.

F+

$\langle\mathrm{F} 1 \mathrm{~F} 2 \ldots \mathrm{F} 3\rangle$

Floating Point Add

F+ adds $F 1$ and F2 to yield F3.

F-

$\langle\mathrm{Fl} \mathrm{F} 2 \ldots \mathrm{F} 3\rangle$

Floating Point Subtract

F- subtracts $F 2$ from $F 1$ to yield F3.

$F^{*}$

$\langle\mathrm{F} 1 \mathrm{~F} 2 \ldots \mathrm{F} 3\rangle$

Floating Point Mutiply

$F^{*}$ multiplies $\mathrm{F} 1$ and $\mathrm{F} 2$ to yield $\mathrm{F}$ 3.

F/

$$
\langle\mathrm{Fl} F 2 \ldots \mathrm{F} 3\rangle
$$

Floating point Divide

F/ divides Fl by F2 to yield F3. 
FABS

$$
\langle\mathrm{F} 1 \ldots \mathrm{F} 2\rangle
$$

Floating Point

Absolute Value

$\mathrm{F} 2$ is equal to the absolute value of $\mathrm{F}$.

FMINUS

$$
\langle\mathrm{F} 1 \ldots \mathrm{F} 2\rangle
$$

Floating Point Minus

$\mathrm{F} 2$ is equal to the negative of $\mathrm{Fl}$.

FSQRT

$$
\langle\mathrm{F} 1 \ldots \mathrm{F} 2\rangle
$$

Floating Point Square Root

F2 is equal to the square root of Fl. Fl must be positive.

FO>

$$
\langle\mathrm{F} \ldots \mathrm{flag}\rangle
$$

Floating Point Zero greater than

FO> leaves a flag on the stack. The flag is true if $F$ is greater than or equal to zero.

$\mathrm{FO}=$

$$
\langle\mathrm{F} \ldots \mathrm{flag}\rangle
$$

Floating Point Zero equal

$\mathrm{FO}=$ leaves a flag on the stack. The flag is true if $\mathrm{F}$ is equal to zero.

FSIN

$$
\langle\mathrm{F} 1 \ldots \mathrm{F} 2\rangle
$$

Floating Point

$$
\text { Sine }
$$

$\mathrm{F} 2$ is equal to $\sin (\mathrm{F} 1) .-1<\mathrm{F} 2<1 . \mathrm{F} 1$ is in degrees.

FCOS

$$
\langle\mathrm{F} 1 \ldots \mathrm{F} 2\rangle
$$

Floating Point

$$
\text { Cosine }
$$

F2 is equal to the cosine of Fl. Fl is in degrees.

FARCTAN

$$
\langle\mathrm{F} 1 \ldots \mathrm{F} 2\rangle
$$

Floating Point

Arctangent

F2 is equal to the arctangent of F1. F2 is in degrees. 
$\mathrm{F} 2$ is equal to $\mathrm{E}^{* * \mathrm{~F} 1}$

FLN

$$
\langle\mathrm{F} 1 \ldots \mathrm{F} 2\rangle
$$

Floating point Natural Iog

F2 is equal to the natural log of Fl, for Fl greater than zero.

$F^{* *}$

$$
\langle\mathrm{Fl} F 2 \ldots \mathrm{F} 3\rangle
$$

Floating Point

Exponentiation

$\mathrm{F} 3$ is equal to $\mathrm{F} 1$ raised to the $\mathrm{F} 2$ power.

FPI

$$
\langle\ldots \mathrm{F} 1\rangle
$$

Floating Point Pi

$\mathrm{Fl}$ is equal to $\mathrm{Pi}, .3146001$

In addition to the Floating Point words, the double precision words 2ROT, 2SWAP, 2DUP, 2OVER, and 2DROP are useful in manipulating floating point numbers. They work just like their single precision counterparts.

Frrors in Floating Point Math can arise from division by zero, taking the square route of a negative number, or simply entering numbers beyond the range of the internal 24 bit representation, among other things. Frrors are passed to the FORTH word ?ERROR. The message numbers for Floating Point errors are listed below. These indicate which operation was in progress when the error occured.

$\begin{array}{lll}\text { Message } & 90 & \text { LN } \\ \text { Message } & 91 & \text { EXP } \\ \text { Message } & 92 & \text { D->F } \\ \text { Message } & 93 & \text { FSQRT } \\ \text { Message } & 94 & \text { F/ } \\ \text { Message } & 95 & \text { F* } \\ \text { Message } & 96 & \text { F- } \\ \text { Message } & 97 & \text { F+ } \\ \text { Message } & 98 & \text { FLOAT } \\ \text { Message } & 99 & \text { F. }\end{array}$


If the user wishes to trap errors, the word (ABORT) can be patched to run a user trap routine.

Screen 223 is a floating point example screen. The sequence:

$\underline{223}$ LOAD (Cr)

will generate the examples.

The file FLOAT.CM must exist on the default drive for floating point to function, except when using FFORTH.CM, which has the file already incorporated. 
DOCUMFNTATION UTILITIES

Woods Hole FORTH includes many screens utilized in documenting a particular application of FORTH. There are two load screens which, by themselves, document the current FORTH environment. Screen 73 will provide complete printed documentation, while screen 74 will provide a brief document. Chapters 11 thru 22 of this manual were printed on a Diablo 620 printer connected to a uart at group E0h with the sequence:

SERPRINTER DIABLO 73 LOAD (cr).

Screens 233 thru 236 contain additional documentation words. A particularly useful word, MEMORY-MAP is contained on screen 235. This word displays the current memory map. An example is given in chapter 20.

The FORTH decompiler, inspired by Ray Duncan of Laboratory Microsystems is compiled into WFORTH.CM because it is extremely useful and simple to use. The sequence:

DECOMPIIE ANY.WORD (cr)

will show the contruction of any word defined by a colon definition, along with memory addresses for every entry in the word. The Decompiler is invaluable when debugging new FORTH words.

WFORTH provides support for a parallel (Centronics type) printer with the RCA 18 S646 card, and a serial printer, using the uart at COMM.GRP. As supplied, WFORTH supports the parallel printer; to use a serial printer, merely invoke the word SERPRINTER. If permanent serial support is desirable, simply execute :

SERPRINTER NOPRINT SAVE WFORTH.CM:I (cr) •

The code for printer support is located on screen 80 . Screen 243 contains code to support a serial printer attached to a Televideo 910+ terminal If this is desired, exchange screens 80 and 243 and then execute the sequence:

65 IOAD SAVE WFORTH.CM:I. (cr)

This will recompile WFORTH and save it. Obviously, any type of printer support can be written to screen 80 and recompiled into WFORTH.CM.

Screen 75 is the master example load screen. The sequence: PRINTER 75 LOAD PAGE TEAR (cr)

will generate the examples listed in Chapter 20. Studying these examples is an excellent way to become familiar with FORTH. 
Disk directories and Cluster Allocation Tables

The disk directories are provided for the two disks which normally occupy drive 0 and drive 1. The distribution disks do not contain any of the files associated with MDOS 1.0. Users must copy these programs from their operating system disk. The cluster allocation tables show how much of the disks are still available; one bit corresponds to one sector. The first disk, ALVIN OPERATING SYSTEM VERSION 1.800, occupies drive 0. The second disk, WOODS HOIE FORTH SCRFEN FILE, occupies drive one. The cluster allocation table for this disk has been marked (with the word CAT-UPDATE) to show that the sectors corresponding to screens 65 through 249 are occupied. Many of these screens are in fact blank; consult the next chapter for a list of utilized screens. The screens do not show in the disk directory since they are not MDOS files. The operating system program SYSGEN.CM provides the best method for copying screen file disks. The sequence:

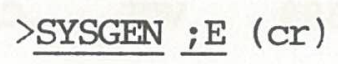

will make an exact copy of a screen file disk. Note that this must be done when running MDOS, rather than when running WFORTH.

Drive 1 is used as the screen file disk so that a complete application or group of application versions of FORTH can be contained on one disk, both as source screens and as .CM files ready to be placed in Eprom or downloaded to a target system, while retaining the operating system on drive 0 . Note that the operating system is not present on the screen file disk, but the MDOS programs SYSGEN.CM and COPY.CM are. The next chapter contains more information on the general subject of screen file organization. 
Disk ID: ALVIN OPERATING SYSTEM VERSION 1.801

Directory Start

\begin{tabular}{|c|c|c|c|c|c|c|c|c|c|c|}
\hline DIR & . $\mathrm{CM}$ & 36 & MEM &. $\mathrm{CM}$ & 50 & NEWDSK.SUB & 53 & GPH &. $\mathrm{CM}$ & 55 \\
\hline DOT &. $\mathrm{CM}$ & 65 & VDL & . SUB & 68 & REPLAY .SUB & 70 & FONT &. $\mathrm{CM}$ & 72 \\
\hline ASM8 &. $\mathrm{CM}$ & 78 & RENAME &. $\mathrm{CM}$ & 103 & ALVIN .DOC & 109 & GRAPH & - BAS & 118 \\
\hline DUMP & . SUB & 127 & ADT & . $\mathrm{CM}$ & 129 & SUBVDL.CM & 132 & SYSGEN & . $\mathrm{CM}$ & 138 \\
\hline FREE &. $\mathrm{CM}$ & 156 & FORMAT & CM & 159 & SER .CM & 163 & ASM4TH & I. $\mathrm{CM}$ & 165 \\
\hline FFORTH &. $\mathrm{CM}$ & 172 & EXAM & . $\mathrm{CM}$ & 238 & MEMTST.CM & 249 & WFORTH & I. $\mathrm{CM}$ & 251 \\
\hline $\mathrm{Z}$ & .TMP & 289 & FSP2 & . $\mathrm{CM}$ & 316 & SERIAL.SUB & 325 & COPY &. $\mathrm{CM}$ & 327 \\
\hline CDSBIN &. $\mathrm{CM}$ & 331 & DELETE & . CM & 335 & UVX4TH.CM & 337 & DUMP & - BAS & 367 \\
\hline $\mathrm{BRP}$ &. $\mathrm{CM}$ & 370 & FORTH &. $\mathrm{CM}$ & 375 & SUBMIT.CM & 389 & DEL &. $\mathrm{CM}$ & 402 \\
\hline $\mathrm{U}$ & . $\mathrm{CM}$ & 406 & DIAG & . $\mathrm{CM}$ & 408 & BASIC2.CM & 412 & GRAPH & . SUB & 439 \\
\hline ORD &. $\mathrm{CM}$ & 441 & VDRI &. $\mathrm{CM}$ & 444 & FTAB .CM & 454 & AFORTH & I. $\mathrm{CM}$ & 457 \\
\hline $\mathrm{OP}$ & . SYS & 10 & VERIFY & CM & 495 & PRINT .CM & 501 & XREF & . $\mathrm{CM}$ & 505 \\
\hline EDIT &. $\mathrm{CM}$ & 517 & PROM25 &. $\mathrm{CM}$ & 530 & WAIT .CM & 536 & SCIVDL & . CM & 539 \\
\hline $\mathrm{NAV} 4 \mathrm{TH}$ & . $\mathrm{CM}$ & 545 & DIVE &. $\mathrm{DOC}$ & 587 & DIVE & 589 & VDL & . $\mathrm{CM}$ & 592 \\
\hline FTABI &. $\mathrm{CM}$ & 602 & & & & & & & & \\
\hline
\end{tabular}

\section{Cluster allocation table}

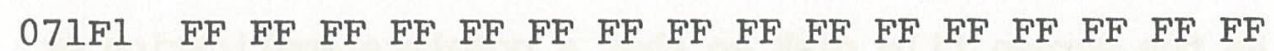

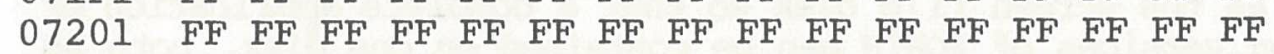

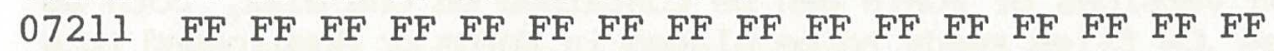

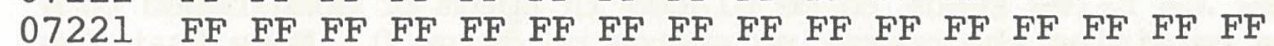

$\begin{array}{lllllllllllllllll}07231 & \text { FF } & \text { FF } & \text { FF } & \text { FF } & \text { FF } & \text { FF } & \text { FF } & \text { FF } & \text { FF } & \text { FF } & \text { FC } & 00 & 00 & 01 & 00 & 00\end{array}$

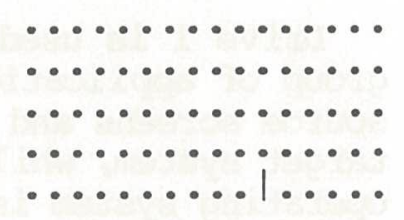

Disk ID: WOODS HOLE FORTH SCREEN FILE VER 1.801 Directory start

$1130 \quad 84$

$\begin{array}{llllllll}\text { FLOAT .CM } & 121 & \text { ASM4TH.CM } & 48 & \text { FFORTH.CM } & 55 & \text { SYSGEN.CM } & 558 \\ \text { WFORTH.CM } & 502 & \text { FORTH .CM } & 540 & \text { COPY .CM } & 554 & \text { AFORTH.CM } & 10\end{array}$

Cluster allocation table

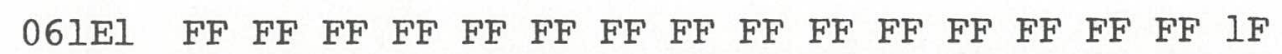

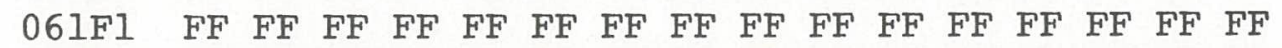

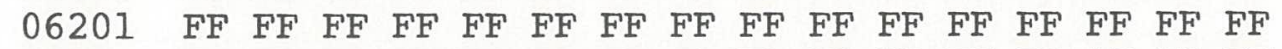

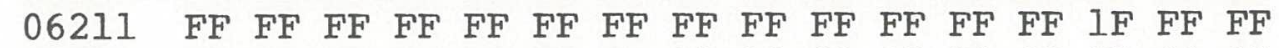

$\begin{array}{lllllllllllllllll}06221 & F F & F F & F F & F F & F F & F F & F F & 80 & 00 & 00 & 00 & 00 & 00 & 01 & 00 & 00\end{array}$

Woods Hole FORTH Version 1.800 Chapter 11 page 38 


\section{Screen Index}

This section contains the index to the sources for all FORTH words which are appended to FORTH.CM. The easiest way to find the source for any of these words is to scan the index until the desired word is found, and then consult the corresponding screen. The index has been generated by the FORTH word INDEX , which prints the first line (line 0) of each screen. This is effective because the following convention has been followed. The first line of each screen is considered the index line. The index line contains the names of all words defined on that screen, as well as any other relevant information, such as the name of the author or the general type of words defined. No actual code is contained on line zero. INDEX uses the word ?INDEX to determine whether line 0 is a valid index line and only valid index lines are printed.

There is a general system of screen organization, as listed below.

\begin{tabular}{|c|c|c|}
\hline Range & Type & Comments \\
\hline $65-79$ & Load screens & All of the load screens are contained here. \\
\hline $80-99$ & FORTH words & Miscellaneous useful forth words \\
\hline $100-111$ & Comm words & $\begin{array}{l}\text { All of the communication words are defined } \\
\text { here, as well as SERPRINTER, which is considered } \\
\text { a Comm word. }\end{array}$ \\
\hline $112-115$ & Decompiler & The decompiler is defined. \\
\hline $116-122$ & Editor & $\begin{array}{l}\text { The fig-Editor and the full screen editor EDIT } \\
\text { are defined. }\end{array}$ \\
\hline $123-130$ & FORTH DOS & The FORTH disk operating system is defined. \\
\hline $131-142$ & Assembler & The FORTH Assembler is defined. \\
\hline $143-163$ & Floating & FORTH floating point is defined. \\
\hline $165-218$ & Applications & $\begin{array}{l}\text { This group of } 54 \text { screens is available to the } \\
\text { user for writing application words and programs. }\end{array}$ \\
\hline $219-224$ & Examples & $\begin{array}{l}\text { This group of screens contains the sources for } \\
\text { the examples in chapter } 20 \text {. }\end{array}$ \\
\hline $225-233$ & Documentation & $\begin{array}{l}\text { This group of screens contains the sources for } \\
\text { FORTH self-documentation words. }\end{array}$ \\
\hline $234-249$ & Reference & $\begin{array}{l}\text { This group of screens is included for reference. } \\
\text { These include additional printer support words, } \\
\text { assembler and editor extensions, teaching words, } \\
\text { and a few screens from the fig-FORTH publication } \\
\text { FORTH DIMENSIONS. }\end{array}$ \\
\hline
\end{tabular}


Screen index for:

Disk ID: WOODS HOLE FORTH SCREEN FILE VER 1.801

65 (Master recursive load screen) (FLOATING) (Akens WHOI)

66 (Load screen used after raw ASM8 output, then 65 LOAD, Akens)

67 (Communications utilities load screen) (Akens, WHOI)

68 (Decompiler load screen QUIT.FLAG WORD.PTR; R Duncan, J Akens)

69 (fig-FORTH portable editor load screen) (William F. Ragsdale)

70 (FORTH-DOS Disk utilities load screen) (Akens, WHOI)

71 (ASSEMBLER load screen) (CODE END-CODE) (Akens, McDaniels)

72 (Floating point load screen) (Stetten, Akens)

73 (Load screen for complete printed documentation, Akens WHOI)

74 (Load screen for brief printed documentation, Akens WHOI)

75 (EXAMPLES load screen) (Akens, WHOI)

76 ( ASM4TH load screen)

77 ( AFORTH load screen)

79 ( parptr serptr) (Parallel and serial printer support)

80 ( PRINTER PARPRINTER NOPRINT)

81 ( U.R LOAD LOADTHRU ASMBR) (incorporated after ASM8 output)

83 ( OVERLAYS) (

84 ( 2DROP 2DUP 2SWAP ZEROES D.RLZ U.RLZ HEX. DEC.)

85 ( NODISK BELL BEEP CMOVE> COPY NOT >IN WORD-79)

86 ( NEXTUSER ALLOTUSER QUOTE BYE CHAPTER) ( USER var utilities)

87 ( ESC ID SEC MSEC CLS NEW-FENCE .VHDR .FOOT ROMIT) (Akens WHOI)

88 ( N. ?Y/N Y? VLIST)

89 ( Match MATCH)

90 (.ASCII DUMP)

91 ( ?EFl-?EF4 WI-W4 WNI-WN4 SETQ RETQ ) ( EF, Q Utilities)

92 ( CASE OF ENDOF ENDCASE ) (Charles Eaker, FORTH DIM. II/3)

93 ( DOS ASSEMBLER)

94 ( DEPTH .S DUMP-TERM SCREEN-DUMP VOCAB?)

95 ( VMOVE VCOPY VSHADOW)

96 ( ?SPACE ?ASCII ?INDEX ?SCREEN INDEX)

97 ( SPREADSCREEN)

98 ( PARPTR PAGE TEAR DISKLIST)

( Akens WHOI)

99 ( )

100 ( COMM.CTL TERM.CTL STATUS CTL $\Rightarrow$ MODEM COMM TERM CONS ESC')

101 ( COMMINIT RCVBRK RCVC RCVCHAR? RSTBRK SENDBRK) (Comm utils 2)

102 ( EMIT' TRC SENDC SENDCHAR? UART U) (Comm utils 3)

103 ( ONHOOK OFFHOOK DIALINIT DIALTONE ?CARRIER TS) (Comm utils 4)

104 ( TS TONE)

105 ( RE-ENTER DIAL ORIGINATE)

( Vram utilities)

106 ( Ansinit ANSWER)

107 (SEND-SCREEN GET-SCREEN) (Screen transfer utils 8 )

108 ( CS CRS 2**N CHANNEL-SELECT CHANNEL-RESET ?KEY ?MONITOR)

109 ( Xmit XMIT) (Comm utils 10)

110 ( CR' U.. ?HEXCHAR CLEAR-KEY SERPRINTER) (Comm utils 11)

111 ( $\mathrm{AH}->\mathrm{B} \quad \mathrm{V}$ V) (Comm utils 12)

112 ( PDOTQ.DSP WORD.DSP BRANCH.DSP CV.DSP CONST.DSP) ( Decompiler)

113 ( USERV.DSP VAR.DSP DOES>.DSP) (Decompiler 2)

114 ( DECOMPILE)

115 ( DECOMPILE, CONTINUED)

116 ( TEXT LINE EDITOR WHERE \#LOCATE \#LEAD)

( Decompiler 3)

117 ( \#LAG -MOVE H E S D M T L)

118 ( R P I TOP CLEAR) (EDITOR 3)

119 ( ILINE FIND DELETE N F)

( Decompiler 4)

120 ( B X TILL C T L)

( EDITOR 1)

( EDITOR 2)

121 ( GOTOXY SETEDIT .CUR !CUR +CUR +.CUR +LIN HOM !BLK S.ERASE)

Woods Hole FORTH Version $1.800 \quad$ Chapter 12

page 40 
122 ( EDIT EDITL)

123 ( EOF IOCB SECTOR NEWSDW)

124 ( GETSEC Open DISKID)

125 (.DIRSEC DIR NEWLSN)

126 ( FNAME FILEID)

127 ( OPEN)

128 ( .AH .BYTE .AHL .AHP)

129 ( F-LEN SAVE SEND)

130 ( DOWNLOAD FILELOAD)

131 ( R0 R1 R2 R3 R4 R5 R6 R7 R8 R9 RA RB RC RD RE RF) ( Registers)

132 ( END-CODE C; ENDCODE)

133 ( IS ?SBERROR)

134 ( T5 T4 T3 T2 T1 NX IP UP SP PC RP IV DMAV) (Register Equates)

135 ( CRN LDN LDA INP PLO ETC)

136 ( $1 \mathrm{BFI}$ 1BSB ETC )

(2BFI LBR LBQ LBZ LBDF LBNQ LBNZ LBNF) ( 1806 ASSEMBLER 7)

138 ( IBOP IDLE IRX ETC) ( 1 byte op codes, 1806 ASSEMBLER 8)

139 ( 2BOP 1BF2BOP 2BF2BOP CR2BOP etC) (1806 ASSEMBLER 9)

140 ( LBLI LBL2 LBL3 LBL4 LAB1: LAB2: LABB3: LAAB4: LAB1 LABB2 ILAB3)

141 ( A.0 A.1 PAGE)

142 ( ?NEXT'PAGE ?PAGESPACE PAGEJUMP PAGEADJUST

143 ( $\mathrm{F} 0>\mathrm{F} 0=$ )

144 ( FLOAT.CM linkage)

145 ( BUFF FSPW) (Floating USER variables)

146 ( [F.])

147 ( F.)

148 ( [FLOAT])

$149([\mathrm{~F}+] \mathrm{F}+)$

$150([\mathrm{~F}-]$ F- FMINUS FABS )

$151\left(\left[\mathrm{~F}^{*}\right] \mathrm{F}^{*}\right)$

$152([\mathrm{~F} /] \mathrm{F} /)$

153 ( [FSQRT] FSQRT)

154 ( 2OVER 2ROT)

155 (FQUAD) (Quadratic solution)

156 ( F@ F!)

157 ( FSIN)

158 ( FCOS)

159 ( FARCTAN)

160 ( [FEXP] FEXP)

161 ( [FLN] FLN)

$162(\mathrm{~F} * *$ FPI)

163 ( FLOAT D->F)

164 ( SCOPY)

219 ( Load screen for file listings)

220 ( ASSEMBLER Examples 2)

221 (Decompiler example screen)

223 (Floating point examples)

224 ( FORTH DOS examples)

225 ( .VHDR3 VLIST3)

226 ( CAT-UPDATE .CAT VLIST2) (Cluster allocation table utilities)

227 (.INDEX DIR' DIRECTORIES INDEX2 DUMP2) (DOCUMENT words)

228 (SHOW.VAR SHOW.USER)

229 ( SHOW.CONST SHOW.VOCAB)

230 ( ZFLAG ZZDICT ZHERE ZZEND ZNAME ZINSERT ZPRINT, Alphabetical )

231 ( ZSORT ZLIST ZZEDLIST)

Woods Hole FORTH Version 1.800
Chapter 12

page 41 
Screen index for:

Disk ID: WOODS HOLE FORTH SCREEN FILE VER 1.801

113084

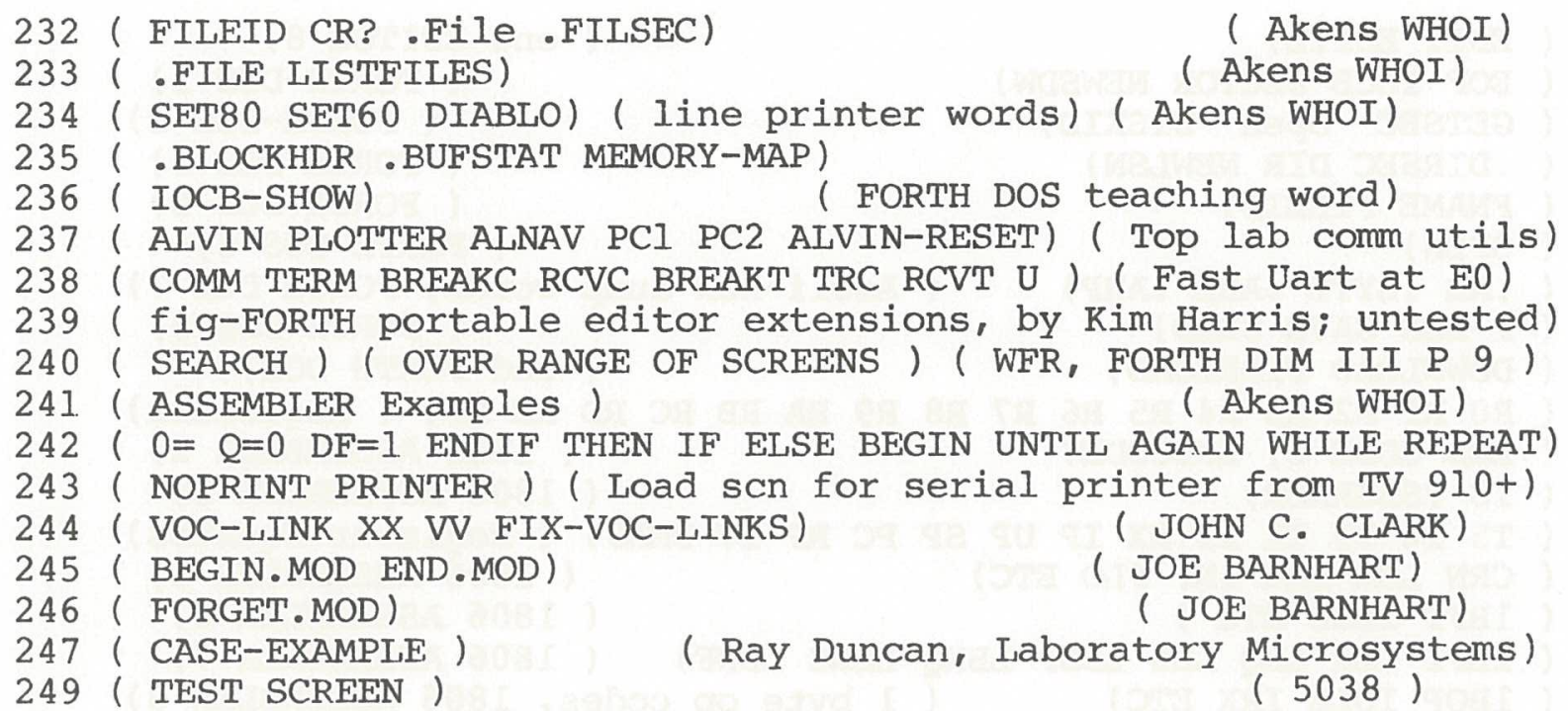


Vocabulary List

This chapter is a list of all words in the FORTH VOCABULARY. The words are listed in the order in which they were defined, most recently defined word first. The name field address (NFA) is also listed. This provides an index to the memory dump if you wish to examine the complete dictionary entry for the word. The complete explanation for each word is given in the Glossary, chapter 25. A diagram explaining the dictionary entry format is shown below. The diagram includes some of the FORTH words which access fields within dictionary entries.

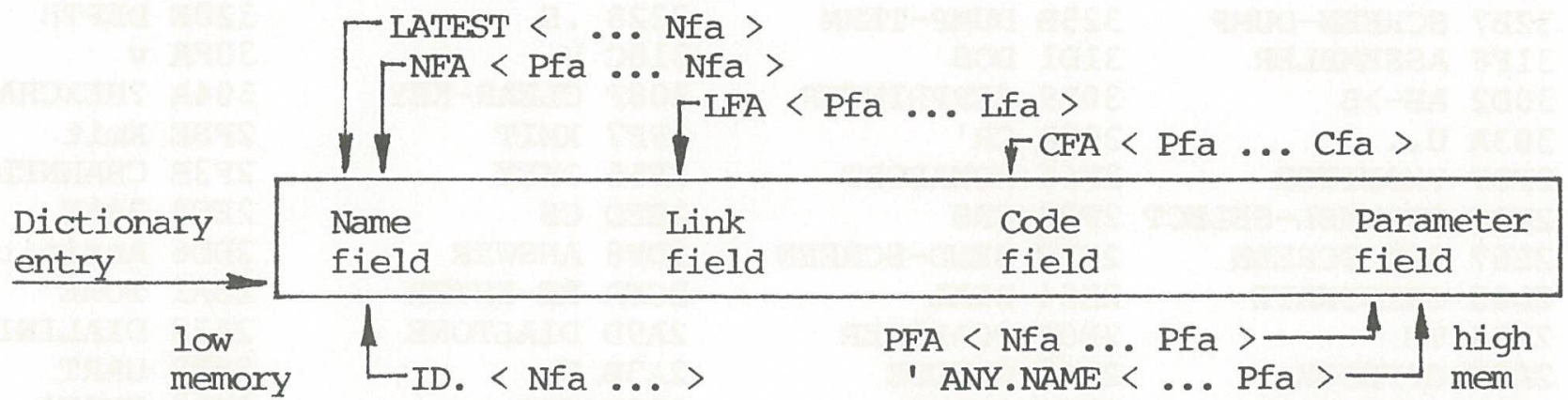

There are 6 pre-defined types of dictionary entries. These are Code definitions, Colon definitions, CONSTANTS, VARIABLES, USER variables and VOCABULARY definitions. Other types, such as string constants and string variables may be defined by the user for specific applications. Refer to the FORTH ENCYCLOPEDIA for complete details.

The lengths of the name field and the parameter field are variable. The link field and the code field are always two bytes long. The link field address points to the name field of the word previously defined in the present VOCABULARY. The code field address points to the code which is actually executed when the word is invoked. The code field of a code word points to the parameter field which contains machine code. For all other types of words, the code field points to code which interprets the entries in the parameter field. The dictionary is composed of a number of dictionary entries threaded together via the link field addresses. The end of the dictionary is signified by a link field address of 0000. Each word which defines a VOCABULARY entry contains a pointer to the most recently defined word in that particulary VOCABULARY (ie FORTH EDITOR ASSEMBLER). 


\begin{tabular}{|c|c|c|c|}
\hline $418 \mathrm{~B}$ & DOCUMENT & 4180 & TASK \\
\hline $410 \mathrm{~F}$ & DISKLIST & 4100 & TEAR \\
\hline $3 E 2 E$ & DECOMPILE & $3 E 06$ & VOCAB.DSP \\
\hline 3D77 & USERV.DSP & $3 \mathrm{D} 4 \mathrm{D}$ & CONST.DSP \\
\hline 3СB6 & WORD.DSP & $3 \mathrm{C} 8 \mathrm{~A}$ & PDOTQ.DSP \\
\hline $3 C 20$ & FILELOAD & $3 B C 9$ & DOWNLOAD \\
\hline $3 \mathrm{~B} 01$ & F-LEN & $3 A D C$ &. AHP \\
\hline $3 A 6 F$ &. $\mathrm{AH}$ & $38 \mathrm{~B} 2$ & OPEN \\
\hline $37 \mathrm{~B} 6$ & NEWLSN & 3775 & DIR \\
\hline $366 \mathrm{D}$ & Open & $35 \mathrm{EC}$ & GETSEC \\
\hline 3547 & IOCB & $353 D$ & $\mathrm{EOF}$ \\
\hline $344 \mathrm{~A}$ & ?SCREEN & 3401 & ?INDEX \\
\hline 3385 & VSHADOW & 3343 & VCOPY \\
\hline $32 \mathrm{~B} 7$ & SCREEN-DUMP & $325 B$ & DUMP-TERM \\
\hline $31 \mathrm{~F} 8$ & ASSEMBLER & 31D1 & DOS \\
\hline 30D2 & $\mathrm{AH}->\mathrm{B}$ & $30 \mathrm{~A} 9$ & SERPRINTER \\
\hline $303 \mathrm{~A}$ & U.. & $302 \mathrm{~A}$ & $\mathrm{CR}^{\prime}$ \\
\hline $2 \mathrm{~F} 87$ & ?MONITOR & $2 \mathrm{~F} 66$ & MONABORT \\
\hline $2 F 20$ & CHANNEL-SELECT & $2 \mathrm{~F} 00$ & CRS \\
\hline 2.E67 & GET-SCREEN & $2 E 2 F$ & SEND-SCREEN \\
\hline 2D85 & ORIGINATE & 2D54 & DIAL \\
\hline $2 B 54$ & $\mathrm{TS}$ & $2 \mathrm{~B} 0 \mathrm{~F}$ & ?CARRIER \\
\hline $2 A 5 E$ & OFFHOOK & $2 \mathrm{~A} 45$ & ONHOOK \\
\hline 29DI & SENDCHAR? & $29 B 3$ & SENDC \\
\hline 2945 & SENDBRK & 2926 & RSTBRK \\
\hline $28 \mathrm{BB}$ & RCVBRK & $28 A 6$ & COMMINIT \\
\hline 2871 & TERM & 2860 & COMM \\
\hline $282 \mathrm{D}$ & STATUS & $281 \mathrm{E}$ & TERM.CTL \\
\hline $27 \mathrm{DD}$ & EDITL & $26 \mathrm{AE}$ & EDIT \\
\hline 2553 & $\mathrm{~T}$ & 2261 & WHERE \\
\hline $220 \mathrm{E}$ & TEXT & $21 D C$ & ENDCASE \\
\hline 2176 & CASE & $216 \mathrm{~B}$ & RETQ \\
\hline 2150 & WN3 & 2148 & WN2 \\
\hline 2132 & W3 & $212 B$ & W2 \\
\hline 2112 & ?EF3 & 2109 & ?EF2 \\
\hline $202 C$ & - ASCII & IFDC & MATCH \\
\hline 1ED0 & Y? & 1E9D & $? Y / N$ \\
\hline 1DC7 & .FOOT & ID93 & .VHDR \\
\hline 1DIE & MSEC & ICFA & SEC \\
\hline 1CB9 & CHAPTER & $1 \mathrm{CAB}$ & BYE \\
\hline $1 C 57$ & NEXTUSER & $1 C 45$ & WORD -79 \\
\hline $1 \mathrm{BF} 8$ & COPY & 1BBD & CMOVE> \\
\hline $1 \mathrm{~B} 80$ & NODISK & $1 \mathrm{~B} 67$ & DEC. \\
\hline $1 \mathrm{Bl} 6$ & D.RLZ & $1 \mathrm{AF} 1$ & ZEROES \\
\hline $1 \mathrm{ABE}$ & 2DROP & $1 \mathrm{AB} 2$ & ASMBR \\
\hline $1 \mathrm{Al} 6$ & $\mathrm{U} \cdot \mathrm{R}$ & $19 \mathrm{E} 8$ & NOPRINT \\
\hline $194 \mathrm{~A}$ & serptr & $192 \mathrm{~A}$ & parptr \\
\hline $18 \mathrm{~A} 4$ & OK & 1892 & (DOS) \\
\hline $182 \mathrm{~F}$ & FORTH & $181 \mathrm{~F}$ & DR0 \\
\hline 17B4 & GROUP & 179D & CDER \\
\hline 16D6 & BLOCK & $168 \mathrm{D}$ & BUFFER \\
\hline 1625 & $+B U F$ & 1613 & . LINE \\
\hline $15 \mathrm{AF}$ & MESSAGE & $15 \mathrm{~A} 2$ & $\mathrm{U}$ \\
\hline $157 \mathrm{~B}$ & D. & $156 \mathrm{~A}$ & $\cdot \mathrm{R}$ \\
\hline 1505 & $\#$ & $14 \mathrm{EC}$ & SIGN \\
\hline
\end{tabular}

416B FLOATING 4156 DOCUMENT

40E3 PAGE

3DDE DOES $>$.DSP

3D2F CV.DSP

3C7B WORD. PTR

3B78 SEND

3AB7 .AHL

388F FILEID

36DF .DIRSEC

359B NEWSDW

34CC SPREADSCREEN

33E0 ?ASCII

332B VMOVE

3228 .S

$318 \mathrm{C} \mathrm{V}$

308F CLEAR-KEY

2FF7 XMIT

2F55 ?KEY

2EED CS

2DF8 ANSWER

2CF'A RE-ENTER

2A9D DIALTONE

2A3B U

298F TRC

2905 RCVCHAR?

2891 ESC'

284E MODEM

280F COMM.CTL

256D CUR

224E EDITOR

21B6 ENDOF

2160 SETQ

2140 WNI

2124 WI

2100 ?EF1

IF9E Match

$1 \mathrm{E} 6 \mathrm{C} \mathrm{N}$.

1D47 NEW-FENCE

ICDI ID

1 C97 QUOTE

IC39 > IN

IBB0 BEEP

IB4E HEX.

IADD 2SWAP

IA95 LOADTHRU

19C5 PARPRINTER

18FE FLUSH

1883 MON

17FD $-\rightarrow$

178C Cder

166F EMPTY-BUFFERS

15EC (LINE)

1596 ?

1546 D.R

14D5 \#>

$1 \mathrm{C86}$ ALLOTU
40BC PARPTR

3DB6 VAR.DSP

3DOI BRANCH.DSP

3C6B QUIT.FLAG

3B35 SAVE

3A99 . BYTE

3851 FNAME

369E DISKID

3552 SECTOR

3494 INDEX

$33 \mathrm{CD}$ ?SPACE

$32 \mathrm{DI}$ VOCAB?

320E DEPTH

30FA V

304A ?HEXCHAR

2FBE xmit

2F3B CHANNEL-RESET

$2 \mathrm{EC} 42 * * \mathrm{~N}$

2DD6 Ansinit

2BAI TONE

2A78 DIALINIT

29F3 UART

2981 EMIT'

28E2 RCVC

2882 CONS

283E CTL $\Rightarrow$

27EF CLEAR

255F L

222D LINE

218D OF

2158 WN4

2139 W4

211B ?EF4

2093 DUMP

IEFE VLIST

1E2E ROMIT

$1 D 37$ CLS

$1 \mathrm{CC} 7 \mathrm{ESC}$

1C2D NOT

1B9F BELL

IB3C U.RLZ

IACE 2DUP

IA26 LOAD

1969 PRINTER

$18 B 3$ LIST

186D DRI

17CA LOAD

$1736 \mathrm{R} / \mathrm{W}$

1650 UPDATE

$15 \mathrm{D} 7 \mathrm{~S}->\mathrm{D}$

158A.

$152 \mathrm{~F}$ \#S

14 C6 <\#

Woods Hole FORTH Version 1.800

Chapter 13

page 44 
Nfa Name Vocabulary list

\begin{tabular}{|c|c|c|c|c|c|c|c|}
\hline $14 \mathrm{~A} 5$ & SPACES & 1495 & WHILE & 1474 & ELSE & $145 \mathrm{~F}$ & IF \\
\hline 1442 & REPEAT & $142 \mathrm{C}$ & AGAIN & 1420 & END & $140 \mathrm{~A}$ & UNTIL \\
\hline $13 F 4$ & +LOOP & $13 \mathrm{DF}$ & LOOP & $13 \mathrm{CE}$ & $\mathrm{DO}$ & $13 \mathrm{Cl}$ & THEN \\
\hline $13 \mathrm{~A} 9$ & ENDIF & 1397 & BEGIN & $138 \mathrm{~A}$ & BACK & $137 \mathrm{~B}$ & */ \\
\hline 1367 & */MOD & 1359 & MOD & $134 \mathrm{~B}$ & 1 & 1338 & /MOD \\
\hline 1311 & $\mathrm{M} /$ & $12 \mathrm{D} 2$ & FORGET & $12 \mathrm{BE}$ & 1 & $12 \mathrm{~B} 0$ & ( \\
\hline 1298 & IMMEDIATE & $127 \AA$ & [COMPILE] & $124 \mathrm{~B}$ & ." = - a n a & 1239 & i \\
\hline $11 \mathrm{DE}$ & ABORT & $11 \mathrm{~B} 3$ & QUIT & 1199 & DEFINITIONS & 1164 & VOCABULARY \\
\hline 1114 & INTERPRET & $10 \mathrm{E} 9$ & ?STACK & $10 \mathrm{CC}$ & DLITERAL & 10B0 & LITERAI \\
\hline $108 D$ & DOES $>$ & $107 \mathrm{~B}$ & $<$ BUILDS & $106 \mathrm{~A}$ & USER & 1055 & VARIABLE \\
\hline 1040 & CONSTANT & 1026 & !CODE & 1002 & : & OF9D & CREATE \\
\hline OF69 & ID. & 0F51 & MIN & $0 F 20$ & ERROR & $0 F 10$ & (ABORT) \\
\hline OEE6 & -FIND & OE8D & NUMBER & 0E40 & (NUMBER) & ODF3 & WORD \\
\hline ODB9 & & OD9D & QUERY & OD2C & EXPECT & $0 \mathrm{DI} 6$ & ;CODE \\
\hline OCFE & $(; \mathrm{CODE})$ & $0 \mathrm{CE} 8$ & DECIMAL & 0CD6 & HEX & $0 \mathrm{CCl}$ & SMUDGE \\
\hline OCBI & ] & $0 C A 3$ & {[} & $0 C 87$ & COMPILE & $0 \mathrm{C} 6 \mathrm{C}$ & ?LOADING \\
\hline $0 C 53$ & ?CSP & $0 C 3 E$ & ?PAIRS & $0 C 28$ & ?EXEC & $0 \mathrm{ClO}$ & ?COMP \\
\hline $0 \mathrm{BF} 5$ & ?ERROR & OBEI & PFA & $\mathrm{OBCB}$ & NFA & 0BB8 & IATEST \\
\hline OB91 & TRAVERSE & OB82 & $\mathrm{D}+-$ & OB74 & +- & 0B57 & $\mathrm{PAD}$ \\
\hline OB3A & (.") & OBI0 & -TRAILING & OAFB & COUNT & OAE7 & !CSP \\
\hline OADF & CFA & $0 A D 3$ & LFA & $0 A C 8$ & $2-$ & $0 \mathrm{AB} 7$ & $1-$ \\
\hline OA9F & $\mathrm{C}$ & $0 A 80$ & , & $0 A 64$ & ALLOT & $0 A 4 C$ & HERE \\
\hline 09FF & $\mathrm{M} / \mathrm{MOD}$ & $09 \mathrm{CD}$ & BLOCK-WRITE & $09 A 5$ & BI,OCK-READ & $095 B$ & SPACE \\
\hline 092C & TYPE & 0904 & $\mathrm{CR}$ & $08 F 5$ & EMIT & 08DE & ?TERMINAL \\
\hline $08 C 9$ & KEY & 08AA & HOLD & $088 \mathrm{~F}$ & BIAANKS & $087 \mathrm{E}$ & ERASE \\
\hline 0861 & FILL & 0835 & INPUT & 0810 & OUTPUT & 0804 & Nextu \\
\hline $07 F 5$ & COMM.GRP & $07 \mathrm{E} 6$ & TERM.GRP & 07D6 & MODEM.GRP & $07 \mathrm{CC}$ & GRP \\
\hline $07 \mathrm{Cl}$ & OUTL & $07 \mathrm{~B} 3$ & SCR/DSK & $07 A 3$ & USER-IOCB & 0798 & PREV \\
\hline $078 \mathrm{E}$ & USE & 0784 & HLD & 077B & R\# & 0771 & CSP \\
\hline 0767 & FLD & $075 \mathrm{D}$ & DPI & 0752 & BASE & 0746 & STATE \\
\hline 0738 & CURRENT & $072 \mathrm{~A}$ & CONTEXT & 071D & OFFSET & 0713 & SCR \\
\hline 0709 & OUT & 0700 & IN & $06 \mathrm{~F} 6$ & BLK & $06 \mathrm{E} 7$ & VOC-LINK \\
\hline $06 \mathrm{DE}$ & $\mathrm{DP}$ & $06 \mathrm{D} 2$ & FENCE & $06 \mathrm{C} 4$ & WARNING & 06B8 & WIDTH \\
\hline 06AE & TIB & $06 \mathrm{~A} 5$ & RO & $069 \mathrm{C}$ & so & $067 \mathrm{E}$ & +ORIGIN \\
\hline 0671 & ORIGIN & 0665 & $\mathrm{~B} / \mathrm{SCR}$ & 0659 & $\mathrm{~B} / \mathrm{BUF}$ & $064 \mathrm{D}$ & LIMIT \\
\hline 0641 & FIRST & 0637 & $\mathrm{~L} / \mathrm{P}$ & $062 \mathrm{D}$ & $\mathrm{C} / \mathrm{L}$ & 0624 & $\mathrm{BL}$ \\
\hline $061 \mathrm{C}$ & 3 & 0614 & 2 & $060 \mathrm{C}$ & 1 & 0604 & 0 \\
\hline 0571 & $\mathrm{M}^{*}$ & 0563 & * & 0541 & MAX & 0532 & DABS \\
\hline 0524 & $\mathrm{ABS}$ & $050 \mathrm{~F}$ & -DUP & $04 \mathrm{~F} 8$ & ROT & $04 \mathrm{~EB}$ & $C !$ \\
\hline 04DC & $!$ & $04 \mathrm{CC}$ & ce & $04 \mathrm{BB}$ & a & $04 \mathrm{~A} 7$ & TOGGLE \\
\hline 0496 & DUP & 0482 & SWAP & 0476 & DROP & 0460 & OVER \\
\hline 0444 & DMINUS & $042 \mathrm{~A}$ & MINUS & 0411 & $D+$ & 0401 & - \\
\hline 03ED & $+!$ & 03E2 & $2+$ & $03 \mathrm{D} 0$ & $1+$ & $03 \mathrm{CO}$ & + \\
\hline $03 A A$ & $>$ & $038 \mathrm{C}$ & $<$ & $036 \mathrm{~F}$ & $=$ & 0362 & $0<$ \\
\hline $034 \mathrm{D}$ & $0=$ & 0342 & $I$ & 0331 & $R>$ & 0323 & $>\mathrm{R}$ \\
\hline 0313 & LEAVE & $02 \mathrm{~F} 4$ & CMOVE & $02 \mathrm{CB}$ & DIGIT & $02 B B$ & iS \\
\hline $02 \mathrm{~A} 5$ & $\mathrm{RP} !$ & 0291 & SP! & 0280 & SP@ & $026 \mathrm{E}$ & XOR \\
\hline $025 D$ & OR & $024 B$ & AND & 0235 & $\mathrm{U}^{*}$ & $01 \mathrm{FF}$ & $\mathrm{U} /$ \\
\hline 01ED & EXECUTE & $018 \mathrm{C}$ & ENCLOSE & 0178 & OBRANCH & 0169 & BRANCH \\
\hline 0154 & $(\mathrm{DO})$ & 0143 & LIT & OOEC & (FIND) & $00 C 9$ & $(+\mathrm{LOOP})$ \\
\hline $00 A 5$ & (LOOP) & 0055 & $\mathrm{EF}$ & $002 \mathrm{D}$ & WARM & OOOD & COLD \\
\hline
\end{tabular}


This chapter contains an alphabetically sorted list of the FORTH VOCABULARY. The first name in the list is the FORTH word NULL, which is a nonprinting character.

A diagram explaining the dictionay entry format is shown below. The diagram includes some of the FORTH words which access fields within the dictionary entry.

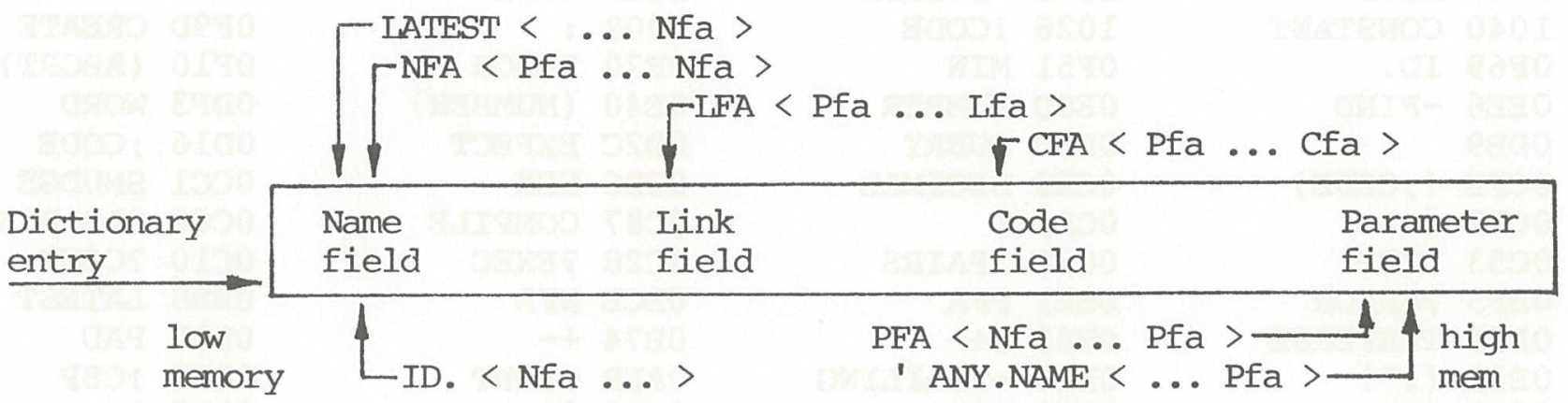

There are 6 pre-defined types of dictionary entries. These are Code definitions, colon definitions, CONSTANTS, VARIABLES, USER variables and VOCABULARY definitions. Other types, such as string constants and string variables may be defined by the user for specific applications. Refer to the FORTH ENCYCLOPEDIA for complete details.

The lengths of the name field and the parameter field are variable. The link field and the code field are always two bytes long. The link field address points to the name field of the word previously defined in the present VOCABULARY. The code field address points to the code which is actually executed when the word is invoked. The code field of a code word points to the parameter field which contains machine code. For all other types of words, the code field points to code which interprets the entries in the parameter field. The dictionary is composed of a number of dictionary entries threaded together via the link field addresses. The end of the dictionary is signified by a link field address of 0000. Each word which defines a VOCABULARY entry contains a pointer to the most recently defined word in that particulary VOCABULARY (ie FORTH EDITOR ASSEMBLER). 


\begin{tabular}{|c|c|c|c|}
\hline ODB9 & & 04DC ! & \\
\hline 1505 & \# & 14D5 & $\#>$ \\
\hline $12 \mathrm{~B} 0$ & ( & $00 c 9$ & $(+\mathrm{L}, \mathrm{OOP})$ \\
\hline OFI0 & (ABORT) & 0154 & (DO) \\
\hline $15 \mathrm{EC}$ & (LINE) & $00 A 5$ & (LOOP) \\
\hline $137 \mathrm{~B}$ & */ & 1367 & */MOD \\
\hline OB74 & +- & 1625 & +BUF \\
\hline $0 A 80$ & , & 0401 & - \\
\hline 0EE6 & -FIND & OBIO & -TRAILING \\
\hline $3 \mathrm{~A} 6 \mathrm{~F}$ &. $\mathrm{AH}$ & $3 \mathrm{AB} 7$ & . AHL \\
\hline 3А999 & . BYTE & $36 \mathrm{DF}$ & .DIRSEC \\
\hline $156 \mathrm{~A}$ & $\cdot \mathrm{R}$ & 3228 &.$S$ \\
\hline 1338 & $/ \mathrm{MOD}$ & 0604 & 0 \\
\hline 0178 & OBRANCH & $060 \mathrm{C}$ & 1 \\
\hline 0614 & 2 & $2 \mathrm{EC} 4$ & $2 * * N$ \\
\hline $1 \mathrm{ABE}$ & 2DROP & $1 \mathrm{ACE}$ & 2DUP \\
\hline 1002 & : & 1239 & ; \\
\hline $038 \mathrm{C}$ & $<$ & $14 \mathrm{C} 6$ & $<\#$ \\
\hline $03 \mathrm{AA}$ & $>$ & $1 C 39$ & $>$ IN \\
\hline $33 \mathrm{E} 0$ & ?ASCII & $2 \mathrm{~B} 0 \mathrm{~F}$ & ?CARRIER \\
\hline 2100 & ?EFI & 2109 & ?EF2 \\
\hline $0 B F 5$ & ?ERROR & $0 C 28$ & ?EXEC \\
\hline $2 F 55$ & ?KEY & $0 \mathrm{C} 6 \mathrm{C}$ & ?LOADING \\
\hline $344 \mathrm{~A}$ & ?SCREEN & $33 \mathrm{CD}$ & ?SPACE \\
\hline 1E9D & ?Y/N & $04 \mathrm{BB}$ & a \\
\hline ] $42 \mathrm{C}$ & AGAIN & $30 \mathrm{D} 2$ & $\mathrm{AH}->\mathrm{B}$ \\
\hline $024 B$ & AND & $2 \mathrm{DF} 8$ & ANSWER \\
\hline 2DD6 & Ansinit & 0659 & $\mathrm{~B} / \mathrm{BUF}$ \\
\hline 0752 & BASE & 1BB0 & BEEP \\
\hline 0624 & $\mathrm{BL}$ & $088 \mathrm{~F}$ & BLANKS \\
\hline 09A5 & BL,OCK-READ & $09 \mathrm{CD}$ & BLOCK-WRITE \\
\hline $168 D$ & BUFFER & $1 \mathrm{CAB}$ & BYE \\
\hline $062 \mathrm{D}$ & $\mathrm{C} / \mathrm{L}$ & $04 \mathrm{CC}$ & c@ \\
\hline OADF & CFA & $2 F 3 B$ & CHANNEL-RESET \\
\hline 27EF & CLEAR & $308 \mathrm{~F}$ & CLEAR-KEY \\
\hline IBBD & CMOVE> & 000D & COLD \\
\hline $07 F 5$ & COMM.GRP & $28 \mathrm{~A} 6$ & COMMINIT \\
\hline 3D4D & CONST.DSP & 1040 & CONSTANT \\
\hline OAFB & COUNT & 0904 & $\mathrm{CR}$ \\
\hline $2 \mathrm{FOO}$ & CRS & 2EED & $\mathrm{CS}$ \\
\hline $256 \mathrm{D}$ & CUR & 0738 & CURRENT' \\
\hline 0411 & $D+$ & OB82 & $\mathrm{D}+-$ \\
\hline $1 B 16$ & D.RLZ & 0532 & $\mathrm{DABS}$ \\
\hline $3 \mathrm{E} 2 \mathrm{E}$ & DECOMPILE & 1199 & DEFINII'IONS \\
\hline $2 \mathrm{~A} 78$ & DIALINIT & 2A9D & DIALTONE \\
\hline $369 E$ & DISKID & $410 \mathrm{~F}$ & DISKLIST \\
\hline $13 \mathrm{CE}$ & DO & $418 B$ & DOCUMENT \\
\hline 3DDE & DOES>.DSP & $31 \mathrm{Dl}$ & DOS \\
\hline $075 \mathrm{D}$ & DPL & $181 \mathrm{~F}$ & DRO \\
\hline 2093 & DUMP & $325 B$ & DUMP-TERM \\
\hline $27 \mathrm{DD}$ & EDITL & $224 E$ & EDITOR \\
\hline $08 F 5$ & EMIT & 2981 & EMIT' \\
\hline 1420 & END & 2IDC & ENDCASE \\
\hline $353 \mathrm{D}$ & $\mathrm{EOF}$ & $087 \mathrm{E}$ & ERASE \\
\hline
\end{tabular}

\begin{tabular}{|c|c|c|c|}
\hline 1026 & CODE & OAE7 ! & CSP \\
\hline $152 \mathrm{~F}$ & $\# S$ & $12 \mathrm{BE}$ & ' \\
\hline OBЗA & $(. ")$ & OCFE & $(; \mathrm{CODE})$ \\
\hline 1892 & (DOS) & OOEC & (FIND) \\
\hline OE40 & (NUMBER) & 0563 & * \\
\hline $03 \mathrm{CO}$ & + & 03ED & $+!$ \\
\hline $13 F 4$ & +LOOP & $067 \mathrm{E}$ & +ORIGIN \\
\hline 17FD & $-->$ & $050 \mathrm{~F}$ & -DUP \\
\hline $158 \mathrm{~A}$ & - & $124 B$ & . " \\
\hline $3 A D C$ & .AHP & $202 \mathrm{C}$ & - ASCII \\
\hline 1DC7 & . FOOT & 1613 & - LINE \\
\hline 1D93 & . VHDR & $134 \mathrm{~B}$ & 1 \\
\hline 0362 & $0<$ & 034D & $0=$ \\
\hline 03D0 & $1+$ & $0 A B 7$ & $1-$ \\
\hline 03E2 & $2+$ & 0AC8 & $2-$ \\
\hline IADD & 2SWAP & $061 \mathrm{C}$ & 3 \\
\hline $0 \mathrm{Dl} 6$ & ;CODE & $02 \mathrm{BB}$ & is \\
\hline $107 \mathrm{~B}$ & <BUILDS & $036 \mathrm{~F}$ & $=$ \\
\hline 0323 & $>\mathrm{R}$ & 1596 & $?$ \\
\hline $0 \mathrm{ClO}$ & ?COMP & $0 \mathrm{C53}$ & ?CSP \\
\hline 2112 & ?EF3 & $211 \mathrm{~B}$ & ?EF4 \\
\hline $304 \mathrm{~A}$ & ?HEXCHAR & 3401 & ?INDEX \\
\hline $2 \mathrm{~F} 87$ & ?MONITOR & OC $3 E$ & ?PAIRS \\
\hline 10E9 & ?STACK & 08DE & ?TERMINAL \\
\hline 11DE & ABORT & 0524 & ABS \\
\hline $0 \mathrm{~A} 64$ & ALLOT & $1 \mathrm{C} 86$ & ALLOTU \\
\hline $1 \mathrm{AB} 2$ & ASMBR & $31 \mathrm{~F} 8$ & ASSEMBLER \\
\hline 0665 & $\mathrm{~B} / \mathrm{SCR}$ & $138 \mathrm{~A}$ & BACK \\
\hline 1397 & BEGIN & $1 \mathrm{~B} 9 \mathrm{~F}$ & BELL \\
\hline $06 \mathrm{~F} 6$ & BLK & 16D6 & BLOCK \\
\hline 0169 & BRANCH & 3D01 & BRANCH.DSP \\
\hline $04 \mathrm{~EB}$ & C! & OA9F & $C_{\text {, }}$ \\
\hline 2176 & CASE & 179D & CDER \\
\hline $2 F 20$ & CHANNEL-SELECT & 1СВ9 & CHAPTER \\
\hline 1D37 & CLS & $02 F 4$ & CMOVE \\
\hline 2860 & COMM & $280 \mathrm{~F}$ & COMM.CTL \\
\hline $0 \subset 87$ & COMPILE & 2882 & CONS \\
\hline $072 \mathrm{~A}$ & CONTEXT & IBF 8 & COPY \\
\hline $302 \mathrm{~A}$ & $\mathrm{CR}^{\prime}$ & OF9D & CREATE \\
\hline 0771 & CSP & $283 E$ & $\mathrm{CTL}=>$ \\
\hline $3 \mathrm{D} 2 \mathrm{~F}$ & CV.DSP & $178 \mathrm{C}$ & Caer \\
\hline $157 \mathrm{~B}$ & D. & 1546 & $\mathrm{D} \cdot \mathrm{R}$ \\
\hline 1B67 & DEC. & OCE8 & DECIMAL \\
\hline $320 E$ & DEPTH & 2D54 & DIAL \\
\hline $02 \mathrm{CB}$ & DIGIT & 3775 & DIR \\
\hline $10 \mathrm{CC}$ & DLITERAL & 0444 & DMINUS \\
\hline 4156 & DOCUMENT & $108 D$ & DOES> \\
\hline $3 \mathrm{BC} 9$ & DOWNLOAD & 06DE & DP \\
\hline $186 \mathrm{D}$ & DRI & 0476 & DROP \\
\hline 0496 & DUP & $26 \mathrm{AE}$ & EDIT \\
\hline 0055 & $\mathrm{EF}$ & 1474 & ELSE \\
\hline $166 \mathrm{~F}$ & EMPTY-BUFFERS & $018 \mathrm{C}$ & ENCLOSE \\
\hline $13 \mathrm{~A} 9$ & ENDIF & $21 B 6$ & ENDOF \\
\hline $0 F 20$ & ERROR & $1 \mathrm{CC} 7$ & ESC \\
\hline
\end{tabular}




\begin{tabular}{|c|c|c|c|c|c|c|c|}
\hline $\mathrm{Nfa}$ & Name & & Vocabulary & & & & \\
\hline 2891 & ESC' & & & & & & \\
\hline 01ED & EXECUTE & OD2C & EXPECT & $3 \mathrm{~B} 0 \mathrm{I}$ & F-LEN & 06D2 & FENCE \\
\hline $388 \mathrm{~F}$ & FILEID & $3 \mathrm{C} 20$ & FILELOAD & 0861 & FILI & 0641 & FIRST \\
\hline 0767 & FLD & $416 \mathrm{~B}$ & FLOATING & $18 \mathrm{FE}$ & FLUSH & 3851 & FNAME \\
\hline $12 \mathrm{D} 2$ & FORGET & $182 \mathrm{~F}$ & FORTH & $2 \mathrm{E} 67$ & GET-SCREEN & $35 \mathrm{EC}$ & GETSEC \\
\hline $17 \mathrm{~B} 4$ & GROUP & $07 \mathrm{CC}$ & GRP & OA4C & HERE & 0CD6 & $\mathrm{HEX}$ \\
\hline $1 B 4 E$ & HEX. & 0784 & HLD & $08 \mathrm{AA}$ & HOLD & 0342 & $I$ \\
\hline $1 \mathrm{CDI}$ & ID & 0F69 & ID. & $145 \mathrm{~F}$ & IF & 1298 & IMMEDIATE \\
\hline 0700 & IN & 3494 & INDEX & 0835 & INPUT & 1114 & INTERPRET \\
\hline 3547 & IOCB & $08 C 9$ & KEY & $255 F$ & L & 0637 & $\mathrm{~L} / \mathrm{P}$ \\
\hline 0BB8 & LATEST & 0313 & LEAVE & $0 \mathrm{AD} 3$ & LFA & $064 \mathrm{D}$ & LIMIT \\
\hline $222 \mathrm{D}$ & LINE & $18 \mathrm{~B} 3$ & LIST & 0143 & LIT & $10 \mathrm{B0}$ & LITERAL \\
\hline $1 \mathrm{~A} 26$ & LOAD & $17 \mathrm{CA}$ & LOAD & 1 A95 & LOADTHRU & $13 \mathrm{DF}$ & LOOP \\
\hline 0571 & $M^{*}$ & 1311 & M/ & $09 \mathrm{FF}$ & $\mathrm{M} / \mathrm{MOD}$ & 1FDC & MATCH \\
\hline 0541 & MAX & $15 \mathrm{AF}$ & MESSAGE & 0F51 & MIN & $042 \mathrm{~A}$ & MINUS \\
\hline 1359 & $\mathrm{MOD}$ & $284 \mathrm{E}$ & MODEM & $07 \mathrm{D} 6$ & MODEM.GRP & 1883 & MON \\
\hline $2 \mathrm{~F} 66$ & MONABORT & $1 \mathrm{D} 1 \mathrm{E}$ & MSEC & $1 F 9 E$ & Match & IE6C & $\mathrm{N}$ \\
\hline $1 D 47$ & NEW-FENCE & $37 \mathrm{~B} 6$ & NEWLSN & $359 B$ & NEWSDW & $1 \mathrm{C} 57$ & NEXTUSER \\
\hline OBCB & NFA & $1 \mathrm{~B} 80$ & NODISK & $19 E 8$ & NOPRINT & $1 \mathrm{C} 2 \mathrm{D}$ & NOT \\
\hline OE8D & NUMBER & 0804 & Nextu & $218 D$ & $\mathrm{OF}$ & $2 \mathrm{~A} 5 \mathrm{E}$ & OFFHOOK \\
\hline $071 D$ & OFFSET & $18 \mathrm{~A} 4$ & $\mathrm{OK}$ & $2 A 45$ & ONHOOK & $38 \mathrm{~B} 2$ & OPEN \\
\hline $025 \mathrm{D}$ & OR & 0671 & ORIGIN & $2 \mathrm{D} 85$ & ORIGINATE & 0709 & OUT \\
\hline $07 \mathrm{Cl}$ & OUTL & 0810 & OUTPUT & 0460 & OVER & $366 \mathrm{D}$ & Open \\
\hline 0B57 & $\mathrm{PAD}$ & $40 \mathrm{E} 3$ & PAGE & $19 \mathrm{C} 5$ & PARPRINTER & $40 B C$ & PARPTR \\
\hline $3 \mathrm{C} 8 \mathrm{~A}$ & PDOTQ.DSP & OBEI & $\mathrm{PFA}$ & 0798 & PREV & 1969 & PRINTER \\
\hline OD9D & QUERY & 11B3 & QUIT & $3 \mathrm{C} 6 \mathrm{~B}$ & QUIT.FLAG & $1 C 97$ & QUOTE \\
\hline $077 \mathrm{~B}$ & R\# & 1736 & $\tilde{R} / W$ & $06 A 5$ & R0 & 0331 & $\tilde{R}>$ \\
\hline $28 \mathrm{BB}$ & RCVBRK & $28 \mathrm{E} 2$ & RCVC & 2905 & RCVCHAR? & $2 \mathrm{CFA}$ & RE-ENTER \\
\hline 1442 & REPEAT & $216 \mathrm{~B}$ & RETQ & $1 \mathrm{E} 2 \mathrm{E}$ & ROMIT & $04 \mathrm{~F} 8$ & ROT \\
\hline $02 A 5$ & $\mathrm{RP} !$ & 2926 & RSTBRK & $15 \mathrm{D} 7$ & $S->D$ & $069 C$ & S0 \\
\hline 3 B35 & SAVE & 0713 & SCR & 07B3 & SCR/DSK & $32 \mathrm{~B} 7$ & SCREEN-DUMP \\
\hline 1CFA & SEC & 3552 & SECTOR & $3 B 78$ & SEND & $2 \mathrm{E} 2 \mathrm{~F}$ & SEND-SCREEN \\
\hline 2945 & SENDBRK & 29B3 & SENDC & 29D1 & SENDCHAR? & $30 A 9$ & SERPRINTER \\
\hline 2160 & SETQ & $14 \mathrm{EC}$ & SIGN & $\mathrm{OCCl}$ & SMUDGE & 0291 & SP! \\
\hline 0280 & SPE & $095 B$ & SPACE & $14 \mathrm{~A} 5$ & SPACES & $34 \mathrm{CC}$ & SPREADSCREEN \\
\hline 0746 & STATE & $282 \mathrm{D}$ & STATUS & 0482 & SWAP & 2553 & $\mathrm{~T}$ \\
\hline 4180 & TASK & 4100 & TEAR & 2871 & TERM & $281 \mathrm{E}$ & TERM.CTL \\
\hline $07 \mathrm{E} 6$ & TERM.GRP & $220 \mathrm{E}$ & TEXT & $13 \mathrm{Cl}$ & THEN & $06 \mathrm{AE}$ & TIB \\
\hline $04 \mathrm{~A} 7$ & TOGGLE & $2 \mathrm{BA} 1$ & TONE & OB91 & TRAVERSE & $298 \mathrm{~F}$ & TRC \\
\hline $2 B 54$ & TS & $092 \mathrm{C}$ & T'YPE & $2 A 3 B$ & $\mathrm{U}$ & 0235 & $U^{*}$ \\
\hline $15 \mathrm{~A} 2$ & U. & $303 \mathrm{~A}$ & U. . & $1 \mathrm{~A} 16$ & $\mathrm{U} \cdot \mathrm{R}$ & IB3C & $\mathrm{U} \cdot \mathrm{RLZ}$ \\
\hline $01 F F$ & $\mathrm{U} /$ & $29 F 3$ & UART & $140 \mathrm{~A}$ & UNTIL & 1650 & UPDATE \\
\hline $078 \mathrm{E}$ & USE & $106 \mathrm{~A}$ & USER & $07 A 3$ & USER-IOCB & $3 \mathrm{D} 77$ & USERV.DSP \\
\hline $318 \mathrm{C}$ & $\mathrm{V}$ & $3 \mathrm{DB} 6$ & VAR.DSP & 1055 & VARIABLE & 3343 & VCOPY \\
\hline $1 \mathrm{EFE}$ & VLIST & $332 B$ & VMOVE & $06 \mathrm{E} 7$ & VOC-LINK & $3 E 06$ & VOCAB.DSP \\
\hline $32 \mathrm{Dl}$ & VOCAB? & 1164 & VOCABULARY & 3385 & VSHADOW & 2124 & WI \\
\hline $212 \mathrm{~B}$ & W2 & 2132 & W3 & 2139 & W4 & $002 \mathrm{D}$ & WARM \\
\hline $06 C 4$ & WARNING & 2261 & WHERE & 1495 & WHILE & $06 B 8$ & WIDTH \\
\hline 2140 & WNI & 2148 & WN2 & 2150 & WN3 & 2158 & WN4 \\
\hline$O D F 3$ & WORD & $1 C 45$ & WORD-79 & $3 \mathrm{CB} 6$ & WORD.DSP & $3 \mathrm{C} 7 \mathrm{~B}$ & WORD.PTR \\
\hline $2 F F 7$ & XMIT & $026 E$ & XOR & $2 \mathrm{FBE}$ & Xmit & IED0 & $Y ?$ \\
\hline $1 A F 1$ & ZEROES & $0 C A 3$ & {[} & $127 \mathrm{~A}$ & [COMPILE] & OCBI & ] \\
\hline $192 \mathrm{~A}$ & parptr & $194 \mathrm{~A}$ & serptr & $30 \mathrm{FA}$ & $\mathrm{v}$ & & \\
\hline
\end{tabular}

Woods Hole FORTH Version 1.800 Chapter 14

page 48 


\section{USER Variables}

This chapter contains a list of all USER variables, including some used for documentation. The offset added to the beginning of the USER variable area (normally 5700h) gives the location where the value of the variable is actually stored. This table was generated by the word SHOW.USER, located on screen 228. Because the compiled code for this word is fairly long, it is not included in WFORTH.CM, however, it is an extremely useful word when developing programs. Therefore the user may find it helpful to compile this word during program development and discard it when the application is working properly. 
USER variables

\begin{tabular}{|c|c|c|c|c|c|c|}
\hline Name & Type & \multicolumn{2}{|c|}{ Offset } & \multicolumn{2}{|c|}{ Value } & \\
\hline WORD.PTR & USER & 96 & $(0060 \mathrm{~h})$, & Currently & $=15494$ & $(3 \mathrm{C} 86 \mathrm{~h})$ \\
\hline QUIT.FLAG & USER & 94 & (005Eh), & Currently & $=1$ & $(0001 \mathrm{~h})$ \\
\hline IOCB & USER & 84 & $(0054 h)$, & Currently & 502 & (01F6h) \\
\hline CUR & USER & 82 & $(0052 h)$, & Currently & 255 & (OOFFh) \\
\hline CHAPTER & USER & 80 & $(0050 h)$, & Currently & 15 & (000Fh) \\
\hline Nextu & USER & 30 & (001Eh), & Currently & 106 & (006Ah) \\
\hline COMM.GRP & USER & 28 & (001Ch), & Currently & 224 & (OOEOh) \\
\hline TERM.GRP & USER & 26 & (001Ah), & Currently & $=$ & $(0001 \mathrm{~h})$ \\
\hline MODEM.GRP & USER & 24 & $(0018 \mathrm{~h})$, & Currently & 240 & (OOFOh) \\
\hline GRP & USER & 68 & $(0044 h)$, & Currently & $=$ & $(000 \mathrm{lh})$ \\
\hline OUTL & USER & 66 & $(0042 \mathrm{~h})$, & Currently & 15 & (OOOFh) \\
\hline SCR/DSK & USER & 22 & (0016h), & Currently & 315 & (013Bh) \\
\hline USER-IOCB & USER & 54 & $(0036 h)$, & Currently & 256 & $(0100 \mathrm{~h})$ \\
\hline PREV & USER & 52 & $(0034 \mathrm{~h})$, & Currently & $=23515$ & (5BDBh) \\
\hline USE & USER & 50 & $(0032 \mathrm{~h})$, & Currently & $=24543$ & (5FDFh) \\
\hline HLD & USER & 48 & (0030h), & Currently & $=19498$ & (4C2Ah) \\
\hline R\# & USER & 46 & (002Eh), & Currently & $=$ & $(0000 \mathrm{~h})$ \\
\hline CSP & USER & 44 & $(002 \mathrm{Ch})$, & Currently & 22008 & (55F8h) \\
\hline FLD & USER & 42 & (002Ah), & Currently & 50 & $(0032 \mathrm{~h})$ \\
\hline DPL & USER & 40 & $(0028 \mathrm{~h})$, & Currently & -1 & (FFFFh) \\
\hline BASE & USER & 38 & $(0026 \mathrm{~h})$, & Currently & 16 & (0010h) \\
\hline STATE & USER & 36 & $(0024 \mathrm{~h})$, & Currently & $=$ & $(0000 h)$ \\
\hline CURRENT & USER & 34 & $(0022 \mathrm{~h})$, & Currently & 6205 & (183Dh) \\
\hline CONTEXT & USER & 32 & (0020h), & Currently & $=16796$ & (419Ch) \\
\hline OFFSET & USER & 78 & (004Eh), & Currently & 315 & (013Bh) \\
\hline $\mathrm{SCR}$ & USER & 76 & $(004 \mathrm{Ch})$, & Currently & 247 & (00F7h) \\
\hline OUT & USER & 74 & $(004 \mathrm{Ah})$, & Currently & 45 & (002Dh) \\
\hline IN & USER & 72 & $(0048 \mathrm{~h})$, & Currently & 658 & (0292h) \\
\hline BLK & USER & 64 & $(0040 \mathrm{~h})$, & Currently & $=73$ & $(0049 \mathrm{~h})$ \\
\hline VOC-LINK & USER & 20 & $(0014 \mathrm{~h})$, & Currently & $=16798$ & (419Eh) \\
\hline DP & USER & 18 & $(0012 \mathrm{~h})$, & Currently & $=19432$ & (4BE8h) \\
\hline FENCE & USER & 16 & $(0010 \mathrm{~h})$, & Currently & $=16779$ & (418Bh) \\
\hline WARNING & USER & 14 & (000Eh), & Currently & $=0$ & $(0000 \mathrm{~h})$ \\
\hline WIDTH & USER & 12 & $(000 \mathrm{Ch})$, & Currently & 31 & (001Fh) \\
\hline TIB & USER & 10 & $(000 \mathrm{Ah})$, & Currently & $=22016$ & (5600h) \\
\hline R0 & USER & 8 & $(0008 \mathrm{~h})$, & Currently & $=22270$ & (56FEh) \\
\hline S0 & USER & 6 & $(0006 \mathrm{~h})$, & Currently & $=22014$ & (55FEh) \\
\hline
\end{tabular}

Woods Hole FORTH Version $1.800 \quad$ Chapter 15

page 50 
CONSTANTS, VARIABIES, and VOCABULARIES

This chapter contains a list of the CONSTANTS, VARIABLES, and VOCABUIARIES. These lists were generated by the words SHOW.CONST, SHOW.VAR , and SHOW.VOCAB respectively. Because the compiled code for these words if quite lengthy, they are not compiled into WFORTH, but the sources for these words are located on screens 228 and 229. Note that very few VARIABLES are used in WFORTH. This is because VARIABIES will not work in an Eprom system, a primary application of Woods Hole FORTH. 
CONSTANTS

\begin{tabular}{|c|c|c|c|c|c|}
\hline Name & Type & & & Value & \\
\hline EOF & CONSTANT & - Currently & $=$ & 19 & $(0013 \mathrm{~h})$ \\
\hline ESC & CONSTANT & , Currently & $=$ & 27 & (001Bh) \\
\hline ORIGIN & CONSTANT & - Currently & $=$ & 6209 & (1841h) \\
\hline $\mathrm{B} / \mathrm{SCR}$ & CONSTANT & - Currently & $=$ & 1 & $(0001 \mathrm{~h})$ \\
\hline $\mathrm{B} / \mathrm{BUF}$ & CONSTANT & - Currently & $=$ & 1024 & $(0400 \mathrm{~h})$ \\
\hline LIMIT & CONSTANT & - Currently & $=$ & 32767 & (7FFFh) \\
\hline FIRST & CONSTANT & , Currently & $=$ & 22487 & (57D7h) \\
\hline $\mathrm{L} / \mathrm{P}$ & CONSTANT & - Currently & $=$ & 60 & (003Ch) \\
\hline $\mathrm{C} / \mathrm{I}$ & CONSTANT & , Currently & $=$ & 64 & $(0040 h)$ \\
\hline BL & CONSTANT & , Currently & $=$ & 32 & $(0020 \mathrm{~h})$ \\
\hline 3 & CONSTANT & , Currently & $=$ & 3 & $(0003 \mathrm{~h})$ \\
\hline 2 & CONSTANT & - Currently & $=$ & 2 & $(0002 \mathrm{~h})$ \\
\hline 1 & CONSTANT & , Currently & $=$ & 1 & $(0001 \mathrm{~h})$ \\
\hline 0 & CONSTANT & , Currently & $=$ & 0 & $(0000 \mathrm{~h})$ \\
\hline EF & CONSTANT & , Currently & $=$ & 94 & (005Eh) \\
\hline
\end{tabular}

VARIABLES

$\begin{array}{lll}\text { Name } & \text { Type } & \text { Value } \\ \text { TERM.CTL } & \text { VARIABLE, Currently }= & 29(001 \mathrm{Dh}) \\ \text { COMM.CTL } & \text { VARIABLE, Currently }= & 29(001 \mathrm{Dh})\end{array}$

VOCABUI.ARIES

VOCABULARY DOCUMENT

VOCABUIAARY EDITOR

VOCABULARY FORTH 


\section{Screen Listings}

This chapter contains listings for all screens used in creating WFORTH.CM, FFORTH.CM, and AFORTH.CM. It is sometimes helpful to examine the source for a word when trying to understand its behavior. In addition, the screens frequent Iy contain comments which provide a better explanation of the word than that in the Glossary.

This chapter was generated by the sequence:

DECIMAL PRINTER $65 \underline{249}$ DISKLIST PAGE TEAR (cr) 


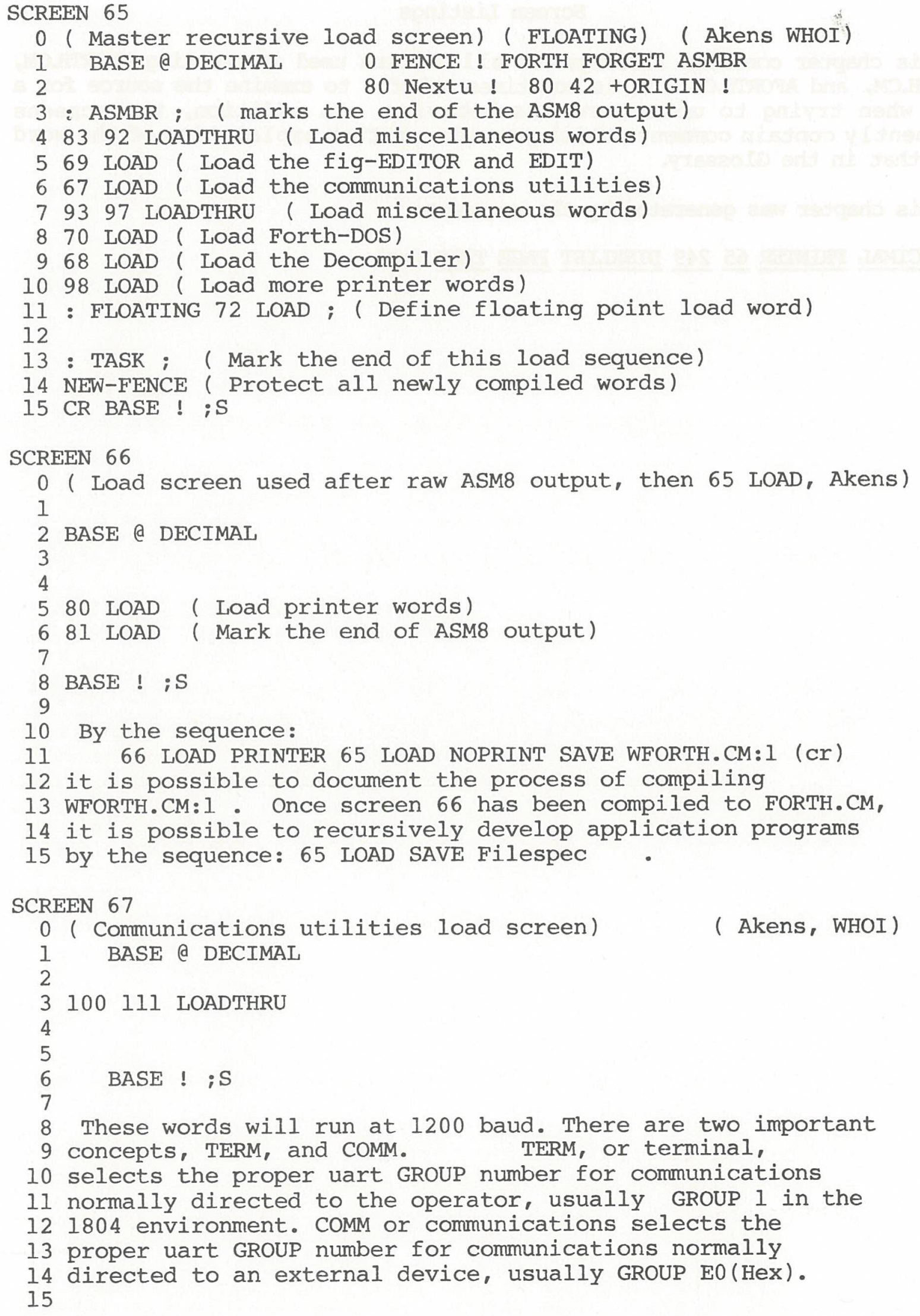




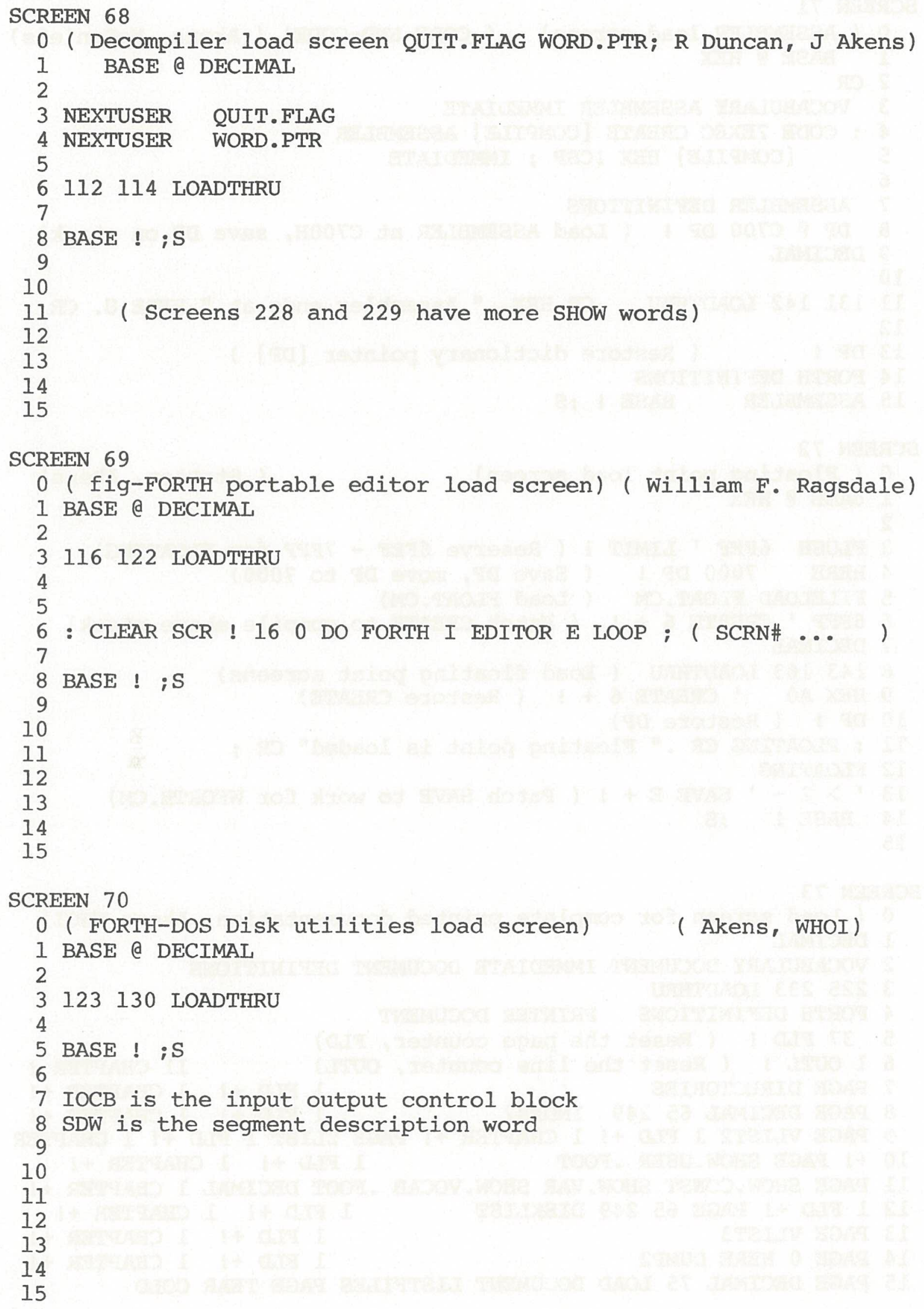


Disk ID: WOODS HOLE FORTH SCREEN FILE VER 1.801

SCREEN 71

0 (ASSEMBLER load screen) ( CODE END-CODE) (Akens, McDaniels)

1 BASE @ HEX

$2 \mathrm{CR}$

3 VOCABUIARY ASSEMBLER IMMEDIATE

4 : CODE ?EXEC CREATE [COMPILE] ASSEMBLER

5 [COMPILE] HEX !CSP ; IMMEDIATE

6

7 ASSEMBLER DEFINITIONS

8 DP a C700 DP ! ( Load ASSEMBLER at C700H, save DP on stack) 9 DECIMAI 10

11131142 LOADTHRU CR HEX ." Assembler ends at " HERE U. CR 12

13 DP ! (Restore dictionary pointer [DP] )

14 FORTH DEFINITIONS

15 ASSEMBLER BASE ! ;S

SCREEN 72

0 (Floating point load screen) (Stetten, Akens)

1 BASE a HEX

2

3 FLUSH 6FEF ' LIMIT ! ( Reserve 6FEF - 7FFF for FLOATING)

4 HERE 7000 DP ! ( Save DP, move DP to 7000)

5 FILELOAD FLOAT.CM (LOad FLOAT.CM)

6 6FFF ' CREATE $6+$ ! ( Patch CREATE to compile above stack)

7 DECIMAL

8143163 LOADTHRU ( Load floating point screens)

9 HEX A0 ' CREATE 6 + ! (Restore CREATE)

$10 \mathrm{DP} !$ ( Restore DP)

11 : FLOATING CR ." Floating point is loaded" $\mathrm{CR}$;

12 FLOATING

13 ' $>2$ - ' SAVE E + ! ( Patch SAVE to work for WFORTH.CM)

14 BASE ! ;S

15

SCREEN 73

0 (Load screen for complete printed documentation, Akens WHOI)

1 DECIMAL

2 VOCABUIARY DOCUMENT IMMEDIATE DOCUMENT DEFINITIONS

3225233 LOADTHRU

4 FORTH DEFINITIONS PRINTER DOCUMENT

537 FLD ! ( Reset the page counter, FLD)

61 OUTL ! (Reset the line counter, OUTL)

7 PAGE DIRECTORIES

8 PAGE DECIMAL 65249 INDEX2

11 CHAPTER !

1 FLD +! 1 CHAPTER +!

9 PAGE VLIST2 1 FLD +! 1 CHAPTER +! PAGE ZLIST 1 FLD +! 1 CHAPTER

$10+$ + PAGE SHOW.USER . FOOT 1 FLD +! 1 CHAPTER +!

11 PAGE SHOW.CONST SHOW.VAR SHOW.VOCAB .FOOT DECIMAL 1 CHAPTER +!

121 FLD +! PAGE 65249 DISKLIST 1 FLD +! 1 CHAPTER +!

13 PAGE VLIST3

14 PAGE 0 HERE DUMP2

15 PAGE DECIMAL 75 LOAD DOCUMENT LISTFILES PAGE TEAR COLD 


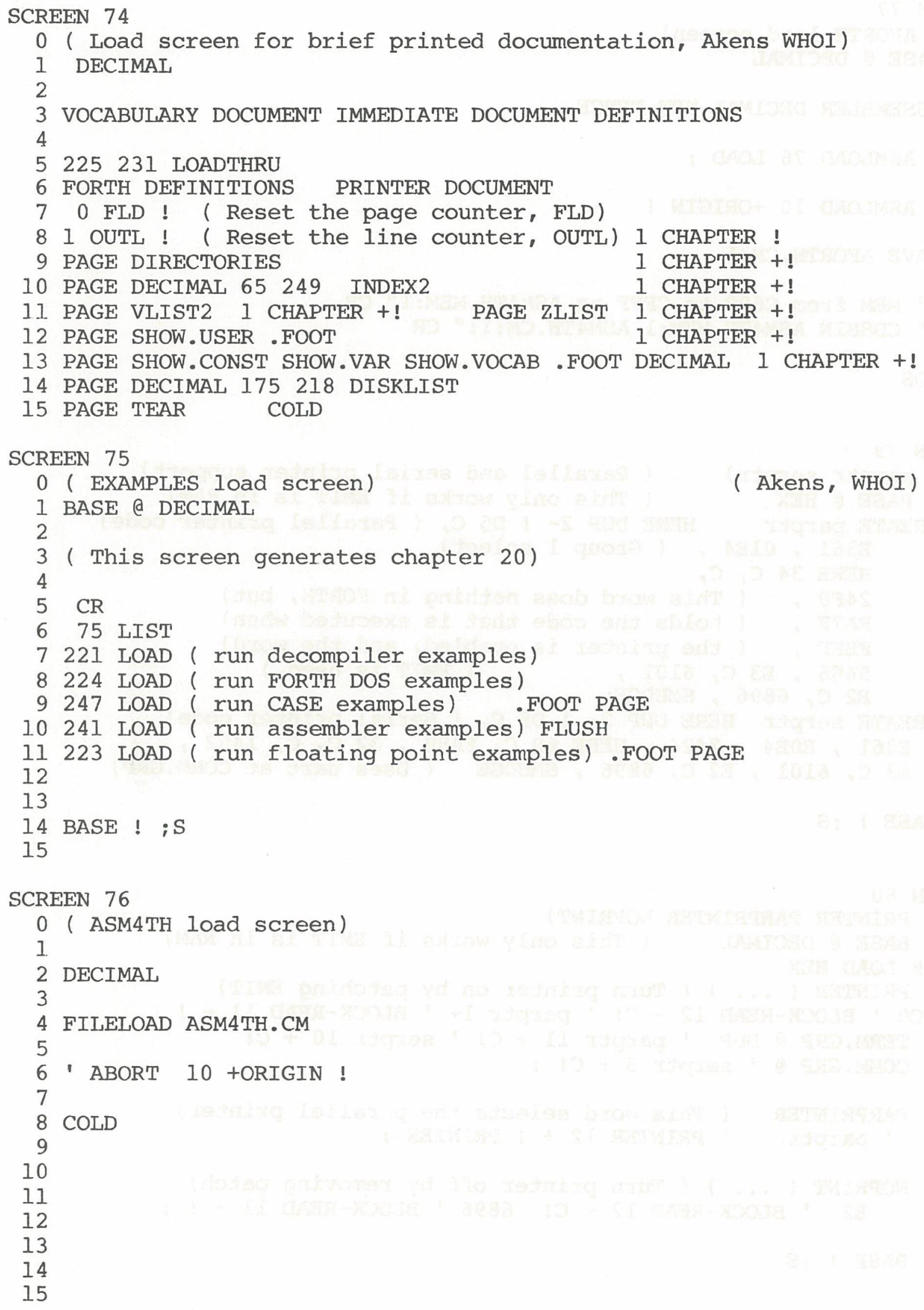




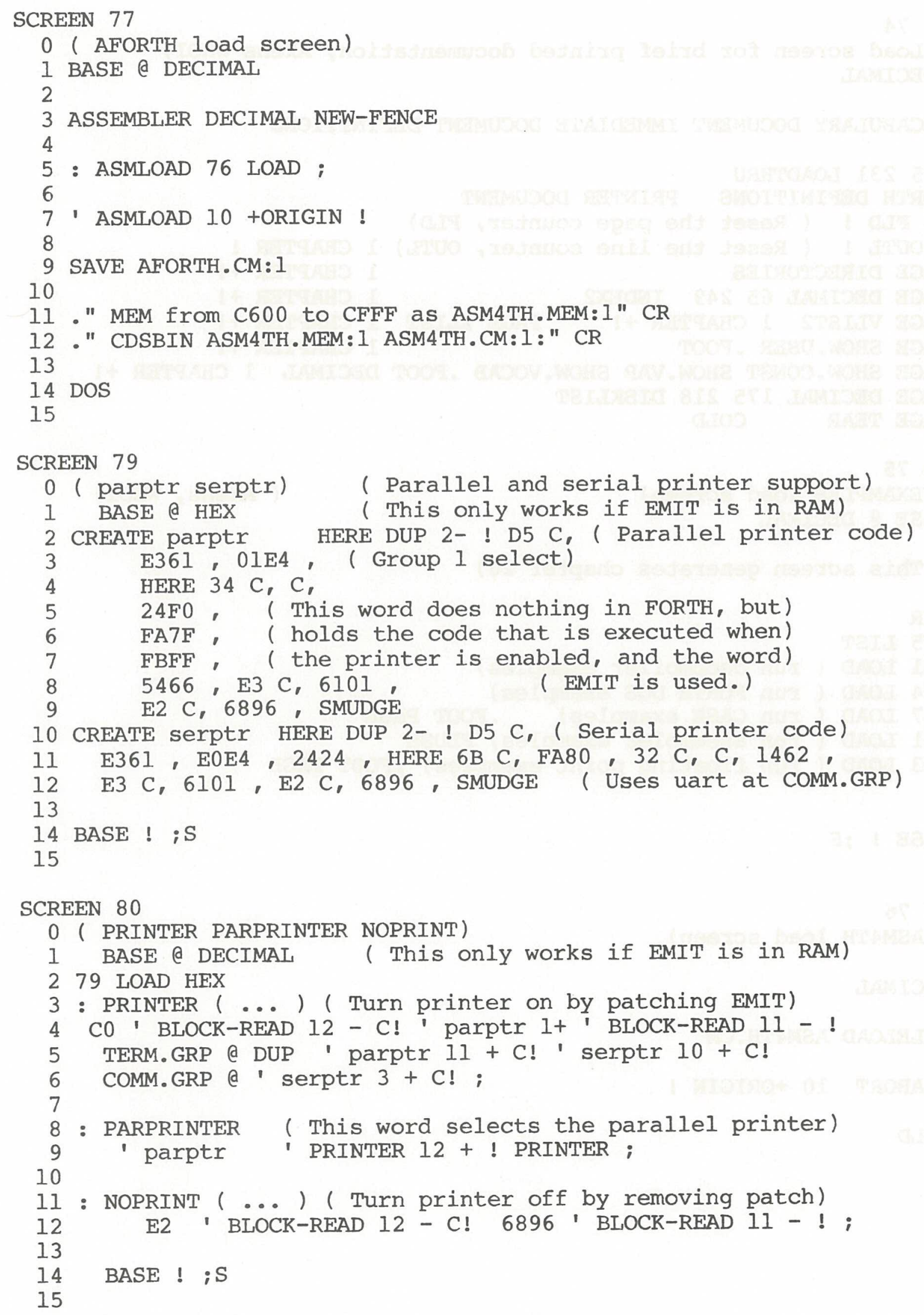




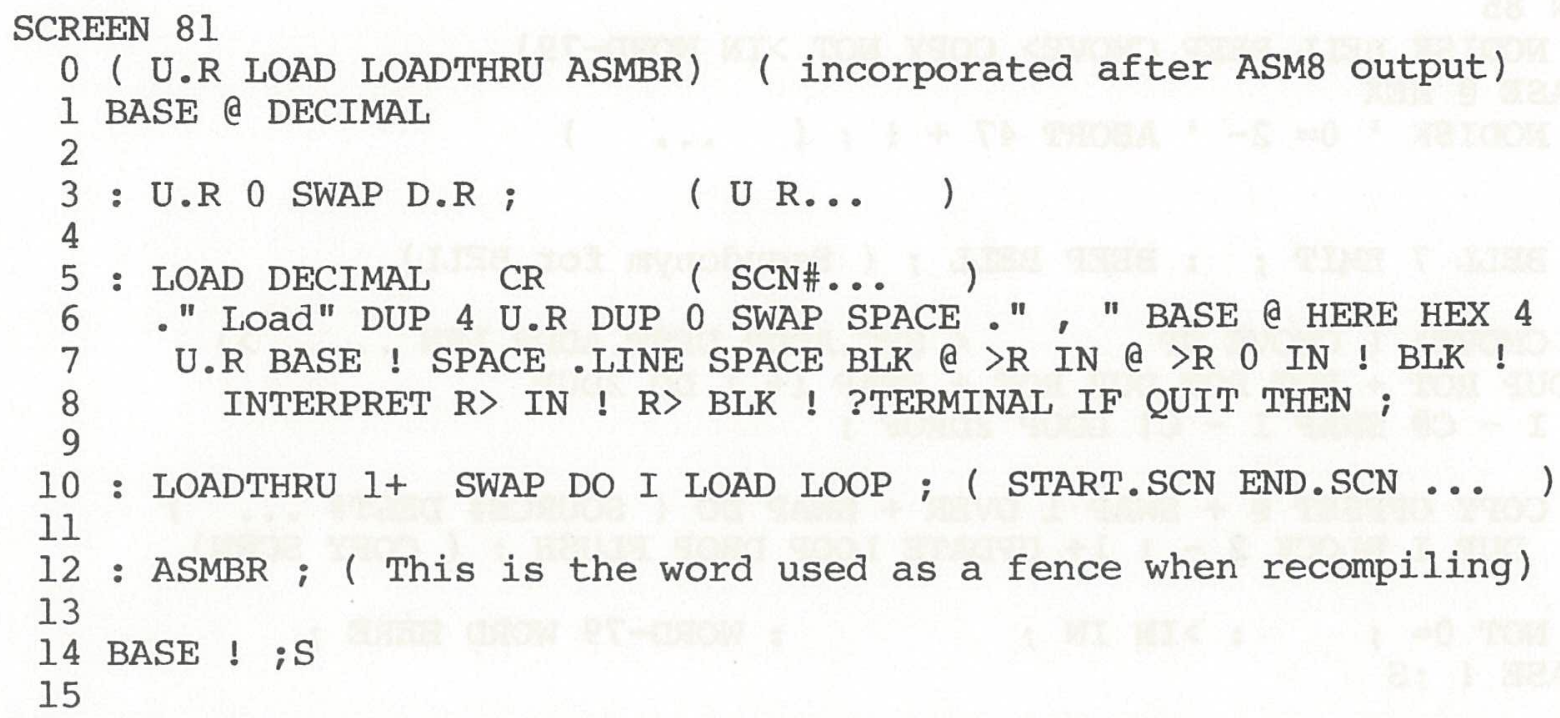




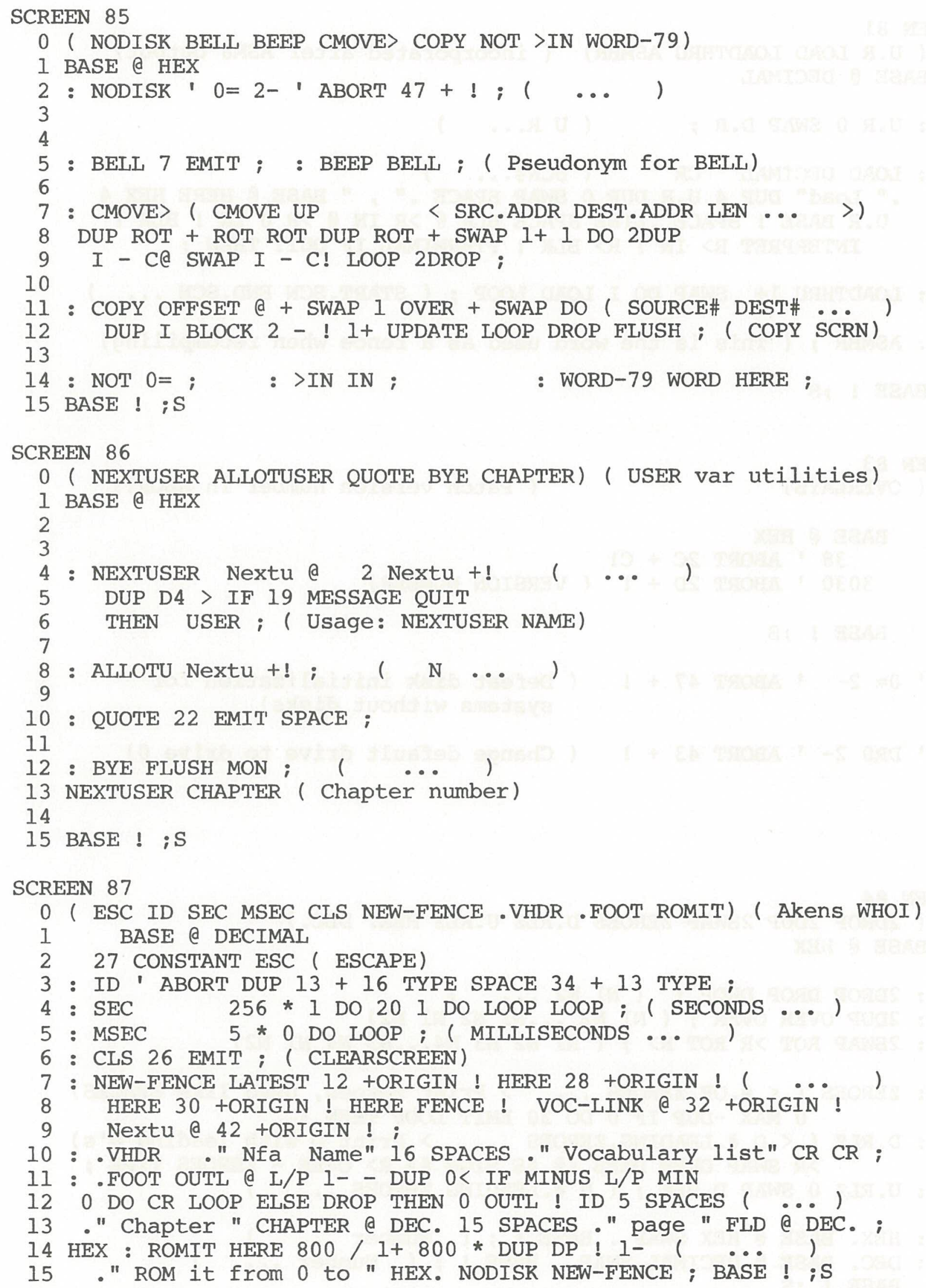


SCREEN 88

0 ( N. ?Y/N Y? VLIST)

1 BASE @ HEX

2 : N. BASE @ >R DECIMAL DUP 5 . R " ( " HEX 4 U.RLZ •" h) " R>

3 BASE ! ;

$4:$ ?Y/N KEY DUP $59=$ SWAP $79=$ OR DUP IF . "Y" ELSE . N" THEN

$5 \quad(\ldots F)$ ( If $Y F=1$; If $\langle>Y$ or $Y F=0$ print $Y$ or $N$ ) ;

6 : Y? ( ... ) (Check KEY, If $\longleftrightarrow Y$ QUIT, otherwise continue)

7 CR ." Type $Y$ to continue" ?Y/N IF ELSE OK QUIT THEN ;

8 : VLIST BASE \& HEX ( ... ) .VHDR

90 OUT ! CONTEXT \& a BEGIN DUP DUP OUT \& $3 \mathrm{C}>$ IF CR 0 OUT !

10 THEN $0<\# \#$ \#\#\# \#> TYPE DUP $1+$ C@ $80-$

11 IF SPACE ID. ELSE DROP THEN

1214 OUT @ OVER MOD DUP IF ELSE DROP 14 THEN - SPACES

13 PFA LFA \& DUP $0=$ ?TERMINAL OR UNTIL DROP CR ID BASE ! ;

BASE ! ; S

SCREEN 89

0 ( Match MATCH)

1 BASE @ DECIMAL

2

3 : Match

5 DO DUP C@ FORTH I C@ -

6 IF $0=$ LEAVE ELSE I+ THEN LOOP ELSE DROP $0=$ THEN ;

7

8 : MATCH ( CUR.ADDR \#BYTES STRING.ADDR STRING.CNT … F CUR.MOV)

$9 \quad>R>R$ 2DUP R>R R 2SWAP OVER + SWAP

10 DO 2DUP FORTH I SWAP Match

11 IF $>$ R 2DROP R> - FORTH I SWAP - 0 SWAP 00 LEAVE

12

13

14 BASE ! ;S

15

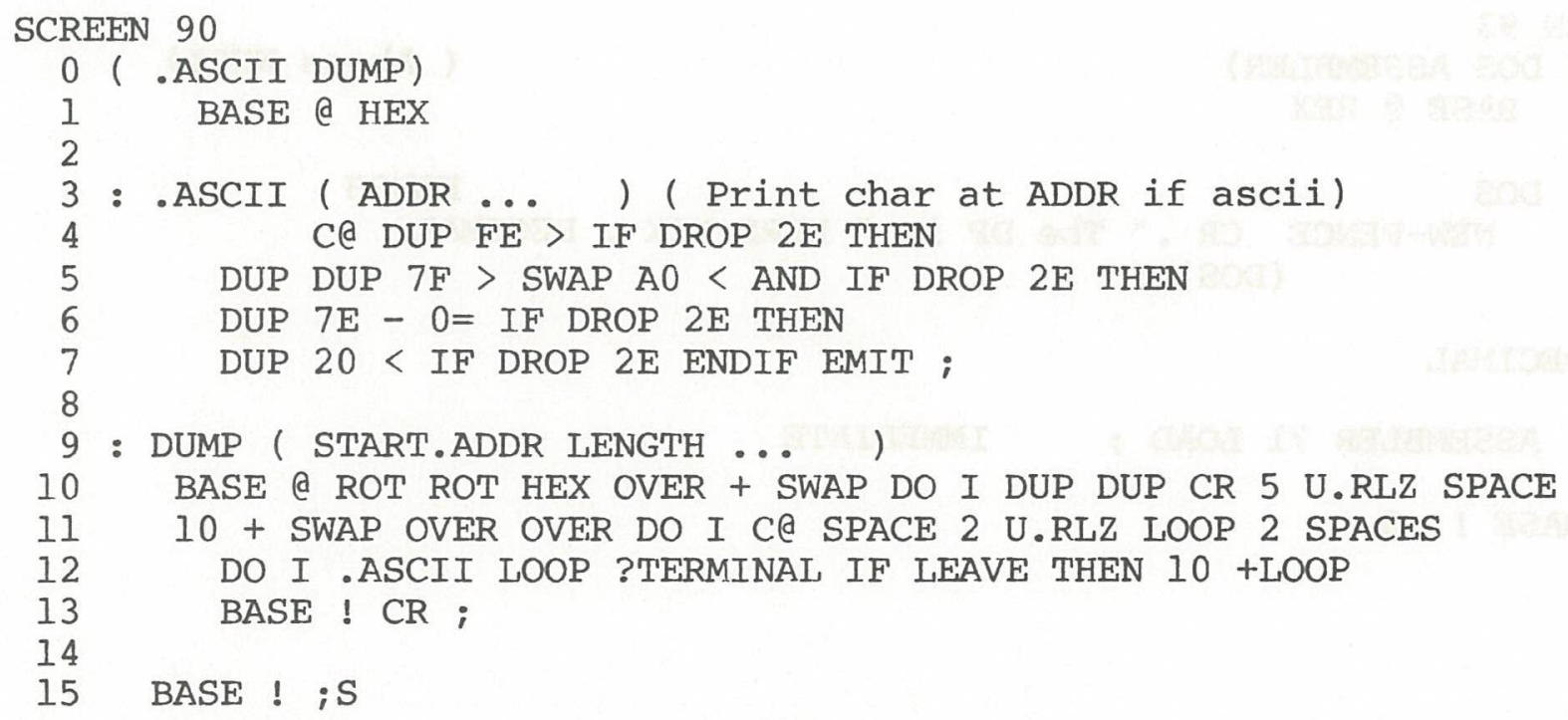




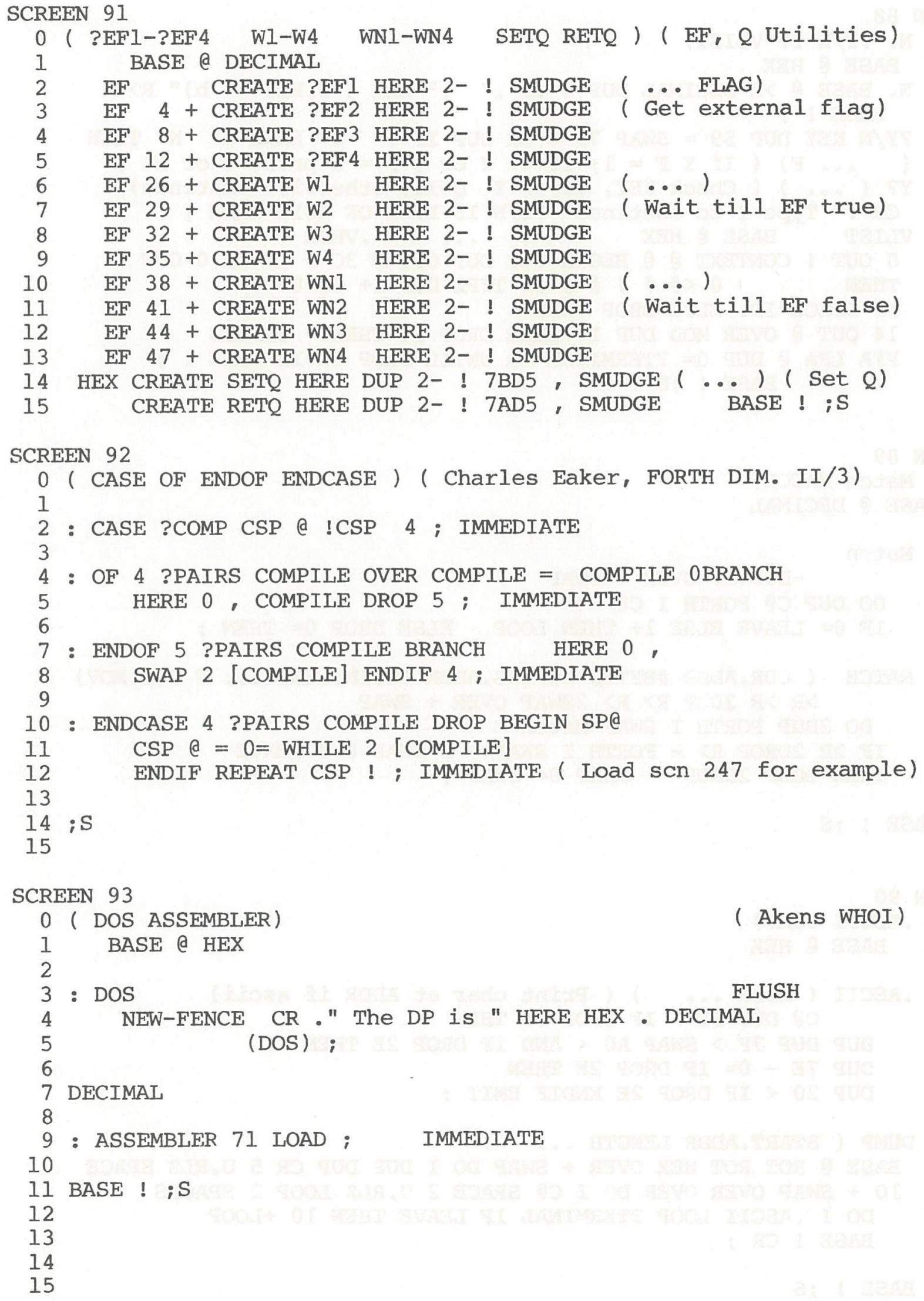




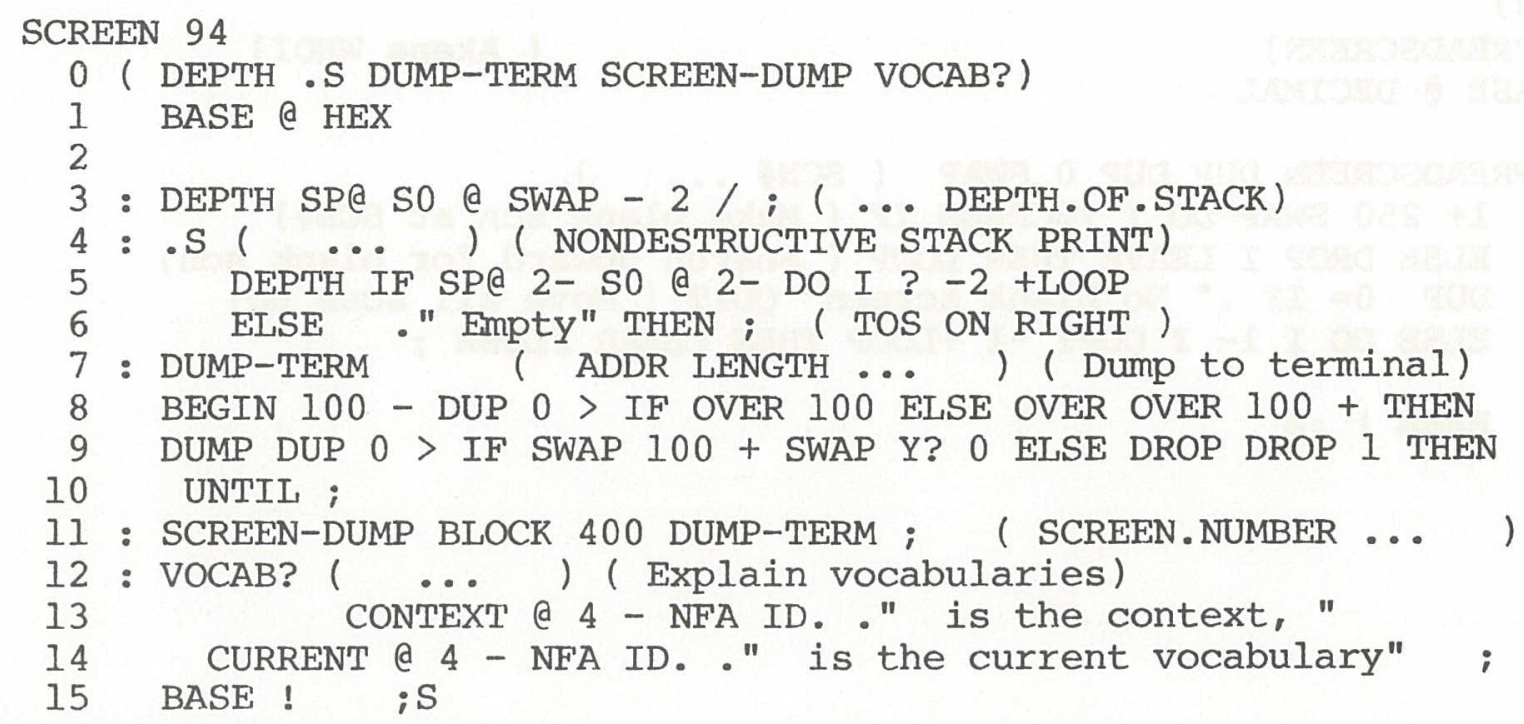




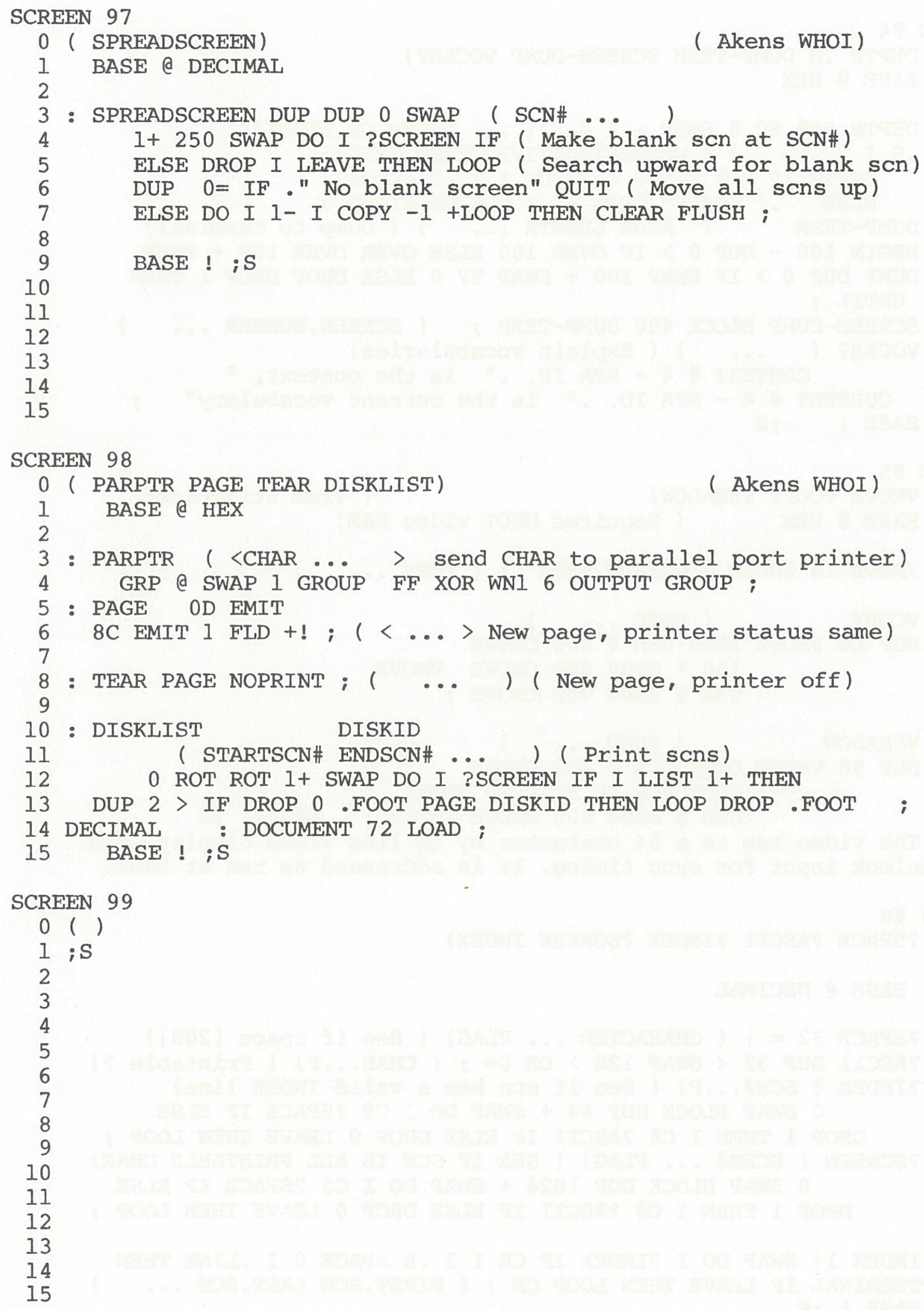




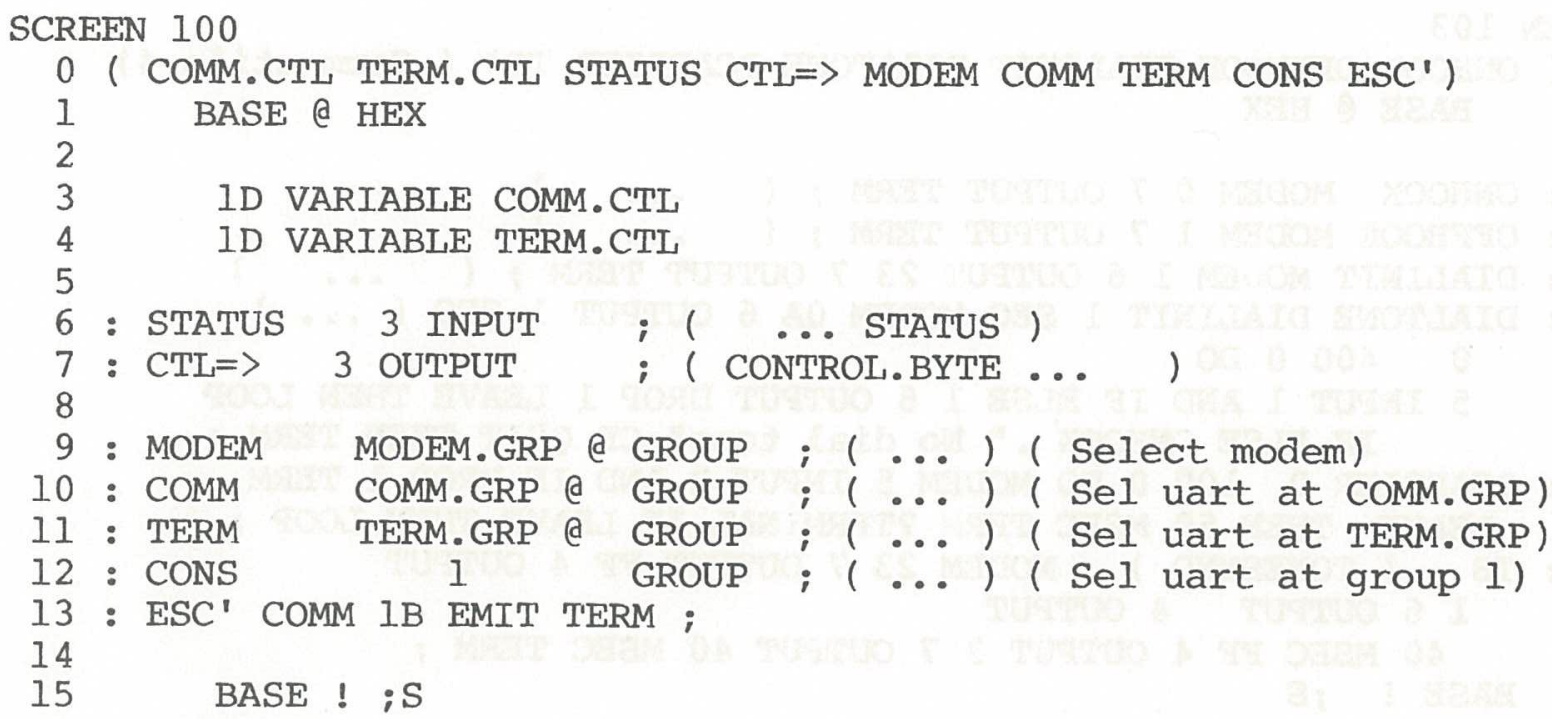




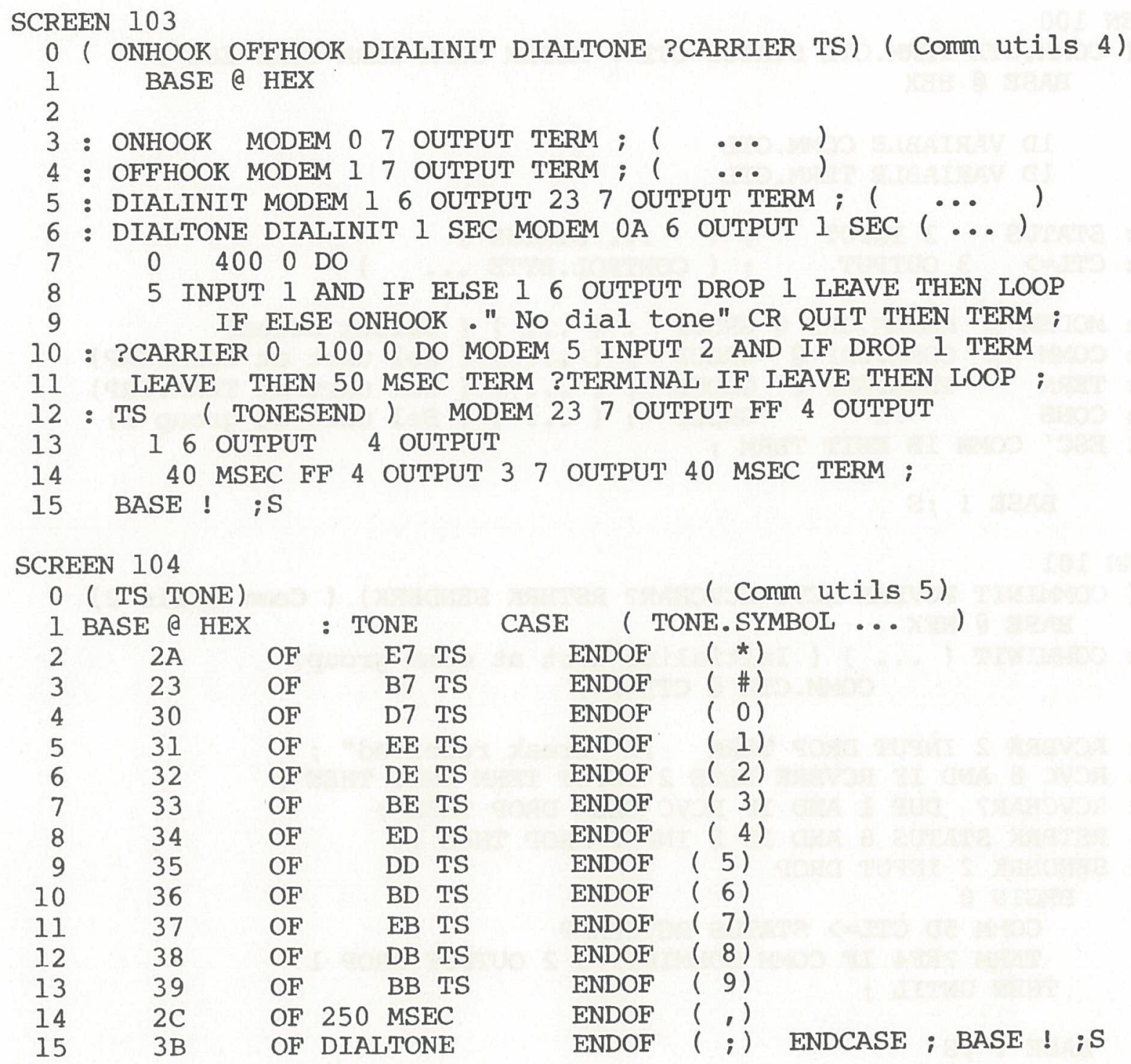

SCREEN 105

0 ( RE-ENTER DIAL ORIGINATE) (Comm utils 6)

1 BASE @ HEX

2

3 : RE-ENTER MODEM.GRP @ COMM.GRP ! UART •" RE-ENTER ? "

4 ?Y/N IF ." Type RE-ENTER when ready" CR ELSE ONHOOK

5 EO COMM.GRP ! THEN ;

6

7 : DIAL ONHOOK 1 SEC

8 DIALTONE 20 WORD HERE CQ I+ 1 DO HERE I + C@ TONE LOOP ; 9

10 : ORIGINATE MODEM 7F 4 OUTPUT 16 OUTPUT 17 OUTPUT

115 SEC ?CARRIER TERM

12 IF ELSE ONHOOK ." NO carrier" QUIT THEN RE-ENTER ;

13

14 BASE ! iS

15

Woods Hole FORTH Version 1.800 


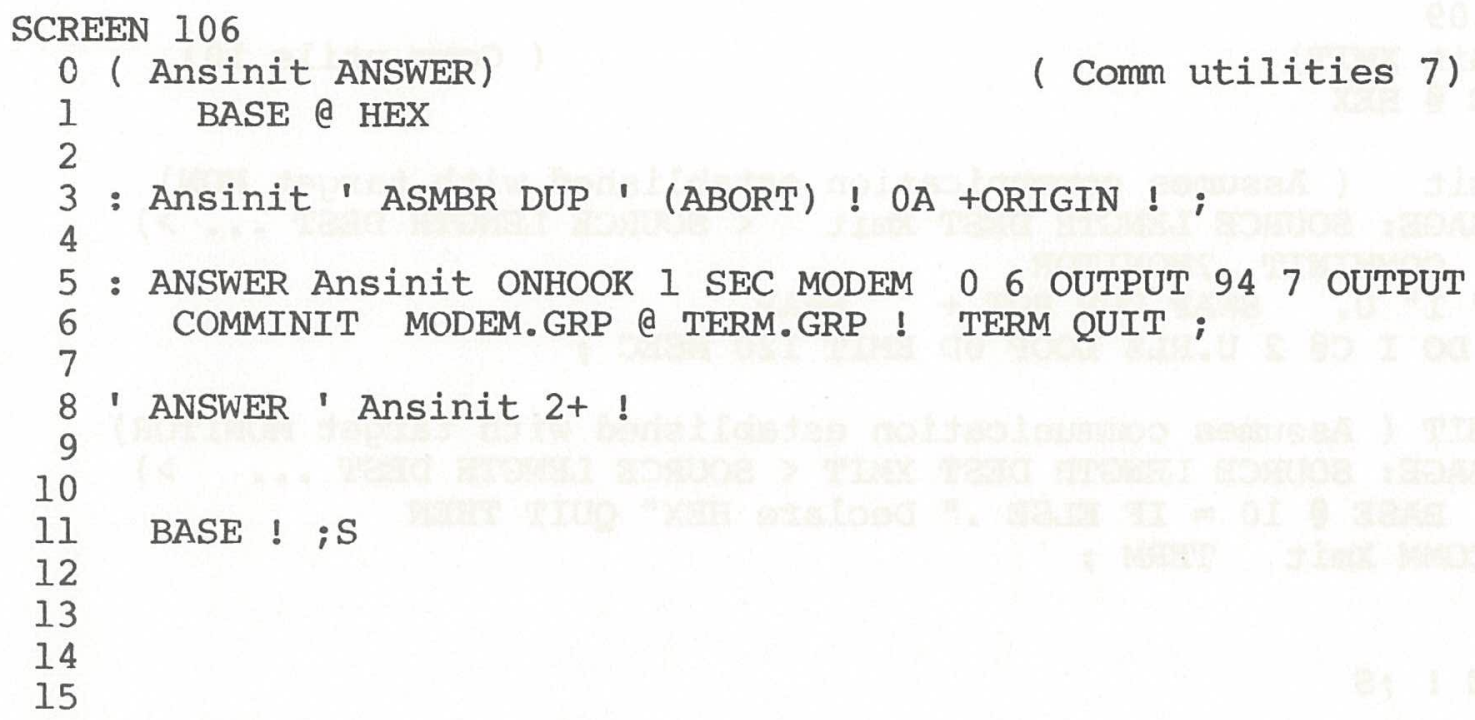




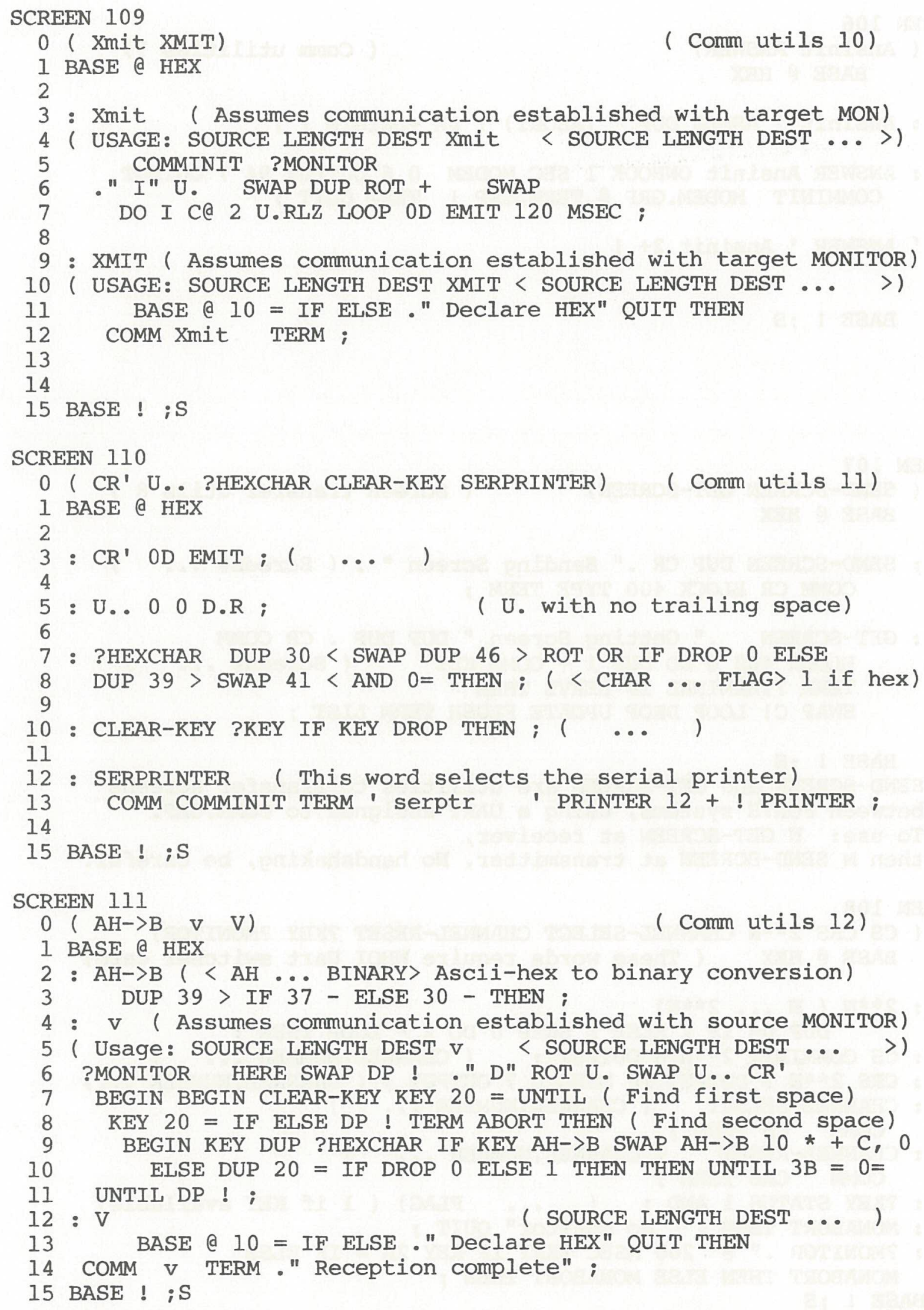




\section{SCREEN 112}

0 ( PDOTQ.DSP WORD.DSP BRANCH.DSP CV.DSP CONST.DSP) (Decompiler)

I BASE @ DECIMAI

2 : PDOTQ.DSP WORD.PTR @ 2+ DUP >R DUP CE + 1- WORD.PTR !

$3 \quad \mathrm{R}>\mathrm{COUNT}$ TYPE ;

5 : WORD.DSP $3--1$ TRAVERSE DUP $1+$ CQ $59=$ IF 1 QUIT.FLAG

6 ! THEN DUP C@ 160 AND 128 = IF ID. ELSE

DROP THEN ;

: BRANCH.DSP ." TO " WORD.PTR @ 2+ DUP WORD.PTR

10 ! a 0 HEX D. DECIMAL ;

11 : CV.DSP ." , Currently = " ;

12

13 : CONST.DSP ' CONSTANT NFA ID. CV.DSP WORD.PTR @

$142+$ a N. I QUIT.FLAG ! ;

15 BASE ! ; S

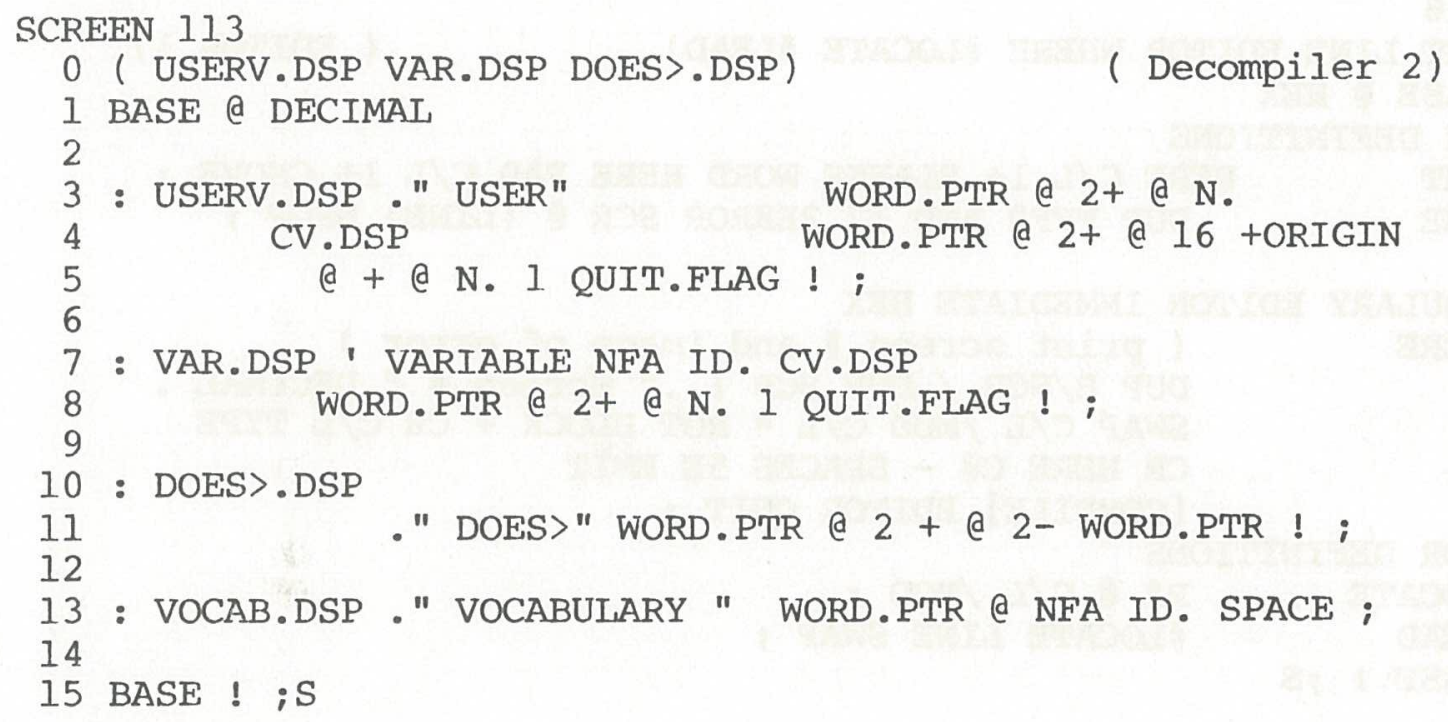




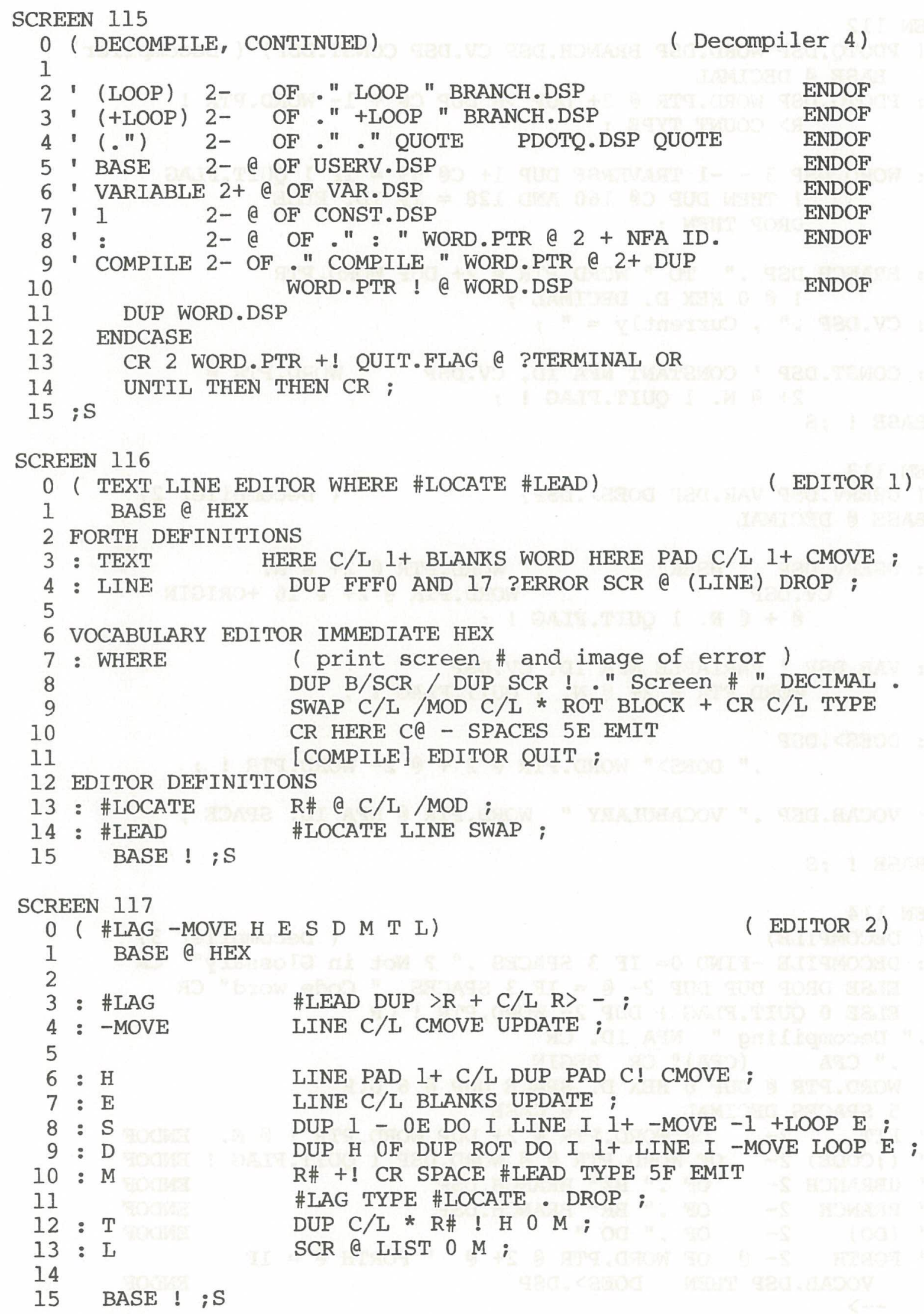




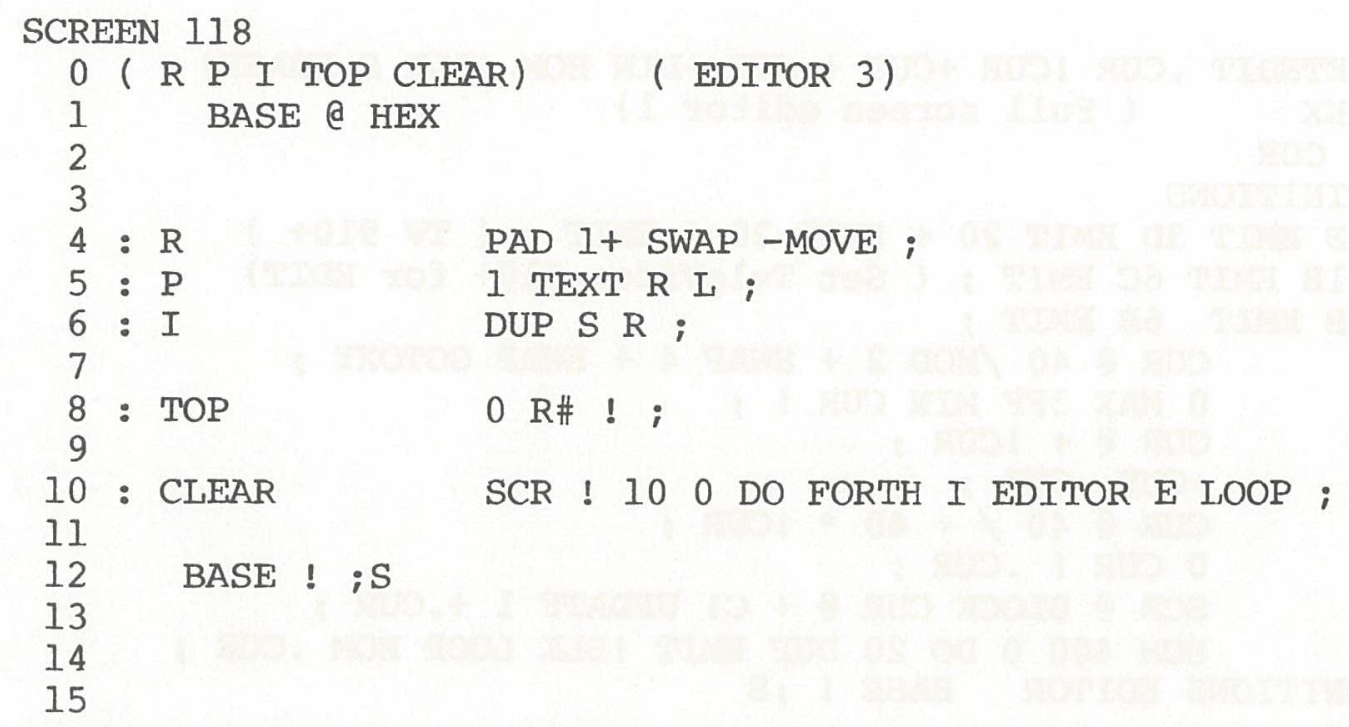

SCREEN 120

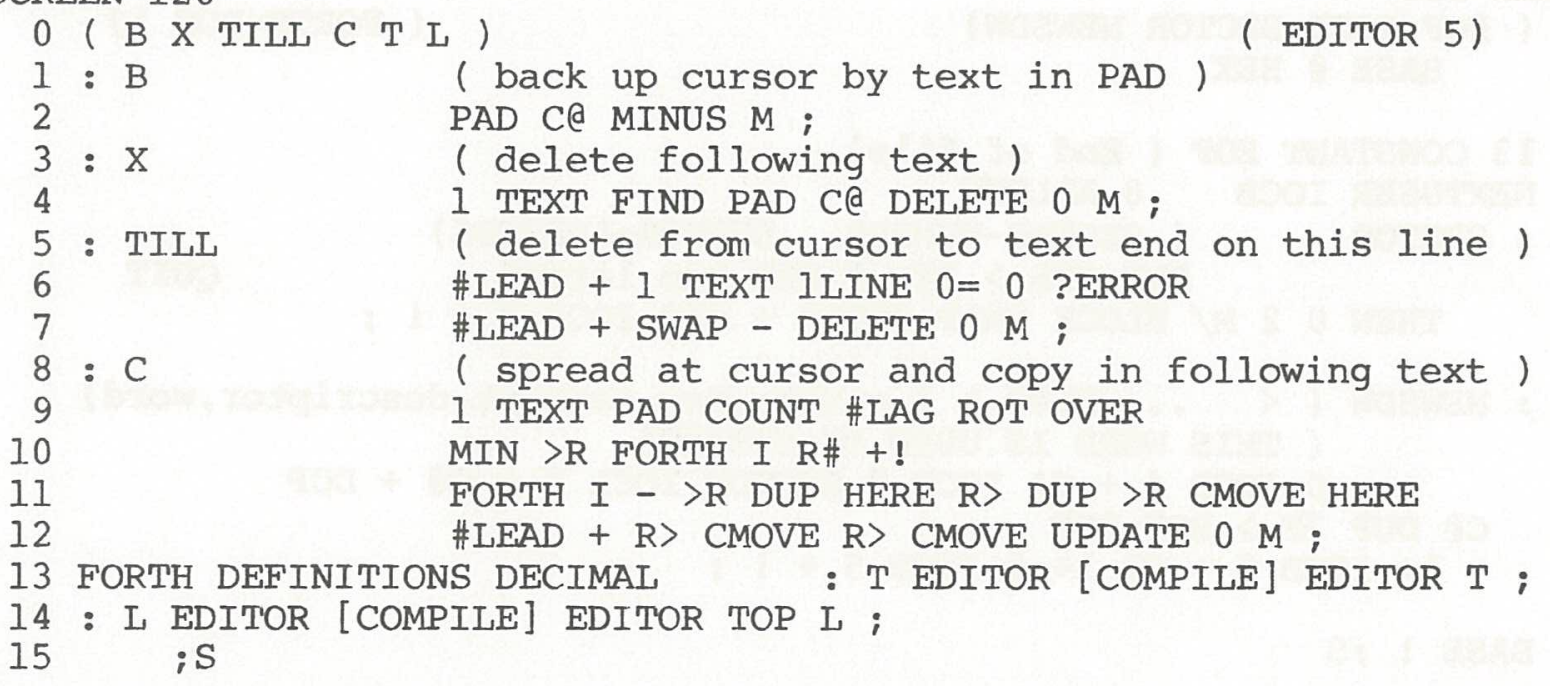




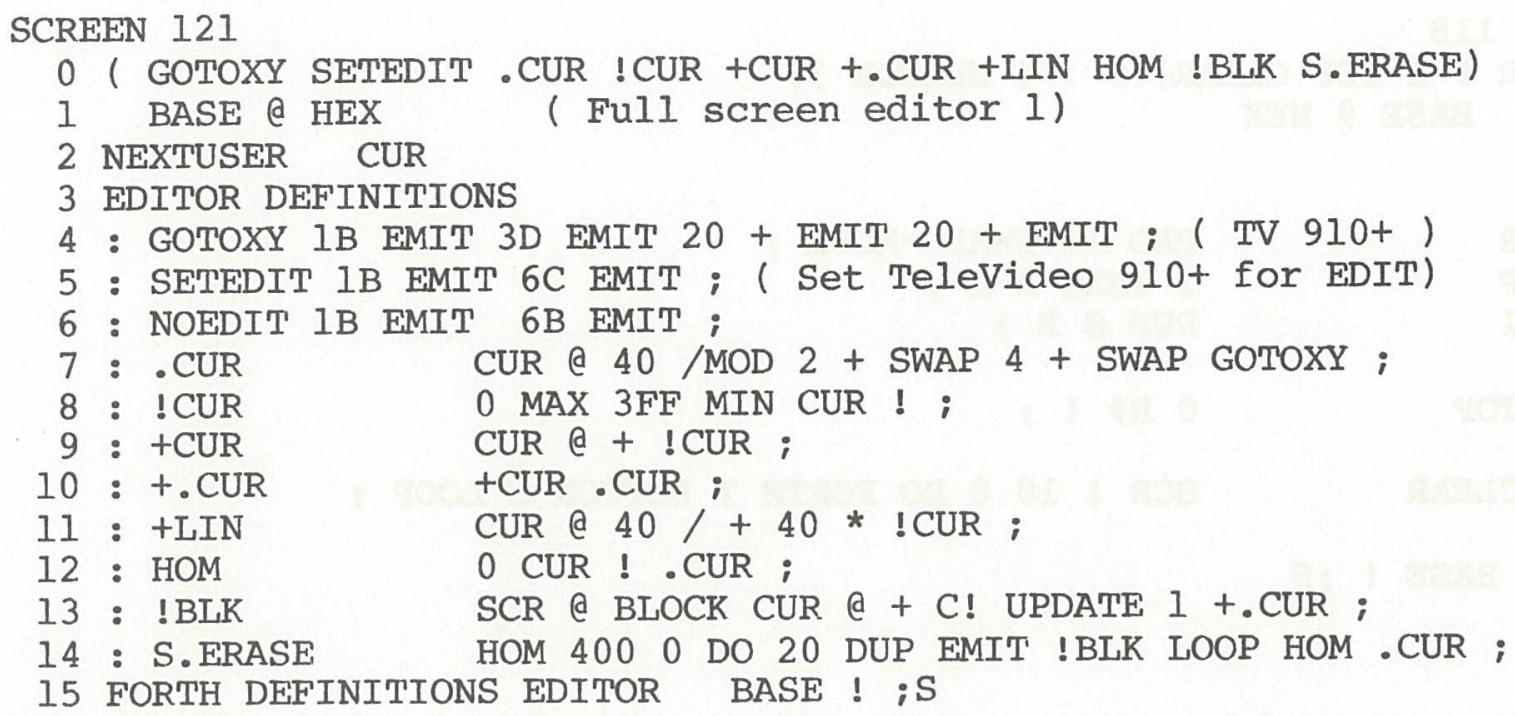




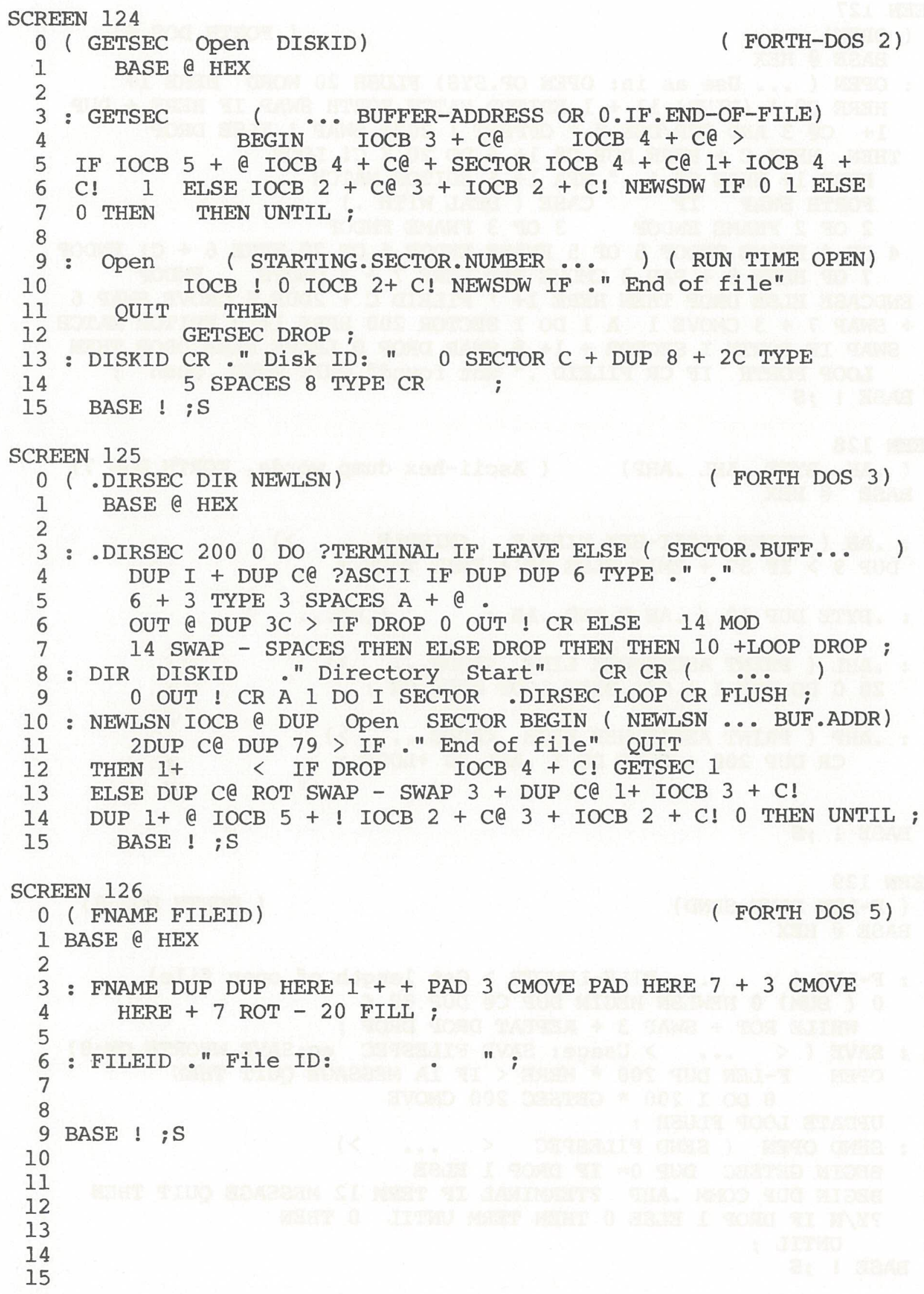




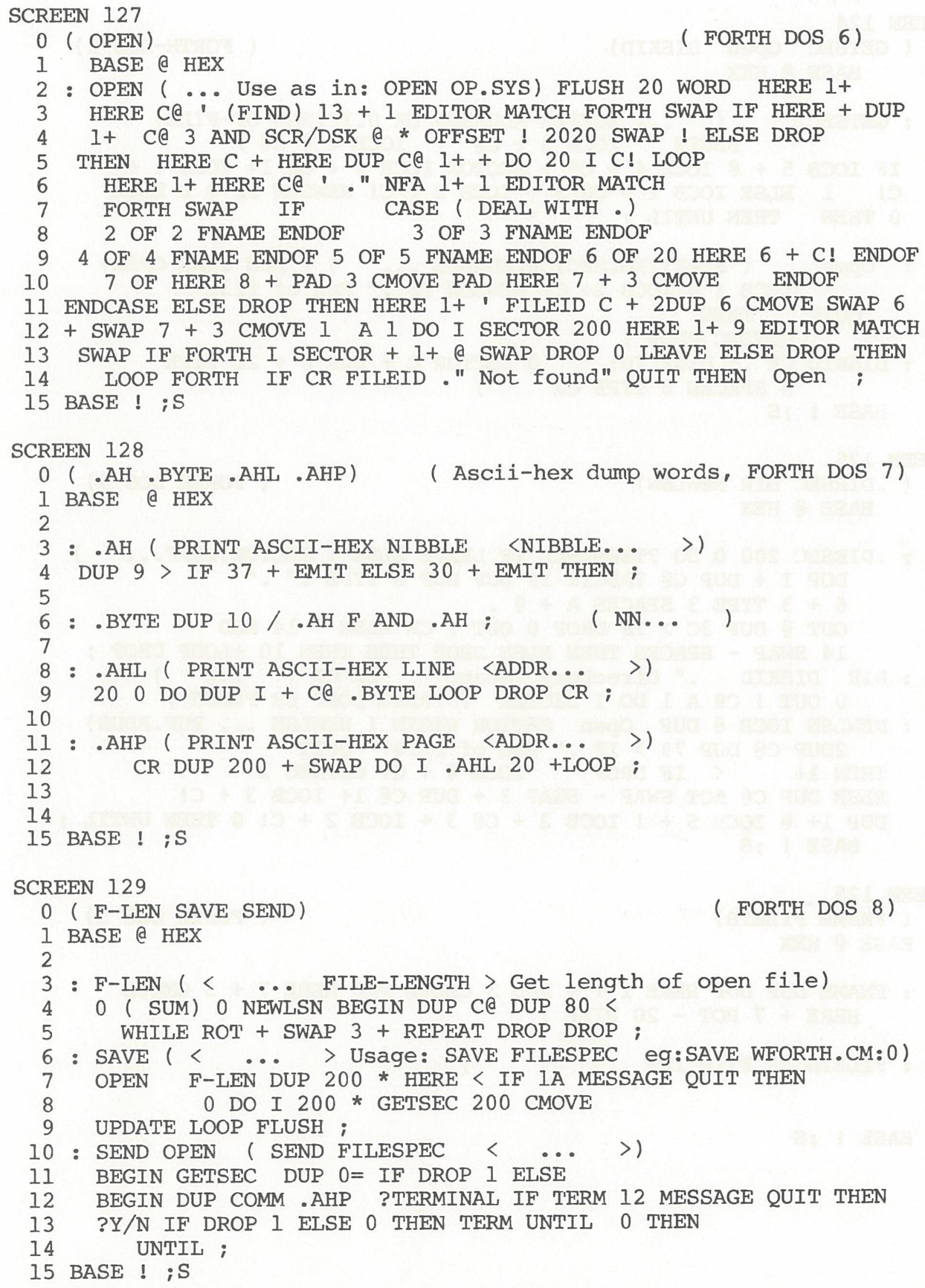




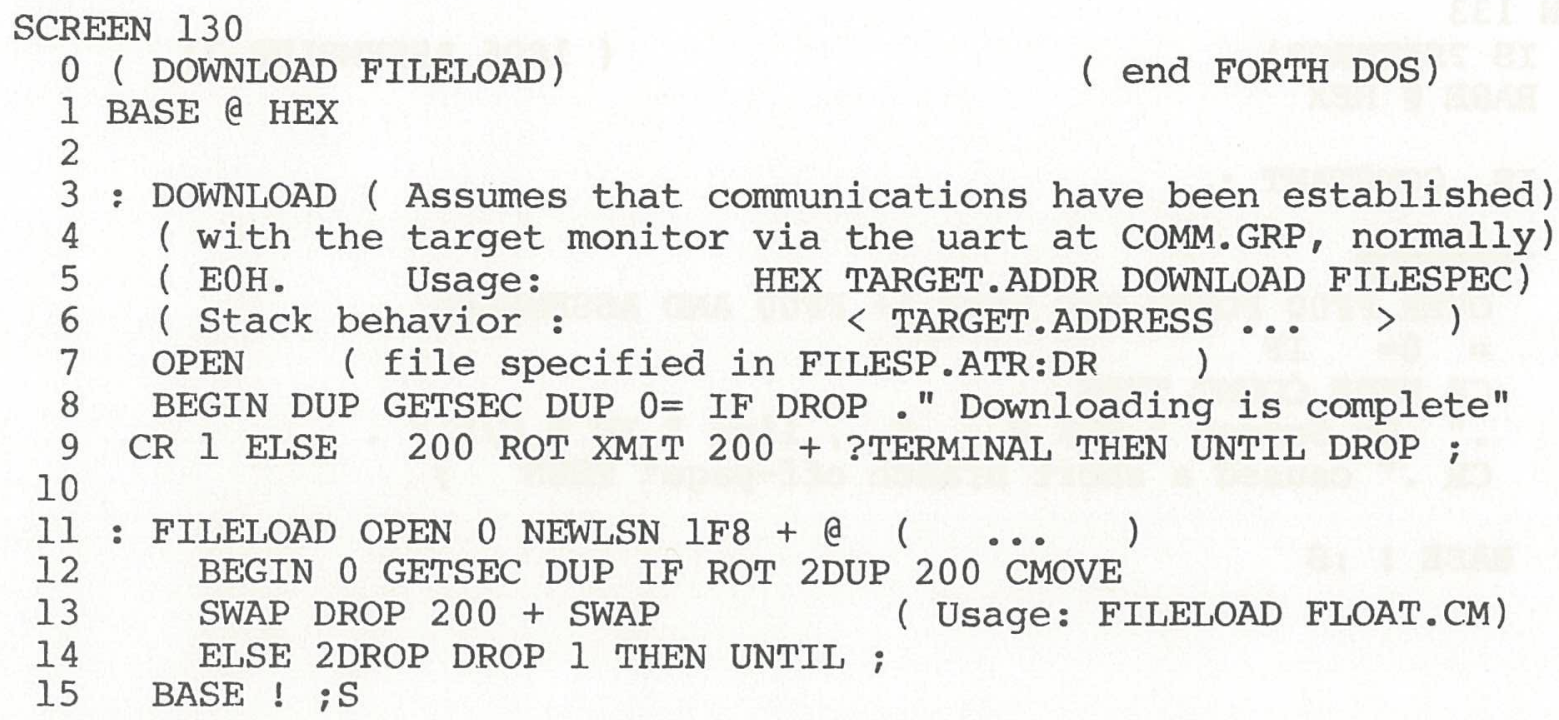




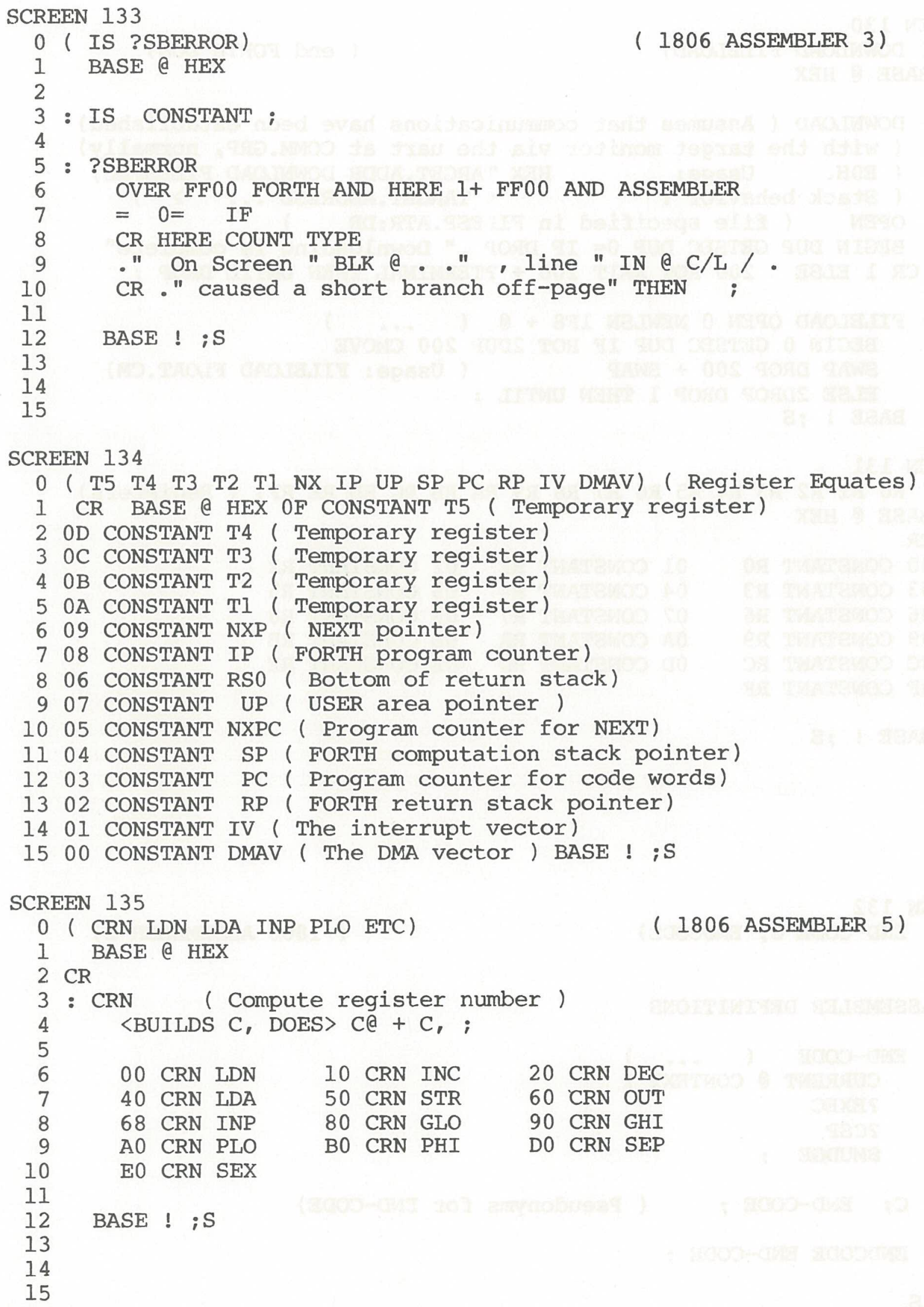




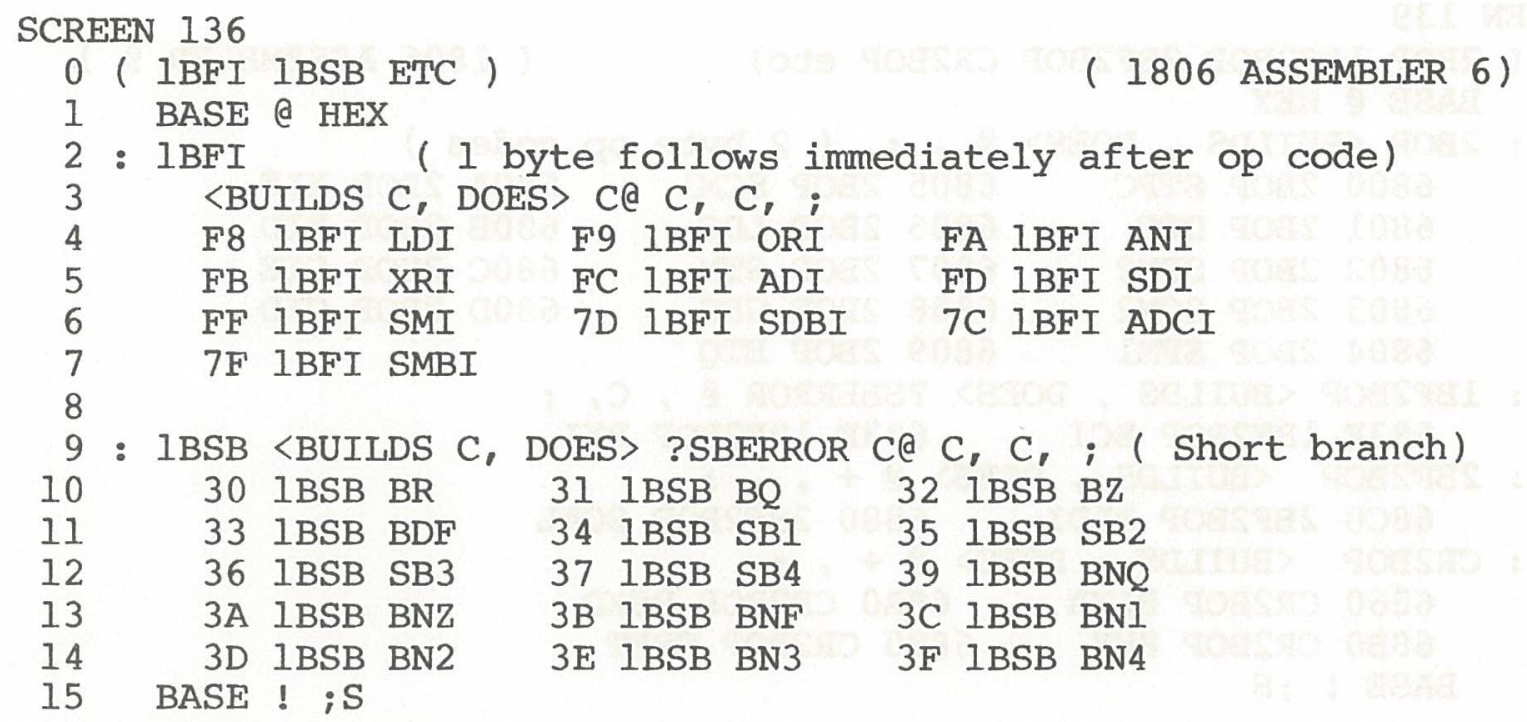




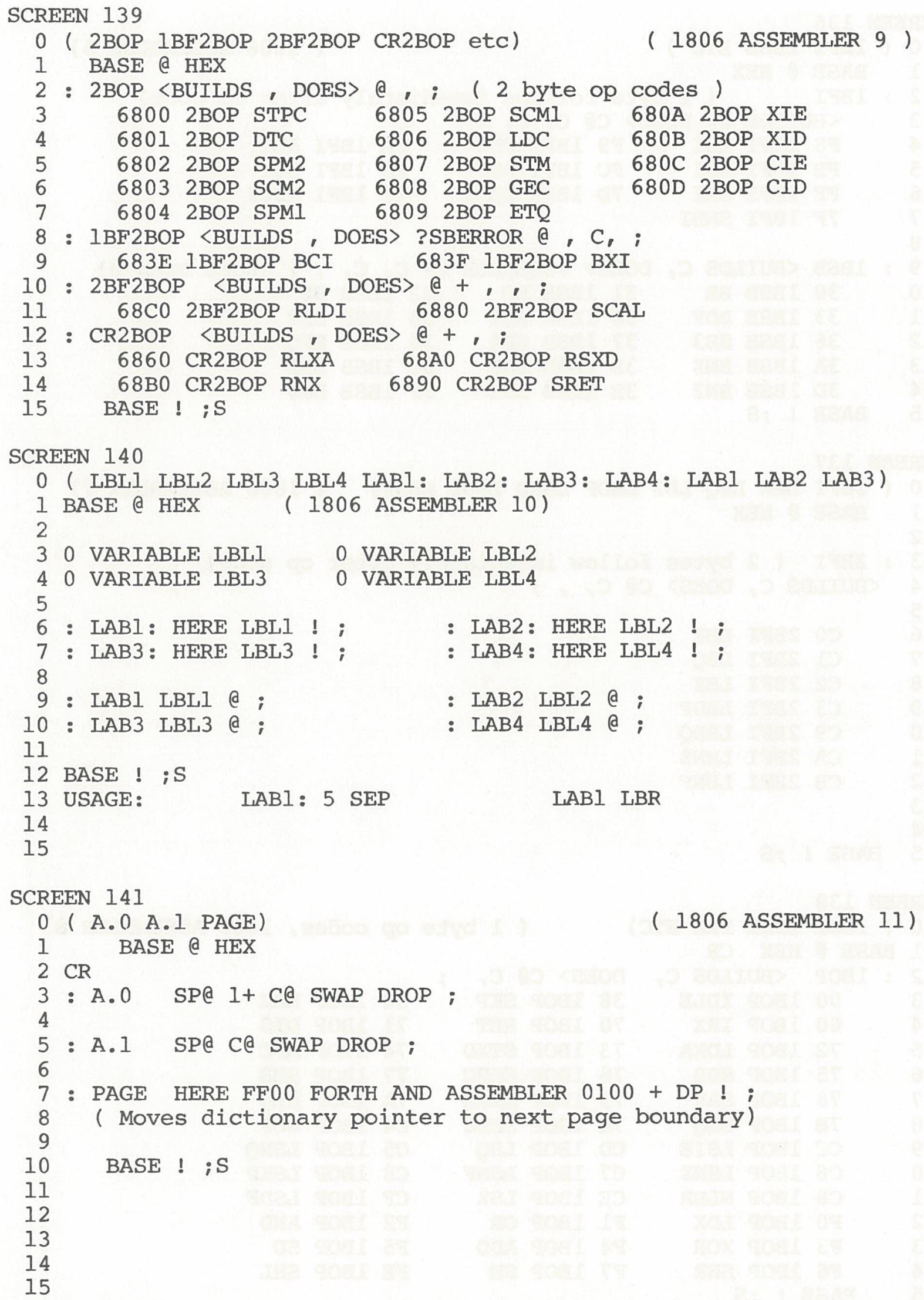




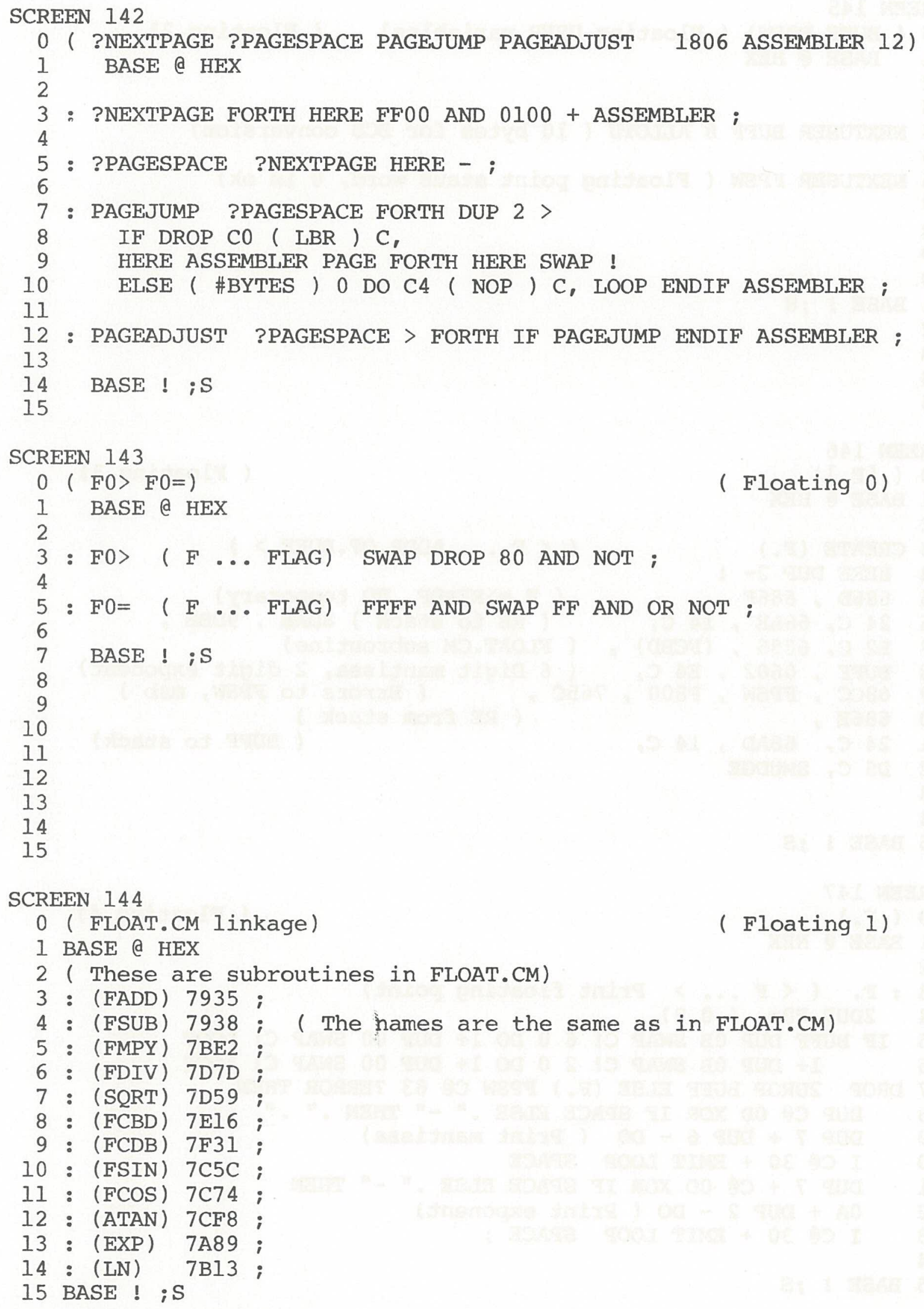




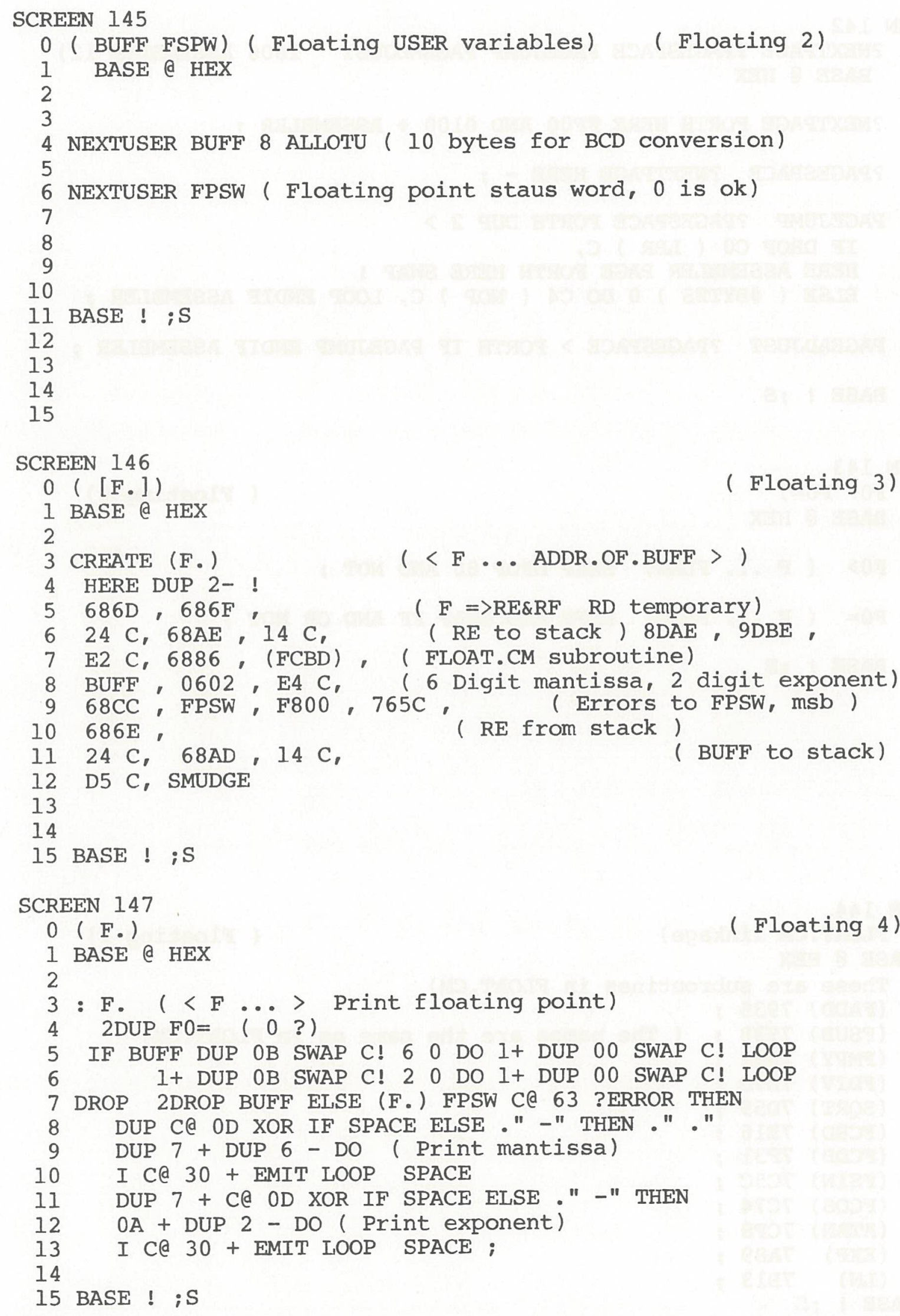




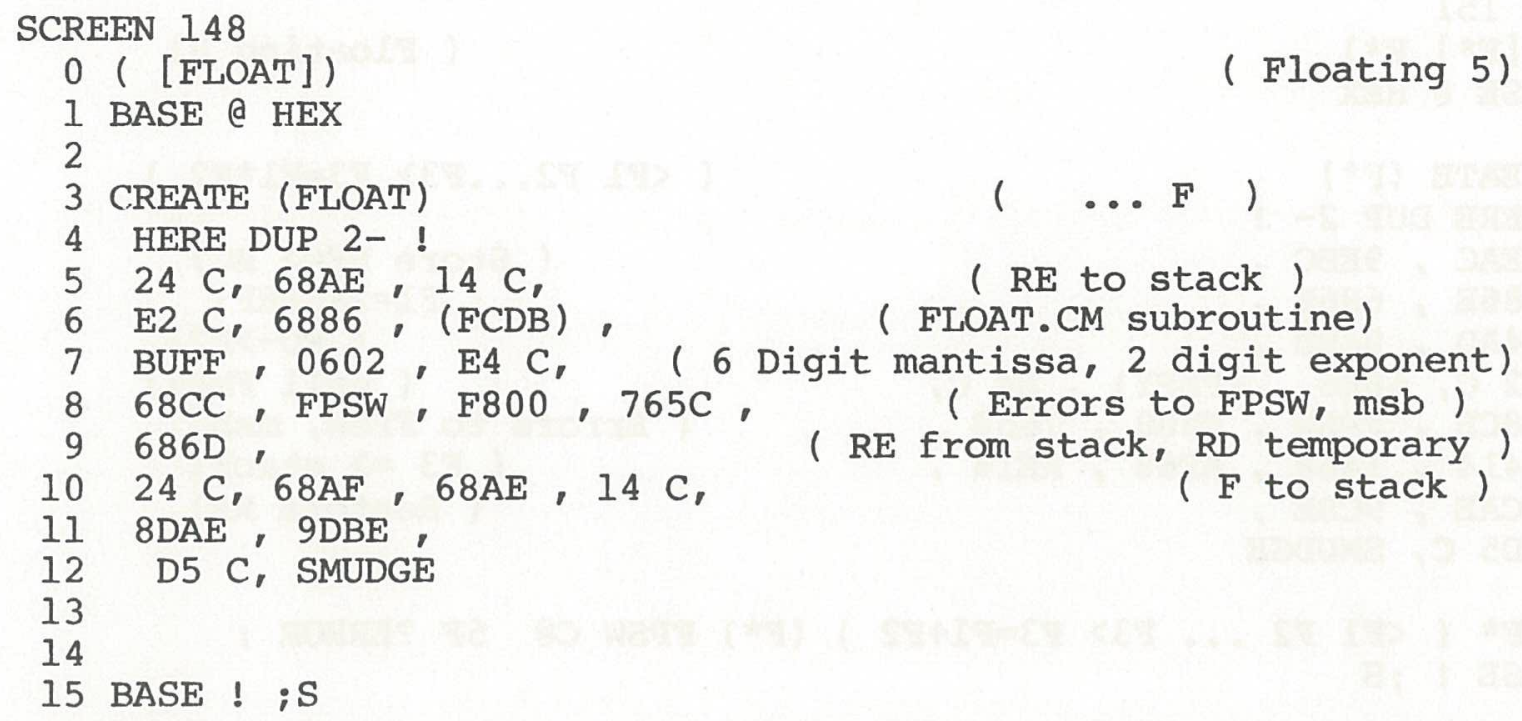




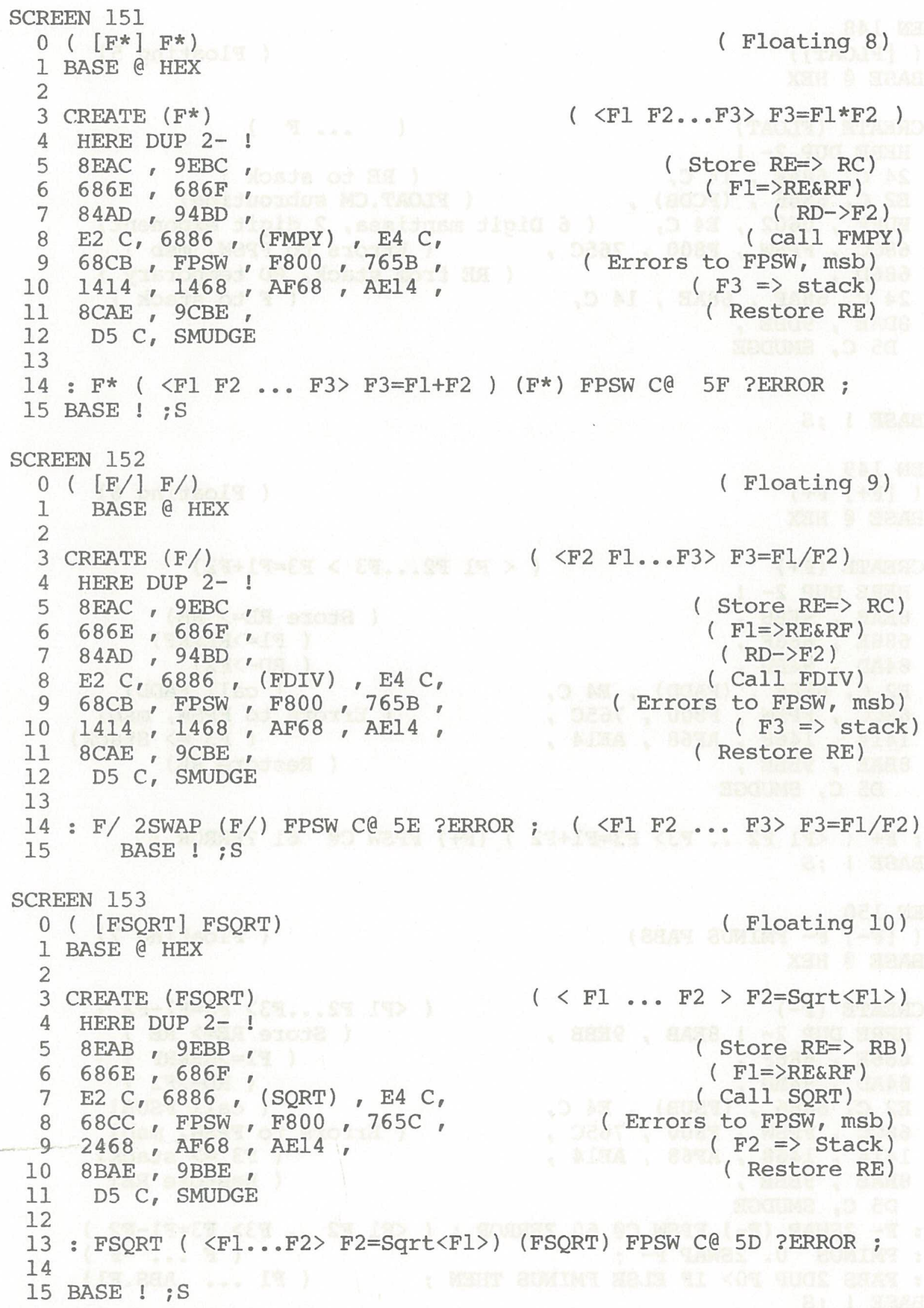




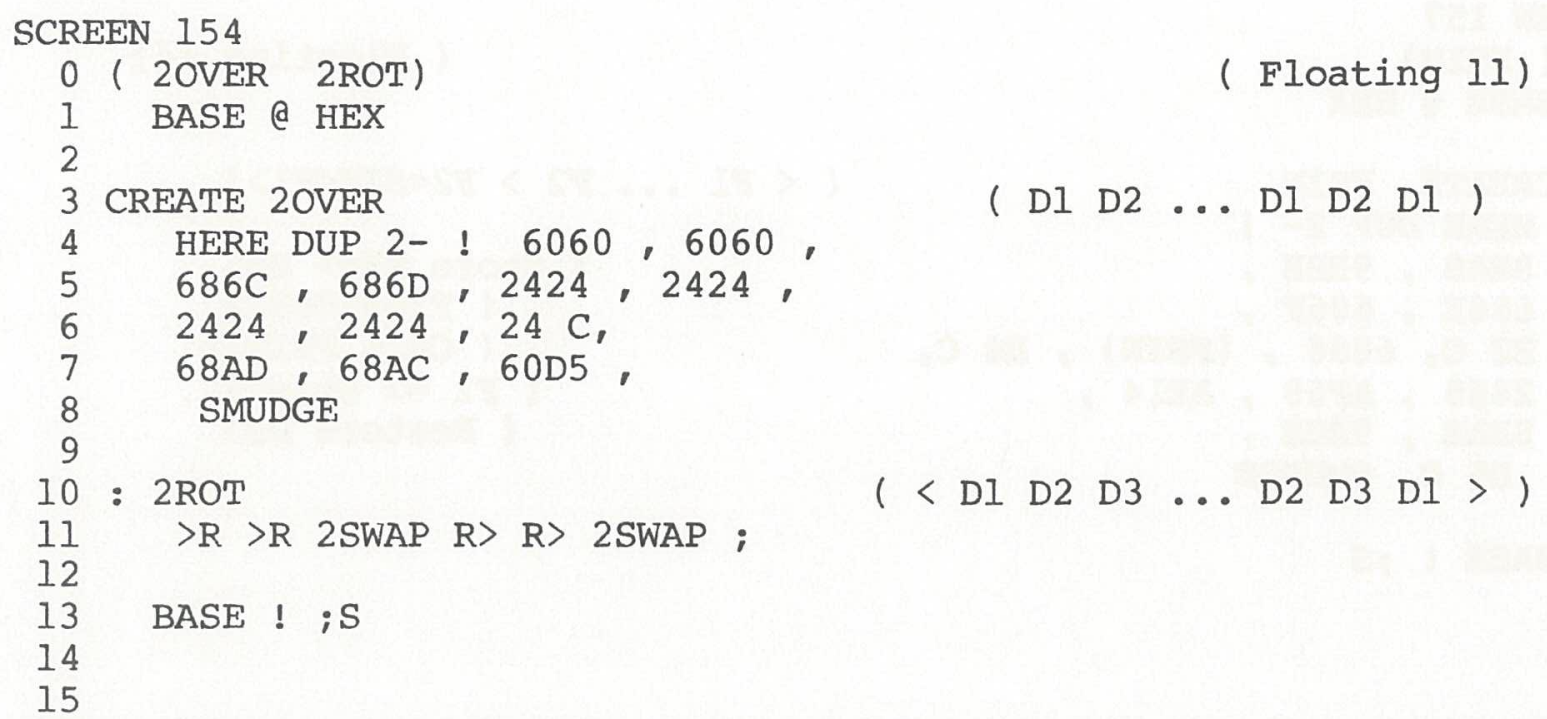




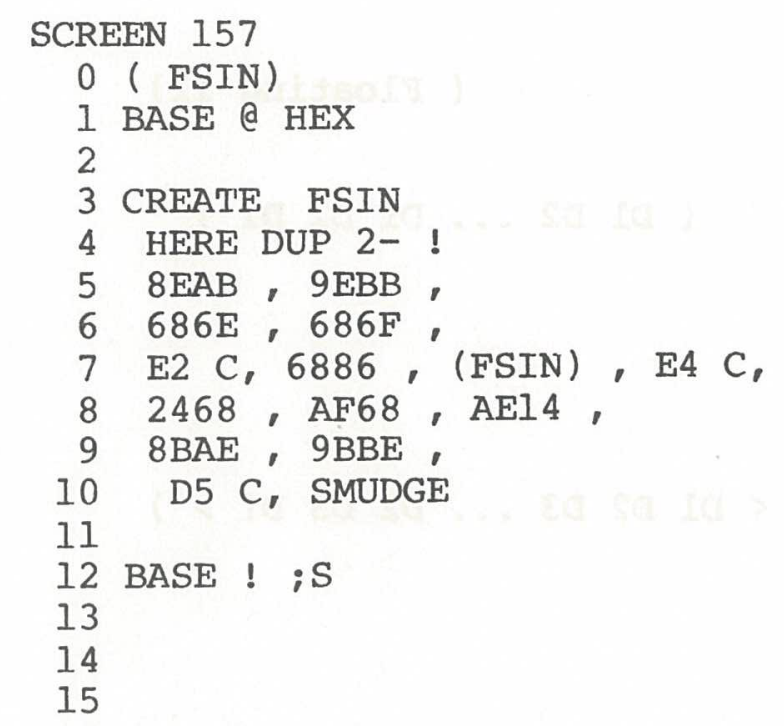

SCREEN 158

0 ( FCOS)

1 BASE @ HEX

2

3 CREATE FCOS

4 HERE DUP 2- !

$58 \mathrm{EAB}$, $9 E B B$,

$6686 \mathrm{E}, 686 \mathrm{~F}$,

$7 \mathrm{E} 2 \mathrm{C}, 6886,(\mathrm{FCOS}), \mathrm{E} 4 \mathrm{C}$,

$82468, A F 68, A E 14$,

9 8BAE , 9BBE ,

10 D5 C, SMUDGE

11

12 BASE ! ; S

13

14

15

SCREEN 159

0 ( FARCTAN)

( Floating 16)

1 BASE \& HEX

2

3 CREATE FACRTAN

4 HERE DUP 2- !

$58 \mathrm{EAB}$, 9EBB ,

$6686 \mathrm{E}, 686 \mathrm{~F}$,

7 E2 C, 6886, (ATAN) , E4 C,

$82468, A F 68, A E 14$,

$98 \mathrm{BAE}, 9 \mathrm{BBE}$,

10 D5 C, SMUDGE

11

12 BASE ! ;

13

14

15

( Floating 15)

( Store $\mathrm{RE} \Rightarrow \mathrm{RB}$ )

( $\mathrm{Fl}=>\mathrm{RE} \& \mathrm{RF}$ )

( Call FCOS )

( $\mathrm{F} 2 \Rightarrow$ Stack)

( Restore RE)

$(\langle\mathrm{F} 1 \ldots \mathrm{F} 2\rangle \mathrm{F} 2=\cos \langle\mathrm{F} 1\rangle)$ 


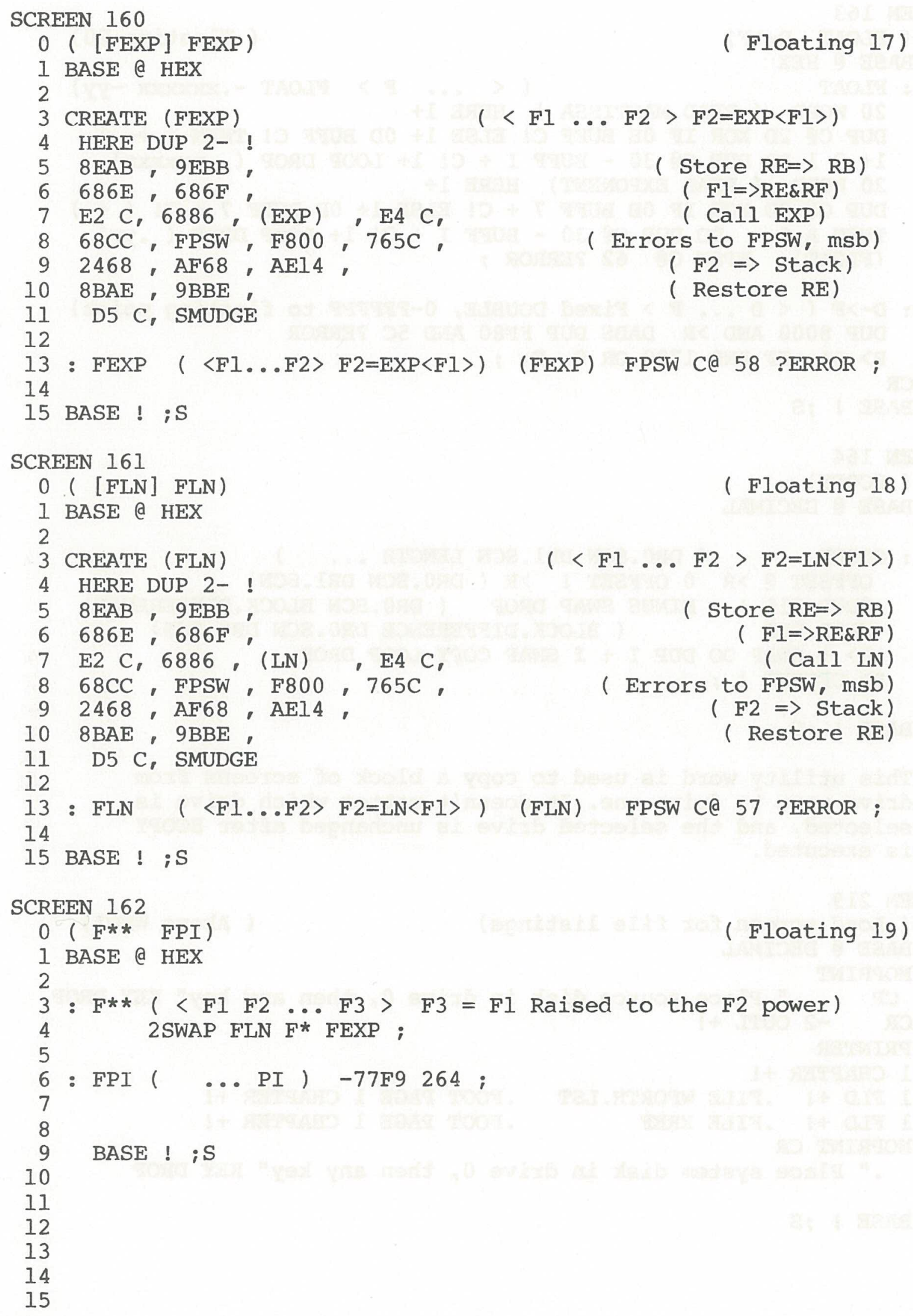




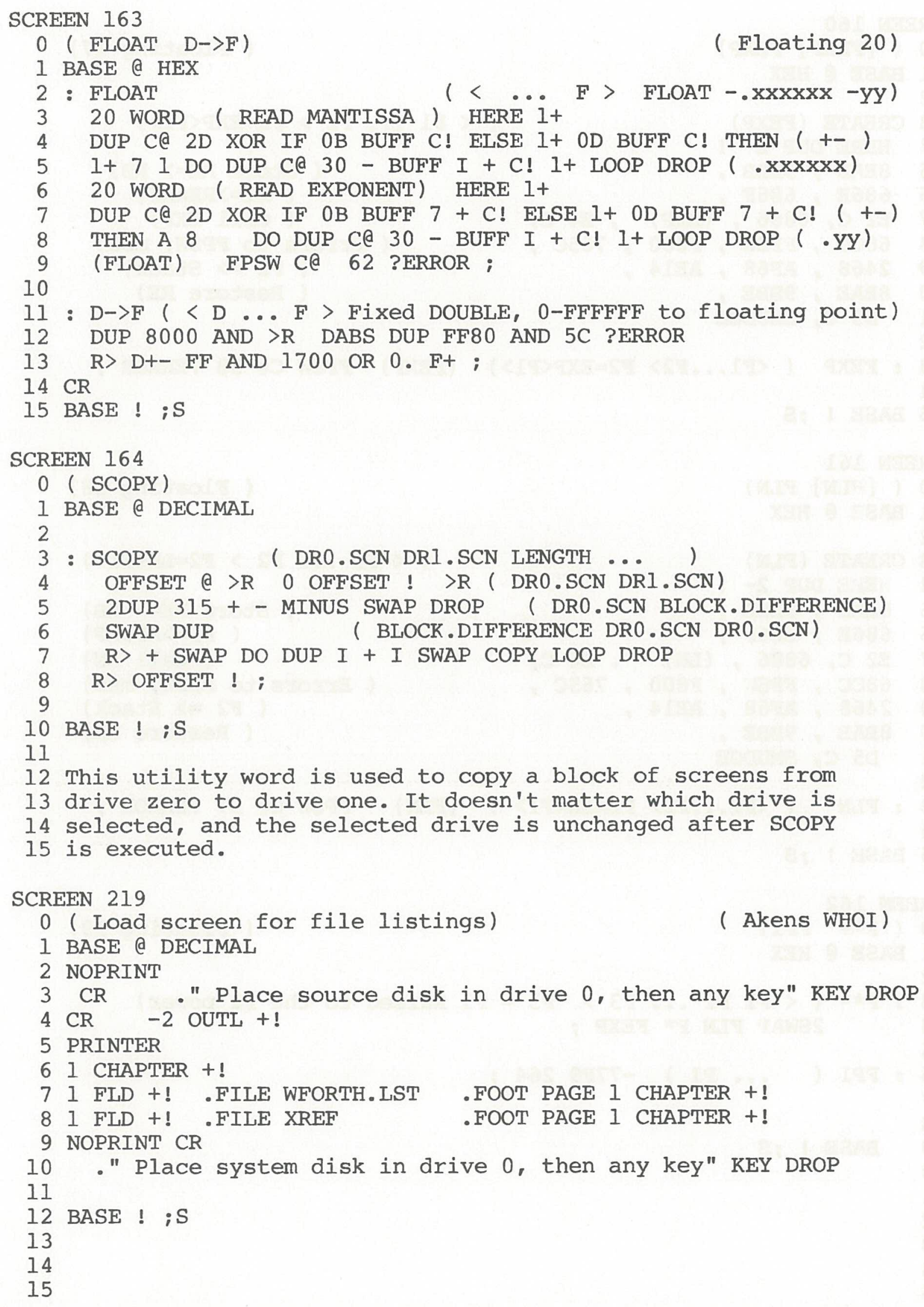




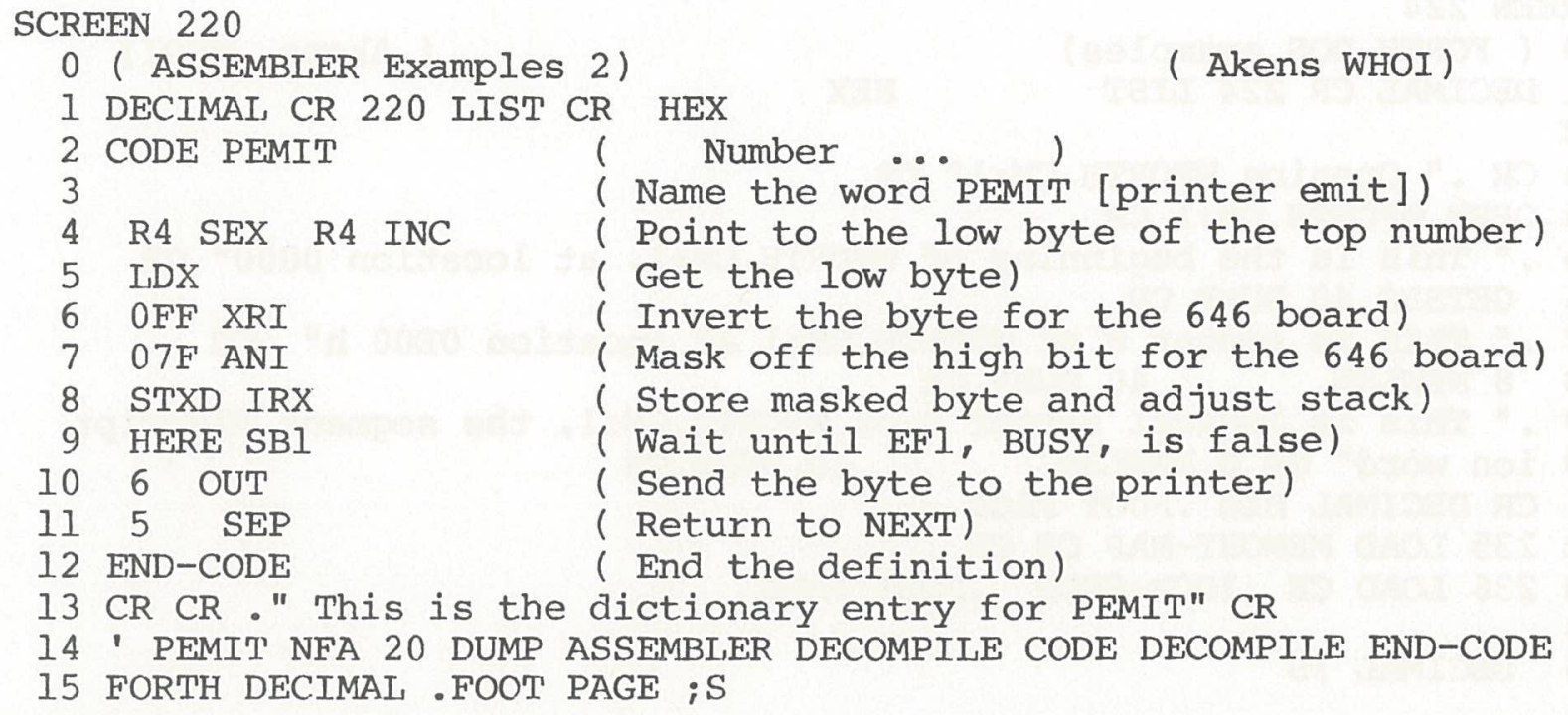


0 ( FORTH DOS examples)

I DECIMAL CR 224 LIST

HEX

( Akens, WHOI)

2

$3 \mathrm{CR}$." Opening WFORTH.CM:I" CR

4 OPEN WFORTH.CM:I CR

5 ." This is the beginning of WFORTH.CM:I, at location 0000 " CR

6 GETSEC 40 DUMP CR

7 ." This is sector 8 of WFORTH.CM:1 at location 0E00 h" CR

88 NEWLSN 40 DUMP CR

9 ." This is logical sector 0 of WFORTH.CM:I, the segment descript

10 ion word" CR 0 NEWLSN 10 DUMP CR

11 CR DECIMAL DIR .FOOT PAGE

12235 LOAD MEMORY-MAP CR CR

13236 LOAD CR IOCB-SHOW .FOOT PAGE

14

15 DECIMAL ;S

\section{SCREEN 225}

0 (.VHDR3 VIIST3)

1 BASE @ HEX

2

3 : .VHDR3 CR ( $\ldots$ ) ) ( Print VLIST3 header)

$4 \quad 10$ SPACES ." Vocabulary list" CR

5 ." NFA Name" OC SPACES ." @LFA CFA @CFA @PFA" CR ;

6 : VLIST3 BASE @ HEX CURRENT a a .VHDR3

7 BEGIN CR 0 OUT ! DUP DUP ( NFA)

85 U.RLZ SPACE ID. OUT \& 16 SWAP - SPACES

9 PFA DUP DUP DUP DUP DUP

10 LFA \& 4 U.RLZ 2 SPACES CFA DUP 5 U.RLZ @ 5 U.R

112 SPACES @ 4 U.RLZ CFA DUP \& 2 - = IF ." Code" THEN NFA CE

1240 AND IF ." Immediate" THEN OUTL \& L/P 3 - < IF

13 ELSE CR .FOOT PAGE .VHDR3 THEN LFA @

14 DUP $0=$ ?TERMINAL OR UNTIL DROP BASE ! CR .FOOT ;

15 BASE ! ;S

SCREEN 226

0 ( CAT-UPDATE .CAT VLIST2) ( Cluster allocation table utilities)

1 BASE a HEX (Cluster allocation table for scrns 65-249)

2

3 : CAT-UPDATE 4 BLOCK 20F + DUP DUP C@ IF OR SWAP C! ( $\ldots$ ) )

4 1+ DUP 2D + SWAP DO FF I C! LOOP 4 BLOCK 23D + DUP

5 SWAP C! UPDATE FLUSH ; ( CLUSTER ALLOCATION TABLE)

6

7 : .CAT 4 BLOCK $200+50$ DUMP ; ( Print cluster allocation tbl)

8 : VLIST2 BASE @ HEX ( ... . VHDR 3 OUTL !

90 OUT ! CURRENT @ a BEGIN DUP DUP OUT @ $3 \mathrm{C}>\mathrm{IF}$ CR 0 OUT !

10 OUTL @ $39>$ IF .FOOT PAGE .VHDR 3 OUTL ! 0 OUT ! THEN

11 THEN $0<\#$ \#\#\#\# \#> TYPE DUP $1+$ CQ 80 -

12 IF SPACE ID. ELSE DROP THEN

1314 OUT @ OVER MOD DUP IF ELSE DROP 14 THEN - SPACES

14 PFA LFA @ DUP $0=$ ?TERMINAL OR UNTIL DROP CR . FOOT BASE ! ;

15 BASE ! ;S 


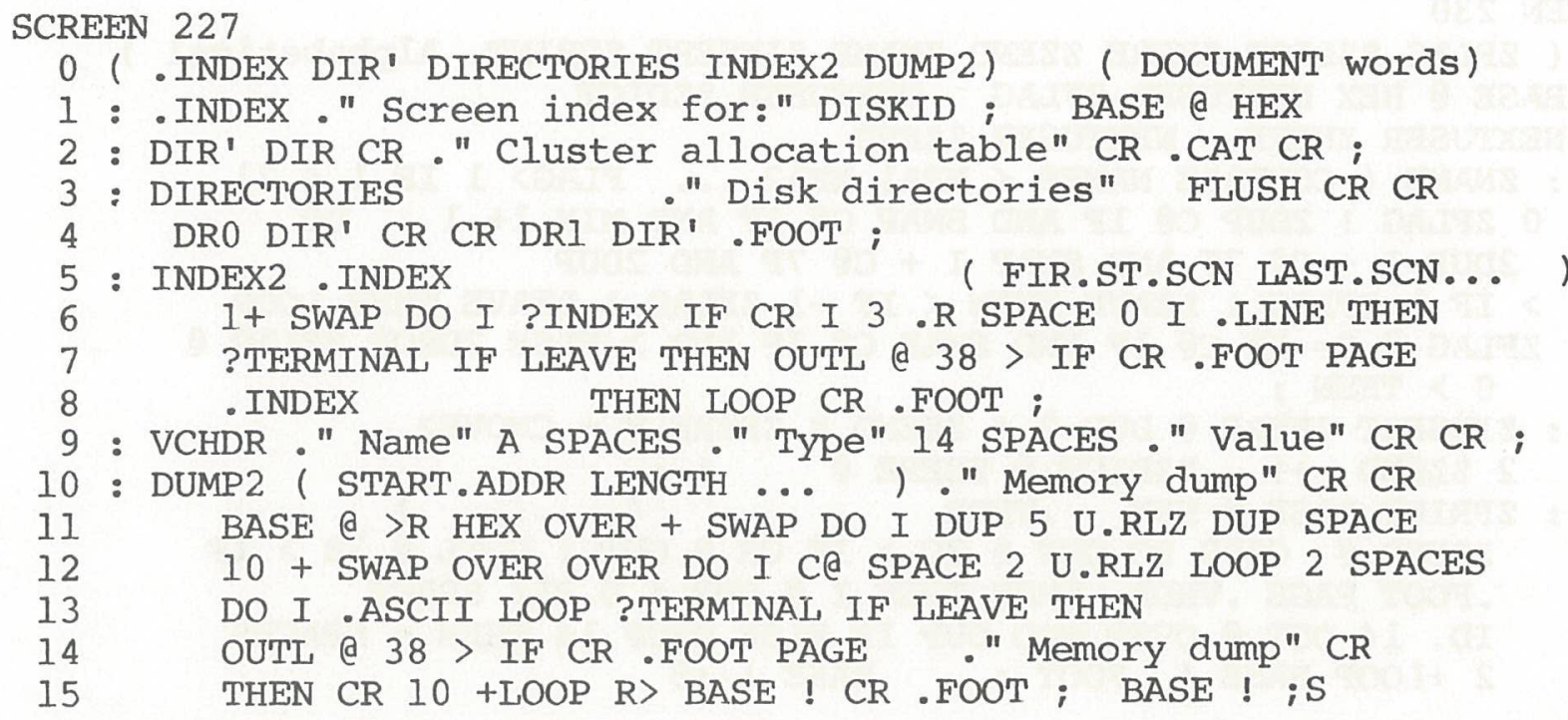




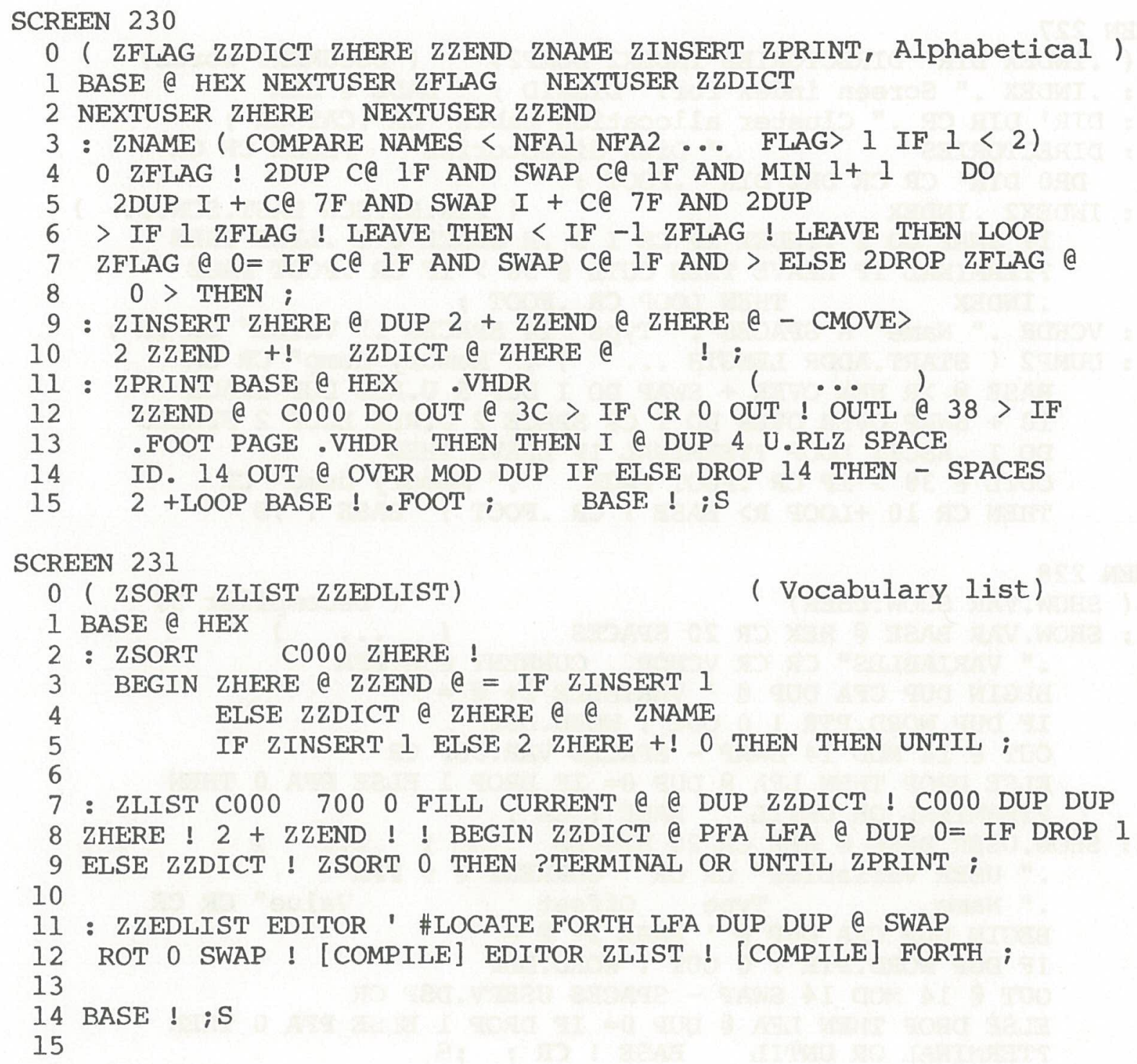




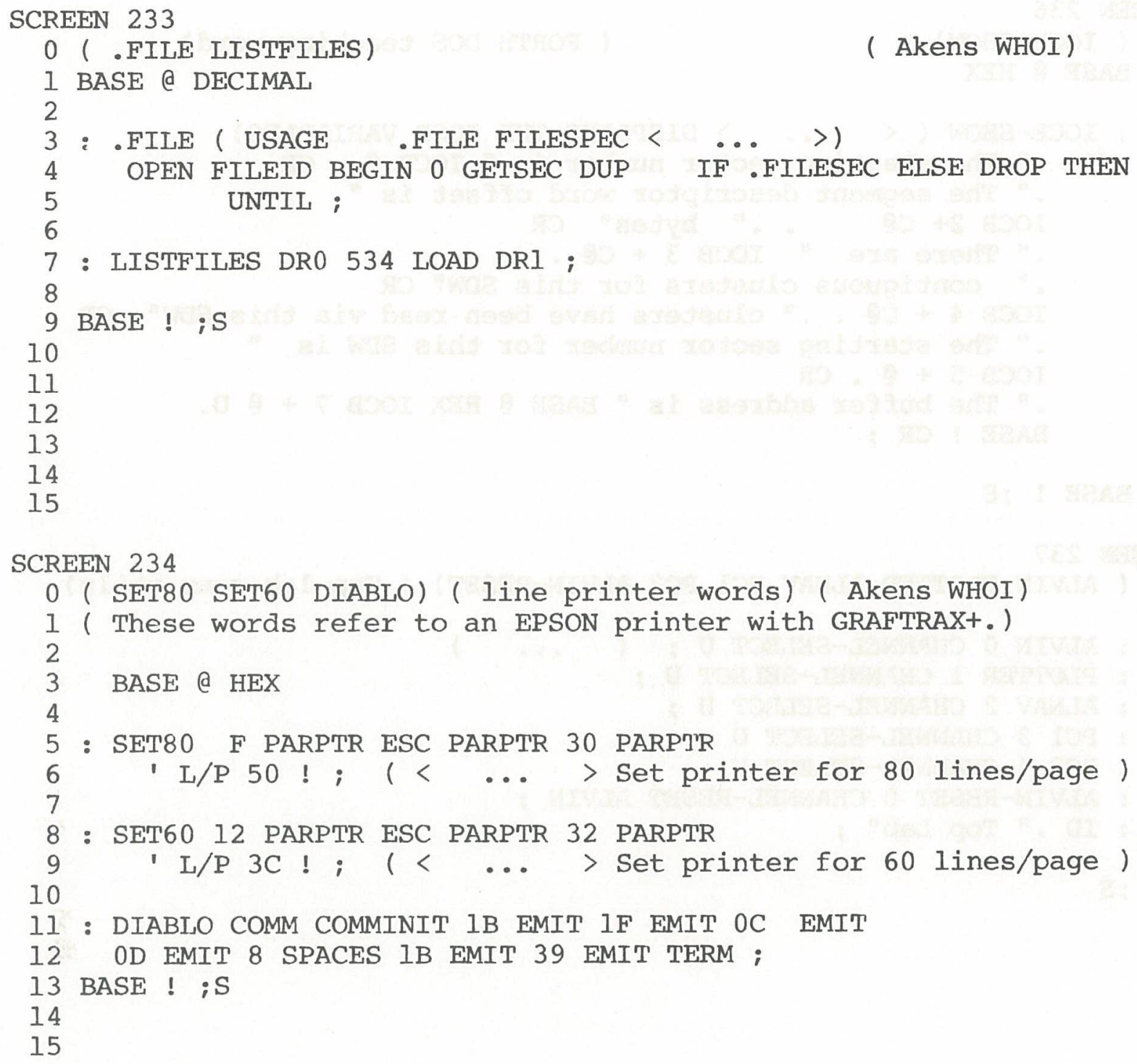




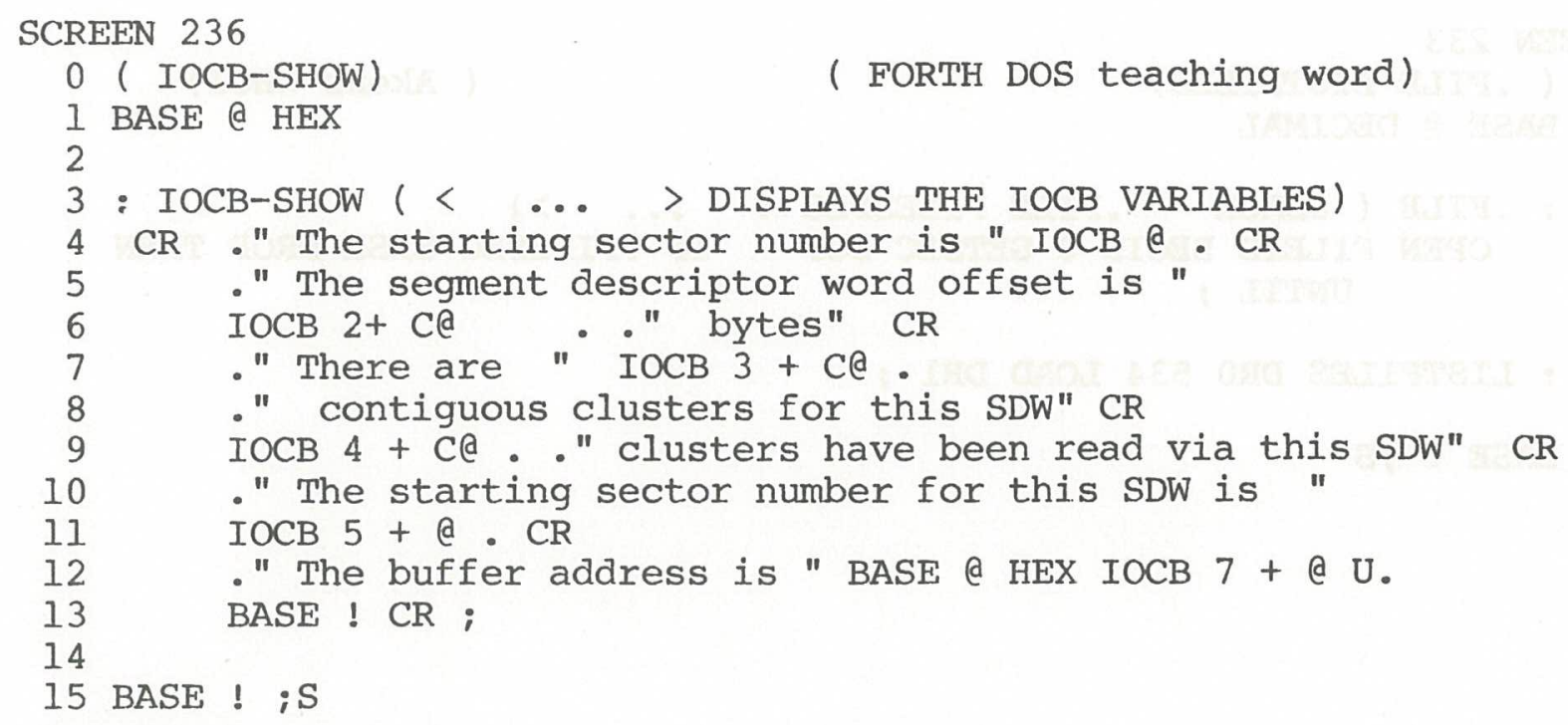




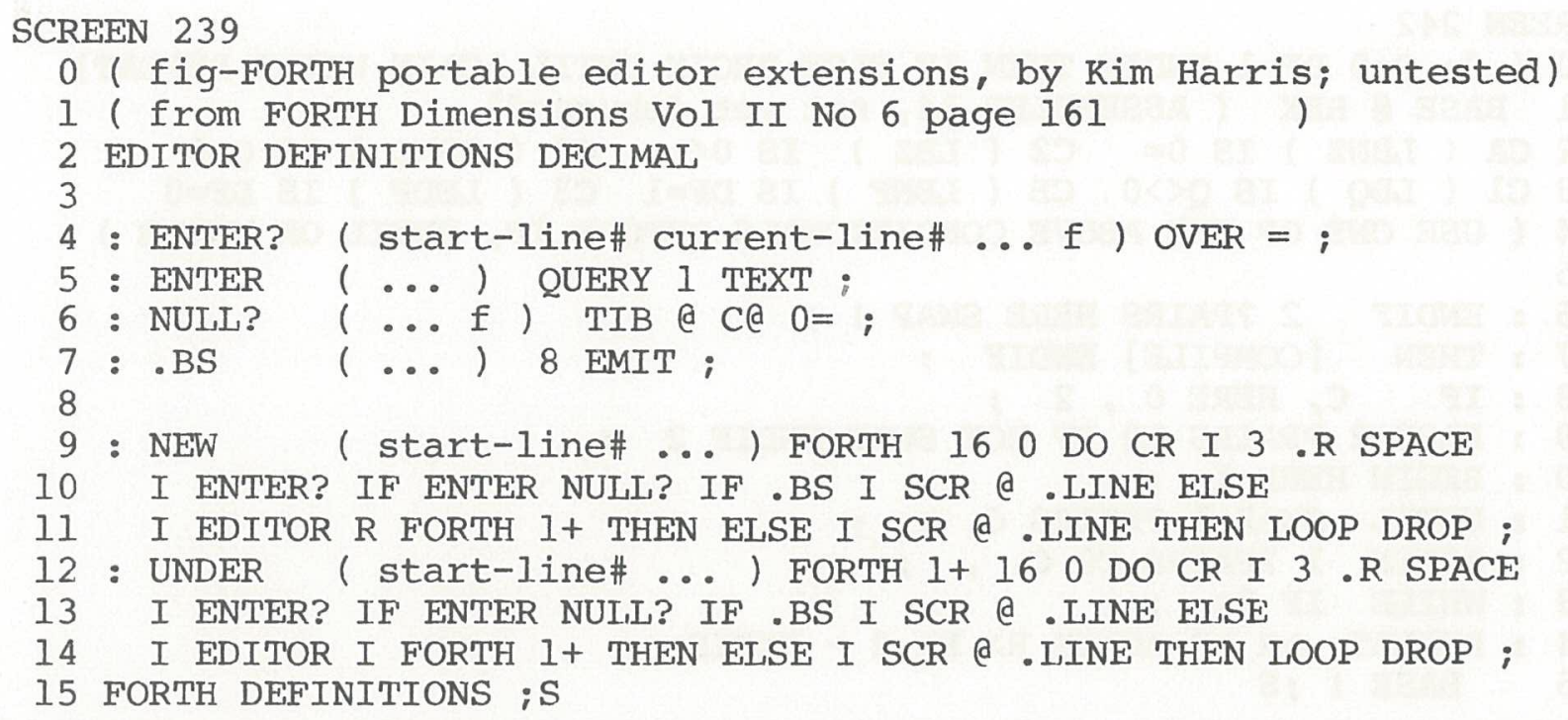

0 ( fig-FORTH portable editor extensions, by Kim Harris; untested)

1 ( from FORTH Dimensions Vol II No 6 page 161 )

2 EDITOR DEFINITIONS DECIMAL

3

4 : ENTER? ( start-line\# current-line\# ... f ) OVER = ;

5 : ENTER ( ...) QUERY I TEXT ;

6 : NULL? ( ... f ) TIB @ CA $0=$;

7 : . BS $\quad(\ldots) \quad 8$ EMIT ;

8

9: NEW (start-line\# ...) FORTH 160 DO CR I 3 .R SPACE

10 I ENTER? IF ENTER NULL? IF .BS I SCR \& .LINE ELSE

11 I EDITOR R FORTH 1+ THEN ELSE I SCR @ . LINE THEN LOOP DROP ;

12 : UNDER (start-line\# ...) FORTH 1+ 160 DO CR I 3 . R SPACE

13 I ENTER? IF ENTER NUIIL? IF .BS I SCR \& .LINE ELSE

14 I EDITOR I FORTH 1+ THEN ELSE I SCR a . LINE THEN LOOP DROP ;

15 FORTH DEFINITIONS ;

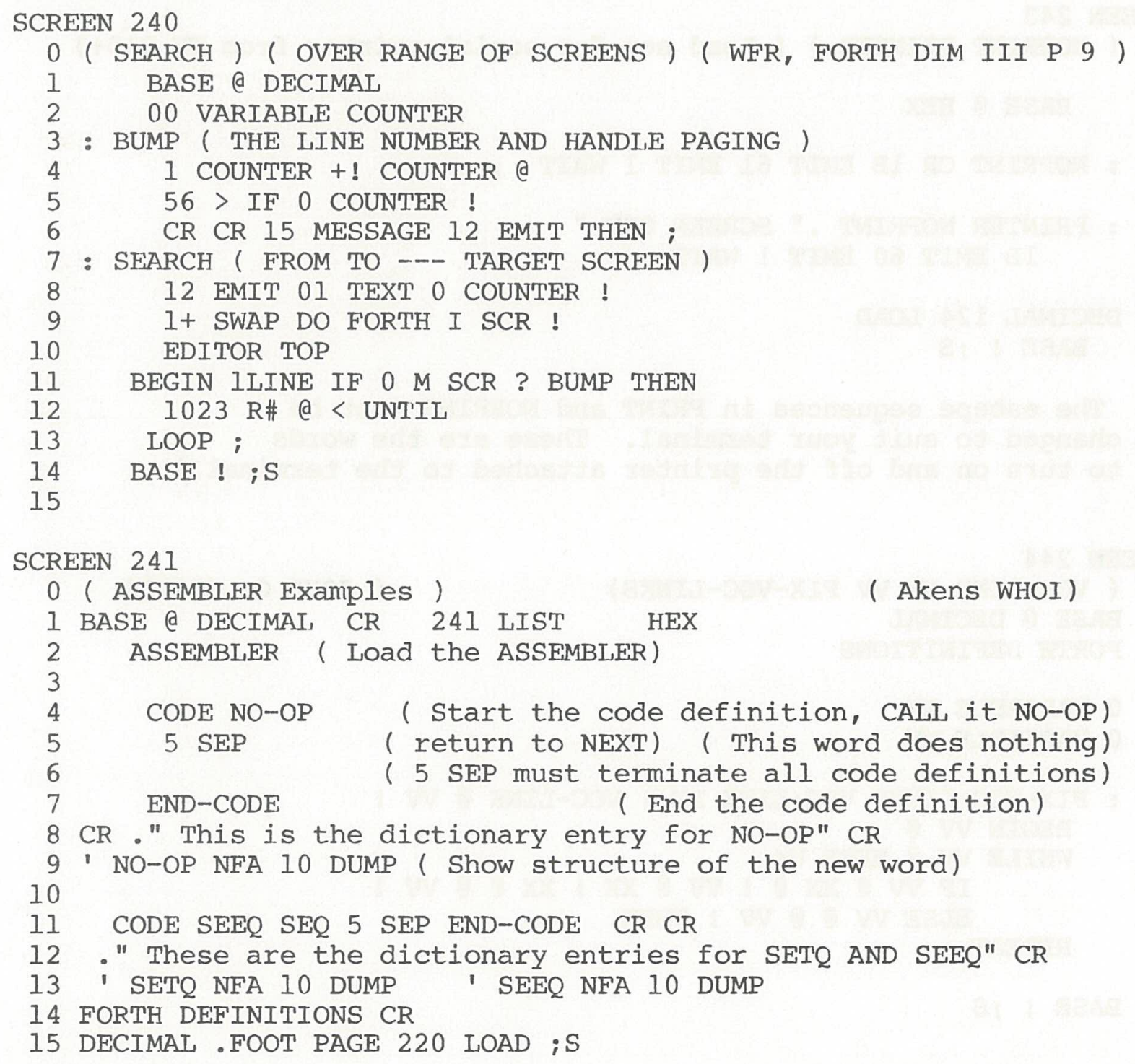




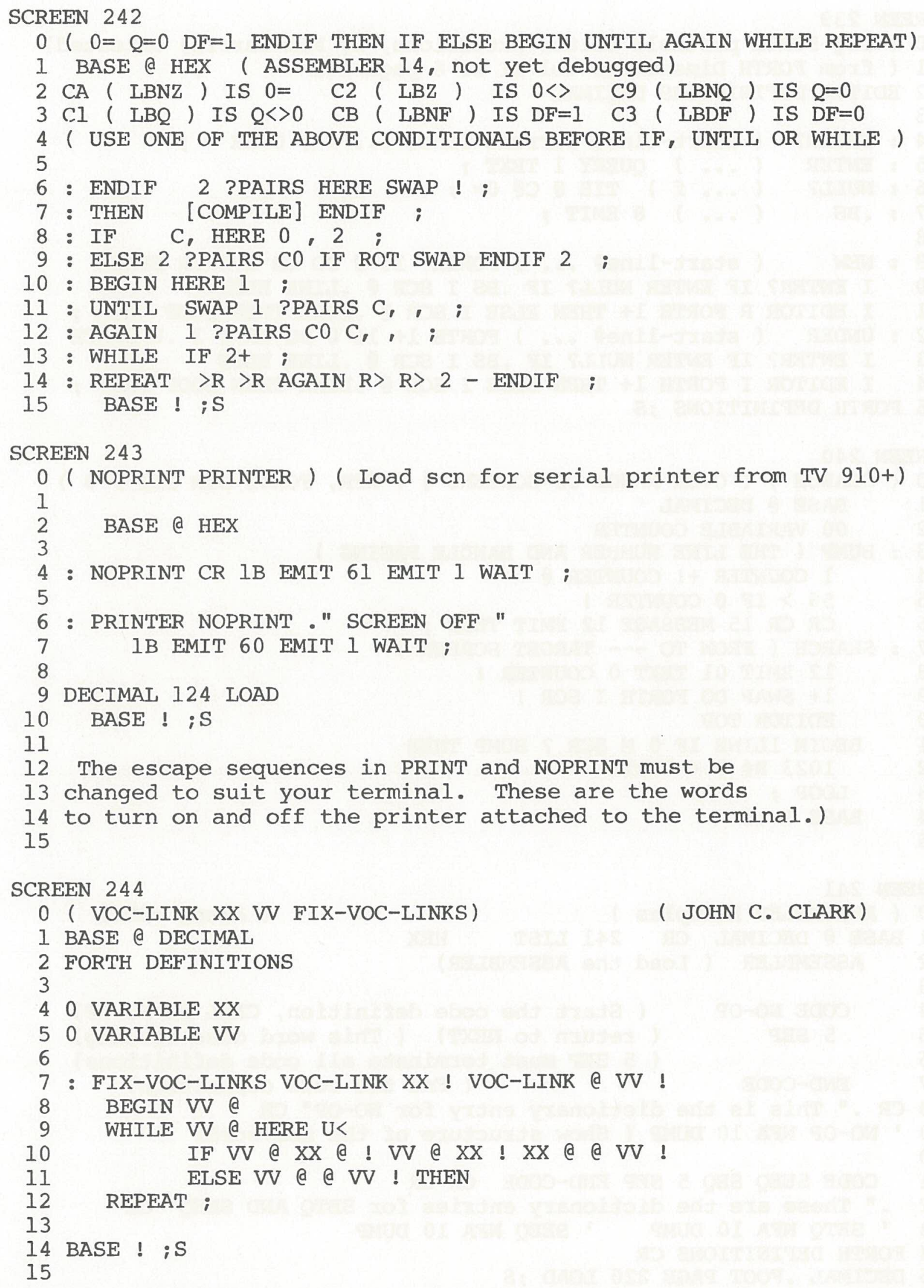




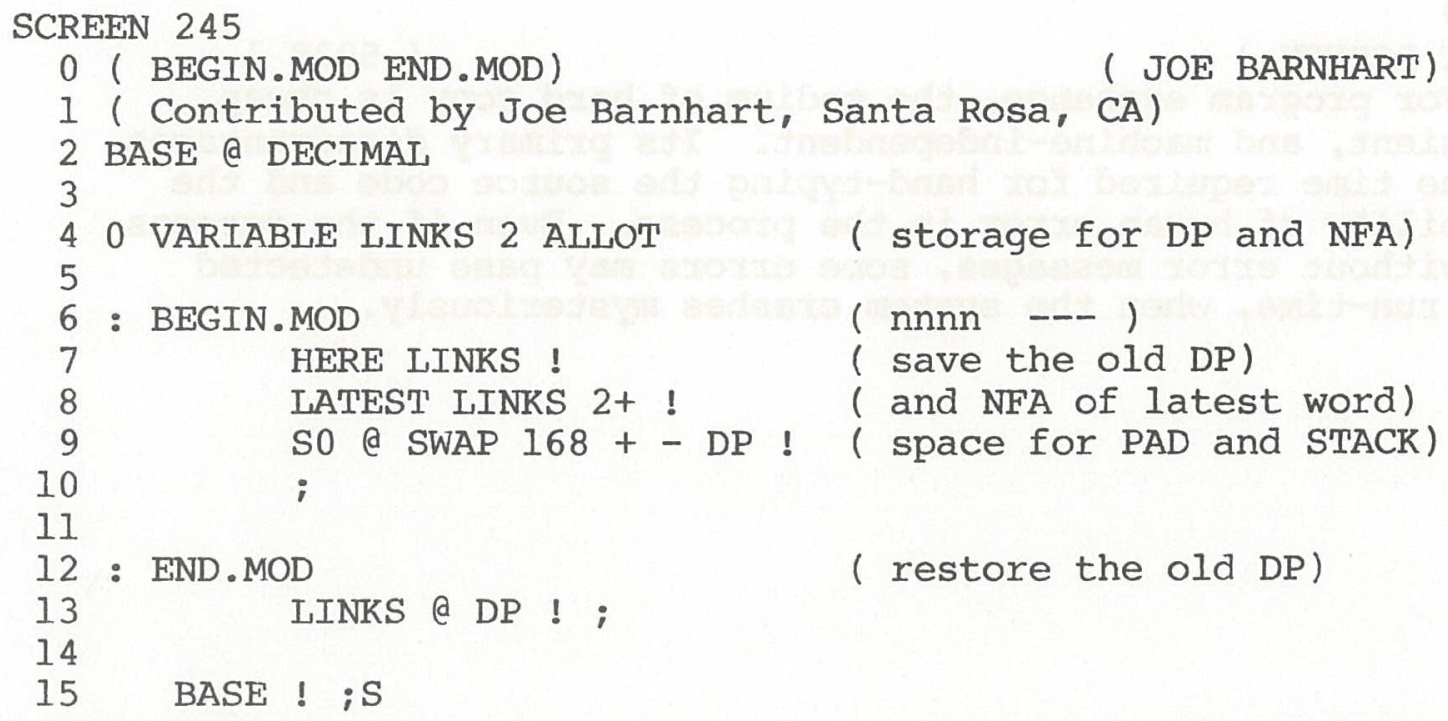




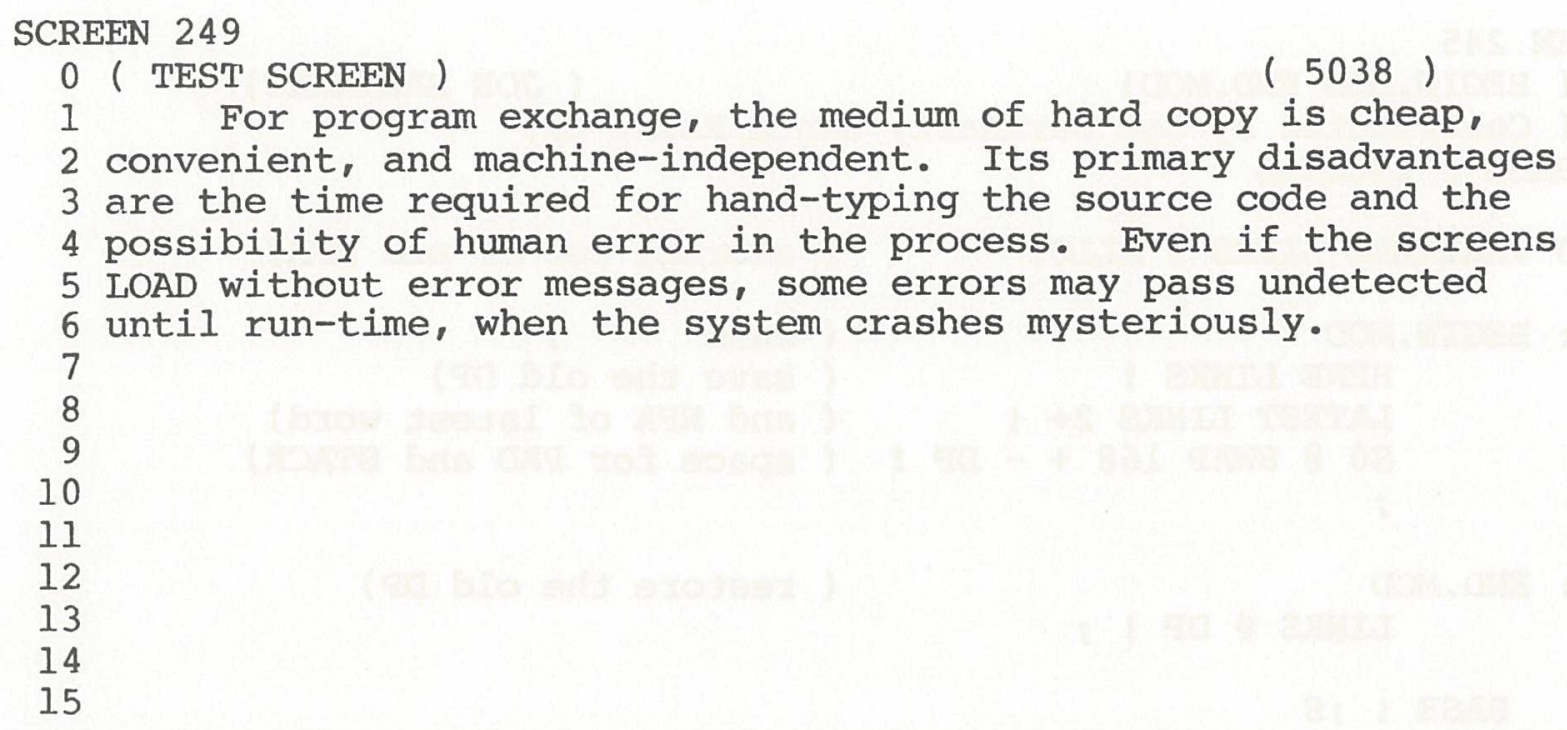


This chapter lists all FORTH words in the order in which they were defined, most recently defined first. Each entry includes the name field address (NFA), the name of the word, the contents of the link field (@LFA), the code field address (CFA), the contents of the code field (@CFA), and the contents of the first byte of the parameter field. In addition, CODE words and IMMEDIATE words are flagged. The order in which each entry is printed corresponds exactly to the actual dictionary entry for each word. The drawing below illustrates the dictionary entry format.

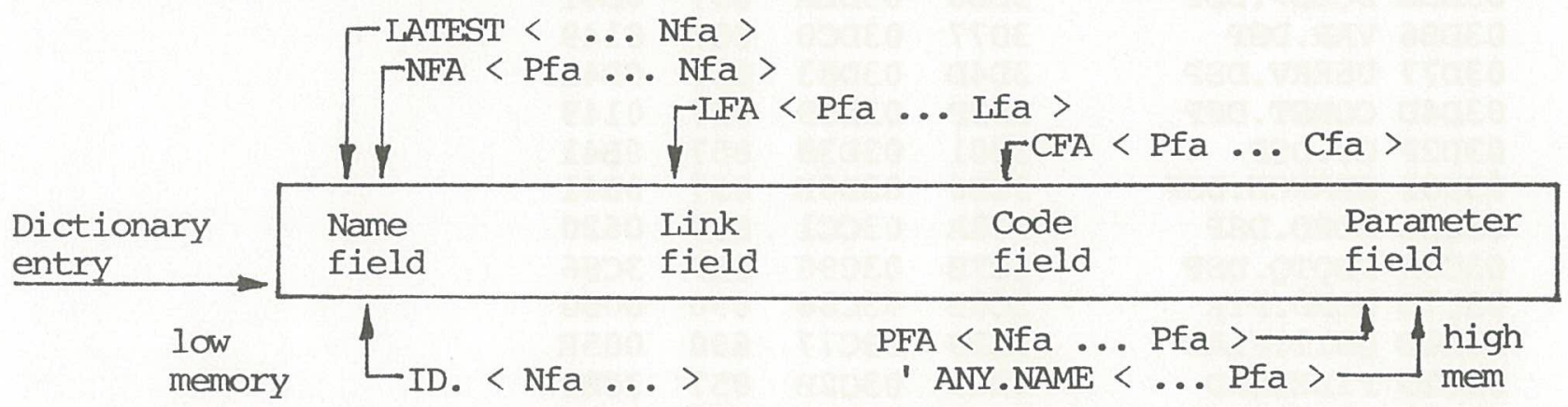

There are 6 pre-defined types of dictionary entries. These are Code definitions, Colon definitions, CONSTANTS, VARIABLES, USER variables and VOCABULARY definitions. Other types, such as string constants and string variables may be defined by the user for specific applications. Refer to the FORTH ENCYCLOPEDIA for complete details.

The lengths of the name field and the parameter field are variable. The link field and the code field are always two bytes long. The link field address points to the name field of the word previously defined in the present VOCABULARY. The code field address points to the code which is actually executed when the word is invoked. The code field of a code word points to the parameter field which contains machine code. For all other types of words, the code field points to code which interprets the entries in the parameter field. The dictionary is composed of a number of dictionary entries threaded together via the link field addresses. The end of the dictionary is signified by a link field address of 0000. Each word which defines a VOCABULARY entry contains a pointer to the most recently defined word in that particulary VOCABULARY (ie FORTH EDITOR ASSEMBIFR).

This chapter was generated by the following sequence:

PRINTER VLIST2 NOPRINT $(\mathrm{cr})$ 


\begin{tabular}{|c|c|c|c|c|c|c|}
\hline NFA & Name & $\begin{array}{l}\text { dary } \\
\text { eLFA }\end{array}$ & $\begin{array}{l}\text { ist } \\
\text { CFA }\end{array}$ & QCFA & @PFA & \\
\hline $0418 B$ & DOCUMENT & 4180 & 04196 & $10 \mathrm{AI}$ & 1191 & Immediate \\
\hline 04180 & TASK & $416 \mathrm{~B}$ & 04187 & 857 & $02 \mathrm{CO}$ & \\
\hline $0416 \mathrm{~B}$ & FLOATING & 4156 & 04176 & 857 & 0149 & \\
\hline 04156 & DOCUMENT & $410 F$ & 04161 & 857 & 0149 & \\
\hline $0410 F$ & DISKIIST & 4100 & $0411 \mathrm{~A}$ & 857 & $36 \mathrm{~A} 7$ & \\
\hline 04100 & TEAR & $40 \mathrm{E} 3$ & 04107 & 857 & $40 E A$ & \\
\hline $040 \mathrm{E} 3$ & PAGE & $40 B C$ & O40EA & 857 & 0149 & \\
\hline $040 B C$ & PARPTR & $3 E 2 E$ & $040 C 5$ & 857 & 07D2 & \\
\hline $03 E 2 E$ & DECOMPILE & $3 \mathrm{E} 06$ & $03 E 3 A$ & 857 & OEEE & \\
\hline $03 \mathrm{E} 06$ & VOCAB.DSP & 3DDE & 03 E12 & 857 & OB41 & \\
\hline 03DDE & DOES $>. D S P$ & 3DB6 & 03DEA & 857 & OB41 & \\
\hline 03DB6 & VAR.DSP & $3 \mathrm{D} 77$ & $03 D C 0$ & 857 & 0149 & \\
\hline $03 \mathrm{D} 77$ & USERV.DSP & $3 D 4 D$ & $03 D 83$ & 857 & OB4 1 & \\
\hline 03D4D & CONST.DSP & $3 \mathrm{D} 2 \mathrm{~F}$ & 03D59 & 857 & 0149 & \\
\hline 03D2F & CV.DSP & $3 \mathrm{D} 01$ & $03 D 38$ & 857 & OB4 1 & \\
\hline 03D0I & BRANCH.DSP & $3 \mathrm{CB} 6$ & O3D0E & 857 & $0 B 41$ & \\
\hline $03 \mathrm{CB} 6$ & WORD.DSP & $3 \mathrm{C} 8 \mathrm{~A}$ & $03 \mathrm{CCl}$ & 857 & 0620 & \\
\hline $03 \mathrm{C} 8 \mathrm{~A}$ & PDOTQ.DSP & $3 \mathrm{C} 7 \mathrm{~B}$ & $03 c 96$ & 857 & $3 C 86$ & \\
\hline $03 \mathrm{C} 7 \mathrm{~B}$ & WORD.PTR & $3 \mathrm{C} 6 \mathrm{~B}$ & $03 c 86$ & 690 & 0060 & \\
\hline $03 \mathrm{C} 6 \mathrm{~B}$ & QUIT.FIAG & $3 C 20$ & $03 C 77$ & 690 & $005 \mathrm{E}$ & \\
\hline $03 \mathrm{C} 20$ & FILELOAD & $3 \mathrm{BC} 9$ & $03 \mathrm{C} 2 \mathrm{~B}$ & 857 & $38 B 9$ & \\
\hline $03 \mathrm{BC} 9$ & DOWNLOAD & $3 B 78$ & 03BD4 & 857 & $38 \mathrm{~B} 9$ & \\
\hline $03 B 78$ & SEND & $3 B 35$ & $03 B 7 F$ & 857 & $38 B 9$ & \\
\hline $03 B 35$ & SAVE & $3 \mathrm{~B} 01$ & $03 B 3 C$ & 857 & $38 B 9$ & \\
\hline $03 \mathrm{~B} 0 \mathrm{I}$ & F-LEN & $3 A D C$ & $03 B 09$ & 857 & 0608 & \\
\hline $03 \mathrm{ADC}$ & . AHP & $3 \mathrm{AB} 7$ & $03 \mathrm{AE} 3$ & 857 & 0909 & \\
\hline $03 \mathrm{AB} 7$ & .AHL & $3 A 99$ & 03ABE & 857 & 0149 & \\
\hline 03 A99 & . BYTE & $3 \mathrm{~A} 6 \mathrm{~F}$ & 03AAI & 857 & $049 \mathrm{C}$ & \\
\hline $03 \mathrm{~A} 6 \mathrm{~F}$ & . $\mathrm{AH}$ & $38 \mathrm{~B} 2$ & $03 \mathrm{~A} 75$ & 857 & $049 \mathrm{C}$ & \\
\hline 038B2 & OPEN & $388 \mathrm{~F}$ & 038B9 & 857 & 1906 & \\
\hline $0388 \mathrm{~F}$ & FILEID & 3851 & 03898 & 857 & OB4 1 & \\
\hline 03851 & FNAME & $37 \mathrm{~B} 6$ & 03859 & 857 & $049 \mathrm{C}$ & \\
\hline $037 \mathrm{~B} 6$ & NEWLSN & 3775 & $037 \mathrm{BF}$ & 857 & $354 \mathrm{E}$ & \\
\hline 03775 & DIR & $36 \mathrm{DF}$ & 0377B & 857 & $36 \mathrm{~A} 7$ & \\
\hline $036 \mathrm{DF}$ & .DIRSEC & $369 E$ & 036E9 & 857 & 0149 & \\
\hline $0369 \mathrm{E}$ & DISKID & $366 \mathrm{D}$ & 036A7 & 857 & 0909 & \\
\hline $0366 \mathrm{D}$ & Open & $35 \mathrm{EC}$ & 03674 & 857 & $354 \mathrm{E}$ & \\
\hline 035EC & GETSEC & $359 B$ & $035 \mathrm{~F} 5$ & 857 & $354 \mathrm{E}$ & \\
\hline 0359B & NEWSDW & 3552 & 035A4 & 857 & 0608 & \\
\hline 03552 & SECTOR & 3547 & $0355 B$ & 857 & $049 \mathrm{C}$ & \\
\hline 03547 & IOCB & $353 D$ & $0354 \mathrm{E}$ & 690 & 0054 & \\
\hline 0353D & EOF & $34 \mathrm{CC}$ & 03543 & $5 \mathrm{FB}$ & 0013 & \\
\hline $034 \mathrm{CC}$ & SPREADSCREEN & 3494 & $034 \mathrm{DB}$ & 857 & $049 \mathrm{C}$ & \\
\hline 03494 & INDEX & $344 \mathrm{~A}$ & $0349 C$ & 857 & $03 \mathrm{D} 5$ & \\
\hline $0344 \mathrm{~A}$ & ?SCREEN & 3401 & 03454 & 857 & 0608 & \\
\hline 03401 & ?INDEX & $33 \mathrm{E} 0$ & $0340 \mathrm{~A}$ & 857 & 0608 & \\
\hline $033 \mathrm{E} 0$ & ?ASCII & $33 \mathrm{CD}$ & $033 \mathrm{E} 9$ & 857 & $049 \mathrm{C}$ & \\
\hline $033 C D$ & ?SPACE & 3385 & 033D6 & 857 & 0149 & \\
\hline 03385 & VSHADOW & 3343 & $0338 \mathrm{~F}$ & 857 & $049 \mathrm{C}$ & \\
\hline 03343 & VCOPY & $332 B$ & $0334 B$ & 857 & $049 \mathrm{C}$ & \\
\hline $0332 B$ & VMOVE & $32 \mathrm{DI}$ & 03333 & 857 & 0149 & \\
\hline 032D1 & VOCAB? & $32 B 7$ & $032 \mathrm{DA}$ & 857 & 0734 & \\
\hline $032 \mathrm{~B} 7$ & SCREEN-DUMP & $325 B$ & $032 \mathrm{C5}$ & 857 & $16 \mathrm{DE}$ & \\
\hline $0325 B$ & DUMP-TERM & 3228 & 03267 & 857 & 0149 & \\
\hline
\end{tabular}

Woods Hole FORTH Version 1.800 Chapter 18

page 98 


\begin{tabular}{|c|c|c|c|c|c|c|}
\hline NFA & Vocal & $\begin{array}{l}\text { alary } \\
\text { aLFA }\end{array}$ & $\begin{array}{l}\text { list } \\
\text { CFA }\end{array}$ & QCFA & @PFA & \\
\hline 03228 &.$S$ & $320 \mathrm{E}$ & 0322D & 857 & 3216 & \\
\hline $0320 \mathrm{E}$ & DEPTH & $31 \mathrm{~F} 8$ & 03216 & 857 & 0286 & \\
\hline $031 \mathrm{~F} 8$ & ASSEMBLER & 31DI & 03204 & 857 & 0149 & Immediate \\
\hline 031D]. & DOS & $318 \mathrm{C}$ & 031D7 & 857 & 1906 & \\
\hline $0318 \mathrm{C}$ & V & 30FA & 03190 & 857 & 0759 & \\
\hline 030FA & $\mathrm{v}$ & 30D2 & 030FE & 857 & $2 F 92$ & \\
\hline 030D2 & $\mathrm{AH}->\mathrm{B}$ & $30 A 9$ & 030DA & 857 & $049 \mathrm{C}$ & \\
\hline 030A9 & SERPRINTER & $308 \mathrm{~F}$ & 030B6 & 857 & 2867 & \\
\hline $0308 \mathrm{~F}$ & CLEAR-KEY & $304 \mathrm{~A}$ & $0309 B$ & 857 & $2 \mathrm{~F} 5 \mathrm{C}$ & \\
\hline $0304 \mathrm{~A}$ & ?HEXCHAR & $303 A$ & 03055 & 857 & $049 C$ & \\
\hline $0303 \mathrm{~A}$ & $\mathrm{U} \ldots$ & $302 \mathrm{~A}$ & 03040 & 857 & 0608 & \\
\hline $0302 \mathrm{~A}$ & $\mathrm{CR}^{\prime}$ & $2 F F 7$ & 03030 & 857 & 0149 & \\
\hline $02 \mathrm{FF} 7$ & XMIT & $2 \mathrm{FBE}$ & $02 \mathrm{FFE}$ & 857 & 0759 & \\
\hline 02FBE & Xmit & $2 \mathrm{~F} 87$ & $02 \mathrm{FC} 5$ & 857 & $28 B 1$ & \\
\hline $02 F 87$ & ?MONITOR & $2 F 66$ & $02 F 92$ & 857 & OB4 I & \\
\hline $02 F 66$ & MONABORT & $2 \mathrm{~F} 55$ & $02 F 71$ & 857 & 2878 & \\
\hline $02 F 55$ & ?KEY & $2 F 3 B$ & $02 \mathrm{~F} 5 \mathrm{C}$ & 857 & 2836 & \\
\hline $02 F 3 B$ & CHANNEL-RESET & $2 \mathrm{~F} 20$ & $02 F 4 B$ & 857 & 2867 & \\
\hline $02 F 20$ & CHANNEL-SELECT' & $2 \mathrm{~F} 00$ & $02 F 31$ & 857 & 2867 & \\
\hline $02 \mathrm{~F} 00$ & CRS & 2EED & $02 \mathrm{~F} 06$ & 857 & 2ECB & \\
\hline 02EED & $\mathrm{CS}$ & $2 \mathrm{EC} 4$ & $02 \mathrm{EF} 2$ & 857 & $28 B 1$ & \\
\hline $02 \mathrm{EC} 4$ & $2 * * N$ & $2 \mathrm{E} 67$ & $02 \mathrm{ECB}$ & 857 & $049 C$ & \\
\hline $02 \mathrm{E} 67$ & GET-SCREEN & $2 \mathrm{E} 2 \mathrm{~F}$ & $02 \mathrm{E} 74$ & 857 & OB41 & \\
\hline $02 \mathrm{E} 2 \mathrm{~F}$ & SEND-SCREEN & $2 \mathrm{DF} 8$ & $02 \mathrm{E} 3 \mathrm{D}$ & 857 & $049 C$ & \\
\hline $02 \mathrm{DF} 8$ & ANSWER & 2DD6 & $02 \mathrm{E} 01$ & 857 & 2DE0 & \\
\hline $02 \mathrm{DD} 6$ & Ansinit & $2 \mathrm{D} 85$ & 02DE0 & 857 & 0149 & \\
\hline $02 \mathrm{D} 85$ & ORIGINATE & $2 D 54$ & 02D91 & 857 & 2856 & \\
\hline $02 \mathrm{D} 54$ & DIAL & $2 \mathrm{CFA}$ & 02D5B & 857 & $2 \mathrm{~A} 4 \mathrm{E}$ & \\
\hline $02 \mathrm{CFA}$ & RE-ENTER & 2BAI & $02 \mathrm{D} 05$ & 857 & $07 \mathrm{E} 2$ & \\
\hline 02BA1 & TONE & $2 B 54$ & 02BA8 & 857 & 0149 & \\
\hline $02 \mathrm{~B} 54$ & $\mathrm{TS}$ & $2 \mathrm{BOF}$ & $02 B 59$ & 857 & 2856 & \\
\hline $02 \mathrm{BOF}$ & ?CARRIER & 2A9D & 02BlA & 857 & 0608 & \\
\hline 02A9D & DIALTONE & $2 \mathrm{~A} 78$ & 02AA8 & 857 & $2 A 83$ & \\
\hline $02 \mathrm{~A} 78$ & DIALINIT & $2 \mathrm{~A} 5 \mathrm{E}$ & $02 A 83$ & 857 & 2856 & \\
\hline $02 \mathrm{~A} 5 \mathrm{E}$ & OFFHOOK & $2 A 45$ & $02 A 68$ & 857 & 2856 & \\
\hline $02 \mathrm{~A} 45$ & ONHOOK & $2 \mathrm{~A} 3 \mathrm{~B}$ & $02 \mathrm{~A} 4 \mathrm{E}$ & 857 & 2856 & \\
\hline $02 \mathrm{~A} 3 \mathrm{~B}$ & $\mathrm{U}$ & $29 F 3$ & $02 \mathrm{~A} 3 \mathrm{~F}$ & 857 & $29 F A$ & \\
\hline $029 F 3$ & UART & 29Dl & 029FA & 857 & OB4I & \\
\hline 029D1 & SENDCHAR? & $29 B 3$ & 029DD & 857 & $049 \mathrm{C}$ & \\
\hline 029B3 & SENDC & $298 F$ & $029 B B$ & 857 & 0149 & \\
\hline $0298 \mathrm{~F}$ & TRC & 2981 & 02995 & 857 & 08CF & \\
\hline 02981 & EMIT' & 2945 & 02989 & $298 B$ & E460 & Code \\
\hline 02945 & SENDBRK & 2926 & $0294 \mathrm{~F}$ & 857 & 0618 & \\
\hline 02926 & RST'BRK & 2905 & $0292 \mathrm{~F}$ & 857 & 2836 & \\
\hline 02905 & RCVCHAR? & $28 \mathrm{E} 2$ & 02910 & 857 & $049 C$ & \\
\hline $028 \mathrm{E} 2$ & RCVC & $28 B B$ & $028 \mathrm{E} 9$ & 857 & 0149 & \\
\hline $028 B B$ & RCVBRK & $28 \mathrm{~A} 6$ & $028 C 4$ & 857 & 0618 & \\
\hline $028 \mathrm{~A} 6$ & COMMINIT & 2891 & $028 \mathrm{BI}$ & 857 & $28 I A$ & \\
\hline 02891 & ESC' & 2882 & 02898 & 857 & 2867 & \\
\hline 02882 & CONS & 2871 & 02889 & 857 & 0610 & \\
\hline 02871 & TERM & 2860 & 02878 & 857 & 07FI & \\
\hline 02860 & COMM & $284 E$ & 02867 & 857 & 0800 & \\
\hline $0284 \mathrm{E}$ & MODEM & $283 E$ & 02856 & 857 & 07E2 & \\
\hline $0283 E$ & $\mathrm{CTL}=>$ & $282 \mathrm{D}$ & 02846 & 857 & 0620 & \\
\hline
\end{tabular}

Woods Hole FORTH Version 1.800 Chapter 18

page 99 


\begin{tabular}{|c|c|c|c|c|c|c|}
\hline NFA & Name & $\begin{array}{l}\operatorname{lary} \\
\text { QLFA }\end{array}$ & $\begin{array}{l}\text { list } \\
\text { CFA }\end{array}$ & QCFA & QPFA & \\
\hline $0282 \mathrm{D}$ & STATUS & $281 \mathrm{E}$ & 02836 & 857 & 0620 & \\
\hline $0281 \mathrm{E}$ & TERM.CTL & $280 F$ & 02829 & $5 F E$ & $001 D$ & \\
\hline $0280 \mathrm{~F}$ & COMM.CTL & 27EF & $0281 \mathrm{~A}$ & $5 \mathrm{FE}$ & $001 \mathrm{D}$ & \\
\hline $027 \mathrm{EF}$ & CLEAR & 27DD & $027 \mathrm{~F} 7$ & 857 & 0719 & \\
\hline $027 \mathrm{DD}$ & EDITL & $26 \mathrm{AE}$ & $027 \mathrm{E} 5$ & 857 & 0719 & \\
\hline 026AE & EDIT & $256 D$ & 026B5 & 857 & 2257 & \\
\hline $0256 \mathrm{D}$ & CUR & $255 \mathrm{~F}$ & 02573 & 690 & 0052 & \\
\hline $0255 \mathrm{~F}$ & L & 2553 & 02563 & 857 & 2257 & \\
\hline 02553 & $\mathrm{~T}$ & 2261 & 02557 & 857 & 2257 & \\
\hline 02261 & WHERE & $224 \mathrm{E}$ & 02269 & 857 & $049 \mathrm{C}$ & \\
\hline $0224 \mathrm{E}$ & EDITOR & $222 \mathrm{D}$ & 02257 & $10 \mathrm{Al}$ & 1191 & Immediate \\
\hline 0222D & LINE & $220 \mathrm{E}$ & 02234 & 857 & $049 \mathrm{C}$ & \\
\hline $0220 \mathrm{E}$ & TEXT & $21 D C$ & 02215 & 857 & $0 A 53$ & \\
\hline $021 \mathrm{DC}$ & ENDCASE & $21 B 6$ & $021 \mathrm{E} 6$ & 857 & 0149 & Immediate \\
\hline $021 \mathrm{~B} 6$ & ENDOF & $218 \mathrm{D}$ & $021 B E$ & 857 & 0149 & Immediate \\
\hline $0218 \mathrm{D}$ & $\mathrm{OF}$ & 2176 & 02192 & 857 & 0149 & Immediate \\
\hline 02176 & CASE & $216 \mathrm{~B}$ & $0217 D$ & 857 & $0 \mathrm{Cl} 18$ & Immediate \\
\hline $0216 \mathrm{~B}$ & RETQ & 2160 & 02172 & 2174 & $7 \mathrm{AD} 5$ & Code \\
\hline 02160 & SETQ & 2158 & 02167 & 2169 & 7BD5 & Code \\
\hline 02158 & WN4 & 2150 & $0215 \mathrm{E}$ & $8 D$ & 8453 & \\
\hline 02150 & WN3 & 2148 & 02156 & $8 \mathrm{~A}$ & 8357 & \\
\hline 02148 & WN2 & 2140 & $0214 \mathrm{E}$ & 87 & 8357 & \\
\hline 02140 & WN1 & 2139 & 02146 & 84 & 8357 & \\
\hline 02139 & W4 & 2132 & $0213 \mathrm{E}$ & 81 & 8357 & \\
\hline 02132 & W3 & $212 B$ & 02137 & $7 E$ & 8257 & \\
\hline $0212 B$ & W2 & 2124 & 02130 & $7 \mathrm{~B}$ & 8257 & \\
\hline 02124 & WI & $211 B$ & 02129 & 78 & 8257 & \\
\hline $0211 \mathrm{~B}$ & ?EF4 & 2112 & 02122 & $6 \mathrm{~A}$ & 8257 & \\
\hline 02112 & ?EF3 & 2109 & 02119 & 66 & $843 F$ & \\
\hline 02109 & ?EF2 & 2100 & 02110 & 62 & $843 F$ & \\
\hline 02100 & ?EFl & 2093 & 02107 & $5 \mathrm{E}$ & $843 F$ & \\
\hline 02093 & DUMP & $202 C$ & 0209A & 857 & 0759 & \\
\hline $0202 \mathrm{C}$ & - ASCII & 1FDC & 02035 & 857 & 04DI & \\
\hline $01 F D C$ & MATCH & IF9E & 0IFE4 & 857 & 0328 & \\
\hline OLF9E & Match & $1 \mathrm{EFE}$ & 01FA6 & 857 & 0516 & \\
\hline 01EFE & VIIST & $1 \mathrm{ED} 0$ & $01 F 06$ & 857 & 0759 & \\
\hline 01ED0 & Y? & 1E9D & 01ED5 & 857 & 0909 & \\
\hline 01E9D & ?Y/N & $1 \mathrm{E} 6 \mathrm{C}$ & 01EA4 & 857 & $08 \mathrm{CF}$ & \\
\hline $01 \mathrm{E} 6 \mathrm{C}$ & N. & $1 \mathrm{E} 2 \mathrm{E}$ & $01 E 71$ & 857 & 0759 & \\
\hline $01 E 2 E$ & ROMIT & $1 \mathrm{DC} 7$ & 01 E36 & 857 & $0 A 53$ & \\
\hline $01 D C 7$ & .FOOT & 1D93 & OIDCF & 857 & $07 C 8$ & \\
\hline $01 D 93$ & . VHDR & $1 D 47$ & OLD9B & 857 & OB4 I & \\
\hline $01 D 47$ & NEW-FENCE & $1 D 37$ & $01 D 53$ & 857 & $\mathrm{OBCl}$ & \\
\hline $01 D 37$ & CLS & IDIE & 0ID3D & 857 & 0149 & \\
\hline OIDIE & MSEC & ICFA & 01D25 & 857 & 0149 & \\
\hline OICFA & SEC & $1 \mathrm{CDI}$ & OID00 & 857 & 0149 & \\
\hline $01 \mathrm{CD} 1$ & ID & $1 \mathrm{CC} 7$ & $01 C D 6$ & 857 & 0149 & \\
\hline $01 \mathrm{CC} 7$ & ESC & 1CB9 & $01 \mathrm{CCD}$ & $5 \mathrm{FB}$ & $001 B$ & \\
\hline $01 \mathrm{CB} 9$ & CHAPTER & $1 \mathrm{CAB}$ & $01 \mathrm{CC} 3$ & 690 & 0050 & \\
\hline $01 C A B$ & BYE & $1 \mathrm{C97}$ & 01CBI & 857 & 1906 & \\
\hline $01 C 97$ & QUOTE & $1 \mathrm{C} 86$ & $01 \mathrm{C} 9 \mathrm{~F}$ & 857 & 0149 & \\
\hline $01 \mathrm{C} 86$ & ALLOTU & $1 C 57$ & $01 \mathrm{C} 8 \mathrm{~F}$ & 857 & $080 \mathrm{C}$ & \\
\hline $01 C 57$ & NEXTUSER & $1 C 45$ & $01 C 62$ & 857 & $080 \mathrm{C}$ & \\
\hline $01 C 45$ & WORD-79 & $1 C 39$ & $0 I C 4 F$ & 857 & ODFA & \\
\hline
\end{tabular}

Woods Hole FORTH Version 1.800 


\begin{tabular}{|c|c|c|c|c|c|c|}
\hline NFA & Name & $\begin{array}{l}\text { alary } \\
\text { QLFA }\end{array}$ & $\begin{array}{l}\text { list } \\
\text { CFA }\end{array}$ & QCFA & QPFA & \\
\hline $01 C 39$ & $>$ IN & $1 \mathrm{C} 2 \mathrm{D}$ & $01 \mathrm{C} 3 \mathrm{~F}$ & 857 & 0705 & \\
\hline $01 C 2 D$ & NOT & $1 \mathrm{BF} 8$ & $01 \mathrm{C} 33$ & 857 & 0352 & \\
\hline 01BF8 & COPY & IBBD & $01 B F F$ & 857 & 0726 & \\
\hline $01 B B D$ & CMOVE $>$ & IBBO & $01 B C 6$ & 857 & $049 C$ & \\
\hline $0 \perp B B 0$ & BEEP & $1 B 9 F$ & $01 B B 7$ & 857 & $1 \mathrm{BA} 6$ & \\
\hline $01 \mathrm{~B} 9 \mathrm{~F}$ & BELL & $1 \mathrm{~B} 80$ & $01 B A 6$ & 857 & 0149 & \\
\hline $01 \mathrm{~B} 80$ & NODISK & $1 \mathrm{~B} 67$ & 01B89 & 857 & 0149 & \\
\hline $01 B 67$ & DEC. & $1 \mathrm{~B} 4 \mathrm{E}$ & 01B6E & 857 & 0759 & \\
\hline $01 B 4 E$ & HEX. & $1 B 3 C$ & 01B55 & 857 & 0759 & \\
\hline $01 B 3 \mathrm{C}$ & U.RLZ & $1 \mathrm{BI} 6$ & 01B44 & 857 & 0608 & \\
\hline $01 \mathrm{~B} 16$ & D.RLZ & $1 \mathrm{AF} I$ & 01BIE & 857 & 0328 & \\
\hline $01 \mathrm{AFI}$ & ZEROES & $1 \mathrm{ADD}$ & 0IAFA & 857 & 0608 & \\
\hline $01 \mathrm{ADD}$ & 2 SWAP & IACE & 0IAE5 & 857 & $04 \mathrm{FE}$ & \\
\hline OIACE & 2DUP & $1 \mathrm{ABE}$ & $01 \mathrm{AD} 5$ & 857 & 0467 & \\
\hline 01ABE & 2DROP & $1 \mathrm{AB} 2$ & 01AC6 & 857 & $047 D$ & \\
\hline $01 \mathrm{AB} 2$ & ASMBR & 1 A95 & $01 \mathrm{ABA}$ & 857 & $02 \mathrm{CO}$ & \\
\hline 01A95 & I,OADTHRU & $1 \mathrm{~A} 26$ & $01 \mathrm{AAO}$ & 857 & 03D5 & \\
\hline $01 A 26$ & I.OAD & $1 \mathrm{~A} 16$ & $01 \mathrm{~A} 2 \mathrm{D}$ & 857 & 0CF2 & \\
\hline $01 \mathrm{~A} 16$ & $\mathrm{U} \cdot \mathrm{R}$ & $19 \mathrm{E} 8$ & 01AIC & 857 & 0608 & \\
\hline $019 \mathrm{E} 8$ & NOPRINT & $19 C 5$ & $019 F 2$ & 857 & 0149 & \\
\hline $019 C 5$ & PARPRINTER & 1969 & 019D2 & 857 & 0149 & \\
\hline 01969 & PRINTER & $194 \mathrm{~A}$ & 01973 & 857 & 0149 & \\
\hline $0194 \mathrm{~A}$ & serptr & $192 \mathrm{~A}$ & 01.953 & 1955 & D5E3 & Code \\
\hline $0192 \mathrm{~A}$ & parptr & $18 \mathrm{FE}$ & 01933 & 1935 & D5E3 & Code \\
\hline $018 \mathrm{FE}$ & FLUSH & $18 \mathrm{~B} 3$ & 01906 & 857 & 0655 & \\
\hline $018 \mathrm{~B} 3$ & LIST & $18 \mathrm{~A} 4$ & $018 \mathrm{BA}$ & 857 & OCF2 & \\
\hline $018 \mathrm{~A} 4$ & OK & 1892 & $018 \mathrm{~A} 9$ & 857 & OB41 & \\
\hline 01892 & (DOS) & 1883 & $0189 A$ & $189 \mathrm{C}$ & F890 & Code \\
\hline 01883 & MON & $186 \mathrm{D}$ & 01889 & $188 \mathrm{~B}$ & 9EFA & Code \\
\hline $0186 \mathrm{D}$ & DRI & $182 \mathrm{~F}$ & 01873 & 857 & $066 \mathrm{D}$ & \\
\hline $0182 \mathrm{~F}$ & FORTH & $181 \mathrm{~F}$ & 01837 & $10 \mathrm{Al}$ & 1191 & Immediate \\
\hline $0181 \mathrm{~F}$ & DRO & $17 \mathrm{FD}$ & 01825 & 857 & 0608 & \\
\hline $017 \mathrm{FD}$ & $-\rightarrow$ & $17 \mathrm{CA}$ & 01803 & 857 & $0 C 77$ & Immediate \\
\hline $017 \mathrm{CA}$ & LOAD & $17 \mathrm{~B} 4$ & $017 \mathrm{DI}$ & 857 & $06 \mathrm{FC}$ & \\
\hline $017 \mathrm{~B} 4$ & GROUP & $179 D$ & $017 \mathrm{BC}$ & $17 \mathrm{BE}$ & F845 & Code \\
\hline 0179D & CDER & $178 \mathrm{C}$ & $017 A 4$ & 857 & 1793 & \\
\hline $0178 \mathrm{C}$ & cder & 1736 & 01793 & 1795 & $248 D$ & Code \\
\hline 01736 & $\mathrm{R} / \mathrm{W}$ & $16 \mathrm{D} 6$ & $0173 \mathrm{C}$ & 857 & 0489 & \\
\hline $016 \mathrm{D} 6$ & BLOCK & $168 \mathrm{D}$ & 016DE & 857 & 0726 & \\
\hline 0168D & BUFFER & $166 \mathrm{~F}$ & 01696 & 857 & 0794 & \\
\hline $0166 \mathrm{~F}$ & EMPTY-BUFFERS & 1650 & $0167 \mathrm{~F}$ & 857 & 0649 & \\
\hline 01650 & UPDATE & 1625 & 01659 & 857 & 079F & \\
\hline 01625 & $+\mathrm{BUF}$ & 1613 & $0162 \mathrm{C}$ & 857 & 0661 & \\
\hline 01613 & . LINE & $15 \mathrm{EC}$ & $0161 \mathrm{~B}$ & 857 & $15 \mathrm{~F} 5$ & \\
\hline $015 \mathrm{EC}$ & (IINE) & $15 \mathrm{D} 7$ & $015 \mathrm{~F} 5$ & 857 & 0328 & \\
\hline 015D7 & $S->D$ & $15 \mathrm{AF}$ & 015DE & $15 \mathrm{E} 0$ & E404 & Code \\
\hline $015 \mathrm{AF}$ & MESSAGE & $15 \mathrm{~A} 2$ & $015 B 9$ & 857 & $0 B 41$ & \\
\hline 015A2 & U. & 1596 & $015 \mathrm{~A} 7$ & 857 & 0608 & \\
\hline 01596 & $?$ & $158 \mathrm{~A}$ & $0159 A$ & 857 & $04 \mathrm{BF}$ & \\
\hline $0158 \mathrm{~A}$ & - & $157 \mathrm{~B}$ & $0158 \mathrm{E}$ & 857 & $15 \mathrm{DE}$ & \\
\hline 0157B & D. & $156 \mathrm{~A}$ & 01580 & 857 & 0608 & \\
\hline $0156 \mathrm{~A}$ & . $\mathrm{R}$ & 1546 & $0156 \mathrm{~F}$ & 857 & 0328 & \\
\hline 01546 & $D \cdot R$ & $152 \mathrm{~F}$ & $0154 \mathrm{C}$ & 857 & 0328 & \\
\hline $0152 \mathrm{~F}$ & $\# S$ & 1505 & 01534 & 857 & 1509 & \\
\hline
\end{tabular}

Woods Hole FORTH Version 1.800

Chapter 18

page 101 


\begin{tabular}{|c|c|c|c|c|c|c|}
\hline NFA & Name & $\begin{array}{l}\text { lary } \\
\text { aLFA }\end{array}$ & $\begin{array}{l}\text { list } \\
\text { CFA }\end{array}$ & QCFA & @PFA & \\
\hline 01505 & \# & $14 \mathrm{EC}$ & 01509 & 857 & 0759 & \\
\hline $014 \mathrm{EC}$ & SIGN & $14 \mathrm{D} 5$ & $014 \mathrm{~F} 3$ & 857 & 04FE & \\
\hline $014 \mathrm{D} 5$ & $\#>$ & $14 \mathrm{C} 6$ & $014 \mathrm{DA}$ & 857 & 047D & \\
\hline $014 \mathrm{C6}$ & $<\#$ & $14 \mathrm{~A} 5$ & $014 \mathrm{CB}$ & 857 & OB5D & \\
\hline $014 \mathrm{~A} 5$ & SPACES & 1495 & OI AAE & 857 & 0608 & \\
\hline 01495 & WHILE & 1474 & $0149 D$ & 857 & 1464 & Immediate \\
\hline 01474 & ELSE & $145 \mathrm{~F}$ & $0147 B$ & 857 & 0618 & Immediate \\
\hline $0145 \mathrm{~F}$ & IF & 1442 & 01464 & 857 & $0 C 91$ & Immediate \\
\hline 01442 & REPEAT & $142 \mathrm{C}$ & $0144 \mathrm{~B}$ & 857 & 0328 & Immediate \\
\hline $0142 \mathrm{C}$ & AGAIN & 1420 & 01434 & 857 & 0610 & Immediate \\
\hline 01420 & END & $140 \mathrm{~A}$ & 01426 & 857 & 1412 & Immediate \\
\hline $0140 \mathrm{~A}$ & UNTIL & $13 F 4$ & 01412 & 857 & 0610 & Immediate \\
\hline $013 F 4$ & +LOOP & $13 \mathrm{DF}$ & $013 F C$ & 857 & 0620 & Immediate \\
\hline 013DF & LOOP & $13 \mathrm{CE}$ & $013 \mathrm{E} 6$ & 857 & 0620 & Immediate \\
\hline $013 \mathrm{CE}$ & DO & $13 \mathrm{Cl}$ & $013 \mathrm{D} 3$ & 857 & $0 C 91$ & Immediat \\
\hline $013 \mathrm{Cl}$ & THEN & 13A9 & $013 \mathrm{C} 8$ & 857 & $13 \mathrm{BI}$ & Immediate \\
\hline $013 A 9$ & ENDIF & 1397 & $013 \mathrm{Bl}$ & 857 & $0 \mathrm{Cl} 18$ & Immediat \\
\hline 01397 & BEGIN & $138 \mathrm{~A}$ & $0139 \mathrm{~F}$ & 857 & $0 \mathrm{Cl} 8$ & Immediat \\
\hline 0138A & BACK & $137 \mathrm{~B}$ & 01391 & 857 & 0 A 84 & \\
\hline 0137B & */ & 1367 & 01380 & 857 & $136 \mathrm{~F}$ & \\
\hline 01367 & */MOD & 1359 & $0136 \mathrm{~F}$ & 857 & 0328 & \\
\hline 01359 & MOD & $134 \mathrm{~B}$ & $0135 \mathrm{~F}$ & 857 & $133 \mathrm{~F}$ & \\
\hline 0134B & I & 1338 & $0] .34 \mathrm{~F}$ & 857 & $133 \mathrm{~F}$ & \\
\hline 01338 & $/ \mathrm{MOD}$ & 1311 & $0133 \mathrm{~F}$ & 857 & 0328 & \\
\hline 01311 & M/ & $12 \mathrm{D} 2$ & 01316 & 857 & 0467 & \\
\hline 012D2 & FORGET & $12 \mathrm{BE}$ & $012 \mathrm{DB}$ & 857 & 0742 & \\
\hline 012BE & 1 & $12 \mathrm{~B} 0$ & $012 \mathrm{C} 2$ & 857 & OEEE & Immediate \\
\hline $012 \mathrm{~B} 0$ & ( & 1298 & 012B4 & 857 & 0149 & Immediat \\
\hline 01298 & IMMEDIATE & $127 \mathrm{~A}$ & $012 \mathrm{~A} 4$ & 857 & $0 \mathrm{BCl}$ & \\
\hline 0127A & [ COMPILE] & $124 \mathrm{~B}$ & 01286 & 857 & OEEE & Immediat \\
\hline 0I24B & •" & 1239 & 01250 & 857 & 0149 & Immediat \\
\hline 01239 & ; & 11DE & $0123 \mathrm{D}$ & 857 & $0 \mathrm{C} 5 \mathrm{~A}$ & Immediat \\
\hline OIIDE & ABORT & $11 B 3$ & $011 \mathrm{E} 6$ & 857 & 0297 & \\
\hline 011B3 & QUIT & 1199 & $011 B A$ & 857 & 0608 & \\
\hline 01199 & DEFINITIONS & 1164 & $011 \mathrm{~A} 7$ & 857 & 0734 & \\
\hline 01164 & VOCABUI_ARY & 1114 & 01171 & 857 & 1085 & \\
\hline 01114 & INTERPRET & $10 \mathrm{E} 9$ & 01120 & 857 & OEEE & \\
\hline 010E9 & ?STACK & $10 \mathrm{CC}$ & $010 F 2$ & 857 & 06Al & \\
\hline $010 \mathrm{CC}$ & DLITERAL & 10B0 & 010D7 & 857 & $074 \mathrm{E}$ & Immediat \\
\hline 010B0 & LITERAL & $108 D$ & $010 \mathrm{BA}$ & 857 & $074 \mathrm{E}$ & Immediat \\
\hline 0108D & DOES> & $107 \mathrm{~B}$ & 01095 & 857 & 0336 & \\
\hline 0107B & <BUILDS & $106 \mathrm{~A}$ & 01085 & 857 & 0608 & \\
\hline $0106 \mathrm{~A}$ & USER & 1055 & 01071 & 857 & 0149 & \\
\hline 01055 & VARIABLE & 1040 & 01060 & 857 & 0149 & \\
\hline 01040 & CONSTANT & 1026 & 0104B & 857 & 0149 & \\
\hline 01026 & !CODE & 1002 & $0102 \mathrm{E}$ & 857 & OFA6 & \\
\hline 01002 & : & OF9D & 01006 & 857 & $0 C 30$ & Immediat \\
\hline OOF9D & CREATE & 0F69 & 00FA6 & 857 & 0286 & \\
\hline 00F69 & ID. & 0F51 & $00 \mathrm{~F} 6 \mathrm{~F}$ & 857 & OB5D & \\
\hline 00F51 & MIN & OF20 & $00 F 57$ & 857 & 0467 & \\
\hline 00F20 & ERROR & 0F10 & $00 F 28$ & 857 & $06 \mathrm{CE}$ & \\
\hline 00F10 & (ABORT) & 0EE6 & $00 F \perp A$ & 857 & $11 \mathrm{E} 6$ & \\
\hline 00EE6 & -FIND & OE8D & OOEEE & 857 & 0629 & \\
\hline 00E8D & NUMBER & OE40 & 00E96 & 857 & 0608 & \\
\hline
\end{tabular}

Woods Hole FORTH Version $1.800 \quad$ Chapter 18

page 102 


\begin{tabular}{|c|c|c|c|c|c|c|}
\hline NFA & Name & $\begin{array}{r}\text { ulary } \\
\text { OLFA }\end{array}$ & $\begin{array}{l}\text { list } \\
\text { CFA }\end{array}$ & @CFA & @PFA & \\
\hline 00E40 & (NUMBER) & ODF3 & $00 E 4 B$ & 857 & $03 D 5$ & \\
\hline 00DF3 & WORD & 0DB9 & OODFA & 857 & $06 \mathrm{FC}$ & \\
\hline 00DB9 & & OD9D & OODBD & 857 & $06 \mathrm{FC}$ & Immediate \\
\hline OOD9D & QUERY & OD2C & 00DA5 & 857 & $06 \mathrm{~B} 4$ & \\
\hline 00D2C & EXPECT & $0 D 16$ & 00D35 & 857 & 0467 & \\
\hline 00D16 & ;CODE & OCFE & 00DIE & 857 & $0 \mathrm{C} 5 \mathrm{~A}$ & Immediate \\
\hline OOCFE & $(; \mathrm{CODE})$ & OCE8 & 00D08 & 857 & 0336 & \\
\hline 00CE8 & DECIMAL & $0 \mathrm{CD} 6$ & $00 \mathrm{CF} 2$ & 857 & 0149 & \\
\hline $00 \mathrm{CD} 6$ & $\mathrm{HEX}$ & $\mathrm{OCCl}$ & $00 \mathrm{CDC}$ & 857 & 0149 & \\
\hline $00 \mathrm{CCI}$ & SMUDGE & OCBI & OOCCA & 857 & OBCI & \\
\hline $00 \mathrm{CBI}$ & ] & $0 \mathrm{CA} 3$ & $00 \mathrm{CB} 5$ & 857 & 0149 & \\
\hline $00 C A 3$ & {[} & $0 C 87$ & 00CA7 & 857 & 0608 & Immediate \\
\hline $00 C 87$ & COMPILE & $0 \mathrm{C} 6 \mathrm{C}$ & $00 C 91$ & 857 & $0 \mathrm{Cl} 18$ & \\
\hline $00 \mathrm{C} 6 \mathrm{C}$ & ?LOADING & $0 C 53$ & $00 C 77$ & 857 & $06 \mathrm{FC}$ & \\
\hline $00 C 53$ & ?CSP & $0 C 3 E$ & $00 \mathrm{C} 5 \mathrm{~A}$ & 857 & 0286 & \\
\hline $00 C 3 E$ & ?PAIRS & $0 C 28$ & $00 C 47$ & 857 & 0405 & \\
\hline $00 C 28$ & ?EXEC & $0 \mathrm{ClO}$ & $00 c 30$ & 857 & $074 \mathrm{E}$ & \\
\hline $00 \mathrm{ClO}$ & ?COMP & $0 \mathrm{BF} 5$ & $00 \mathrm{Cl} 18$ & 857 & $074 \mathrm{E}$ & \\
\hline 00BF5 & ?ERROR & OBEI & OOBFE & 857 & 0489 & \\
\hline 00BEI. & PFA & $O B C B$ & 00BE7 & 857 & 0610 & \\
\hline OOBCB & NFA & OBB8 & 00BDI & 857 & 0149 & \\
\hline 00BB8 & LATEST & OB91 & $00 \mathrm{BCl}$ & 857 & 0742 & \\
\hline 00B91 & TRAVERSE & 0B82 & 00B9C & 857 & 0489 & \\
\hline 00B82 & $\mathrm{D}+-$ & $0 B 74$ & 00B88 & B8A & 4414 & Code \\
\hline OOB74 & +- & $0 B 57$ & 00B79 & B7B & 4414 & Code \\
\hline 00B57 & $\mathrm{PAD}$ & OB3A & 00B5D & B5F & E2F8 & Code \\
\hline OOB3A & $(. ")$ & OBIO & 00B4I & B43 & 4852 & Code \\
\hline OOBIO & -TRAILING & OAFB & 0OBIC & $\mathrm{BlE}$ & $686 \mathrm{~B}$ & Code \\
\hline OOAFB & COUNT & OAE7 & $00 \mathrm{~B} 03$ & B05 & $686 \mathrm{~B}$ & Code \\
\hline 00AE7 & !CSP & OADF & OOAEE & AFO & E2F8 & Code \\
\hline 00ADF & CFA & $0 A D 3$ & 00AE5 & $\mathrm{ACF}$ & 8421 & \\
\hline $00 A D 3$ & LFA & $0 A C 8$ & 00AD9 & $\mathrm{ADB}$ & F804 & Code \\
\hline 00AC8 & $2-$ & $0 A B 7$ & OOACD & $\mathrm{ACF}$ & $\mathrm{F} 802$ & Code \\
\hline $00 \mathrm{AB} 7$ & $1-$ & $0 A 9 F$ & OOABC & $A B E$ & F801 & Code \\
\hline 00A9F & $\mathrm{C}_{\text {r }}$ & $0 A 80$ & 00AA4 & AA 6 & E2F 8 & Code \\
\hline 00A80 & , & $0 \AA 64$ & $00 A 84$ & A86 & E2F8 & Code \\
\hline 00A64 & ALLOT & OA $4 \mathrm{C}$ & $00 A 6 C$ & $\mathrm{~A} 6 \mathrm{E}$ & E2F 8 & Code \\
\hline $00 \mathrm{~A} 4 \mathrm{C}$ & HERE & $09 F F$ & 00A53 & A55 & E2F8 & Code \\
\hline 009FF & $\mathrm{M} / \mathrm{MOD}$ & $09 \mathrm{CD}$ & $00 A 07$ & A09 & $686 \mathrm{~F}$ & Code \\
\hline 009CD & BLOCK-WRITE & $09 A 5$ & 009DB & 9DD & $686 \mathrm{~F}$ & Code \\
\hline 009A5 & BLOCK-READ & $095 B$ & 009B2 & $9 B 4$ & $686 \mathrm{~F}$ & Code \\
\hline 0095B & SPACE & $092 \mathrm{C}$ & 00963 & 965 & $24 \mathrm{~F} 8$ & Code \\
\hline $0092 \mathrm{C}$ & TYPE & 0904 & 00933 & 935 & E2F8 & Code \\
\hline 00904 & $\mathrm{CR}$ & $08 F 5$ & 00909 & $90 B$ & $24 \mathrm{~F} 8$ & Code \\
\hline $008 \mathrm{F5}$ & EMIT & $08 D E$ & $008 \mathrm{FC}$ & $8 \mathrm{FE}$ & E268 & Code \\
\hline $008 \mathrm{DE}$ & ?TERMINAL & $08 C 9$ & 008EA & $8 \mathrm{EC}$ & $246 B$ & Code \\
\hline $008 C 9$ & KEY & 08AA & $008 \mathrm{CF}$ & 8D1 & $246 B$ & Code \\
\hline 008AA & HOLD & $088 \mathrm{~F}$ & 008BI & $8 B 3$ & $\mathrm{E} 2 \mathrm{~F} 8$ & Code \\
\hline $0088 \mathrm{~F}$ & BI_ANKS & $087 \mathrm{E}$ & 00898 & $89 A$ & F820 & Code \\
\hline $0087 \mathrm{E}$ & ERASE & 0861 & 00886 & 888 & F800 & Code \\
\hline 00861 & FIII & 0835 & 00868 & $86 A$ & $686 \mathrm{~F}$ & Code \\
\hline 00835 & INPUT & 0810 & 0083D & $83 F$ & E222 & Code \\
\hline 00810 & OUTPUT & 0804 & 00819 & $81 \mathrm{~B}$ & 1468 & Code \\
\hline 00804 & Nextu & $07 \mathrm{~F} 5$ & $0080 \mathrm{C}$ & 690 & $001 \mathrm{E}$ & \\
\hline
\end{tabular}

Woods Hole FORTH Version 1.800 Chapter 18

page 103 


\begin{tabular}{|c|c|c|c|c|c|}
\hline NFA & Name & $\begin{array}{l}\text { lary } \\
\text { QLFA }\end{array}$ & $\begin{array}{l}\text { list } \\
\text { CFA }\end{array}$ & QCFA & QPFA \\
\hline $007 F 5$ & COMM.GRP & $07 \mathrm{E} 6$ & 00800 & 690 & $001 \mathrm{C}$ \\
\hline $007 \mathrm{E} 6$ & TERM.GRP & 07D6 & $007 \mathrm{Fl}$ & 690 & $001 \mathrm{~A}$ \\
\hline $007 D 6$ & MODEM.GRP & $07 \mathrm{CC}$ & $007 \mathrm{E} 2$ & 690 & 0018 \\
\hline $007 \mathrm{CC}$ & GRP & $07 \mathrm{Cl}$ & 007D2 & 690 & 0044 \\
\hline $007 \mathrm{CI}$ & OUTL & $07 \mathrm{~B} 3$ & $007 C 8$ & 690 & 0042 \\
\hline $007 \mathrm{~B} 3$ & SCR/DSK & $07 \mathrm{~A} 3$ & $007 B D$ & 690 & 0016 \\
\hline $007 A 3$ & USER-IOCB & 0798 & $007 \mathrm{AF}$ & 690 & 0036 \\
\hline 00798 & PREV & $078 \mathrm{E}$ & $0079 \mathrm{~F}$ & 690 & 0034 \\
\hline $0078 \mathrm{E}$ & USE & 0784 & 00794 & 690 & 0032 \\
\hline 00784 & HLD & $077 \mathrm{~B}$ & $0078 \mathrm{~A}$ & 690 & 0030 \\
\hline $0077 \mathrm{~B}$ & R\# & 0771 & 00780 & 690 & $002 \mathrm{E}$ \\
\hline 00771 & CSP & 0767 & 00777 & 690 & $002 \mathrm{C}$ \\
\hline 00767 & FLD & $075 \mathrm{D}$ & $0076 \mathrm{D}$ & 690 & $002 \mathrm{~A}$ \\
\hline $0075 \mathrm{D}$ & $\mathrm{DPL}$ & 0752 & 00763 & 690 & 0028 \\
\hline 00752 & BASE & 0746 & 00759 & 690 & 0026 \\
\hline 00746 & STATE & 0738 & $0074 \mathrm{E}$ & 690 & 0024 \\
\hline 00738 & CURRENT & $072 \mathrm{~A}$ & 00742 & 690 & 0022 \\
\hline $0072 \mathrm{~A}$ & CONTEXT & $071 D$ & 00734 & 690 & 0020 \\
\hline $0071 \mathrm{D}$ & OFFSET & 0713 & 00726 & 690 & $004 \mathrm{E}$ \\
\hline 00713 & $\mathrm{SCR}$ & 0709 & 00719 & 690 & $004 \mathrm{C}$ \\
\hline 00709 & OUT & 0700 & $0070 \mathrm{~F}$ & 690 & $004 \mathrm{~A}$ \\
\hline 00700 & IN & $06 \mathrm{~F} 6$ & 00705 & 690 & 0048 \\
\hline $006 \mathrm{~F} 6$ & BLK & $06 \mathrm{E7}$ & $006 \mathrm{FC}$ & 690 & 0040 \\
\hline 006E7 & VOC-LINK & $06 \mathrm{DE}$ & $006 F 2$ & 690 & 0014 \\
\hline $006 \mathrm{DE}$ & $\mathrm{DP}$ & $06 \mathrm{D} 2$ & $006 E 3$ & 690 & 0012 \\
\hline 006D2 & FENCE & $06 C 4$ & $006 \mathrm{DA}$ & 690 & 0010 \\
\hline $006 C 4$ & WARNING & $06 \mathrm{~B} 8$ & $006 \mathrm{CE}$ & 690 & OOOE \\
\hline $006 \mathrm{~B} 8$ & WIDTH & $06 \mathrm{AE}$ & $006 \mathrm{C} 0$ & 690 & $000 \mathrm{C}$ \\
\hline $006 \mathrm{AE}$ & TIB & $06 A 5$ & 006B4 & 690 & OOOA \\
\hline $006 A 5$ & RO & $069 \mathrm{C}$ & $006 A A$ & 690 & 0008 \\
\hline $0069 \mathrm{C}$ & so & $067 \mathrm{E}$ & $006 \mathrm{Al}$ & 690 & 0006 \\
\hline $0067 \mathrm{E}$ & +ORIGIN & 0671 & 00688 & 857 & $067 \mathrm{~A}$ \\
\hline 00671 & ORIGIN & 0665 & $0067 A$ & $5 \mathrm{FB}$ & 1841 \\
\hline 00665 & $\mathrm{~B} / \mathrm{SCR}$ & 0659 & $0066 \mathrm{D}$ & $5 \mathrm{FB}$ & 0001 \\
\hline 00659 & B/BUF & $064 \mathrm{D}$ & 00661 & $5 \mathrm{FB}$ & 0400 \\
\hline $0064 \mathrm{D}$ & LIMIT & 0641 & 00655 & $5 \mathrm{FB}$ & $7 \mathrm{FFF}$ \\
\hline 00641 & FIRST & 0637 & 00649 & $5 \mathrm{FB}$ & $57 D 7$ \\
\hline 00637 & $\mathrm{~L} / \mathrm{P}$ & $062 \mathrm{D}$ & $0063 \mathrm{D}$ & $5 \mathrm{FB}$ & $003 C$ \\
\hline $0062 \mathrm{D}$ & $\mathrm{C} / \mathrm{L}$ & 0624 & 00633 & $5 \mathrm{FB}$ & 0040 \\
\hline 00624 & $\mathrm{BL}$ & $061 C$ & 00629 & $5 \mathrm{FB}$ & 0020 \\
\hline $0061 \mathrm{C}$ & 3 & 0614 & 00620 & $5 \mathrm{FB}$ & 0003 \\
\hline 00614 & 2 & $060 \mathrm{C}$ & 00618 & $5 \mathrm{FB}$ & 0002 \\
\hline $0060 \mathrm{C}$ & 1 & 0604 & 00610 & $5 \mathrm{FB}$ & 0001 \\
\hline 00604 & 0 & 0571 & 00608 & $5 \mathrm{FB}$ & 0000 \\
\hline 00571 & $M^{*}$ & 0563 & 00576 & 578 & E268 \\
\hline 00563 & * & 0541 & 00567 & 569 & E268 \\
\hline 00541 & MAX & 0532 & 00547 & 549 & $686 \mathrm{~A}$ \\
\hline 00532 & DABS & 0524 & 00539 & $53 \mathrm{~B}$ & $04 \mathrm{FE}$ \\
\hline 00524 & ABS & $050 \mathrm{~F}$ & $0052 \mathrm{~A}$ & $52 \mathrm{C}$ & $04 \mathrm{FE}$ \\
\hline $0050 \mathrm{~F}$ & -DUP & $04 \mathrm{~F} 8$ & 00516 & 518 & $686 \mathrm{~A}$ \\
\hline $004 \mathrm{~F} 8$ & ROT & $04 \mathrm{~EB}$ & $004 \mathrm{FE}$ & 500 & $686 A$ \\
\hline $004 \mathrm{~EB}$ & C! & 04DC & $004 \mathrm{~F} 0$ & $4 F 2$ & $686 A$ \\
\hline $004 \mathrm{DC}$ & $!$ & $04 \mathrm{CC}$ & $004 \mathrm{E} 0$ & $4 \mathrm{E} 2$ & $686 A$ \\
\hline $004 C C$ & $\mathrm{c}$ & $04 \mathrm{BB}$ & $004 \mathrm{DI}$ & 4D3 & $686 \mathrm{~A}$ \\
\hline
\end{tabular}




\begin{tabular}{|c|c|c|c|c|c|c|}
\hline NFA & Name & $\begin{array}{l}\text { גary } \\
\text { @LFA }\end{array}$ & $\begin{array}{l}\text { ist } \\
\text { CFA }\end{array}$ & @CFA & QPFA & \\
\hline $004 \mathrm{BB}$ & a & $04 A 7$ & $004 \mathrm{BF}$ & $4 \mathrm{Cl}$ & $686 \mathrm{~A}$ & Code \\
\hline $004 \mathrm{~A} 7$ & TOGGLE & 0496 & $004 \mathrm{BO}$ & $4 B 2$ & $686 \mathrm{~A}$ & Code \\
\hline 00496 & DUP & 0482 & $0049 C$ & $49 \mathrm{E}$ & $686 \mathrm{~A}$ & Code \\
\hline 00482 & SWAP & 0476 & 00489 & $48 B$ & $686 A$ & Code \\
\hline 00476 & DROP & 0460 & $0047 D$ & $47 F$ & 1414 & Code \\
\hline 00460 & OVER & 0444 & 00467 & 469 & EB68 & Code \\
\hline 00444 & DMINUS & $042 \mathrm{~A}$ & $0044 \mathrm{D}$ & $44 F$ & 1414 & Code \\
\hline $0042 \mathrm{~A}$ & MINUS & 0411 & 00432 & 434 & $14 \mathrm{FF}$ & Code \\
\hline 00411 & $\mathrm{D}+$ & 0401 & 00416 & 418 & $686 A$ & Code \\
\hline 00401 & - & 03ED & 00405 & 407 & $686 A$ & Code \\
\hline 003ED & $+!$ & 03E2 & $003 F 2$ & $3 F 4$ & $686 B$ & Code \\
\hline $003 \mathrm{E} 2$ & $2+$ & 03D0 & $003 E 7$ & $3 \mathrm{E} 9$ & $\mathrm{~F} 802$ & Code \\
\hline 003D0 & It & $03 \mathrm{CO}$ & $003 D 5$ & $3 \mathrm{D} 7$ & F801 & Code \\
\hline $003 C 0$ & + & 03AA & $003 C 4$ & $3 \mathrm{C} 6$ & $686 \mathrm{~A}$ & Code \\
\hline 003AA & $>$ & $038 \mathrm{C}$ & 003AE & $3 \mathrm{~B} 0$ & $686 \mathrm{~A}$ & Code \\
\hline $0038 \mathrm{C}$ & $<$ & $036 \mathrm{~F}$ & 00390 & 392 & $686 \mathrm{~A}$ & Code \\
\hline $0036 \mathrm{~F}$ & $=$ & 0362 & 00373 & 375 & $686 \mathrm{~A}$ & Code \\
\hline 00362 & $0<$ & $034 \mathrm{D}$ & 00367 & 369 & $44 \mathrm{FE}$ & Code \\
\hline 0034D & $0=$ & 0342 & 00352 & 354 & $44 \mathrm{FI}$ & Code \\
\hline 00342 & $I$ & 0331 & 00346 & 348 & 2468 & Code \\
\hline 00331 & $R>$ & 0323 & 00336 & 338 & 2468 & Code \\
\hline 00323 & $>\mathrm{R}$ & 0313 & 00328 & $32 \mathrm{~A}$ & E268 & Code \\
\hline 00313 & LEAVE & $02 \mathrm{~F} 4$ & $0031 \mathrm{~B}$ & 3ID & E212 & Code \\
\hline $002 \mathrm{~F} 4$ & CMOVE & $02 \mathrm{CB}$ & $002 \mathrm{FC}$ & $2 \mathrm{FE}$ & $44 F C$ & Code \\
\hline $002 \mathrm{CB}$ & DIGIT & $02 B B$ & $002 D 3$ & 2D5 & $686 \mathrm{~A}$ & Code \\
\hline $002 \mathrm{BB}$ & ;S & $02 \mathrm{~A} 5$ & $002 \mathrm{CO}$ & $2 \mathrm{C} 2$ & E868 & Code \\
\hline 002A5 & RP! & 0291 & $002 A B$ & $2 A D$ & $87 \mathrm{FC}$ & code \\
\hline 00291 & SP! & 0280 & 00297 & 299 & $87 \mathrm{FC}$ & Code \\
\hline 00280 & SPC & $026 \mathrm{E}$ & 00286 & 288 & EA68 & Code \\
\hline $0026 \mathrm{E}$ & XOR & $025 \mathrm{D}$ & 00274 & 276 & $686 A$ & Code \\
\hline $0025 \mathrm{D}$ & $\mathrm{OR}$ & $024 \mathrm{~B}$ & 00262 & 264 & $686 \mathrm{~A}$ & Code \\
\hline $0024 B$ & AND & 0235 & 00251 & 253 & $686 A$ & Code \\
\hline 00235 & $\mathrm{U}^{*}$ & OLFF & $0023 \mathrm{~A}$ & $23 c$ & $686 A$ & Code \\
\hline $001 \mathrm{FF}$ & $\mathrm{U} /$ & 01ED & 00204 & 206 & $686 \mathrm{~F}$ & Code \\
\hline 001ED & EXECUTE & $018 \mathrm{C}$ & $001 F 7$ & $1 \mathrm{~F} 9$ & 6869 & Code \\
\hline $0018 \mathrm{C}$ & ENCLOSE & 0178 & 00196 & 198 & 1414 & Code \\
\hline 00178 & OBRANCH & 0169 & 00182 & 184 & $44 \mathrm{Fl}$ & Code \\
\hline 00169 & BRANCH & 0154 & 00172 & 174 & E868 & Code \\
\hline 00154 & (DO) & 0143 & $0015 B$ & $15 \mathrm{D}$ & E268 & Code \\
\hline 00143 & LIT & 00EC & 00149 & $14 \mathrm{~B}$ & E868 & Code \\
\hline $000 \mathrm{EC}$ & (FIND) & $00 C 9$ & $000 \mathrm{~F} 5$ & F7 & $686 \mathrm{~A}$ & Code \\
\hline $000 C 9$ & $(+\mathrm{LOOP})$ & 00A5 & 000D3 & D5 & 1486 & Code \\
\hline 000A5 & (LOOP) & 0055 & $000 \mathrm{AE}$ & B0 & $16 \mathrm{E} 2$ & Code \\
\hline 00055 & $\mathrm{EF}$ & $002 \mathrm{D}$ & $0005 A$ & $5 \mathrm{FB}$ & $005 \mathrm{E}$ & \\
\hline $0002 \mathrm{D}$ & WARM & 000D & 00034 & 36 & F810 & Code \\
\hline $0000 \mathrm{D}$ & COLD & 0000 & 00014 & 16 & $68 \mathrm{CB}$ & Code \\
\hline
\end{tabular}




\section{Memory Dump}

This chapter consists of a memory dump of the program WFORTH.CM, including additional documentation words. It is generated by the following sequence:

PRINTER $\underline{0}$ HERE DUMP NOPRINT (cr) 
$\begin{array}{lllllllllllllllll}00000 & \mathrm{C} 0 & 00 & 05 & 30 & 36 & 71 & 00 & 61 & 01 & 63 & 1 \mathrm{D} & 30 & 16 & 84 & 43 & 4 \mathrm{~F}\end{array}$ $\begin{array}{llllllllllllllllll}00010 & 4 \mathrm{C} & \mathrm{C} 4 & 00 & 00 & 00 & 16 & 68 & \mathrm{CB} & 18 & 3 \mathrm{D} & 68 & \mathrm{CA} & 18 & 4 \mathrm{D} & 4 \mathrm{~A} & 5 \mathrm{~B}\end{array}$ $\begin{array}{lllllllllllllllll}00020 & \text { IB } & 4 \mathrm{~A} & 5 \mathrm{~B} & \text { 1B } & \mathrm{F} 8 & 00 & 5 \mathrm{~B} & \text { IB } & 5 \mathrm{~B} & \mathrm{~F} 8 & 20 & 30 & 38 & 84 & 57 & 41\end{array}$ $\begin{array}{lllllllllllllllll}00030 & 52 & \mathrm{CD} & 00 & 0 \mathrm{D} & 00 & 36 & \mathrm{~F} 8 & 10 & \mathrm{AF} & 68 & \mathrm{CA} & 18 & 41 & \mathrm{FA} & 68 & 62\end{array}$ $\begin{array}{llllllllllllllllll}00040 & 68 & 64 & 68 & 65 & 68 & 66 & 68 & 67 & 68 & 68 & \mathrm{~EB} & 68 & \mathrm{~B} 7 & 4 \mathrm{~A} & 5 \mathrm{~B} & 1 \mathrm{~B}\end{array}$ $\begin{array}{lllllllllllllllllllllll}00050 & 2 \mathrm{~F} & 8 \mathrm{~F} & 3 \mathrm{~A} & 4 \mathrm{D} & \mathrm{D} 5 & 82 & 45 & \mathrm{C} 6 & 00 & 2 \mathrm{D} & 05 & \mathrm{FB} & 00 & 5 \mathrm{E} & 3 \mathrm{C} & 6 \mathrm{~F}\end{array}$ $\begin{array}{lllllllllllllllll}00060 & 30 & 6 \mathrm{C} & 3 \mathrm{D} & 6 \mathrm{~F} & 30 & 6 \mathrm{C} & 3 \mathrm{E} & 6 \mathrm{~F} & 30 & 6 \mathrm{C} & 3 \mathrm{~F} & 6 \mathrm{~F} & \mathrm{~F} 8 & 01 & \mathrm{C} 8 & \mathrm{~F} 8\end{array}$ $\begin{array}{lllllllllllllllll}00070 & 00 & 24 & 54 & 24 & \mathrm{~F} 8 & 00 & 54 & \mathrm{D} 5 & 3 \mathrm{C} & 78 & \mathrm{D} 5 & 3 \mathrm{D} & 7 \mathrm{~B} & \mathrm{D} 5 & 3 \mathrm{E} & 7 \mathrm{E}\end{array}$ $\begin{array}{lllllllllllllllll}00080 & \text { D5 } & 3 \mathrm{~F} & 81 & \mathrm{D} 5 & 34 & 84 & \mathrm{D} 5 & 35 & 87 & \mathrm{D} 5 & 36 & 8 \mathrm{~A} & \mathrm{D} 5 & 37 & 8 \mathrm{D} & \mathrm{D} 5\end{array}$ $\begin{array}{lllllllllllllllll}00090 & \text { E8 } & 68 & 69 & \text { E9 } & 68 & 63 & \text { E4 } & \text { D3 } & 30 & 90 & \text { E3 } & 68 & \text { B6 } & \text { E2 } & 12 & 68\end{array}$ $\begin{array}{lllllllllllllllll}000 A 0 & 66 & 22 & \text { D3 } & 30 & 9 A & 86 & 28 & 4 \mathrm{C} & 4 \mathrm{~F} & 4 \mathrm{~F} & 50 & \mathrm{~A} 9 & 00 & 55 & 00 & \mathrm{~B} 0\end{array}$ $\begin{array}{lllllllllllllllll}000 B 0 & 16 & E 2 & 12 & 12 & 86 & F 7 & 22 & 96 & 77 & F E & 3 B & C 1 & 22 & E 8 & 68 & 68\end{array}$ $\begin{array}{lllllllllllllllll}000 \mathrm{C} 0 & \mathrm{D} 5 & 18 & 18 & 12 & 12 & 68 & 66 & 22 & \mathrm{D} 5 & 87 & 28 & 2 \mathrm{~B} & 4 \mathrm{C} & 4 \mathrm{~F} & 4 \mathrm{~F} & 50\end{array}$ 000D0 A9 00 A5 00 D5 $14 \quad 86$ F4 $146 \begin{array}{llllllll}24 & 96 & 74 & \text { B6 } & 44 & 14 & \text { E2 }\end{array}$

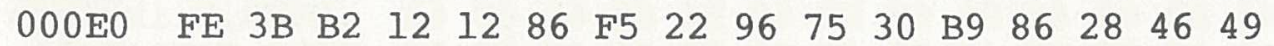
000F0 4E 44 A9 00 C9 00 F7 68 6A 68 6B 24 FA $0 A \begin{array}{llllll}52 & 4 B\end{array}$

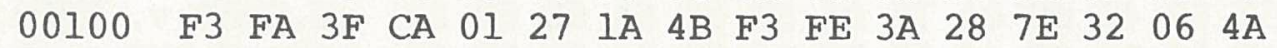
$\begin{array}{lllllllllllllllll}00110 & \text { FA } & 80 & 32 & \text { OF } & \text { E4 } & 8 A & \text { FC } & 04 & 73 & 9 A & 7 C & 00 & 73 & 02 & 73 & \text { F8 }\end{array}$ $\begin{array}{lllllllllllllllll}00120 & 00 & 73 & \text { F8 } & \text { FF } & 73 & 54 & \text { D5 } & 1 A & 4 A & F A & 80 & 32 & 28 & 0 A & 3 A & 3 B\end{array}$ $\begin{array}{lllllllllllllllll}00130 & 1 A & 0 A & 2 A & 3 A & 3 B & F 8 & 00 & E 4 & 73 & 54 & D 5 & E A & 68 & 6 A & 24 & E 4\end{array}$ $\begin{array}{lllllllllllllllll}00140 & \text { C0 } & 00 & \text { F9 } & 83 & 4 \mathrm{C} & 49 & \text { D4 } & 00 & \text { EC } & 01 & 4 B & \text { E8 } & 68 & 6 A & \text { E4 } & 24\end{array}$ $\begin{array}{lllllllllllllllll}00150 & 68 & A A & 14 & D 5 & 84 & 28 & 44 & 4 F & A 9 & 01 & 43 & 01 & 5 D & E 2 & 68 & \text { A6 }\end{array}$ $\begin{array}{llllllllllllllllll}00160 & \mathrm{E} 4 & 68 & 66 & 68 & 6 \mathrm{~B} & \mathrm{E} 2 & 68 & \mathrm{AB} & \mathrm{D} 5 & 86 & 42 & 52 & 41 & 4 \mathrm{E} & 43 & \mathrm{C} 8\end{array}$ $\begin{array}{lllllllllllllllll}00170 & 01 & 54 & 01 & 74 & \mathrm{E} 8 & 68 & 68 & \mathrm{D} 5 & 87 & 30 & 42 & 52 & 41 & 4 \mathrm{E} & 43 & \mathrm{C} 8\end{array}$ $\begin{array}{lllllllllllllllll}00180 & 01 & 69 & 01 & 84 & 44 & \mathrm{FI} & 14 & 32 & 74 & 18 & 18 & \mathrm{D} 5 & 87 & 45 & 4 \mathrm{E} & 43\end{array}$ $\begin{array}{lllllllllllllllll}00190 & 4 \mathrm{C} & 4 \mathrm{~F} & 53 & \mathrm{C} 5 & 01 & 78 & 01 & 98 & 14 & 14 & 68 & 6 \mathrm{~B} & 24 & 24 & 24 & 04\end{array}$ 001A0 52 E2 F8 00 AA BA BC $0 B$ F7 $3 A$ AF $1 B$ IA 30 A7 $\begin{array}{lllllllllllllllll}001 B 0 & 8 A & 73 & A C & 9 A & 73 & B C & F 8 & 00 & 73 & 73 & 73 & 54 & 14 & E 2 & 0 B & 32\end{array}$ $\begin{array}{lllllllllllllllll}001 \mathrm{C} 0 & \mathrm{C} 8 & \mathrm{~F} 7 & 32 & \mathrm{E} 3 & 1 \mathrm{~B} & 1 \mathrm{~A} & 30 & \mathrm{BE} & 8 \mathrm{~A} & \mathrm{E} 4 & 73 & 9 \mathrm{~A} & 54 & 60 & 8 \mathrm{C} & \mathrm{F} 7\end{array}$ $\begin{array}{lllllllllllllllll}001 D 0 & 3 A & D 9 & 24 & 9 C & F 7 & 14 & 3 A & D 9 & 1 A & 9 A & 14 & 54 & 14 & 8 A & 54 & 24\end{array}$ $\begin{array}{lllllllllllllllll}001 E 0 & 24 & 24 & \text { D5 } & 1 A & 8 A & 54 & 24 & 9 A & 54 & 14 & 2 A & 30 & \text { D9 } & 87 & 45 & 58\end{array}$ $\begin{array}{lllllllllllllllll}001 F 0 & 45 & 43 & 55 & 54 & \mathrm{C} 5 & 01 & 8 \mathrm{C} & 01 & \mathrm{~F} 9 & 68 & 69 & \mathrm{~F} 8 & 93 & \mathrm{~A} 5 & \mathrm{D} 5 & 82\end{array}$ $\begin{array}{lllllllllllllllll}00200 & 55 & \mathrm{AF} & 01 & \mathrm{ED} & 02 & 06 & 68 & 6 \mathrm{~F} & 68 & 6 \mathrm{~B} & 68 & 6 \mathrm{~A} & 24 & 68 & \mathrm{AF} & 14\end{array}$ $\begin{array}{lllllllllllllllllllllll}00210 & 14 & \mathrm{~F} 8 & 11 & \mathrm{AF} & \mathrm{FE} & 8 \mathrm{~A} & 7 \mathrm{E} & \mathrm{AA} & 9 \mathrm{~A} & 7 \mathrm{E} & \mathrm{BA} & 2 \mathrm{~F} & 8 \mathrm{~F} & 32 & 45 & 8 \mathrm{~B}\end{array}$ $\begin{array}{lllllllllllllllll}00220 & 7 \mathrm{E} & \mathrm{AB} & 9 \mathrm{~B} & 7 \mathrm{E} & \mathrm{BB} & 8 \mathrm{~B} & \mathrm{~F} 7 & \mathrm{BF} & 24 & 9 \mathrm{~B} & 77 & 14 & \mathrm{CB} & 02 & 15 & \mathrm{BB}\end{array}$ $\begin{array}{lllllllllllllllll}00230 & 9 \mathrm{~F} & \mathrm{AB} & \mathrm{C} 0 & 02 & 15 & 82 & 55 & \mathrm{AA} & 01 & \mathrm{FF} & 02 & 3 \mathrm{C} & 68 & 6 \mathrm{~A} & 14 & \mathrm{E} 2\end{array}$ $\begin{array}{lllllllllllllllll}00240 & 68 & 86 & 05 & \mathrm{CE} & \mathrm{E} 4 & 68 & \mathrm{AB} & 68 & \mathrm{AA} & 14 & \mathrm{D} 5 & 83 & 41 & 4 \mathrm{E} & \mathrm{C} 4 & 02\end{array}$ $\begin{array}{lllllllllllllllll}00250 & 35 & 02 & 53 & 68 & 6 A & 14 & 8 A & F 2 & 73 & 9 A & F 2 & 54 & \text { D5 } & 82 & 4 F & \text { D2 }\end{array}$ $\begin{array}{lllllllllllllllll}00260 & 02 & 4 \mathrm{~B} & 02 & 64 & 68 & 6 \mathrm{~A} & 14 & 8 \mathrm{~A} & \mathrm{Fl} & 73 & 9 \mathrm{~A} & \mathrm{Fl} & 54 & \mathrm{D} 5 & 83 & 58\end{array}$

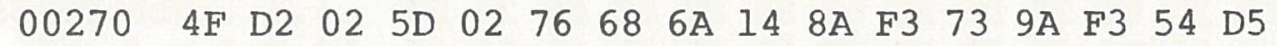
$\begin{array}{lllllllllllllllll}00280 & 83 & 53 & 50 & \mathrm{C} 0 & 02 & 6 \mathrm{E} & 02 & 88 & \mathrm{EA} & 68 & \mathrm{~B} 4 & \mathrm{E} 4 & 24 & 68 & \mathrm{AA} & 14\end{array}$ $\begin{array}{lllllllllllllllll}00290 & \text { D5 } & 83 & 53 & 50 & \text { Al } & 02 & 80 & 02 & 99 & 87 & \text { FC } & 06 & \text { AA } & 97 & 7 C & 00\end{array}$ $\begin{array}{lllllllllllllllll}002 A 0 & B A & E A & 68 & 64 & D 5 & 83 & 52 & 50 & \text { AI } & 02 & 91 & 02 & A D & 87 & \text { FC } & 08\end{array}$

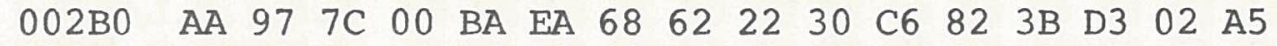

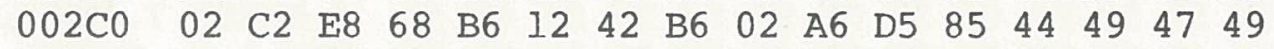
$\begin{array}{lllllllllllllllll}002 \mathrm{D} 0 & \mathrm{D} 4 & 02 & \mathrm{BB} & 02 & \mathrm{D} 5 & 68 & 6 \mathrm{~A} & 14 & 04 & \mathrm{FF} & 30 & \mathrm{CB} & 03 & 58 & \mathrm{FF} & 11\end{array}$ $\begin{array}{lllllllllllllllll}002 \mathrm{E} 0 & 33 & \mathrm{E} 7 & \mathrm{FF} & \mathrm{F} 9 & \mathrm{C} 3 & 03 & 58 & \mathrm{FC} & 0 \mathrm{~A} & 73 & 24 & \mathrm{~F} 7 & \mathrm{CB} & 03 & 5 \mathrm{~B} & 14\end{array}$ $\begin{array}{lllllllllllllllll}002 \mathrm{~F} 0 & 14 & \mathrm{C} 0 & 03 & 58 & 85 & 43 & 4 \mathrm{D} & 4 \mathrm{~F} & 56 & \mathrm{C} 5 & 02 & \mathrm{CB} & 02 & \mathrm{FE} & 44 & \mathrm{FC}\end{array}$ $\begin{array}{lllllllllllllllll}00300 & 01 & B F & 44 & A F & 2 F & 68 & 6 A & 68 & 6 B & 9 F & 32 & 12 & 4 B & 5 A & 1 A & 2 F\end{array}$ $\begin{array}{lllllllllllllllll}00310 & 30 & 09 & D 5 & 85 & 4 \mathrm{C} & 45 & 41 & 56 & \mathrm{C} 5 & 02 & \mathrm{~F} 4 & 03 & 1 \mathrm{D} & \mathrm{E} 2 & 12 & 12\end{array}$ $\begin{array}{lllllllllllllllll}00320 & 68 & \text { A6 } & \text { D5 } & 82 & 3 E & \text { D2 } & 03 & 13 & 03 & 2 A & E 2 & 68 & \text { A6 } & \text { E4 } & 68 & 66\end{array}$ $\begin{array}{lllllllllllllllll}00330 & \text { D5 } & 82 & 52 & \text { BE } & 03 & 23 & 03 & 38 & 24 & 68 & \text { A6 } & 14 & 12 & 42 & \text { B6 } & 02\end{array}$ $\begin{array}{lllllllllllllllll}00340 & \text { A6 } & \text { D5 } & 81 & \text { C9 } & 03 & 31 & 03 & 48 & 24 & 68 & \text { A6 } & 14 & \text { D5 } & 82 & 30 & \text { BD }\end{array}$ $\begin{array}{lllllllllllllllll}00350 & 03 & 42 & 03 & 54 & 44 & \text { F1 } & 32 & 5 B & \text { F8 } & 00 & \text { C8 } & \text { F8 } & 01 & 73 & \text { F8 } & 00\end{array}$ $\begin{array}{lllllllllllllllll}00360 & 54 & \mathrm{D} 5 & 82 & 30 & \mathrm{BC} & 03 & 4 \mathrm{D} & 03 & 69 & 44 & \mathrm{FE} & 33 & 5 \mathrm{~B} & 30 & 58 & 81\end{array}$ $\begin{array}{lllllllllllllllll}00370 & \mathrm{BD} & 03 & 62 & 03 & 75 & 68 & 6 \mathrm{~A} & 9 \mathrm{~A} & \mathrm{~F} 3 & \mathrm{BA} & 14 & 8 \mathrm{~A} & \mathrm{~F} 3 & 54 & 9 \mathrm{~A} & \mathrm{Fl}\end{array}$ a.. 06q.a.c.0..co $L D \ldots . . h K_{0}=h J . M J[$ .J[ . $x_{0}[.[\mathrm{x}$ 08.WA RM...6x./hJ.Ajhb hahehfhghhkh7J [ . /.:MU.EF.-. $\left\{.{ }^{\wedge}<0\right.$ $01=001>001$ ? $0 \mathrm{x} \cdot \mathrm{Hx}$ . $\$ T \$ x . T U<x U=\{U>$. U?.U4.U5.U6.U7.U hhiihcds 0. ch $6 \mathrm{~b} \cdot \mathrm{h}$ f"SO .. ( IOOP) .U. 0 .b....w" $\cdot w^{\sim} ; A^{\prime \prime h h h}$ U....hf "U. (+LOOP ).. U. . t \& \$.t6D.b ;2...u".u09. (FI ND) . I. whjhk $\$ j \cdot R K$ sz?J.'. $\mathrm{Ks}^{\sim}:(.2 . \mathrm{J}$ z.2.d.l.s.l.s.sx .sx.sTU.Jz.2 (.: ; ..*:;x.dsTUjhj\$d a.Y.IIT.1.Khhja\$ h*.U. (DO) .C. ]bh\& dhfhkbh+U. BRANCH .T.thhhU. OBRANCH .i...Dq.2t..U.ENC LOSE. $x$....hk\$\$\$. Rbx.*:<. $w_{0} / \ldots 0$ 'd . $\mathrm{s}, \mathrm{s}<\mathrm{x}$.sssT.b. 2 Hw2c..0 .ds.T' .w :Y\$.W.:Y...T.TS \$\$..T\$.T.*OY.EX ECUTE...yhix. $\%$ U. U/.m. .hohkhj\$h/. .x./ ..*..: :/.2E. . + . i .w?\$.w.K.. ; . + a... U*...<hj.b h..Nah+h*.U.AND. 5.Shj..rs.rTU.OR .K.dhj..qs.qTU.x OR.].vhj..ss.sTU .SP@.n..jh4d\$h*. U.SP! .........।. : jhdU.RP!...-.।. *.1 . : jhb"OF.;S. \% .Bhh6.B6.\&U.DIGI T. ..Uhj...OK.X.. $3 \mathrm{~g} \cdot \mathrm{YC} \cdot \mathrm{X} \mid \cdot \mathrm{s} \$ \mathrm{wK} \cdot[$. - A.X.CMOVE.K. D .?D//hjhk.2.KZ./ 0.U.LEAVE.t..b.. h\&U.>R ...*bh\&dhf U.R>.\#. 8 \$h\& . B6. \&U.I.I.H\$h\&.U. $0=$ . B. TDq2 [x.Hx . sx. TU. $0<. M_{0}$ iD $3[0 \mathrm{X}$. =.b.uhj.s:..sT.q 
$\begin{array}{lllllllllllllllll}00380 & 32 & 85 & \text { F8 } & 00 & \text { C8 } & \text { F8 } & 01 & 73 & \text { F8 } & 00 & 54 & \text { D5 } & 81 & \text { BC } & 03 & 6 \mathrm{~F}\end{array}$ $\begin{array}{lllllllllllllllll}00390 & 03 & 92 & 68 & 6 A & 14 & 8 A & F 5 & 24 & 04 & F B & 80 & 54 & 9 A & F B & 80 & 75\end{array}$ $\begin{array}{lllllllllllllllll}003 \mathrm{~A} 0 & 14 & \mathrm{~F} 8 & 00 & 7 \mathrm{E} & \mathrm{FB} & 01 & 73 & \mathrm{~F} 6 & 54 & \mathrm{D} 5 & 81 & \mathrm{BE} & 03 & 8 \mathrm{C} & 03 & \mathrm{~B} 0\end{array}$ $\begin{array}{lllllllllllllllllllllllll}003 \mathrm{~B} 0 & 68 & 6 \mathrm{~A} & 14 & 8 \mathrm{~A} & \mathrm{~F} 7 & 24 & 04 & \mathrm{FB} & 80 & 54 & 9 \mathrm{~A} & \mathrm{FB} & 80 & 77 & 30 & \mathrm{~A} 0\end{array}$ $\begin{array}{lllllllllllllllll}003 \mathrm{C} 0 & 81 & \mathrm{AB} & 03 & \mathrm{AA} & 03 & \mathrm{C} 6 & 68 & 6 \mathrm{~A} & 14 & 8 \mathrm{~A} & \mathrm{~F} 4 & 73 & 9 \mathrm{~A} & 74 & 54 & \mathrm{D} 5\end{array}$ $\begin{array}{lllllllllllllllll}003 \mathrm{D} 0 & 82 & 31 & \mathrm{AB} & 03 & \mathrm{C} 0 & 03 & \mathrm{D} 7 & \mathrm{~F} 8 & 01 & \mathrm{E} 4 & 14 & \mathrm{~F} 4 & 73 & \mathrm{~F} 8 & 00 & 74\end{array}$ $\begin{array}{lllllllllllllllll}003 \mathrm{E} 0 & 54 & \mathrm{D} 5 & 82 & 32 & \mathrm{AB} & 03 & \mathrm{D} 0 & 03 & \mathrm{E} 9 & \mathrm{~F} 8 & 02 & 30 & \mathrm{D} 9 & 82 & 2 \mathrm{~B} & \mathrm{Al}\end{array}$

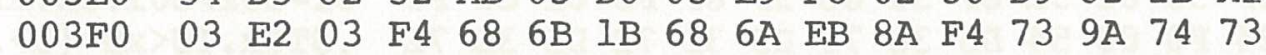
$\begin{array}{lllllllllllllllll}00400 & D 5 & 81 & A D & 03 & E D & 04 & 07 & 68 & 6 A & 14 & 8 A & F 5 & 73 & 9 A & 75 & 54\end{array}$ $\begin{array}{lllllllllllllllll}00410 & \mathrm{D} 5 & 82 & 44 & \mathrm{AB} & 04 & 01 & 04 & 18 & 68 & 6 \mathrm{~A} & 68 & 6 \mathrm{~B} & 14 & 14 & 14 & 8 \mathrm{~B}\end{array}$ $\begin{array}{lllllllllllllllll}00420 & \text { F4 } & 73 & 9 B & 74 & 73 & 8 A & 74 & \text { CO } & 03 & \text { CB } & 85 & 4 \mathrm{D} & 49 & 4 \mathrm{E} & 55 & \mathrm{D} 3\end{array}$ $\begin{array}{lllllllllllllllll}00430 & 04 & 11 & 04 & 34 & 14 & \mathrm{FF} & 00 & 04 & \mathrm{FB} & \mathrm{FF} & 7 \mathrm{C} & 00 & 73 & 04 & \mathrm{FB} & \mathrm{FF}\end{array}$ $\begin{array}{lllllllllllllllll}00440 & 7 \mathrm{C} & 00 & 54 & \mathrm{D} 5 & 86 & 44 & 4 \mathrm{D} & 49 & 4 \mathrm{E} & 55 & \mathrm{D} 3 & 04 & 2 \mathrm{~A} & 04 & 4 \mathrm{~F} & 14\end{array}$ $\begin{array}{llllllllllllllllll}00450 & 14 & 14 & 04 & \text { FB } & \text { FF } & \text { FC } & 01 & 73 & 04 & \text { FB } & \text { FF } & 7 C & 00 & 73 & 30 & 37\end{array}$ $\begin{array}{lllllllllllllllll}00460 & 84 & 4 \mathrm{~F} & 56 & 45 & \mathrm{D} 2 & 04 & 44 & 04 & 69 & \mathrm{~EB} & 68 & \mathrm{~B} 4 & 68 & 6 \mathrm{~A} & 68 & 6 \mathrm{~A}\end{array}$ $\begin{array}{lllllllllllllllll}00470 & \text { E4 } & 24 & 68 & \text { AA } & 14 & \text { D5 } & 84 & 44 & 52 & 4 \mathrm{~F} & \text { D0 } & 04 & 60 & 04 & 7 \mathrm{~F} & 14\end{array}$ $\begin{array}{lllllllllllllllll}00480 & 14 & \mathrm{D} 5 & 84 & 53 & 57 & 41 & \mathrm{D} 0 & 04 & 76 & 04 & 8 \mathrm{~B} & 68 & 6 \mathrm{~A} & 68 & 6 \mathrm{~B} & 24\end{array}$ $\begin{array}{lllllllllllllllll}00490 & 68 & \mathrm{AA} & 68 & \mathrm{AB} & 14 & \mathrm{D} 5 & 83 & 44 & 55 & \mathrm{D} 0 & 04 & 82 & 04 & 9 \mathrm{E} & 68 & 6 \mathrm{~A}\end{array}$ $\begin{array}{lllllllllllllllll}004 \mathrm{~A} 0 & 24 & 24 & 24 & 68 & \mathrm{AA} & 14 & \mathrm{D} 5 & 86 & 54 & 4 \mathrm{~F} & 47 & 47 & 4 \mathrm{C} & \mathrm{C} 5 & 04 & 96\end{array}$ $\begin{array}{llllllllllllllllll}004 \mathrm{~B} 0 & 04 & \mathrm{~B} 2 & 68 & 6 \mathrm{~A} & 68 & 6 \mathrm{~B} & \mathrm{~EB} & 8 \mathrm{~A} & \mathrm{~F} 3 & 5 \mathrm{~B} & \mathrm{D} 5 & 81 & \mathrm{C} 0 & 04 & \mathrm{~A} 7 & 04\end{array}$ $\begin{array}{lllllllllllllllll}004 \mathrm{C} 0 & \mathrm{Cl} & 68 & 6 \mathrm{~A} & 24 & \mathrm{EA} & 68 & 6 \mathrm{~A} & \mathrm{E} 4 & 68 & \mathrm{AA} & 14 & \mathrm{D} 5 & 82 & 43 & \mathrm{C} 0 & 04\end{array}$ 004D0 BB $04 \begin{array}{lllllllllllllll} & \text { D3 } & 68 & 6 \mathrm{~A} & 24 & 0 \mathrm{~A} & 73 & \mathrm{~F} 8 & 00 & 54 & \mathrm{D} 5 & 81 & \mathrm{Al} & 04 & \mathrm{CC}\end{array}$ $\begin{array}{llllllllllllllllll}004 \mathrm{E} 0 & 04 & \mathrm{E} 2 & 68 & 6 \mathrm{~A} & 68 & 6 \mathrm{~B} & \mathrm{EA} & 1 \mathrm{~A} & 68 & \mathrm{AB} & \mathrm{D} 5 & 82 & 43 & \mathrm{Al} & 04 & \mathrm{DC}\end{array}$ $\begin{array}{lllllllllllllllll}004 \mathrm{~F} 0 & 04 & \mathrm{~F} 2 & 68 & 6 \mathrm{~A} & 14 & 44 & 5 \mathrm{~A} & \mathrm{D} 5 & 83 & 52 & 4 \mathrm{~F} & \mathrm{D} 4 & 04 & \mathrm{~EB} & 05 & 00\end{array}$ $\begin{array}{lllllllllllllllll}00500 & 68 & 6 \mathrm{~A} & 68 & 6 \mathrm{~B} & 68 & 6 \mathrm{C} & 24 & 68 & \mathrm{AB} & 68 & \mathrm{AA} & 68 & \mathrm{AC} & 14 & \mathrm{D} 5 & 84\end{array}$ $\begin{array}{lllllllllllllllll}00510 & 2 \mathrm{D} & 44 & 55 & \mathrm{D} 0 & 04 & \mathrm{~F} 8 & 05 & 18 & 68 & 6 \mathrm{~A} & 24 & 9 \mathrm{~A} & \mathrm{Fl} & 24 & 24 & \mathrm{CE}\end{array}$ $\begin{array}{lllllllllllllllll}00520 & 68 & \mathrm{AA} & 14 & \mathrm{D} 5 & 83 & 41 & 42 & \mathrm{D} 3 & 05 & \text { OF } & 05 & 2 \mathrm{C} & 04 & \mathrm{FE} & \mathrm{C} 3 & 04\end{array}$ $\begin{array}{lllllllllllllllll}00530 & 34 & \mathrm{D} 5 & 84 & 44 & 41 & 42 & \mathrm{D} 3 & 05 & 24 & 05 & 3 \mathrm{~B} & 04 & \mathrm{FE} & \mathrm{C} 3 & 04 & 4 \mathrm{~F}\end{array}$ $\begin{array}{lllllllllllllllll}00540 & \text { D5 } & 83 & 4 \mathrm{D} & 41 & \mathrm{D} 8 & 05 & 32 & 05 & 49 & 68 & 6 \mathrm{~A} & 14 & 8 \mathrm{~A} & \mathrm{~F} 5 & 24 & 04\end{array}$ $\begin{array}{llllllllllllllllll}00550 & \text { FB } & 80 & 54 & 9 A & F B & 80 & \text { BA } & 75 & 33 & 5 \mathrm{E} & 14 & 68 & \mathrm{AA} & 14 & 04 & \mathrm{FB}\end{array}$ $\begin{array}{lllllllllllllllll}00560 & 80 & 54 & D 5 & 81 & A A & 05 & 41 & 05 & 69 & \mathrm{E} 2 & 68 & 86 & 05 & 7 \mathrm{E} & 14 & 14\end{array}$ $\begin{array}{lllllllllllllllll}00570 & \text { D5 } & 82 & 4 D & A A & 05 & 63 & 05 & 78 & \text { E2 } & 68 & 86 & 05 & 7 E & \text { D5 } & \text { E4 } & 68\end{array}$ $\begin{array}{lllllllllllllllll}00580 & 6 \mathrm{~A} & \mathrm{~F} 8 & 00 & \mathrm{AF} & 44 & \mathrm{FE} & 3 \mathrm{~B} & 96 & 04 & \mathrm{FB} & \mathrm{FF} & \mathrm{FC} & 01 & 73 & 04 & \mathrm{FB}\end{array}$

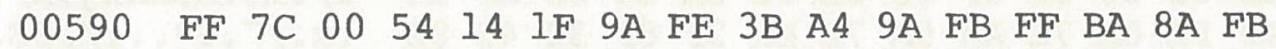
005A0 FF AA $1 A$ IF E2 $68 \quad 86 \quad 05 \quad \mathrm{CE}$ E4 $8 \mathrm{~F}$ F6 $3 \mathrm{~B}$ C6 $8 \mathrm{~B}$ FB $005 B 0$ FF FC 01 AB 9B FB FF 7C 00 BB $8 A$ FB FF $7 C$ C0 AA $\begin{array}{lllllllllllllllll}005 C 0 & 9 A & F B & F F & 7 C & 00 & B A & 68 & A B & 68 & A A & 14 & E 2 & 68 & 96 & E 4 & F 8\end{array}$

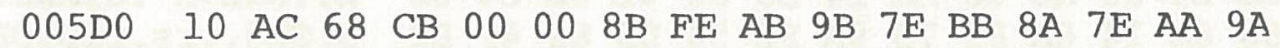

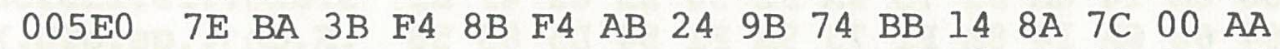
$\begin{array}{llllllllllllllllll}005 \mathrm{~F} 0 & 9 \mathrm{~A} & 7 \mathrm{C} & 00 & \mathrm{BA} & 2 \mathrm{C} & 8 \mathrm{C} & 3 \mathrm{~A} & \mathrm{D} 6 & \mathrm{E} 2 & 68 & 96 & \mathrm{E} 9 & 68 & 69 & 24 & \mathrm{E} 4\end{array}$ $\begin{array}{lllllllllllllllll}00600 & 68 & \mathrm{~A} 9 & 14 & \mathrm{D} 5 & 81 & \mathrm{~B} 0 & 05 & 71 & 05 & \mathrm{FB} & 00 & 00 & 81 & \mathrm{BI} & 06 & 04\end{array}$ $\begin{array}{lllllllllllllllll}00610 & 05 & \mathrm{FB} & 00 & 01 & 81 & \mathrm{~B} 2 & 06 & 0 \mathrm{C} & 05 & \mathrm{FB} & 00 & 02 & 81 & \mathrm{~B} 3 & 06 & 14\end{array}$ $\begin{array}{llllllllllllllllll}00620 & 05 & \mathrm{FB} & 00 & 03 & 82 & 42 & \mathrm{CC} & 06 & 1 \mathrm{C} & 05 & \mathrm{FB} & 00 & 20 & 83 & 43 & 2 \mathrm{~F}\end{array}$ $\begin{array}{lllllllllllllllll}00630 & \mathrm{CC} & 06 & 24 & 05 & \mathrm{FB} & 00 & 40 & 83 & 4 \mathrm{C} & 2 \mathrm{~F} & \mathrm{D} 0 & 06 & 2 \mathrm{D} & 05 & \mathrm{FB} & 00\end{array}$ $\begin{array}{lllllllllllllllll}00640 & 3 \mathrm{C} & 85 & 46 & 49 & 52 & 53 & \mathrm{D} 4 & 06 & 37 & 05 & \mathrm{FB} & 57 & \mathrm{D} 7 & 85 & 4 \mathrm{C} & 49\end{array}$ $\begin{array}{lllllllllllllllll}00650 & 4 D & 49 & D 4 & 06 & 41 & 05 & F B & 7 F & F F & 85 & 42 & 2 F & 42 & 55 & C 6 & 06\end{array}$ $\begin{array}{lllllllllllllllll}00660 & 4 \mathrm{D} & 05 & \mathrm{FB} & 04 & 00 & 85 & 42 & 2 \mathrm{~F} & 53 & 43 & \mathrm{D} 2 & 06 & 59 & 05 & \mathrm{FB} & 00\end{array}$ $\begin{array}{lllllllllllllllll}00670 & 01 & 86 & 4 \mathrm{~F} & 52 & 49 & 47 & 49 & \mathrm{CE} & 06 & 65 & 05 & \mathrm{FB} & 18 & 41 & 87 & 2 \mathrm{~B}\end{array}$ $\begin{array}{lllllllllllllllll}00680 & 4 \mathrm{~F} & 52 & 49 & 47 & 49 & \mathrm{CE} & 06 & 71 & 08 & 57 & 06 & 7 \mathrm{~A} & 03 & \mathrm{C} 4 & 02 & \mathrm{C} 0\end{array}$ $\begin{array}{lllllllllllllllll}00690 & 24 & \mathrm{E} 9 & 19 & 87 & \mathrm{~F} 4 & 54 & 24 & 29 & 97 & 74 & 54 & \mathrm{D} 5 & 82 & 53 & \text { B0 } & 06\end{array}$ $\begin{array}{lllllllllllllllll}006 \mathrm{AO} & 7 \mathrm{E} & 06 & 90 & 00 & 06 & 82 & 52 & \mathrm{~B} 0 & 06 & 9 \mathrm{C} & 06 & 90 & 00 & 08 & 83 & 54\end{array}$ $\begin{array}{lllllllllllllllll}006 \mathrm{~B} 0 & 49 & \mathrm{C} 2 & 06 & \mathrm{~A} 5 & 06 & 90 & 00 & 0 \mathrm{~A} & 85 & 57 & 49 & 44 & 54 & \mathrm{C} 8 & 06 & \mathrm{AE}\end{array}$ $\begin{array}{lllllllllllllllll}006 \mathrm{C} 0 & 06 & 90 & 00 & 0 \mathrm{C} & 87 & 57 & 41 & 52 & 4 \mathrm{E} & 49 & 4 \mathrm{E} & \mathrm{C} 7 & 06 & \mathrm{~B} 8 & 06 & 90\end{array}$

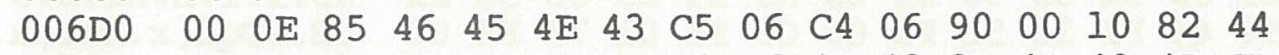
$\begin{array}{lllllllllllllllll}006 \mathrm{E} 0 & \mathrm{D} 0 & 06 & \mathrm{D} 2 & 06 & 90 & 00 & 12 & 88 & 56 & 4 \mathrm{~F} & 43 & 2 \mathrm{D} & 4 \mathrm{C} & 49 & 4 \mathrm{E} & \mathrm{CB}\end{array}$ $\begin{array}{lllllllllllllllll}006 \mathrm{~F} 0 & 06 & \mathrm{DE} & 06 & 90 & 00 & 14 & 83 & 42 & 4 \mathrm{C} & \mathrm{CB} & 06 & \mathrm{E} 7 & 06 & 90 & 00 & 40\end{array}$
S.X.Hx.SX.TU. . $_{0} \mathrm{O}$ ..hj..u\$.\{.T.\{.u .X.. . . SvTU.>...0 hj...w\$. \{.T. \{.w0 .t.*.Fhj..ts.tTU $.1+$. a.Wx.d.tsx.t TU.2+.P.ix.OY.+! .b.thk.hjk.ts.ts U.-.m..hj..us.uT $\mathrm{U} . \mathrm{D}+\ldots . . \mathrm{hjhk} \ldots .$. ts.ts.te.K.MINUS ...4.... . . . s. \{. I.TU.DMINUS.*.O. ... \{. . .s. \{.|.s07 .OVER.D.ikh4hjhj d\$h*.U.DROP. ... .U.SWAP.V..hjhk\$ $h * h+. U . D U P \ldots . . h j$ \$\$h*.U.TOGGLE. . .2hjhkk.s[u.@.' . Ahj\$jhjdh*.U.C@. i.Shj\$.Sx.TU.!.L . bhjhkj.h+U.C!. I . rhj.DZU.ROT.k. . $h j h k h l \$ h+h * h, . U$. -DUP.x..hj\$.q\$\$N $h *$.U.ABS.... C . 4U.DABS. $\$ . ; \sim C . O$ U.MAX.2.Ihj..u\$. \{.T. $\left\{.: u 3^{\wedge} . h^{*} \ldots\{\right.$ .TU.*.A.ibh..... $\mathrm{U} \cdot \mathrm{M}^{*}$. C. xbh... Udh $\mathrm{jx} . / \mathrm{D}^{\sim} ; . .\{. \mid . s .\{$ .|.T... ; $\$$. . : \{ .*..bh...Nd.v;F. \{ . . $_{0}\left\{.1\right.$. . $_{0}\left\{. I_{.}\right.$* . $\{.1 .: h+h * . b h \cdot d x$ ,hK... ${ }^{+}+\ldots i \ldots *$ . : ; t. $t+\$ . t ; . .1 . *$ . 1.:, .:Vbh.ihi\$d h).U.0.q. \{.... . . \{...2... \{... . \{...BL... . . C / L.\$.\{.Q.L/P.-. \{. <.FIRST.7. \{WW.II MIT.A. \{ . B/BUF . M. $\{\ldots B / S C R . Y .\{$. ..ORIGIN.e.\{.A.t ORIGIN.q.W.z.D.Q \$i..tT\$).tTU.SO .

$\ldots . . . R 0 \ldots . . . T$ IB..... WIDTH . .....WARNING.8. ...FENCE.D..... D P.R.....VOC-LINK .^....BLK.g... a page 108 
$\begin{array}{lllllllllllllllll}00700 & 82 & 49 & \mathrm{CE} & 06 & \mathrm{~F} 6 & 06 & 90 & 00 & 48 & 83 & 4 \mathrm{~F} & 55 & \mathrm{D} 4 & 07 & 00 & 06\end{array}$ $\begin{array}{lllllllllllllllll}00710 & 90 & 00 & 4 \mathrm{~A} & 83 & 53 & 43 & \mathrm{D} 2 & 07 & 09 & 06 & 90 & 00 & 4 \mathrm{C} & 86 & 4 \mathrm{~F} & 46\end{array}$ $\begin{array}{lllllllllllllllll}00720 & 46 & 53 & 45 & \mathrm{D} 4 & 07 & 13 & 06 & 90 & 00 & 4 \mathrm{E} & 87 & 43 & 4 \mathrm{~F} & 4 \mathrm{E} & 54 & 45\end{array}$ $\begin{array}{lllllllllllllllll}00730 & 58 & \text { D4 } & 07 & \text { ID } & 06 & 90 & 00 & 20 & 87 & 43 & 55 & 52 & 52 & 45 & 4 \mathrm{E} & \mathrm{D} 4\end{array}$ $\begin{array}{lllllllllllllllll}00740 & 07 & 2 \mathrm{~A} & 06 & 90 & 00 & 22 & 85 & 53 & 54 & 41 & 54 & \mathrm{C} 5 & 07 & 38 & 06 & 90\end{array}$ $\begin{array}{lllllllllllllllll}00750 & 00 & 24 & 84 & 42 & 41 & 53 & C 5 & 07 & 46 & 06 & 90 & 00 & 26 & 83 & 44 & 50\end{array}$ $\begin{array}{lllllllllllllllll}00760 & \text { CC } & 07 & 52 & 06 & 90 & 00 & 28 & 83 & 46 & 4 \mathrm{C} & \mathrm{C} 4 & 07 & 5 \mathrm{D} & 06 & 90 & 00\end{array}$ $\begin{array}{lllllllllllllllll}00770 & 2 A & 83 & 43 & 53 & \text { D0 } & 07 & 67 & 06 & 90 & 00 & 2 C & 82 & 52 & \text { A3 } & 07 & 71\end{array}$ $\begin{array}{lllllllllllllllll}00780 & 06 & 90 & 00 & 2 \mathrm{E} & 83 & 48 & 4 \mathrm{C} & \mathrm{C} 4 & 07 & 7 \mathrm{~B} & 06 & 90 & 00 & 30 & 83 & 55\end{array}$ $\begin{array}{lllllllllllllllll}00790 & 53 & C 5 & 07 & 84 & 06 & 90 & 00 & 32 & 84 & 50 & 52 & 45 & \text { D6 } & 07 & 8 \mathrm{E} & 06\end{array}$ $\begin{array}{lllllllllllllllll}007 A 0 & 90 & 00 & 34 & 89 & 55 & 53 & 45 & 52 & 2 D & 49 & 4 F & 43 & C 2 & 07 & 98 & 06\end{array}$ $\begin{array}{lllllllllllllllll}007 \mathrm{~B} 0 & 90 & 00 & 36 & 87 & 53 & 43 & 52 & 2 \mathrm{~F} & 44 & 53 & \mathrm{CB} & 07 & \mathrm{~A} 3 & 06 & 90 & 00\end{array}$ $\begin{array}{lllllllllllllllll}007 \mathrm{C} 0 & 16 & 84 & 4 \mathrm{~F} & 55 & 54 & \mathrm{CC} & 07 & \mathrm{~B} 3 & 06 & 90 & 00 & 42 & 83 & 47 & 52 & \mathrm{D} 0\end{array}$ $\begin{array}{lllllllllllllllll}007 \mathrm{D} 0 & 07 & \mathrm{Cl} & 06 & 90 & 00 & 44 & 89 & 4 \mathrm{D} & 4 \mathrm{~F} & 44 & 45 & 4 \mathrm{D} & 2 \mathrm{E} & 47 & 52 & \mathrm{D} 0\end{array}$ $\begin{array}{lllllllllllllllll}007 \mathrm{E} 0 & 07 & \mathrm{CC} & 06 & 90 & 00 & 18 & 88 & 54 & 45 & 52 & 4 \mathrm{D} & 2 \mathrm{E} & 47 & 52 & \mathrm{D} 0 & 07\end{array}$

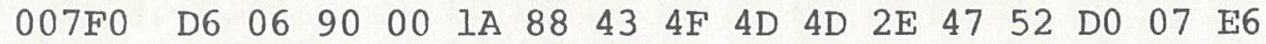
$\begin{array}{lllllllllllllllll}00800 & 06 & 90 & 00 & 1 \mathrm{C} & 85 & 4 \mathrm{E} & 65 & 78 & 74 & \mathrm{~F} 5 & 07 & \mathrm{~F} 5 & 06 & 90 & 00 & 1 \mathrm{E}\end{array}$ $\begin{array}{lllllllllllllllll}00810 & 86 & 4 \mathrm{~F} & 55 & 54 & 50 & 55 & \mathrm{D} 4 & 08 & 04 & 08 & 1 \mathrm{~B} & 14 & 68 & 6 \mathrm{~A} & 9 \mathrm{~A} & \mathrm{FA}\end{array}$

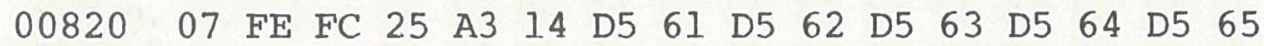
$\begin{array}{lllllllllllllllll}00830 & \text { D5 } & 66 & \text { D5 } & 67 & \text { D5 } & 85 & 49 & 4 \mathrm{E} & 50 & 55 & \text { D4 } & 08 & 10 & 08 & 3 \mathrm{~F} & \mathrm{E} 2\end{array}$ $\begin{array}{lllllllllllllllll}00840 & 22 & \text { F8 } & \text { D5 } & 73 & \text { F8 } & 24 & 73 & \text { E4 } & 04 & \text { F3 } & 54 & 14 & 04 & \text { FA } & 07 & 3 A\end{array}$ $\begin{array}{lllllllllllllllll}00850 & 53 & 24 & \text { D5 } & \text { F9 } & 68 & 52 & \text { D2 } & \text { E2 } & 68 & \text { A6 } & \text { E6 } & 68 & \text { B8 } & \text { E8 } & 68 & \text { B9 }\end{array}$ $\begin{array}{lllllllllllllllll}00860 & \mathrm{D} 5 & 84 & 46 & 49 & 4 \mathrm{C} & \mathrm{CC} & 08 & 35 & 08 & 6 \mathrm{~A} & 68 & 6 \mathrm{~F} & 68 & 6 \mathrm{~B} & 68 & 6 \mathrm{~A}\end{array}$ $\begin{array}{lllllllllllllllll}00870 & \text { E2 } & 8 B & 52 & 9 B & F 1 & 32 & 7 D & 8 F & 5 A & 1 A & 2 B & 30 & 71 & D 5 & 85 & 45\end{array}$ $\begin{array}{lllllllllllllllll}00880 & 52 & 41 & 53 & C 5 & 08 & 61 & 08 & 88 & \text { F8 } & 00 & 24 & 73 & \text { C0 } & 08 & 6 A & 86\end{array}$ $\begin{array}{lllllllllllllllll}00890 & 42 & 4 \mathrm{C} & 41 & 4 \mathrm{E} & 4 \mathrm{~B} & \mathrm{D} 3 & 08 & 7 \mathrm{E} & 08 & 9 \mathrm{~A} & \mathrm{~F} 8 & 20 & \mathrm{C} 0 & 08 & 8 \mathrm{~A} & 52\end{array}$ $\begin{array}{lllllllllllllllll}008 \mathrm{~A} 0 & 87 & \mathrm{~F} 4 & \mathrm{AB} & 97 & 7 \mathrm{C} & 00 & \mathrm{BB} & 1 \mathrm{~B} & 68 & 96 & 84 & 48 & 4 \mathrm{~F} & 4 \mathrm{C} & \mathrm{C} 4 & 08\end{array}$

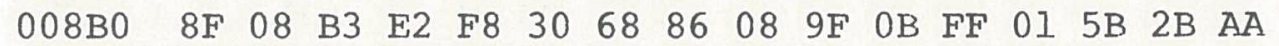
$\begin{array}{lllllllllllllllll}008 \mathrm{C} 0 & 0 \mathrm{~B} & 7 \mathrm{~F} & 00 & 5 \mathrm{~B} & \mathrm{BA} & 14 & 44 & 5 \mathrm{~A} & \mathrm{D} 5 & 83 & 4 \mathrm{~B} & 45 & \mathrm{D} 9 & 08 & \mathrm{AA} & 08\end{array}$

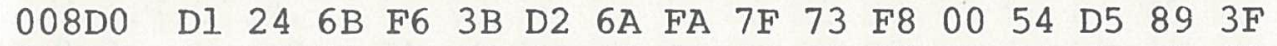
$\begin{array}{lllllllllllllllll}008 \mathrm{E} 0 & 54 & 45 & 52 & 4 \mathrm{D} & 49 & 4 \mathrm{E} & 41 & \mathrm{CC} & 08 & \mathrm{C} 9 & 08 & \mathrm{EC} & 24 & 6 \mathrm{~B} & \mathrm{FA} & 08\end{array}$ 008F0 73 6A F3 54 D5 $84 \quad 45$ 4D 49 D4 08 DE 08 FE E2 68 $\begin{array}{llllllllllllllllll}00900 & 86 & 09 & 8 \mathrm{C} & \mathrm{D} 5 & 82 & 43 & \mathrm{D} 2 & 08 & \mathrm{~F} 5 & 09 & 0 \mathrm{~B} & 24 & \mathrm{~F} 8 & \text { OD } & 54 & \mathrm{E} 2\end{array}$ $\begin{array}{lllllllllllllllll}00910 & 68 & 86 & 09 & 9 \mathrm{C} & 24 & \mathrm{~F} 8 & 0 \mathrm{~A} & 54 & 68 & 86 & 09 & 9 \mathrm{C} & \mathrm{F} 8 & 42 & 68 & 86\end{array}$ $\begin{array}{lllllllllllllllll}00920 & 08 & 9 \mathrm{~F} & 0 \mathrm{~B} & \mathrm{FC} & 01 & 5 \mathrm{~B} & 2 \mathrm{~B} & \text { OB } & 7 \mathrm{C} & 00 & 5 \mathrm{~B} & \mathrm{D} 5 & 84 & 54 & 59 & 50\end{array}$ $\begin{array}{lllllllllllllllll}00930 & \mathrm{C} 5 & 09 & 04 & 09 & 35 & \mathrm{E} 2 & \mathrm{~F} 8 & 4 \mathrm{~A} & 68 & 86 & 08 & 9 \mathrm{~F} & \mathrm{E} 4 & 68 & 6 \mathrm{~A} & 68\end{array}$ 00940 6D EB 8A F4 73 9A $74 \quad 73$ E2 $8 A$ 52 $9 A$ F1 C2 09 2B $\begin{array}{lllllllllllllllll}00950 & 24 & 4 \mathrm{D} & 54 & 68 & 86 & 09 & 9 \mathrm{C} & 2 \mathrm{~A} & \mathrm{C} 0 & 09 & 49 & 85 & 53 & 50 & 41 & 43\end{array}$ $\begin{array}{lllllllllllllllll}00960 & \mathrm{C} 5 & 09 & 2 \mathrm{C} & 09 & 65 & 24 & \mathrm{~F} 8 & 20 & 73 & \mathrm{C} 0 & 08 & \mathrm{FE} & 68 & \mathrm{~A} 4 & 68 & \mathrm{C} 4\end{array}$ $\begin{array}{lllllllllllllllll}00970 & 09 & 77 & 15 & 15 & 68 & 96 & \mathrm{D} 3 & \mathrm{E} 2 & 68 & \text { A6 } & \mathrm{E} 6 & 68 & \mathrm{~B} 3 & 68 & 63 & \text { E2 }\end{array}$ $\begin{array}{lllllllllllllllll}00980 & 30 & 76 & 12 & 68 & 64 & 22 & 68 & \mathrm{C} 5 & 00 & 98 & 68 & 96 & \mathrm{~F} 8 & 4 \mathrm{~A} & 68 & 86\end{array}$

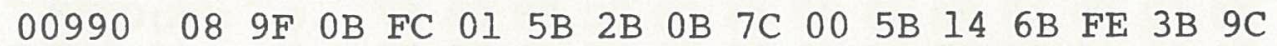
009A0 E4 62 C0 $19 \begin{array}{lllllllllllll}56 & 8 \mathrm{~A} & 42 & 4 \mathrm{C} & 4 \mathrm{~F} & 43 & 4 \mathrm{~B} & 2 \mathrm{D} & 52 & 45 & 41 & \mathrm{C} 4\end{array}$ 009B0 $09 \begin{array}{llllllllllllllll}5 \mathrm{~B} & 09 & \mathrm{~B} 4 & 68 & 6 \mathrm{~F} & \mathrm{E} 2 & 68 & 86 & 09 & 6 \mathrm{C} & 68 & \mathrm{~A} 8 & 68 & \mathrm{~A} 7 & \mathrm{D} 4\end{array}$ $\begin{array}{lllllllllllllllll}009 \mathrm{C} 0 & 87 & \mathrm{~F} 6 & \mathrm{D} 4 & 87 & \mathrm{~F} 9 & \mathrm{D} 4 & 17 & 78 & \mathrm{D} 4 & 87 & \mathrm{~F} 9 & 30 & \mathrm{~F} 4 & 8 \mathrm{~B} & 42 & 4 \mathrm{C}\end{array}$

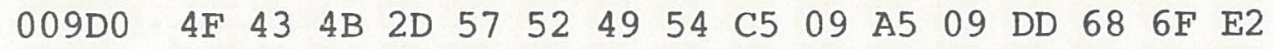
009E0 $68 \begin{array}{llllllllllllllll} & 86 & 09 & 6 \mathrm{C} & 68 & \text { A8 } & 68 & \text { A7 } & \text { D4 } & 87 & \text { F6 } & \text { D4 } & 87 & \text { FC } & \text { D4 } & 17\end{array}$ $\begin{array}{lllllllllllllllll}009 F 0 & 78 & \text { D4 } & 87 & \text { FC } & 12 & 68 & 67 & 68 & 68 & 22 & 68 & 86 & 09 & 82 & \text { D5 } & 85\end{array}$ $\begin{array}{lllllllllllllllll}00 \mathrm{~A} 00 & 4 \mathrm{D} & 2 \mathrm{~F} & 4 \mathrm{D} & 4 \mathrm{~F} & \mathrm{C} 4 & 09 & \mathrm{CD} & 0 \mathrm{~A} & 09 & 68 & 6 \mathrm{~F} & 68 & 6 \mathrm{~B} & 68 & 6 \mathrm{~A} & 68\end{array}$

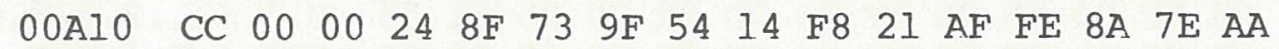
$\begin{array}{lllllllllllllllll}00 A 20 & 9 A & 7 E & B A & 8 B & 7 E & A B & 9 B & 7 E & B B & 2 F & 8 F & C 2 & 0 A & 44 & 8 C & 7 E\end{array}$ $\begin{array}{lllllllllllllllll}00 A 30 & A C & 9 C & 7 E & B C & 8 C & F 7 & B F & 24 & 9 C & 77 & 14 & C B & 0 A & 1 D & B C & 9 F\end{array}$ 00A40 AC C0 OA ID 68 AC 68 AA 68 AB $14 \quad$ D5 $84 \quad 48 \quad 45 \quad 52$ 00A50 C5 09 FF $0 A \begin{array}{llllllllllll}55 & \text { E2 } & \text { F8 } & 12 & 68 & 86 & 08 & 9 \mathrm{~F} & 24 & \text { OB } & 54 & 24\end{array}$ $\begin{array}{lllllllllllllllll}00 A 60 & 2 B & 0 B & 54 & D 5 & 85 & 41 & 4 C & 4 C & 4 F & D 4 & 0 A & 4 C & 0 A & 6 E & E 2 & F\end{array}$ $\begin{array}{lllllllllllllllll}00 A 70 & 12 & 68 & 86 & 08 & 9 \mathrm{~F} & \mathrm{E} 4 & 68 & 6 \mathrm{~A} & \mathrm{~EB} & 8 \mathrm{~A} & \mathrm{~F} 4 & 73 & 9 \mathrm{~A} & 74 & 73 & \mathrm{D} 5\end{array}$
. IN.V...H.OUT... .J.SCR ....... I. OF FSET....... NONTE XT..... CURRENT ....".STATE.8.. .\$.BASE.F..\&.DP L.R... (.FLD.]... *.CSP.g.... R\#. $q$ $\ldots$....HL. $\{\ldots 0 . \mathrm{U}$ SE..... 2. PREV ... .4.USER-IOCB... .6. . SCR/DSK.\# ... . OUTI.3 ... B. GRP .A... D. MODEM . GRP . L.... TERM.GRP. V.... COMM.GRP.f ..... Nextu.u.... . OUTPUT.....h . . z - \% \& . UaUbucuaUe UfUgU. INPUT. . . ?b "xUsx\$sd.sT..z.: S\$UyhRRbh\&fh8hh9 U.FILL.5.jhohkhj b.R.q2\}.Z.+oqu.E RASE.a..x.\$s@.j. BI.ANKS.... $\mathrm{x}$ (...R .t+. . . . h. . HOLD. ..3bx0h..... [t* .. [ ..DZU.KEY.*. $\mathrm{Q} \$ \mathrm{kV} ; \mathrm{Rjzsx} . \mathrm{TU} . ?$ TERMINAL. I. $1 \$ \mathrm{kz}$. sjsTU.EMIT. ^ $\mathrm{bh}$ ...U.CR.u..\$x.Tb h...\$x.Th... $\mathrm{xBh}$. ... . . [+.1.[U.TYP E...5bxJh ...dhjh mk.ts.tsb.R.qB.t \$MTh...*a.I.SPAC E...e\$x s@. $\mathrm{h} \$ \mathrm{hD}$ .w. h. Sbh\&fh3hcb Ov.hd"hE..h.xJh. ... I. [+. . . [. $\mathrm{k}^{\sim}$; . abl.V.BLOCK-READ . [.4hobh.. Ih ( $h^{\prime} \mathrm{T}$ -vT.YT.XT.YOt.BL OCK-WRITE. \% ] hob h... Ih ( h'T.VT. I . xT. I.hghh"h...U. M/MOD.M..hohkhjh L..\$.S.T. X!/ ..* $\ldots: \ldots+\ldots ; / . B . D$.

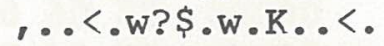
, d.. h, h*h+.U.HER E...Ubx.h...\$.T\$ +.TU.AILOT.I. $\mathrm{nbx}$ .h...dhjk.ts.tsU 


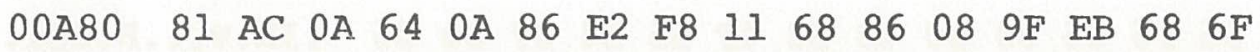
00A90 2B $44 \begin{array}{lllllllllllllll} & \text { IF } & \text { IF } & \text { F8 } & 02 & \text { AA } & 44 & 5 \mathrm{~F} & \mathrm{~F} 8 & 00 & \mathrm{BA} & \mathrm{C} 0 & 0 \mathrm{~A} & 78 & 82\end{array}$ $\begin{array}{lllllllllllllllll}00 \mathrm{AA} 0 & 43 & \mathrm{AC} & 0 \mathrm{~A} & 80 & 0 \mathrm{~A} & \mathrm{~A} 6 & \mathrm{E} 2 & \mathrm{~F} 8 & 11 & 68 & 86 & 08 & 9 \mathrm{~F} & \mathrm{~EB} & 68 & 6 \mathrm{~F}\end{array}$

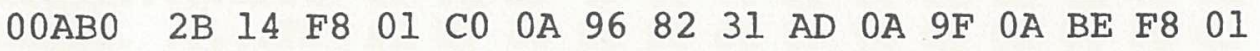
$\begin{array}{lllllllllllllllll}00 A C 0 & 14 & F 5 & 73 & F 8 & 00 & 75 & 54 & D 5 & 82 & 32 & A D & 0 A & B 7 & 0 A & C F & F 8\end{array}$

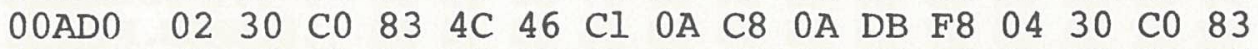

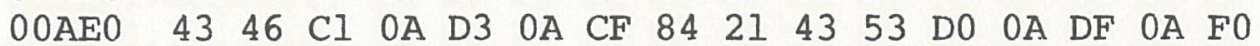

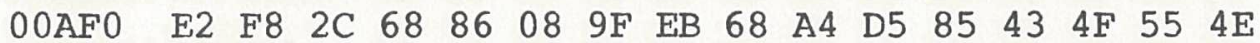
$\begin{array}{llllllllllllllllll}00 B 00 & D 4 & O A & E 7 & 0 B & 05 & 68 & 6 B & 24 & 4 B & 68 & A B & 73 & F 8 & 00 & 54 & D 5\end{array}$ $\begin{array}{lllllllllllllllll}00 B 10 & 89 & 2 D & 54 & 52 & 41 & 49 & 4 \mathrm{C} & 49 & 4 \mathrm{E} & \mathrm{C} 7 & 0 \mathrm{~A} & \mathrm{FB} & 0 \mathrm{~B} & 1 \mathrm{E} & 68 & 6 \mathrm{~B}\end{array}$

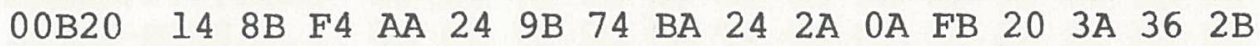

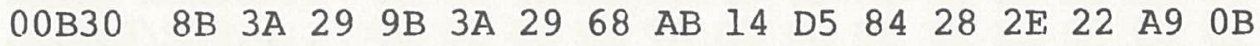
OOB40 10 OB $43 \begin{array}{llllllllllllll}48 & 52 & 24 & 68 & \text { A8 } & 73 & \text { F8 } & 00 & 54 & \text { E2 } & 88 & \text { F4 } & \text { A8 }\end{array}$ $\begin{array}{lllllllllllllllll}00 B 50 & 98 & 7 C & 00 & B 8 & C 0 & 09 & 35 & 83 & 50 & 41 & C 4 & \text { OB } & 3 A & 0 B & 5 F & E 2\end{array}$ $\begin{array}{lllllllllllllllll}00 B 60 & \text { F8 } & 11 & 68 & 86 & 08 & 9 \mathrm{~F} & \mathrm{~EB} & 68 & 6 \mathrm{~F} & \mathrm{E} 4 & 24 & 8 \mathrm{~F} & \mathrm{FC} & 44 & 73 & 9 \mathrm{~F}\end{array}$ $\begin{array}{lllllllllllllllll}00 \mathrm{~B} 70 & 7 \mathrm{C} & 00 & 54 & \mathrm{D} 5 & 82 & 2 \mathrm{~B} & \mathrm{AD} & 0 \mathrm{~B} & 57 & 0 \mathrm{~B} & 7 \mathrm{~B} & 44 & 14 & \mathrm{FE} & \mathrm{C} 3 & 04\end{array}$ $\begin{array}{lllllllllllllllll}00 \mathrm{~B} 80 & 34 & \mathrm{D} 5 & 83 & 44 & 2 \mathrm{~B} & \mathrm{AD} & 0 \mathrm{~B} & 74 & \text { OB } & 8 \mathrm{~A} & 44 & 14 & \mathrm{FE} & \mathrm{C} 3 & 04 & 4 \mathrm{~F}\end{array}$

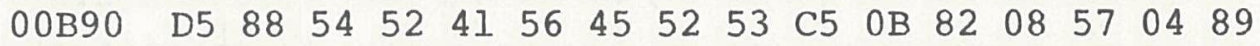
$\begin{array}{lllllllllllllllll}\text { 00BA0 } & 04 & 67 & 03 & \mathrm{C} 4 & 01 & 49 & 00 & 7 \mathrm{~F} & 04 & 67 & 04 & \mathrm{Dl} & 03 & 90 & 01 & 82\end{array}$

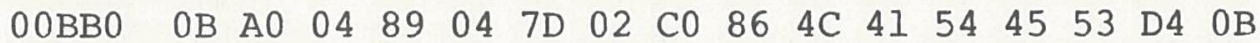
$\begin{array}{lllllllllllllllll}00 B C 0 & 91 & 08 & 57 & 07 & 42 & 04 & \mathrm{BF} & 04 & \mathrm{BF} & 02 & \mathrm{C} 0 & 83 & 4 \mathrm{E} & 46 & \mathrm{Cl} & \mathrm{OB}\end{array}$ $\begin{array}{lllllllllllllllll}00 B D 0 & B 8 & 08 & 57 & 01 & 49 & 00 & 05 & 04 & 05 & 01 & 49 & F F & F F & 0 B & 9 C & 02\end{array}$ $\begin{array}{lllllllllllllllll}00 B E 0 & C 0 & 83 & 50 & 46 & C l & 0 B & C B & 08 & 57 & 06 & 10 & 0 B & 9 C & 01 & 49 & 00\end{array}$ $\begin{array}{lllllllllllllllll}00 B F 0 & 05 & 03 & C 4 & 02 & C 0 & 86 & 3 F & 45 & 52 & 52 & 4 F & D 2 & 0 B & E 1 & 08 & 57\end{array}$ $\begin{array}{lllllllllllllllll}00 C 00 & 04 & 89 & 01 & 82 & 0 C & 0 C & 0 F & 28 & 01 & 72 & 0 C & 0 E & 04 & 7 D & 02 & C 0\end{array}$ $\begin{array}{lllllllllllllllll}00 \mathrm{Cl} 10 & 85 & 3 \mathrm{~F} & 43 & 4 \mathrm{~F} & 4 \mathrm{D} & \mathrm{D} 0 & 0 \mathrm{~B} & \mathrm{~F} 5 & 08 & 57 & 07 & 4 \mathrm{E} & 04 & \mathrm{BF} & 03 & 52\end{array}$ $\begin{array}{lllllllllllllllll}00 C 20 & 01 & 49 & 00 & 11 & 0 B & F E & 02 & C 0 & 85 & 3 F & 45 & 58 & 45 & C 3 & 0 C & 10\end{array}$ $\begin{array}{lllllllllllllllll}00 \mathrm{C} 30 & 08 & 57 & 07 & 4 \mathrm{E} & 04 & \mathrm{BF} & 01 & 49 & 00 & 12 & 0 \mathrm{~B} & \mathrm{FE} & 02 & \mathrm{C} 0 & 86 & 3 \mathrm{~F}\end{array}$ $\begin{array}{lllllllllllllllll}00 C 40 & 50 & 41 & 49 & 52 & \text { D3 } & 0 \mathrm{C} & 28 & 08 & 57 & 04 & 05 & 01 & 49 & 00 & 13 & 0 \mathrm{~B}\end{array}$

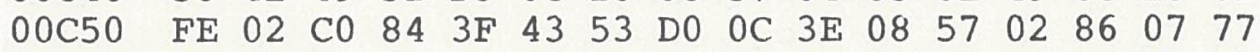
$\begin{array}{lllllllllllllllll}00 C 60 & 04 & \mathrm{BF} & 04 & 05 & 01 & 49 & 00 & 14 & 0 \mathrm{~B} & \mathrm{~F} E & 02 & \mathrm{C} 0 & 88 & 3 \mathrm{~F} & 4 \mathrm{C} & 4 \mathrm{~F}\end{array}$ $\begin{array}{lllllllllllllllll}00 C 70 & 41 & 44 & 49 & 4 \mathrm{E} & \mathrm{C} 7 & 0 \mathrm{C} & 53 & 08 & 57 & 06 & \mathrm{FC} & 04 & \mathrm{BF} & 03 & 52 & 01\end{array}$ $\begin{array}{lllllllllllllllll}00 C 80 & 49 & 00 & 16 & 0 B & F E & 02 & C 0 & 87 & 43 & 4 F & 4 D & 50 & 49 & 4 \mathrm{C} & C 5 & 0 C\end{array}$ $\begin{array}{lllllllllllllllll}00 C 90 & 6 \mathrm{C} & 08 & 57 & 0 \mathrm{C} & 18 & 03 & 36 & 04 & 9 \mathrm{C} & 03 & \mathrm{E} 7 & 03 & 28 & 04 & \mathrm{BF} & 0 \mathrm{~A}\end{array}$ $\begin{array}{lllllllllllllllll}00 C A 0 & 84 & 02 & \mathrm{C} 0 & \mathrm{Cl} & \mathrm{DB} & 0 \mathrm{C} & 87 & 08 & 57 & 06 & 08 & 07 & 4 \mathrm{E} & 04 & \mathrm{E} 0 & 02\end{array}$ $\begin{array}{lllllllllllllllll}00 C B 0 & C 0 & 81 & D D & 0 C & A 3 & 08 & 57 & 01 & 49 & 00 & \text { C0 } & 07 & 4 \mathrm{E} & 04 & \mathrm{E} 0 & 02\end{array}$ $\begin{array}{lllllllllllllllll}00 C C 0 & \mathrm{C} 0 & 86 & 53 & 4 \mathrm{D} & 55 & 44 & 47 & \mathrm{C} 5 & 0 \mathrm{C} & \mathrm{Bl} & 08 & 57 & 0 \mathrm{~B} & \mathrm{Cl} & 01 & 49\end{array}$ 00CD0 $00 \begin{array}{llllllllllllllll}20 & 04 & \mathrm{~B} 0 & 02 & \mathrm{C} 0 & 83 & 48 & 45 & \mathrm{D} 8 & 0 \mathrm{C} & \mathrm{Cl} & 08 & 57 & 01 & 49\end{array}$ OOCE0 $00 \begin{array}{llllllllllllllll}10 & 07 & 59 & 04 & \mathrm{E} 0 & 02 & \mathrm{C} 0 & 87 & 44 & 45 & 43 & 49 & 4 \mathrm{D} & 41 & \mathrm{CC}\end{array}$ $\begin{array}{lllllllllllllllll}00 C F 0 & \text { OC } & \text { D6 } & 08 & 57 & 01 & 49 & 00 & 0 A & 07 & 59 & 04 & \text { E0 } & 02 & \text { C0 } & 87 & 28\end{array}$ $\begin{array}{lllllllllllllllll}00 D 00 & 3 B & 43 & 4 \mathrm{~F} & 44 & 45 & \mathrm{~A} 9 & 0 \mathrm{C} & \mathrm{E} 8 & 08 & 57 & 03 & 36 & 0 \mathrm{~B} & \mathrm{Cl} & 0 \mathrm{~B} & \mathrm{E} 7\end{array}$

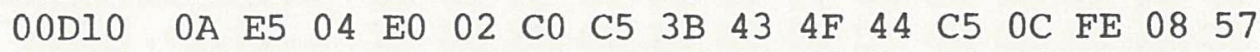

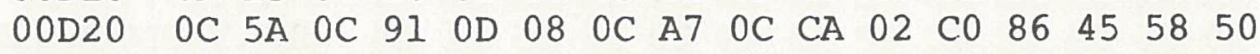
$\begin{array}{lllllllllllllllll}00 D 30 & 45 & 43 & D 4 & 0 D & 16 & 08 & 57 & 04 & 67 & 03 & C 4 & 04 & 67 & 01 & 5 B & 08\end{array}$

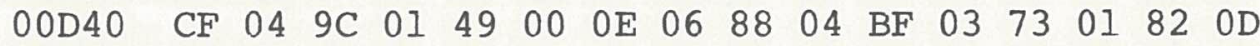
$\begin{array}{lllllllllllllllll}00 D 50 & 6 \mathrm{D} & 04 & 7 \mathrm{D} & 01 & 49 & 00 & 08 & 04 & 67 & 03 & 46 & 03 & 73 & 04 & 9 \mathrm{C} & 03\end{array}$ $\begin{array}{lllllllllllllllll}00 D 60 & 36 & 0 A & C D & 03 & C 4 & 03 & 28 & 04 & 05 & 01 & 72 & \text { OD } & 93 & 04 & 9 \mathrm{C} & 01\end{array}$ $\begin{array}{lllllllllllllllll}00 D 70 & 49 & 00 & \text { OD } & 03 & 73 & 01 & 82 & \text { OD } & 85 & 03 & \text { 1B } & 04 & 7 \mathrm{D} & 06 & 29 & 06\end{array}$ $\begin{array}{llllllllllllllllll}00 D 80 & 08 & 01 & 72 & 0 D & 87 & 04 & 9 C & 03 & 46 & 04 & F 0 & 06 & 08 & 03 & 46 & 03\end{array}$ 00D90 D5 $04 \begin{array}{lllllllllllllll} & \text { E0 } & 08 & \text { FC } & 00 & \text { AE } & 0 D & 3 F & 04 & 7 D & 02 & \text { C0 } & 85 & 51 & 55\end{array}$ $\begin{array}{lllllllllllllllll}00 D A 0 & 45 & 52 & D 9 & 0 D & 2 C & 08 & 57 & 06 & B 4 & 04 & B F & 01 & 49 & 00 & 80 & 0 D\end{array}$ $\begin{array}{lllllllllllllllll}00 \mathrm{DB} 0 & 35 & 06 & 08 & 07 & 05 & 04 & \mathrm{E} 0 & 02 & \mathrm{C} 0 & \mathrm{Cl} & 80 & 0 \mathrm{D} & 9 \mathrm{D} & 08 & 57 & 06\end{array}$ 00DC0 $\quad$ FC $04 \quad$ BF 01 82 0 OD ED $06 \begin{array}{lllllllll}10 & 06 & \text { FC } & 03 & \text { F2 } & 06 & 08 & 07\end{array}$

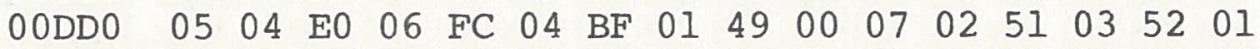

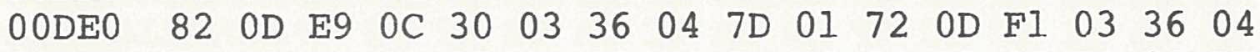

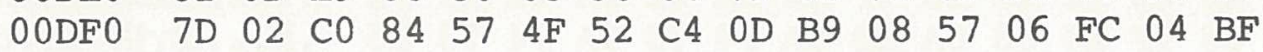

...d..bx.h...kho $+D_{-} \cdot x_{0} * D_{1} x_{0}:$ e.x. C, ... dbx.h...kho +.x. ....1-... >x. . usx.uTU.2-.7.0x .0@.LFA.H. [x.0@. CFA.S.O.!CSP._. $\mathrm{bx}, \mathrm{h} . . . \mathrm{kh} \$ \mathrm{~K} . \mathrm{CO} U \mathrm{UN}$ T.g..hk\$Kh+sX.TU .-TRAILING. \{..hk ..t*\$.t:\$*. $\{: 6+$ .:).: )h+.U.(."). .. CHR\$h (sx.Tb.t) ( $.1 .80 .5 . \mathrm{PAD} .:-\mathrm{b}$ x.h...khods.|Ds. I.TU.t-.W. \{D. C . 4 U.D+-.t..D. C.O U.TRAVERSE...W.. .g.D.I..g.Q.... - ... . a.LATEST. ..W.B.?.?.Q.NFA. 8.W.I........... @.PFA.K.W......I. ..D. . ?ERROR.a.W ............ . . a -?COMP.U.W.N.?.R .I... . . ? EXEC.. .W.N.?.I... . . ? PAIRS. (.W...I... . . . ?CSP.>.W...W .?...I... ^. . ?LO ADING.S.W. I ?.R. I... . . .COMPILE. 1.W...6...g. (.?. .. AA [...W...N. a. ].\#.W.I.@.N.' @.SMUDGE.I.W.A.I - 0. Q.HEX.A.W.I ..... . . DECIMAL -V.W.I...Y.'.a.( ;CODE).h.W.6.A.g .e.'. $A E ; C O D E$. .W .Z.....'.J.a.EXP ECT...W.g.D.g. [ O...I.....?.S...

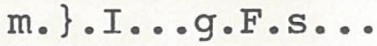
6.M.D. (...r..... I...s.......\}.). .......F.p...F. U. $.1 \ldots ?.\} . \mathrm{a} \cdot \mathrm{QU}$ ERY.,.W.4.?.I... $5 \ldots . .$. . $A$....W. I.?...m...l.r... .. . .?.I...Q.R. i. 0.6.$\} \cdot r \cdot q \cdot 6$. \}. . WORD.9.W. . .? 
$\begin{array}{lllllllllllllllll}00 E 00 & 01 & 82 & 0 E & 0 E & 06 & F C & 04 & B F & 16 & \text { DE } & 01 & 72 & 0 E & 12 & 06 & B\end{array}$ $\begin{array}{lllllllllllllllll}00 E 10 & 04 & \text { BF } & 07 & 05 & 04 & \text { BF } & 03 & \text { C4 } & 04 & 89 & 01 & 96 & 0 A & 53 & 01 & 49\end{array}$ 00E20 $00 \begin{array}{llllllllllllllll}0 & 22 & 08 & 98 & 07 & 05 & 03 & \text { F2 } & 04 & 67 & 04 & 05 & 03 & 28 & 03 & 46\end{array}$

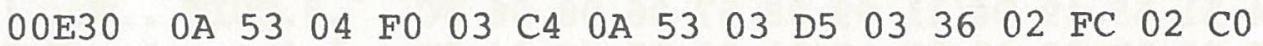
$\begin{array}{lllllllllllllllll}00 E 40 & 88 & 28 & 4 E & 55 & 4 D & 42 & 45 & 52 & \text { A9 } & \text { OD } & \text { F3 } & 08 & 57 & 03 & \text { D5 } & 04\end{array}$ $\begin{array}{lllllllllllllllll}00 \mathrm{E} 50 & 9 \mathrm{C} & 03 & 28 & 04 & \mathrm{DI} & 07 & 59 & 04 & \mathrm{BF} & 02 & \mathrm{D} 3 & 01 & 82 & 0 \mathrm{E} & 89 & 04\end{array}$ $\begin{array}{lllllllllllllllll}00 \mathrm{E} 60 & 89 & 07 & 59 & 04 & \mathrm{BF} & 02 & 3 \mathrm{~A} & 04 & 7 \mathrm{D} & 04 & \mathrm{FE} & 07 & 59 & 04 & \mathrm{BF} & 02\end{array}$ $\begin{array}{lllllllllllllllll}00 E 70 & 3 A & 04 & 16 & 07 & 63 & 04 & \text { BF } & 03 & \text { D5 } & 01 & 82 & 0 \mathrm{E} & 83 & 06 & 10 & 07\end{array}$ $\begin{array}{lllllllllllllllll}00 \mathrm{E} 80 & 63 & 03 & \mathrm{~F} 2 & 03 & 36 & 01 & 72 & 0 \mathrm{E} & 4 \mathrm{D} & 03 & 36 & 02 & \mathrm{C} 0 & 86 & 4 \mathrm{E} & 55\end{array}$

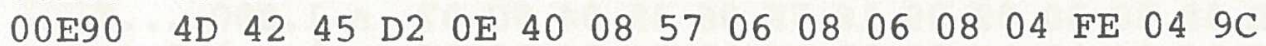
$\begin{array}{lllllllllllllllll}00 E A 0 & 03 & D 5 & 04 & D 1 & 01 & 49 & 00 & 2 D & 03 & 73 & 04 & 9 C & 03 & 28 & 03 & C 4\end{array}$

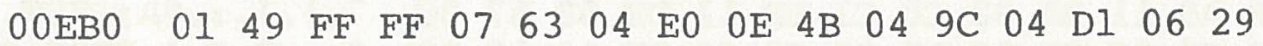
$\begin{array}{lllllllllllllllll}00 E C 0 & 04 & 05 & 01 & 82 & \text { OE } & \text { DA } & 04 & 9 \mathrm{C} & 04 & \mathrm{DI} & 01 & 49 & 00 & 2 \mathrm{E} & 04 & 05\end{array}$ $\begin{array}{lllllllllllllllll}00 E D 0 & 06 & 08 & 0 B & F E & 06 & 08 & 01 & 72 & 0 E & B 4 & 04 & 7 D & 03 & 36 & 01 & 82\end{array}$

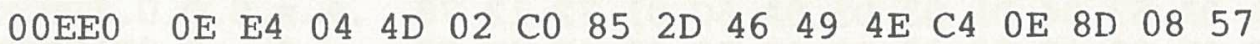
OOEFO $06 \quad 29$ OD FA $0 A \begin{array}{lllllllllll}53 & 07 & 34 & 04 & \text { BF } & 04 & \text { BF } & 00 & \text { F5 } & 04 & 9 \mathrm{C}\end{array}$ $\begin{array}{lllllllllllllllll}00 F 00 & 03 & 52 & 01 & 82 & \text { OF } & 0 \mathrm{E} & 04 & 7 \mathrm{D} & 0 \mathrm{~A} & 53 & 0 \mathrm{~B} & \mathrm{Cl} & 00 & \mathrm{~F} 5 & 02 & \mathrm{C} 0\end{array}$ $\begin{array}{lllllllllllllllll}00 \mathrm{~F} 10 & 87 & 28 & 41 & 42 & 4 \mathrm{~F} & 52 & 54 & \mathrm{~A} 9 & 0 \mathrm{E} & \mathrm{E} 6 & 08 & 57 & 11 & \mathrm{E} 6 & 02 & \mathrm{C} 0\end{array}$ $\begin{array}{lllllllllllllllll}00 \mathrm{~F} 20 & 85 & 45 & 52 & 52 & 4 \mathrm{~F} & \mathrm{D} 2 & 0 \mathrm{~F} & 10 & 08 & 57 & 06 & \mathrm{CE} & 04 & \mathrm{BF} & 03 & 67\end{array}$ $\begin{array}{lllllllllllllllll}00 F 30 & 01 & 82 & \text { OF } & 36 & \text { OF } & \text { IA } & 0 A & 53 & \text { OB } & 03 & 09 & 33 & 0 B & 41 & 02 & 20\end{array}$ $\begin{array}{lllllllllllllllll}00 \mathrm{~F} 40 & 3 \mathrm{~F} & 15 & \mathrm{~B} 9 & 02 & 97 & 07 & 05 & 04 & \mathrm{BF} & 06 & \mathrm{FC} & 04 & \mathrm{BF} & 11 & \mathrm{BA} & 02\end{array}$ $\begin{array}{lllllllllllllllll}00 F 50 & \mathrm{C} 0 & 83 & 4 \mathrm{D} & 49 & \mathrm{CE} & 0 \mathrm{~F} & 20 & 08 & 57 & 04 & 67 & 04 & 67 & 03 & \mathrm{AE} & 01\end{array}$ $\begin{array}{lllllllllllllllll}00 \mathrm{~F} 60 & 82 & \text { OF } & 65 & 04 & 89 & 04 & 7 \mathrm{D} & 02 & \mathrm{C} 0 & 83 & 49 & 44 & \mathrm{AE} & 0 \mathrm{~F} & 51 & 08\end{array}$ $\begin{array}{lllllllllllllllll}00 \mathrm{~F} 70 & 57 & 0 \mathrm{~B} & 5 \mathrm{D} & 01 & 49 & 00 & 20 & 01 & 49 & 00 & 5 \mathrm{~F} & 08 & 68 & 04 & 9 \mathrm{C} & 0 \mathrm{~B}\end{array}$ $\begin{array}{lllllllllllllllll}00 F 80 & E 7 & 0 A & D 9 & 04 & 67 & 04 & 05 & 0 B & 5 D & 04 & 89 & 02 & F C & 0 B & 5 D & 0 B\end{array}$ $\begin{array}{lllllllllllllllll}00 F 90 & 03 & 01 & 49 & 00 & 1 \mathrm{~F} & 02 & 51 & 09 & 33 & 09 & 63 & 02 & \mathrm{C} 0 & 86 & 43 & 52\end{array}$ $\begin{array}{lllllllllllllllll}\text { OOFA0 } & 45 & 41 & 54 & \mathrm{C} 5 & \text { OF } & 69 & 08 & 57 & 02 & 86 & 0 A & 53 & 01 & 49 & 00 & A 0\end{array}$

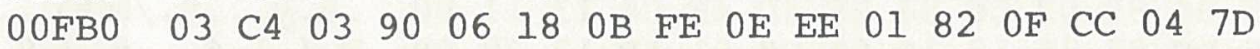
$\begin{array}{lllllllllllllllll}00 F C 0 & 0 B & D 1 & 0 F & 6 F & 01 & 49 & 00 & 04 & 15 & B 9 & 09 & 63 & 0 A & 53 & 04 & 9 C\end{array}$

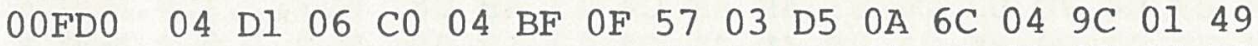
OOFEO 00 A0 04 BO $0 A \begin{array}{llllllllllll}53 & 06 & 10 & 04 & 05 & 01 & 49 & 00 & 80 & 04 & \text { B0 }\end{array}$ $\begin{array}{llllllllllllllllllllll}00 F F O & 0 B & C l & 0 A & 84 & 07 & 42 & 04 & B F & 04 & E 0 & 0 A & 53 & 03 & \text { E7 } & 0 A & 84\end{array}$ $\begin{array}{lllllllllllllllll}01000 & 02 & \mathrm{C} 0 & \mathrm{Cl} & \mathrm{BA} & 0 \mathrm{~F} & 9 \mathrm{D} & 08 & 57 & 0 \mathrm{C} & 30 & 0 \mathrm{~A} & \mathrm{EE} & 07 & 42 & 04 & \mathrm{BF}\end{array}$ $\begin{array}{llllllllllllllllll}01010 & 07 & 34 & 04 & \text { E0 } & 0 F & A 6 & 0 C & B 5 & 01 & 49 & \text { FF } & \text { FE } & 06 & \text { E3 } & 03 & \text { F2 }\end{array}$ $\begin{array}{lllllllllllllllll}01020 & 0 \mathrm{C} & 91 & 08 & 57 & 02 & \mathrm{C} 0 & 85 & 21 & 43 & 4 \mathrm{~F} & 44 & \mathrm{C} 5 & 10 & 02 & 08 & 57\end{array}$

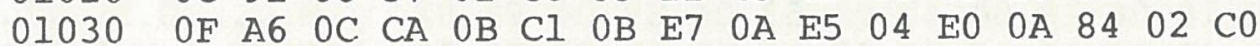
$\begin{array}{lllllllllllllllll}01040 & 88 & 43 & 4 \mathrm{~F} & 4 \mathrm{E} & 53 & 54 & 41 & 4 \mathrm{E} & \mathrm{D} 4 & 10 & 26 & 08 & 57 & 01 & 49 & 05\end{array}$ $\begin{array}{lllllllllllllllll}01050 & \text { FB } & 10 & 2 E & 02 & \text { C0 } & 88 & 56 & 41 & 52 & 49 & 41 & 42 & 4 C & C 5 & 10 & 40\end{array}$ $\begin{array}{lllllllllllllllll}01060 & 08 & 57 & 01 & 49 & 05 & \mathrm{FE} & 10 & 2 \mathrm{E} & 02 & \mathrm{C} 0 & 84 & 55 & 53 & 45 & \mathrm{D} 2 & 10\end{array}$ $\begin{array}{lllllllllllllllll}01070 & 55 & 08 & 57 & 01 & 49 & 06 & 90 & 10 & 2 \mathrm{E} & 02 & \mathrm{C} 0 & 87 & 3 \mathrm{C} & 42 & 55 & 49\end{array}$ $\begin{array}{lllllllllllllllll}01080 & 4 \mathrm{C} & 44 & \mathrm{D} 3 & 10 & 6 \mathrm{~A} & 08 & 57 & 06 & 08 & 10 & 4 \mathrm{~B} & 02 & \mathrm{C} 0 & 85 & 44 & 4 \mathrm{~F}\end{array}$ $\begin{array}{lllllllllllllllll}01090 & 45 & 53 & \mathrm{BE} & 10 & 7 \mathrm{~B} & 08 & 57 & 03 & 36 & \mathrm{OB} & \mathrm{Cl} & \text { OB } & \mathrm{E} 7 & 04 & \mathrm{E} & 0 \mathrm{OD}\end{array}$

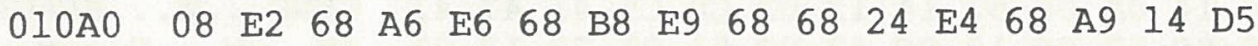
010B0 $\quad$ C7 $7 \begin{array}{lllllllllllllll}4 \mathrm{C} & 49 & 54 & 45 & 52 & 41 & \mathrm{CC} & 10 & 8 \mathrm{D} & 08 & 57 & 07 & 4 \mathrm{E} & 04 & \mathrm{BF}\end{array}$ $\begin{array}{lllllllllllllllll}010 C 0 & 01 & 82 & 10 & C A & 0 C & 91 & 01 & 49 & 0 A & 84 & 02 & C 0 & C 8 & 44 & 4 \mathrm{C} & 49\end{array}$ 010D0 $54 \begin{array}{llllllllllllllll} & 45 & 52 & 41 & \mathrm{CC} & 10 & \mathrm{~B} 0 & 08 & 57 & 07 & 4 \mathrm{E} & 04 & \mathrm{BF} & 01 & 82 & 10\end{array}$ OIOE0 E7 $04 \begin{array}{lllllllllllllll} & 10 & \mathrm{BA} & 10 & \mathrm{BA} & 02 & \mathrm{C} 0 & 86 & 3 \mathrm{~F} & 53 & 54 & 41 & 43 & \mathrm{CB}\end{array}$ 010F0 $10 \begin{array}{lllllllllllllll} & 08 & 57 & 06 & \mathrm{Al} & 04 & \mathrm{BF} & 04 & 9 \mathrm{C} & 02 & 86 & 03 & 90 & 06 & 10\end{array}$ $\begin{array}{lllllllllllllllll}01100 & 0 B & F E & 01 & 49 & 01 & 00 & 04 & 05 & 02 & 86 & 03 & A E & 01 & 49 & 00 & 07\end{array}$ $\begin{array}{lllllllllllllllll}01110 & 0 \mathrm{~B} & \mathrm{FE} & 02 & \mathrm{C} 0 & 89 & 49 & 4 \mathrm{E} & 54 & 45 & 52 & 50 & 52 & 45 & \mathrm{D} 4 & 10 & \mathrm{E} 9\end{array}$ $\begin{array}{lllllllllllllllll}01120 & 08 & 57 & 0 E & E E & 01 & 82 & 11 & 44 & 07 & 4 \mathrm{E} & 04 & \mathrm{BF} & 03 & 90 & 01 & 82\end{array}$ $\begin{array}{lllllllllllllllll}01130 & 11 & 3 \mathrm{~A} & 0 \mathrm{~A} & \mathrm{E} 5 & 0 \mathrm{~A} & 84 & 01 & 72 & 11 & 3 \mathrm{E} & 0 \mathrm{~A} & \mathrm{E} 5 & 01 & \mathrm{~F} 7 & 10 & \mathrm{~F} 2\end{array}$ $\begin{array}{lllllllllllllllll}01140 & 01 & 72 & 11 & 5 E & 0 A & 53 & 0 E & 96 & 07 & 63 & 04 & \text { BF } & 03 & \text { D5 } & 01 & 82\end{array}$ $\begin{array}{lllllllllllllllll}01150 & 11 & 58 & 10 & \text { D7 } & 01 & 72 & 11 & 5 C & 04 & 7 D & 10 & \text { BA } & 10 & \text { F2 } & 01 & 72\end{array}$ $\begin{array}{lllllllllllllllll}01160 & 11 & 22 & 02 & \mathrm{C} 0 & 8 \mathrm{~A} & 56 & 4 \mathrm{~F} & 43 & 41 & 42 & 55 & 4 \mathrm{C} & 41 & 52 & \mathrm{D} 9 & 11\end{array}$ $\begin{array}{lllllllllllllllll}01170 & 14 & 08 & 57 & 10 & 85 & 01 & 49 & 81 & A 0 & O A & 84 & 07 & 42 & 04 & B F & 0 A\end{array}$
.......?. . . ...4 ?...?.D.....S.I ............ (.F .S.P.D.S.U.6.1. a - (NUMBER) . S.W.U. .. (.Q.Y.?.S..... ..Y.?...\}. . Y.?. ¿... . . U...... c.r.6.r.M.6.d.NU MBER. C.W..... .. .U.Q.I. . . . ... (.D .I...C.'.K...Q.)

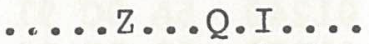
$\ldots \sim \ldots r .4\} ..6 \ldots$ .d.M. . -FIND...W .).z.S.4.?.?.U. .R..... . . S.A.u. Q - (ABORT) .f.W.f. a .ERROR ...W.N.?.g ..6... . . 3.A. ?.9....?.।.?. : a.MIN. .W.g.g... ..e...\}. Q.ID..Q. W. ].I. .I._.h... g.Y.g...]...... . ..I...Q.3.C.Q.CR EATE.i.W...S.I. .D..... . . ...... .Q.O.I...9.C.S. .Q.Q.?.W.U.I...I . $0 . S_{\ldots} \ldots . . . .0$ . A...B.?.S.g. . QA: ...W. $0 . n \cdot B . ?$ .4. .\&.5.I. .C.r ...W. . . !CODE...W .\&.J.A.g.e.'... ( . CONSTANT.\&.W.I. \{.....VARIABLE. a .W.I. ... a.USER. U.W.I....... LDS. j.W...K. @.DO ES>. \{.W.6.A.g.' . bh\&fh8ihh\$ah).U GLITERAL...W.N.? ...J...... AHDLI TERAL. 0.W.N.?... g.........?STACK . L.W. . ?........ $\sim$. .......... - 〜.INTERPRET.i .W.n...D.N.?... . . e...r. >.e.w.r .r.^.S...c.?.U. .X.W.r. .. $\}$ : . r. r .". @ VOCABULARY. ..W........?.
Woods Hole FORTH Version 1.800 
$\begin{array}{lllllllllllllllll}01180 & E 5 & 0 A & 84 & 0 A & 53 & 06 & F 2 & 04 & B F & 0 A & 84 & 06 & F 2 & 04 & E 0 & 10\end{array}$ $\begin{array}{lllllllllllllllll}01190 & 95 & 03 & \mathrm{E} 7 & 07 & 34 & 04 & \mathrm{E} 0 & 02 & \mathrm{C} 0 & 8 \mathrm{~B} & 44 & 45 & 46 & 49 & 4 \mathrm{E} & 49\end{array}$ $\begin{array}{lllllllllllllllll}011 \mathrm{~A} 0 & 54 & 49 & 4 \mathrm{~F} & 4 \mathrm{E} & \mathrm{D} 3 & 11 & 64 & 08 & 57 & 07 & 34 & 04 & \mathrm{BF} & 07 & 42 & 04\end{array}$ $\begin{array}{lllllllllllllllll}011 \mathrm{~B} 0 & \mathrm{E} 0 & 02 & \mathrm{C} 0 & 84 & 51 & 55 & 49 & \mathrm{D} 4 & 11 & 99 & 08 & 57 & 06 & 08 & 06 & \mathrm{FC}\end{array}$

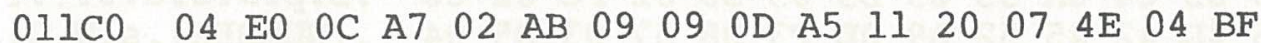
$\begin{array}{lllllllllllllllll}011 D 0 & 03 & 52 & 01 & 82 & 11 & \text { D8 } & 18 & \text { A9 } & 01 & 72 & 11 & \text { C4 } & 02 & \text { C0 } & 85 & 41 .\end{array}$ $\begin{array}{lllllllllllllllll}011 \mathrm{E} 0 & 42 & 4 \mathrm{~F} & 52 & \mathrm{D} 4 & 11 & \mathrm{~B} 3 & 08 & 57 & 02 & 97 & 0 \mathrm{C} & \mathrm{F} 2 & 06 & 10 & 17 & \mathrm{BC}\end{array}$ $\begin{array}{lllllllllllllllll}011 \mathrm{~F} 0 & 09 & 09 & 0 \mathrm{~B} & 41 & 10 & 57 & 6 \mathrm{~F} & 6 \mathrm{~F} & 64 & 73 & 20 & 48 & 6 \mathrm{~F} & 6 \mathrm{C} & 65 & 20\end{array}$ $\begin{array}{lllllllllllllllll}01200 & 46 & 4 \mathrm{~F} & 52 & 54 & 48 & 09 & 09 & \text { OB } & 41 & 0 \mathrm{D} & 56 & 65 & 72 & 73 & 69 & 6 \mathrm{~F}\end{array}$ $\begin{array}{lllllllllllllllll}01210 & 6 \mathrm{E} & 20 & 31 & 2 \mathrm{E} & 38 & 30 & 30 & 09 & 09 & 16 & 7 \mathrm{~F} & 06 & 49 & 04 & 9 \mathrm{C} & 07\end{array}$ $\begin{array}{lllllllllllllllll}01220 & 9 \mathrm{~F} & 04 & \mathrm{E} 0 & 07 & 94 & 04 & \mathrm{E} 0 & 18 & 37 & 11 & \mathrm{~A} 7 & 18 & 73 & 06 & 10 & 16\end{array}$

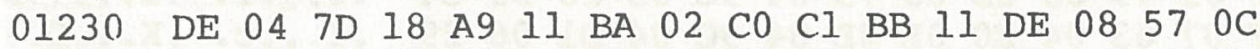
$\begin{array}{lllllllllllllllll}01240 & 5 A & 0 C & 91 & 02 & C 0 & 0 C & C A & 0 C & A 7 & 02 & C 0 & C 2 & 2 E & A 2 & 12 & 39\end{array}$ $\begin{array}{lllllllllllllllll}01250 & 08 & 57 & 01 & 49 & 00 & 22 & 07 & 4 \mathrm{E} & 04 & \mathrm{BF} & 01 & 82 & 12 & 70 & 0 \mathrm{C} & 91\end{array}$ $\begin{array}{lllllllllllllllll}01260 & 0 B & 41 & O D & F A & 0 A & 53 & 04 & D 1 & 03 & D 5 & 0 A & 6 C & 01 & 72 & 12 & 78\end{array}$ $\begin{array}{lllllllllllllllll}01270 & 0 D & F A & 0 A & 53 & 0 B & 03 & 09 & 33 & 02 & \text { C0 } & \text { C9 } & 5 B & 43 & 4 \mathrm{~F} & 4 \mathrm{D} & 50\end{array}$ $\begin{array}{lllllllllllllllll}01280 & 49 & 4 \mathrm{C} & 45 & \mathrm{DD} & 12 & 4 \mathrm{~B} & 08 & 57 & 0 \mathrm{E} & \mathrm{EE} & 03 & 52 & 06 & 08 & 0 \mathrm{~B} & \mathrm{FE}\end{array}$ $\begin{array}{lllllllllllllllll}01290 & 04 & 7 \mathrm{D} & 0 \mathrm{~A} & \mathrm{E} 5 & 0 \mathrm{~A} & 84 & 02 & \mathrm{C} 0 & 89 & 49 & 4 \mathrm{D} & 4 \mathrm{D} & 45 & 44 & 49 & 41\end{array}$ $\begin{array}{lllllllllllllllll}012 \mathrm{~A} 0 & 54 & \mathrm{C} 5 & 12 & 7 \mathrm{~A} & 08 & 57 & 0 \mathrm{~B} & \mathrm{Cl} & 01 & 49 & 00 & 40 & 04 & \mathrm{~B} 0 & 02 & \mathrm{C} 0\end{array}$

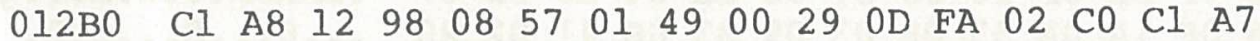
$\begin{array}{lllllllllllllllll}012 \mathrm{C} 0 & 12 & \mathrm{~B} 0 & 08 & 57 & 0 \mathrm{E} & \mathrm{EE} & 03 & 52 & 06 & 08 & 0 \mathrm{~B} & \mathrm{FE} & 04 & 7 \mathrm{D} & 10 & \mathrm{BA}\end{array}$ $\begin{array}{lllllllllllllllll}012 \mathrm{D} 0 & 02 & \mathrm{C} 0 & 86 & 46 & 4 \mathrm{~F} & 52 & 47 & 45 & \mathrm{D} 4 & 12 & \mathrm{BE} & 08 & 57 & 07 & 42 & 04\end{array}$ $\begin{array}{lllllllllllllllll}012 \mathrm{E} 0 & \mathrm{BF} & 07 & 34 & 04 & \mathrm{BF} & 04 & 05 & 01 & 49 & 00 & 18 & \text { OB } & \mathrm{FE} & 12 & \mathrm{C} 2 & 04\end{array}$ $\begin{array}{lllllllllllllllll}012 \mathrm{~F} 0 & 9 \mathrm{C} & 06 & \mathrm{DA} & 04 & \mathrm{BF} & 03 & 90 & 01 & 49 & 00 & 15 & 0 \mathrm{~B} & \mathrm{FE} & 04 & 9 \mathrm{C} & 0 \mathrm{~B}\end{array}$ $\begin{array}{lllllllllllllllll}01300 & \mathrm{D} 1 & 06 & \mathrm{E} 3 & 04 & \mathrm{E} 0 & 0 \mathrm{~A} & \mathrm{D} 9 & 04 & \mathrm{BF} & 07 & 34 & 04 & \mathrm{BF} & 04 & \mathrm{E} 0 & 02\end{array}$ $\begin{array}{lllllllllllllllll}01310 & \text { C0 } & 82 & 4 \mathrm{D} & \mathrm{AF} & 12 & \mathrm{D} 2 & 08 & 57 & 04 & 67 & 03 & 28 & 03 & 28 & 05 & 39\end{array}$ $\begin{array}{lllllllllllllllll}01320 & 03 & 46 & 05 & 2 \mathrm{~A} & 02 & 04 & 03 & 36 & 03 & 46 & 02 & 74 & 0 \mathrm{~B} & 79 & 04 & 89\end{array}$ $\begin{array}{lllllllllllllllll}01330 & 03 & 36 & 0 B & 79 & 04 & 89 & 02 & \text { C0 } & 84 & 2 \mathrm{~F} & 4 \mathrm{D} & 4 \mathrm{~F} & \mathrm{C} 4 & 13 & 11 & 08\end{array}$ $\begin{array}{lllllllllllllllll}01340 & 57 & 03 & 28 & 15 & \mathrm{DE} & 03 & 36 & 13 & 16 & 02 & \mathrm{C} 0 & 81 & \mathrm{AF} & 13 & 38 & 08\end{array}$ $\begin{array}{lllllllllllllllll}01350 & 57 & 13 & 3 \mathrm{~F} & 04 & 89 & 04 & 7 \mathrm{D} & 02 & \mathrm{C} 0 & 83 & 4 \mathrm{D} & 4 \mathrm{~F} & \mathrm{C} 4 & 13 & 4 \mathrm{~B} & 08\end{array}$ $\begin{array}{lllllllllllllllll}01360 & 57 & 13 & 3 \mathrm{~F} & 04 & 7 \mathrm{D} & 02 & \mathrm{C} 0 & 85 & 2 \mathrm{~A} & 2 \mathrm{~F} & 4 \mathrm{D} & 4 \mathrm{~F} & \mathrm{C} 4 & 13 & 59 & 08\end{array}$ $\begin{array}{lllllllllllllllll}01370 & 57 & 03 & 28 & 05 & 76 & 03 & 36 & 13 & 16 & 02 & \mathrm{C} 0 & 82 & 2 \mathrm{~A} & \mathrm{AF} & 13 & 67\end{array}$ $\begin{array}{lllllllllllllllll}01380 & 08 & 57 & 13 & 6 \mathrm{~F} & 04 & 89 & 04 & 7 \mathrm{D} & 02 & \mathrm{C} 0 & 84 & 42 & 41 & 43 & \mathrm{CB} & 13\end{array}$ $\begin{array}{lllllllllllllllll}01390 & 7 \mathrm{~B} & 08 & 57 & 0 \mathrm{~A} & 84 & 02 & \mathrm{C} 0 & \mathrm{C} 5 & 42 & 45 & 47 & 49 & \mathrm{CE} & 13 & 8 \mathrm{~A} & 08\end{array}$ $\begin{array}{lllllllllllllllll}013 \mathrm{~A} 0 & 57 & \text { OC } & 18 & \text { OA } & 53 & 06 & 10 & 02 & \mathrm{C} 0 & \mathrm{C} 5 & 45 & 4 \mathrm{E} & 44 & 49 & \mathrm{C} 6 & 13\end{array}$ $\begin{array}{lllllllllllllllll}013 \mathrm{~B} 0 & 97 & 08 & 57 & 0 \mathrm{C} & 18 & 06 & 18 & 0 \mathrm{C} & 47 & 0 \mathrm{~A} & 53 & 04 & 89 & 04 & \mathrm{E} 0 & 02\end{array}$ $\begin{array}{lllllllllllllllll}013 \mathrm{C} 0 & \mathrm{C} 0 & \mathrm{C} 4 & 54 & 48 & 45 & \mathrm{CE} & 13 & \mathrm{~A} 9 & 08 & 57 & 13 & \mathrm{Bl} & 02 & \mathrm{C} 0 & \mathrm{C} 2 & 44\end{array}$ $\begin{array}{lllllllllllllllll}013 \mathrm{D} 0 & \mathrm{CF} & 13 & \mathrm{Cl} & 08 & 57 & 0 \mathrm{C} & 91 & 01 & 5 \mathrm{~B} & 0 \mathrm{~A} & 53 & 06 & 20 & 02 & \mathrm{C} 0 & \mathrm{C} 4\end{array}$ $\begin{array}{lllllllllllllllll}013 \mathrm{E} 0 & 4 \mathrm{C} & 4 \mathrm{~F} & 4 \mathrm{~F} & \mathrm{D} 0 & 13 & \mathrm{CE} & 08 & 57 & 06 & 20 & 0 \mathrm{C} & 47 & 0 \mathrm{C} & 91 & 00 & \mathrm{AE}\end{array}$ $\begin{array}{lllllllllllllllll}013 \mathrm{~F} 0 & 13 & 91 & 02 & \mathrm{C} 0 & \mathrm{C} 5 & 2 \mathrm{~B} & 4 \mathrm{C} & 4 \mathrm{~F} & 4 \mathrm{~F} & \mathrm{D} 0 & 13 & \mathrm{DF} & 08 & 57 & 06 & 20\end{array}$ $\begin{array}{lllllllllllllllll}01400 & 0 \mathrm{C} & 47 & 0 \mathrm{C} & 91 & 00 & \mathrm{D} 3 & 13 & 91 & 02 & \mathrm{C} 0 & \mathrm{C} 5 & 55 & 4 \mathrm{E} & 54 & 49 & \mathrm{CC}\end{array}$ $\begin{array}{lllllllllllllllll}01410 & 13 & \mathrm{~F} 4 & 08 & 57 & 06 & 10 & 0 \mathrm{C} & 47 & 0 \mathrm{C} & 91 & 01 & 82 & 13 & 91 & 02 & \mathrm{CO}\end{array}$ $\begin{array}{lllllllllllllllll}01420 & \text { C3 } & 45 & 4 \mathrm{E} & \text { C4 } 4 & 14 & 0 \mathrm{~A} & 08 & 57 & 14 & 12 & 02 & \mathrm{C} 0 & \mathrm{C} 5 & 41 & 47 & 41\end{array}$ $\begin{array}{lllllllllllllllll}01430 & 49 & \mathrm{CE} & 14 & 20 & 08 & 57 & 06 & 10 & 0 \mathrm{C} & 47 & 0 \mathrm{C} & 91 & 01 & 72 & 13 & 91\end{array}$ $\begin{array}{lllllllllllllllll}01440 & 02 & \text { C0 } & \text { C6 } & 52 & 45 & 50 & 45 & 41 & \text { D4 } & 14 & 2 \mathrm{C} & 08 & 57 & 03 & 28 & 03\end{array}$ $\begin{array}{lllllllllllllllll}01450 & 28 & 14 & 34 & 03 & 36 & 03 & 36 & 06 & 18 & 04 & 05 & 13 & \mathrm{Bl} & 02 & \mathrm{C} 0 & \mathrm{C} 2\end{array}$ $\begin{array}{lllllllllllllllll}01460 & 49 & \mathrm{C} 6 & 14 & 42 & 08 & 57 & 0 \mathrm{C} & 91 & 01 & 82 & 0 \mathrm{~A} & 53 & 06 & 08 & 0 \mathrm{~A} & 84\end{array}$ $\begin{array}{lllllllllllllllll}01470 & 06 & 18 & 02 & \mathrm{C} 0 & \mathrm{C} 4 & 45 & 4 \mathrm{C} & 53 & \mathrm{C} 5 & 14 & 5 \mathrm{~F} & 08 & 57 & 06 & 18 & 0 \mathrm{C}\end{array}$ $\begin{array}{lllllllllllllllll}01480 & 47 & \text { OC } & 91 & 01 & 72 & \text { OA } & 53 & 06 & 08 & 0 A & 84 & 04 & 89 & 06 & 18 & 13\end{array}$ $\begin{array}{lllllllllllllllll}01490 & \text { B1 } & 06 & 18 & 02 & \text { C0 } & \text { C5 } & 57 & 48 & 49 & 4 \mathrm{C} & \text { C5 } & 14 & 74 & 08 & 57 & 14\end{array}$ $\begin{array}{lllllllllllllllll}014 \mathrm{~A} 0 & 64 & 03 & \mathrm{E} 7 & 02 & \mathrm{C} 0 & 86 & 53 & 50 & 41 & 43 & 45 & \mathrm{D} 3 & 1.4 & 95 & 08 & 57\end{array}$ $\begin{array}{llllllllllllllllll}014 \mathrm{~B} 0 & 06 & 08 & 05 & 47 & 05 & 16 & 01 & 82 & 14 & \mathrm{C} 4 & 06 & 08 & 01 & 5 \mathrm{~B} & 09 & 63\end{array}$ $\begin{array}{lllllllllllllllll}014 \mathrm{C} 0 & 00 & \mathrm{AE} & 14 & \mathrm{BE} & 02 & \mathrm{C} 0 & 82 & 3 \mathrm{C} & \mathrm{A} 3 & 14 & \mathrm{~A} 5 & 08 & 57 & 0 \mathrm{~B} & 5 \mathrm{D} & 07\end{array}$ $\begin{array}{lllllllllllllllll}014 \mathrm{D} 0 & 8 \mathrm{~A} & 04 & \mathrm{E} 0 & 02 & \mathrm{C} 0 & 82 & 23 & \mathrm{BE} & 14 & \mathrm{C} 6 & 08 & 57 & 04 & 7 \mathrm{D} & 04 & 7 \mathrm{D}\end{array}$ $\begin{array}{lllllllllllllllll}014 \mathrm{E} 0 & 07 & 8 \mathrm{~A} & 04 & \mathrm{BF} & 0 \mathrm{~B} & 5 \mathrm{D} & 04 & 67 & 04 & 05 & 02 & \mathrm{C} 0 & 84 & 53 & 49 & 47\end{array}$ $\begin{array}{lllllllllllllllll}014 \mathrm{~F} 0 & \mathrm{CE} & 14 & \mathrm{D} 5 & 08 & 57 & 04 & \mathrm{FE} & 03 & 67 & 01 & 82 & 15 & 03 & 01 & 49 & 00\end{array}$ e...s.r.?...r.'. ..9.4. . Q.DEFINI TIONS.d.W.4.?.B. .Q.QUIT....W...। $\therefore$ '. ... \% . N.? .R....X.).r.D. (.A BORT.3.W...r...< ....A.Woods Hole FORTH...A. Versio n $1.800 \ldots . .$. . ..... $7 . '$. S... ๑).).:. QA ; . ^.W. Z....... . . B.".9 .W.I.".N.?.... . . .A.z.S.Q.U.I.r.x .z.S...3. a I [ COMP ILE].K.W.n.R... .\}.e... a. IMMEDIA TE.z.W.A.I.Q.0. a $A\left(\ldots W . I_{0}\right) . z . A^{\prime}$ . O.W.n.R... . . : . (a.FORGET.>.W.B. ?.4.?...I... . B. ..Z.?...I... ... Q.C.'Y.?.4.?.' a.M/.R.W.g. (. (.9 .F.*...6.F.t.Y.. .6.Y... এ./MOD... W. $(. \wedge 6 \ldots . . .8$. W.?... . @.MOD.K. W.?.\}. a.*/MOD.Y. W. $(. v \cdot 6 \ldots . * / . g$ .W.O... . . . BACK. \{.W.... AEBEGIN... W...S... QEEENDIF. ..W........... QDTHEN.).W.1. @BD O.A.W.... . . . . QD LOOP.N.W. .G.... ... QEE+LOOP..W. .G...S... QEUTNTIL .t.W...G....... CEND...W... EEAGA IN. .W...G...r.. . QFREPEAT. ..W. (. (.4.6.6..... . . B IF.B.W.....S... ... QDELSE._.W... G...r.s........ 1...@EWHILE.t.W. d.g. . SPACES...W ... G..... D... . C ... >. [.<\#.8.W.]. .....\#>.F.W.\}.\} ...?.].g...e.sIG N.U.W. . . .....I. 
$\begin{array}{lllllllllllllllll}01500 & 2 \mathrm{D} & 08 & \mathrm{Bl} & 02 & \mathrm{C} 0 & 81 & \mathrm{~A} 3 & 14 & \mathrm{EC} & 08 & 57 & 07 & 59 & 04 & \mathrm{BF} & 0 \mathrm{~A}\end{array}$ $\begin{array}{lllllllllllllllll}01510 & 07 & 04 & \mathrm{FE} & 01 & 49 & 00 & 09 & 04 & 67 & 03 & 90 & 01 & 82 & 15 & 25 & 01\end{array}$ $\begin{array}{lllllllllllllllll}01520 & 49 & 00 & 07 & 03 & \text { C4 } & 01 & 49 & 00 & 30 & 03 & \text { C4 } & 08 & \text { Bl } & 02 & \text { C0 } & 82\end{array}$ $\begin{array}{lllllllllllllllll}01530 & 23 & \text { D3 } & 15 & 05 & 08 & 57 & 15 & 09 & 04 & 67 & 04 & 67 & 02 & 62 & 03 & 52\end{array}$ $\begin{array}{lllllllllllllllll}01540 & 01 & 82 & 15 & 36 & 02 & \mathrm{C} 0 & 83 & 44 & 2 \mathrm{E} & \mathrm{D} 2 & 15 & 2 \mathrm{~F} & 08 & 57 & 03 & 28\end{array}$ $\begin{array}{llllllllllllllllll}01550 & 04 & 89 & 04 & 67 & 05 & 39 & 14 & \mathrm{CB} & 15 & 34 & 14 & \mathrm{~F} 3 & 14 & \mathrm{DA} & 03 & 36\end{array}$ $\begin{array}{lllllllllllllllll}01560 & 04 & 67 & 04 & 05 & 14 & \mathrm{AE} & 09 & 33 & 02 & \mathrm{C} 0 & 82 & 2 \mathrm{E} & \mathrm{D} 2 & 15 & 46 & 08\end{array}$ $\begin{array}{lllllllllllllllll}01570 & 57 & 03 & 28 & 15 & \mathrm{DE} & 03 & 36 & 15 & 4 \mathrm{C} & 02 & \mathrm{C} 0 & 82 & 44 & \mathrm{AE} & 15 & 6 \mathrm{~A}\end{array}$ $\begin{array}{llllllllllllllllll}01580 & 08 & 57 & 06 & 08 & 15 & 4 \mathrm{C} & 09 & 63 & 02 & \mathrm{C} 0 & 81 & \mathrm{AE} & 15 & 7 \mathrm{~B} & 08 & 57\end{array}$ $\begin{array}{lllllllllllllllll}01590 & 15 & \mathrm{DE} & 15 & 80 & 02 & \mathrm{C} 0 & 81 & \mathrm{BF} & 15 & 8 \mathrm{~A} & 08 & 57 & 04 & \mathrm{BF} & 15 & 8 \mathrm{E}\end{array}$ $\begin{array}{lllllllllllllllll}015 \mathrm{~A} 0 & 02 & \mathrm{C} 0 & 82 & 55 & \mathrm{AE} & 15 & 96 & 08 & 57 & 06 & 08 & 15 & 80 & 02 & \mathrm{C} 0 & 87\end{array}$ $\begin{array}{lllllllllllllllll}015 \mathrm{~B} 0 & 4 \mathrm{D} & 45 & 53 & 53 & 41 & 47 & \mathrm{C} 5 & 15 & \mathrm{~A} 2 & 08 & 57 & 0 \mathrm{~B} & 41 & 09 & 20 & 4 \mathrm{D}\end{array}$ $\begin{array}{lllllllllllllllll}015 \mathrm{C} 0 & 65 & 73 & 73 & 61 & 67 & 65 & 20 & 07 & 59 & 04 & \mathrm{BF} & 04 & 89 & 0 \mathrm{C} & \mathrm{F} 2 & 15\end{array}$ $\begin{array}{lllllllllllllllll}015 \mathrm{D} 0 & 8 \mathrm{E} & 07 & 59 & 04 & \mathrm{E} 0 & 02 & \mathrm{C} 0 & 84 & 53 & 2 \mathrm{D} & 3 \mathrm{E} & \mathrm{C} 4 & 15 & \mathrm{AF} & 15 & \mathrm{E} 0\end{array}$

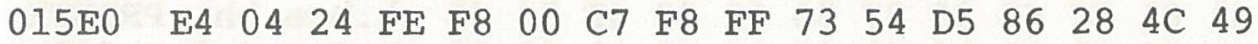
$\begin{array}{lllllllllllllllll}015 \mathrm{~F} 0 & 4 \mathrm{E} & 45 & \text { A9 } & 15 & \mathrm{D} 7 & 08 & 57 & 03 & 28 & 01 & 49 & 00 & 40 & 06 & 61 & 13\end{array}$ $\begin{array}{lllllllllllllllll}01600 & 6 \mathrm{~F} & 03 & 36 & 06 & 6 \mathrm{D} & 05 & 67 & 03 & \mathrm{C} 4 & 16 & \mathrm{DE} & 03 & \mathrm{C} 4 & 01 & 49 & 00\end{array}$ $\begin{array}{lllllllllllllllll}01610 & 40 & 02 & \mathrm{C} 0 & 85 & 2 \mathrm{E} & 4 \mathrm{C} & 49 & 4 \mathrm{E} & \mathrm{C} 5 & 15 & \mathrm{EC} & 08 & 57 & 15 & \mathrm{~F} 5 & 0 \mathrm{~B}\end{array}$ $\begin{array}{lllllllllllllllll}01620 & 1 \mathrm{C} & 09 & 33 & 02 & \mathrm{C} 0 & 84 & 2 \mathrm{~B} & 42 & 55 & \mathrm{C} 6 & 16 & 13 & 08 & 57 & 06 & 61\end{array}$ $\begin{array}{lllllllllllllllll}01630 & 01 & 49 & 00 & 04 & 03 & C 4 & 03 & C 4 & 04 & 9 C & 06 & 55 & 03 & 73 & 01 & 82\end{array}$ $\begin{array}{lllllllllllllllll}01640 & 16 & 46 & 04 & 7 \mathrm{D} & 06 & 49 & 04 & 9 \mathrm{C} & 07 & 9 \mathrm{~F} & 04 & \mathrm{BF} & 04 & 05 & 02 & \mathrm{C} 0\end{array}$ $\begin{array}{lllllllllllllllll}01650 & 86 & 55 & 50 & 44 & 41 & 54 & \mathrm{C} 5 & 16 & 25 & 08 & 57 & 07 & 9 \mathrm{~F} & 04 & \mathrm{BF} & 04\end{array}$ $\begin{array}{lllllllllllllllll}01660 & \mathrm{BF} & 01 & 49 & 80 & 00 & 02 & 62 & 07 & 9 \mathrm{~F} & 04 & \mathrm{BF} & 04 & \mathrm{E} 0 & 02 & \mathrm{C} 0 & 8 \mathrm{D}\end{array}$ $\begin{array}{lllllllllllllllll}01670 & 45 & 4 \mathrm{D} & 50 & 54 & 59 & 2 \mathrm{D} & 42 & 55 & 46 & 46 & 45 & 52 & \mathrm{D} 3 & 16 & 50 & 08\end{array}$ $\begin{array}{lllllllllllllllll}01680 & 57 & 06 & 49 & 06 & 55 & 04 & 67 & 04 & 05 & 08 & 86 & 02 & \mathrm{C} 0 & 86 & 42 & 55\end{array}$ $\begin{array}{lllllllllllllllll}01690 & 46 & 46 & 45 & \mathrm{D} 2 & 16 & 6 \mathrm{~F} & 08 & 57 & 07 & 94 & 04 & \mathrm{BF} & 04 & 9 \mathrm{C} & 03 & 28\end{array}$ $\begin{array}{lllllllllllllllll}016 \mathrm{~A} 0 & 16 & 2 \mathrm{C} & 01 & 82 & 16 & \mathrm{~A} 0 & 07 & 94 & 04 & \mathrm{E} 0 & 03 & 46 & 04 & \mathrm{BF} & 03 & 67\end{array}$ $\begin{array}{lllllllllllllllll}016 \mathrm{~B} 0 & 01 & 82 & 16 & \mathrm{C} 6 & 03 & 46 & 03 & \mathrm{E} 7 & 03 & 46 & 04 & \mathrm{BF} & 01 & 49 & 7 \mathrm{~F} & \mathrm{FF}\end{array}$ $\begin{array}{lllllllllllllllll}016 \mathrm{C} 0 & 02 & 51 & 06 & 08 & 17 & 3 \mathrm{C} & 03 & 46 & 04 & \mathrm{E} 0 & 03 & 46 & 07 & 9 \mathrm{~F} & 04 & \mathrm{E} 0\end{array}$ $\begin{array}{lllllllllllllllll}016 \mathrm{D} 0 & 03 & 36 & 03 & \mathrm{E} 7 & 02 & \mathrm{C} 0 & 85 & 42 & 4 \mathrm{C} & 4 \mathrm{~F} & 43 & \mathrm{CB} & 16 & 8 \mathrm{D} & 08 & 57\end{array}$ $\begin{array}{lllllllllllllllll}016 \mathrm{E} 0 & 07 & 26 & 04 & \mathrm{BF} & 03 & \mathrm{C} 4 & 03 & 28 & 07 & 9 \mathrm{~F} & 04 & \mathrm{BF} & 04 & 9 \mathrm{C} & 04 & \mathrm{BF}\end{array}$ $\begin{array}{lllllllllllllllll}016 \mathrm{~F} 0 & 03 & 46 & 04 & 05 & 04 & 9 \mathrm{C} & 03 & \mathrm{C} 4 & 01 & 82 & 17 & 2 \mathrm{E} & 16 & 2 \mathrm{C} & 03 & 52\end{array}$ $\begin{array}{lllllllllllllllll}01700 & 01 & 82 & 17 & 16 & 04 & 7 \mathrm{D} & 03 & 46 & 16 & 96 & 04 & 9 \mathrm{C} & 03 & 46 & 06 & 10\end{array}$ $\begin{array}{lllllllllllllllll}01710 & 17 & 3 \mathrm{C} & 06 & 18 & 04 & 05 & 04 & 9 \mathrm{C} & 04 & \mathrm{BF} & 03 & 46 & 04 & 05 & 04 & 9 \mathrm{C}\end{array}$ $\begin{array}{lllllllllllllllll}01720 & 03 & C 4 & 03 & 52 & 01 & 82 & 16 & \text { FC } & 04 & 9 \mathrm{C} & 07 & 9 \mathrm{~F} & 04 & \mathrm{E} 0 & 03 & 36\end{array}$ $\begin{array}{lllllllllllllllll}01730 & 04 & 7 \mathrm{D} & 03 & \mathrm{E} 7 & 02 & \mathrm{C} 0 & 83 & 52 & 2 \mathrm{~F} & \mathrm{D} 7 & 16 & \mathrm{D} 6 & 08 & 57 & 04 & 89\end{array}$ $\begin{array}{lllllllllllllllll}01740 & 07 & \mathrm{BD} & 04 & \mathrm{BF} & 13 & 3 \mathrm{~F} & 07 & \mathrm{AF} & 04 & \mathrm{~F} 0 & 06 & 18 & 05 & 67 & 07 & \mathrm{AF}\end{array}$ $\begin{array}{lllllllllllllllll}01750 & 03 & \mathrm{D} 5 & 04 & \mathrm{E} 0 & 04 & 89 & 07 & \mathrm{AF} & 06 & 20 & 03 & \mathrm{C} 4 & 04 & \mathrm{E} 0 & 07 & \mathrm{AF}\end{array}$ $\begin{array}{lllllllllllllllll}01760 & 04 & 89 & 01 & 82 & 17 & 6 \mathrm{C} & 09 & \mathrm{~B} 2 & 01 & 72 & 17 & 6 \mathrm{E} & 09 & \mathrm{DB} & 07 & \mathrm{D} 2\end{array}$ $\begin{array}{lllllllllllllllll}01770 & 04 & \mathrm{BF} & 17 & \mathrm{BC} & 17 & \mathrm{~A} 4 & 02 & \mathrm{C} 0 & \mathrm{EF} & 1 \mathrm{~F} & 1 \mathrm{~F} & 1 \mathrm{~F} & 0 \mathrm{~F} & \mathrm{FC} & 02 & 73\end{array}$ $\begin{array}{lllllllllllllllll}01780 & \text { OF } & \text { FC } & 01 & 73 & \text { OF } & 7 C & 00 & 73 & \text { D4 } & 87 & \text { F6 } & \text { D5 } & 84 & 43 & 64 & 65\end{array}$ $\begin{array}{lllllllllllllllll}01790 & \text { F2 } & 17 & 36 & 17 & 95 & 24 & 8 D & 54 & 24 & \text { F8 } & 00 & 54 & \text { D5 } & 84 & 43 & 44\end{array}$ $\begin{array}{lllllllllllllllll}017 \mathrm{~A} 0 & 45 & \mathrm{D} 2 & 17 & 8 \mathrm{C} & 08 & 57 & 17 & 93 & 01 & 82 & 17 & \mathrm{~B} 2 & 01 & 49 & 00 & 08\end{array}$

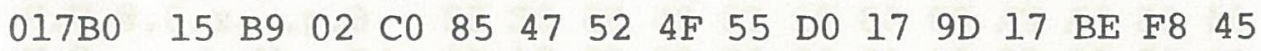
$\begin{array}{lllllllllllllllll}017 \mathrm{C} 0 & \mathrm{~A} 7 & 14 & 04 & 57 & \mathrm{~F} 8 & 00 & \mathrm{~A} 7 & \mathrm{E} 4 & 61 & \mathrm{D} 5 & 84 & 4 \mathrm{C} & 4 \mathrm{~F} & 41 & \mathrm{C} 4 & 17\end{array}$ 017D0 B4 $08 \begin{array}{lllllllllllllll}57 & 06 & \mathrm{FC} & 04 & \mathrm{BF} & 03 & 28 & 07 & 05 & 04 & \mathrm{BF} & 03 & 28 & 06\end{array}$ $\begin{array}{lllllllllllllllll}017 \mathrm{E} 0 & 08 & 07 & 05 & 04 & \mathrm{E} 0 & 06 & 6 \mathrm{D} & 05 & 67 & 06 & \mathrm{FC} & 04 & \mathrm{E} 0 & 11 & 20 & 03\end{array}$ $\begin{array}{lllllllllllllllll}017 \mathrm{~F} 0 & 36 & 07 & 05 & 04 & \mathrm{E} 0 & 03 & 36 & 06 & \mathrm{FC} & 04 & \mathrm{E} 0 & 02 & \mathrm{CO} & \mathrm{C} 3 & 2 \mathrm{D} & 2 \mathrm{D}\end{array}$ $\begin{array}{lllllllllllllllll}01800 & \mathrm{BE} & 17 & \mathrm{CA} & 08 & 57 & 0 \mathrm{C} & 77 & 06 & 08 & 07 & 05 & 04 & \mathrm{E} 0 & 06 & 6 \mathrm{D} & 06\end{array}$ $\begin{array}{lllllllllllllllll}01810 & \mathrm{FC} & 04 & \mathrm{BF} & 04 & 67 & 13 & 5 \mathrm{~F} & 04 & 05 & 06 & \mathrm{FC} & 03 & \mathrm{~F} 2 & 02 & \mathrm{C} 0 & 83\end{array}$ $\begin{array}{lllllllllllllllll}01820 & 44 & 52 & \text { B0 } & 17 & \text { FD } & 08 & 57 & 06 & 08 & 07 & 26 & 04 & \text { E0 } & 02 & \text { C0 } & \text { C5 }\end{array}$ $\begin{array}{lllllllllllllllll}01830 & 46 & 4 \mathrm{~F} & 52 & 54 & \mathrm{C} 8 & 18 & \mathrm{IF} & 10 & \mathrm{Al} & 11 & 91 & 81 & \mathrm{~A} 0 & 41 & 8 \mathrm{~B} & 00\end{array}$ $\begin{array}{lllllllllllllllll}01840 & 00 & 56 & \mathrm{FE} & 55 & \mathrm{FE} & 00 & 98 & 00 & 16 & 57 & 00 & 11 & \mathrm{E} 8 & 41 & 80 & 00\end{array}$ $\begin{array}{lllllllllllllllll}01850 & 7 \mathrm{~F} & 57 & 00 & 55 & \mathrm{FE} & 56 & \mathrm{FE} & 56 & 00 & 00 & 1 \mathrm{~F} & 00 & 00 & 41 & 8 \mathrm{~B} & 41\end{array}$ $\begin{array}{lllllllllllllllll}01860 & 8 \mathrm{~B} & 22 & 5 \mathrm{~F} & 01 & 3 \mathrm{~B} & 00 & \mathrm{~F} 0 & 00 & 01 & 00 & \mathrm{E} 0 & 00 & 62 & 83 & 44 & 52\end{array}$ $\begin{array}{lllllllllllllllll}01870 & \mathrm{BI} & 18 & 2 \mathrm{~F} & 08 & 57 & 06 & 6 \mathrm{D} & 07 & \mathrm{BD} & 04 & \mathrm{BF} & 05 & 67 & 07 & 26 & 04\end{array}$
-.1.@.\#.1.W.Y.?. .. . I......... I...D.I.0.D.1.Q. \#S....W...g.g.b.R -..6. Q.D.R./.W. ( -...g.9.K.4.s.Z.6 .g..........R.F. W. (.^.6.L.Q.D..j .W...L.c. Q... \{.W ....e.?.... W.?.. . a.U.......... a. MESSAGE." W.A. M essage .Y.?...r. ..Y.'. E.S-D./. d. $\$$ X.GX.sTU. (LI NE).W.W. (.I.a.a. 0.6.m.g.D. ^.D.I. Q. Q..LINE.I.W.u. ..3. a.+BUF... W.a. .I...D.D...U.S. . .F.\}.I....?.... .UPDATE. $\%$.W...? ?.I...b...?. a. EMPTY-BUFFERS.P. W.I.U.g..... A.BU FFER.O.W...?...( , .......?.? ...F.F.g.F.?.I. . Q...<.F。'.F.. .6.g. A.BLOCK...W .\&.?.D. (...?...? .F..........R ..... . F....F.. .<......?.F... .D.R... . .... 6 .\} \cdot g \cdot a \cdot R / W \cdot V \cdot W$. .=.?.?./.p...g./ .U.'.../. . D.'./ ....1.2.r.n. [.R .?.<.\$. do....।. .s . |.S. I.sT.vU.Cde r.6..\$.T\$X.TU.CD ER...W.....2.I.. .9. a.GROUP... > XE ' ..Wx. 'daU. LOAD. 4.W.1.?. ( ...?. ( .

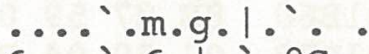
6... 6.1. . ec->.J.W.W......... I.?.g.... I.r.a. DRO.\}. $\bar{W}_{\ldots} \ldots \&_{0}$. $\mathrm{AEE}$ FORTH....... A. $. V^{\sim} U^{\sim} \ldots . . W_{.} . h A \ldots$ W. $U^{\sim} V^{\sim} V \ldots . . A . A$ . $1 . T_{\cdot} \cdot \mathrm{m}_{0}=\cdot ? \cdot g \cdot \&$. 


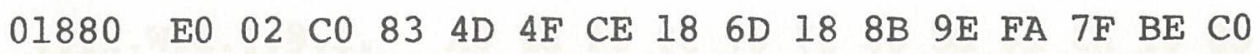
$\begin{array}{lllllllllllllllll}01890 & 87 & \mathrm{~F} 0 & 85 & 28 & 44 & 4 \mathrm{~F} & 53 & \mathrm{~A} 9 & 18 & 83 & 18 & 9 \mathrm{C} & \mathrm{F} 8 & 90 & \mathrm{~B} 0 & \mathrm{~F} 8\end{array}$ $\begin{array}{lllllllllllllllll}018 \mathrm{~A} 0 & 05 & \mathrm{~A} 0 & \mathrm{E} 0 & \mathrm{D} 0 & 82 & 4 \mathrm{~F} & \mathrm{CB} & 18 & 92 & 08 & 57 & 0 \mathrm{~B} & 41 & 03 & 20 & 4 \mathrm{~F}\end{array}$ $\begin{array}{lllllllllllllllll}018 \mathrm{~B} 0 & 4 \mathrm{~B} & 02 & \mathrm{C} 0 & 84 & 4 \mathrm{C} & 49 & 53 & \mathrm{D} 4 & 18 & \mathrm{~A} 4 & 08 & 57 & \text { 0C } & \mathrm{F} 2 & 09 & 09\end{array}$ $\begin{array}{lllllllllllllllll}018 \mathrm{C} 0 & 04 & 9 \mathrm{C} & 07 & 19 & 04 & \mathrm{E} 0 & 0 \mathrm{~B} & 41 & 07 & 53 & 43 & 52 & 45 & 45 & 4 \mathrm{E} & 20\end{array}$

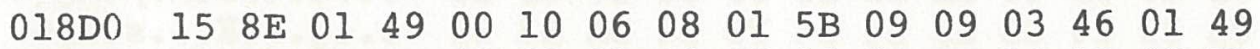
$\begin{array}{lllllllllllllllll}018 \mathrm{E} 0 & 00 & 03 & 15 & 6 \mathrm{~F} & 09 & 63 & 03 & 46 & 07 & 19 & 04 & \mathrm{BF} & 16 & 1 \mathrm{~B} & 08 & \mathrm{EA}\end{array}$ $\begin{array}{lllllllllllllllll}018 \mathrm{~F} 0 & 01 & 82 & 18 & \mathrm{~F} 6 & 03 & 1 \mathrm{~B} & 00 & \mathrm{AE} & 18 & \mathrm{DA} & 09 & 09 & 02 & \mathrm{C} 0 & 85 & 46\end{array}$ $\begin{array}{lllllllllllllllll}01900 & 4 \mathrm{C} & 55 & 53 & \mathrm{C} 8 & 18 & \mathrm{~B} 3 & 08 & 57 & 06 & 55 & 06 & 49 & 04 & 05 & 06 & 61\end{array}$ $\begin{array}{lllllllllllllllll}01910 & 01 & 49 & 00 & 04 & 03 & C 4 & 13 & 4 F & 06 & 08 & 01 & 5 B & 01 & 49 & 7 F & F F\end{array}$ $\begin{array}{lllllllllllllllll}01920 & 16 & 96 & 04 & 7 \mathrm{D} & 00 & \mathrm{AE} & 19 & 1 \mathrm{C} & 02 & \mathrm{C} 0 & 86 & 70 & 61 & 72 & 70 & 74\end{array}$

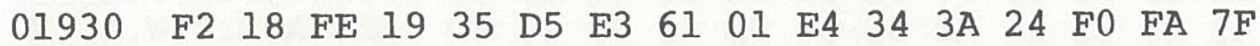
$\begin{array}{lllllllllllllllll}01940 & \text { FB } & F F & 54 & 66 & \text { E3 } & 61 & 01 & \text { E2 } & 68 & 96 & 86 & 73 & 65 & 72 & 70 & 74\end{array}$ $\begin{array}{lllllllllllllllll}01950 & \text { F2 } & 19 & 2 A & 19 & 55 & \text { D5 } & \text { E3 } & 61 & \text { E0 } & \text { E4 } & 24 & 24 & 6 B & \text { FA } & 80 & 32\end{array}$ $\begin{array}{lllllllllllllllll}01960 & 5 \mathrm{C} & 14 & 62 & \mathrm{E} 3 & 61 & 01 & \mathrm{E} 2 & 68 & 96 & 87 & 50 & 52 & 49 & 4 \mathrm{E} & 54 & 45\end{array}$ $\begin{array}{lllllllllllllllll}01970 & \text { D2 } & 19 & 4 \mathrm{~A} & 08 & 57 & 01 & 49 & 00 & \mathrm{C} 0 & 01 & 49 & 09 & \mathrm{~B} 4 & 01 & 49 & 00\end{array}$ $\begin{array}{lllllllllllllllll}01980 & 12 & 04 & 05 & 04 & \text { F0 } & 01 & 49 & 19 & 55 & 03 & \text { D5 } & 01 & 49 & 09 & \text { B4 } & 01\end{array}$ $\begin{array}{lllllllllllllllll}01990 & 49 & 00 & 11 & 04 & 05 & 04 & \mathrm{E} 0 & 07 & \mathrm{Fl} & 04 & \mathrm{BF} & 04 & 9 \mathrm{C} & 01 & 49 & 19\end{array}$

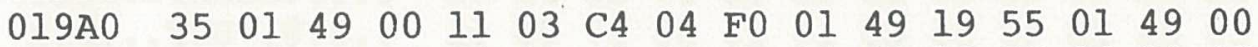
019B0 $10 \begin{array}{llllllllllllllll}03 & \mathrm{C} 4 & 04 & \mathrm{~F} 0 & 08 & 00 & 04 & \mathrm{BF} & 01 & 49 & 19 & 55 & 06 & 20 & 03\end{array}$

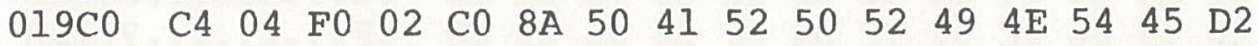
019D0 $19 \begin{array}{llllllllllllllll}19 & 69 & 08 & 57 & 01 & 49 & 19 & 35 & 01 & 49 & 19 & 75 & 01 & 49 & 00 & 12\end{array}$ 019E0 $03 \quad$ C4 $04 \begin{array}{llllllllllllll} & 019 & 19 & 73 & 02 & \mathrm{C} 0 & 87 & 4 \mathrm{E} & 4 \mathrm{~F} & 50 & 52 & 49 & 4 \mathrm{E} & \mathrm{D} 4\end{array}$

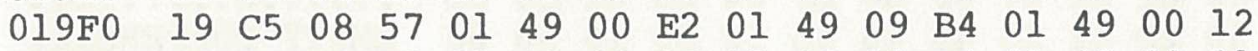
01A00 $04 \begin{array}{llllllllllllllll}05 & 04 & \mathrm{~F} 0 & 01 & 49 & 68 & 96 & 01 & 49 & 09 & \mathrm{~B} 4 & 01 & 49 & 00 & 11\end{array}$ $\begin{array}{lllllllllllllllll}01 \mathrm{AlO} & 04 & 05 & 04 & \mathrm{E} 0 & 02 & \mathrm{C} 0 & 83 & 55 & 2 \mathrm{E} & \mathrm{D} 2 & 19 & \mathrm{E} 8 & 08 & 57 & 06 & 08\end{array}$ $\begin{array}{lllllllllllllllll}01 \mathrm{~A} 20 & 04 & 89 & 15 & 4 \mathrm{C} & 02 & \mathrm{C} 0 & 84 & 4 \mathrm{C} & 4 \mathrm{~F} & 41 & \mathrm{C} 4 & 1 \mathrm{~A} & 16 & 08 & 57 & 0 \mathrm{C}\end{array}$ $\begin{array}{lllllllllllllllll}01 \mathrm{~A} 30 & \mathrm{~F} 2 & 09 & 09 & 0 \mathrm{~B} & 41 & 04 & 4 \mathrm{C} & 6 \mathrm{~F} & 61 & 64 & 04 & 9 \mathrm{C} & 01 & 49 & 00 & 04\end{array}$ $\begin{array}{lllllllllllllllll}01 \mathrm{~A} 40 & 1 \mathrm{~A} & 1 \mathrm{C} & 04 & 9 \mathrm{C} & 06 & 08 & 04 & 89 & 09 & 63 & 0 \mathrm{~B} & 41 & 02 & 2 \mathrm{C} & 20 & 07\end{array}$ $\begin{array}{lllllllllllllllll}01 A 50 & 59 & 04 & \text { BF } & 0 A & 53 & 0 C & \text { DC } & 01 & 49 & 00 & 04 & \text { IA } & \text { IC } & 07 & 59 & 04\end{array}$ $\begin{array}{lllllllllllllllll}01 \mathrm{~A} 60 & \mathrm{E} 0 & 09 & 63 & 16 & 1 \mathrm{~B} & 09 & 63 & 06 & \mathrm{FC} & 04 & \mathrm{BF} & 03 & 28 & 07 & 05 & 04\end{array}$ $\begin{array}{lllllllllllllllll}01 \mathrm{~A} 70 & \mathrm{BF} & 03 & 28 & 06 & 08 & 07 & 05 & 04 & \mathrm{E} 0 & 06 & \mathrm{FC} & 04 & \mathrm{E} 0 & 11 & 20 & 03\end{array}$ $\begin{array}{lllllllllllllllll}01 A 80 & 36 & 07 & 05 & 04 & \mathrm{E} 0 & 03 & 36 & 06 & \mathrm{FC} & 04 & \mathrm{E} 0 & 08 & \mathrm{EA} & 01 & 82 & 1 \mathrm{~A}\end{array}$ $\begin{array}{lllllllllllllllll}01 A 90 & 93 & 11 & \text { BA } & 02 & \text { C0 } & 88 & 4 \mathrm{C} & 4 \mathrm{~F} & 41 & 44 & 54 & 48 & 52 & \text { D5 } & 1 \mathrm{~A} & 26\end{array}$ $\begin{array}{lllllllllllllllll}01 \mathrm{AAO} & 08 & 57 & 03 & \mathrm{D} 5 & 04 & 89 & 01 & 5 \mathrm{~B} & 03 & 46 & 1 \mathrm{~A} & 2 \mathrm{D} & 00 & \mathrm{AE} & \text { IA } & \mathrm{A} 8\end{array}$ $\begin{array}{lllllllllllllllll}01 \mathrm{AB} 0 & 02 & \mathrm{C} 0 & 85 & 41 & 53 & 4 \mathrm{D} & 42 & \mathrm{D} 2 & 1 \mathrm{~A} & 95 & 08 & 57 & 02 & \mathrm{C} 0 & 85 & 32\end{array}$ $\begin{array}{lllllllllllllllll}01 A C 0 & 44 & 52 & 4 F & \text { D0 } & 1 A & B 2 & 08 & 57 & 04 & 7 D & 04 & 7 D & 02 & \text { C0 } & 84 & 32\end{array}$ $\begin{array}{lllllllllllllllll}01 \mathrm{ADO} & 44 & 55 & \mathrm{D} 0 & \text { IA } & \mathrm{BE} & 08 & 57 & 04 & 67 & 04 & 67 & 02 & \mathrm{C} 0 & 85 & 32 & 53\end{array}$ $\begin{array}{lllllllllllllllll}01 \mathrm{AEO} & 57 & 41 & \mathrm{D} 0 & 1 \mathrm{~A} & \mathrm{CE} & 08 & 57 & 04 & \mathrm{FE} & 03 & 28 & 04 & \mathrm{FE} & 03 & 36 & 02\end{array}$ $\begin{array}{lllllllllllllllll}01 \mathrm{AF} 0 & \mathrm{C} 0 & 86 & 5 \mathrm{~A} & 45 & 52 & 4 \mathrm{~F} & 45 & \mathrm{D} 3 & \text { IA } & \mathrm{DD} & 08 & 57 & 06 & 08 & 05 & 47\end{array}$ $\begin{array}{lllllllllllllllll}01 \mathrm{~B} 00 & 05 & 16 & 01 & 82 & 1 \mathrm{~B} & 14 & 06 & 08 & 01 & 5 \mathrm{~B} & 01 & 49 & 00 & 30 & 08 & \mathrm{FC}\end{array}$ $\begin{array}{lllllllllllllllll}01 \mathrm{BI0} & 00 & \mathrm{AE} & 1 \mathrm{~B} & 0 \mathrm{~A} & 02 & \mathrm{C} 0 & 85 & 44 & 2 \mathrm{E} & 52 & 4 \mathrm{C} & \mathrm{DA} & 1 \mathrm{~A} & \mathrm{Fl} & 08 & 57\end{array}$ $\begin{array}{lllllllllllllllll}01 B 20 & 03 & 28 & 04 & 89 & 04 & 67 & 05 & 39 & 14 & \mathrm{CB} & 15 & 34 & 14 & \mathrm{~F} 3 & 1.4 & \mathrm{DA}\end{array}$ $\begin{array}{lllllllllllllllll}01 B 30 & 03 & 36 & 04 & 67 & 04 & 05 & \text { IA } & \text { FA } & 09 & 33 & 02 & \text { C0 } & 85 & 55 & 2 \mathrm{E} & 52\end{array}$ $\begin{array}{lllllllllllllllll}01 B 40 & 4 \mathrm{C} & \mathrm{DA} & \text { IB } & 16 & 08 & 57 & 06 & 08 & 04 & 89 & \text { IB } & \text { IE } & 02 & \mathrm{C} 0 & 84 & 48\end{array}$ $\begin{array}{lllllllllllllllll}01 B 50 & 45 & 58 & \mathrm{AE} & \text { IB } & 3 \mathrm{C} & 08 & 57 & 07 & 59 & 04 & \mathrm{BF} & \text { OC } & \mathrm{DC} & 04 & 89 & 15\end{array}$ $\begin{array}{lllllllllllllllll}01 \mathrm{~B} 60 & 8 \mathrm{E} & 07 & 59 & 04 & \mathrm{E} 0 & 02 & \mathrm{C} 0 & 84 & 44 & 45 & 43 & \mathrm{AE} & 1 \mathrm{~B} & 4 \mathrm{E} & 08 & 57\end{array}$ $\begin{array}{lllllllllllllllll}01 B 70 & 07 & 59 & 04 & \mathrm{BF} & 0 \mathrm{C} & \mathrm{F} 2 & 04 & 89 & 15 & 8 \mathrm{E} & 07 & 59 & 04 & \mathrm{E} 0 & 02 & \mathrm{C} 0\end{array}$ $\begin{array}{lllllllllllllllll}01 B 80 & 86 & 4 \mathrm{E} & 4 \mathrm{~F} & 44 & 49 & 53 & \mathrm{CB} & 1 \mathrm{~B} & 67 & 08 & 57 & 01 & 49 & 03 & 54 & 0 \mathrm{~A}\end{array}$ $\begin{array}{lllllllllllllllll}01 \mathrm{~B} 90 & \mathrm{CD} & 01 & 49 & 11 & \mathrm{E} 8 & 01 & 49 & 00 & 47 & 03 & \mathrm{C} 4 & 04 & \mathrm{E} 0 & 02 & \mathrm{C} 0 & 84\end{array}$ $\begin{array}{lllllllllllllllll}01 B A 0 & 42 & 45 & 4 C & C C & 1 B & 80 & 08 & 57 & 01 & 49 & 00 & 07 & 08 & \text { FC } & 02 & C 0\end{array}$ $\begin{array}{lllllllllllllllll}01 \mathrm{BB} 0 & 84 & 42 & 45 & 45 & \mathrm{D} 0 & \text { IB } & 9 \mathrm{~F} & 08 & 57 & 1 \mathrm{~B} & \mathrm{~A} 6 & 02 & \mathrm{C} 0 & 86 & 43 & 4 \mathrm{D}\end{array}$ $\begin{array}{lllllllllllllllll}01 \mathrm{BC} 0 & 4 \mathrm{~F} & 56 & 45 & \mathrm{BE} & 1 \mathrm{~B} & \mathrm{~B} 0 & 08 & 57 & 04 & 9 \mathrm{C} & 04 & \mathrm{FE} & 03 & \mathrm{C} 4 & 04 & \mathrm{FE}\end{array}$

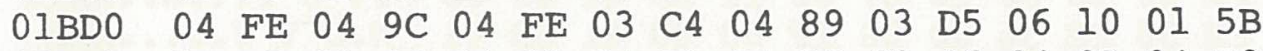

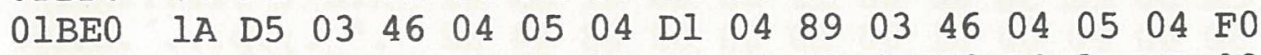
$\begin{array}{lllllllllllllllll}01 \mathrm{BF} 0 & 00 & \mathrm{AE} & 1 \mathrm{~B} & \mathrm{E} 0 & 1 \mathrm{~A} & \mathrm{C} 6 & 02 & \mathrm{C} 0 & 84 & 43 & 4 \mathrm{~F} & 50 & \mathrm{D} 9 & \text { IB } & \mathrm{BD} & 08\end{array}$ $\because$ MON.m...z $>$ a .p. (DOS) ....x.0x -P.OK...W.A. O K. Q.LIST.\$.W.r.. ....... SCREEN ..I..... [ ..F.I ...o.c.F...?...j .... ............ . F LUSH.3.W.U.I... a .I...D.O... [.I. ...\}...... a.parpt r. . 5Uca.d4:\$pz

\{. Tfca.bh...serpt r.*.UUca' $\mathrm{d} \$ \$ \mathrm{kz} .2$ \.bca.bh..PRINTE R.J.W.I.Q.I.4.I. ....p.I.U.U.I.4. I........... 5.I...D.P.I.U.I. ..D.p...?.I.U. D.p. A.PARPRINTER .i.W.I.5.I.U.I.. .D.'.s.@.NOPRINT .E.W.I.b.I.4.I.. ...p.Ih..I.4.I.. ... . . U.R.h.W.. ....L. . .LOAD...W. r...A.Load....... …........... Y.?.S. \.I....Y. .c...c.l.?. (...

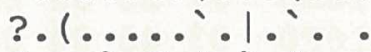
$6 \ldots .6 .1 . ` j \ldots$ ... . . . LOADTHRU \& \& .W.U... . . F. - ... ( . a.ASMBR....W. a.2 DROP.2.W.\}.\}.@. 2 DUP.>.W.g.g. Q. 2S WAP.N.W. . (. . 6 . [. ZEROES. ].W...G ............. 0.1 .......D.RLZ.q.W . (...g.9.K.4.s.Z .6.g...z.3.9.U.R LZ...W........H EX..<.W.Y.?.।... ..Y. .Q.DEC..N.W .Y.?.r...... . . -NODISK.g.W.I.T. M.I.h.I.G.D. . BELI...W.I...I. . .BEEP....W.\&.Q.CM OVE $>.0 . W . . \sim . D . \sim$ . ... .D...U... [ .U.F...Q...F...P $\ldots \mathrm{F} \mathrm{Q}_{\cdot} \mathrm{COPY}=$. 
$\begin{array}{lllllllllllllllll}01 \mathrm{C} 00 & 57 & 07 & 26 & 04 & \mathrm{BF} & 03 & \mathrm{C} 4 & 04 & 89 & 06 & 10 & 04 & 67 & 03 & \mathrm{C} 4 & 04\end{array}$ $\begin{array}{lllllllllllllllll}01 \mathrm{Cl} 0 & 89 & 01 & 5 \mathrm{~B} & 04 & 9 \mathrm{C} & 03 & 46 & 16 & \mathrm{DE} & 06 & 18 & 04 & 05 & 04 & \mathrm{E} 0 & 03\end{array}$ $\begin{array}{lllllllllllllllll}01 \mathrm{C} 20 & \mathrm{D} 5 & 16 & 59 & 00 & \mathrm{AE} & 1 \mathrm{C} & 13 & 04 & 7 \mathrm{D} & 19 & 06 & 02 & \mathrm{C} 0 & 83 & 4 \mathrm{E} & 4 \mathrm{~F}\end{array}$ $\begin{array}{lllllllllllllllll}01 C 30 & D 4 & 1 B & F 8 & 08 & 57 & 03 & 52 & 02 & C 0 & 83 & 3 E & 49 & C E & 1 C & 2 D & 08\end{array}$ $\begin{array}{lllllllllllllllll}01 C 40 & 57 & 07 & 05 & 02 & \mathrm{C} 0 & 87 & 57 & 4 \mathrm{~F} & 52 & 44 & 2 \mathrm{D} & 37 & \mathrm{~B} 9 & 1 \mathrm{C} & 39 & 08\end{array}$

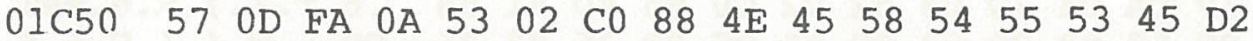

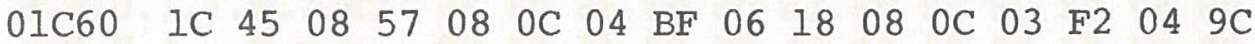
$\begin{array}{lllllllllllllllll}01 C 70 & 01 & 49 & 00 & \mathrm{D} 4 & 03 & \mathrm{AE} & 01 & 82 & 1 \mathrm{C} & 82 & 01 & 49 & 00 & 19 & 15 & \mathrm{~B} 9\end{array}$ $\begin{array}{lllllllllllllllll}01 \mathrm{C} 80 & 11 & \mathrm{BA} & 10 & 71 & 02 & \mathrm{C} 0 & 86 & 41 & 4 \mathrm{C} & 4 \mathrm{C} & 4 \mathrm{~F} & 54 & \mathrm{D} 5 & 1 \mathrm{C} & 57 & 08\end{array}$ $\begin{array}{lllllllllllllllll}01 C 90 & 57 & 08 & 0 \mathrm{C} & 03 & \mathrm{~F} 2 & 02 & \mathrm{C} 0 & 85 & 51 & 55 & 4 \mathrm{~F} & 54 & \mathrm{C} 5 & \text { IC } & 86 & 08\end{array}$ $\begin{array}{lllllllllllllllll}01 C A 0 & 57 & 01 & 49 & 00 & 22 & 08 & \text { FC } & 09 & 63 & 02 & \text { C0 } & 83 & 42 & 59 & \text { C5 } & 1 \mathrm{C}\end{array}$ $\begin{array}{lllllllllllllllll}01 \mathrm{CB} 0 & 97 & 08 & 57 & 19 & 06 & 18 & 89 & 02 & \mathrm{C} 0 & 87 & 43 & 48 & 41 & 50 & 54 & 45\end{array}$ $\begin{array}{lllllllllllllllll}01 \mathrm{CC} 0 & \mathrm{D} 2 & 1 \mathrm{C} & \mathrm{AB} & 06 & 90 & 00 & 50 & 83 & 45 & 53 & \mathrm{C} 3 & 1 \mathrm{C} & \mathrm{B} 9 & 05 & \mathrm{FB} & 00\end{array}$ $\begin{array}{lllllllllllllllll}01 \mathrm{CD} 0 & 1 \mathrm{~B} & 82 & 49 & \mathrm{C} 4 & \text { IC } & \mathrm{C} 7 & 08 & 57 & 01 & 49 & 11 & \mathrm{E} 8 & 04 & 9 \mathrm{C} & 01 & 49\end{array}$

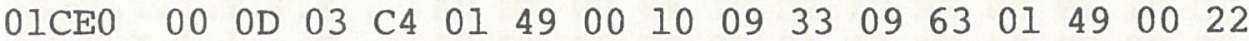
$\begin{array}{lllllllllllllllll}01 C F 0 & 03 & C 4 & 01 & 49 & 00 & 0 D & 09 & 33 & 02 & \text { C0 } & 83 & 53 & 45 & \text { C3 } & \text { IC } & \text { D] }\end{array}$ $\begin{array}{lllllllllllllllll}01 D 00 & 08 & 57 & 01 & 49 & 01 & 00 & 05 & 67 & 06 & 10 & 01 & 5 \mathrm{~B} & 01 & 49 & 00 & 14\end{array}$ $\begin{array}{lllllllllllllllll}01 D 10 & 06 & 10 & 01 & 5 \mathrm{~B} & 00 & \mathrm{AE} & 1 \mathrm{D} & 14 & 00 & \mathrm{AE} & 1 \mathrm{D} & 0 \mathrm{C} & 02 & \mathrm{C} 0 & 84 & 4 \mathrm{D}\end{array}$ $\begin{array}{lllllllllllllllll}01 D 20 & 53 & 45 & C 3 & 1 C & F A & 08 & 57 & 01 & 49 & 00 & 05 & 05 & 67 & 06 & 08 & 01\end{array}$ $\begin{array}{lllllllllllllllll}01 D 30 & 5 \mathrm{~B} & 00 & \mathrm{AE} & 1 \mathrm{D} & 31 & 02 & \mathrm{C} 0 & 83 & 43 & 4 \mathrm{C} & \mathrm{D} 3 & 1 \mathrm{D} & 1 \mathrm{E} & 08 & 57 & 01\end{array}$ $\begin{array}{lllllllllllllllll}01 D 40 & 49 & 00 & 1 A & 08 & F C & 02 & C 0 & 89 & 4 E & 45 & 57 & 2 D & 46 & 45 & 4 E & 43\end{array}$ $\begin{array}{lllllllllllllllll}01 D 50 & \text { C5 } & \text { ID } & 37 & 08 & 57 & \text { OB } & \text { Cl } & 01 & 49 & 00 & 0 \mathrm{C} & 06 & 88 & 04 & \text { E0 } & 0 A\end{array}$ $\begin{array}{lllllllllllllllll}01 D 60 & 53 & 01 & 49 & 00 & 1 C & 06 & 88 & 04 & E 0 & 0 A & 53 & 01 & 49 & 00 & \text { IE } & 06\end{array}$ $\begin{array}{lllllllllllllllll}01 D 70 & 88 & 04 & E 0 & 0 A & 53 & 06 & D A & 04 & E 0 & 06 & F 2 & 04 & \text { BF } & 01 & 49 & 00\end{array}$ $\begin{array}{lllllllllllllllll}01 D 80 & 20 & 06 & 88 & 04 & \mathrm{E} 0 & 08 & 0 \mathrm{C} & 04 & \mathrm{BF} & 01 & 49 & 00 & 2 \mathrm{~A} & 06 & 88 & 04\end{array}$ $\begin{array}{lllllllllllllllll}01 D 90 & \mathrm{E} 0 & 02 & \mathrm{C} 0 & 85 & 2 \mathrm{E} & 56 & 48 & 44 & \mathrm{D} 2 & \text { ID } & 47 & 08 & 57 & 0 \mathrm{~B} & 41 & 09\end{array}$ $\begin{array}{lllllllllllllllll}01 D A 0 & 4 \mathrm{E} & 66 & 61 & 20 & 20 & 4 \mathrm{E} & 61 & 6 \mathrm{D} & 65 & 01 & 49 & 00 & 10 & 14 & \mathrm{AE} & 0 \mathrm{~B}\end{array}$ $\begin{array}{lllllllllllllllll}01 D B 0 & 41 & 0 F & 56 & 6 \mathrm{~F} & 63 & 61 & 62 & 75 & 6 \mathrm{C} & 61 & 72 & 79 & 20 & 6 \mathrm{C} & 69 & 73\end{array}$ $\begin{array}{lllllllllllllllll}01 D C 0 & 74 & 09 & 09 & 09 & 09 & 02 & \mathrm{C} 0 & 85 & 2 \mathrm{E} & 46 & 4 \mathrm{~F} & 4 \mathrm{~F} & \mathrm{D} 4 & \text { ID } & 93 & 08\end{array}$ $\begin{array}{lllllllllllllllll}01 D D 0 & 57 & 07 & \mathrm{C} 8 & 04 & \mathrm{BF} & 06 & 3 \mathrm{D} & 0 \mathrm{~A} & \mathrm{BC} & 04 & 05 & 04 & 9 \mathrm{C} & 03 & 67 & 01\end{array}$ $\begin{array}{lllllllllllllllll}01 D E 0 & 82 & \text { ID } & \mathrm{F} 7 & 04 & 32 & 06 & 3 \mathrm{D} & 0 \mathrm{~F} & 57 & 06 & 08 & 01 & 5 \mathrm{~B} & 09 & 09 & 00\end{array}$ $\begin{array}{lllllllllllllllll}01 D F 0 & A E & 1 D & E D & 01 & 72 & \text { ID } & \text { F9 } & 04 & 7 D & 06 & 08 & 07 & \text { C8 } & 04 & \text { E0 } & \text { IC }\end{array}$ 01E00 D6 $01 \quad 49 \begin{array}{llllllllllllll} & 00 & 05 & 14 & \mathrm{AE} & 0 \mathrm{~B} & 41 & 08 & 43 & 68 & 61 & 70 & 74 & 65\end{array}$ $\begin{array}{lllllllllllllllll}01 \mathrm{E} I 0 & 72 & 20 & \text { IC } & \mathrm{C} 3 & 04 & \mathrm{BF} & 1 \mathrm{~B} & 6 \mathrm{E} & 01 & 49 & 00 & 0 \mathrm{~F} & 14 & \mathrm{AE} & 0 \mathrm{~B} & 41\end{array}$ $\begin{array}{lllllllllllllllll}01 \mathrm{E} 20 & 05 & 70 & 61 & 67 & 65 & 20 & 07 & 6 \mathrm{D} & 04 & \mathrm{BF} & 1 \mathrm{~B} & 6 \mathrm{E} & 02 & \mathrm{C} 0 & 85 & 52\end{array}$

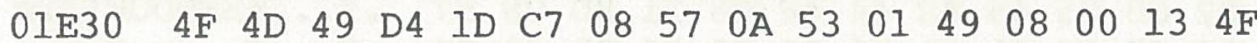
$\begin{array}{lllllllllllllllll}01 E 40 & 03 & D 5 & 01 & 49 & 08 & 00 & 05 & 67 & 04 & 9 C & 06 & \text { E3 } & 04 & \text { E0 } & 0 A & \text { BC }\end{array}$ $\begin{array}{lllllllllllllllll}01 E 50 & 0 B & 41 & 11 & 52 & 4 \mathrm{~F} & 4 \mathrm{D} & 20 & 69 & 74 & 20 & 66 & 72 & 6 \mathrm{~F} & 6 \mathrm{D} & 20 & 30\end{array}$ $\begin{array}{lllllllllllllllll}01 \mathrm{E} 60 & 20 & 74 & 6 \mathrm{~F} & 20 & 1 \mathrm{~B} & 55 & \mathrm{IB} & 89 & \text { ID } & 53 & 02 & \mathrm{C} 0 & 82 & 4 \mathrm{E} & \mathrm{AE} & 1 \mathrm{E}\end{array}$ $\begin{array}{lllllllllllllllll}01 E 70 & 2 E & 08 & 57 & 07 & 59 & 04 & B F & 03 & 28 & 0 C & F 2 & 04 & 9 C & 01 & 49 & 00\end{array}$ $\begin{array}{lllllllllllllllll}01 \mathrm{E} 80 & 05 & 15 & 6 \mathrm{~F} & 0 \mathrm{~B} & 41 & 02 & 20 & 28 & 0 \mathrm{C} & \mathrm{DC} & 01 & 49 & 00 & 04 & 1 \mathrm{~B} & 44\end{array}$ $\begin{array}{lllllllllllllllll}01 E 90 & 0 B & 41 & 02 & 68 & 29 & 03 & 36 & 07 & 59 & 04 & \mathrm{E} 0 & 02 & \mathrm{C} 0 & 84 & 3 \mathrm{~F} & 59\end{array}$

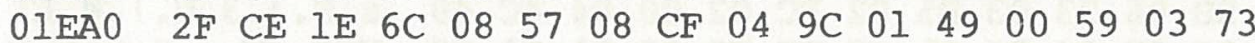
$\begin{array}{lllllllllllllllll}01 E B O & 04 & 89 & 01 & 49 & 00 & 79 & 03 & 73 & 02 & 62 & 04 & 9 \mathrm{C} & 01 & 82 & \text { IE } & C 9\end{array}$ $\begin{array}{lllllllllllllllll}01 E C O & 0 B & 41 & 02 & 20 & 59 & 01 & 72 & 1 E & C E & 0 B & 41 & 02 & 20 & 4 E & 02 & C 0\end{array}$

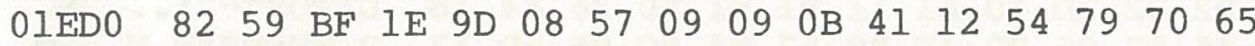
$\begin{array}{lllllllllllllllll}\text { OIEE0 } & 20 & 59 & 20 & 74 & 6 \mathrm{~F} & 20 & 63 & 6 \mathrm{~F} & 6 \mathrm{E} & 74 & 69 & 6 \mathrm{E} & 75 & 65 & 1 \mathrm{E} & \mathrm{A} 4\end{array}$ $\begin{array}{lllllllllllllllll}01 E F 0 & 01 & 82 & 1 E & F 8 & 01 & 72 & 1 E & F C & 18 & A 9 & 11 & B A & 02 & C 0 & 85 & 56\end{array}$

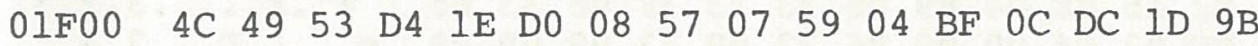
$\begin{array}{lllllllllllllllll}01 \mathrm{~F} 10 & 06 & 08 & 07 & 0 \mathrm{~F} & 04 & \mathrm{E} 0 & 07 & 34 & 04 & \mathrm{BF} & 04 & \mathrm{BF} & 04 & 9 \mathrm{C} & 04 & 9 \mathrm{C}\end{array}$ $\begin{array}{lllllllllllllllll}01 F 20 & 07 & 0 F & 04 & \mathrm{BF} & 01 & 49 & 00 & 3 \mathrm{C} & 03 & \mathrm{AE} & 01 & 82 & 1 \mathrm{~F} & 36 & 09 & 09\end{array}$ $\begin{array}{lllllllllllllllll}01 \mathrm{~F} 30 & 06 & 08 & 07 & 0 \mathrm{~F} & 04 & \mathrm{E} 0 & 06 & 08 & 14 & \mathrm{CB} & 15 & 09 & 15 & 09 & 15 & 09\end{array}$ $\begin{array}{lllllllllllllllll}01 \mathrm{~F} 40 & 15 & 09 & 14 & \mathrm{DA} & 09 & 33 & 04 & 9 \mathrm{C} & 03 & \mathrm{D} 5 & 04 & \mathrm{DI} & 01 & 49 & 00 & 80\end{array}$ $\begin{array}{lllllllllllllllll}01 F 50 & 04 & 05 & 01 & 82 & 1 F & 5 E & 09 & 63 & \text { OF } & 6 \mathrm{~F} & 01 & 72 & \text { IF } & 60 & 04 & 7 \mathrm{D}\end{array}$ $\begin{array}{lllllllllllllllll}01 \mathrm{~F} 60 & 01 & 49 & 00 & 14 & 07 & 0 \mathrm{~F} & 04 & \mathrm{BF} & 04 & 67 & 13 & 5 \mathrm{~F} & 04 & 9 \mathrm{C} & 01 & 82\end{array}$ $\begin{array}{lllllllllllllllll}01 \mathrm{~F} 70 & 1 \mathrm{~F} & 76 & 01 & 72 & 1 \mathrm{~F} & 7 \mathrm{C} & 04 & 7 \mathrm{D} & 01 & 49 & 00 & 14 & 04 & 05 & 14 & \mathrm{AE}\end{array}$
W.\&.?.D....g.D. ... [ . F. ...... U.Y............NO T.X.W.R. (..>IN.-. W... Q.WORD-79.9. W.Z.S. a.NEXTUSER .E.W...?....... .I.T......... 9 $\therefore$. . A.ALLOTU.W. W...r. a.QUOTE... W.I.".।.C.Q.BYE. ..W....... CHAPTE R.t...P.ESC.9.\{. ..ID.G.W.I.h...I ...D.I...3.C.I." .D.I...3. Q.SEC.Q .W.I...g... [.I.. ... [ ............ SEC. $z . W . I \ldots g . .$. [...1. a.CLS ...W. I...I. . . NEW-FENC E.7.W.A.I..... S.I..... .S.I... ..'.S.Z.'.r.?.I. ......?.I.*... a. VHDR.G.W.A. $\mathrm{Nfa}$ Name.I..... A. Vocabulary lis t........FOOT... $W_{0} H_{0} ?_{0}=.<\ldots$. g.

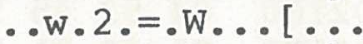
..m.r.y.\}...H. V.I.....A.Chapte $r$.C.?.n.I.....A -page .m.?.n. A.R OMIT.G.W.S.I...O .U.I...g....C. '< -A.ROM it from 0 to .U...s. ..... ..W.Y.?. (.r...I. ..O.A. (.).I...D .A.h).6.Y.'.Q.?Y /N.I.W.O...I.Y.S ...I.Y.s.b.....I .A. Y.r.N.A. N. a .Y?...W...A. Type $Y$ to continue. ....x.r.l.).:. a.v LIST.P.W.Y.?.।.. ......4.?.?... ...?.I.<....6. .............. ...Z.3...U.Q.I. .........o.r.'.\} .I.....?.g._... .v.r.|.\}.I..... 


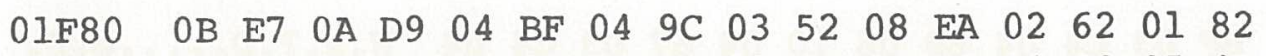
$\begin{array}{lllllllllllllllll}01 \mathrm{~F} 90 & \text { IF } & \text { IC } & 04 & 7 \mathrm{D} & 09 & 09 & \text { IC } & \mathrm{D} 6 & 07 & 59 & 04 & \mathrm{E} 0 & 02 & \mathrm{C} 0 & 85 & 4 \mathrm{D}\end{array}$ $\begin{array}{lllllllllllllllll}01 F A 0 & 61 & 74 & 63 & \text { E8 } & \text { IE } & \text { FE } & 08 & 57 & 05 & 16 & 01 & 82 & \text { IF } & \text { D6 } & 04 & 67\end{array}$

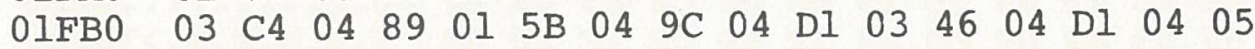
$\begin{array}{lllllllllllllllll}01 \mathrm{FC} 0 & 01 & 82 & 1 \mathrm{~F} & \mathrm{CC} & 03 & 52 & 03 & \text { IB } & 01 & 72 & \mathrm{IF} & \mathrm{CE} & 03 & \mathrm{D} 5 & 00 & \mathrm{AE}\end{array}$ $\begin{array}{lllllllllllllllll}01 F D 0 & \text { IF } & \text { B6 } & 01 & 72 & \text { IF } & \text { DA } & 04 & 7 D & 03 & 52 & 02 & \text { C0 } & 85 & 4 D & 41 & 54\end{array}$ $\begin{array}{lllllllllllllllll}01 \mathrm{FE} 0 & 43 & \mathrm{C} 8 & 1 \mathrm{~F} & 9 \mathrm{E} & 08 & 57 & 03 & 28 & 03 & 28 & \text { AA } & \text { D5 } & 03 & 36 & 03 & 36\end{array}$ 0IFFO IA E5 $04 \quad 67 \quad 03 \quad$ C4 $04 \begin{array}{llllllllll} & 09 & 01 & 5 B & 1 A & \text { D5 } & 03 & 46 & 04 & 89\end{array}$ $\begin{array}{lllllllllllllllll}02000 & \text { IF } & A 6 & 01 & 82 & 20 & 1 \mathrm{E} & 03 & 28 & 1 \mathrm{~A} & \mathrm{C} 6 & 03 & 36 & 04 & 05 & 03 & 46\end{array}$ $\begin{array}{lllllllllllllllll}02010 & 04 & 89 & 04 & 05 & 06 & 08 & 04 & 89 & 06 & 08 & 06 & 08 & 03 & 1 B & 00 & A E\end{array}$ $\begin{array}{lllllllllllllllll}02020 & \text { IF } & F A & 1 A & C 6 & 04 & 89 & 03 & 52 & 04 & 89 & 02 & \text { C0 } & 86 & 2 \mathrm{E} & 41 & 53\end{array}$ $\begin{array}{lllllllllllllllll}02030 & 43 & 49 & \text { C9 } & \text { IF } & \text { DC } & 08 & 57 & 04 & \text { Dl } & 04 & 9 C & 01 & 49 & 00 & \text { FE } & 03\end{array}$ $\begin{array}{lllllllllllllllll}02040 & \mathrm{AE} & 01 & 82 & 20 & 4 \mathrm{~B} & 04 & 7 \mathrm{D} & 01 & 49 & 00 & 2 \mathrm{E} & 04 & 9 \mathrm{C} & 04 & 9 \mathrm{C} & 01\end{array}$ $\begin{array}{lllllllllllllllll}02050 & 49 & 00 & 7 \mathrm{~F} & 03 & \mathrm{AE} & 04 & 89 & 01 & 49 & 00 & \mathrm{~A} 0 & 03 & 90 & 02 & 51 & 01\end{array}$ $\begin{array}{lllllllllllllllll}02060 & 82 & 20 & 69 & 04 & 7 \mathrm{D} & 01 & 49 & 00 & 2 \mathrm{E} & 04 & 9 \mathrm{C} & 01 & 49 & 00 & 7 \mathrm{E} & 04\end{array}$ $\begin{array}{lllllllllllllllll}02070 & 05 & 03 & 52 & 01 & 82 & 20 & 7 \mathrm{D} & 04 & 7 \mathrm{D} & 01 & 49 & 00 & 2 \mathrm{E} & 04 & 9 \mathrm{C} & 01\end{array}$ $\begin{array}{lllllllllllllllll}02080 & 49 & 00 & 20 & 03 & 90 & 01 & 82 & 20 & 8 \mathrm{~F} & 04 & 7 \mathrm{D} & 01 & 49 & 00 & 2 \mathrm{E} & 08\end{array}$ $\begin{array}{lllllllllllllllll}02090 & \text { FC } & 02 & \text { C0 } & 84 & 44 & 55 & 4 \mathrm{D} & \mathrm{D} 0 & 20 & 2 \mathrm{C} & 08 & 57 & 07 & 59 & 04 & \mathrm{BF}\end{array}$ $\begin{array}{lllllllllllllllll}020 \mathrm{AO} & 04 & \mathrm{FE} & 04 & \mathrm{FE} & 0 \mathrm{C} & \mathrm{DC} & 04 & 67 & 03 & \mathrm{C} 4 & 04 & 89 & 01 & 5 \mathrm{~B} & 03 & 46\end{array}$

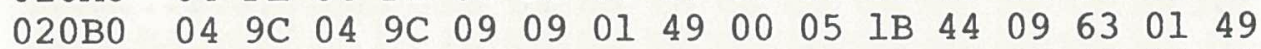
$\begin{array}{lllllllllllllllll}020 \mathrm{C} 0 & 00 & 10 & 03 & \mathrm{C} 4 & 04 & 89 & 04 & 67 & 04 & 67 & 01 & 5 \mathrm{~B} & 03 & 46 & 04 & \mathrm{Dl}\end{array}$ 020D0 $09 \begin{array}{llllllllllllllll}63 & 06 & 18 & 1 \mathrm{~B} & 44 & 00 & \mathrm{AE} & 20 & \mathrm{CC} & 06 & 18 & 14 & \mathrm{AE} & 01 & 5 \mathrm{~B}\end{array}$ $020 \mathrm{E} 0 \quad 03 \quad 46 \quad 20 \quad 35 \quad 00$ AE 20 E0 08 EA $01 \quad 82 \quad 20$ F0 03 1B $\begin{array}{lllllllllllllllll}020 \mathrm{~F} 0 & 01 & 49 & 00 & 10 & 00 & \mathrm{D} 3 & 20 & \mathrm{AE} & 07 & 59 & 04 & \mathrm{E} 0 & 09 & 09 & 02 & \mathrm{C} 0\end{array}$ $\begin{array}{lllllllllllllllll}02100 & 84 & 3 \mathrm{~F} & 45 & 46 & \mathrm{Bl} & 20 & 93 & 00 & 5 \mathrm{E} & 84 & 3 \mathrm{~F} & 45 & 46 & \mathrm{~B} 2 & 21 & 00\end{array}$ $\begin{array}{lllllllllllllllll}02110 & 00 & 62 & 84 & 3 \mathrm{~F} & 45 & 46 & \mathrm{~B} 3 & 21 & 09 & 00 & 66 & 84 & 3 \mathrm{~F} & 45 & 46 & \mathrm{~B} 4\end{array}$ $\begin{array}{lllllllllllllllll}02120 & 21 & 12 & 00 & 6 \mathrm{~A} & 82 & 57 & \mathrm{~B} 1 & 21 & 1 \mathrm{~B} & 00 & 78 & 82 & 57 & \mathrm{~B} 2 & 21 & 24\end{array}$ $\begin{array}{lllllllllllllllll}02130 & 00 & 7 \mathrm{~B} & 82 & 57 & \mathrm{~B} 3 & 21 & 2 \mathrm{~B} & 00 & 7 \mathrm{E} & 82 & 57 & \mathrm{~B} 4 & 21 & 32 & 00 & 81\end{array}$ $\begin{array}{lllllllllllllllll}02140 & 83 & 57 & 4 \mathrm{E} & \mathrm{B} 1 & 21 & 39 & 00 & 84 & 83 & 57 & 4 \mathrm{E} & \mathrm{B} 2 & 21 & 40 & 00 & 87\end{array}$ $\begin{array}{lllllllllllllllll}02150 & 83 & 57 & 4 \mathrm{E} & \mathrm{B} 3 & 21 & 48 & 00 & 8 \mathrm{~A} & 83 & 57 & 4 \mathrm{E} & \mathrm{B} 4 & 21 & 50 & 00 & 8 \mathrm{D}\end{array}$ $\begin{array}{lllllllllllllllll}02160 & 84 & 53 & 45 & 54 & \mathrm{Dl} & 21 & 58 & 21 & 69 & 7 \mathrm{~B} & \mathrm{D} 5 & 84 & 52 & 45 & 54 & \mathrm{Dl}\end{array}$ $\begin{array}{lllllllllllllllll}02170 & 21 & 60 & 21 & 74 & 7 \mathrm{~A} & \mathrm{D} 5 & \mathrm{C} 4 & 43 & 41 & 53 & \mathrm{C} 5 & 21 & 6 \mathrm{~B} & 08 & 57 & 0 \mathrm{C}\end{array}$ $\begin{array}{lllllllllllllllll}02180 & 18 & 07 & 77 & 04 & \text { BF } & 0 A & \text { EE } & 01 & 49 & 00 & 04 & 02 & \text { C0 } & \text { C2 } & 4 \mathrm{~F} & \text { C6 } 6\end{array}$ $\begin{array}{lllllllllllllllll}02190 & 21 & 76 & 08 & 57 & 01 & 49 & 00 & 04 & 0 \mathrm{C} & 47 & 0 \mathrm{C} & 91 & 04 & 67 & 0 \mathrm{C} & 91\end{array}$ $\begin{array}{lllllllllllllllll}021 A 0 & 03 & 73 & 0 C & 91 & 01 & 82 & \text { OA } & 53 & 06 & 08 & 0 A & 84 & 0 C & 91 & 04 & 7 D\end{array}$ $\begin{array}{lllllllllllllllll}021 \mathrm{~B} 0 & 01 & 49 & 00 & 05 & 02 & \mathrm{C} 0 & \mathrm{C} 5 & 45 & 4 \mathrm{E} & 44 & 4 \mathrm{~F} & \mathrm{C} 6 & 21 & 8 \mathrm{D} & 08 & 57\end{array}$ $\begin{array}{lllllllllllllllll}021 \mathrm{C} 0 & 01 & 49 & 00 & 05 & 0 \mathrm{C} & 47 & 0 \mathrm{C} & 91 & 01 & 72 & 0 A & 53 & 06 & 08 & 0 \mathrm{~A} & 84\end{array}$ $\begin{array}{lllllllllllllllll}021 \mathrm{D} 0 & 04 & 89 & 06 & 18 & 13 & \mathrm{Bl} & 01 & 49 & 00 & 04 & 02 & \mathrm{C} 0 & \mathrm{C} 7 & 45 & 4 \mathrm{E} & 44\end{array}$ $\begin{array}{lllllllllllllllll}021 \mathrm{E} 0 & 43 & 41 & 53 & \mathrm{C} 5 & 21 & \mathrm{~B} 6 & 08 & 57 & 01 & 49 & 00 & 04 & 0 \mathrm{C} & 47 & 0 \mathrm{C} & 91\end{array}$ $\begin{array}{lllllllllllllllll}021 \mathrm{~F} 0 & 04 & 7 \mathrm{D} & 02 & 86 & 07 & 77 & 04 & \mathrm{BF} & 03 & 73 & 03 & 52 & 01 & 82 & 22 & 08\end{array}$ $\begin{array}{lllllllllllllllll}02200 & 06 & 18 & 13 & \mathrm{BI} & 01 & 72 & 21 & \mathrm{~F} 2 & 07 & 77 & 04 & \mathrm{EO} & 02 & \mathrm{C} 0 & 84 & 54\end{array}$ $\begin{array}{lllllllllllllllll}02210 & 45 & 58 & \text { D4 } & 21 & \text { DC } & 08 & 57 & 0 A & 53 & 06 & 33 & 03 & \text { D5 } & 08 & 98 & 0 D\end{array}$ $\begin{array}{lllllllllllllllll}02220 & \text { FA } & 0 A & 53 & 0 B & 5 D & 06 & 33 & 03 & \text { D5 } & 02 & \text { FC } & 02 & \text { C0 } & 84 & 4 C & 49\end{array}$ $\begin{array}{lllllllllllllllll}02230 & 4 \mathrm{E} & \mathrm{C} 5 & 22 & \text { OE } & 08 & 57 & 04 & 9 \mathrm{C} & 01 & 49 & \mathrm{FF} & \mathrm{F} 0 & 02 & 51 & 01 & 49\end{array}$ $\begin{array}{lllllllllllllllll}02240 & 00 & 17 & 0 B & F E & 07 & 19 & 04 & \text { BF } & 15 & \text { F5 } & 04 & 7 D & 02 & \text { C0 } & \text { C6 } & 45\end{array}$ $\begin{array}{lllllllllllllllll}02250 & 44 & 49 & 54 & 4 \mathrm{~F} & \mathrm{D} 2 & 22 & 2 \mathrm{D} & 10 & \mathrm{Al} & 11 & 91 & 81 & \mathrm{~A} 0 & 26 & 84 & 18\end{array}$ $\begin{array}{lllllllllllllllll}02260 & 3 \mathrm{~F} & 85 & 57 & 48 & 45 & 52 & \mathrm{C} 5 & 22 & 4 \mathrm{E} & 08 & 57 & 04 & 9 \mathrm{C} & 06 & 6 \mathrm{D} & 13\end{array}$ $\begin{array}{lllllllllllllllll}02270 & 4 \mathrm{~F} & 04 & 9 \mathrm{C} & 07 & 19 & 04 & \mathrm{E} 0 & 0 \mathrm{~B} & 41 & 09 & 53 & 63 & 72 & 65 & 65 & 6 \mathrm{E}\end{array}$ $\begin{array}{lllllllllllllllll}02280 & 20 & 23 & 20 & 0 \mathrm{C} & \mathrm{F} 2 & 15 & 8 \mathrm{E} & 04 & 89 & 06 & 33 & 13 & 3 \mathrm{~F} & 06 & 33 & 05\end{array}$ $\begin{array}{lllllllllllllllll}02290 & 67 & 04 & \mathrm{FE} & 16 & \mathrm{DE} & 03 & \mathrm{C} 4 & 09 & 09 & 06 & 33 & 09 & 33 & 09 & 09 & 0 \mathrm{~A}\end{array}$ $\begin{array}{lllllllllllllllll}022 \mathrm{~A} 0 & 53 & 04 & \mathrm{Dl} & 04 & 05 & 14 & \mathrm{AE} & 01 & 49 & 00 & 5 \mathrm{E} & 08 & \mathrm{FC} & 22 & 57 & 11\end{array}$ $\begin{array}{lllllllllllllllll}022 \mathrm{~B} 0 & \mathrm{BA} & 02 & \mathrm{C} 0 & 87 & 23 & 4 \mathrm{C} & 4 \mathrm{~F} & 43 & 41 & 54 & \mathrm{C} 5 & 18 & 3 \mathrm{~B} & 08 & 57 & 07\end{array}$ $\begin{array}{lllllllllllllllll}022 \mathrm{C} 0 & 80 & 04 & \mathrm{BF} & 06 & 33 & 13 & 3 \mathrm{~F} & 02 & \mathrm{C} 0 & 85 & 23 & 4 \mathrm{C} & 45 & 41 & \mathrm{C} 4 & 22\end{array}$ $\begin{array}{lllllllllllllllll}022 \mathrm{D} 0 & \mathrm{~B} 3 & 08 & 57 & 22 & \mathrm{BD} & 22 & 34 & 04 & 89 & 02 & \mathrm{C} 0 & 84 & 23 & 4 \mathrm{C} & 41 & \mathrm{C} 7\end{array}$ $\begin{array}{lllllllllllllllll}022 \mathrm{E} 0 & 22 & \mathrm{C} 9 & 08 & 57 & 22 & \mathrm{Dl} & 04 & 9 \mathrm{C} & 03 & 28 & 03 & \mathrm{C} 4 & 06 & 33 & 03 & 36\end{array}$ $\begin{array}{lllllllllllllllll}022 \mathrm{~F} 0 & 04 & 05 & 02 & \mathrm{C} 0 & 85 & 2 \mathrm{D} & 4 \mathrm{D} & 4 \mathrm{~F} & 56 & \mathrm{C} 5 & 22 & \mathrm{DB} & 08 & 57 & 22 & 34\end{array}$ $\cdot g \cdot Y . ? . . . R \cdot j \cdot b .$. ........v.Y.'. a.M atch. .W.....V.g .D... [ ...Q.F.Q.. ...L.R...r.N.U.. .6.r.Z.\}.R. Q.MAT CH...W. . . .U.6.6 .e.g.D... [.U.F.. .$\&_{\ldots} \ldots(. F .6 \ldots F$ .z.F...R.....AS CII. \.W.Q...I. . ... K.\}.I....... $I \ldots \ldots I$. . . Q Q . i.\}.I........ ..R..\}.\}.I.... I. ....... . . I... I.Q.DUMP ,.W.Y.? . $\sim$. . g.D... [.F ..........D.C.I W.....g.g. [.F. Q . C...D.. L..... [ .F $5 \ldots$. j.. $^{\circ}$. .I...S ..Y.'... .?EF1 .. .?EF2! . .b.?EF3! ..f.?EF4 !.j.W1!..x.W2! \$ . \{.W3!+...W4!2.. .WN1 ! 9...WN2! @. .WN3!H...WN4 ! P.. .SETQ!X! I \{U.RETQ !' ! tzUDCASE!k.W. ..w.?.n.I... @BOF !v.W.I...G...g. . .S............ . I... AEENDOF!..W .I...G...r.S... .......... QGEND CASE! 6.W.I...G.. .\}...w.?.s.R.." ...l.r!r.w. . EXT!\.W.S.3.U... z.S.].3.U.1.Q.II NE"..W...I.p.Q.I ... …?.u.\}.QFE DITOR"-.!... \&.. ?.WHERE"N.W...m. O..... A. Screen \# .r.....3.?.3. g. . .D...3.3... S.Q........। "W. :.@.\#LOCATE.; .W. ..?.3.?. @.\#LEAD" $3 . W "=" 4 \ldots$... \#LAG "I.W"Q... (.D.3.6 .....-MOVE" [ .W"4 
$\begin{array}{lllllllllllllllll}02300 & 06 & 33 & 02 & \mathrm{FC} & 16 & 59 & 02 & \mathrm{C} 0 & 81 & \mathrm{C} 8 & 22 & \mathrm{~F} 4 & 08 & 57 & 22 & 34\end{array}$ $\begin{array}{llllllllllllllllll}02310 & 0 B & 5 D & 03 & D 5 & 06 & 33 & 04 & 9 C & 0 B & 5 D & 04 & F 0 & 02 & F C & 02 & C 0\end{array}$ $\begin{array}{lllllllllllllllll}02320 & 81 & \mathrm{C} 5 & 23 & 08 & 08 & 57 & 22 & 34 & 06 & 33 & 08 & 98 & 16 & 59 & 02 & \mathrm{C} 0\end{array}$ $\begin{array}{lllllllllllllllll}02330 & 81 & \text { D3 } & 23 & 20 & 08 & 57 & 04 & 9 \mathrm{C} & 06 & 10 & 04 & 05 & 01 & 49 & 00 & 0 \mathrm{E}\end{array}$ $\begin{array}{lllllllllllllllll}02340 & 01 & 5 B & 03 & 46 & 22 & 34 & 03 & 46 & 03 & \text { D5 } & 22 & \text { FC } & 01 & 49 & \mathrm{FF} & \mathrm{FF}\end{array}$ $\begin{array}{lllllllllllllllll}02350 & 00 & \text { D3 } & 23 & 42 & 23 & 24 & 02 & \text { C0 } & 81 & \text { C4 } & 23 & 30 & 08 & 57 & 04 & 9 \mathrm{C}\end{array}$ $\begin{array}{lllllllllllllllll}02360 & 23 & \text { OC } & 01 & 49 & 00 & \text { OF } & 04 & 9 \mathrm{C} & 04 & \mathrm{FE} & 01 & 5 \mathrm{~B} & 03 & 46 & 03 & \mathrm{D} 5\end{array}$ $\begin{array}{lllllllllllllllll}02370 & 22 & 34 & 03 & 46 & 22 & \mathrm{FC} & 00 & \mathrm{AE} & 23 & 6 \mathrm{C} & 23 & 24 & 02 & \mathrm{C} 0 & 81 & \mathrm{CD}\end{array}$ $\begin{array}{lllllllllllllllll}02380 & 23 & 58 & 08 & 57 & 07 & 80 & 03 & \text { F2 } & 09 & 09 & 09 & 63 & 22 & \text { D1 } & 09 & 33\end{array}$ $\begin{array}{lllllllllllllllll}02390 & 01 & 49 & 00 & 5 F & 08 & F C & 22 & E 2 & 09 & 33 & 22 & \text { BD } & 15 & 8 E & 04 & 7 D\end{array}$ $\begin{array}{lllllllllllllllll}023 \mathrm{~A} 0 & 02 & \mathrm{C} 0 & 81 & \mathrm{D} 4 & 23 & 7 \mathrm{E} & 08 & 57 & 04 & 9 \mathrm{C} & 06 & 33 & 05 & 67 & 07 & 80\end{array}$ $\begin{array}{lllllllllllllllll}023 \mathrm{~B} 0 & 04 & \mathrm{E} 0 & 23 & 0 \mathrm{C} & 06 & 08 & 23 & 82 & 02 & \mathrm{C} 0 & 81 & \mathrm{CC} & 23 & \mathrm{~A} 2 & 08 & 57\end{array}$ $\begin{array}{lllllllllllllllll}023 \mathrm{C} 0 & 07 & 19 & 04 & \mathrm{BF} & 18 & \mathrm{BA} & 06 & 08 & 23 & 82 & 02 & \mathrm{C} 0 & 81 & \mathrm{D} 2 & 23 & \mathrm{BA}\end{array}$ $\begin{array}{llllllllllllllllll}023 \mathrm{D} 0 & 08 & 57 & 0 \mathrm{~B} & 5 \mathrm{D} & 03 & \mathrm{D} 5 & 04 & 89 & 22 & \mathrm{FC} & 02 & \mathrm{C} 0 & 81 & \mathrm{D} 0 & 23 & \mathrm{CC}\end{array}$ $\begin{array}{llllllllllllllllll}023 \mathrm{E} 0 & 08 & 57 & 06 & 10 & 22 & 15 & 23 & \mathrm{D} 0 & 23 & \mathrm{BE} & 02 & \mathrm{C} 0 & 81 & \mathrm{C} 9 & 23 & \mathrm{DC}\end{array}$ $\begin{array}{lllllllllllllllll}023 \mathrm{~F} 0 & 08 & 57 & 04 & 9 \mathrm{C} & 23 & 34 & 23 & \mathrm{D} 0 & 02 & \mathrm{C} 0 & 83 & 54 & 4 \mathrm{~F} & \mathrm{D} 0 & 23 & \mathrm{EC}\end{array}$ $\begin{array}{lllllllllllllllll}02400 & 08 & 57 & 06 & 08 & 07 & 80 & 04 & \mathrm{E} 0 & 02 & \mathrm{C} 0 & 85 & 43 & 4 \mathrm{C} & 45 & 41 & \mathrm{D} 2\end{array}$ $\begin{array}{lllllllllllllllll}02410 & 23 & \mathrm{FA} & 08 & 57 & 07 & 19 & 04 & \mathrm{E} 0 & 01 & 49 & 00 & 10 & 06 & 08 & 01 & 5 \mathrm{~B}\end{array}$ $\begin{array}{lllllllllllllllll}02420 & 03 & 46 & 23 & 24 & 00 & \mathrm{AE} & 24 & 20 & 02 & \mathrm{C} 0 & 85 & 31 & 4 \mathrm{C} & 49 & 4 \mathrm{E} & \mathrm{C} 5\end{array}$ $\begin{array}{lllllllllllllllll}02430 & 24 & \text { OA } & 08 & 57 & 22 & \mathrm{E} 2 & \text { OB } & 5 \mathrm{D} & \mathrm{OB} & 03 & \text { IF } & \mathrm{E} 4 & 07 & 80 & 03 & \mathrm{~F} 2\end{array}$ $\begin{array}{lllllllllllllllll}02440 & 02 & \mathrm{C} 0 & 84 & 46 & 49 & 4 \mathrm{E} & \mathrm{C} 4 & 24 & 2 \mathrm{~A} & 08 & 57 & 01 & 49 & 03 & \mathrm{FF} & 07\end{array}$ $\begin{array}{lllllllllllllllll}02450 & 80 & 04 & \mathrm{BF} & 03 & 90 & 01 & 82 & 24 & 69 & 24 & 00 & \text { 0B } & 5 \mathrm{D} & 0 \mathrm{~A} & 53 & 06\end{array}$ $\begin{array}{lllllllllllllllll}02460 & 33 & 03 & \text { D5 } & 02 & \text { FC } & 06 & 08 & \text { OF } & 28 & 24 & 32 & 01 & 82 & 24 & 4 \mathrm{~B} & 02\end{array}$ $\begin{array}{lllllllllllllllll}02470 & \text { C0 } & 86 & 44 & 45 & 4 \mathrm{C} & 45 & 54 & \mathrm{C} 5 & 24 & 42 & 08 & 57 & 03 & 28 & 22 & \mathrm{E} 2\end{array}$ $\begin{array}{lllllllllllllllll}02480 & 03 & \mathrm{C} 4 & 03 & 46 & 04 & 05 & 22 & \mathrm{E} 2 & 03 & 46 & 04 & 32 & 07 & 80 & 03 & \mathrm{~F} 2\end{array}$ $\begin{array}{lllllllllllllllll}02490 & 22 & \mathrm{D} 1 & 03 & \mathrm{C} 4 & 04 & 89 & 02 & \mathrm{FC} & 03 & 36 & 08 & 98 & 16 & 59 & 02 & \mathrm{C} 0\end{array}$

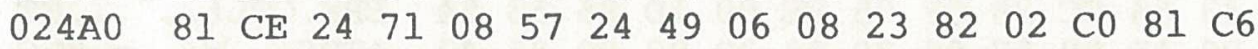
$\begin{array}{lllllllllllllllll}024 \mathrm{~B} 0 & 24 & \mathrm{~A} 0 & 08 & 57 & 06 & 10 & 22 & 15 & 24 & \mathrm{~A} 4 & 02 & \mathrm{C} 0 & 81 & \mathrm{C} 2 & 24 & \mathrm{AE}\end{array}$ $\begin{array}{lllllllllllllllll}024 \mathrm{C} 0 & 08 & 57 & 0 \mathrm{~B} & 5 \mathrm{D} & 04 & \mathrm{Dl} & 04 & 32 & 23 & 82 & 02 & \mathrm{C} 0 & 81 & \mathrm{D} 8 & 24 & \mathrm{BC}\end{array}$ $\begin{array}{lllllllllllllllll}024 \mathrm{D} 0 & 08 & 57 & 06 & 10 & 22 & 15 & 24 & 49 & 0 \mathrm{~B} & 5 \mathrm{D} & 04 & \mathrm{Dl} & 24 & 7 \mathrm{~A} & 06 & 08\end{array}$ $\begin{array}{lllllllllllllllll}024 \mathrm{E} 0 & 23 & 82 & 02 & \mathrm{C} 0 & 84 & 54 & 49 & 4 \mathrm{C} & \mathrm{CC} & 24 & \mathrm{CC} & 08 & 57 & 22 & \mathrm{Dl} & 03\end{array}$

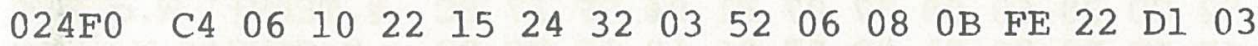
$\begin{array}{lllllllllllllllll}02500 & \mathrm{C} 4 & 04 & 89 & 04 & 05 & 24 & 7 \mathrm{~A} & 06 & 08 & 23 & 82 & 02 & \mathrm{C} 0 & 81 & \mathrm{C} 3 & 24\end{array}$ $\begin{array}{lllllllllllllllll}02510 & \text { E4 } & 08 & 57 & 06 & 10 & 22 & 15 & \text { OB } & 5 \mathrm{D} & \text { OB } & 03 & 22 & \mathrm{E} 2 & 04 & \mathrm{FE} & 04\end{array}$ $\begin{array}{lllllllllllllllll}02520 & 67 & 0 F & 57 & 03 & 28 & 03 & 46 & 07 & 80 & 03 & \text { F2 } & 03 & 46 & 04 & 05 & 03\end{array}$ $\begin{array}{lllllllllllllllll}02530 & 28 & 04 & 9 \mathrm{C} & 0 \mathrm{~A} & 53 & 03 & 36 & 04 & 9 \mathrm{C} & 03 & 28 & 02 & \mathrm{FC} & 0 \mathrm{~A} & 53 & 22\end{array}$ $\begin{array}{lllllllllllllllll}02540 & \text { D1 } & 03 & \text { C4 } & 03 & 36 & 02 & \text { FC } & 03 & 36 & 02 & \text { FC } & 16 & 59 & 06 & 08 & 23\end{array}$ $\begin{array}{lllllllllllllllll}02550 & 82 & 02 & \text { C0 } & 81 & \text { D4 } & 22 & 61 & 08 & 57 & 22 & 57 & 23 & \text { A6 } & 02 & \text { C0 } & 81\end{array}$ $\begin{array}{lllllllllllllllll}02560 & \text { CC } & 25 & 53 & 08 & 57 & 22 & 57 & 24 & 00 & 23 & \text { BE } & 02 & \text { C0 } & 83 & 43 & 55\end{array}$ $\begin{array}{lllllllllllllllll}02570 & \text { D2 } & 25 & 5 \mathrm{~F} & 06 & 90 & 00 & 52 & 86 & 47 & 4 \mathrm{~F} & 54 & 4 \mathrm{~F} & 58 & \mathrm{D} 9 & 25 & 0 \mathrm{D}\end{array}$ $\begin{array}{llllllllllllllllll}02580 & 08 & 57 & 01 & 49 & 00 & 1 B & 08 & \text { FC } & 01 & 49 & 00 & 3 \mathrm{D} & 08 & \text { FC } & 01 & 49\end{array}$ $\begin{array}{lllllllllllllllll}02590 & 00 & 20 & 03 & \text { C4 } & 08 & \text { FC } & 01 & 49 & 00 & 20 & 03 & \text { C4 } & 08 & \text { FC } & 02 & \text { C0 }\end{array}$ $\begin{array}{lllllllllllllllll}025 \mathrm{~A} 0 & 87 & 53 & 45 & 54 & 45 & 44 & 49 & \mathrm{D} 4 & 25 & 77 & 08 & 57 & 01 & 49 & 00 & 1 \mathrm{~B}\end{array}$

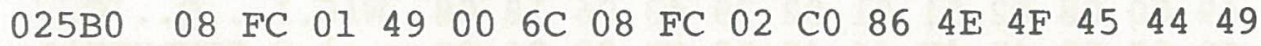
$\begin{array}{lllllllllllllllll}025 \mathrm{C} 0 & \mathrm{D} 4 & 25 & \mathrm{~A} 0 & 08 & 57 & 01 & 49 & 00 & 1 \mathrm{~B} & 08 & \mathrm{FC} & 01 & 49 & 00 & 6 \mathrm{~B} & 08\end{array}$ $\begin{array}{lllllllllllllllll}025 \mathrm{D} 0 & \mathrm{FC} & 02 & \mathrm{C} 0 & 84 & 2 \mathrm{E} & 43 & 55 & \mathrm{D} 2 & 25 & \mathrm{BA} & 08 & 57 & 25 & 73 & 04 & \mathrm{BF}\end{array}$ $\begin{array}{lllllllllllllllll}025 \mathrm{E} 0 & 01 & 49 & 00 & 40 & 13 & 3 \mathrm{~F} & 06 & 18 & 03 & \mathrm{C} 4 & 04 & 89 & 01 & 49 & 00 & 04\end{array}$ $\begin{array}{lllllllllllllllll}025 \mathrm{~F} 0 & 03 & \mathrm{C} 4 & 04 & 89 & 25 & 80 & 02 & \mathrm{C} 0 & 84 & 21 & 43 & 55 & \mathrm{D} 2 & 25 & \mathrm{D} 3 & 08\end{array}$ $\begin{array}{lllllllllllllllll}02600 & 57 & 06 & 08 & 05 & 47 & 01 & 49 & 03 & \mathrm{FF} & 0 \mathrm{~F} & 57 & 25 & 73 & 04 & \mathrm{E} 0 & 02\end{array}$ $\begin{array}{lllllllllllllllll}02610 & \mathrm{C} 0 & 84 & 2 \mathrm{~B} & 43 & 55 & \mathrm{D} 2 & 25 & \mathrm{~F} 8 & 08 & 57 & 25 & 73 & 04 & \mathrm{BF} & 03 & \mathrm{C} 4\end{array}$ $\begin{array}{lllllllllllllllll}02620 & 25 & \mathrm{FF} & 02 & \mathrm{C} 0 & 85 & 2 \mathrm{~B} & 2 \mathrm{E} & 43 & 55 & \mathrm{D} 2 & 26 & 11 & 08 & 57 & 26 & 18\end{array}$ $\begin{array}{lllllllllllllllll}02630 & 25 & \mathrm{DA} & 02 & \mathrm{C} 0 & 84 & 2 \mathrm{~B} & 4 \mathrm{C} & 49 & \mathrm{CE} & 26 & 24 & 08 & 57 & 25 & 73 & 04\end{array}$ $\begin{array}{lllllllllllllllll}02640 & \text { BF } & 01 & 49 & 00 & 40 & 13 & 4 \mathrm{~F} & 03 & \mathrm{C} 4 & 01 & 49 & 00 & 40 & 05 & 67 & 25\end{array}$ $\begin{array}{lllllllllllllllll}02650 & \mathrm{FF} & 02 & \mathrm{C} 0 & 83 & 48 & 4 \mathrm{~F} & \mathrm{CD} & 26 & 34 & 08 & 57 & 06 & 08 & 25 & 73 & 04\end{array}$ $\begin{array}{lllllllllllllllll}02660 & \text { E0 } & 25 & \mathrm{DA} & 02 & \mathrm{C} 0 & 84 & 21 & 42 & 4 \mathrm{C} & \mathrm{CB} & 26 & 53 & 08 & 57 & 07 & 19\end{array}$ $\begin{array}{lllllllllllllllll}02670 & 04 & \mathrm{BF} & 16 & \mathrm{DE} & 25 & 73 & 04 & \mathrm{BF} & 03 & \mathrm{C} 4 & 04 & \mathrm{~F} 0 & 16 & 59 & 06 & 10\end{array}$
.3.1.Y.@.H"t.W"4 .].U.3...].p.1.a .E\#..W"4.3...Y. Q .S\# .W......... . [.F"4.F.U"|.I.. . S\#B\#\$.Q.D\#0.W.. \#..I..... 〜. [.F.U "4.F"|..\#I\#\$.Q.M $\# X . W \ldots r \ldots . . . c^{\prime} Q .3$ .I._. | "b. $3 "=\ldots$. \} . . T\#..W...3.g.. -\#...\#.....L\#".W ...?.........R\# : .W.].U.."। . A.P\#L .W...".\#P\#>. (a.I\# .W..\# 4 \#P. A.TOP\# I .W....... CLEAR \#z.W... .I..... [ .F\#\$..\$ . C.ILINE \$.. W"b.]...d...r . a.FIND\$*.W.I... ..?....\$i\$..].S. 3.U.।... \$ $\$ 2$. \$K. a.DELETE\$B.W. ( "b .D.F.."b.F.2...r "Q.D...।.6...Y. Q . N\$q.W\$I...\# . . . . F \$.W..".\$\$.@. B\$. .W.].Q.2\#.....XS< .W..".\$I.].Q\$z . \# .. ..TILLSL.W"Q. D..".\$2.R... "Q. D....\$z..\# ....C\$ d.W.."... .. "b. 。 g.W. (.F...r.F... (...S. .... (.). . S" Q.D.6.1.6.1.Y..\# ....T"a.W"W\#\&.@. L\&S.W"W\$.\#>. Q.CU R\% ....R.GOTOXY\%. .W.I...I.I. =. I.I -.D.I.I. .D.I. a . SETEDIT'\%.W.I.. -1.I.1.1. . NOEDI To .W.I...I.I.k. I. Q.. CUR\%:.W\%s.? .I.Q.?...D...I.. .D.. 8... a. !CURS. W...G.I...W W S. $^{\prime}$ a.+CUR\&X.W\%s.?.D \%......CUR\&..W\&. \&Z. . + LIN\&\$.W\%S. ?.I.Q.O.D.I.A.g\% .... HOM\&4.W.. 85 . \%Z. @.!BLK\&S.W.. .?. ^ 8 s.?.D.p.Y.. 
$\begin{array}{lllllllllllllllll}02680 & 26 & 2 \mathrm{C} & 02 & \mathrm{C} 0 & 87 & 53 & 2 \mathrm{E} & 45 & 52 & 41 & 53 & \mathrm{C} 5 & 26 & 65 & 08 & 57\end{array}$ $\begin{array}{lllllllllllllllll}02690 & 26 & 59 & 01 & 49 & 04 & 00 & 06 & 08 & 01 & 5 B & 01 & 49 & 00 & 20 & 04 & 9 \mathrm{C}\end{array}$ $\begin{array}{lllllllllllllllll}026 \mathrm{~A} 0 & 08 & \mathrm{FC} & 26 & 6 \mathrm{C} & 00 & \mathrm{AE} & 26 & 9 \mathrm{~A} & 26 & 59 & 25 & \mathrm{DA} & 02 & \mathrm{C} 0 & 84 & 45\end{array}$ $\begin{array}{lllllllllllllllll}026 \mathrm{BO} & 44 & 49 & \mathrm{D} 4 & 25 & 6 \mathrm{D} & 08 & 57 & 22 & 57 & 25 & \mathrm{AA} & \text { ID } & 3 \mathrm{D} & 18 & \mathrm{BA} & 26\end{array}$ $\begin{array}{lllllllllllllllll}026 \mathrm{C} 0 & 59 & 25 & \mathrm{DA} & 06 & 08 & 08 & \mathrm{CF} & 01 & 49 & 00 & 05 & 04 & 67 & 03 & 73 & 01\end{array}$ $\begin{array}{lllllllllllllllll}026 \mathrm{D} 0 & 82 & 26 & \mathrm{DB} & 04 & 7 \mathrm{D} & 26 & 8 \mathrm{E} & 01 & 72 & 27 & \mathrm{D} 7 & \text { IC } & \mathrm{CD} & 04 & 67 & 03\end{array}$ $\begin{array}{lllllllllllllllll}026 \mathrm{E} 0 & 73 & 01 & 82 & 26 & \mathrm{FD} & 04 & 7 \mathrm{D} & 06 & 08 & 01 & 49 & 00 & 11 & 25 & 80 & 25\end{array}$ $\begin{array}{lllllllllllllllll}026 \mathrm{~F} 0 & \mathrm{C} 3 & 09 & 09 & 09 & 09 & 04 & 7 \mathrm{D} & 06 & 10 & 01 & 72 & 27 & \mathrm{D} 7 & 01 & 49 & 00\end{array}$ 02700

02710

02720

02730

02740

02750

02760

02770

02780

02790

027A0

027B0

$027 \mathrm{C} 0$

027D0

027E0

027F0

02800

02810

02820

02830

02840

02850

02860

02870

02880

02890

028A0

028B0

$028 \mathrm{C} 0$

028D0

028E0

$028 \mathrm{~F} 0$

02900

02910

02920

02930

02940

02950

02960

02970

02980

02990

029A0

029B0

029C0

029D0

029E0

$029 \mathrm{FO}$

$\begin{array}{llllllllllllllll}0 A & 04 & 67 & 03 & 73 & 01 & 82 & 27 & 15 & 04 & 7 D & 01 & 49 & 00 & 40 & 26\end{array}$ $\begin{array}{llllllllllllllll}2 \mathrm{C} & 01 & 72 & 27 & \mathrm{D} 7 & 01 & 49 & 00 & 7 \mathrm{~F} & 04 & 67 & 03 & 73 & 01 & 82 & 27\end{array}$ $\begin{array}{llllllllllllllll}2 \mathrm{D} & 04 & 7 \mathrm{D} & 01 & 49 & \mathrm{FF} & \mathrm{FF} & 26 & 2 \mathrm{C} & 01 & 72 & 27 & \mathrm{D} 7 & 01 & 49 & 00\end{array}$ $\begin{array}{llllllllllllllll}08 & 04 & 67 & 03 & 73 & 01 & 82 & 27 & 45 & 04 & 7 D & 01 & 49 & F F & F F & 26\end{array}$ $\begin{array}{llllllllllllllll}2 \mathrm{C} & 01 & 72 & 27 & \mathrm{D} 7 & 01 & 49 & 00 & 0 \mathrm{D} & 04 & 67 & 03 & 73 & 01 & 82 & 27\end{array}$ $\begin{array}{llllllllllllllll}5 \mathrm{D} & 04 & 7 \mathrm{D} & 06 & 10 & 26 & 3 \mathrm{~B} & 25 & \mathrm{DA} & 01 & 72 & 27 & \mathrm{D} 7 & 01 & 49 & 00\end{array}$ $\begin{array}{llllllllllllllll}0 B & 04 & 67 & 03 & 73 & 01 & 82 & 27 & 75 & 04 & 7 D & 01 & 49 & F F & C 0 & 26\end{array}$ $\begin{array}{llllllllllllllll}2 \mathrm{C} & 01 & 72 & 27 & \mathrm{D} 7 & 01 & 49 & 00 & 0 \mathrm{C} & 04 & 67 & 03 & 73 & 01 & 82 & 27\end{array}$ $\begin{array}{llllllllllllllll}8 \mathrm{~B} & 04 & 7 \mathrm{D} & 06 & 10 & 26 & 2 \mathrm{C} & 01 & 72 & 27 & \mathrm{D} 7 & 01 & 49 & 00 & 09 & 04\end{array}$ $\begin{array}{llllllllllllllll}67 & 03 & 73 & 01 & 82 & 27 & \text { B7 } & 04 & 7 D & 25 & 73 & 04 & \text { BF } & 01 & 49 & 00\end{array}$ $\begin{array}{llllllllllllllll}08 & 13 & 4 \mathrm{~F} & 01 & 49 & 00 & 08 & 05 & 67 & 01 & 49 & 00 & 08 & 03 & \mathrm{C} 4 & 25\end{array}$ $\begin{array}{llllllllllllllll}\text { FF } & 25 & \text { DA } & 01 & 72 & 27 & \text { D7 } & 04 & 9 C & 01 & 49 & 00 & 20 & 03 & 90 & 01\end{array}$ $\begin{array}{llllllllllllllll}82 & 27 & \mathrm{CD} & 01 & 49 & 00 & 07 & 08 & \text { FC } & 01 & 72 & 27 & \mathrm{D} 5 & 04 & 9 \mathrm{C} & 04\end{array}$ $\begin{array}{llllllllllllllll}9 \mathrm{C} & 08 & \mathrm{FC} & 26 & 6 \mathrm{C} & 04 & 7 \mathrm{D} & 01 & 82 & 26 & \mathrm{C} 3 & 02 & \mathrm{C} 0 & 85 & 45 & 44\end{array}$ $\begin{array}{llllllllllllllll}49 & 54 & \mathrm{CC} & 26 & \mathrm{AE} & 08 & 57 & 07 & 19 & 04 & \mathrm{BF} & 26 & \mathrm{~B} 5 & 02 & \mathrm{C} 0 & 85\end{array}$ $\begin{array}{llllllllllllllll}43 & 4 \mathrm{C} & 45 & 41 & \mathrm{D} 2 & 27 & \mathrm{DD} & 08 & 57 & 07 & 19 & 04 & \mathrm{E} 0 & 01 & 49 & 00\end{array}$ $\begin{array}{llllllllllllllll}10 & 06 & 08 & 01 & 5 \mathrm{~B} & 03 & 46 & 23 & 24 & 00 & \mathrm{AE} & 28 & 05 & 02 & \mathrm{C} 0 & 88\end{array}$ $\begin{array}{llllllllllllllll}43 & 4 \mathrm{~F} & 4 \mathrm{D} & 4 \mathrm{D} & 2 \mathrm{E} & 43 & 54 & \mathrm{CC} & 27 & \mathrm{EF} & 05 & \mathrm{FE} & 00 & 1 \mathrm{D} & 88 & 54\end{array}$ $\begin{array}{llllllllllllllll}45 & 52 & 4 \mathrm{D} & 2 \mathrm{E} & 43 & 54 & \mathrm{CC} & 28 & \text { OF } & 05 & \mathrm{FE} & 00 & \text { ID } & 86 & 53 & 54\end{array}$ $\begin{array}{llllllllllllllll}41 & 54 & 55 & \mathrm{D} 3 & 28 & 1 \mathrm{E} & 08 & 57 & 06 & 20 & 08 & 3 \mathrm{D} & 02 & \mathrm{C} 0 & 85 & 43\end{array}$ $\begin{array}{llllllllllllllll}54 & 4 \mathrm{C} & 3 \mathrm{D} & \mathrm{BE} & 28 & 2 \mathrm{D} & 08 & 57 & 06 & 20 & 08 & 19 & 02 & \mathrm{C} 0 & 85 & 4 \mathrm{D}\end{array}$ $\begin{array}{llllllllllllllll}4 \mathrm{~F} & 44 & 45 & \mathrm{CD} & 28 & 3 \mathrm{E} & 08 & 57 & 07 & \mathrm{E} 2 & 04 & \mathrm{BF} & 17 & \mathrm{BC} & 02 & \mathrm{C} 0\end{array}$ $\begin{array}{lllllllllllllllll}84 & 43 & 4 \mathrm{~F} & 4 \mathrm{D} & \mathrm{CD} & 28 & 4 \mathrm{E} & 08 & 57 & 08 & 00 & 04 & \mathrm{BF} & 17 & \mathrm{BC} & 02\end{array}$ $\begin{array}{llllllllllllllll}\mathrm{C} 0 & 84 & 54 & 45 & 52 & \mathrm{CD} & 28 & 60 & 08 & 57 & 07 & \mathrm{Fl} & 04 & \mathrm{BF} & 17 & \mathrm{BC}\end{array}$ $\begin{array}{llllllllllllllll}02 & \mathrm{C} 0 & 84 & 43 & 4 \mathrm{~F} & 4 \mathrm{E} & \mathrm{D} 3 & 28 & 71 & 08 & 57 & 06 & 10 & 17 & \mathrm{BC} & 02\end{array}$ $\begin{array}{llllllllllllllll}\mathrm{C} 0 & 84 & 45 & 53 & 43 & \mathrm{~A} 7 & 28 & 82 & 08 & 57 & 28 & 67 & 01 & 49 & 00 & \text { IB }\end{array}$ $\begin{array}{llllllllllllllll}08 & \text { FC } & 28 & 78 & 02 & \text { C0 } & 88 & 43 & 4 \mathrm{~F} & 4 \mathrm{D} & 4 \mathrm{D} & 49 & 4 \mathrm{E} & 49 & \mathrm{D} 4 & 28\end{array}$ $\begin{array}{llllllllllllllll}91 & 08 & 57 & 28 & 1 \mathrm{~A} & 04 & \mathrm{BF} & 28 & 46 & 02 & \mathrm{C} 0 & 86 & 52 & 43 & 56 & 42\end{array}$ $\begin{array}{llllllllllllllll}52 & \mathrm{CB} & 28 & \mathrm{~A} 6 & 08 & 57 & 06 & 18 & 08 & 3 \mathrm{D} & 04 & 7 \mathrm{D} & 28 & 78 & 0 \mathrm{~B} & 41\end{array}$ $\begin{array}{llllllllllllllll}0 \mathrm{~F} & 20 & 42 & 72 & 65 & 61 & 6 \mathrm{~B} & 20 & 72 & 65 & 63 & 65 & 69 & 76 & 65 & 64\end{array}$ $\begin{array}{llllllllllllllll}02 & \mathrm{C} 0 & 84 & 52 & 43 & 56 & \mathrm{C} 3 & 28 & \mathrm{BB} & 08 & 57 & 01 & 49 & 00 & 08 & 02\end{array}$ $\begin{array}{llllllllllllllll}51 & 01 & 82 & 28 & \text { FB } & 28 & \text { C4 } & 01 & 72 & 29 & 03 & 06 & 18 & 08 & 3 D & 28\end{array}$ $\begin{array}{llllllllllllllll}78 & 08 & \text { FC } & 02 & \text { C0 } & 88 & 52 & 43 & 56 & 43 & 48 & 41 & 52 & \text { BF } & 28 & \text { E2 }\end{array}$ $\begin{array}{llllllllllllllll}08 & 57 & 04 & 9 \mathrm{C} & 06 & 10 & 02 & 51 & 01 & 82 & 29 & 22 & 28 & \text { E9 } & 01 & 72\end{array}$ $\begin{array}{llllllllllllllll}29 & 24 & 04 & 7 \mathrm{D} & 02 & \mathrm{C} 0 & 86 & 52 & 53 & 54 & 42 & 52 & \mathrm{CB} & 29 & 05 & 08\end{array}$ $\begin{array}{llllllllllllllll}57 & 28 & 36 & 01 & 49 & 00 & 08 & 02 & 51 & 01 & 82 & 29 & 43 & 06 & 18 & 08\end{array}$ $\begin{array}{llllllllllllllll}3 \mathrm{D} & 04 & 7 \mathrm{D} & 02 & \mathrm{C} 0 & 87 & 53 & 45 & 4 \mathrm{E} & 44 & 42 & 52 & \mathrm{CB} & 29 & 26 & 08\end{array}$ $\begin{array}{llllllllllllllll}57 & 06 & 18 & 08 & 3 \mathrm{D} & 04 & 7 \mathrm{D} & 06 & 08 & 28 & 67 & 01 & 49 & 00 & 5 \mathrm{D} & 28\end{array}$ $\begin{array}{lllllllllllllllll}46 & 28 & 36 & 29 & 10 & 28 & 78 & 21 & 22 & 01 & 82 & 29 & 7 \mathrm{~B} & 28 & 67 & 28\end{array}$ $\begin{array}{llllllllllllllll}\mathrm{B} 1 & 06 & 08 & 06 & 18 & 08 & 19 & 04 & 7 \mathrm{D} & 06 & 10 & 01 & 82 & 29 & 57 & 02\end{array}$ $\begin{array}{llllllllllllllll}\text { C0 } & 85 & 45 & 4 \mathrm{D} & 49 & 54 & \mathrm{~A} 7 & 29 & 45 & 29 & 8 \mathrm{~B} & \mathrm{E} 4 & 60 & 62 & \mathrm{D} 5 & 83\end{array}$ $\begin{array}{llllllllllllllll}54 & 52 & \mathrm{C} 3 & 29 & 81 & 08 & 57 & 08 & \mathrm{CF} & 04 & 9 \mathrm{C} & 1 \mathrm{C} & \mathrm{CD} & 03 & 73 & 01\end{array}$ $\begin{array}{llllllllllllllll}82 & 29 & \mathrm{AD} & 04 & 7 \mathrm{D} & 04 & 7 \mathrm{D} & 06 & 10 & 01 & 72 & 29 & \mathrm{BI} & 28 & 67 & 08\end{array}$ $\begin{array}{llllllllllllllll}\text { FC } & 02 & \text { C0 } & 85 & 53 & 45 & 4 \mathrm{E} & 44 & \mathrm{C} 3 & 29 & 8 \mathrm{~F} & 08 & 57 & 01 & 49 & 00\end{array}$ $\begin{array}{llllllllllllllll}08 & 02 & 51 & 01 & 82 & 29 & \mathrm{CD} & 29 & 4 \mathrm{~F} & 01 & 72 & 29 & \mathrm{CF} & 29 & 95 & 02\end{array}$ $\begin{array}{llllllllllllllll}\mathrm{C} 0 & 89 & 53 & 45 & 4 \mathrm{E} & 44 & 43 & 48 & 41 & 52 & \mathrm{BF} & 29 & \mathrm{~B} 3 & 08 & 57 & 04\end{array}$ $\begin{array}{llllllllllllllll}9 \mathrm{C} & 06 & 10 & 02 & 51 & 01 & 82 & 29 & \mathrm{EF} & 29 & \mathrm{BB} & 01 & 72 & 29 & \mathrm{Fl} & 04\end{array}$ $\begin{array}{llllllllllllllll}7 \mathrm{D} & 02 & \mathrm{C} 0 & 84 & 55 & 41 & 52 & \mathrm{D} 4 & 29 & \mathrm{DI} & 08 & 57 & \text { OB } & 41 & \text { OE } & 55\end{array}$
\&, . A.S.ERASE\&E.W $\& Y . I \ldots . . .[. I . \ldots$ . $\mid \& I \ldots \& . \& Y \& Z . Q . E$ DIT\&m.W"W요. =. :\& Yoz,...O.I...g.s. $. \&[\}. \& . . r^{\prime} W \cdot M \cdot g \bullet$ S..\&\}.\}...I...8.8 C......... $r^{\prime} W . I$. ..g.s..... . I. Qd \& ,.r'W.I..g.s..' -.\}.I..\&,..r'W.I. ..g.S..'E.\}.I..\& ,.. Y'W.I...g.S..' ].\}..\&; $8 Z . r^{\prime} W . I$. ..g.s..'u.\}.I. $a \&$ ,.r'W.I...g.s..' ..\}..\&, . r'W.I... g.s..'7.\}\%s.?.I. ..O.I...g.I...D斿 . $8 \mathrm{z}$. $x^{\prime} W \ldots I . .$. .'M.I...l.r'U... $\ldots \mid \& 1.\} \ldots \& C$. Q.ED ITL\&..W...?\&5.@. CLEAR' ].W...'.I. ..... [.F\#\$.. (... . COMM.CTL'O. ...T ERM.CTL ( . . . . ST $\operatorname{ATUS}(\ldots \mathrm{W}, .=$. a.C $T L=>(-. W . \quad \ldots$.. M $\operatorname{ODEM}(>\cdot$ W.b.?.<. . $\operatorname{COMM}(N . W . . . ? .<$. Q.TERM( .W. $\mathrm{q} \cdot ? .<$ . a. CONS ( $q \cdot w . .<<$. a.ESC' ( ..W (g.I.. - I x. a.commINIT) ..W( ..? (F. A.RCVB $\mathrm{RK}$ (\&.W. ... =. ) ( X.A - Break received . a.RCVC ( ; .W.I... Q.. $(\{(D, r) \ldots .=$ ( $\mathrm{x}$. . . . RCVCHAR? (b .W.........)"(i.r )\$.\}. (..RSTBRK).. $W(6 . I \ldots Q \ldots) C \ldots$ $=$. $\}$. . SENDBRK ) \& 。 W...... $\}. .\left(g . I_{0}\right]$ ( $F(6) \cdot(x ! " \ldots)\{(g($ 1....... ....)W. (..EMIT')E). d'bU. TRC) ..W.O...M.S. .) -. . . . . r r) I ( $g$. I. ..SENDC) ..W.I. ..Q..)MOO.r)O).. (a.SENDCHAR? ) $3 . W$. ....Q..)ol; .rlq. \}.@.UART) Q.W.A.U 
$\begin{array}{lllllllllllllllll}02 \mathrm{~A} 00 & 61 & 72 & 74 & 20 & 61 & 74 & 20 & 67 & 72 & 6 \mathrm{~F} & 75 & 70 & 20 & 07 & 59 & 04\end{array}$ $\begin{array}{lllllllllllllllll}02 \mathrm{~A} 10 & \mathrm{BF} & 0 \mathrm{C} & \mathrm{DC} & 08 & 00 & 04 & \mathrm{BF} & 15 & 8 \mathrm{E} & 09 & 09 & 07 & 59 & 04 & \mathrm{E} 0 & 28\end{array}$ $\begin{array}{lllllllllllllllll}02 \mathrm{~A} 20 & 67 & 28 & \mathrm{BI} & 29 & 2 \mathrm{~F} & 06 & 08 & 28 & 67 & 28 & 36 & 29 & 10 & 28 & 78 & 28\end{array}$ $\begin{array}{lllllllllllllllll}02 \mathrm{~A} 30 & 36 & 29 & \mathrm{DD} & 01 & 82 & 2 \mathrm{~A} & 25 & 09 & 09 & 02 & \mathrm{C} 0 & 81 & \mathrm{D} 5 & 29 & \mathrm{~F} 3 & 08\end{array}$ $\begin{array}{lllllllllllllllll}02 \mathrm{~A} 40 & 57 & 29 & \mathrm{FA} & 02 & \mathrm{CO} & 86 & 4 \mathrm{~F} & 4 \mathrm{E} & 48 & 4 \mathrm{~F} & 4 \mathrm{~F} & \mathrm{CB} & 2 \mathrm{~A} & 3 \mathrm{~B} & 08 & 57\end{array}$ $\begin{array}{lllllllllllllllll}02 \mathrm{~A} 50 & 28 & 56 & 06 & 08 & 01 & 49 & 00 & 07 & 08 & 19 & 28 & 78 & 02 & \mathrm{C} 0 & 87 & 4 \mathrm{~F}\end{array}$ $\begin{array}{lllllllllllllllll}02 \mathrm{~A} 60 & 46 & 46 & 48 & 4 \mathrm{~F} & 4 \mathrm{~F} & \mathrm{CB} & 2 \mathrm{~A} & 45 & 08 & 57 & 28 & 56 & 06 & 10 & 01 & 49\end{array}$ $\begin{array}{llllllllllllllllll}02 \mathrm{~A} 70 & 00 & 07 & 08 & 19 & 28 & 78 & 02 & \mathrm{C} 0 & 88 & 44 & 49 & 41 & 4 \mathrm{C} & 49 & 4 \mathrm{E} & 49\end{array}$

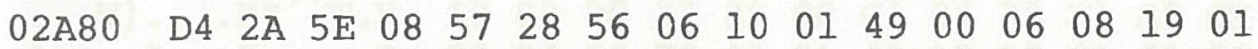
$\begin{array}{lllllllllllllllll}02 \mathrm{~A} 90 & 49 & 00 & 23 & 01 & 49 & 00 & 07 & 08 & 19 & 28 & 78 & 02 & \mathrm{C} 0 & 88 & 44 & 49\end{array}$ $\begin{array}{lllllllllllllllll}02 \mathrm{AAO} & 41 & 4 \mathrm{C} & 54 & 4 \mathrm{~F} & 4 \mathrm{E} & \mathrm{C} 5 & 2 \mathrm{~A} & 78 & 08 & 57 & 2 \mathrm{~A} & 83 & 06 & 10 & \text { ID } & 00\end{array}$ $\begin{array}{lllllllllllllllll}02 \mathrm{ABO} & 28 & 56 & 01 & 49 & 00 & 0 \mathrm{~A} & 01 & 49 & 00 & 06 & 08 & 19 & 06 & 10 & 1 \mathrm{D} & 00\end{array}$ $\begin{array}{llllllllllllllllll}02 \mathrm{AC} 0 & 06 & 08 & 01 & 49 & 04 & 00 & 06 & 08 & 01 & 5 \mathrm{~B} & 01 & 49 & 00 & 05 & 08 & 3 \mathrm{D}\end{array}$ $\begin{array}{lllllllllllllllll}02 A D 0 & 06 & 10 & 02 & 51 & 01 & 82 & 2 A & D C & 01 & 72 & 2 A & E A & 06 & 10 & 01 & 49\end{array}$ $\begin{array}{lllllllllllllllll}02 \mathrm{AE} 0 & 00 & 06 & 08 & 19 & 04 & 7 \mathrm{D} & 06 & 10 & 03 & 1 \mathrm{~B} & 00 & \mathrm{AE} & 2 \mathrm{~A} & \mathrm{CA} & 01 & 82\end{array}$ $\begin{array}{lllllllllllllllll}02 \mathrm{AFO} & 2 \mathrm{~A} & \mathrm{~F} 6 & 01 & 72 & 2 \mathrm{~B} & 0 \mathrm{~B} & 2 \mathrm{~A} & 4 \mathrm{E} & 0 \mathrm{~B} & 41 & 0 \mathrm{C} & 4 \mathrm{E} & 6 \mathrm{~F} & 20 & 64 & 69\end{array}$ $\begin{array}{lllllllllllllllll}02 \mathrm{~B} 00 & 61 & 6 \mathrm{C} & 20 & 74 & 6 \mathrm{~F} & 6 \mathrm{E} & 65 & 09 & 09 & 11 & \mathrm{BA} & 28 & 78 & 02 & \mathrm{C} 0 & 88\end{array}$ $\begin{array}{lllllllllllllllll}02 \mathrm{~B} 10 & 3 \mathrm{~F} & 43 & 41 & 52 & 52 & 49 & 45 & \mathrm{D} 2 & 2 \mathrm{~A} & 9 \mathrm{D} & 08 & 57 & 06 & 08 & 01 & 49\end{array}$ $\begin{array}{lllllllllllllllll}02 \mathrm{~B} 20 & 01 & 00 & 06 & 08 & 01 & 5 \mathrm{~B} & 28 & 56 & 01 & 49 & 00 & 05 & 08 & 3 \mathrm{D} & 06 & 18\end{array}$ $\begin{array}{lllllllllllllllll}02 \mathrm{~B} 30 & 02 & 51 & 01 & 82 & 2 \mathrm{~B} & 3 \mathrm{E} & 04 & 7 \mathrm{D} & 06 & 10 & 28 & 78 & 03 & 1 \mathrm{~B} & 01 & 49\end{array}$ $\begin{array}{lllllllllllllllll}02 \mathrm{~B} 40 & 00 & 50 & 1 \mathrm{D} & 25 & 28 & 78 & 08 & \mathrm{EA} & 01 & 82 & 2 \mathrm{~B} & 4 \mathrm{E} & 03 & 1 \mathrm{~B} & 00 & \mathrm{AE}\end{array}$ $\begin{array}{lllllllllllllllll}02 \mathrm{~B} 50 & 2 \mathrm{~B} & 26 & 02 & \mathrm{C} 0 & 82 & 54 & \mathrm{D} 3 & 2 \mathrm{~B} & 0 \mathrm{~F} & 08 & 57 & 28 & 56 & 01 & 49 & 00\end{array}$ $\begin{array}{lllllllllllllllll}02 \mathrm{~B} 60 & 23 & 01 & 49 & 00 & 07 & 08 & 19 & 01 & 49 & 00 & \mathrm{FF} & 01 & 49 & 00 & 04 & 08\end{array}$ $\begin{array}{llllllllllllllllll}02 \mathrm{~B} 70 & 19 & 06 & 10 & 01 & 49 & 00 & 06 & 08 & 19 & 01 & 49 & 00 & 04 & 08 & 19 & 01\end{array}$ $\begin{array}{lllllllllllllllll}02 \mathrm{~B} 80 & 49 & 00 & 40 & 1 \mathrm{D} & 25 & 01 & 49 & 00 & \mathrm{FF} & 01 & 49 & 00 & 04 & 08 & 19 & 06\end{array}$ $\begin{array}{lllllllllllllllll}02 B 90 & 20 & 01 & 49 & 00 & 07 & 08 & 19 & 01 & 49 & 00 & 40 & 1 D & 25 & 28 & 78 & 02\end{array}$ $\begin{array}{lllllllllllllllll}02 \mathrm{BA} 0 & \mathrm{C} 0 & 84 & 54 & 4 \mathrm{~F} & 4 \mathrm{E} & \mathrm{C} 5 & 2 \mathrm{~B} & 54 & 08 & 57 & 01 & 49 & 00 & 2 \mathrm{~A} & 04 & 67\end{array}$ $\begin{array}{lllllllllllllllll}02 \mathrm{BB} 0 & 03 & 73 & 01 & 82 & 2 \mathrm{~B} & \mathrm{C} 2 & 04 & 7 \mathrm{D} & 01 & 49 & 00 & \mathrm{E} 7 & 2 \mathrm{~B} & 59 & 01 & 72\end{array}$ $\begin{array}{lllllllllllllllll}02 \mathrm{BC} 0 & 2 \mathrm{C} & \mathrm{F} 8 & 01 & 49 & 00 & 23 & 04 & 67 & 03 & 73 & 01 & 82 & 2 \mathrm{~B} & \mathrm{DA} & 04 & 7 \mathrm{D}\end{array}$ $\begin{array}{lllllllllllllllll}02 \mathrm{BD} 0 & 01 & 49 & 00 & \mathrm{~B} 7 & 2 \mathrm{~B} & 59 & 01 & 72 & 2 \mathrm{C} & \mathrm{F} 8 & 01 & 49 & 00 & 30 & 04 & 67\end{array}$ $\begin{array}{lllllllllllllllll}02 \mathrm{BE} 0 & 03 & 73 & 01 & 82 & 2 \mathrm{~B} & \mathrm{~F} 2 & 04 & 7 \mathrm{D} & 01 & 49 & 00 & \mathrm{D} 7 & 2 \mathrm{~B} & 59 & 01 & 72\end{array}$ $\begin{array}{lllllllllllllllll}02 \mathrm{BFO} & \text { 2C } & \mathrm{F} 8 & 01 & 49 & 00 & 31 & 04 & 67 & 03 & 73 & 01 & 82 & 2 \mathrm{C} & 0 \mathrm{~A} & 04 & 7 \mathrm{D}\end{array}$ $\begin{array}{lllllllllllllllll}02 \mathrm{C} 00 & 01 & 49 & 00 & \mathrm{EE} & 2 \mathrm{~B} & 59 & 01 & 72 & 2 \mathrm{C} & \mathrm{F} 8 & 01 & 49 & 00 & 32 & 04 & 67\end{array}$ $\begin{array}{lllllllllllllllll}02 \mathrm{C} 10 & 03 & 73 & 01 & 82 & 2 \mathrm{C} & 22 & 04 & 7 \mathrm{D} & 01 & 49 & 00 & \mathrm{DE} & 2 \mathrm{~B} & 59 & 01 & 72\end{array}$ $\begin{array}{lllllllllllllllll}02 \mathrm{C} 20 & 2 \mathrm{C} & \mathrm{F} 8 & 01 & 49 & 00 & 33 & 04 & 67 & 03 & 73 & 01 & 82 & 2 \mathrm{C} & 3 \mathrm{~A} & 04 & 7 \mathrm{D}\end{array}$ $\begin{array}{lllllllllllllllll}02 \mathrm{C} 30 & 01 & 49 & 00 & \mathrm{BE} & 2 \mathrm{~B} & 59 & 01 & 72 & 2 \mathrm{C} & \mathrm{F} 8 & 01 & 49 & 00 & 34 & 04 & 67\end{array}$ $\begin{array}{lllllllllllllllll}02 \mathrm{C} 40 & 03 & 73 & 01 & 82 & 2 \mathrm{C} & 52 & 04 & 7 \mathrm{D} & 01 & 49 & 00 & \mathrm{ED} & 2 \mathrm{~B} & 59 & 01 & 72\end{array}$ $\begin{array}{lllllllllllllllll}02 \mathrm{C} 50 & 2 \mathrm{C} & \mathrm{F} 8 & 01 & 49 & 00 & 35 & 04 & 67 & 03 & 73 & 01 & 82 & 2 \mathrm{C} & 6 \mathrm{~A} & 04 & 7 \mathrm{D}\end{array}$ $\begin{array}{lllllllllllllllll}02 \mathrm{C} 60 & 01 & 49 & 00 & \mathrm{DD} & 2 \mathrm{~B} & 59 & 01 & 72 & 2 \mathrm{C} & \mathrm{F} 8 & 01 & 49 & 00 & 36 & 04 & 67\end{array}$ $\begin{array}{lllllllllllllllll}02 \mathrm{C} 70 & 03 & 73 & 01 & 82 & 2 \mathrm{C} & 82 & 04 & 7 \mathrm{D} & 01 & 49 & 00 & \mathrm{BD} & 2 \mathrm{~B} & 59 & 01 & 72\end{array}$ $\begin{array}{lllllllllllllllll}02 \mathrm{C} 80 & 2 \mathrm{C} & \mathrm{F} 8 & 01 & 49 & 00 & 37 & 04 & 67 & 03 & 73 & 01 & 82 & 2 \mathrm{C} & 9 \mathrm{~A} & 04 & 7 \mathrm{D}\end{array}$ $\begin{array}{lllllllllllllllll}02 \mathrm{C} 90 & 01 & 49 & 00 & \mathrm{~EB} & 2 \mathrm{~B} & 59 & 01 & 72 & 2 \mathrm{C} & \mathrm{F} 8 & 01 & 49 & 00 & 38 & 04 & 67\end{array}$ $\begin{array}{lllllllllllllllll}02 \mathrm{CA} 0 & 03 & 73 & 01 & 82 & 2 \mathrm{C} & \mathrm{B} 2 & 04 & 7 \mathrm{D} & 01 & 49 & 00 & \mathrm{DB} & 2 \mathrm{~B} & 59 & 01 & 72\end{array}$ $\begin{array}{lllllllllllllllll}02 \mathrm{CB} 0 & 2 \mathrm{C} & \mathrm{F} 8 & 01 & 49 & 00 & 39 & 04 & 67 & 03 & 73 & 01 & 82 & 2 \mathrm{C} & \mathrm{CA} & 04 & 7 \mathrm{D}\end{array}$ $\begin{array}{lllllllllllllllll}02 \mathrm{CC} 0 & 01 & 49 & 00 & \mathrm{BB} & 2 \mathrm{~B} & 59 & 01 & 72 & 2 \mathrm{C} & \mathrm{F} 8 & 01 & 49 & 00 & 2 \mathrm{C} & 04 & 67\end{array}$ $\begin{array}{lllllllllllllllll}02 \mathrm{CD} 0 & 03 & 73 & 01 & 82 & 2 \mathrm{C} & \mathrm{E} 2 & 04 & 7 \mathrm{D} & 01 & 49 & 02 & 50 & \text { ID } & 25 & 01 & 72\end{array}$ $\begin{array}{lllllllllllllllll}02 \mathrm{CE0} & 2 \mathrm{C} & \mathrm{F} 8 & 01 & 49 & 00 & 3 \mathrm{~B} & 04 & 67 & 03 & 73 & 01 & 82 & 2 \mathrm{C} & \mathrm{F} 6 & 04 & 7 \mathrm{D}\end{array}$ $\begin{array}{lllllllllllllllll}02 \mathrm{CFO} & 2 \mathrm{~A} & \mathrm{~A} 8 & 01 & 72 & 2 \mathrm{C} & \mathrm{F} 8 & 04 & 7 \mathrm{D} & 02 & \mathrm{C} 0 & 88 & 52 & 45 & 2 \mathrm{D} & 45 & 4 \mathrm{E}\end{array}$ $\begin{array}{lllllllllllllllll}02 \mathrm{D} 00 & 54 & 45 & \mathrm{D} 2 & 2 \mathrm{~B} & \mathrm{~A} 1 & 08 & 57 & 07 & \mathrm{E} 2 & 04 & \mathrm{BF} & 08 & 00 & 04 & \mathrm{E} 0 & 29\end{array}$ $\begin{array}{lllllllllllllllll}02 \mathrm{D} 10 & \mathrm{FA} & 0 \mathrm{~B} & 41 & 0 \mathrm{~B} & 52 & 45 & 2 \mathrm{D} & 45 & 4 \mathrm{E} & 54 & 45 & 52 & 20 & 3 \mathrm{~F} & 20 & 1 \mathrm{E}\end{array}$ $\begin{array}{lllllllllllllllll}02 \mathrm{D} 20 & \mathrm{~A} 4 & 01 & 82 & 2 \mathrm{D} & 48 & 0 \mathrm{~B} & 41 & 1 \mathrm{~A} & 20 & 54 & 79 & 70 & 65 & 20 & 52 & 45\end{array}$ $\begin{array}{lllllllllllllllll}02 \mathrm{D} 30 & 2 \mathrm{D} & 45 & 4 \mathrm{E} & 54 & 45 & 52 & 20 & 20 & 77 & 68 & 65 & 6 \mathrm{E} & 20 & 72 & 65 & 61\end{array}$ $\begin{array}{lllllllllllllllll}02 \mathrm{D} 40 & 64 & 79 & 09 & 09 & 01 & 72 & 2 \mathrm{D} & 52 & 2 \mathrm{~A} & 4 \mathrm{E} & 01 & 49 & 00 & \mathrm{E} 0 & 08 & 00\end{array}$ $\begin{array}{lllllllllllllllll}02 \mathrm{D} 50 & 04 & \mathrm{E} 0 & 02 & \mathrm{C} 0 & 84 & 44 & 49 & 41 & \mathrm{CC} & 2 \mathrm{C} & \mathrm{FA} & 08 & 57 & 2 \mathrm{~A} & 4 \mathrm{E} & 06\end{array}$ $\begin{array}{lllllllllllllllll}02 D 60 & 10 & 1 D & 00 & 2 A & A 8 & 01 & 49 & 00 & 20 & \text { OD } & F A & 0 A & 53 & 04 & \text { DI } & 03\end{array}$ $\begin{array}{llllllllllllllllllllllllll}02 D 70 & D 5 & 06 & 10 & 01 & 5 B & 0 A & 53 & 03 & 46 & 03 & C 4 & 04 & D 1 & 2 B & A 8 & 00\end{array}$ art at group .Y. ?.1...?...... Y.' ( $g(1) / \ldots(g(6) .(x)$ 6) ] ..*\% ...(a.U) s. W) $z$. a. ONHOOK*; $\cdot W$ (V......... (x. Q. O FFHOOK*E.W $(\mathrm{V}$...I -.... $x \cdot$. DIALINI $T^{* \wedge} \cdot W(V . \ldots I \ldots \ldots$ I.\#.I.... (x.Q.DI ALTONE* $\mathrm{X} \cdot \mathrm{W}^{*} \ldots \ldots$ (V.I........... $\ldots I \ldots \ldots\left[. I_{\ldots} \ldots=\right.$ $\ldots . . . * \backslash . r * j \ldots I$ $\ldots . ..\} \ldots *$ J... *V.r+.*N.A. No di al tone....: (x. $a_{\text {. }}$ ?CARRIER* ..W...I $\ldots . .[$ (V.I. . . $=$. $\left.. Q .+\rangle_{.}\right\} \ldots$ (x...I . P. 8 (x. j... +N... $+\&$. Q.TSt...W V.I. \#.I.....I...I... ....I.......... I. $. .8 . I \ldots I \ldots \ldots$ . I........ Q. 8 (x. a. TONE+T.W.I.*.g . S... B. \}.I.g+Y.r , X.I.\#.g.s..+Z.\} .I.7+Y.r, X.I.0.g .S... + . $\} . I . W+Y . r$ $, \mathrm{x} \cdot I \cdot \mathrm{l} \cdot \mathrm{g} \cdot \mathrm{S} \ldots \ldots\}$ . I. $n+Y \cdot r, x \cdot I \cdot 2 \cdot g$ .S...".\}.I.^+Y.r , X.I.3.g.S...:..\} .I.>+Y.r, X.I.4.g .S..,R.\}.I.m+Y.r , x.I.5.g.s...j.\} .I. ]+Y.I, X.I.6.g $\left.. S_{0}, \ldots\right\} \cdot I_{0}=+Y \cdot r$ , X.I.7.g.S..... - I.k+Y.r, x.I.8.g .S...,2.].I. [+Y.r , X.I.9.g.S..,J.\} .I. $;+Y . r, X . I ., . g$ .s..,b.\}.I.P. \&. , x.I.i.g.s.., v. * $\left(. r, x_{0}\right\},(\mathrm{C} \cdot \mathrm{RE}-\mathrm{EN}$ TER+!.W.b.?...') Z.A.RE-ENTER ? \$..-H.A. Type RE -ENTER when rea dy...r $-R^{*} N . I$. .. . Q.DIAL, $z \cdot W * N$. ...* (.I. .Z.S.Q. U... [.S.F.D.Q+ . 
$\begin{array}{lllllllllllllllll}02 \mathrm{D} 80 & \mathrm{AE} & 2 \mathrm{D} & 75 & 02 & \mathrm{C} 0 & 89 & 4 \mathrm{~F} & 52 & 49 & 47 & 49 & 4 \mathrm{E} & 41 & 54 & \mathrm{C} 5 & 2 \mathrm{D}\end{array}$ $\begin{array}{lllllllllllllllll}02 \mathrm{D} 90 & 54 & 08 & 57 & 28 & 56 & 01 & 49 & 00 & 7 \mathrm{~F} & 01 & 49 & 00 & 04 & 08 & 19 & 06\end{array}$ 02DA0 $10 \begin{array}{llllllllllllllll}10 & 01 & 49 & 00 & 06 & 08 & 19 & 06 & 10 & 01 & 49 & 00 & 07 & 08 & 19 & 01\end{array}$ $\begin{array}{lllllllllllllllll}02 D B 0 & 49 & 00 & 05 & 1 D & 00 & 2 B & 1 A & 28 & 78 & 01 & 82 & 2 D & C 1 & 01 & 72 & 2 D\end{array}$ $\begin{array}{lllllllllllllllll}02 D C 0 & D 2 & 2 A & 4 E & 0 B & 41 & 0 A & 4 E & 6 F & 20 & 63 & 61 & 72 & 72 & 69 & 65 & 72\end{array}$

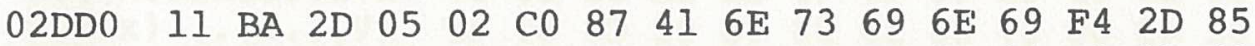
$\begin{array}{lllllllllllllllll}02 \mathrm{DE} 0 & 08 & 57 & 01 & 49 & 2 \mathrm{E} & 03 & 04 & 9 \mathrm{C} & 01 & 49 & 0 \mathrm{~F} & 1 \mathrm{C} & 04 & \mathrm{E} 0 & 01 & 49\end{array}$ $\begin{array}{lllllllllllllllll}02 \mathrm{DF} 0 & 00 & 0 \mathrm{~A} & 06 & 88 & 04 & \mathrm{E} 0 & 02 & \mathrm{C} 0 & 86 & 41 & 4 \mathrm{E} & 53 & 57 & 45 & \mathrm{D} 2 & 2 \mathrm{D}\end{array}$ $\begin{array}{lllllllllllllllll}02 \mathrm{E} 00 & \mathrm{D} 6 & 08 & 57 & 2 \mathrm{D} & \mathrm{E} 0 & 2 \mathrm{~A} & 4 \mathrm{E} & 06 & 10 & 1 \mathrm{D} & 00 & 28 & 56 & 06 & 08 & 01\end{array}$ $\begin{array}{llllllllllllllllll}02 \mathrm{E} 10 & 49 & 00 & 06 & 08 & 19 & 01 & 49 & 00 & 94 & 01 & 49 & 00 & 07 & 08 & 19 & 28\end{array}$ $\begin{array}{lllllllllllllllll}02 \mathrm{E} 20 & \mathrm{~B} 1 & 07 & \mathrm{E} 2 & 04 & \mathrm{BF} & 07 & \mathrm{~F} 1 & 04 & \mathrm{E} 0 & 28 & 78 & 11 & \mathrm{BA} & 02 & \mathrm{C} 0 & 8 \mathrm{~B}\end{array}$ $\begin{array}{lllllllllllllllll}02 E 30 & 53 & 45 & 4 E & 44 & 2 D & 53 & 43 & 52 & 45 & 45 & C E & 2 D & F 8 & 08 & 57 & 04\end{array}$

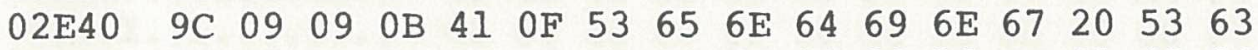
$\begin{array}{lllllllllllllllll}02 \mathrm{E} 50 & 72 & 65 & 65 & 6 \mathrm{E} & 20 & 15 & 8 \mathrm{E} & 28 & 67 & 09 & 09 & 16 & \mathrm{DE} & 01 & 49 & 04\end{array}$ $\begin{array}{lllllllllllllllll}02 \mathrm{E} 60 & 00 & 09 & 33 & 28 & 78 & 02 & \mathrm{C} 0 & 8 \mathrm{~A} & 47 & 45 & 54 & 2 \mathrm{D} & 53 & 43 & 52 & 45\end{array}$ $\begin{array}{lllllllllllllllll}02 \mathrm{E} 70 & 45 & \mathrm{CE} & 2 \mathrm{E} & 2 \mathrm{~F} & 08 & 57 & \mathrm{OB} & 41 & 0 \mathrm{~F} & 47 & 65 & 74 & 74 & 69 & 6 \mathrm{E} & 67\end{array}$ $\begin{array}{lllllllllllllllll}02 \mathrm{E} 80 & 20 & 53 & 63 & 72 & 65 & 65 & 6 \mathrm{E} & 20 & 04 & 9 \mathrm{C} & 04 & 9 \mathrm{C} & 15 & 8 \mathrm{E} & 09 & 09\end{array}$ $\begin{array}{lllllllllllllllll}02 \mathrm{E} 90 & 28 & 67 & 16 & \mathrm{DE} & 01 & 49 & 04 & 00 & 06 & 08 & 01 & 5 \mathrm{~B} & 04 & 9 \mathrm{C} & 03 & 46\end{array}$ 02EA0 03 C4 $28 \begin{array}{llllllllllllll} & 67 & 08 & \mathrm{CF} & 28 & 78 & 08 & \mathrm{EA} & 01 & 82 & 2 \mathrm{E} & \mathrm{B} 0 & 03 & 1 \mathrm{~B}\end{array}$ $\begin{array}{lllllllllllllllll}02 \mathrm{~EB} 0 & 04 & 89 & 04 & \mathrm{~F} 0 & 00 & \mathrm{AE} & 2 \mathrm{E} & 9 \mathrm{C} & 04 & 7 \mathrm{D} & 16 & 59 & 19 & 06 & 28 & 78\end{array}$ $\begin{array}{lllllllllllllllll}02 \mathrm{EC} 0 & 18 & \mathrm{BA} & 02 & \mathrm{C} 0 & 84 & 32 & 2 \mathrm{~A} & 2 \mathrm{~A} & \mathrm{CE} & 2 \mathrm{E} & 67 & 08 & 57 & 04 & 9 \mathrm{C} & 03\end{array}$ $\begin{array}{lllllllllllllllll}02 \mathrm{ED} 0 & 52 & 01 & 82 & 2 \mathrm{E} & \mathrm{DB} & 06 & 10 & 01 & 72 & 2 \mathrm{E} & \mathrm{EB} & 06 & 10 & 04 & 89 & 06\end{array}$ $\begin{array}{lllllllllllllllll}02 \mathrm{EE} 0 & 08 & 01 & 5 \mathrm{~B} & 06 & 18 & 05 & 67 & 00 & \mathrm{AE} & 2 \mathrm{E} & \mathrm{E} 3 & 02 & \mathrm{C} 0 & 82 & 43 & \mathrm{D} 3\end{array}$ $\begin{array}{lllllllllllllllll}02 \mathrm{EF} 0 & 2 \mathrm{E} & \mathrm{C} 4 & 08 & 57 & 28 & \mathrm{Bl} & 2 \mathrm{E} & \mathrm{CB} & 01 & 49 & 00 & 06 & 08 & 19 & 02 & \mathrm{C} 0\end{array}$ $\begin{array}{lllllllllllllllll}02 \mathrm{~F} 00 & 83 & 43 & 52 & \mathrm{D} 3 & 2 \mathrm{E} & \mathrm{ED} & 08 & 57 & 2 \mathrm{E} & \mathrm{CB} & 01 & 49 & 00 & 07 & 08 & 19\end{array}$ $\begin{array}{lllllllllllllllll}02 \mathrm{~F} 10 & 01 & 49 & 00 & 10 & 1 \mathrm{D} & 25 & 06 & 08 & 01 & 49 & 00 & 07 & 08 & 19 & 02 & \mathrm{C} 0\end{array}$ $\begin{array}{lllllllllllllllll}02 \mathrm{~F} 20 & 8 \mathrm{E} & 43 & 48 & 41 & 4 \mathrm{E} & 4 \mathrm{E} & 45 & 4 \mathrm{C} & 2 \mathrm{D} & 53 & 45 & 4 \mathrm{C} & 45 & 43 & \mathrm{D} 4 & 2 \mathrm{~F}\end{array}$ $\begin{array}{llllllllllllllllll}02 \mathrm{~F} 30 & 00 & 08 & 57 & 28 & 67 & 2 \mathrm{E} & \mathrm{F} 2 & 28 & 78 & 02 & \mathrm{C} 0 & 8 \mathrm{D} & 43 & 48 & 41 & 4 \mathrm{E}\end{array}$ $\begin{array}{lllllllllllllllll}02 \mathrm{~F} 40 & 4 \mathrm{E} & 45 & 4 \mathrm{C} & 2 \mathrm{D} & 52 & 45 & 53 & 45 & \mathrm{D} 4 & 2 \mathrm{~F} & 20 & 08 & 57 & 28 & 67 & 2 \mathrm{~F}\end{array}$ $\begin{array}{llllllllllllllllll}02 \mathrm{~F} 50 & 06 & 28 & 78 & 02 & \mathrm{C} 0 & 84 & 3 \mathrm{~F} & 4 \mathrm{~B} & 45 & \mathrm{D} 9 & 2 \mathrm{~F} & 3 \mathrm{~B} & 08 & 57 & 28 & 36\end{array}$ $\begin{array}{lllllllllllllllll}02 \mathrm{~F} 60 & 06 & 10 & 02 & 51 & 02 & \mathrm{C} 0 & 88 & 4 \mathrm{D} & 4 \mathrm{~F} & 4 \mathrm{E} & 41 & 42 & 4 \mathrm{~F} & 52 & \mathrm{D} 4 & 2 \mathrm{~F}\end{array}$ $\begin{array}{lllllllllllllllll}02 \mathrm{~F} 70 & 55 & 08 & 57 & 28 & 78 & 0 \mathrm{~B} & 41 & 0 \mathrm{~B} & 20 & 4 \mathrm{E} & 6 \mathrm{~F} & 20 & 6 \mathrm{D} & 6 \mathrm{~F} & 6 \mathrm{E} & 69\end{array}$ $\begin{array}{lllllllllllllllll}02 \mathrm{~F} 80 & 74 & 6 \mathrm{~F} & 72 & 11 & \mathrm{BA} & 02 & \mathrm{C} 0 & 88 & 3 \mathrm{~F} & 4 \mathrm{D} & 4 \mathrm{~F} & 4 \mathrm{E} & 49 & 54 & 4 \mathrm{~F} & \mathrm{D} 2\end{array}$ $\begin{array}{lllllllllllllllll}02 \mathrm{~F} 90 & 2 \mathrm{~F} & 66 & 08 & 57 & 0 \mathrm{~B} & 41 & 01 & 40 & 01 & 49 & 02 & 00 & 1 D & 25 & 2 \mathrm{~F} & 5 \mathrm{C}\end{array}$ $\begin{array}{lllllllllllllllll}02 \mathrm{FA0} & 01 & 82 & 2 \mathrm{~F} & \mathrm{BA} & 08 & \mathrm{CF} & 01 & 49 & 00 & 2 \mathrm{~A} & 03 & 73 & 01 & 82 & 2 \mathrm{~F} & \mathrm{~B} 4\end{array}$ $\begin{array}{lllllllllllllllll}02 \mathrm{FB} 0 & 01 & 72 & 2 \mathrm{~F} & \mathrm{~B} 6 & 2 \mathrm{~F} & 71 & 01 & 72 & 2 \mathrm{~F} & \mathrm{BC} & 2 \mathrm{~F} & 71 & 02 & \mathrm{C} 0 & 84 & 58\end{array}$ $\begin{array}{lllllllllllllllll}02 \mathrm{FC} 0 & 6 \mathrm{D} & 69 & \mathrm{~F} 4 & 2 \mathrm{~F} & 87 & 08 & 57 & 28 & \mathrm{Bl} & 2 \mathrm{~F} & 92 & 0 \mathrm{~B} & 41 & 01 & 49 & 15\end{array}$ $\begin{array}{lllllllllllllllll}02 \mathrm{FD} 0 & \mathrm{~A} 7 & 04 & 89 & 04 & 9 \mathrm{C} & 04 & \mathrm{FE} & 03 & \mathrm{C} 4 & 04 & 89 & 01 & 5 \mathrm{~B} & 03 & 46 & 04\end{array}$

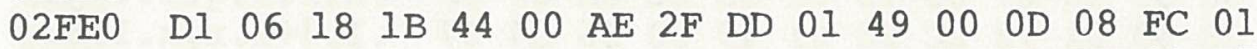
$\begin{array}{lllllllllllllllll}02 \mathrm{FFO} & 49 & 01 & 20 & 1 \mathrm{D} & 25 & 02 & \mathrm{C} 0 & 84 & 58 & 4 \mathrm{D} & 49 & \mathrm{D} 4 & 2 \mathrm{~F} & \mathrm{BE} & 08 & 57\end{array}$ $\begin{array}{lllllllllllllllll}03000 & 07 & 59 & 04 & \mathrm{BF} & 01 & 49 & 00 & 10 & 03 & 73 & 01 & 82 & 30 & 12 & 01 & 72\end{array}$ $\begin{array}{lllllllllllllllll}03010 & 30 & 22 & \text { OB } & 41 & \text { OB } & 44 & 65 & 63 & 6 \mathrm{C} & 61 & 72 & 65 & 20 & 48 & 45 & 58\end{array}$ $\begin{array}{lllllllllllllllll}03020 & 11 & \mathrm{BA} & 28 & 67 & 2 \mathrm{~F} & \mathrm{C} 5 & 28 & 78 & 02 & \mathrm{C} 0 & 83 & 43 & 52 & \mathrm{~A} 7 & 2 \mathrm{~F} & \mathrm{~F} 7\end{array}$ $\begin{array}{lllllllllllllllll}03030 & 08 & 57 & 01 & 49 & 00 & 0 D & 08 & F C & 02 & C 0 & 83 & 55 & 2 E & A E & 30 & 2 A\end{array}$ $\begin{array}{llllllllllllllllll}03040 & 08 & 57 & 06 & 08 & 06 & 08 & 15 & 4 \mathrm{C} & 02 & \mathrm{C} 0 & 88 & 3 \mathrm{~F} & 48 & 45 & 58 & 43\end{array}$ $\begin{array}{lllllllllllllllll}03050 & 48 & 41 & \text { D2 } & 30 & 3 A & 08 & 57 & 04 & 9 C & 01 & 49 & 00 & 30 & 03 & 90 & 04\end{array}$ $\begin{array}{lllllllllllllllll}03060 & 89 & 04 & 9 \mathrm{C} & 01 & 49 & 00 & 46 & 03 & \mathrm{AE} & 04 & \mathrm{FE} & 02 & 62 & 01 & 82 & 30\end{array}$ $\begin{array}{lllllllllllllllll}03070 & 79 & 04 & 7 \mathrm{D} & 06 & 08 & 01 & 72 & 30 & 8 \mathrm{D} & 04 & 9 \mathrm{C} & 01 & 49 & 00 & 39 & 03\end{array}$ $\begin{array}{lllllllllllllllll}03080 & \mathrm{AE} & 04 & 89 & 01 & 49 & 00 & 41 & 03 & 90 & 02 & 51 & 03 & 52 & 02 & \mathrm{C} 0 & 89\end{array}$ $\begin{array}{lllllllllllllllll}03090 & 43 & 4 \mathrm{C} & 45 & 41 & 52 & 2 \mathrm{D} & 4 \mathrm{~B} & 45 & \mathrm{D} 9 & 30 & 4 \mathrm{~A} & 08 & 57 & 2 \mathrm{~F} & 5 \mathrm{C} & 01\end{array}$ $\begin{array}{lllllllllllllllll}030 A 0 & 82 & 30 & A 7 & 08 & C F & 04 & 7 D & 02 & C 0 & 8 A & 53 & 45 & 52 & 50 & 52 & 49\end{array}$

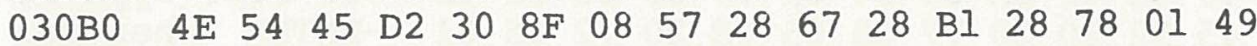
$\begin{array}{lllllllllllllllll}030 \mathrm{C} 0 & 19 & 55 & 01 & 49 & 19 & 75 & 01 & 49 & 00 & 12 & 03 & \mathrm{C} 4 & 04 & \text { E0 } & 19 & 73\end{array}$ $\begin{array}{lllllllllllllllll}030 D 0 & 02 & C 0 & 85 & 41 & 48 & 2 D & 3 E & C 2 & 30 & A 9 & 08 & 57 & 04 & 9 C & 01 & 49\end{array}$ $\begin{array}{lllllllllllllllll}030 \mathrm{E} 0 & 00 & 39 & 03 & \mathrm{AE} & 01 & 82 & 30 & \mathrm{~F} 2 & 01 & 49 & 00 & 37 & 04 & 05 & 01 & 72\end{array}$ $\begin{array}{lllllllllllllllll}030 \mathrm{F0} & 30 & \mathrm{~F} 8 & 01 & 49 & 00 & 30 & 04 & 05 & 02 & \text { C0 } & 81 & \text { F6 } & 30 & \text { D2 } & 08 & 57\end{array}$
- -u. a.ORIGINATE-

T.W $(V . I \ldots I \ldots \ldots$

..............

I...... (x..-A.r-

$R^{*} N . A$. No carrier .:-....Ansinit-.

.W.I.....I... . I .... ...ANSWER$\mathrm{V} . \mathrm{W}-{ }^{\prime}{ }^{\mathrm{N}} \ldots . . \mathrm{V}$ (V... I.....I...I.... ( l.b.?.q.' (x.:. a. SEND-SCREEN-X.W. ....A. Sending Sc reen ... (g...). . . ..3 (x. a.GET-SCRE EN./.W.A. Getting Screen ........ (g. . I..... [ ...F . $\mathrm{D}(\mathrm{g} . \mathrm{O}(\mathrm{x} . \mathrm{j} \ldots \mathrm{0} \ldots$

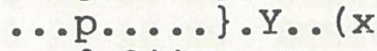
. . . . 2**N.g.W... R... [...r.k.... ... ...g...c. @. CS .D.W(I.K.I..... Q .CRS.M.W.K.I.... .I...8...I..... a - CHANNEL-SELECT/ ..W (g.r (x. a.CHAN NEL-RESET/ .W ( g/ -(x. a.?KEY/; .W (6 ...Q. a.MONABORT/ U.W(x.A. No moni tor.:.@.?MONITOR /f.W.A. Q.I... $8 /$ ../:.O.I.*.s../4 . $r / 6 / q \cdot r /</ q$. a.X $\mathrm{mit} / \ldots \mathrm{W}(\mathrm{l} / \ldots \mathrm{A} . \mathrm{I}$. ..... . D... [.F. Q...D../].I...I.

I. 왕. Q.XMIT/>.W .Y.?.I...s..0..r $0 "$. A. Declare HEX . : $\left(\mathrm{g} / \mathrm{E}\left(\mathrm{x}, \mathrm{e} \cdot \mathrm{CR}^{\prime} / \mathrm{w}\right.\right.$ .W.I... . . A.U.. O* .W....... @. ?HEXC HARO : .W...I. $0 \ldots$ ...I.F... . . . . 0 Y. $\ldots . . r 0 \ldots I .9$. ....I.A...Q.R. . CLEAR-KEYOJ.W/. $\left..0^{\prime} .0.\right\}$. Q.SERPRI NTERO ..W $/ g(1$ (x.I .U.I.U.I...D.'.S . (a. $\mathrm{AH}->\mathrm{BO}$ ) .W...I .9...0r.I.7...r Ox.I.0... Q.vOR.W 
$\begin{array}{lllllllllllllllll}03100 & 2 \mathrm{~F} & 92 & 0 \mathrm{~A} & 53 & 04 & 89 & 06 & \mathrm{E} 3 & 04 & \mathrm{E} 0 & 0 \mathrm{~B} & 41 & 01 & 44 & 04 & \mathrm{FE}\end{array}$ $\begin{array}{lllllllllllllllll}03110 & 15 & \mathrm{~A} 7 & 04 & 89 & 30 & 40 & 30 & 30 & 30 & 9 \mathrm{~B} & 08 & \mathrm{CF} & 01 & 49 & 00 & 20\end{array}$ $\begin{array}{lllllllllllllllll}03120 & 03 & 73 & 01 & 82 & 31 & 18 & 08 & \mathrm{CF} & 01 & 49 & 00 & 20 & 03 & 73 & 01 & 82\end{array}$ $\begin{array}{lllllllllllllllll}03130 & 31 & 36 & 01 & 72 & 31 & 3 \mathrm{E} & 06 & \mathrm{E} 3 & 04 & \mathrm{E} 0 & 28 & 78 & 11 & \mathrm{E} 6 & 08 & \mathrm{CF}\end{array}$ $\begin{array}{lllllllllllllllll}03140 & 04 & 9 \mathrm{C} & 30 & 55 & 01 & 82 & 31 & 60 & 08 & \mathrm{CF} & 30 & \mathrm{DA} & 04 & 89 & 30 & \mathrm{DA}\end{array}$ $\begin{array}{lllllllllllllllll}03150 & 01 & 49 & 00 & 10 & 05 & 67 & 03 & C 4 & 0 A & A 4 & 06 & 08 & 01 & 72 & 31 & 76\end{array}$ $\begin{array}{lllllllllllllllll}03160 & 04 & 9 \mathrm{C} & 01 & 49 & 00 & 20 & 03 & 73 & 01 & 82 & 31 & 74 & 04 & 7 \mathrm{D} & 06 & 08\end{array}$ $\begin{array}{lllllllllllllllll}03170 & 01 & 72 & 31 & 76 & 06 & 10 & 01 & 82 & 31 & 3 \mathrm{E} & 01 & 49 & 00 & 3 \mathrm{~B} & 03 & 73\end{array}$ $\begin{array}{lllllllllllllllll}03180 & 03 & 52 & 01 & 82 & 31 & 18 & 06 & \mathrm{E} 3 & 04 & \mathrm{E} 0 & 02 & \mathrm{C} 0 & 81 & \mathrm{D} 6 & 30 & \mathrm{FA}\end{array}$ $\begin{array}{lllllllllllllllll}03190 & 08 & 57 & 07 & 59 & 04 & \mathrm{BF} & 01 & 49 & 00 & 10 & 03 & 73 & 01 & 82 & 31 & \mathrm{~A} 4\end{array}$ $\begin{array}{lllllllllllllllll}031 \mathrm{AO} & 01 & 72 & 31 & \mathrm{~B} 4 & \mathrm{OB} & 41 & \mathrm{OB} & 44 & 65 & 63 & 6 \mathrm{C} & 61 & 72 & 65 & 20 & 48\end{array}$ $\begin{array}{lllllllllllllllll}031 \mathrm{~B} 0 & 45 & 58 & 11 & \mathrm{BA} & 28 & 67 & 30 & \mathrm{FE} & 28 & 78 & 0 \mathrm{~B} & 41 & 12 & 52 & 65 & 63\end{array}$ $\begin{array}{lllllllllllllllll}031 \mathrm{C} 0 & 65 & 70 & 74 & 69 & 6 \mathrm{~F} & 6 \mathrm{E} & 20 & 63 & 6 \mathrm{~F} & 6 \mathrm{D} & 70 & 6 \mathrm{C} & 65 & 74 & 65 & 02\end{array}$ $\begin{array}{lllllllllllllllll}031 D 0 & C 0 & 83 & 44 & 4 F & D 3 & 31 & 8 C & 08 & 57 & 19 & 06 & 1 D & 53 & 09 & 09 & 0 B\end{array}$ $\begin{array}{llllllllllllllllll}031 E 0 & 41 & O A & 54 & 68 & 65 & 20 & 44 & 50 & 20 & 69 & 73 & 20 & 0 A & 53 & \text { OC } & \text { DC }\end{array}$ $\begin{array}{lllllllllllllllll}031 \mathrm{~F} 0 & 15 & 8 \mathrm{E} & 0 \mathrm{C} & \mathrm{F} 2 & 18 & 9 \mathrm{~A} & 02 & \mathrm{C} 0 & \mathrm{C} 9 & 41 & 53 & 53 & 45 & 4 \mathrm{D} & 42 & 4 \mathrm{C}\end{array}$ $\begin{array}{lllllllllllllllll}03200 & 45 & D 2 & 31 & D 1 & 08 & 57 & 01 & 49 & 00 & 47 & 1 A & 2 D & 02 & C 0 & 85 & 44\end{array}$ $\begin{array}{lllllllllllllllll}03210 & 45 & 50 & 54 & \text { C8 } & 31 & \text { F8 } & 08 & 57 & 02 & 86 & 06 & \text { Al } & 04 & \text { BF } & 04 & 89\end{array}$ $\begin{array}{lllllllllllllllll}03220 & 04 & 05 & 06 & 18 & 13 & 4 \mathrm{~F} & 02 & \mathrm{C} 0 & 82 & 2 \mathrm{E} & \mathrm{D} 3 & 32 & 0 \mathrm{E} & 08 & 57 & 32\end{array}$ $\begin{array}{lllllllllllllllll}03230 & 16 & 01 & 82 & 32 & 51 & 02 & 86 & 0 A & C D & 06 & A 1 & 04 & B F & 0 A & C D & 01\end{array}$ $\begin{array}{lllllllllllllllll}03240 & 5 \mathrm{~B} & 03 & 46 & 15 & 9 \mathrm{~A} & 01 & 49 & \mathrm{FF} & \mathrm{FE} & 00 & \mathrm{D} 3 & 32 & 41 & 01 & 72 & 32\end{array}$ $\begin{array}{lllllllllllllllll}03250 & 59 & 0 \mathrm{~B} & 41 & 05 & 45 & 6 \mathrm{D} & 70 & 74 & 79 & 02 & \mathrm{C} 0 & 89 & 44 & 55 & 4 \mathrm{D} & 50\end{array}$ $\begin{array}{lllllllllllllllll}03260 & 2 \mathrm{D} & 54 & 45 & 52 & \mathrm{CD} & 32 & 28 & 08 & 57 & 01 & 49 & 01 & 00 & 04 & 05 & 04\end{array}$ $\begin{array}{lllllllllllllllll}03270 & 9 \mathrm{C} & 06 & 08 & 03 & \mathrm{AE} & 01 & 82 & 32 & 83 & 04 & 67 & 01 & 49 & 01 & 00 & 01\end{array}$ $\begin{array}{lllllllllllllllll}03280 & 72 & 32 & 8 \mathrm{D} & 04 & 67 & 04 & 67 & 01 & 49 & 01 & 00 & 03 & \mathrm{C} 4 & 20 & 9 \mathrm{~A} & 04\end{array}$ $\begin{array}{lllllllllllllllll}03290 & 9 \mathrm{C} & 06 & 08 & 03 & \mathrm{AE} & 01 & 82 & 32 & \mathrm{AB} & 04 & 89 & 01 & 49 & 01 & 00 & 03\end{array}$ $\begin{array}{lllllllllllllllll}032 \mathrm{AO} & \mathrm{C} 4 & 04 & 89 & \text { IE } & \mathrm{D} 5 & 06 & 08 & 01 & 72 & 32 & \mathrm{Bl} & 04 & 7 \mathrm{D} & 04 & 7 \mathrm{D} & 06\end{array}$ $\begin{array}{lllllllllllllllll}032 \mathrm{~B} 0 & 10 & 01 & 82 & 32 & 69 & 02 & \mathrm{C} 0 & 8 \mathrm{~B} & 53 & 43 & 52 & 45 & 45 & 4 \mathrm{E} & 2 \mathrm{D} & 44\end{array}$ $\begin{array}{lllllllllllllllll}032 \mathrm{C} 0 & 55 & 4 \mathrm{D} & \mathrm{D} 0 & 32 & 5 \mathrm{~B} & 08 & 57 & 16 & \mathrm{DE} & 01 & 49 & 04 & 00 & 32 & 67 & 02\end{array}$ $\begin{array}{lllllllllllllllll}032 \mathrm{DO} & \mathrm{C} 0 & 86 & 56 & 4 \mathrm{~F} & 43 & 41 & 42 & \mathrm{BF} & 32 & \mathrm{~B} 7 & 08 & 57 & 07 & 34 & 04 & \mathrm{BF}\end{array}$ $\begin{array}{lllllllllllllllll}032 \mathrm{E} 0 & 01 & 49 & 00 & 04 & 04 & 05 & 0 B & \mathrm{Dl} & 0 \mathrm{~F} & 6 \mathrm{~F} & 0 \mathrm{~B} & 41 & 11 & 20 & 69 & 73\end{array}$ $\begin{array}{lllllllllllllllll}032 \mathrm{FO} & 20 & 74 & 68 & 65 & 20 & 63 & 6 \mathrm{~F} & 6 \mathrm{E} & 74 & 65 & 78 & 74 & 2 \mathrm{C} & 20 & 07 & 42\end{array}$ $\begin{array}{lllllllllllllllll}03300 & 04 & \mathrm{BF} & 01 & 49 & 00 & 04 & 04 & 05 & 0 \mathrm{~B} & \mathrm{DI} & 0 \mathrm{~F} & 6 \mathrm{~F} & 0 \mathrm{~B} & 41 & 1 \mathrm{~A} & 20\end{array}$ $\begin{array}{lllllllllllllllll}03310 & 69 & 73 & 20 & 74 & 68 & 65 & 20 & 63 & 75 & 72 & 72 & 65 & 6 \mathrm{E} & 74 & 20 & 76\end{array}$ $\begin{array}{lllllllllllllllll}03320 & 6 \mathrm{~F} & 63 & 61 & 62 & 75 & 6 \mathrm{C} & 61 & 72 & 79 & 02 & \mathrm{C} 0 & 85 & 56 & 4 \mathrm{D} & 4 \mathrm{~F} & 56\end{array}$ $\begin{array}{lllllllllllllllll}03330 & \mathrm{C} 5 & 32 & \mathrm{DI} & 08 & 57 & 01 & 49 & 00 & 10 & 17 & \mathrm{BC} & 06 & 18 & 08 & 19 & 28\end{array}$ $\begin{array}{lllllllllllllllll}03340 & 78 & 02 & \mathrm{C} 0 & 85 & 56 & 43 & 4 \mathrm{~F} & 50 & \mathrm{D} 9 & 33 & 2 \mathrm{~B} & 08 & 57 & 04 & 9 \mathrm{C} & 01\end{array}$ $\begin{array}{lllllllllllllllll}03350 & 49 & 00 & \text { D0 } & 33 & 33 & 01 & 49 & \text { D0 } & 00 & 07 & 94 & 04 & \text { BF } & 01 & 49 & 04\end{array}$ $\begin{array}{lllllllllllllllll}03360 & 00 & 02 & \text { FC } & 01 & 49 & 01 & 00 & 05 & 67 & 01 & 49 & \text { D0 } & 00 & 01 & 49 & 04\end{array}$ $\begin{array}{lllllllllllllllll}03370 & 00 & 02 & \text { FC } & 33 & 33 & 07 & 94 & 04 & \text { BF } & 01 & 49 & \text { D0 } & 00 & 01 & 49 & 04\end{array}$

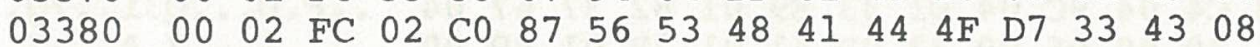
$\begin{array}{lllllllllllllllll}03390 & 57 & 04 & 9 \mathrm{C} & 01 & 49 & 00 & 90 & 33 & 33 & 01 & 49 & \text { D0 } & 00 & 07 & 94 & 04\end{array}$ $\begin{array}{lllllllllllllllll}033 \mathrm{AO} & \mathrm{BF} & 01 & 49 & 04 & 00 & 02 & \mathrm{FC} & 01 & 49 & \mathrm{D} 0 & 00 & 01 & 49 & 04 & 00 & 01\end{array}$ $\begin{array}{lllllllllllllllll}033 \mathrm{~B} 0 & 49 & 00 & 20 & 08 & 68 & 01 & 49 & 00 & \mathrm{~F} 0 & 33 & 33 & 07 & 94 & 04 & \mathrm{BF} & 01\end{array}$ $\begin{array}{lllllllllllllllll}033 \mathrm{C} 0 & 49 & \mathrm{D} 0 & 00 & 01 & 49 & 04 & 00 & 02 & \mathrm{FC} & 33 & 33 & 02 & \mathrm{C} 0 & 86 & 3 \mathrm{~F} & 53\end{array}$ $\begin{array}{lllllllllllllllll}033 D 0 & 50 & 41 & 43 & C 5 & 33 & 85 & 08 & 57 & 01 & 49 & 00 & 20 & 03 & 73 & 02 & C 0\end{array}$ $\begin{array}{lllllllllllllllll}033 \mathrm{E} 0 & 86 & 3 \mathrm{~F} & 41 & 53 & 43 & 49 & \mathrm{C} 9 & 33 & \mathrm{CD} & 08 & 57 & 04 & 9 \mathrm{C} & 01 & 49 & 00\end{array}$ $\begin{array}{lllllllllllllllll}033 \mathrm{~F} 0 & 20 & 03 & 90 & 04 & 89 & 01 & 49 & 00 & 7 \mathrm{E} & 03 & \mathrm{AE} & 02 & 62 & 03 & 52 & 02\end{array}$ $\begin{array}{lllllllllllllllll}03400 & \mathrm{C} 0 & 86 & 3 \mathrm{~F} & 49 & 4 \mathrm{E} & 44 & 45 & \mathrm{D} 8 & 33 & \mathrm{E} 0 & 08 & 57 & 06 & 08 & 04 & 89\end{array}$ $\begin{array}{lllllllllllllllll}03410 & 16 & \mathrm{DE} & 04 & 9 \mathrm{C} & 01 & 49 & 00 & 40 & 03 & \mathrm{C} 4 & 04 & 89 & 01 & 5 \mathrm{~B} & 03 & 46\end{array}$ $\begin{array}{lllllllllllllllll}03420 & 04 & \mathrm{D} 1 & 33 & \mathrm{D} 6 & 01 & 82 & 34 & 2 \mathrm{C} & 01 & 72 & 34 & 30 & 04 & 7 \mathrm{D} & 06 & 10\end{array}$ $\begin{array}{llllllllllllllllll}03430 & 03 & 46 & 04 & \mathrm{Dl} & 33 & \mathrm{E} 9 & 01 & 82 & 34 & 3 \mathrm{E} & 01 & 72 & 34 & 44 & 04 & 7 \mathrm{D}\end{array}$ $\begin{array}{lllllllllllllllll}03440 & 06 & 08 & 03 & I B & 00 & A E & 34 & 1 E & 02 & C 0 & 87 & 3 F & 53 & 43 & 52 & 45\end{array}$ $\begin{array}{lllllllllllllllll}03450 & 45 & \mathrm{CE} & 34 & 01 & 08 & 57 & 06 & 08 & 04 & 89 & 16 & \mathrm{DE} & 04 & 9 \mathrm{C} & 01 & 49\end{array}$ $\begin{array}{lllllllllllllllll}03460 & 04 & 00 & 03 & \text { C4 } & 04 & 89 & 01 & 5 B & 03 & 46 & 04 & \text { D1 } & 33 & \text { D6 } & 01 & 82\end{array}$ $\begin{array}{lllllllllllllllll}03470 & 34 & 76 & 01 & 72 & 34 & 7 \mathrm{~A} & 04 & 7 \mathrm{D} & 06 & 10 & 03 & 46 & 04 & \mathrm{Dl} & 33 & \mathrm{E} 9\end{array}$
/......C. '.A.D. . . .0@000..O.I. .S.....O.I..S.. 16.rI>.c.'(x.f.O ..0U..1 .00Z..0Z .I...g.D.\$...rIv ...I. .s..It.\}.. . $r I v_{0} . . .1>$.I. i.s .R..1... . . Q.V0z .W.Y.?.I...S..I\$ - rl4.A. Declare H $\mathrm{EX} .:\left(\mathrm{g} 0^{\sim}(\mathrm{x} . \mathrm{A} \cdot \mathrm{Rec}\right.$ eption complete. a.DOSI...W...s... A. The DP is .S.। ...r... AIASSEMBL ERIQ.W.I.G.-.Q.D EPTHIX.W...!.?.. .........s2 ..w2 ...2Q...M.!.?.M. [.F...I. .S2A.r2 Y.A.Empty. Q.DUMP -TERM2 (.W.I..... .......2..g.I... r2..g.g.I...D .. .............. D...U...r21.\}.\}. ...2i. a.SCREEN-D UMP2 [.W.^. I..2g. @.VOCAB?27.W.4.? ........0.A. is the context, .B .?.I.....Q.O.A. is the current $V$ ocabulary. a. VMOV E2Q.W.I...<.... ( $\mathrm{x}$. @.VCOPY $3+. \mathrm{W} . .$. I.P33.IP....?.I. ..I.I...g.IP..I. ..133...?.IP..I. .. I. Q.VSHADOW3C. W...I..33.IP.... ?.I...I.IP..I... I. .h.I.p33...? IP..I...|33.a.?S PACE3..W.I. . S. @ .?ASCII3M.W...I. .........b.R. @.?INDEX3 -...I. Q.D... [.F $. Q 3 V . .4, . r 40.\} \ldots$ .F.Q3i..4>.r4D.\} .....4...?SCRE EN4 ..W........I ...D... [.F.Q3V.. $4 \mathrm{v} \cdot r 4 \mathrm{z} \cdot\} \ldots \mathrm{F} \cdot \mathrm{Q} 3 \mathrm{i}$ 
$\begin{array}{lllllllllllllllll}03480 & 01 & 82 & 34 & 88 & 01 & 72 & 34 & 8 \mathrm{E} & 04 & 7 \mathrm{D} & 06 & 08 & 03 & 1 \mathrm{~B} & 00 & \mathrm{AE}\end{array}$ $\begin{array}{llllllllllllllllll}03490 & 34 & 68 & 02 & \mathrm{C} 0 & 85 & 49 & 4 \mathrm{E} & 44 & 45 & \mathrm{D} 8 & 34 & 4 \mathrm{~A} & 08 & 57 & 03 & \mathrm{D} 5\end{array}$ $\begin{array}{lllllllllllllllll}034 \mathrm{~A} 0 & 04 & 89 & 01 & 5 \mathrm{~B} & 03 & 46 & 34 & 0 \mathrm{~A} & 01 & 82 & 34 & \mathrm{BC} & 09 & 09 & 03 & 46\end{array}$ $\begin{array}{lllllllllllllllll}034 \mathrm{BO} & 06 & 20 & 15 & 6 \mathrm{~F} & 09 & 63 & 06 & 08 & 03 & 46 & 16 & 1 \mathrm{~B} & 08 & \mathrm{EA} & 01 & 82\end{array}$ $\begin{array}{lllllllllllllllll}034 \mathrm{C} 0 & 34 & \mathrm{C} 4 & 03 & 1 \mathrm{~B} & 00 & \mathrm{AE} & 34 & \mathrm{~A} 4 & 09 & 09 & 02 & \mathrm{C} 0 & 8 \mathrm{C} & 53 & 50 & 52\end{array}$ $\begin{array}{lllllllllllllllll}034 \mathrm{DC} & 45 & 41 & 44 & 53 & 43 & 52 & 45 & 45 & \mathrm{CE} & 34 & 94 & 08 & 57 & 04 & 9 \mathrm{C} & 04\end{array}$ $\begin{array}{lllllllllllllllll}034 \mathrm{E} 0 & 9 \mathrm{C} & 06 & 08 & 04 & 89 & 03 & \mathrm{D} 5 & 01 & 49 & 00 & \mathrm{FA} & 04 & 89 & 01 & 5 \mathrm{~B} & 03\end{array}$ $\begin{array}{lllllllllllllllll}034 \mathrm{FO} & 46 & 34 & 54 & 01 & 82 & 34 & \mathrm{FB} & 01 & 72 & 35 & 01 & 04 & 7 \mathrm{D} & 03 & 46 & 03\end{array}$ $\begin{array}{lllllllllllllllll}03500 & 1 \mathrm{~B} & 00 & \mathrm{AE} & 34 & \mathrm{EF} & 04 & 9 \mathrm{C} & 03 & 52 & 01 & 82 & 35 & 25 & 0 \mathrm{~B} & 41 & 0 \mathrm{~F}\end{array}$ $\begin{array}{lllllllllllllllll}03510 & 4 \mathrm{E} & 6 \mathrm{~F} & 20 & 62 & 6 \mathrm{C} & 61 & 6 \mathrm{E} & 6 \mathrm{~B} & 20 & 73 & 63 & 72 & 65 & 65 & 6 \mathrm{E} & 11\end{array}$ $\begin{array}{lllllllllllllllll}03520 & \mathrm{BA} & 01 & 72 & 35 & 37 & 01 & 5 \mathrm{~B} & 03 & 46 & \text { OA } & \mathrm{BC} & 03 & 46 & \text { IB } & \mathrm{FF} & 01\end{array}$ $\begin{array}{lllllllllllllllll}03530 & 49 & \mathrm{FF} & \mathrm{FF} & 00 & \mathrm{D} 3 & 35 & 27 & 27 & \mathrm{~F} 7 & 19 & 06 & 02 & \mathrm{C} 0 & 83 & 45 & 4 \mathrm{~F}\end{array}$ $\begin{array}{lllllllllllllllll}03540 & \mathrm{C} 6 & 34 & \mathrm{CC} & 05 & \mathrm{FB} & 00 & 13 & 84 & 49 & 4 \mathrm{~F} & 43 & \mathrm{C} 2 & 35 & 3 \mathrm{D} & 06 & 90\end{array}$ $\begin{array}{lllllllllllllllll}03550 & 00 & 54 & 86 & 53 & 45 & 43 & 54 & 4 \mathrm{~F} & \mathrm{D} 2 & 35 & 47 & 08 & 57 & 04 & 9 \mathrm{C} & 01 .\end{array}$ $\begin{array}{lllllllllllllllll}03560 & 49 & 02 & 75 & 03 & \mathrm{AE} & 01 & 82 & 35 & 7 \mathrm{~B} & 0 \mathrm{~B} & 41 & \text { OD } & 50 & 53 & 4 \mathrm{E} & 20\end{array}$ $\begin{array}{lllllllllllllllll}03570 & 74 & 6 \mathrm{~F} & 6 \mathrm{~F} & 20 & 6 \mathrm{C} & 61 & 72 & 67 & 65 & 11 & \mathrm{BA} & 06 & 08 & 06 & 18 & 13\end{array}$ $\begin{array}{lllllllllllllllll}03580 & 16 & 16 & \mathrm{DE} & 04 & 89 & 01 & 49 & 02 & 00 & 05 & 67 & 03 & \mathrm{C} 4 & 04 & 9 \mathrm{C} & 35\end{array}$ $\begin{array}{lllllllllllllllll}03590 & 4 \mathrm{E} & 01 & 49 & 00 & 07 & 03 & \mathrm{C} 4 & 04 & \mathrm{E} 0 & 02 & \mathrm{C} 0 & 86 & 4 \mathrm{E} & 45 & 57 & 53\end{array}$ $\begin{array}{lllllllllllllllll}035 \mathrm{AO} & 44 & \mathrm{D} 7 & 35 & 52 & 08 & 57 & 06 & 08 & 35 & 4 \mathrm{E} & 01 & 49 & 00 & 04 & 03 & \mathrm{C} 4\end{array}$ 035B0 $04 \begin{array}{llllllllllllllll}\mathrm{F} 0 & 35 & 4 \mathrm{E} & 04 & \mathrm{BF} & 35 & 5 \mathrm{~B} & 35 & 4 \mathrm{E} & 06 & 18 & 03 & \mathrm{C} 4 & 04 & \mathrm{Dl}\end{array}$ $\begin{array}{lllllllllllllllll}035 \mathrm{C} 0 & 03 & \mathrm{C} 4 & 04 & 9 \mathrm{C} & 04 & \mathrm{Dl} & 04 & 9 \mathrm{C} & 01 & 49 & 00 & 7 \mathrm{~F} & 03 & \mathrm{AE} & 04 & \mathrm{FE}\end{array}$

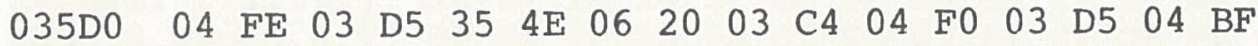
$\begin{array}{lllllllllllllllll}035 \mathrm{E} 0 & 35 & 4 \mathrm{E} & 01 & 49 & 00 & 05 & 03 & \mathrm{C} 4 & 04 & \mathrm{E} 0 & 02 & \mathrm{C} 0 & 86 & 47 & 45 & 54\end{array}$ $\begin{array}{lllllllllllllllll}035 \mathrm{~F} 0 & 53 & 45 & \mathrm{C} 3 & 35 & 9 \mathrm{~B} & 08 & 57 & 35 & 4 \mathrm{E} & 06 & 20 & 03 & \mathrm{C} 4 & 04 & \mathrm{Dl} & 35\end{array}$ $\begin{array}{lllllllllllllllll}03600 & 4 \mathrm{E} & 01 & 49 & 00 & 04 & 03 & \mathrm{C} 4 & 04 & \mathrm{Dl} & 03 & \mathrm{AE} & 01 & 82 & 36 & 43 & 35\end{array}$ $\begin{array}{lllllllllllllllll}03610 & 4 \mathrm{E} & 01 & 49 & 00 & 05 & 03 & \mathrm{C} 4 & 04 & \mathrm{BF} & 35 & 4 \mathrm{E} & 01 & 49 & 00 & 04 & 03\end{array}$ $\begin{array}{lllllllllllllllll}03620 & \mathrm{C} 4 & 04 & \mathrm{Dl} & 03 & \mathrm{C} 4 & 35 & 5 \mathrm{~B} & 35 & 4 \mathrm{E} & 01 & 49 & 00 & 04 & 03 & \mathrm{C} 4 & 04\end{array}$ $\begin{array}{lllllllllllllllll}03630 & \text { D1 } & 03 & \text { D5 } & 35 & 4 \mathrm{E} & 01 & 49 & 00 & 04 & 03 & \mathrm{C} 4 & 04 & \mathrm{~F} 0 & 06 & 10 & 01\end{array}$ $\begin{array}{lllllllllllllllll}03640 & 72 & 36 & 67 & 35 & 4 \mathrm{E} & 06 & 18 & 03 & \mathrm{C} 4 & 04 & \mathrm{Dl} & 06 & 20 & 03 & \mathrm{C} 4 & 35\end{array}$ $\begin{array}{lllllllllllllllll}03650 & 4 \mathrm{E} & 06 & 18 & 03 & \mathrm{C} 4 & 04 & \mathrm{~F} 0 & 35 & \mathrm{~A} 4 & 01 & 82 & 36 & 65 & 06 & 08 & 06\end{array}$ $\begin{array}{lllllllllllllllll}03660 & 10 & 01 & 72 & 36 & 67 & 06 & 08 & 01 & 82 & 35 & \mathrm{~F} 7 & 02 & \mathrm{C} 0 & 84 & 4 \mathrm{~F} & 70\end{array}$ $\begin{array}{lllllllllllllllll}03670 & 65 & \mathrm{EE} & 35 & \mathrm{EC} & 08 & 57 & 35 & 4 \mathrm{E} & 04 & \mathrm{E} 0 & 06 & 08 & 35 & 4 \mathrm{E} & 03 & \mathrm{E} 7\end{array}$ $\begin{array}{lllllllllllllllll}03680 & 04 & \mathrm{~F} 0 & 35 & \mathrm{~A} 4 & 01 & 82 & 36 & 98 & \text { OB } & 41 & \text { OB } & 45 & 6 \mathrm{E} & 64 & 20 & 6 \mathrm{~F}\end{array}$ $\begin{array}{lllllllllllllllll}03690 & 66 & 20 & 66 & 69 & 6 \mathrm{C} & 65 & 11 & \mathrm{BA} & 35 & \mathrm{~F} 5 & 04 & 7 \mathrm{D} & 02 & \mathrm{C} 0 & 86 & 44\end{array}$ $\begin{array}{lllllllllllllllll}036 \mathrm{AO} & 49 & 53 & 4 \mathrm{~B} & 49 & \mathrm{C} 4 & 36 & 6 \mathrm{D} & 08 & 57 & 09 & 09 & 0 \mathrm{~B} & 41 & 09 & 44 & 69\end{array}$ $\begin{array}{lllllllllllllllll}036 \mathrm{~B} 0 & 73 & 6 \mathrm{~B} & 20 & 49 & 44 & 3 \mathrm{~A} & 20 & 06 & 08 & 35 & 5 \mathrm{~B} & 01 & 49 & 00 & 0 \mathrm{C} & 03\end{array}$ $\begin{array}{lllllllllllllllll}036 \mathrm{C} 0 & \mathrm{C} 4 & 04 & 9 \mathrm{C} & 01 & 49 & 00 & 08 & 03 & \mathrm{C} 4 & 01 & 49 & 00 & 2 \mathrm{C} & 09 & 33 & 01\end{array}$ $\begin{array}{lllllllllllllllll}036 \mathrm{D} 0 & 49 & 00 & 05 & 14 & \mathrm{AE} & 01 & 49 & 00 & 08 & 09 & 33 & 09 & 09 & 02 & \mathrm{C} 0 & 87\end{array}$ $\begin{array}{lllllllllllllllll}036 \mathrm{E} 0 & 2 \mathrm{E} & 44 & 49 & 52 & 53 & 45 & \mathrm{C} 3 & 36 & 9 \mathrm{E} & 08 & 57 & 01 & 49 & 02 & 00 & 06\end{array}$ $\begin{array}{lllllllllllllllll}036 \mathrm{~F} 0 & 08 & 01 & 5 \mathrm{~B} & 08 & \mathrm{EA} & 01 & 82 & 36 & \mathrm{FF} & 03 & 1 \mathrm{~B} & 01 & 72 & 37 & 69 & 04\end{array}$ $\begin{array}{lllllllllllllllll}03700 & 9 \mathrm{C} & 03 & 46 & 03 & \mathrm{C} 4 & 04 & 9 \mathrm{C} & 04 & \mathrm{Dl} & 33 & \mathrm{E} 9 & 01 & 82 & 37 & 67 & 04\end{array}$ $\begin{array}{lllllllllllllllll}03710 & 9 \mathrm{C} & 04 & 9 \mathrm{C} & 01 & 49 & 00 & 06 & 09 & 33 & 0 \mathrm{~B} & 41 & 01 & 2 \mathrm{E} & 01 & 49 & 00\end{array}$ $\begin{array}{lllllllllllllllll}03720 & 06 & 03 & C 4 & 06 & 20 & 09 & 33 & 06 & 20 & 14 & \mathrm{AE} & 01 & 49 & 00 & 0 \mathrm{~A} & 03\end{array}$ $\begin{array}{lllllllllllllllll}03730 & \mathrm{C} 4 & 04 & \mathrm{BF} & 15 & 8 \mathrm{E} & 07 & 0 \mathrm{~F} & 04 & \mathrm{BF} & 04 & 9 \mathrm{C} & 01 & 49 & 00 & 3 \mathrm{C} & 03\end{array}$ $\begin{array}{lllllllllllllllll}03740 & A E & 01 & 82 & 37 & 53 & 04 & 7 D & 06 & 08 & 07 & 0 F & 04 & E 0 & 09 & 09 & 01\end{array}$ $\begin{array}{lllllllllllllllll}03750 & 72 & 37 & 63 & 01 & 49 & 00 & 14 & 13 & 5 \mathrm{~F} & 01 & 49 & 00 & 14 & 04 & 89 & 04\end{array}$ $\begin{array}{lllllllllllllllll}03760 & 05 & 14 & \mathrm{AE} & 01 & 72 & 37 & 69 & 04 & 7 \mathrm{D} & 01 & 49 & 00 & 10 & 00 & \mathrm{D} 3 & 36\end{array}$ $\begin{array}{lllllllllllllllll}03770 & F 3 & 04 & 7 D & 02 & C 0 & 83 & 44 & 49 & D 2 & 36 & D F & 08 & 57 & 36 & \text { A7 } & 0 B\end{array}$ $\begin{array}{lllllllllllllllll}03780 & 41 & 10 & 44 & 69 & 72 & 65 & 63 & 74 & 6 \mathrm{~F} & 72 & 79 & 20 & 20 & 53 & 74 & 61\end{array}$ $\begin{array}{lllllllllllllllll}03790 & 72 & 74 & 09 & 09 & 09 & 09 & 06 & 08 & 07 & 0 \mathrm{~F} & 04 & \mathrm{E} 0 & 09 & 09 & 01 & 49\end{array}$ $\begin{array}{lllllllllllllllll}037 \mathrm{AO} & 00 & 0 \mathrm{~A} & 06 & 10 & 01 & 5 \mathrm{~B} & 03 & 46 & 35 & 5 \mathrm{~B} & 36 & \mathrm{E} 9 & 00 & \mathrm{AE} & 37 & \mathrm{~A} 6\end{array}$ $\begin{array}{lllllllllllllllll}037 \mathrm{~B} 0 & 09 & 09 & 19 & 06 & 02 & \mathrm{C} 0 & 86 & 4 \mathrm{E} & 45 & 57 & 4 \mathrm{C} & 53 & \mathrm{CE} & 37 & 75 & 08\end{array}$ $\begin{array}{lllllllllllllllll}037 \mathrm{C} 0 & 57 & 35 & 4 \mathrm{E} & 04 & \mathrm{BF} & 04 & 9 \mathrm{C} & 36 & 74 & 35 & 5 \mathrm{~B} & \text { IA } & \mathrm{D} 5 & 04 & \mathrm{Dl} & 04\end{array}$ $\begin{array}{lllllllllllllllll}037 \mathrm{D} 0 & 9 \mathrm{C} & 01 & 49 & 00 & 79 & 03 & \mathrm{AE} & 01 & 82 & 37 & \mathrm{~EB} & 0 \mathrm{~B} & 41 & 0 \mathrm{~B} & 45 & 6 \mathrm{E}\end{array}$ $\begin{array}{lllllllllllllllll}037 \mathrm{E} 0 & 64 & 20 & 6 \mathrm{~F} & 66 & 20 & 66 & 69 & 6 \mathrm{C} & 65 & 11 & \mathrm{BA} & 03 & \mathrm{D} 5 & 03 & 90 & 01\end{array}$ $\begin{array}{llllllllllllllllll}037 \mathrm{~F} 0 & 82 & 38 & 07 & 04 & 7 \mathrm{D} & 35 & 4 \mathrm{E} & 01 & 49 & 00 & 04 & 03 & \mathrm{C} 4 & 04 & \mathrm{~F} 0 & 35\end{array}$ $\ldots 4 \ldots r 4 \ldots\} \ldots \ldots$ 4h. a.INDEX4J.W.U $\ldots[. F 4 \ldots 4<\ldots . . F$ . .O.C...F...j.. $4 \mathrm{D} \ldots 4$.......SPR EADSCREEN4 . .W... .....U.I.Z... [ F 4 T.. $4\{. r 5 \ldots\} . F$. ...40...R.. 5\%.A. No blank screen. :.r57.[.F.<.F... I...S5' 'w...e.EO F4L. $\{\ldots$. IOCB5 $=$. .T.SECTOR5G.W... I.U....5 .A.PSN too large. :..... .........g.D..5 N.I...D. @.NEWS DW5R.W. .5N.I...D .p5N.?5[5N...D.Q .D...Q...I...

- .U5N. .D.p.U.? 5N.I...D. . ..GET SEC5..W5N. .D.Q5 N.I...D.Q...6 6C5 N.I...D.?5N.I... D.Q.D5 [ 5N.I...D. Q.U5N.I...D.P... r6g5N...D.Q. .D5 N...D.p $5 \$ .6$ e... ..r6g.... 5w. a. op en51.W5N. $\ldots 5 \mathrm{~N} . \mathrm{g}$ .p5\$..6... End o f file.: $\left.5 u_{0}\right\}$. a.D ISKID6m.W...A.Di sk ID: ..5[.I... D...I...D.I... 3 . I............. .DIRSEC6..W.I... ..[.j..6...r7i. ..F.D...Q3i..7g. ....I...3.A...I. ..D. .3. ...I... D.?.....?...I. $<$. ...7S.\}........ r7c.I......... ...r.ri.\}.I...s6 s.\}.@.DIR6_.W6'。 A.Directory Sta rt............ ..... [.F5[6i.. 7\& ..... @. NEWLSN7u. W5N.?. . 6t5 [.U.Q. ..I.Y...7k.A.En d of file.:.U... .8..\}5N.I...D.p5 
$\begin{array}{lllllllllllllllll}03800 & \text { F5 } & 06 & 10 & 01 & 72 & 38 & 4 \mathrm{~B} & 04 & 9 \mathrm{C} & 04 & \mathrm{Dl} & 04 & \mathrm{FE} & 04 & 89 & 04\end{array}$ $\begin{array}{lllllllllllllllll}03810 & 05 & 04 & 89 & 06 & 20 & 03 & \mathrm{C} 4 & 04 & 9 \mathrm{C} & 04 & \mathrm{DI} & 03 & \mathrm{D} 5 & 35 & 4 \mathrm{E} & 06\end{array}$ $\begin{array}{lllllllllllllllll}03820 & 20 & 03 & \text { C4 } & 04 & \text { F0 } & 04 & 9 \mathrm{C} & 03 & \text { D5 } & 04 & \text { BF } & 35 & 4 \mathrm{E} & 01 & 49 & 00\end{array}$ $\begin{array}{lllllllllllllllll}03830 & 05 & 03 & \mathrm{C} 4 & 04 & \mathrm{E} 0 & 35 & 4 \mathrm{E} & 06 & 18 & 03 & \mathrm{C} 4 & 04 & \mathrm{Dl} & 06 & 20 & 03\end{array}$ $\begin{array}{lllllllllllllllll}03840 & \mathrm{C} 4 & 35 & 4 \mathrm{E} & 06 & 18 & 03 & \mathrm{C} 4 & 04 & \mathrm{~F} 0 & 06 & 08 & 01 & 82 & 37 & \mathrm{CB} & 02\end{array}$ $\begin{array}{lllllllllllllllll}03850 & \mathrm{C} 0 & 85 & 46 & 4 \mathrm{E} & 41 & 4 \mathrm{D} & \mathrm{C} 5 & 37 & \mathrm{~B} 6 & 08 & 57 & 04 & 9 \mathrm{C} & 04 & 9 \mathrm{C} & 0 \mathrm{~A}\end{array}$ $\begin{array}{lllllllllllllllll}03860 & 53 & 06 & 10 & 03 & \mathrm{C} 4 & 03 & \mathrm{C} 4 & 0 \mathrm{~B} & 5 \mathrm{D} & 06 & 20 & 02 & \mathrm{FC} & 0 \mathrm{~B} & 5 \mathrm{D} & 0 \mathrm{~A}\end{array}$ $\begin{array}{lllllllllllllllll}03870 & 53 & 01 & 49 & 00 & 07 & 03 & \mathrm{C} 4 & 06 & 20 & 02 & \mathrm{FC} & 0 \mathrm{~A} & 53 & 03 & \mathrm{C} 4 & 01\end{array}$ $\begin{array}{lllllllllllllllll}03880 & 49 & 00 & 07 & 04 & \mathrm{FE} & 04 & 05 & 01 & 49 & 00 & 20 & 08 & 68 & 02 & \mathrm{C} 0 & 86\end{array}$ $\begin{array}{lllllllllllllllll}03890 & 46 & 49 & 4 \mathrm{C} & 45 & 49 & \mathrm{C} 4 & 38 & 51 & 08 & 57 & 0 \mathrm{~B} & 41 & 13 & 46 & 69 & 6 \mathrm{C}\end{array}$ $\begin{array}{lllllllllllllllll}038 \mathrm{~A} 0 & 65 & 20 & 49 & 44 & 3 \mathrm{~A} & 20 & 57 & 46 & 4 \mathrm{~F} & 52 & 54 & 48 & 2 \mathrm{E} & 43 & 4 \mathrm{D} & 20\end{array}$ $\begin{array}{lllllllllllllllll}038 \mathrm{~B} 0 & 02 & \mathrm{C} 0 & 84 & 4 \mathrm{~F} & 50 & 45 & \mathrm{CE} & 38 & 8 \mathrm{~F} & 08 & 57 & 19 & 06 & 01 & 49 & 00\end{array}$ $\begin{array}{lllllllllllllllll}038 C 0 & 20 & 0 D & F A & 0 A & 53 & 03 & \text { D5 } & \text { OA } & 53 & 04 & \text { DI } & 01 & 49 & 00 & \text { F7 } & 01\end{array}$ $\begin{array}{lllllllllllllllll}038 D 0 & 49 & 00 & 13 & 03 & C 4 & 06 & 10 & \text { IF } & E 4 & 04 & 89 & 01 & 82 & 39 & 03 & 0 A\end{array}$ $\begin{array}{lllllllllllllllll}038 E 0 & 53 & 03 & \text { C4 } & 04 & 9 \mathrm{C} & 03 & \mathrm{D} 5 & 04 & \mathrm{Dl} & 06 & 20 & 02 & 51 & 07 & \mathrm{BD} & 04\end{array}$ $\begin{array}{lllllllllllllllll}038 \mathrm{FO} & \mathrm{BF} & 05 & 67 & 07 & 26 & 04 & \mathrm{E} 0 & 01 & 49 & 20 & 20 & 04 & 89 & 04 & \mathrm{E} 0 & 01\end{array}$ $\begin{array}{lllllllllllllllll}03900 & 72 & 39 & 05 & 04 & 7 \mathrm{D} & 0 \mathrm{~A} & 53 & 01 & 49 & 00 & 0 \mathrm{C} & 03 & \mathrm{C} 4 & 0 \mathrm{~A} & 53 & 04\end{array}$ $\begin{array}{lllllllllllllllll}03910 & 9 \mathrm{C} & 04 & \mathrm{DI} & 03 & \mathrm{D} 5 & 03 & \mathrm{C} 4 & 01 & 5 \mathrm{~B} & 01 & 49 & 00 & 20 & 03 & 46 & 04\end{array}$ $\begin{array}{lllllllllllllllll}03920 & \text { F0 } & 00 & A E & 39 & 19 & \text { OA } & 53 & 03 & \text { D5 } & \text { OA } & 53 & 04 & \text { DI } & 01 & 49 & 12\end{array}$ $\begin{array}{lllllllllllllllll}03930 & 52 & 0 B & D 1 & 03 & D 5 & 06 & 10 & \text { IF } & \text { E4 } & 04 & 89 & 01 & 82 & 39 & \text { EB } & 06\end{array}$ $\begin{array}{lllllllllllllllll}03940 & 18 & 04 & 67 & 03 & 73 & 01 & 82 & 39 & 53 & 04 & 7 \mathrm{D} & 06 & 18 & 38 & 59 & 01\end{array}$ $\begin{array}{lllllllllllllllll}03950 & 72 & 39 & \mathrm{E} 7 & 06 & 20 & 04 & 67 & 03 & 73 & 01 & 82 & 39 & 67 & 04 & 7 \mathrm{D} & 06\end{array}$ $\begin{array}{lllllllllllllllll}03960 & 20 & 38 & 59 & 01 & 72 & 39 & \mathrm{E} 7 & 01 & 49 & 00 & 04 & 04 & 67 & 03 & 73 & 01\end{array}$ $\begin{array}{lllllllllllllllll}03970 & 82 & 39 & 7 \mathrm{~F} & 04 & 7 \mathrm{D} & 01 & 49 & 00 & 04 & 38 & 59 & 01 & 72 & 39 & \mathrm{E} 7 & 01\end{array}$ $\begin{array}{lllllllllllllllll}03980 & 49 & 00 & 05 & 04 & 67 & 03 & 73 & 01 & 82 & 39 & 97 & 04 & 7 \mathrm{D} & 01 & 49 & 00\end{array}$ $\begin{array}{lllllllllllllllll}03990 & 05 & 38 & 59 & 01 & 72 & 39 & \mathrm{E} 7 & 01 & 49 & 00 & 06 & 04 & 67 & 03 & 73 & 01\end{array}$ $\begin{array}{lllllllllllllllll}039 A 0 & 82 & 39 & \text { B7 } & 04 & 7 D & 01 & 49 & 00 & 20 & 0 A & 53 & 01 & 49 & 00 & 06 & 03\end{array}$

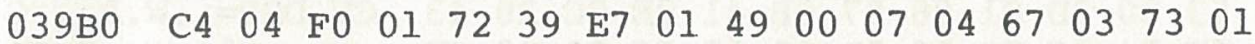
$\begin{array}{lllllllllllllllll}039 \mathrm{C} 0 & 82 & 39 & \mathrm{E} 5 & 04 & 7 \mathrm{D} & 0 \mathrm{~A} & 53 & 01 & 49 & 00 & 08 & 03 & \mathrm{C} 4 & 0 \mathrm{~B} & 5 \mathrm{D} & 06\end{array}$ $\begin{array}{lllllllllllllllll}039 D 0 & 20 & 02 & \text { FC } & 0 B & 5 D & 0 A & 53 & 01 & 49 & 00 & 07 & 03 & \text { C4 } & 06 & 20 & 02\end{array}$ $\begin{array}{lllllllllllllllll}039 E 0 & F C & 01 & 72 & 39 & E 7 & 04 & 7 D & 01 & 72 & 39 & E D & 04 & 7 D & 0 A & 53 & 03\end{array}$

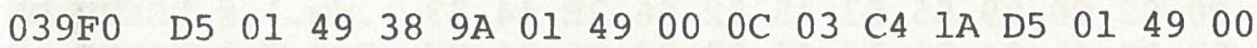
$\begin{array}{lllllllllllllllll}03 \mathrm{~A} 00 & 06 & 02 & \mathrm{FC} & 04 & 89 & 01 & 49 & 00 & 06 & 03 & \mathrm{C} 4 & 04 & 89 & 01 & 49 & 00\end{array}$ $\begin{array}{lllllllllllllllll}03 \mathrm{Al} 0 & 07 & 03 & \mathrm{C} 4 & 06 & 20 & 02 & \mathrm{FC} & 06 & 10 & 01 & 49 & 00 & 0 \mathrm{~A} & 06 & 10 & 01\end{array}$ $\begin{array}{lllllllllllllllll}03 \mathrm{~A} 20 & 5 \mathrm{~B} & 03 & 46 & 35 & 5 \mathrm{~B} & 01 & 49 & 02 & 00 & 0 \mathrm{~A} & 53 & 03 & \mathrm{D} 5 & 01 & 49 & 00\end{array}$ $\begin{array}{lllllllllllllllll}03 A 30 & 09 & 1 F & E 4 & 04 & 89 & 01 & 82 & 3 A & 4 F & 03 & 46 & 35 & 5 B & 03 & C 4 & 03\end{array}$ $\begin{array}{lllllllllllllllll}03 \mathrm{~A} 40 & \mathrm{D} 5 & 04 & \mathrm{BF} & 04 & 89 & 04 & 7 \mathrm{D} & 06 & 08 & 03 & 1 \mathrm{~B} & 01 & 72 & 3 \mathrm{~A} & 51 & 04\end{array}$ $\begin{array}{lllllllllllllllll}03 A 50 & 7 D & 00 & A E & 3 A & 21 & 01 & 82 & 3 A & 6 B & 09 & 09 & 38 & 98 & 0 B & 41 & 09\end{array}$ $\begin{array}{lllllllllllllllll}03 A 60 & 4 \mathrm{E} & 6 \mathrm{~F} & 74 & 20 & 66 & 6 \mathrm{~F} & 75 & 6 \mathrm{E} & 64 & 11 & \mathrm{BA} & 36 & 74 & 02 & \mathrm{C} 0 & 83\end{array}$ $\begin{array}{lllllllllllllllll}03 \mathrm{~A} 70 & 2 \mathrm{E} & 41 & \mathrm{C} 8 & 38 & \mathrm{~B} 2 & 08 & 57 & 04 & 9 \mathrm{C} & 01 & 49 & 00 & 09 & 03 & \mathrm{AE} & 01\end{array}$ $\begin{array}{lllllllllllllllll}03 \mathrm{~A} 80 & 82 & 3 \mathrm{~A} & 8 \mathrm{~F} & 01 & 49 & 00 & 37 & 03 & \mathrm{C} 4 & 08 & \mathrm{FC} & 01 & 72 & 3 \mathrm{~A} & 97 & 01\end{array}$ 03A90 $49 \begin{array}{llllllllllllllll}00 & 30 & 03 & \mathrm{C} 4 & 08 & \mathrm{FC} & 02 & \mathrm{C} 0 & 85 & 2 \mathrm{E} & 42 & 59 & 54 & \mathrm{C} 5 & 3 \mathrm{~A}\end{array}$ $\begin{array}{lllllllllllllllll}03 A A 0 & 6 \mathrm{~F} & 08 & 57 & 04 & 9 \mathrm{C} & 01 & 49 & 00 & 10 & 13 & 4 \mathrm{~F} & 3 \mathrm{~A} & 75 & 01 & 49 & 00\end{array}$ $\begin{array}{lllllllllllllllll}03 \mathrm{ABO} & 0 \mathrm{~F} & 02 & 51 & 3 \mathrm{~A} & 75 & 02 & \mathrm{C} 0 & 84 & 2 \mathrm{E} & 41 & 48 & \mathrm{CC} & 3 \mathrm{~A} & 99 & 08 & 57\end{array}$ $\begin{array}{lllllllllllllllll}03 A C 0 & 01 & 49 & 00 & 20 & 06 & 08 & 01 & 5 B & 04 & 9 C & 03 & 46 & 03 & C 4 & 04 & D 1\end{array}$

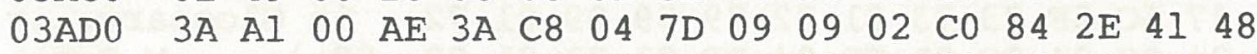

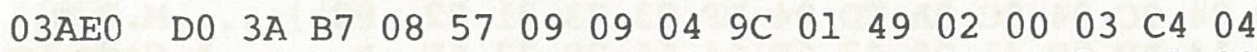
$\begin{array}{lllllllllllllllll}03 A F 0 & 89 & 01 & 5 B & 03 & 46 & 3 A & B E & 01 & 49 & 00 & 20 & 00 & D 3 & 3 A & F 3 & 02\end{array}$ $\begin{array}{lllllllllllllllll}03 \mathrm{~B} 00 & \mathrm{C} 0 & 85 & 46 & 2 \mathrm{D} & 4 \mathrm{C} & 45 & \mathrm{CE} & 3 \mathrm{~A} & \mathrm{DC} & 08 & 57 & 06 & 08 & 06 & 08 & 37\end{array}$ $\begin{array}{lllllllllllllllll}03 \mathrm{Bl} 0 & \mathrm{BF} & 04 & 9 \mathrm{C} & 04 & \mathrm{Dl} & 04 & 9 \mathrm{C} & 01 & 49 & 00 & 80 & 03 & 90 & 01 & 82 & 3 \mathrm{~B}\end{array}$ $\begin{array}{lllllllllllllllll}03 \mathrm{~B} 20 & 2 \mathrm{~F} & 04 & \mathrm{FE} & 03 & \mathrm{C} 4 & 04 & 89 & 06 & 20 & 03 & \mathrm{C} 4 & 01 & 72 & 3 \mathrm{~B} & 11 & 04\end{array}$ $\begin{array}{lllllllllllllllll}03 \mathrm{~B} 30 & 7 \mathrm{D} & 04 & 7 \mathrm{D} & 02 & \mathrm{C} 0 & 84 & 53 & 41 & 56 & \mathrm{C} 5 & 3 \mathrm{~B} & 01 & 08 & 57 & 38 & \mathrm{~B} 9\end{array}$ $\begin{array}{lllllllllllllllll}03 \mathrm{~B} 40 & 3 \mathrm{~B} & 09 & 04 & 9 \mathrm{C} & 01 & 49 & 02 & 00 & 05 & 67 & 0 \mathrm{~A} & 53 & 03 & 90 & 01 & 82\end{array}$ $\begin{array}{lllllllllllllllll}03 \mathrm{~B} 50 & 3 \mathrm{~B} & 5 \mathrm{~A} & 01 & 49 & 00 & 1 \mathrm{~A} & 15 & \mathrm{~B} 9 & 11 & \mathrm{BA} & 06 & 08 & 01 & 5 \mathrm{~B} & 03 & 46\end{array}$ $\begin{array}{lllllllllllllllll}03 B 60 & 01 & 49 & 02 & 00 & 05 & 67 & 35 & F 5 & 01 & 49 & 02 & 00 & 02 & \text { FC } & 16 & 59\end{array}$ $\begin{array}{lllllllllllllllll}03 \mathrm{~B} 70 & 00 & \mathrm{AE} & 3 \mathrm{~B} & 5 \mathrm{E} & 19 & 06 & 02 & \mathrm{C} 0 & 84 & 53 & 45 & 4 \mathrm{E} & \mathrm{C} 4 & 3 \mathrm{~B} & 35 & 08\end{array}$
u...r8K...Q. $\ldots$ ........Q.U5N. .D.p...U. ?5N.I. ..D. 5 N...D.Q. D5N...D.P....7K. (a.FNAME76.W..... S...D.D.]. .1.]. S.I...D. I.S.D. I... ......h. . FILEID8Q.W.A.Fil e ID: WFORTH.CM . Q.OPEN8..W.... . .Z.S.U.S.Q.I.W. I...D...d....9. S.D...U.Q. $\cdot Q \cdot=$ ?.g.\&.'.I $\ldots$.. r9... .S.I...D.S. ..Q.U.D.[.I. .F. P..9..S.U.S.Q.I. R.Q.U...d....9k . ..g.s..9S.\}..8Y. r9g. .g.s..9g.\} . $8 Y . r 9 g . I \ldots g . s$. .9.\} . I_{0} .8 Y . r 9 g$.

I...g.s..9..\}.I. .8Y.r9g.I...g.s. .97.\} . I$. .S.I... D.p.r9g.I...g.s. .9e.\}.S.I...D.]. . I.].S.I...D. \{.rgg.\}.r9m.\}.S. U.I8..I...D.U.I. ..I...I...D...I. ..D. . I...I.... [.F5 [.I...S.U.I. ..d....: : O.F5[.D. U.?...\}.....r:Q. \}..: !..:k..8..A. Not found.: 6t. .AH82.W...I..... . :.I.7.D.|.r:.. I.0.D.1.A..BYTE: O.W...I...O:U.I. ..Q:u.Q..AHL:...W .I. ... [ . . F.D.Q $: ! \ldots: \mathrm{H}.\} \ldots$...AH P: 7.W.....I...D. ..[.F:>.I. .S:S. Q.F-LEN : \.W....7 ?...Q...I......; /. .D.... . . r; . \}.\}.@.SAVE; ..W89 i.........g.S.... ;Z.I...9.:... . . F .I...g5u.I.... .Y ..; ......SEND;5. 
$\begin{array}{lllllllllllllllll}03 B 80 & 57 & 38 & B 9 & 35 & F 5 & 04 & 9 C & 03 & 52 & 01 & 82 & 3 B & 95 & 04 & 7 D & 06\end{array}$ $\begin{array}{lllllllllllllllll}03 B 90 & 10 & 01 & 72 & 3 B & C 3 & 04 & 9 C & 28 & 67 & 3 A & E 3 & 08 & E A & 01 & 82 & 3 B\end{array}$ $\begin{array}{lllllllllllllllll}03 \mathrm{BA0} & \mathrm{AB} & 28 & 78 & 01 & 49 & 00 & 12 & 15 & \mathrm{~B} 9 & 11 & \mathrm{BA} & 1 \mathrm{E} & \mathrm{A} 4 & 01 & 82 & 3 \mathrm{~B}\end{array}$ $\begin{array}{lllllllllllllllll}03 \mathrm{BB} 0 & \mathrm{~B} 9 & 04 & 7 \mathrm{D} & 06 & 10 & 01 & 72 & 3 \mathrm{~B} & \mathrm{BB} & 06 & 08 & 28 & 78 & 01 & 82 & 3 \mathrm{~B}\end{array}$ $\begin{array}{lllllllllllllllll}03 \mathrm{BC} 0 & 95 & 06 & 08 & 01 & 82 & 3 \mathrm{~B} & 83 & 02 & \mathrm{C} 0 & 88 & 44 & 4 \mathrm{~F} & 57 & 4 \mathrm{E} & 4 \mathrm{C} & 4 \mathrm{~F}\end{array}$ $\begin{array}{lllllllllllllllll}03 \mathrm{BD} 0 & 41 & \mathrm{C} 4 & 3 \mathrm{~B} & 78 & 08 & 57 & 38 & \mathrm{~B} 9 & 04 & 9 \mathrm{C} & 35 & \mathrm{~F} 5 & 04 & 9 \mathrm{C} & 03 & 52\end{array}$ $\begin{array}{lllllllllllllllll}03 \mathrm{BE} 0 & 01 & 82 & 3 \mathrm{C} & 08 & 04 & 7 \mathrm{D} & 0 \mathrm{~B} & 41 & 17 & 44 & 6 \mathrm{~F} & 77 & 6 \mathrm{E} & 6 \mathrm{C} & 6 \mathrm{~F} & 61\end{array}$ $\begin{array}{lllllllllllllllll}03 \mathrm{BF} 0 & 64 & 69 & 6 \mathrm{E} & 67 & 20 & 69 & 73 & 20 & 63 & 6 \mathrm{~F} & 6 \mathrm{D} & 70 & 6 \mathrm{C} & 65 & 74 & 65\end{array}$ $\begin{array}{lllllllllllllllll}03 \mathrm{C} 00 & 09 & 09 & 06 & 10 & 01 & 72 & 3 \mathrm{C} & 18 & 01 & 49 & 02 & 00 & 04 & \mathrm{FE} & 2 \mathrm{~F} & \mathrm{FE}\end{array}$ $\begin{array}{lllllllllllllllll}03 \mathrm{Cl} 0 & 01 & 49 & 02 & 00 & 03 & \mathrm{C} 4 & 08 & \mathrm{EA} & 01 & 82 & 3 \mathrm{~B} & \mathrm{D} 8 & 04 & 7 \mathrm{D} & 02 & \mathrm{C} 0\end{array}$ $\begin{array}{llllllllllllllllll}03 \mathrm{C} 20 & 88 & 46 & 49 & 4 \mathrm{C} & 45 & 4 \mathrm{C} & 4 \mathrm{~F} & 41 & \mathrm{C} 4 & 3 \mathrm{~B} & \mathrm{C} 9 & 08 & 57 & 38 & \mathrm{~B} 9 & 06\end{array}$ $\begin{array}{lllllllllllllllll}03 \mathrm{C} 30 & 08 & 37 & \mathrm{BF} & 01 & 49 & 01 & \mathrm{~F} 8 & 03 & \mathrm{C} 4 & 04 & \mathrm{BF} & 06 & 08 & 35 & \mathrm{~F} 5 & 04\end{array}$ $\begin{array}{lllllllllllllllll}03 C 40 & 9 C & 01 & 82 & 3 C & 5 F & 04 & F E & \text { IA } & \text { D5 } & 01 & 49 & 02 & 00 & 02 & \text { FC } & 04\end{array}$ $\begin{array}{lllllllllllllllll}03 \mathrm{C} 50 & 89 & 04 & 7 \mathrm{D} & 01 & 49 & 02 & 00 & 03 & \mathrm{C} 4 & 04 & 89 & 01 & 72 & 3 \mathrm{C} & 65 & 1 \mathrm{~A}\end{array}$ $\begin{array}{lllllllllllllllll}03 \mathrm{C} 60 & \mathrm{C} 6 & 04 & 7 \mathrm{D} & 06 & 10 & 01 & 82 & 3 \mathrm{C} & 3 \mathrm{~B} & 02 & \mathrm{C} 0 & 89 & 51 & 55 & 49 & 54\end{array}$ $\begin{array}{lllllllllllllllll}03 \mathrm{C} 70 & 2 \mathrm{E} & 46 & 4 \mathrm{C} & 41 & \mathrm{C} 7 & 3 \mathrm{C} & 20 & 06 & 90 & 00 & 5 \mathrm{E} & 88 & 57 & 4 \mathrm{~F} & 52 & 44\end{array}$ $\begin{array}{lllllllllllllllll}03 \mathrm{C} 80 & 2 \mathrm{E} & 50 & 54 & \mathrm{D} 2 & 3 \mathrm{C} & 6 \mathrm{~B} & 06 & 90 & 00 & 60 & 89 & 50 & 44 & 4 \mathrm{~F} & 54 & 51\end{array}$ $\begin{array}{lllllllllllllllll}03 C 90 & 2 \mathrm{E} & 44 & 53 & \mathrm{D} 0 & 3 \mathrm{C} & 7 \mathrm{~B} & 08 & 57 & 3 \mathrm{C} & 86 & 04 & \mathrm{BF} & 03 & \mathrm{E} 7 & 04 & 9 \mathrm{C}\end{array}$ $\begin{array}{lllllllllllllllll}03 C A 0 & 03 & 28 & 04 & 9 \mathrm{C} & 04 & \mathrm{Dl} & 03 & \mathrm{C} 4 & \mathrm{OA} & \mathrm{BC} & 3 \mathrm{C} & 86 & 04 & \mathrm{E} 0 & 03 & 36\end{array}$ $\begin{array}{lllllllllllllllll}03 \mathrm{CB} 0 & \text { OB } & 03 & 09 & 33 & 02 & \mathrm{C} 0 & 88 & 57 & 4 \mathrm{~F} & 52 & 44 & 2 \mathrm{E} & 44 & 53 & \mathrm{D} 0 & 3 \mathrm{C}\end{array}$ $\begin{array}{lllllllllllllllll}03 \mathrm{CC} 0 & 8 \mathrm{~A} & 08 & 57 & 06 & 20 & 04 & 05 & 01 & 49 & \mathrm{FF} & \mathrm{FF} & 0 \mathrm{~B} & 9 \mathrm{C} & 04 & 9 \mathrm{C} & 03\end{array}$ $\begin{array}{lllllllllllllllll}03 C D 0 & D 5 & 04 & D 1 & 01 & 49 & 00 & 3 B & 03 & 73 & 01 & 82 & 3 C & E 3 & 06 & 10 & 3 C\end{array}$ $\begin{array}{lllllllllllllllll}03 \mathrm{CE} 0 & 77 & 04 & \mathrm{E} 0 & 04 & 9 \mathrm{C} & 04 & \mathrm{DI} & 01 & 49 & 00 & \mathrm{~A} 0 & 02 & 51 & 01 & 49 & 00\end{array}$ $\begin{array}{llllllllllllllllll}03 \mathrm{CF} 0 & 80 & 03 & 73 & 01 & 82 & 3 \mathrm{C} & \mathrm{FD} & 0 \mathrm{~F} & 6 \mathrm{~F} & 01 & 72 & 3 \mathrm{C} & \mathrm{FF} & 04 & 7 \mathrm{D} & 02\end{array}$ $\begin{array}{lllllllllllllllll}03 \mathrm{D} 00 & \mathrm{C} 0 & 8 \mathrm{~A} & 42 & 52 & 41 & 4 \mathrm{E} & 43 & 48 & 2 \mathrm{E} & 44 & 53 & \mathrm{D} 0 & 3 \mathrm{C} & \mathrm{B} 6 & 08 & 57\end{array}$ $\begin{array}{lllllllllllllllll}03 \mathrm{DI0} & \mathrm{OB} & 41 & 04 & 20 & 54 & 4 \mathrm{~F} & 20 & 3 \mathrm{C} & 86 & 04 & \mathrm{BF} & 03 & \mathrm{E} 7 & 04 & 9 \mathrm{C} & 3 \mathrm{C}\end{array}$ $\begin{array}{lllllllllllllllll}03 \mathrm{D} 20 & 86 & 04 & \mathrm{E} 0 & 04 & \mathrm{BF} & 06 & 08 & \text { OC } & \text { DC } & 15 & 80 & \text { OC } & \text { F2 } & 02 & \text { C0 } & 86\end{array}$ $\begin{array}{lllllllllllllllll}03 \mathrm{D} 30 & 43 & 56 & 2 \mathrm{E} & 44 & 53 & \mathrm{D} 0 & 3 \mathrm{D} & 01 & 08 & 57 & 0 \mathrm{~B} & 41 & \text { OE } & 2 \mathrm{C} & 20 & 43\end{array}$ $\begin{array}{lllllllllllllllll}03 \mathrm{D} 40 & 75 & 72 & 72 & 65 & 6 \mathrm{E} & 74 & 6 \mathrm{C} & 79 & 20 & 3 \mathrm{D} & 20 & 02 & \mathrm{C} 0 & 89 & 43 & 4 \mathrm{~F}\end{array}$ $\begin{array}{lllllllllllllllll}03 \mathrm{D} 50 & 4 \mathrm{E} & 53 & 54 & 2 \mathrm{E} & 44 & 53 & \mathrm{D} 0 & 3 \mathrm{D} & 2 \mathrm{~F} & 08 & 57 & 01 & 49 & 10 & 4 \mathrm{D} & 0 \mathrm{~B}\end{array}$ $\begin{array}{lllllllllllllllll}03 \mathrm{D} 60 & \mathrm{D} 1 & 0 \mathrm{~F} & 6 \mathrm{~F} & 3 \mathrm{D} & 38 & 3 \mathrm{C} & 86 & 04 & \mathrm{BF} & 03 & \mathrm{E} 7 & 04 & \mathrm{BF} & 1 \mathrm{E} & 71 & 06\end{array}$ $\begin{array}{lllllllllllllllll}03 \mathrm{D} 70 & 10 & 3 \mathrm{C} & 77 & 04 & \mathrm{E} 0 & 02 & \mathrm{C} 0 & 89 & 55 & 53 & 45 & 52 & 56 & 2 \mathrm{E} & 44 & 53\end{array}$ $\begin{array}{lllllllllllllllll}03 D 80 & \text { D0 } & 3 \mathrm{D} & 4 \mathrm{D} & 08 & 57 & 0 \mathrm{~B} & 41 & 04 & 55 & 53 & 45 & 52 & 3 \mathrm{C} & 86 & 04 & \mathrm{BF}\end{array}$ $\begin{array}{lllllllllllllllll}03 \mathrm{D} 90 & 03 & \mathrm{E} 7 & 04 & \mathrm{BF} & 1 \mathrm{E} & 71 & 3 \mathrm{D} & 38 & 3 \mathrm{C} & 86 & 04 & \mathrm{BF} & 03 & \mathrm{E} 7 & 04 & \mathrm{BF}\end{array}$ $\begin{array}{lllllllllllllllll}03 \mathrm{DA} 0 & 01 & 49 & 00 & 10 & 06 & 88 & 04 & \mathrm{BF} & 03 & \mathrm{C} 4 & 04 & \mathrm{BF} & \text { IE } & 71 & 06 & 10\end{array}$ $\begin{array}{lllllllllllllllll}03 \mathrm{DB} 0 & 3 \mathrm{C} & 77 & 04 & \mathrm{E} 0 & 02 & \mathrm{C} 0 & 87 & 56 & 41 & 52 & 2 \mathrm{E} & 44 & 53 & \mathrm{D} 0 & 3 \mathrm{D} & 77\end{array}$ $\begin{array}{lllllllllllllllll}03 \mathrm{DC} 0 & 08 & 57 & 01 & 49 & 10 & 62 & 0 \mathrm{~B} & \mathrm{DI} & 0 \mathrm{~F} & 6 \mathrm{~F} & 3 \mathrm{D} & 38 & 3 \mathrm{C} & 86 & 04 & \mathrm{BF}\end{array}$ $\begin{array}{lllllllllllllllll}03 \mathrm{DD} 0 & 03 & \mathrm{E} 7 & 04 & \mathrm{BF} & 1 \mathrm{E} & 71 & 06 & 10 & 3 \mathrm{C} & 77 & 04 & \mathrm{E} 0 & 02 & \mathrm{C} 0 & 89 & 44\end{array}$ $\begin{array}{lllllllllllllllll}03 \mathrm{DE} 0 & 4 \mathrm{~F} & 45 & 53 & 3 \mathrm{E} & 2 \mathrm{E} & 44 & 53 & \mathrm{D} 0 & 3 \mathrm{D} & \mathrm{B} 6 & 08 & 57 & 0 \mathrm{~B} & 41 & 05 & 44\end{array}$ $\begin{array}{lllllllllllllllllllll}03 \mathrm{DF} 0 & 4 \mathrm{~F} & 45 & 53 & 3 \mathrm{E} & 3 \mathrm{C} & 86 & 04 & \mathrm{BF} & 06 & 18 & 03 & \mathrm{C} 4 & 04 & \mathrm{BF} & 0 \mathrm{~A} & \mathrm{CD}\end{array}$ $\begin{array}{lllllllllllllllll}03 \mathrm{E} 00 & 3 \mathrm{C} & 86 & 04 & \mathrm{E} 0 & 02 & \mathrm{C} 0 & 89 & 56 & 4 \mathrm{~F} & 43 & 41 & 42 & 2 \mathrm{E} & 44 & 53 & \mathrm{D} 0\end{array}$ $\begin{array}{lllllllllllllllll}03 E 10 & 3 \mathrm{D} & \mathrm{DE} & 08 & 57 & 0 \mathrm{~B} & 41 & 0 \mathrm{~B} & 56 & 4 \mathrm{~F} & 43 & 41 & 42 & 55 & 4 \mathrm{C} & 41 & 52\end{array}$ $\begin{array}{lllllllllllllllll}03 E 20 & 59 & 20 & 3 C & 86 & 04 & B F & 0 B & D 1 & 0 F & 6 F & 09 & 63 & 02 & C 0 & 89 & 44\end{array}$ $\begin{array}{lllllllllllllllll}03 E 30 & 45 & 43 & 4 \mathrm{~F} & 4 \mathrm{D} & 50 & 49 & 4 \mathrm{C} & \mathrm{C} 5 & 3 \mathrm{E} & 06 & 08 & 57 & 0 \mathrm{E} & \mathrm{EE} & 03 & 52\end{array}$ $\begin{array}{lllllllllllllllll}03 E 40 & 01 & 82 & 3 E & 62 & 06 & 20 & 14 & A E & 0 B & 41 & 11 & 3 F & 20 & 4 E & 6 F & 74\end{array}$ $\begin{array}{lllllllllllllllll}03 \mathrm{E} 50 & 20 & 69 & 6 \mathrm{E} & 20 & 47 & 6 \mathrm{C} & 6 \mathrm{~F} & 73 & 73 & 61 & 72 & 79 & 09 & 09 & 01 & 72\end{array}$ $\begin{array}{lllllllllllllllllll}03 E 60 & 40 & B 8 & 04 & 7 D & 04 & 9 C & 04 & 9 C & 0 A & C D & 04 & B F & 03 & 73 & 01 & 82\end{array}$ $\begin{array}{lllllllllllllllll}03 \mathrm{E} 70 & 3 \mathrm{E} & 88 & 06 & 20 & 14 & \mathrm{AE} & 0 \mathrm{~B} & 41 & 09 & 43 & 6 \mathrm{~F} & 64 & 65 & 20 & 77 & 6 \mathrm{~F}\end{array}$

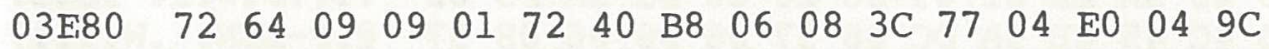

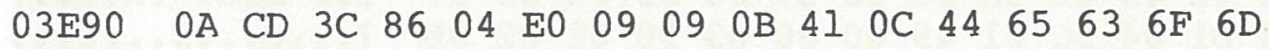

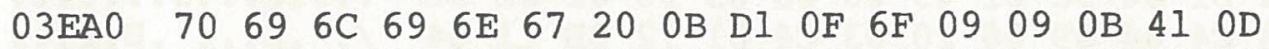

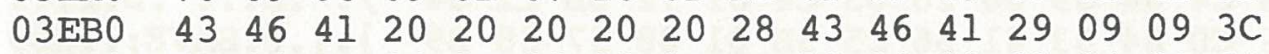
$\begin{array}{lllllllllllllllll}03 \mathrm{ECO} & 86 & 04 & \mathrm{BF} & 04 & 9 \mathrm{C} & 06 & 08 & \text { OC } & \text { DC } & 15 & 80 & 09 & 63 & 04 & 9 \mathrm{C} & 04\end{array}$

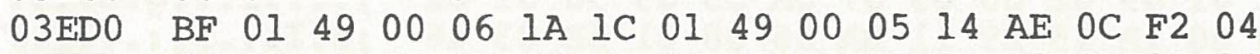
$\begin{array}{lllllllllllllllll}03 E E 0 & B F & 01 & 49 & 01 & 4 B & 0 A & C D & 04 & 67 & 03 & 73 & 01 & 82 & 3 F & 05 & 04\end{array}$

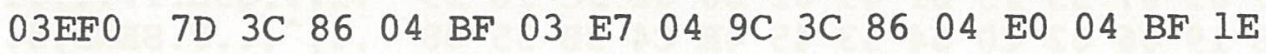

W895u...R..... $\ldots r ; C . .(g: C \cdot j \ldots ;$ $+\left(x . I_{0} . .9 .: \$ \ldots ;\right.$ 9. $\} \ldots r i ; \ldots\left(x_{0} \ldots ;\right.$ ......... D. DOWNLO $A D ; x . W 89 \ldots 5 u \ldots R$ ......... Downloa ding is complete .....r $r$...... $/ \sim$ .I....D.j..; X. $\}$. -FILEILOAD; I.W89. .7?.I.x.D.?..5u. ...<_. .U.I...l. $\ldots\} . \bar{I} \ldots$....r<e. F. $\ldots . . .<;$. Q.QUIT . FLAG $\ldots \wedge$.WORD . $P T R<k$... .PDOTQ . $D S P<\{. W<\ldots$. . . . . ( . . Q. D. < _. . 6 ...3. @.WORD.DSP $<$ ..W. ...I...... U.Q.I. $;. S_{.}$< < . . < w.'..Q.I. .Q.I. ..s. . < $.0 . r<\ldots\}$. a. BRANCH. DSP $<6 . W$ . A. TO $<\ldots$. . g..< ............ CV.DSP $=$..W.A., C urrently $=$. Q.CO NST . DSP $=/$.W.I.M. Q. $O=8<\ldots$ ?.g.?.q. .<w. . @.USERV.DS $\mathrm{P}=\mathrm{M} \cdot \mathrm{W} . \mathrm{A}$. USER $<\ldots$ ? .g.?. $q=8<\ldots$ ?.g.? .I.....?.D.?.q.. $<w . \ddots Q \cdot V A R \cdot D S P=w$ .W.I.b.Q. $O=8<\ldots$ ? $.9 . ? \cdot q . .<w_{0} \cdot$. Q. D OES $>\cdot D S P=6 \cdot W \cdot A \cdot D$ OES><..?...D.?.M $<$.. a.VOCAB.DSP $=$.W.A.VOCABULAR $Y<$..?.Q.O.C.Q.D ECOMPILE $>$..W. $n \cdot R$ ..>b. ...A.? Not in Glossary...r Q8.\}........?.s.. $>$....A. Code wo rd...rere. <w. '.. . $M<$.....A.Decom piling $\cdot Q \cdot O$...A. CFA $\quad(\mathrm{CFA}) \ldots<$ ........................ ?.I........... ?.I.K.M.g.S..?.. \}$<\ldots$. . . . . <..? ? 
$\begin{array}{lllllllllllllllll}03 F 00 & 71 & 01 & 72 & 40 & \text { A4 } & 01 & 49 & \text { OD } & \text { OA } & \text { OA } & \text { CD } & 04 & 67 & 03 & 73 & 01\end{array}$ $\begin{array}{lllllllllllllllll}03 \mathrm{~F} 10 & 82 & 3 \mathrm{~F} & 27 & 04 & 7 \mathrm{D} & 3 \mathrm{C} & 86 & 04 & \mathrm{BF} & 04 & \mathrm{BF} & 3 \mathrm{C} & \mathrm{Cl} & 06 & 10 & 3 \mathrm{C}\end{array}$ $\begin{array}{lllllllllllllllll}03 F 20 & 77 & 04 & \mathrm{E} 0 & 01 & 72 & 40 & \mathrm{~A} 4 & 01 & 49 & 01 & 84 & 0 \mathrm{~A} & \mathrm{CD} & 04 & 67 & 03\end{array}$ $\begin{array}{lllllllllllllllllllll}03 F 30 & 73 & 01 & 82 & 3 F & 42 & 04 & 7 D & 0 B & 41 & 02 & 42 & 5 A & 3 D & 0 E & 01 & 72\end{array}$ $\begin{array}{lllllllllllllllll}03 \mathrm{~F} 40 & 40 & \mathrm{~A} 4 & 01 & 49 & 01 & 74 & 0 \mathrm{~A} & \mathrm{CD} & 04 & 67 & 03 & 73 & 01 & 82 & 3 \mathrm{~F} & 5 \mathrm{D}\end{array}$ $\begin{array}{lllllllllllllllll}03 F 50 & 04 & 7 D & 0 B & 41 & 02 & 42 & 52 & 3 D & 0 E & 01 & 72 & 40 & A 4 & 01 & 49 & 01\end{array}$ $\begin{array}{lllllllllllllllll}03 F 60 & 5 D & 0 A & C D & 04 & 67 & 03 & 73 & 01 & 82 & 3 F & 77 & 04 & 7 D & 0 B & 41 & 03\end{array}$ $\begin{array}{lllllllllllllllll}03 \mathrm{~F} 70 & 44 & 4 \mathrm{~F} & 20 & 01 & 72 & 40 & \mathrm{~A} 4 & 01 & 49 & 18 & 39 & 0 \mathrm{~A} & \mathrm{CD} & 04 & \mathrm{BF} & 04\end{array}$ $\begin{array}{lllllllllllllllll}03 F 80 & 67 & 03 & 73 & 01 & 82 & 3 F & A 5 & 04 & 7 D & 3 C & 86 & 04 & B F & 03 & E 7 & 04\end{array}$ $\begin{array}{lllllllllllllllll}03 \mathrm{~F} 90 & \mathrm{BF} & 01 & 49 & 18 & 39 & 04 & \mathrm{BF} & 03 & 73 & 01 & 82 & 3 \mathrm{~F} & 9 \mathrm{~F} & 3 \mathrm{E} & 12 & 3 \mathrm{D}\end{array}$ $\begin{array}{lllllllllllllllll}03 F A 0 & E A & 01 & 72 & 40 & A 4 & 01 & 49 & 00 & \text { BO } & 0 A & C D & 04 & 67 & 03 & 73 & 01\end{array}$ $\begin{array}{lllllllllllllllll}03 F B 0 & 82 & 3 F & C & 04 & 7 D & 0 B & 41 & 05 & 4 C & 4 F & 4 F & 50 & 20 & 3 D & 0 E & 01\end{array}$ $\begin{array}{lllllllllllllllll}03 \mathrm{FC} 0 & 72 & 40 & \mathrm{~A} 4 & 01 & 49 & 00 & \mathrm{D} 5 & 0 \mathrm{~A} & \mathrm{CD} & 04 & 67 & 03 & 73 & 01 & 82 & 3 \mathrm{~F}\end{array}$ $\begin{array}{lllllllllllllllll}03 F D 0 & E 2 & 04 & 7 D & 0 B & 4 I & 06 & 2 B & 4 C & 4 F & 4 F & 50 & 20 & 3 D & 0 E & 01 & 72\end{array}$

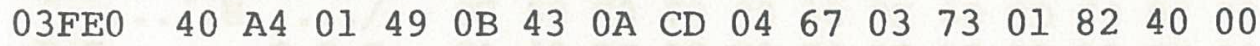
$\begin{array}{lllllllllllllllll}03 F F 0 & 04 & 7 D & 0 B & 41 & 01 & 2 E & 1 C & 9 F & 3 C & 96 & \text { IC } & 9 F & 01 & 72 & 40 & \text { A4 }\end{array}$ $\begin{array}{lllllllllllllllll}04000 & 01 & 49 & 07 & 5 \mathrm{~B} & 0 \mathrm{~A} & \mathrm{CD} & 04 & \mathrm{BF} & 04 & 67 & 03 & 73 & 01 & 82 & 40 & 18\end{array}$ $\begin{array}{lllllllllllllllll}04010 & 04 & 7 D & 3 D & 83 & 01 & 72 & 40 & A 4 & 01 & 49 & 10 & 62 & 03 & \text { E7 } & 04 & B F\end{array}$ $\begin{array}{llllllllllllllllll}04020 & 04 & 67 & 03 & 73 & 01 & 82 & 40 & 30 & 04 & 7 D & 3 D & C 0 & 01 & 72 & 40 & \text { A4 }\end{array}$ $\begin{array}{lllllllllllllllll}04030 & 01 & 49 & 06 & 12 & 0 A & C D & 04 & \text { BF } & 04 & 67 & 03 & 73 & 01 & 82 & 40 & 48\end{array}$ $\begin{array}{lllllllllllllllll}04040 & 04 & 7 D & 3 D & 59 & 01 & 72 & 40 & A 4 & 01 & 49 & 10 & 08 & 0 A & C D & 04 & B F\end{array}$ $\begin{array}{lllllllllllllllll}04050 & 04 & 67 & 03 & 73 & 01 & 82 & 40 & 6 \mathrm{~F} & 04 & 7 \mathrm{D} & 0 \mathrm{~B} & 41 & 02 & 3 \mathrm{~A} & 20 & 3 \mathrm{C}\end{array}$ $\begin{array}{lllllllllllllllll}04060 & 86 & 04 & \mathrm{BF} & 06 & 18 & 03 & \mathrm{C} 4 & 0 \mathrm{~B} & \mathrm{Dl} & 0 \mathrm{~F} & 6 \mathrm{~F} & 01 & 72 & 40 & \mathrm{~A} 4 & 01\end{array}$ $\begin{array}{lllllllllllllllll}04070 & 49 & 0 \mathrm{C} & 93 & 0 \mathrm{~A} & \mathrm{CD} & 04 & 67 & 03 & 73 & 01 & 82 & 40 & 9 \mathrm{E} & 04 & 7 \mathrm{D} & 0 \mathrm{~B}\end{array}$ $\begin{array}{lllllllllllllllll}04080 & 41 & 08 & 43 & 4 \mathrm{~F} & 4 \mathrm{D} & 50 & 49 & 4 \mathrm{C} & 45 & 20 & 3 \mathrm{C} & 86 & 04 & \mathrm{BF} & 03 & \mathrm{E} 7\end{array}$ $\begin{array}{lllllllllllllllll}04090 & 04 & 9 \mathrm{C} & 3 \mathrm{C} & 86 & 04 & \mathrm{E} 0 & 04 & \mathrm{BF} & 3 \mathrm{C} & \mathrm{Cl} & 01 & 72 & 40 & \mathrm{~A} 4 & 04 & 9 \mathrm{C}\end{array}$ $\begin{array}{lllllllllllllllll}040 \mathrm{~A} 0 & 3 \mathrm{C} & \mathrm{Cl} & 04 & 7 \mathrm{D} & 09 & 09 & 06 & 18 & 3 \mathrm{C} & 86 & 03 & \mathrm{~F} 2 & 3 \mathrm{C} & 77 & 04 & \mathrm{BF}\end{array}$ $\begin{array}{lllllllllllllllll}040 \mathrm{~B} 0 & 08 & \mathrm{EA} & 02 & 62 & 01 & 82 & 3 \mathrm{E} & \mathrm{BF} & 09 & 09 & 02 & \mathrm{C} 0 & 86 & 50 & 41 & 52\end{array}$ $\begin{array}{lllllllllllllllll}040 \mathrm{C} 0 & 50 & 54 & \mathrm{D} 2 & 3 \mathrm{E} & 2 \mathrm{E} & 08 & 57 & 07 & \mathrm{D} 2 & 04 & \mathrm{BF} & 04 & 89 & 06 & 10 & 17\end{array}$ $\begin{array}{lllllllllllllllll}040 \mathrm{DO} & \mathrm{BC} & 01 & 49 & 00 & \mathrm{FF} & 02 & 74 & 21 & 46 & 01 & 49 & 00 & 06 & 08 & 19 & 17\end{array}$ $\begin{array}{lllllllllllllllll}040 \mathrm{E} 0 & \mathrm{BC} & 02 & \mathrm{C} 0 & 84 & 50 & 41 & 47 & \mathrm{C} 5 & 40 & \mathrm{BC} & 08 & 57 & 01 & 49 & 00 & 0 \mathrm{D}\end{array}$ $\begin{array}{lllllllllllllllll}040 F 0 & 08 & F C & 01 & 49 & 00 & 8 C & 08 & F C & 06 & 10 & 07 & 6 D & 03 & F 2 & 02 & C 0\end{array}$ $\begin{array}{lllllllllllllllll}04100 & 84 & 54 & 45 & 41 & \text { D2 } & 40 & \text { E3 } & 08 & 57 & 40 & \text { EA } & 19 & \text { F2 } & 02 & \text { C0 } & 88\end{array}$ $\begin{array}{llllllllllllllllll}04110 & 44 & 49 & 53 & 4 \mathrm{~B} & 4 \mathrm{C} & 49 & 53 & \mathrm{D} 4 & 41 & 00 & 08 & 57 & 36 & \mathrm{~A} 7 & 06 & 08\end{array}$ $\begin{array}{lllllllllllllllll}04120 & 04 & \mathrm{FE} & 04 & \mathrm{FE} & 03 & \mathrm{D} 5 & 04 & 89 & 01 & 5 \mathrm{~B} & 03 & 46 & 34 & 54 & 01 & 82\end{array}$ $\begin{array}{lllllllllllllllll}04130 & 41 & 38 & 03 & 46 & 18 & \mathrm{BA} & 03 & \mathrm{D} 5 & 04 & 9 \mathrm{C} & 06 & 18 & 03 & \mathrm{AE} & 01 & 82\end{array}$ $\begin{array}{lllllllllllllllll}04140 & 41 & 4 \mathrm{C} & 04 & 7 \mathrm{D} & 06 & 08 & \text { ID } & \mathrm{CF} & 40 & \mathrm{EA} & 36 & \mathrm{~A} 7 & 00 & \mathrm{AE} & 41 & 2 \mathrm{~A}\end{array}$ $\begin{array}{lllllllllllllllll}04150 & 04 & 7 D & \text { ID } & C F & 02 & C 0 & 88 & 44 & 4 \mathrm{~F} & 43 & 55 & 4 \mathrm{D} & 45 & 4 \mathrm{E} & \mathrm{D} 4 & 41\end{array}$ $\begin{array}{lllllllllllllllll}04160 & 0 \mathrm{~F} & 08 & 57 & 01 & 49 & 00 & 48 & 1 \mathrm{~A} & 2 \mathrm{D} & 02 & \mathrm{C} 0 & 88 & 46 & 4 \mathrm{C} & 4 \mathrm{~F} & 41\end{array}$ $\begin{array}{lllllllllllllllll}04170 & 54 & 49 & 4 \mathrm{E} & \mathrm{C} 7 & 41 & 56 & 08 & 57 & 01 & 49 & 00 & 48 & 1 \mathrm{~A} & 2 \mathrm{D} & 02 & \mathrm{C} 0\end{array}$ $\begin{array}{lllllllllllllllll}04180 & 84 & 54 & 41 & 53 & \mathrm{CB} & 41 & 6 \mathrm{~B} & 08 & 57 & 02 & \mathrm{C} 0 & \mathrm{C} 8 & 44 & 4 \mathrm{~F} & 43 & 55\end{array}$

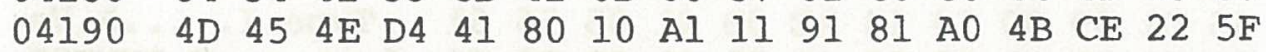
$\begin{array}{lllllllllllllllll}041 \mathrm{AO} & 86 & 2 \mathrm{E} & 56 & 48 & 44 & 52 & \mathrm{~B} 3 & 18 & 3 \mathrm{~B} & 08 & 57 & 09 & 09 & 01 & 49 & 00\end{array}$ $\begin{array}{lllllllllllllllll}041 \mathrm{BO} & 10 & 14 & \mathrm{AE} & 0 \mathrm{~B} & 41 & 0 \mathrm{~F} & 56 & 6 \mathrm{~F} & 63 & 61 & 62 & 75 & 6 \mathrm{C} & 61 & 72 & 79\end{array}$ $\begin{array}{lllllllllllllllll}041 \mathrm{C} 0 & 20 & 6 \mathrm{C} & 69 & 73 & 74 & 09 & 09 & 0 \mathrm{~B} & 41 & 0 \mathrm{~A} & 20 & 20 & 4 \mathrm{E} & 46 & 41 & 20\end{array}$ $\begin{array}{lllllllllllllllll}041 D 0 & 4 \mathrm{E} & 61 & 6 \mathrm{D} & 65 & 01 & 49 & 00 & 0 \mathrm{C} & 14 & \mathrm{AE} & 0 \mathrm{~B} & 41 & 16 & 40 & 4 \mathrm{C} & 46\end{array}$

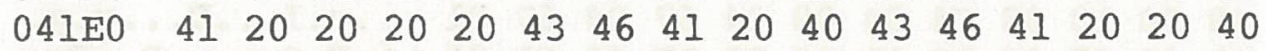
04IF0 $50 \quad 46 \quad 41 \quad 0909 \quad 02$ C0 $86 \quad 56 \quad 4$ C $49 \begin{array}{llllll}53 & 54 & \text { B3 } & 41 & \text { A0 }\end{array}$ $\begin{array}{lllllllllllllllll}04200 & 08 & 57 & 07 & 59 & 04 & \mathrm{BF} & 0 \mathrm{C} & \mathrm{DC} & 07 & 42 & 04 & \mathrm{BF} & 04 & \mathrm{BF} & 41 & \mathrm{~A} 9\end{array}$ $\begin{array}{lllllllllllllllll}04210 & 09 & 09 & 06 & 08 & 07 & 0 \mathrm{~F} & 04 & \mathrm{E} 0 & 04 & 9 \mathrm{C} & 04 & 9 \mathrm{C} & 01 & 49 & 00 & 05\end{array}$ $\begin{array}{lllllllllllllllll}04220 & 1 \mathrm{~B} & 44 & 09 & 63 & 0 \mathrm{~F} & 6 \mathrm{~F} & 07 & 0 \mathrm{~F} & 04 & \mathrm{BF} & 01 & 49 & 00 & 16 & 04 & 89\end{array}$ $\begin{array}{lllllllllllllllll}04230 & 04 & 05 & 14 & \mathrm{AE} & 0 \mathrm{~B} & \mathrm{E} 7 & 04 & 9 \mathrm{C} & 04 & 9 \mathrm{C} & 04 & 9 \mathrm{C} & 04 & 9 \mathrm{C} & 04 & 9 \mathrm{C}\end{array}$

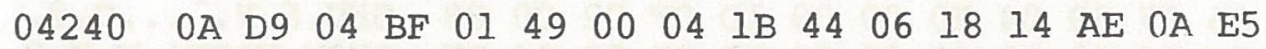
$\begin{array}{llllllllllllllllll}04250 & 04 & 9 \mathrm{C} & 01 & 49 & 00 & 05 & 1 \mathrm{~B} & 44 & 04 & \mathrm{BF} & 01 & 49 & 00 & 05 & \text { IA } & \text { IC }\end{array}$ $\begin{array}{lllllllllllllllll}04260 & 06 & 18 & 14 & \mathrm{AE} & 04 & \mathrm{BF} & 01 & 49 & 00 & 04 & 1 \mathrm{~B} & 44 & 0 \mathrm{~A} & \mathrm{E} 5 & 04 & 9 \mathrm{C}\end{array}$ $\begin{array}{lllllllllllllllll}04270 & 04 & \mathrm{BF} & 06 & 18 & 04 & 05 & 03 & 73 & 01 & 82 & 42 & 84 & 0 \mathrm{~B} & 41 & 05 & 20\end{array}$
q.re\$.I...M.g.s. .? . $\}<$. $^{2}$.? $<\mathrm{A}_{0} .<<$ w. .res.I...M.g. S..?B.\}.A.BZ=..r as.I.t.M.g.s..?] .\}.A.BR=..res.I. ].M.g.S..? ?. $\} \cdot A$. DO .re\$.I.9.M.?. g.s..? $\left.8^{\circ}\right\}<\ldots ? \cdot g$. ?.I.9.?.S..?. $\rangle_{.}=$ j.r@\$.I.0.M.g.s. .?C. $. A \cdot L O O P=$. r@\$.I.U.M.g.S..? b. $. A_{0}+L O O P=\ldots r$ as.I.C.M.g.s... .\}.A.... ....ras .I. [.M.?.g.s... .\}=\ldots r$ a.$I \cdot b \cdot g \cdot ?$ -g.s.. @0. $\}=$ a.res .I...M.?.g.S... .\}=Y . r @ \$ . I \ldots M_{0}$ ? .g.s..do.\}.A.: < ..?...D.Q.O.r@s. I...M.g.s.....\}. A. COMPILE <..?.g ..<.. . ?<A.r@\$. $\left.<A_{0}\right\} \ldots . . .<<<w_{0}$ ? .j.b..>?...e.PAR PTR> ..W.R.?..... <.I...t!F.I..... $<$. a.PAGEQ<.W.I.. . I.I...l...m.r. a - TEAR@c.W@j.r. a. DISKLISTA..W6' .. . . .U... [.F4T'. A8.F.:.U........ AL. ...O@j6' ...A* .\}.0.@.DOCUMENTA ..W.I.H.-.a.FLOA TINGAV.W.I.H.-.@ - TASKAK.W. @HDOCU MENTA. . . ... KN" ..VHDR3 • ;.W..... ....A. Vocabulary list...A. NFA Name.I.....A. QLF A CFA QCFA Q PFA.... @.VLIST3A .W.Y.?.(.B.?.?A) .............. .D.C.O...?.I.... .............. .Y.?.I...D...e ...I...D.?.I.... ........... D.e.. .?....s.....A. page 125 
$\begin{array}{lllllllllllllllll}04280 & 43 & 6 \mathrm{~F} & 64 & 65 & \text { OB } & \mathrm{Dl} & 04 & \mathrm{Dl} & 01 & 49 & 00 & 40 & 02 & 51 & 01 & 82\end{array}$ $\begin{array}{lllllllllllllllll}04290 & 42 & 9 F & 0 B & 41 & 0 A & 20 & 49 & 6 D & 6 D & 65 & 64 & 69 & 61 & 74 & 65 & 07\end{array}$ $\begin{array}{lllllllllllllllll}042 \mathrm{~A} 0 & \mathrm{C} 8 & 04 & \mathrm{BF} & 06 & 3 \mathrm{D} & 06 & 20 & 04 & 05 & 03 & 90 & 01 & 82 & 42 & \mathrm{~B} 3 & 01\end{array}$ $\begin{array}{lllllllllllllllll}042 \mathrm{~B} 0 & 72 & 42 & \mathrm{BB} & 09 & 09 & \text { ID } & \mathrm{CF} & 40 & \mathrm{EA} & 41 & \mathrm{~A} 9 & 0 \mathrm{~A} & \mathrm{D} 9 & 04 & \mathrm{BF} & 04\end{array}$ $\begin{array}{lllllllllllllllll}042 \mathrm{C} 0 & 9 \mathrm{C} & 03 & 52 & 08 & \mathrm{EA} & 02 & 62 & 01 & 82 & 42 & 10 & 04 & 7 \mathrm{D} & 07 & 59 & 04\end{array}$ $\begin{array}{lllllllllllllllll}042 \mathrm{DO} & \mathrm{E} 0 & 09 & 09 & 1 \mathrm{D} & \mathrm{CF} & 02 & \mathrm{C} 0 & 8 \mathrm{~A} & 43 & 41 & 54 & 2 \mathrm{D} & 55 & 50 & 44 & 41\end{array}$ $\begin{array}{lllllllllllllllll}042 \mathrm{E} 0 & 54 & \mathrm{C} 5 & 41 & \mathrm{~F} 7 & 08 & 57 & 01 & 49 & 00 & 04 & 16 & \mathrm{DE} & 01 & 49 & 02 & 0 \mathrm{~F}\end{array}$ $\begin{array}{lllllllllllllllll}042 \mathrm{~F} 0 & 03 & \mathrm{C} 4 & 04 & 9 \mathrm{C} & 04 & 9 \mathrm{C} & 04 & \mathrm{Dl} & 01 & 49 & 00 & \text { IF } & 02 & 62 & 04 & 89\end{array}$ $\begin{array}{lllllllllllllllll}04300 & 04 & \text { F0 } & 03 & \text { D5 } & 04 & 9 \mathrm{C} & 01 & 49 & 00 & 2 \mathrm{D} & 03 & \mathrm{C} 4 & 04 & 89 & 01 & 5 \mathrm{~B}\end{array}$ $\begin{array}{lllllllllllllllll}04310 & 01 & 49 & 00 & \mathrm{FF} & 03 & 46 & 04 & \mathrm{~F} 0 & 00 & \mathrm{AE} & 43 & 10 & 01 & 49 & 00 & 04\end{array}$ $\begin{array}{lllllllllllllllll}04320 & 16 & \mathrm{DE} & 01 & 49 & 02 & 3 \mathrm{D} & 03 & \mathrm{C} 4 & 04 & 9 \mathrm{C} & 04 & 89 & 04 & \mathrm{~F} 0 & 16 & 59\end{array}$ $\begin{array}{lllllllllllllllll}04330 & 19 & 06 & 02 & \mathrm{C} 0 & 84 & 2 \mathrm{E} & 43 & 41 & \mathrm{D} 4 & 42 & \mathrm{D} 7 & 08 & 57 & 01 & 49 & 00\end{array}$ $\begin{array}{lllllllllllllllll}04340 & 04 & 16 & \mathrm{DE} & 01 & 49 & 02 & 00 & 03 & \mathrm{C} 4 & 01 & 49 & 00 & 50 & 20 & 9 \mathrm{~A} & 02\end{array}$ $\begin{array}{lllllllllllllllll}04350 & \mathrm{C} 0 & 86 & 56 & 4 \mathrm{C} & 49 & 53 & 54 & \mathrm{~B} 2 & 43 & 34 & 08 & 57 & 07 & 59 & 04 & \mathrm{BF}\end{array}$ $\begin{array}{llllllllllllllllll}04360 & 0 C & D C & \text { ID } & 9 B & 06 & 20 & 07 & \text { C8 } & 04 & \text { E0 } & 06 & 08 & 07 & 0 F & 04 & \text { E0 }\end{array}$ $\begin{array}{lllllllllllllllll}04370 & 07 & 42 & 04 & \mathrm{BF} & 04 & \mathrm{BF} & 04 & 9 \mathrm{C} & 04 & 9 \mathrm{C} & 07 & 0 \mathrm{~F} & 04 & \mathrm{BF} & 01 & 49\end{array}$ $\begin{array}{lllllllllllllllll}04380 & 00 & 3 \mathrm{C} & 03 & \mathrm{AE} & 01 & 82 & 43 & \mathrm{~B} 0 & 09 & 09 & 06 & 08 & 07 & 0 \mathrm{~F} & 04 & \mathrm{E} 0\end{array}$ $\begin{array}{lllllllllllllllll}04390 & 07 & \mathrm{C} 8 & 04 & \mathrm{BF} & 01 & 49 & 00 & 39 & 03 & \mathrm{AE} & 01 & 82 & 43 & \mathrm{~B} 0 & \text { ID } & \mathrm{CF}\end{array}$ $\begin{array}{lllllllllllllllll}043 \mathrm{~A} 0 & 40 & \mathrm{EA} & \text { ID } & 9 \mathrm{~B} & 06 & 20 & 07 & \mathrm{C} 8 & 04 & \mathrm{E} 0 & 06 & 08 & 07 & 0 \mathrm{~F} & 04 & \mathrm{E} 0\end{array}$ $\begin{array}{lllllllllllllllll}043 \mathrm{~B} 0 & 06 & 08 & 14 & \mathrm{CB} & 15 & 09 & 15 & 09 & 15 & 09 & 15 & 09 & 14 & \mathrm{DA} & 09 & 33\end{array}$ $\begin{array}{lllllllllllllllll}043 \mathrm{C} 0 & 04 & 9 \mathrm{C} & 03 & \mathrm{D} 5 & 04 & \mathrm{D} 1 & 01 & 49 & 00 & 80 & 04 & 05 & 01 & 82 & 43 & \text { D8 }\end{array}$ $\begin{array}{lllllllllllllllll}043 \mathrm{D} 0 & 09 & 63 & 0 \mathrm{~F} & 6 \mathrm{~F} & 01 & 72 & 43 & \mathrm{DA} & 04 & 7 \mathrm{D} & 01 & 49 & 00 & 14 & 07 & 0 \mathrm{~F}\end{array}$ $\begin{array}{lllllllllllllllll}043 \mathrm{E} 0 & 04 & \mathrm{BF} & 04 & 67 & 13 & 5 \mathrm{~F} & 04 & 9 \mathrm{C} & 01 & 82 & 43 & \mathrm{~F} 0 & 01 & 72 & 43 & \mathrm{~F} 6\end{array}$ $\begin{array}{lllllllllllllllll}043 \mathrm{~F} 0 & 04 & 7 \mathrm{D} & 01 & 49 & 00 & 14 & 04 & 05 & 14 & \mathrm{AE} & 0 \mathrm{~B} & \mathrm{E} 7 & 0 \mathrm{~A} & \mathrm{D} 9 & 04 & \mathrm{BF}\end{array}$ $\begin{array}{lllllllllllllllll}04400 & 04 & 9 \mathrm{C} & 03 & 52 & 08 & \mathrm{EA} & 02 & 62 & 01 & 82 & 43 & 76 & 04 & 7 \mathrm{D} & 09 & 09\end{array}$ $\begin{array}{lllllllllllllllll}04410 & 1 \mathrm{D} & \mathrm{CF} & 07 & 59 & 04 & \mathrm{E} 0 & 02 & \mathrm{C} 0 & 86 & 2 \mathrm{E} & 49 & 4 \mathrm{E} & 44 & 45 & \mathrm{D} 8 & 43\end{array}$ $\begin{array}{lllllllllllllllll}04420 & 51 & 08 & 57 & 0 B & 41 & 11 & 53 & 63 & 72 & 65 & 65 & 6 \mathrm{E} & 20 & 69 & 6 \mathrm{E} & 64\end{array}$ $\begin{array}{lllllllllllllllll}04430 & 65 & 78 & 20 & 66 & 6 \mathrm{~F} & 72 & 3 \mathrm{~A} & 36 & \mathrm{~A} 7 & 02 & \mathrm{C} 0 & 84 & 44 & 49 & 52 & \mathrm{~A} 7\end{array}$ $\begin{array}{lllllllllllllllll}04440 & 44 & 18 & 08 & 57 & 37 & 7 \mathrm{~B} & 09 & 09 & \text { OB } & 41 & 18 & 43 & 6 \mathrm{C} & 75 & 73 & 74\end{array}$ $\begin{array}{lllllllllllllllll}04450 & 65 & 72 & 20 & 61 & 6 \mathrm{C} & 6 \mathrm{C} & 6 \mathrm{~F} & 63 & 61 & 74 & 69 & 6 \mathrm{~F} & 6 \mathrm{E} & 20 & 74 & 61\end{array}$ $\begin{array}{lllllllllllllllll}04460 & 62 & 6 \mathrm{C} & 65 & 09 & 09 & 43 & 3 \mathrm{~B} & 09 & 09 & 02 & \mathrm{C} 0 & 8 \mathrm{~B} & 44 & 49 & 52 & 45\end{array}$ $\begin{array}{lllllllllllllllll}04470 & 43 & 54 & 4 \mathrm{~F} & 52 & 49 & 45 & \mathrm{D} 3 & 44 & 3 \mathrm{~B} & 08 & 57 & 0 \mathrm{~B} & 41 & 10 & 44 & 69\end{array}$ $\begin{array}{lllllllllllllllll}04480 & 73 & 6 \mathrm{~B} & 20 & 64 & 69 & 72 & 65 & 63 & 74 & 6 \mathrm{~F} & 72 & 69 & 65 & 73 & 19 & 06\end{array}$ $\begin{array}{lllllllllllllllll}04490 & 09 & 09 & 09 & 09 & 18 & 25 & 44 & 42 & 09 & 09 & 09 & 09 & 18 & 73 & 44 & 42\end{array}$ $\begin{array}{lllllllllllllllll}044 \mathrm{~A} 0 & 1 \mathrm{D} & \mathrm{CF} & 02 & \mathrm{C} 0 & 86 & 49 & 4 \mathrm{E} & 44 & 45 & 58 & \mathrm{~B} 2 & 44 & 6 \mathrm{~B} & 08 & 57 & 44\end{array}$ $\begin{array}{lllllllllllllllll}044 \mathrm{~B} 0 & 21 & 03 & \mathrm{D} 5 & 04 & 89 & 01 & 5 \mathrm{~B} & 03 & 46 & 34 & 0 \mathrm{~A} & 01 & 82 & 44 & \mathrm{CF} & 09\end{array}$ $\begin{array}{lllllllllllllllll}044 \mathrm{C} 0 & 09 & 03 & 46 & 06 & 20 & 15 & 6 \mathrm{~F} & 09 & 63 & 06 & 08 & 03 & 46 & 16 & 1 \mathrm{~B} & 08\end{array}$ $\begin{array}{lllllllllllllllll}044 \mathrm{D} 0 & \mathrm{EA} & 01 & 82 & 44 & \mathrm{D} 7 & 03 & \text { BB } & 07 & \mathrm{C} 8 & 04 & \mathrm{BF} & 01 & 49 & 00 & 38 & 03\end{array}$

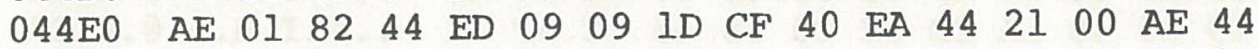
$\begin{array}{lllllllllllllllll}044 \mathrm{~F} 0 & \mathrm{~B} 7 & 09 & 09 & 1 \mathrm{D} & \mathrm{CF} & 02 & \mathrm{C} 0 & 85 & 56 & 43 & 48 & 44 & \mathrm{D} 2 & 44 & \mathrm{~A} 4 & 08\end{array}$ $\begin{array}{lllllllllllllllll}04500 & 57 & 0 B & 41 & 04 & 4 E & 61 & 6 D & 65 & 01 & 49 & 00 & 0 A & 14 & A E & 0 B & 41\end{array}$ $\begin{array}{llllllllllllllllll}04510 & 04 & 54 & 79 & 70 & 65 & 01 & 49 & 00 & 14 & 14 & \mathrm{AE} & 0 \mathrm{~B} & 41 & 05 & 56 & 61\end{array}$ $\begin{array}{lllllllllllllllll}04520 & 6 \mathrm{C} & 75 & 65 & 09 & 09 & 09 & 09 & 02 & \mathrm{C} 0 & 85 & 44 & 55 & 4 \mathrm{D} & 50 & \mathrm{~B} 2 & 44\end{array}$ $\begin{array}{lllllllllllllllll}04530 & \text { F7 } & 08 & 57 & \text { OB } & 41 & \text { OB } & 4 D & 65 & 6 D & 6 F & 72 & 79 & 20 & 64 & 75 & 6 D\end{array}$ $\begin{array}{lllllllllllllllll}04540 & 70 & 09 & 09 & 09 & 09 & 07 & 59 & 04 & \mathrm{BF} & 03 & 28 & 0 \mathrm{C} & \mathrm{DC} & 04 & 67 & 03\end{array}$ $\begin{array}{lllllllllllllllll}04550 & \mathrm{C} 4 & 04 & 89 & 01 & 5 \mathrm{~B} & 03 & 46 & 04 & 9 \mathrm{C} & 01 & 49 & 00 & 05 & 1 \mathrm{~B} & 44 & 04\end{array}$ $\begin{array}{lllllllllllllllll}04560 & 9 \mathrm{C} & 09 & 63 & 01 & 49 & 00 & 10 & 03 & \mathrm{C} 4 & 04 & 89 & 04 & 67 & 04 & 67 & 01\end{array}$ $\begin{array}{lllllllllllllllll}04570 & 5 \mathrm{~B} & 03 & 46 & 04 & \mathrm{DI} & 09 & 63 & 06 & 18 & 1 \mathrm{~B} & 44 & 00 & \mathrm{AE} & 45 & 71 & 06\end{array}$ $\begin{array}{lllllllllllllllll}04580 & 18 & 14 & \mathrm{AE} & 01 & 5 \mathrm{~B} & 03 & 46 & 20 & 35 & 00 & \mathrm{AE} & 45 & 85 & 08 & \mathrm{EA} & 01\end{array}$ $\begin{array}{lllllllllllllllll}04590 & 82 & 45 & 95 & 03 & \mathrm{BB} & 07 & \mathrm{C} 8 & 04 & \mathrm{BF} & 01 & 49 & 00 & 38 & 03 & \mathrm{AE} & 01\end{array}$ $\begin{array}{lllllllllllllllllll}045 \mathrm{~A} 0 & 82 & 45 & \mathrm{~B} 9 & 09 & 09 & \text { ID } & \mathrm{CF} & 40 & \mathrm{EA} & 0 \mathrm{~B} & 41 & 0 \mathrm{~B} & 4 \mathrm{D} & 65 & 6 \mathrm{D} & 6 \mathrm{~F}\end{array}$ $\begin{array}{lllllllllllllllll}045 \mathrm{~B} 0 & 72 & 79 & 20 & 64 & 75 & 6 \mathrm{D} & 70 & 09 & 09 & 09 & 09 & 01 & 49 & 00 & 10 & 00\end{array}$ $\begin{array}{lllllllllllllllll}045 \mathrm{C} 0 & \mathrm{D} 3 & 45 & 55 & 03 & 36 & 07 & 59 & 04 & \mathrm{E} 0 & 09 & 09 & \text { ID } & \mathrm{CF} & 02 & \mathrm{C} 0 & 88\end{array}$ $\begin{array}{lllllllllllllllll}045 \mathrm{D} 0 & 53 & 48 & 4 \mathrm{~F} & 57 & 2 \mathrm{E} & 56 & 41 & \mathrm{D} 2 & 45 & 29 & 08 & 57 & 07 & 59 & 04 & \mathrm{BF}\end{array}$ $\begin{array}{lllllllllllllllll}045 E 0 & 0 C & D C & 09 & 09 & 01 & 49 & 00 & 14 & 14 & A E & 0 B & 41 & 09 & 56 & 41 & 52\end{array}$ $\begin{array}{lllllllllllllllll}045 F 0 & 49 & 41 & 42 & 4 \mathrm{C} & 45 & 53 & 09 & 09 & 09 & 09 & 44 & \mathrm{FF} & 07 & 42 & 04 & \mathrm{BF}\end{array}$
Code.Q.Q.I.Q.Q.. B..A. Immediate. $\mathrm{H} . ? .=. . . \mathrm{B} 3$. $r B ; \ldots .0 @ j A) . Y . ?$. ..R.j.b..B..\}.Y. $\because$ O...CAT-UPDA TEAW.W.I...^.I.. .D.............. .P.U....I.-.D... [ .I...F.P........

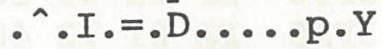
.......CATBW.W.I. ..^.I...D.I.P .. Q.VLIST2C4.W.Y.? $. \backslash \ldots . \mathrm{H}$...... .B.?.?......?.I .$<\ldots . \mathrm{C} 0 \ldots . . .$. .H.?.I.9.... C0.O aj........... .............. 3 ...U.Q.I......CX .C.O.rCZ.\}.I.... .?.g._.... Cp.rCv . . I. ........ Y.? ......j.b...cv.\}.. .O.Y. . ... INDEXC Q.W.A.Screen ind ex for:6'.@.DIR' D..W7\{...A.Clust er allocation ta ble..ci.....DIRE CTORIESD; .W.A.Di sk directories.. ..... \&DB..... sDB .0. @. INDEX2Dk.WD !.U... [.F4 ...DO. ..F. . O.C...F... j..DW...H.?.I.8. ....Dm...OQjD!... 7.......VCHDRD\$. W.A.Name. I ......A .Type.I.....A.Va lue.......DUMP2D w.W.A. Memory dum p.....Y.?.(.).g. D... [.F...I...D. ..C.I...D...g.g. [ .F.Q.C...D..Eq. .... [.F 5..E..j. .E....H.?.I.8... .E9...o@j.A.Memo ry dump......... SEU.6.Y. …0.@. SHOW.VARE) .W.Y.? . $1 \ldots$. . . ...... VAR IABLES....D..B.? 
$\begin{array}{lllllllllllllllll}04600 & 04 & \text { BF } & 0 B & E 7 & 04 & 9 C & 0 A & \text { E5 } & 04 & 9 \mathrm{C} & 04 & \text { BF } & 01 & 49 & 10 & 62\end{array}$ $\begin{array}{lllllllllllllllll}04610 & 03 & \mathrm{E} 7 & 04 & \mathrm{BF} & 03 & 73 & 01 & 82 & 46 & 44 & 04 & 9 \mathrm{C} & 3 \mathrm{C} & 86 & 04 & \mathrm{E} 0\end{array}$ $\begin{array}{lllllllllllllllll}04620 & 06 & 08 & 07 & 0 \mathrm{~F} & 04 & \mathrm{E} 0 & 3 \mathrm{C} & \mathrm{Cl} & 07 & \mathrm{OF} & 04 & \mathrm{BF} & 01 & 49 & 00 & 0 \mathrm{E}\end{array}$ $\begin{array}{lllllllllllllllll}04630 & 13 & 5 \mathrm{~F} & 01 & 49 & 00 & 0 \mathrm{E} & 04 & 89 & 04 & 05 & 14 & \mathrm{AE} & 3 \mathrm{D} & \mathrm{C} 0 & 09 & 09\end{array}$ $\begin{array}{lllllllllllllllll}04640 & 01 & 72 & 46 & 46 & 04 & 7 \mathrm{D} & 0 \mathrm{~A} & \mathrm{D} 9 & 04 & \mathrm{BF} & 04 & 9 \mathrm{C} & 03 & 52 & 01 & 82\end{array}$ $\begin{array}{lllllllllllllllll}04650 & 46 & 5 \mathrm{~A} & 04 & 7 \mathrm{D} & 06 & 10 & 01 & 72 & 46 & 5 \mathrm{E} & 0 \mathrm{~B} & \mathrm{E} 7 & 06 & 08 & 08 & \mathrm{EA}\end{array}$ $\begin{array}{lllllllllllllllll}04660 & 02 & 62 & 01 & 82 & 46 & 04 & 07 & 59 & 04 & \mathrm{E} 0 & 09 & 09 & 02 & \mathrm{C} 0 & 89 & 53\end{array}$ $\begin{array}{lllllllllllllllll}04670 & 48 & 4 \mathrm{~F} & 57 & 2 \mathrm{E} & 55 & 53 & 45 & \mathrm{D} 2 & 45 & \mathrm{CF} & 08 & 57 & 07 & 59 & 04 & \mathrm{BF}\end{array}$

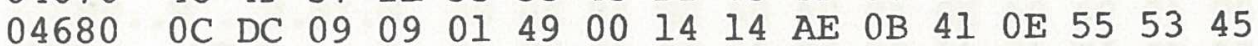
$\begin{array}{lllllllllllllllll}04690 & 52 & 20 & 76 & 61 & 72 & 69 & 61 & 62 & 6 \mathrm{C} & 65 & 73 & 09 & 09 & 09 & 09 & 07\end{array}$ $\begin{array}{lllllllllllllllll}046 \mathrm{AO} & 42 & 04 & \mathrm{BF} & 04 & \mathrm{BF} & 0 \mathrm{~B} & \mathrm{E} 7 & 0 \mathrm{~B} & 41 & 2 \mathrm{C} & 4 \mathrm{E} & 61 & 6 \mathrm{D} & 65 & 20 & 20\end{array}$

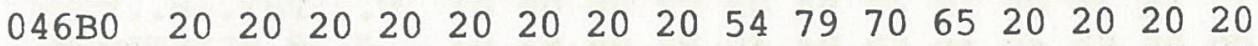

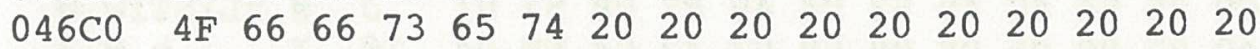
$\begin{array}{lllllllllllllllll}046 \mathrm{D} 0 & 20 & 56 & 61 & 6 \mathrm{C} & 75 & 65 & 09 & 09 & 09 & 09 & 04 & 9 \mathrm{C} & 0 \mathrm{~A} & \mathrm{E} 5 & 04 & 9 \mathrm{C}\end{array}$ $\begin{array}{lllllllllllllllll}046 \mathrm{E} 0 & 04 & \mathrm{BF} & 01 & 49 & 07 & 5 \mathrm{~B} & 0 \mathrm{~A} & \mathrm{CD} & 04 & \mathrm{BF} & 03 & 73 & 01 & 82 & 47 & 1 \mathrm{~A}\end{array}$ $\begin{array}{lllllllllllllllll}046 \mathrm{~F} 0 & 04 & 9 \mathrm{C} & 3 \mathrm{C} & 86 & 04 & \mathrm{E} 0 & 06 & 08 & 07 & 0 \mathrm{~F} & 04 & \mathrm{E} 0 & 3 \mathrm{C} & \mathrm{Cl} & 07 & 0 \mathrm{~F}\end{array}$ $\begin{array}{lllllllllllllllll}04700 & 04 & \mathrm{BF} & 01 & 49 & 00 & 0 \mathrm{E} & 13 & 5 \mathrm{~F} & 01 & 49 & 00 & 0 \mathrm{E} & 04 & 89 & 04 & 05\end{array}$ $\begin{array}{lllllllllllllllll}04710 & 14 & \mathrm{AE} & 3 \mathrm{D} & 83 & 09 & 09 & 01 & 72 & 47 & 1 \mathrm{C} & 04 & 7 \mathrm{D} & 0 \mathrm{~A} & \mathrm{D} 9 & 04 & \mathrm{BF}\end{array}$ $\begin{array}{lllllllllllllllll}04720 & 04 & 9 \mathrm{C} & 03 & 52 & 01 & 82 & 47 & 30 & 04 & 7 \mathrm{D} & 06 & 10 & 01 & 72 & 47 & 34\end{array}$ $\begin{array}{lllllllllllllllll}04730 & 0 B & E 7 & 06 & 08 & 08 & E A & 02 & 62 & 01 & 82 & 46 & D A & 07 & 59 & 04 & E 0\end{array}$ $\begin{array}{lllllllllllllllll}04740 & 09 & 09 & 02 & \mathrm{C} 0 & 8 \mathrm{~A} & 53 & 48 & 4 \mathrm{~F} & 57 & 2 \mathrm{E} & 43 & 4 \mathrm{~F} & 4 \mathrm{E} & 53 & \mathrm{D} 4 & 46\end{array}$ $\begin{array}{lllllllllllllllll}04750 & 6 \mathrm{E} & 08 & 57 & 07 & 59 & 04 & \mathrm{BF} & 0 \mathrm{C} & \mathrm{DC} & 09 & 09 & 01 & 49 & 00 & 14 & 14\end{array}$ $\begin{array}{lllllllllllllllll}04760 & \mathrm{AE} & 0 \mathrm{~B} & 41 & 09 & 43 & 4 \mathrm{~F} & 4 \mathrm{E} & 53 & 54 & 41 & 4 \mathrm{E} & 54 & 53 & 09 & 09 & 09\end{array}$ $\begin{array}{lllllllllllllllll}04770 & 09 & 44 & \mathrm{FF} & 07 & 42 & 04 & \mathrm{BF} & 04 & \mathrm{BF} & 0 \mathrm{~B} & \mathrm{E} 7 & 04 & 9 \mathrm{C} & 0 \mathrm{~A} & \mathrm{E} 5 & 04\end{array}$ $\begin{array}{lllllllllllllllll}04780 & 9 \mathrm{C} & 04 & \mathrm{BF} & 01 & 49 & 06 & \text { IA } & 0 \mathrm{~A} & \mathrm{CD} & 04 & \mathrm{BF} & 03 & 73 & 01 & 82 & 47\end{array}$ $\begin{array}{lllllllllllllllll}04790 & \mathrm{BB} & 04 & 9 \mathrm{C} & 3 \mathrm{C} & 86 & 04 & \mathrm{E} 0 & 06 & 08 & 07 & 0 \mathrm{~F} & 04 & \mathrm{E} 0 & 3 \mathrm{C} & \mathrm{Cl} & 07\end{array}$ $\begin{array}{lllllllllllllllll}047 \mathrm{AO} & 0 \mathrm{~F} & 04 & \mathrm{BF} & 01 & 49 & 00 & 0 \mathrm{E} & 13 & 5 \mathrm{~F} & 01 & 49 & 00 & 0 \mathrm{E} & 04 & 89 & 04\end{array}$ $\begin{array}{lllllllllllllllll}047 \mathrm{~B} 0 & 05 & 14 & \mathrm{AE} & 3 \mathrm{D} & 59 & 09 & 09 & 01 & 72 & 47 & \mathrm{BD} & 04 & 7 \mathrm{D} & 0 \mathrm{~A} & \mathrm{D} 9 & 04\end{array}$ $\begin{array}{lllllllllllllllll}047 \mathrm{C} 0 & \mathrm{BF} & 04 & 9 \mathrm{C} & 03 & 52 & 01 & 82 & 47 & \mathrm{D} 1 & 04 & 7 \mathrm{D} & 06 & 10 & 01 & 72 & 47\end{array}$ $\begin{array}{lllllllllllllllll}047 D 0 & \text { D5 } & 0 B & E 7 & 06 & 08 & 08 & E A & 02 & 62 & 01 & 82 & 47 & 7 B & 07 & 59 & 04\end{array}$ $\begin{array}{lllllllllllllllll}047 \mathrm{E} 0 & \mathrm{E} 0 & 09 & 09 & 02 & \mathrm{C} 0 & 8 \mathrm{~A} & 53 & 48 & 4 \mathrm{~F} & 57 & 2 \mathrm{E} & 56 & 4 \mathrm{~F} & 43 & 41 & \mathrm{C} 2\end{array}$ $\begin{array}{lllllllllllllllll}047 \mathrm{~F} 0 & 47 & 44 & 08 & 57 & 07 & 59 & 04 & \mathrm{BF} & 0 \mathrm{C} & \mathrm{DC} & 09 & 09 & 01 & 49 & 00 & 14\end{array}$ $\begin{array}{lllllllllllllllll}04800 & 14 & \mathrm{AE} & 0 \mathrm{~B} & 41 & 0 \mathrm{C} & 56 & 4 \mathrm{~F} & 43 & 41 & 42 & 55 & 4 \mathrm{C} & 41 & 52 & 49 & 45\end{array}$ $\begin{array}{lllllllllllllllll}04810 & 53 & 09 & 09 & 09 & 09 & 07 & 42 & 04 & \mathrm{BF} & 04 & \mathrm{BF} & 0 \mathrm{~B} & \mathrm{E} 7 & 04 & 9 \mathrm{C} & 0 \mathrm{~A}\end{array}$ $\begin{array}{lllllllllllllllll}04820 & \mathrm{E} 5 & 04 & 9 \mathrm{C} & 04 & \mathrm{BF} & 01 & 49 & 18 & 39 & 0 \mathrm{~A} & \mathrm{CD} & 04 & \mathrm{BF} & 03 & 73 & 01\end{array}$ $\begin{array}{lllllllllllllllll}04830 & 82 & 48 & 45 & 04 & 9 \mathrm{C} & 3 \mathrm{C} & 86 & 04 & \mathrm{E} 0 & 3 \mathrm{E} & 12 & 09 & 09 & 06 & 18 & 03\end{array}$ $\begin{array}{lllllllllllllllll}04840 & \mathrm{C} 4 & 01 & 72 & 48 & 47 & 04 & 7 \mathrm{D} & 0 \mathrm{~A} & \mathrm{D} 9 & 04 & \mathrm{BF} & 04 & 9 \mathrm{C} & 03 & 52 & 01\end{array}$ $\begin{array}{lllllllllllllllll}04850 & 82 & 48 & 5 \mathrm{~F} & 04 & 7 \mathrm{D} & 04 & 7 \mathrm{D} & 04 & 7 \mathrm{D} & 06 & 10 & 01 & 72 & 48 & 63 & 0 \mathrm{~B}\end{array}$ $\begin{array}{lllllllllllllllll}04860 & \text { E7 } & 06 & 08 & 08 & \text { EA } & 02 & 62 & 01 & 82 & 48 & 1 D & 07 & 59 & 04 & \text { E0 } & 09\end{array}$ $\begin{array}{lllllllllllllllll}04870 & 09 & 02 & \text { C0 } & 85 & 5 A & 46 & 4 C & 41 & \text { C7 } & 47 & \text { E5 } & 06 & 90 & 00 & 62 & 86\end{array}$ $\begin{array}{lllllllllllllllll}04880 & 5 \mathrm{~A} & 5 \mathrm{~A} & 44 & 49 & 43 & \mathrm{D} 4 & 48 & 73 & 06 & 90 & 00 & 64 & 85 & 5 \mathrm{~A} & 48 & 45\end{array}$ $\begin{array}{lllllllllllllllll}04890 & 52 & \mathrm{C} 5 & 48 & 7 \mathrm{~F} & 06 & 90 & 00 & 66 & 85 & 5 \mathrm{~A} & 5 \mathrm{~A} & 45 & 4 \mathrm{E} & \mathrm{C} 4 & 48 & 8 \mathrm{C}\end{array}$ $\begin{array}{lllllllllllllllll}048 \mathrm{~A} 0 & 06 & 90 & 00 & 68 & 85 & 5 \mathrm{~A} & 4 \mathrm{E} & 41 & 4 \mathrm{D} & \mathrm{C} 5 & 48 & 98 & 08 & 57 & 06 & 08\end{array}$ $\begin{array}{lllllllllllllllll}048 \mathrm{~B} 0 & 48 & 7 \mathrm{~B} & 04 & \mathrm{E} 0 & 1 \mathrm{~A} & \mathrm{D} 5 & 04 & \mathrm{Dl} & 01 & 49 & 00 & 1 \mathrm{~F} & 02 & 51 & 04 & 89\end{array}$ $\begin{array}{lllllllllllllllll}048 \mathrm{C} 0 & 04 & \mathrm{Dl} & 01 & 49 & 00 & \mathrm{IF} & 02 & 51 & \text { OF } & 57 & 03 & \mathrm{D} 5 & 06 & 10 & 01 & 5 \mathrm{~B}\end{array}$ $\begin{array}{lllllllllllllllll}048 \mathrm{D} 0 & 1 \mathrm{~A} & \mathrm{D} 5 & 03 & 46 & 03 & \mathrm{C} 4 & 04 & \mathrm{Dl} & 01 & 49 & 00 & 7 \mathrm{~F} & 02 & 51 & 04 & 89\end{array}$ $\begin{array}{lllllllllllllllll}048 \mathrm{E} 0 & 03 & 46 & 03 & \mathrm{C} 4 & 04 & \mathrm{DI} & 01 & 49 & 00 & 7 \mathrm{~F} & 02 & 51 & \text { IA } & \mathrm{D} 5 & 03 & \mathrm{AE}\end{array}$ $\begin{array}{lllllllllllllllll}048 \mathrm{~F} 0 & 01 & 82 & 48 & \mathrm{FC} & 06 & 10 & 48 & 7 \mathrm{~B} & 04 & \mathrm{E} 0 & 03 & 1 \mathrm{~B} & 03 & 90 & 01 & 82\end{array}$ $\begin{array}{lllllllllllllllll}04900 & 49 & 0 \mathrm{C} & 01 & 49 & \mathrm{FF} & \mathrm{FF} & 48 & 7 \mathrm{~B} & 04 & \mathrm{E} 0 & 03 & 1 \mathrm{~B} & 00 & \mathrm{AE} & 48 & \mathrm{D} 0\end{array}$ $\begin{array}{lllllllllllllllll}04910 & 48 & 7 \mathrm{~B} & 04 & \mathrm{BF} & 03 & 52 & 01 & 82 & 49 & 32 & 04 & \mathrm{Dl} & 01 & 49 & 00 & 1 \mathrm{~F}\end{array}$ $\begin{array}{lllllllllllllllll}04920 & 02 & 51 & 04 & 89 & 04 & \mathrm{DI} & 01 & 49 & 00 & \text { IF } & 02 & 51 & 03 & \mathrm{AE} & 01 & 72\end{array}$ $\begin{array}{lllllllllllllllll}04930 & 49 & 3 \mathrm{C} & 1 \mathrm{~A} & \mathrm{C} 6 & 48 & 7 \mathrm{~B} & 04 & \mathrm{BF} & 06 & 08 & 03 & \mathrm{AE} & 02 & \mathrm{C} 0 & 87 & 5 \mathrm{~A}\end{array}$ $\begin{array}{lllllllllllllllll}04940 & 49 & 4 \mathrm{E} & 53 & 45 & 52 & \mathrm{D} 4 & 48 & \mathrm{~A} 4 & 08 & 57 & 48 & 94 & 04 & \mathrm{BF} & 04 & 9 \mathrm{C}\end{array}$ $\begin{array}{lllllllllllllllll}04950 & 06 & 18 & 03 & \mathrm{C} 4 & 48 & \mathrm{~A} 0 & 04 & \mathrm{BF} & 48 & 94 & 04 & \mathrm{BF} & 04 & 05 & 1 \mathrm{~B} & \mathrm{C} 6\end{array}$ $\begin{array}{lllllllllllllllll}04960 & 06 & 18 & 48 & \mathrm{~A} 0 & 03 & \mathrm{~F} 2 & 48 & 88 & 04 & \mathrm{BF} & 48 & 94 & 04 & \mathrm{BF} & 04 & \mathrm{E} 0\end{array}$ $\begin{array}{lllllllllllllllll}04970 & 02 & \text { C0 } & 86 & 5 A & 50 & 52 & 49 & 4 E & D 4 & 49 & 3 E & 08 & 57 & 07 & 59 & 04\end{array}$
.?.g...e...?.I.b . 9. ?.S..FD..... ..... $<$ A...?.I..

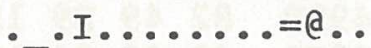
. $\overline{r F F}.\} . Y . ? \ldots R$... FZ.\}...rF^.g...j .b..F...Y. '...... HOW. USEREO.W.Y.? . $. . . . . . . . A . U S E$ $\mathrm{R}$ variables.... B.?.?.g.A, Name Type

Offset

Value......e... .?.I.[.M.?.S..G. ... $<$..... $<$ A. $^{\circ}$ .?.I..._.I..... $\left.\ldots=\ldots . \bar{r} G_{0}.\right\} . Y . ?$ ...R..G0.\} ...rG4 .g...j.b..FZ.Y. ......SHOW. CONSTF n.W.Y.?.\...I... ..A.CONSTANTS... .D..B.?.?.g...e. ..?.I...M.?.S..G $i \ldots<\ldots . . .<<$. ..?.I..._.I.... $\left.\ldots=Y_{\ldots} . \bar{r} G=.\right\} . Y$.

?...R..GQ. $\}$...rG U.g...j.b..G\{.Y. ...... SHOW. VOCAB GD.W.Y.?. \...I.. ...A. VOCABULARIE S......?.?.g... e...?.I.9.M.?.s. $. \mathrm{HE} .<\ldots>\ldots .$. D.rHG..$Y_{\bullet} ? \ldots R$. . $\left.\left.\left.\mathrm{H}_{-}\right\}_{\bullet}\right\}.\right\} \ldots \mathrm{rHC}$. g...j.b....... ....ZFLAGGe...b. ZZDICTHS ...d.ZHE REH.... .ZZENDH. ...h. ZNAMEH ..W. . $\mathrm{H}\{$ ○.U.Q.I...Q. .Q.I...Q.W.U... [ .U.F.D.Q.I..Q. .F.D.Q.I..Q.U.. $\ldots H \mid \ldots H\{. \ldots .$.

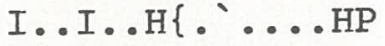
$H\{$. .R..I2 .Q.I.. .Q...Q.I...Q...r $I<. F H\{. ? . . .$. Q.Z INSERTHS.WH..?.. $\ldots$..DH . ?H..?...F . $\mathrm{H} . \mathrm{rH} . . ? \mathrm{H} \ldots ?$. . . ZPRINTI>.W.Y. 
$\begin{array}{lllllllllllllllll}04980 & \text { BF } & \text { OC } & \text { DC } & \text { ID } & 9 B & 48 & \text { A0 } & 04 & \text { BF } & 01 & 49 & \text { C0 } & 00 & 01 & 5 B & 07\end{array}$ $\begin{array}{lllllllllllllllll}04990 & 0 \mathrm{~F} & 04 & \mathrm{BF} & 01 & 49 & 00 & 3 \mathrm{C} & 03 & \mathrm{AE} & 01 & 82 & 49 & \mathrm{~B} 9 & 09 & 09 & 06\end{array}$ $\begin{array}{lllllllllllllllll}049 \mathrm{~A} 0 & 08 & 07 & 0 \mathrm{~F} & 04 & \mathrm{E} 0 & 07 & \mathrm{C} 8 & 04 & \mathrm{BF} & 01 & 49 & 00 & 38 & 03 & \mathrm{AE} & 01\end{array}$

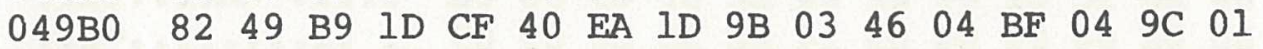
$\begin{array}{lllllllllllllllll}049 \mathrm{C} 0 & 49 & 00 & 04 & 1 \mathrm{~B} & 44 & 09 & 63 & \text { OF } & 6 \mathrm{~F} & 01 & 49 & 00 & 14 & 07 & \text { OF } & 04\end{array}$ $\begin{array}{lllllllllllllllll}049 \mathrm{DC} & \mathrm{BF} & 04 & 67 & 13 & 5 \mathrm{~F} & 04 & 9 \mathrm{C} & 01 & 82 & 49 & \mathrm{DF} & 01 & 72 & 49 & \mathrm{E} 5 & 04\end{array}$ $\begin{array}{lllllllllllllllll}049 \mathrm{E} 0 & 7 \mathrm{D} & 01 & 49 & 00 & 14 & 04 & 05 & 14 & \mathrm{AE} & 06 & 18 & 00 & \mathrm{D} 3 & 49 & 8 \mathrm{~F} & 07\end{array}$ $\begin{array}{lllllllllllllllll}049 \mathrm{~F} 0 & 59 & 04 & \mathrm{E} 0 & 1 \mathrm{D} & \mathrm{CF} & 02 & \mathrm{C} 0 & 85 & 5 \mathrm{~A} & 53 & 4 \mathrm{~F} & 52 & \mathrm{D} 4 & 49 & 72 & 08\end{array}$ $\begin{array}{lllllllllllllllll}04 \mathrm{~A} 00 & 57 & 01 & 49 & \mathrm{C} 0 & 00 & 48 & 94 & 04 & \mathrm{E} 0 & 48 & 94 & 04 & \mathrm{BF} & 48 & \mathrm{~A} 0 & 04\end{array}$ $\begin{array}{lllllllllllllllll}04 \mathrm{Al} 0 & \mathrm{BF} & 03 & 73 & 01 & 82 & 4 \mathrm{~A} & \text { IF } & 49 & 48 & 06 & 10 & 01 & 72 & 4 \mathrm{~A} & 3 \mathrm{~F} & 48\end{array}$ $\begin{array}{lllllllllllllllll}04 \mathrm{~A} 20 & 88 & 04 & \mathrm{BF} & 48 & 94 & 04 & \mathrm{BF} & 04 & \mathrm{BF} & 48 & \mathrm{AC} & 01 & 82 & 4 \mathrm{~A} & 37 & 49\end{array}$ $\begin{array}{llllllllllllllllll}04 \mathrm{~A} 30 & 48 & 06 & 10 & 01 & 72 & 4 \mathrm{~A} & 3 \mathrm{~F} & 06 & 18 & 48 & 94 & 03 & \text { F2 } & 06 & 08 & 01\end{array}$ $\begin{array}{lllllllllllllllll}04 \mathrm{~A} 40 & 82 & 4 \mathrm{~A} & 09 & 02 & \mathrm{C} 0 & 85 & 5 \mathrm{~A} & 4 \mathrm{C} & 49 & 53 & \mathrm{D} 4 & 49 & \text { F7 } & 08 & 57 & 01\end{array}$ $\begin{array}{lllllllllllllllll}04 \mathrm{~A} 50 & 49 & \mathrm{C} 0 & 00 & 01 & 49 & 07 & 00 & 06 & 08 & 08 & 68 & 07 & 42 & 04 & \mathrm{BF} & 04\end{array}$ $\begin{array}{lllllllllllllllll}04 \mathrm{~A} 60 & \mathrm{BF} & 04 & 9 \mathrm{C} & 48 & 88 & 04 & \mathrm{E} 0 & 01 & 49 & \mathrm{C} 0 & 00 & 04 & 9 \mathrm{C} & 04 & 9 \mathrm{C} & 48\end{array}$ $\begin{array}{llllllllllllllllll}04 \mathrm{~A} 70 & 94 & 04 & \mathrm{E} 0 & 06 & 18 & 03 & \mathrm{C} 4 & 48 & \mathrm{A0} & 04 & \mathrm{E} 0 & 04 & \mathrm{E} 0 & 48 & 88 & 04\end{array}$ $\begin{array}{lllllllllllllllll}04 \mathrm{~A} 80 & \mathrm{BF} & 0 \mathrm{~B} & \mathrm{E} 7 & 0 \mathrm{~A} & \mathrm{D} 9 & 04 & \mathrm{BF} & 04 & 9 \mathrm{C} & 03 & 52 & 01 & 82 & 4 \mathrm{~A} & 97 & 04\end{array}$ $\begin{array}{lllllllllllllllll}04 \mathrm{~A} 90 & 7 \mathrm{D} & 06 & 10 & 01 & 72 & 4 \mathrm{~A} & 9 \mathrm{~F} & 48 & 88 & 04 & \mathrm{E} 0 & 49 & \mathrm{FF} & 06 & 08 & 08\end{array}$ $\begin{array}{lllllllllllllllll}04 A A 0 & E A & 02 & 62 & 01 & 82 & 4 A & 7 D & 49 & 7 B & 02 & C 0 & 88 & 5 A & 5 A & 45 & 44\end{array}$ $\begin{array}{lllllllllllllllll}04 \mathrm{ABO} & 4 \mathrm{C} & 49 & 53 & \mathrm{D} 4 & 4 \mathrm{~A} & 45 & 08 & 57 & 01 & 49 & 22 & \mathrm{BF} & 0 \mathrm{~A} & \mathrm{D} 9 & 04 & 9 \mathrm{C}\end{array}$ $\begin{array}{lllllllllllllllll}04 \mathrm{AC} 0 & 04 & 9 \mathrm{C} & 04 & \mathrm{BF} & 04 & 89 & 04 & \mathrm{FE} & 06 & 08 & 04 & 89 & 04 & \mathrm{E} 0 & 22 & 57\end{array}$

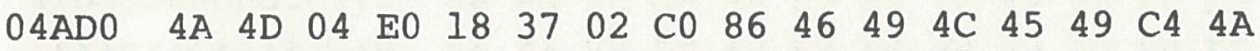
$\begin{array}{lllllllllllllllll}04 \mathrm{AE} 0 & \mathrm{AB} & 08 & 57 & 38 & 98 & 36 & \mathrm{~A} 7 & 09 & 09 & 02 & \mathrm{C} 0 & 83 & 43 & 52 & \mathrm{BF} & 4 \mathrm{~A}\end{array}$ $\begin{array}{lllllllllllllllll}04 A F 0 & D 8 & 08 & 57 & 09 & 09 & 07 & C 8 & 04 & B F & 01 & 49 & 00 & 04 & 03 & \text { C4 } & 06\end{array}$

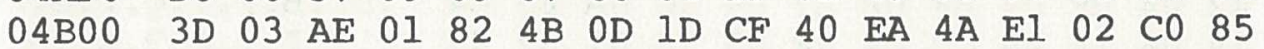
$\begin{array}{lllllllllllllllll}04 \mathrm{~B} 10 & 2 \mathrm{E} & 46 & 69 & 6 \mathrm{C} & \mathrm{E} 5 & 4 \mathrm{~A} & \mathrm{~EB} & 08 & 57 & 04 & 9 \mathrm{C} & 01 & 49 & 00 & 0 \mathrm{C} & 03\end{array}$ $\begin{array}{lllllllllllllllll}04 \mathrm{~B} 20 & 73 & 01 & 82 & 4 \mathrm{~B} & 2 \mathrm{~B} & 04 & 7 \mathrm{D} & 01 & 72 & 4 \mathrm{~B} & 69 & 04 & 9 \mathrm{C} & 01 & 49 & 00\end{array}$ $\begin{array}{lllllllllllllllll}04 \mathrm{~B} 30 & 0 \mathrm{~A} & 03 & 73 & 01 & 82 & 4 \mathrm{~B} & 3 \mathrm{D} & 04 & 7 \mathrm{D} & 01 & 72 & 4 \mathrm{~B} & 69 & 04 & 9 \mathrm{C} & 01 .\end{array}$ $\begin{array}{lllllllllllllllll}04 \mathrm{~B} 40 & 49 & 00 & 13 & 03 & 73 & 01 & 82 & 4 \mathrm{~B} & 53 & 04 & 7 \mathrm{D} & 04 & 7 \mathrm{D} & 06 & 10 & 01\end{array}$ $\begin{array}{lllllllllllllllll}04 \mathrm{~B} 50 & 72 & 4 \mathrm{~B} & 69 & 04 & 9 \mathrm{C} & 01 & 49 & 00 & 0 \mathrm{D} & 03 & 73 & 01 & 82 & 4 \mathrm{~B} & 67 & 4 \mathrm{~A}\end{array}$ $\begin{array}{lllllllllllllllll}04 \mathrm{~B} 60 & \mathrm{~F} 1 & 04 & 7 \mathrm{D} & 01 & 72 & 4 \mathrm{~B} & 69 & 08 & \mathrm{FC} & 02 & \mathrm{C} 0 & 88 & 2 \mathrm{E} & 46 & 49 & 4 \mathrm{C}\end{array}$ $\begin{array}{lllllllllllllllll}04 \mathrm{~B} 70 & 45 & 53 & 45 & \mathrm{C} 3 & 4 \mathrm{~B} & 0 \mathrm{~F} & 08 & 57 & 04 & 9 \mathrm{C} & 01 & 49 & 02 & 00 & 03 & \mathrm{C} 4\end{array}$ $\begin{array}{llllllllllllllllll}04 \mathrm{~B} 80 & 04 & 89 & 01 & 5 \mathrm{~B} & 03 & 46 & 04 & \mathrm{Dl} & 04 & 9 \mathrm{C} & 35 & 43 & 03 & 73 & 08 & \mathrm{EA}\end{array}$ $\begin{array}{lllllllllllllllll}04 \mathrm{~B} 90 & 02 & 62 & 01 & 82 & 4 \mathrm{~B} & \mathrm{~A} 0 & 04 & 7 \mathrm{D} & 06 & 10 & 03 & 1 \mathrm{~B} & 01 & 72 & 4 \mathrm{~B} & \mathrm{~A} 2\end{array}$ $\begin{array}{lllllllllllllllll}04 \mathrm{BA0} & 4 \mathrm{~B} & 17 & 00 & \mathrm{AE} & 4 \mathrm{~B} & 84 & 02 & \mathrm{C} 0 & 85 & 2 \mathrm{E} & 46 & 49 & 4 \mathrm{C} & \mathrm{C} 5 & 4 \mathrm{~B} & 6 \mathrm{~B}\end{array}$ $\begin{array}{lllllllllllllllll}04 \mathrm{BB} 0 & 08 & 57 & 38 & \mathrm{~B} 9 & 4 \mathrm{~A} & \mathrm{E} 1 & 06 & 08 & 35 & \mathrm{~F} 5 & 04 & 9 \mathrm{C} & 01 & 82 & 4 \mathrm{~B} & \mathrm{C} 6\end{array}$ $\begin{array}{lllllllllllllllll}04 \mathrm{BC} 0 & 4 \mathrm{~B} & 76 & 01 & 72 & 4 \mathrm{~B} & \mathrm{C} 8 & 04 & 7 \mathrm{D} & 01 & 82 & 4 \mathrm{~B} & \mathrm{~B} 6 & 02 & \mathrm{C} 0 & 89 & 4 \mathrm{C}\end{array}$ $\begin{array}{lllllllllllllllll}04 \mathrm{BD0} & 49 & 53 & 54 & 46 & 49 & 4 \mathrm{C} & 45 & \mathrm{D} 3 & 4 \mathrm{~B} & \mathrm{~A} 8 & 08 & 57 & 18 & 25 & 01 & 49\end{array}$ $\begin{array}{lllllllllllllllll}04 \mathrm{BEO} & 02 & 16 & 1 \mathrm{~A} & 2 \mathrm{D} & 18 & 73 & 02 & \mathrm{C} 0 & 05 & 44 & 55 & 4 \mathrm{D} & 50 & 32 & 20 & 20\end{array}$
?.】... H ?.I@... [ ..?.I.<....I9 ... ……?.I.8... .I9.0@j....F.?... I...D.C.O.I..... ?.g._.......rIe. \}. I. .........SI. Y. .0.@.ZSORTIr. W.I@.H.. 'H..?H . ?.S..J.IH...rJ?H ..?H..?.?H, ..J7I H...rJ?......... .J..@.ZLISTIw.W. I ... I.....h.B.?. ?..H...I@.....H $\ldots \ldots$ DH $\because$ H... ?.g.Y.?...R... J. \}...rJ.H...... j.b..J\} I . a.ZZED LISTJE.W.I"?.Y.. ..?... .... "W JM. .7.@.FILEIDJ +.W8. $6^{\prime} \ldots$... C.CR?J X.W...H.?.I...D. =....K..o@jJa.@. .FileJk.W....... s..K+.\}.rKi...I. ..s...K=.\}.rKi... I.......KS.\}.\}... rKi...I...s...KgJ q.\}.rKi.1.Q..FIL ESECK..W...I...D ... [.F.Q..5C.S.j .b..K.\}....r.rk" K...K..@..FILEKk .W89Ja..5u .... KF Kv.rKH.\}..K6.@.I ISTFILESK (.W.\%.I ...-.s.a.DUMP2 


\section{Examples}

This chapter is a collection of WFORTH examples. They are designed to be self-explanatory although not necessarily obvious. The examples are grouped in five general catagories: decompiler, FORTH DOs, the CASE statement, the FORTH Assembler, and Floating point examples. The load screen for all of the examples is screen 75, and each example first lists its individual load screen and then executes. A brief explanation of each catagory is given below.

Decompiler examples: The load screen for these examples is screen 221. The examples themselves are simply a decompilation of key FORTH words which in turn provide insights into how the words work. For instance, the word ABORT contains the FORTH sign on message. The word ID uses information contained in the word ABORT to print the task ID. The word ROMIT changes the dictionary pointer and calls NODISK and NEW-FENCE. The word MESSAGE prints the message number always in decimal without changing the number base.

FORTH Dos examples: The load screen for these examples is screen 224. The FORTH Dos examples are intended to show how to access files. First the file WFORTH.CM is opened, and GETSEC is executed, bringing in the first sector. The first 40 bytes are then dumped (note that this is the same as shown in chapter 19 for locations 0-003Fh). The next example illustrates the random access feature, using the word NEWLSN. The third example shows logical sector 0 of the file, which contains the file chaining information (refer to MPM 201, appendix E for more details) and the disk directory. Finally, two teaching words, MEMORY-MAP and IOCB-SHOW are compiled and executed.

CASE examples: The load screen for these examples is screen 247. The decompiler and full screen editor also use the CASE statement.

Assembler examples: The load screen for these examples is screen 241. The first assembler example shows the invocation procedure for the assembler. It defines a no-operation word, the simplest word possible. The next example defines a word called SEEQ (set q). Compare this with the WFORTH word SETQ, which has been defined using the CREATE statement. The equivalence of assembled words and created words is thus shown. Following this, a nontrivial example is provided by assembling the word PEMIT (printer emit). This example demonstrates how to deal with the computation stack and hardware I/O. Finally the words CODE and END-CODE are decompiled.

Floating point examples: The load screen for these examples is screen 223. These examples print the value of $\mathrm{Pi}$, take the square root of 3 , and solve two quadratic equations. 


\section{SCREEN 75}

0 ( EXAMPLES load screen)

( Akens, WHOI)

1 BASE @ DECIMAL

$$
2
$$

3 ( This screen generates chapter 20)

4

$5 \mathrm{CR}$

675 IIST

7221 LOAD (run decompiler examples)

8224 LOAD (run FORTH DOS examples)

9247 LOAD (run CASE examples) .FOOT PAGE

10241 LOAD ( run assembler examples) FLUSH

11223 LOAD ( run floating point examples) .FOOT PAGE

12

13

14 BASE ! ;S

15

Load 221 , 4BE8 (Decompiler example screen)

( Akens, WHOI)

SCREEN 221

0 (Decompiler example screen)

1 DECIMAL 221 LIST DECOMPIIE ID .FOOT PAGE

2 DECOMPILE ABORT DECOMPILE ROMIT .FOOT PAGE

3 DECOMPIILE MESSAGE

4 DECOMPILE ERROR

5 DECOMPILE >IN

6 DECOMPILE NOT

7 DECOMPILE WORD-79 .FOOT PAGE

8 DECOMPILE INDEX

9 DECOMPILE CMOVE>

10 DECOMPILE GRP .FOOT PAGE

11 DECOMPILE UART DECOMPILE TERM DECOMPILE TERM.GRP

12 DECOMPILE COMM DECOMPILE COMM.GRP .FOOT PAGE

13 DECOMPILE NEW-FENCE DECOMPILE NODISK .FOOT PAGE

14 DECOMPILE . FOOT DECOMPILE PAGE .FOOT PAGE

15 DECIMAL is

Decompiling ID

\begin{tabular}{|c|c|c|c|}
\hline CFA & (CFA) & & \\
\hline 1CD6 & 857 & : ID & \\
\hline $1 \mathrm{CD} 8$ & 149 & 4584 & (11E8h) \\
\hline ICDC & $49 C$ & DUP & \\
\hline $1 \mathrm{CDE}$ & 149 & 13 & (000Dh) \\
\hline 1CE2 & $3 C 4$ & + & \\
\hline 1CE4 & 149 & 16 & (0010h) \\
\hline 1CE8 & 933 & TYPE & \\
\hline ICEA & 963 & SPACE & \\
\hline 1CEC & 149 & 34 & $(0022 \mathrm{~h})$ \\
\hline $1 \mathrm{CF} 0$ & $3 C 4$ & + & \\
\hline ICF2 & 149 & 13 & (000Dh) \\
\hline $1 \mathrm{CF} 6$ & 933 & TYPE & \\
\hline ICF8 & $2 \mathrm{CO}$ & is & \\
\hline
\end{tabular}




\begin{tabular}{|c|c|c|}
\hline \multicolumn{3}{|c|}{ Decompiling ABORT } \\
\hline $11 \mathrm{E} 6$ & 857 & : $\mathrm{ABORT}$ \\
\hline $11 \mathrm{E} 8$ & 297 & SP! \\
\hline 11EA & CF2 & DECIMAL \\
\hline $11 \mathrm{EC}$ & 610 & 1 \\
\hline $11 \mathrm{EE}$ & $17 \mathrm{BC}$ & GROUP \\
\hline $11 \mathrm{~F} 0$ & 909 & $\mathrm{CR}$ \\
\hline $11 \mathrm{~F} 2$ & B41 & ."Woods Hole FORTH" \\
\hline 1205 & 909 & CR \\
\hline 1207 & B41 & ."Version 1.800" \\
\hline 1217 & 909 & CR \\
\hline 1219 & $167 \mathrm{~F}$ & EMPTY-BUFFERS \\
\hline $121 \mathrm{~B}$ & 649 & FIRST \\
\hline ]. 21D & $49 \mathrm{C}$ & DUP \\
\hline $121 \mathrm{~F}$ & $79 \mathrm{~F}$ & PREV \\
\hline 1221 & $4 \mathrm{E} 0$ & ! \\
\hline 1223 & 794 & USE \\
\hline 1225 & $4 \mathrm{E} 0$ & ! \\
\hline 1227 & 1837 & FORTH \\
\hline 1229 & $11 \mathrm{~A} 7$ & DEFINITIONS \\
\hline $122 \mathrm{~B}$ & 1873 & DRI \\
\hline $122 \mathrm{D}$ & 610 & 1 \\
\hline $122 \mathrm{~F}$ & $16 \mathrm{DE}$ & BLOCK \\
\hline 1231 & $47 \mathrm{D}$ & DROP \\
\hline 1233 & 18A9 & OK \\
\hline 1235 & $11 \mathrm{BA}$ & QUIT \\
\hline 1237 & $2 \mathrm{CO}$ & is \\
\hline
\end{tabular}

\begin{tabular}{|c|c|c|}
\hline CFA & (CFA) & Decompiling $R$ \\
\hline 1 E36 & 857 & : ROMIT \\
\hline 1E38 & A53 & HERE \\
\hline $1 \mathrm{E} 3 \mathrm{~A}$ & 149 & $2048(0800 \mathrm{~h})$ \\
\hline $1 \mathrm{E} 3 \mathrm{E}$ & $134 \mathrm{~F}$ & $/$ \\
\hline $1 \mathrm{E} 40$ & 3D5 & $1+$ \\
\hline $1 \mathrm{E} 42$ & 149 & $2048(0800 \mathrm{~h})$ \\
\hline $1 \mathrm{E} 46$ & 567 & * \\
\hline $1 \mathrm{E} 48$ & $49 \mathrm{C}$ & DUP \\
\hline $1 \mathrm{E} 4 \mathrm{~A}$ & 6E3 & DP \\
\hline $1 \mathrm{E} 4 \mathrm{C}$ & $4 \mathrm{EO}$ & $!$ \\
\hline $1 \mathrm{E} 4 \mathrm{E}$ & $\mathrm{ABC}$ & $1-$ \\
\hline $1 \mathrm{E} 50$ & B41 & . " ROM it from 0 to " \\
\hline $1 \mathrm{E} 64$ & $1 \mathrm{~B} 55$ & $\mathrm{HEX}$ \\
\hline $1 \mathrm{E} 66$ & $1 B 89$ & NODISK \\
\hline $1 \mathrm{E} 68$ & 1D53 & NEW-FENCE \\
\hline $1 \mathrm{E} 6 \mathrm{~A}$ & $2 \mathrm{C} 0$ & is \\
\hline
\end{tabular}




\begin{tabular}{|c|c|c|}
\hline \multicolumn{3}{|c|}{ Decompiling MESSAGE } \\
\hline CFA & \multicolumn{2}{|c|}{ (CFA) } \\
\hline $15 \mathrm{~B} 9$ & 857 & : MESSAGE \\
\hline $15 B B$ & B41 & " Message \\
\hline $15 \mathrm{C} 7$ & 759 & BASE \\
\hline $15 C 9$ & $4 \mathrm{BF}$ & a \\
\hline $15 \mathrm{CB}$ & 489 & SWAP \\
\hline $15 \mathrm{CD}$ & CF2 & DECIMAL \\
\hline $15 \mathrm{CF}$ & $158 \mathrm{E}$ & - \\
\hline $15 \mathrm{Dl}$ & 759 & BASE \\
\hline $15 \mathrm{D} 3$ & $4 \mathrm{E} 0$ & $!$ \\
\hline 15D5 & $2 \mathrm{CO}$ & ;S \\
\hline \multicolumn{3}{|c|}{ Decompiling ERROR } \\
\hline CFA & (CFA) & \\
\hline F28 & 857 & : ERROR \\
\hline F2A & $6 \mathrm{CE}$ & WARNING \\
\hline F2C & $4 \mathrm{BF}$ & a \\
\hline F2E & 367 & $0<$ \\
\hline F30 & 182 & BZ TO F36 \\
\hline F34 & $F \perp A$ & $(\mathrm{ABORT})$ \\
\hline F36 & A53 & HERE \\
\hline F38 & B03 & COUNT \\
\hline F3A & 933 & TYPE \\
\hline F3C & B4I & " ?" \\
\hline F41 & $15 \mathrm{~B} 9$ & MESSAGE \\
\hline F43 & 297 & SP! \\
\hline F45 & 705 & IN \\
\hline F47 & $4 \mathrm{BF}$ & a \\
\hline F49 & $6 \mathrm{FC}$ & BLK \\
\hline F 4B & $4 \mathrm{BF}$ & a \\
\hline F4D & $11 \mathrm{BA}$ & QUIT \\
\hline $\mathrm{F} 4 \mathrm{~F}$ & $2 \mathrm{CO}$ & is \\
\hline \multicolumn{3}{|c|}{ Decompiling >IN } \\
\hline CFA & (CFA) & \\
\hline $1 \mathrm{C} 3 \mathrm{~F}$ & 857 & $:>$ IN \\
\hline $1 C 41$ & 705 & IN \\
\hline $1 C 43$ & $2 \mathrm{CO}$ & ; $S$ \\
\hline \multicolumn{3}{|c|}{ Decompiling NOT } \\
\hline CFA & (CFA) & \\
\hline 1 C33 & 857 & : NOT \\
\hline $1 C 35$ & 352 & $0=$ \\
\hline $1 C 37$ & $2 \mathrm{CO}$ & is \\
\hline \multicolumn{3}{|c|}{ Decompiling WORD-79 } \\
\hline CFA & (CFA) & \\
\hline $1 \mathrm{C} 4 \mathrm{~F}$ & 857 & : WORD-79 \\
\hline $1 \mathrm{C} 51$ & $\mathrm{DFA}$ & WORD \\
\hline $1 C 53$ & A53 & HERE \\
\hline $1 C 55$ & $2 \mathrm{CO}$ & ;S \\
\hline
\end{tabular}




\begin{tabular}{|c|c|c|}
\hline \multicolumn{3}{|c|}{ Decompiling INDEX } \\
\hline CFA & $(\mathrm{CFA})$ & \\
\hline $349 C$ & 857 & : INDEX \\
\hline $349 \mathrm{E}$ & 3D5 & $1+$ \\
\hline $34 \mathrm{AO}$ & 489 & SWAP \\
\hline $34 \mathrm{~A} 2$ & $15 \mathrm{~B}$ & DO \\
\hline $34 \mathrm{~A} 4$ & 346 & I \\
\hline $34 \mathrm{~A} 6$ & $340 \mathrm{~A}$ & ? INDEX \\
\hline $34 \mathrm{~A} 8$ & 182 & BZ TO $34 \mathrm{BC}$ \\
\hline $34 \mathrm{AC}$ & 909 & $\mathrm{CR}$ \\
\hline $34 \mathrm{AE}$ & 346 & I \\
\hline $34 \mathrm{~B} 0$ & 620 & 3 \\
\hline $34 \mathrm{~B} 2$ & $156 \mathrm{~F}$ & . $\mathrm{R}$ \\
\hline $34 \mathrm{~B} 4$ & 963 & SPACE \\
\hline 34B6 & 608 & 0 \\
\hline $34 \mathrm{~B} 8$ & 346 & I \\
\hline $34 \mathrm{BA}$ & $161 \mathrm{~B}$ & . LINE \\
\hline $34 \mathrm{BC}$ & 8EA & ?TERMINAI \\
\hline 34BE & 182 & BZ TO $34 \mathrm{C} 4$ \\
\hline $34 \mathrm{C} 2$ & $31 \mathrm{~B}$ & LEAVE \\
\hline $34 \mathrm{C4}$ & $\mathrm{AE}$ & LOOP TO $34 A 4$ \\
\hline $34 \mathrm{C} 8$ & 909 & CR \\
\hline $34 \mathrm{CA}$ & $2 \mathrm{CO}$ & ;S \\
\hline
\end{tabular}

\begin{tabular}{|c|c|c|}
\hline \multicolumn{3}{|c|}{ Decompiling CMOVE> } \\
\hline CFA & (CFA) & \\
\hline $1 \mathrm{BC} 6$ & 857 & : CMOVE> \\
\hline $1 \mathrm{BC} 8$ & $49 C$ & DUP \\
\hline $1 \mathrm{BCA}$ & $4 \mathrm{FE}$ & ROT \\
\hline IBCC & $3 C 4$ & + \\
\hline IBCE & $4 F E$ & ROT \\
\hline $1 \mathrm{BD} 0$ & $4 \mathrm{FE}$ & ROT \\
\hline $1 \mathrm{BD} 2$ & $49 C$ & DUP \\
\hline 1BD4 & $4 \mathrm{FE}$ & ROT \\
\hline $1 \mathrm{BD} 6$ & $3 C 4$ & + \\
\hline 1BD8 & 489 & SWAP \\
\hline IBDA & 3D5 & $1+$ \\
\hline IBDC & 610 & 1 \\
\hline IBDE & $15 \mathrm{~B}$ & DO \\
\hline 1BEO & $1 \mathrm{AD} 5$ & 2DUP \\
\hline $1 \mathrm{BE} 2$ & 346 & $I$ \\
\hline 1BE4 & 405 & - \\
\hline 1BE6 & $4 \mathrm{Dl}$ & c@ \\
\hline 1BE8 & 489 & SWAP \\
\hline 1BEA & 346 & I \\
\hline 1BEC & 405 & - \\
\hline 1BEE & $4 \mathrm{FO}$ & $C !$ \\
\hline $1 \mathrm{BF} 0$ & $\mathrm{AE}$ & LOOP TO $1 \mathrm{BE} 0$ \\
\hline 1BF4 & $1 \mathrm{AC} 6$ & 2DROP \\
\hline $1 \mathrm{BF} 6$ & $2 \mathrm{CO}$ & ;S \\
\hline
\end{tabular}

Decompiling GRP

$\begin{array}{llll}\text { CFA } & \text { (CFA) } \\ \text { 7D2 } & 690 & \text { USER } & 68(0044 \mathrm{~h}), \text { Currently }= \\ & & 1(000 \mathrm{~h})\end{array}$

Woods Hole FORTH Version 1.800 Chapter 20

page 133 


\begin{tabular}{|c|c|c|}
\hline $\begin{array}{l}\text { Decon } \\
\text { CFA }\end{array}$ & (CFA) & \\
\hline 29FA & 857 & : UART \\
\hline $29 F C$ & B41 & ." Uart at group \\
\hline $2 \mathrm{AOD}$ & 759 & BASE \\
\hline $2 \mathrm{AOF}$ & $4 \mathrm{BF}$ & a \\
\hline $2 \mathrm{~A} 11$ & $\mathrm{CDC}$ & HEX \\
\hline $2 \mathrm{~A} 13$ & 800 & COMM.GRP \\
\hline $2 \mathrm{~A} 15$ & $4 \mathrm{BF}$ & a \\
\hline $2 \mathrm{~A} 17$ & $158 \mathrm{E}$ & . \\
\hline $2 \mathrm{~A} 19$ & 909 & $\mathrm{CR}$ \\
\hline $2 A \perp B$ & 759 & BASE \\
\hline 2AID & $4 \mathrm{E} 0$ & ! \\
\hline $2 \mathrm{~A} \perp \mathrm{F}$ & 2867 & COMM \\
\hline $2 \mathrm{~A} 21$ & $28 B 1$ & COMMINIT \\
\hline $2 \mathrm{~A} 23$ & $292 \mathrm{~F}$ & RSTBRK \\
\hline $2 \mathrm{~A} 25$ & 608 & 0 \\
\hline $2 \mathrm{~A} 27$ & 2867 & COMM \\
\hline $2 \mathrm{~A} 29$ & 2836 & STATUS \\
\hline $2 \mathrm{~A} 2 \mathrm{~B}$ & 2910 & RCVCHAR? \\
\hline 2.A2D & 2878 & TERM \\
\hline $2 \mathrm{~A} 2 \mathrm{~F}$ & 2836 & STATUS \\
\hline $2 \mathrm{~A} 31$ & 29DD & SENDCHAR? \\
\hline $2 \mathrm{~A} 33$ & 182 & BZ TO 2 A25 \\
\hline $2 \mathrm{~A} 37$ & 909 & $\mathrm{CR}$ \\
\hline $2 \mathrm{~A} 39$ & $2 \mathrm{CO}$ & ;S \\
\hline
\end{tabular}

\begin{tabular}{lcl}
\multicolumn{3}{l}{ Decompiling TERM } \\
CFA & (CFA) & \\
2878 & 857 & : TERM \\
$287 \mathrm{~A}$ & $7 \mathrm{~F} 1$ & T'ERM.GRP \\
$287 \mathrm{C}$ & $4 \mathrm{BF}$ & C \\
$287 \mathrm{E}$ & $17 \mathrm{BC}$ & GROUP \\
2880 & $2 \mathrm{C} 0$ & iS
\end{tabular}

Decompiling TERM.GRP

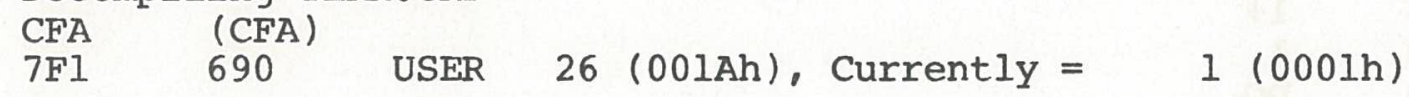

\begin{tabular}{|c|c|c|}
\hline \multicolumn{3}{|c|}{ Decompiling COMM } \\
\hline CFA & (CFA) & \\
\hline 2867 & 857 & : COMM \\
\hline 2869 & 800 & COMM.GRP \\
\hline $286 B$ & $4 \mathrm{BF}$ & a \\
\hline $286 D$ & $17 \mathrm{BC}$ & GROUP \\
\hline $286 \mathrm{~F}$ & $2 \mathrm{CO}$ & ; $S$ \\
\hline
\end{tabular}

Decompiling COMM.GRP
CFA $($ CFA
$800 \quad 690$ USER $28(001 \mathrm{Ch})$, Currently $=224$ (OOEOh) 


\begin{tabular}{|c|c|c|}
\hline \multicolumn{3}{|c|}{ Decompiling NEW-FENCE } \\
\hline CFA & $(\mathrm{CFA})$ & \\
\hline 1D53 & 857 & : NEW-FENCE \\
\hline ID55 & $\mathrm{BCl}$ & LATEST \\
\hline 1D57 & 149 & $12(000 \mathrm{Ch})$ \\
\hline ID5B & 688 & +ORIGIN \\
\hline 1D5D & $4 \mathrm{E} 0$ & $!$ \\
\hline ID5F & A53 & HERE \\
\hline ID6I & 149 & $28(001 \mathrm{Ch})$ \\
\hline 1D65 & 688 & +ORIGIN \\
\hline $1 D 67$ & $4 \mathrm{E} 0$ & ! \\
\hline 1D69 & A53 & HERE \\
\hline 1D6B & 149 & 30 (001Eh) \\
\hline ID6F & 688 & +ORIGIN \\
\hline $1 \mathrm{D} 71$ & $4 \mathrm{E} 0$ & ! \\
\hline $1 \mathrm{D} 73$ & A53 & HERE \\
\hline 1D75 & 6DA & FENCE \\
\hline 1D77 & $4 \mathrm{E} 0$ & $!$ \\
\hline $1 D 79$ & $6 \mathrm{~F} 2$ & VOC-LINK \\
\hline $1 \mathrm{D} 7 \mathrm{~B}$ & $4 \mathrm{BF}$ & a \\
\hline ID7D & 149 & $32(0020 \mathrm{~h})$ \\
\hline 1D81 & 688 & +ORIGIN \\
\hline 1D83 & $4 \mathrm{E} 0$ & $!$ \\
\hline 1D85 & $80 \mathrm{C}$ & Nextu \\
\hline 1D87 & $4 \mathrm{BF}$ & \\
\hline 1D89 & 149 & 42 (002Ah) \\
\hline 1D8D & 688 & +ORIGIN \\
\hline 1D8F & $4 \mathrm{E} 0$ & ! \\
\hline $1 \mathrm{D} 91$ & $2 \mathrm{CO}$ & ; $\mathrm{S}$ \\
\hline Decor & ing 1 & \\
\hline CFA & (CFA) & \\
\hline $1 \mathrm{~B} 89$ & 857 & : NODISK \\
\hline 1B8B & 149 & $852(0354 h)$ \\
\hline $1 \mathrm{~B} 8 \mathrm{~F}$ & ACD & $2-$ \\
\hline 1B91 & 149 & 4584 (11E8h) \\
\hline IB95 & 149 & $71(0047 \mathrm{~h})$ \\
\hline 1B99 & $3 C 4$ & + \\
\hline IB9B & $4 \mathrm{EO}$ & ! \\
\hline 1B9D & $2 \mathrm{CO}$ & ; S \\
\hline
\end{tabular}




\begin{tabular}{|c|c|c|}
\hline \multicolumn{3}{|c|}{ Decompiling .FOOT } \\
\hline CFA & $(\mathrm{CFA})$ & \\
\hline IDCF & 857 & : .FOOT \\
\hline IDDI & $7 C 8$ & OUTL \\
\hline 1DD3 & $4 B F$ & a \\
\hline 1DD5 & $63 D$ & $\mathrm{~L} / \mathrm{P}$ \\
\hline IDD7 & $\mathrm{ABC}$ & $1-$ \\
\hline 1DD9 & 405 & - \\
\hline IDDB & $49 C$ & DUP \\
\hline 1DDD & 367 & $0<$ \\
\hline IDDF & 182 & BZ TO 1DF7 \\
\hline 1DE3 & 432 & MINUS \\
\hline IDE5 & $63 \mathrm{D}$ & $\mathrm{L} / \mathrm{P}$ \\
\hline 1DE7 & F57 & MIN \\
\hline 1DE9 & 608 & 0 \\
\hline 1DEB & $15 \mathrm{~B}$ & DO \\
\hline IDED & 909 & $\mathrm{CR}$ \\
\hline IDEF & $\mathrm{AE}$ & LOOP TO IDED \\
\hline 1DF3 & 172 & BR TO 1DF9 \\
\hline IDF7 & $47 \mathrm{D}$ & DROP \\
\hline IDF9 & 608 & 0 \\
\hline 1DFB & $7 \mathrm{C} 8$ & OUTL \\
\hline IDFD & $4 \mathrm{E} 0$ & $!$ \\
\hline IDFF & $1 \mathrm{CD} 6$ & ID \\
\hline $1 \mathrm{E} 01$ & 149 & $5(0005 h)$ \\
\hline $1 \mathrm{E} 05$ & $14 \mathrm{AE}$ & SPACES \\
\hline 1E07 & B41 & ." Chapter " \\
\hline $1 \mathrm{E} 12$ & $1 \mathrm{CC} 3$ & CHAPTER \\
\hline $1 \mathrm{El} 4$ & $4 B F$ & a \\
\hline $1 \mathrm{E} 16$ & 1B6E & DEC. \\
\hline 1E18 & 149 & 15 (000Fh) \\
\hline IEIC & $14 \mathrm{AE}$ & SPACES \\
\hline 1ElE & B41 & ." page " \\
\hline $1 \mathrm{E} 26$ & $76 \mathrm{D}$ & FLD \\
\hline $1 \mathrm{E} 28$ & $4 \mathrm{BF}$ & a \\
\hline $1 \mathrm{E} 2 \mathrm{~A}$ & 1B6E & DEC. \\
\hline $1 \mathrm{E} 2 \mathrm{C}$ & $2 \mathrm{CO}$ & is \\
\hline Decom & ling $P$ & \\
\hline CFA & (CFA) & \\
\hline 40EA & 857 & : PAGE \\
\hline 40EC & 149 & $13(000 \mathrm{Dh})$ \\
\hline $40 F 0$ & $8 \mathrm{FC}$ & EMIT \\
\hline $40 F 2$ & 149 & $140(008 \mathrm{Ch})$ \\
\hline $40 F 6$ & $8 \mathrm{FC}$ & EMIT \\
\hline $40 F 8$ & 610 & 1 \\
\hline $40 F A$ & $76 \mathrm{D}$ & FLD \\
\hline $40 F C$ & $3 F 2$ & $+!$ \\
\hline $40 F E$ & $2 \mathrm{CO}$ & ; $S$ \\
\hline
\end{tabular}


SCREEN 224

0 ( FORTH DOS examples)

1 DECIMAL CR 224 LIST

HEX

( Akens, WHOI)

2

$3 \mathrm{CR}$." Opening WFORTH.CM:I" CR

4 OPEN WFORTH. CM:I CR

5 ." This is the beginning of WFORTH.CM:I, at location 0000 " CR

6 GETSEC 40 DUMP CR

7 ." This is sector 8 of WFORTH.CM:l at location OEO0 h" CR

88 NEWLSN 40 DUMP CR

9 ." This is logical sector 0 of WFORTH.CM:I, the segment descript

10 ion word" CR 0 NEWLSN 10 DUMP CR

11 CR DECIMAL DIR .FOOT PAGE

12235 LOAD MEMORY-MAP CR CR

13236 LOAD CR IOCB-SHOW .FOOT PAGE

14

15 DECIMAL ;S

Opening WFORTH. CM:1

This is the beginning of WFORTH.CM:1, at location 0000

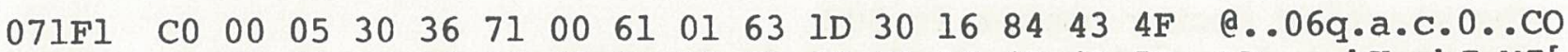

07201 4C C4 $000000016 \quad 68$ CB 18 3D 68 CA 18 4D 4 A $5 B$ LD....hK. =hJ.MJ [

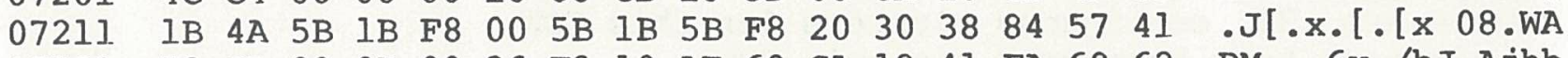

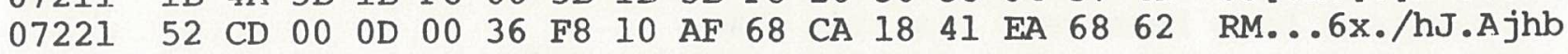

This is sector 8 of WFORTH.CM:1 at location 0E00 h

073F5 0I 82 OE 0 0E 06 FC 04 BF 16 DE 0172 OE 1206 B4

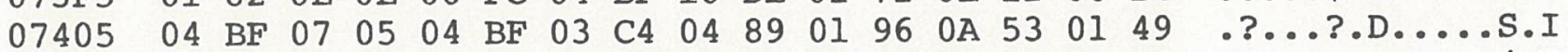

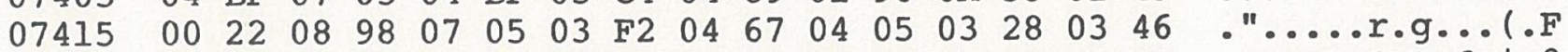

$\begin{array}{llllllllllllllllll}07425 & 0 A & 53 & 04 & \text { F0 } & 03 & \text { C4 } & \text { OA } & 53 & 03 & \text { D5 } & 03 & 36 & 02 & \text { FC } & 02 & \text { C0 } & \text {.S.p.D.S.U.6.I. A }\end{array}$

This is logical sector 0 of WFORTH.CM:I, the segment description word

06FF1 $2501 \quad$ F6 $80 \quad 2500 \quad 00000000000000000000 \quad 8 . v .8 \ldots \ldots \ldots$

Disk ID: WOODS HOLE FORTH SCREEN FILE VER 1.801

$1130 \quad 84$

Directory start

$\begin{array}{llllllll}\text { FLOAT } . C M & 121 & \text { ASM4TH.CM } & 48 & \text { FFORTH.CM } & 55 & \text { SYSGEN.CM } & 558\end{array}$

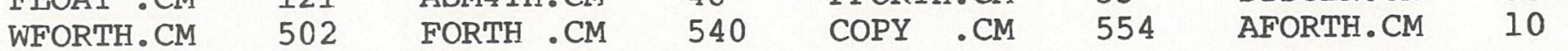


Load 235 , 4BE8 (.BLOCKHDR .BUFSTAT MEMORY-MAP)

Address block update

7BFB $-1 \quad$ Yes

$77 \mathrm{~F} 7 \quad-1 \quad$ Yes

$73 \mathrm{~F} 3 \quad-1 \quad$ Yes

6FEF -1 Yes

6BEB -1 Yes

$67 E 7 \quad-1 \quad$ Yes

63E3 -1 Yes

5FDF $-1 \quad$ Yes

$5 B D B \quad 550 \quad$ Yes

57D7 $539 \quad$ Yes

5700 User area starts here

56FE Return stack starts down from here

5600 Terminal input buffer

55FE Computation stack starts down here

4D8B Dictionary pointer

418B FENCE

Load 236,4 D8B ( IOCB-SHOW)

The starting sector number is 502

The segment descriptor word offset is 0 bytes

There are 38 contiguous clusters for this SDW

1 clusters have been read via this SDW

The starting sector number for this SDW is 502

The buffer address is 7DFD 


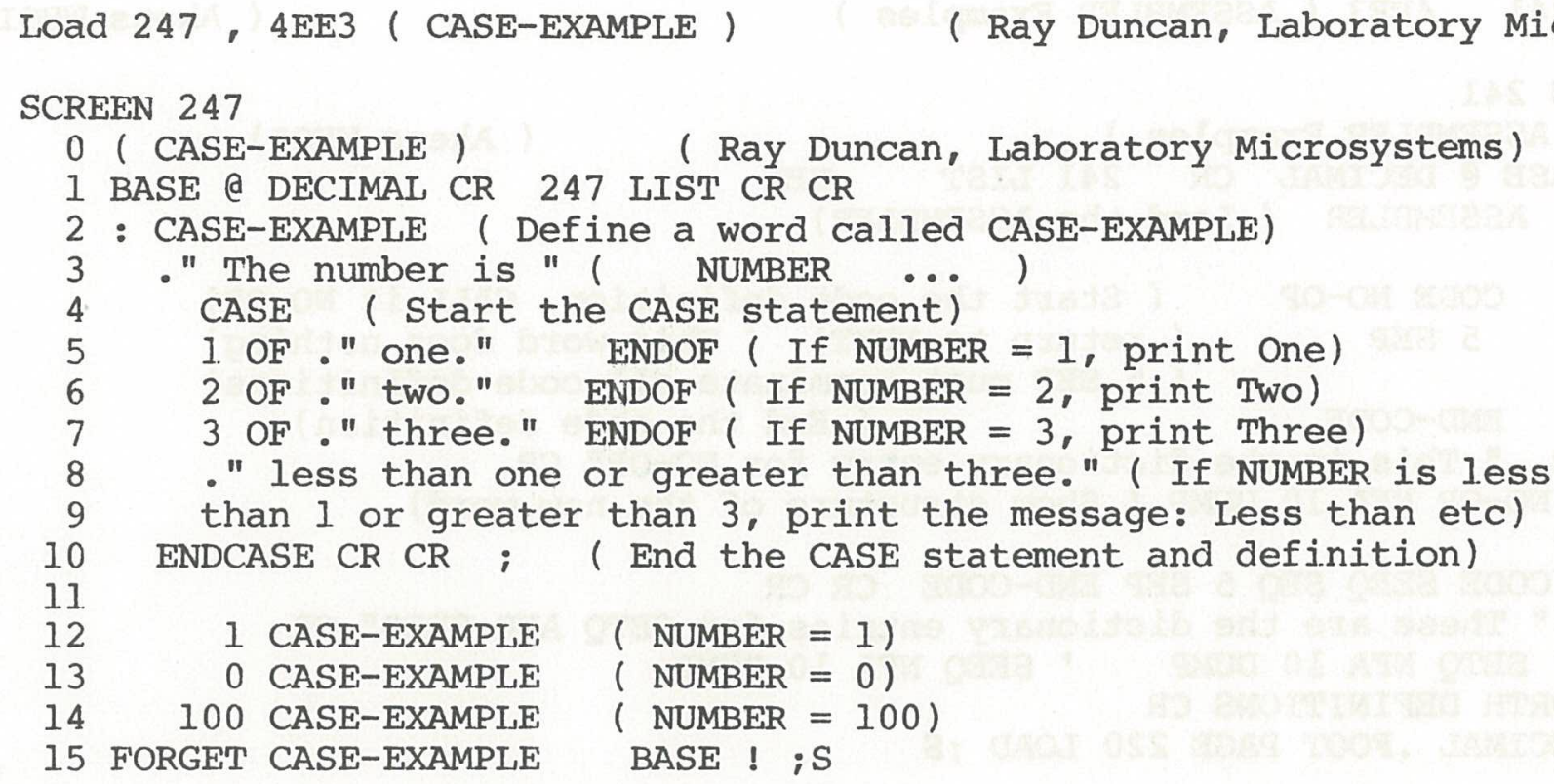

The number is one.

The number is less than one or greater than three.

The number is less than one or greater than three. 


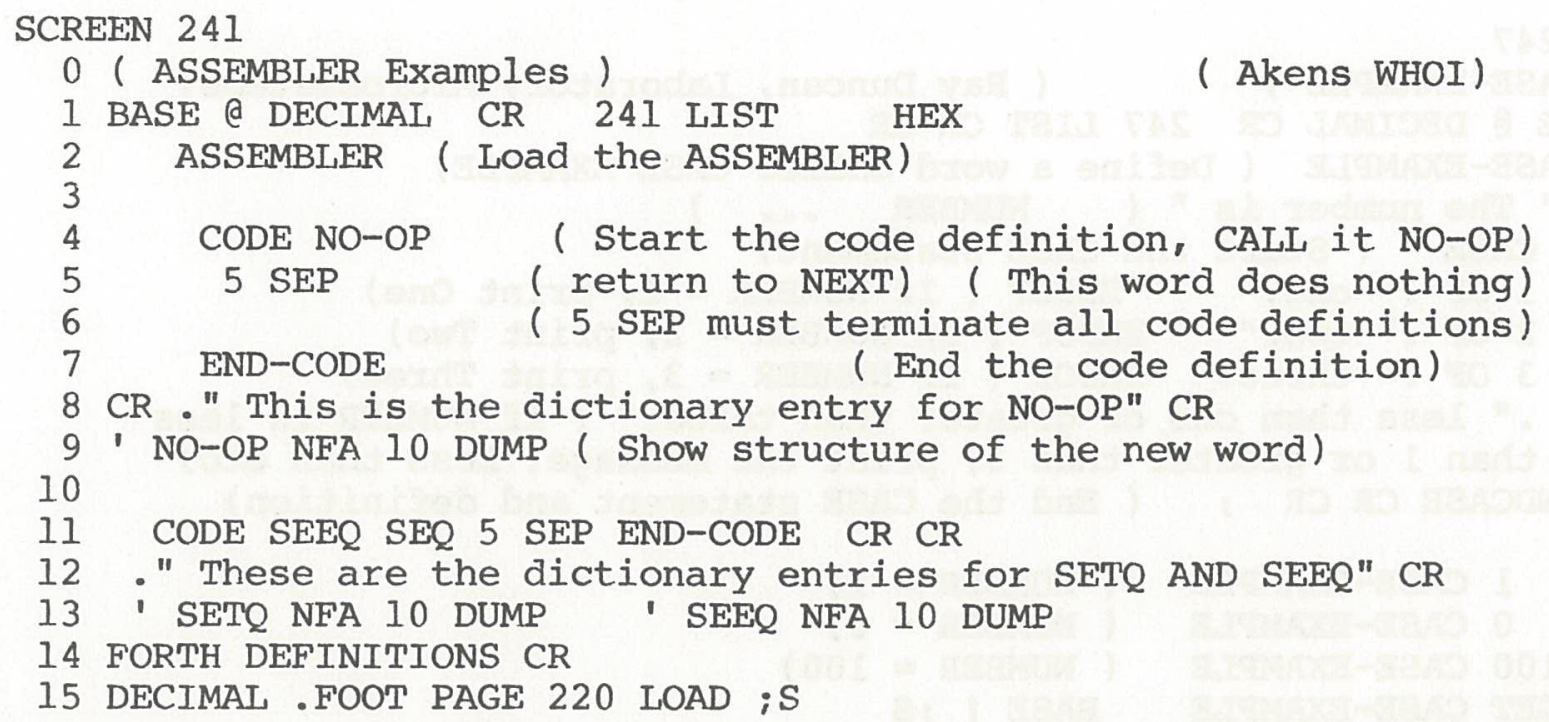

Load 71 , 4EE3 (ASSEMBLER load screen) ( CODE END-CODE) (Akens, McDaniels) ASSEMBI.ER Message 4

Load 131, C700 ( R0 R1 R2 R3 R4 R5 R6 R7 R8 R9 RA RB RC RD RE RF) ( Registers) R0 Message 4

Load 132 , C790 ( END-CODE C; ENDCODE)

Load 133 , C7C8 ( IS ?SBERROR)

( 1806 ASSEMBLER 2)

LOad 133 , C7C8 ( IS ?SBERROR) (1806 ASSEMBLER 3)

Load 134, C850 ( T5 T4 T3 T2 T1 NX IP UP SP PC RP IV DMAV) ( Register Equates)

UP Message 4

Load 135 , C8DD ( CRN LDN LDA INP PLO ETC) ( 1806 ASSEMBLER 5)

OUT Message 4

Load 136 , C982 ( 1BFI 1BSB ETC ) ( 1806 ASSEMBLER 6)

Load 137 , CAC5 ( 2BFI LBR LBQ LBZ LBDF LBNQ LBNZ LBNF) ( 1806 ASSEMBLER 7)

Load 138 , CB2D ( IBOP IDLE IRX ETC) ( 1 byte op codes, 1806 ASSEMBLER 8) OR Message 4 AND Message 4 XOR Message 4

Load 139, CCD8 (2BOP 1BF2BOP 2BF2BOP CR2BOP etc) ( 1806 ASSEMBLER 9)

Load 140, CE50 ( LBL1 LBL2 LBL3 LBL4 IAB1: LAAB2: LAB3: LAB4: LABB1 LAB2 LAB3)

Load 141 , CF00 (A.0 A.1 PAGE)

( 1806 ASSEMBLER 11)

PAGE Message 4

Load 142 , CF43 ( ?NEXTPAGE ?PAGESPACE PAGEJUMP PAGEADJUST 1806 ASSEMBLER 12)

Assembler ends at CFD2

This is the dictionary entry for NO-OP

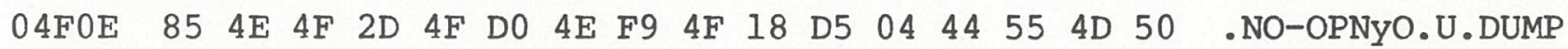

These are the dictionary entries for SETQ AND SEEQ

$\begin{array}{llllllllllllllllll}02160 & 84 & 53 & 45 & 54 & \mathrm{Dl} & 21 & 58 & 21 & 69 & 7 \mathrm{~B} & \mathrm{D} 5 & 84 & 52 & 45 & 54 & \mathrm{Dl} & \text {.SETQ!X!I }\end{array}$

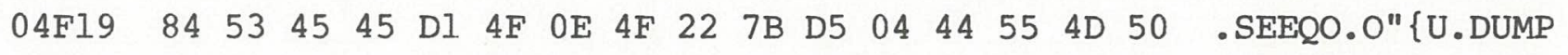

Woods Hole FORTH Version 1.800 Chapter 20 page 140 


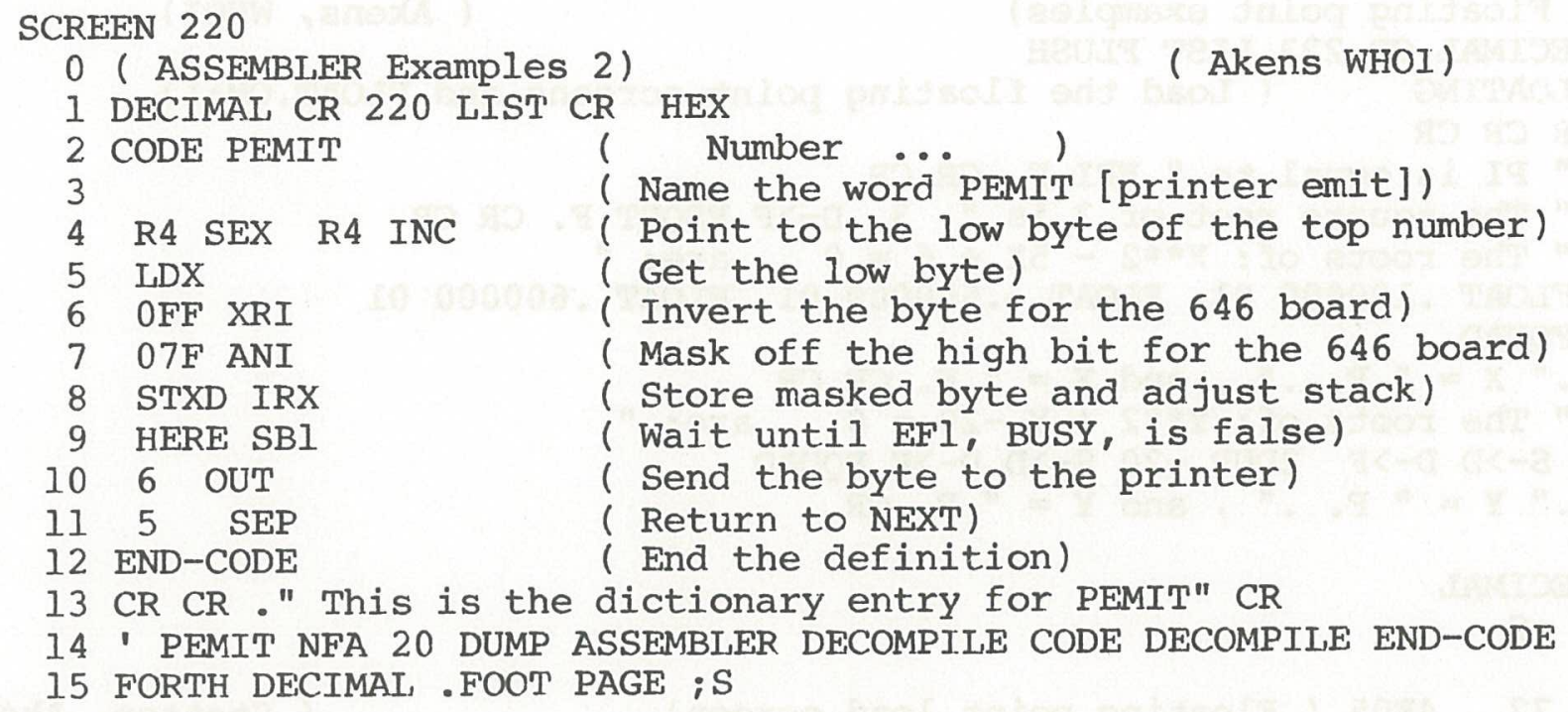

This is the dictionary entry for PEMIT

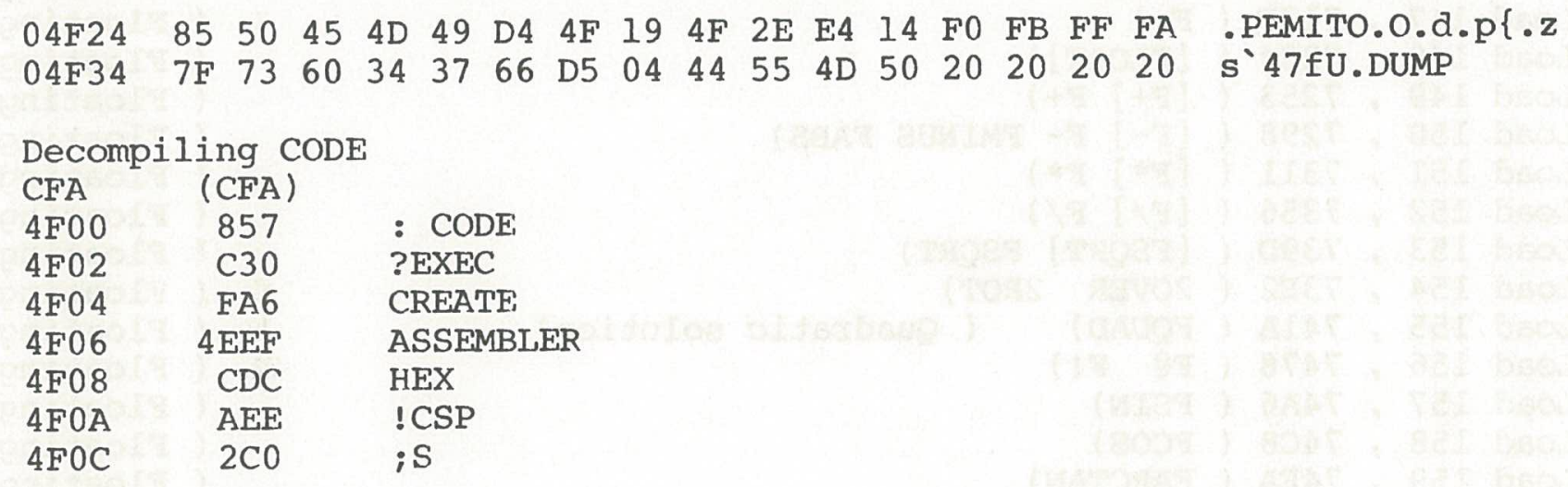

Decompiling END-CODE

CFA (CFA)

C79B 857 : END-CODE

C79D $742 \quad$ CURRENT

C79F $4 \mathrm{BF} \quad$ a

C7AI $734 \quad$ CONTEXT

C7A3 $4 \mathrm{E} 0 \quad$ !

C7A5 C30 ?EXEC

C7A7 C5A ?CSP

C7A9 CCA SMUDGE

$\mathrm{C} 7 \mathrm{AB} \quad 2 \mathrm{CO}$; $\mathrm{S}$ 


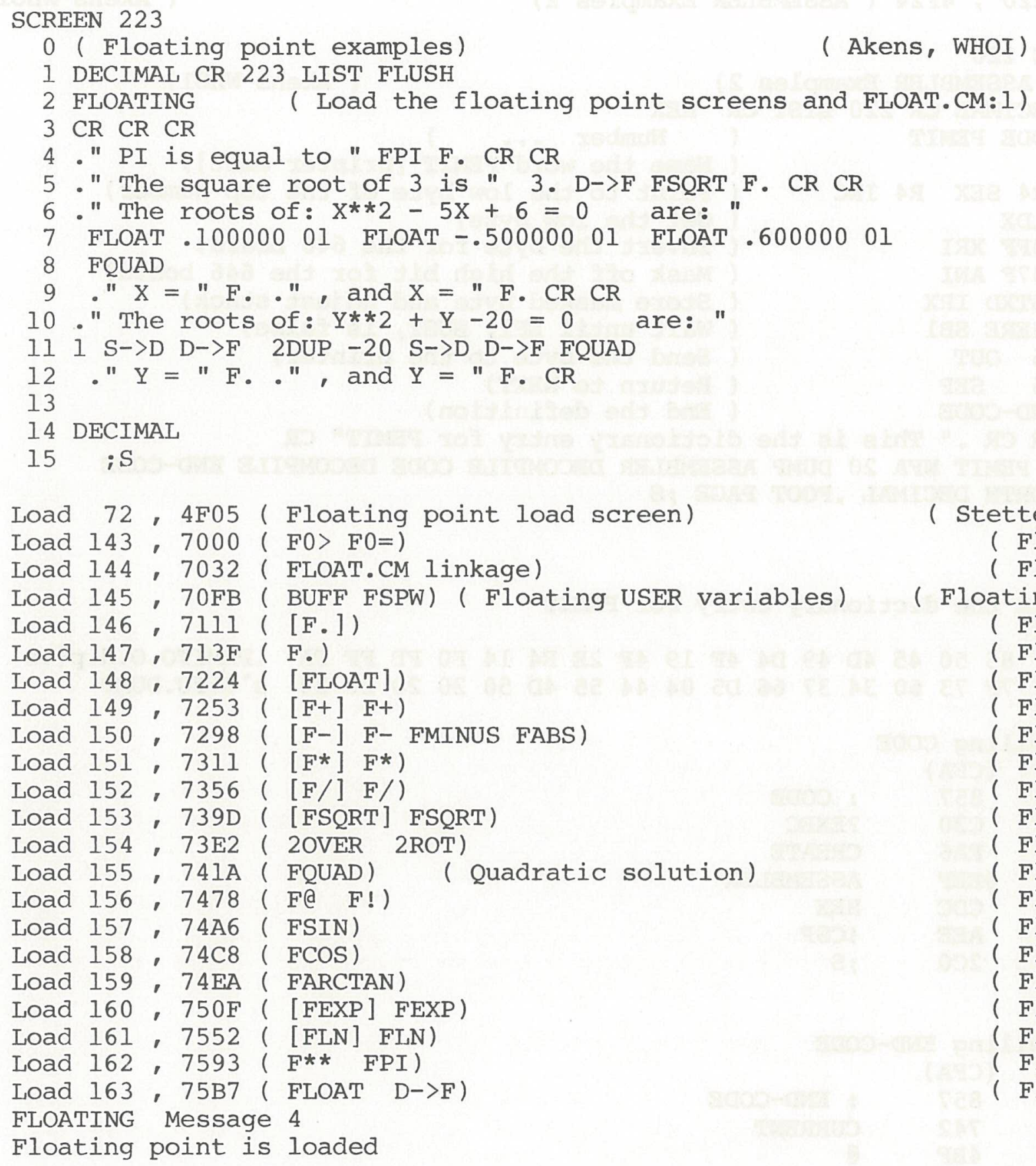

$\mathrm{PI}$ is equal to .31416001

The square root of 3 is .173205 01

The roots of: $\mathrm{X} * * 2-5 \mathrm{X}+6=0$ are: $\mathrm{X}=.29999401$, and $\mathrm{X}=.20000401$ The roots of: $Y * * 2+Y-20=0$ are: $Y=.40000001$, and $Y=-.50000001$ 


\section{Source Listing}

The source listing (WFORTH.SRC) for the program FORTH.CM follows. This listing was generated from within FORTH by the word .FILE. The sources for this word are located on screens 232 and 233. 
File ID: WFORTH.LST

Disk ID: WOODS HOLE FORTH SOURCE \& LIST VER 1.800

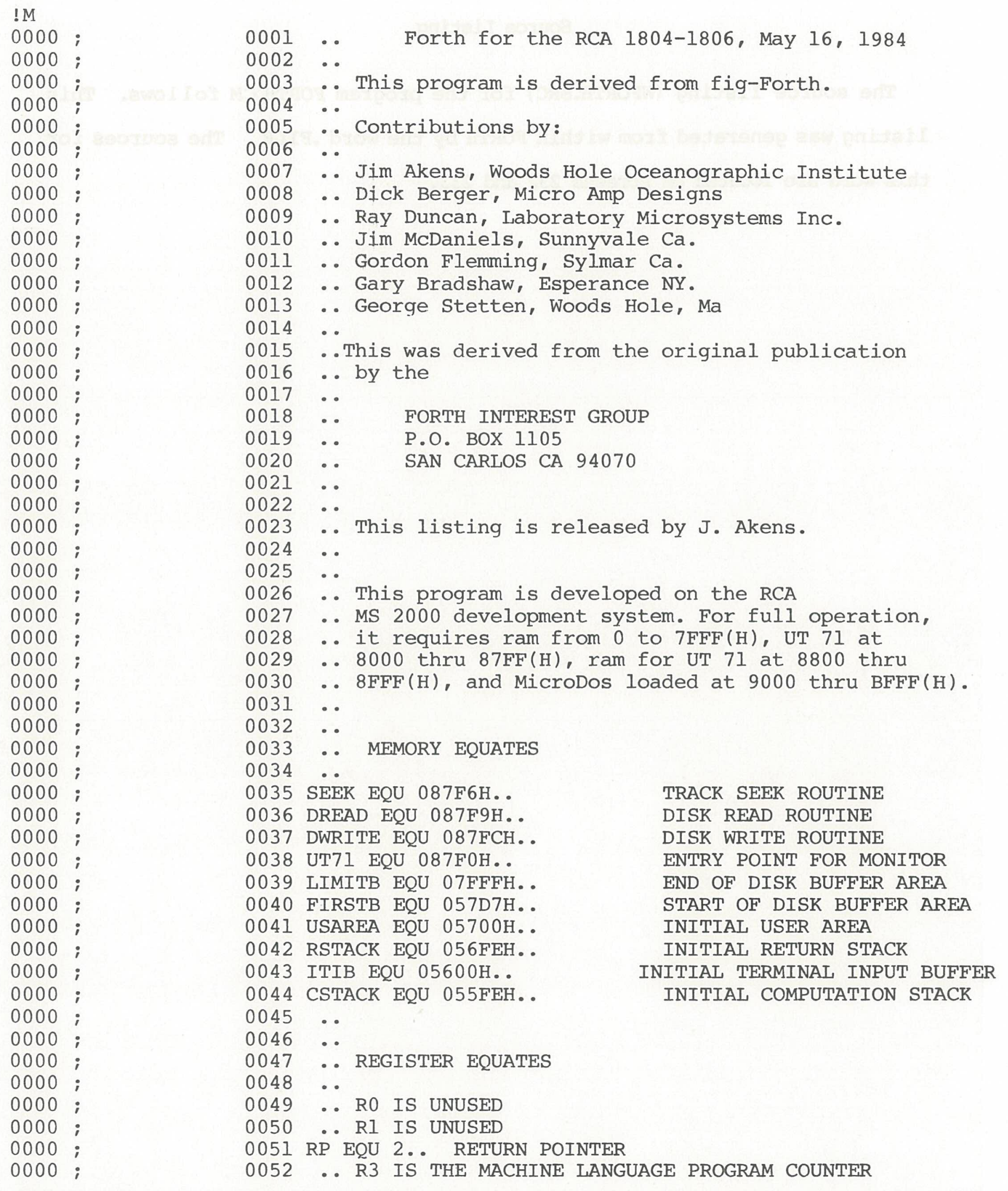

Woods Hole FORTH Version $1.800 \quad$ Chapter 21

page 144 
File ID: WFORTH.LST

Disk ID: WOODS HOLE FORTH SOURCE \& LIST VER 1.800

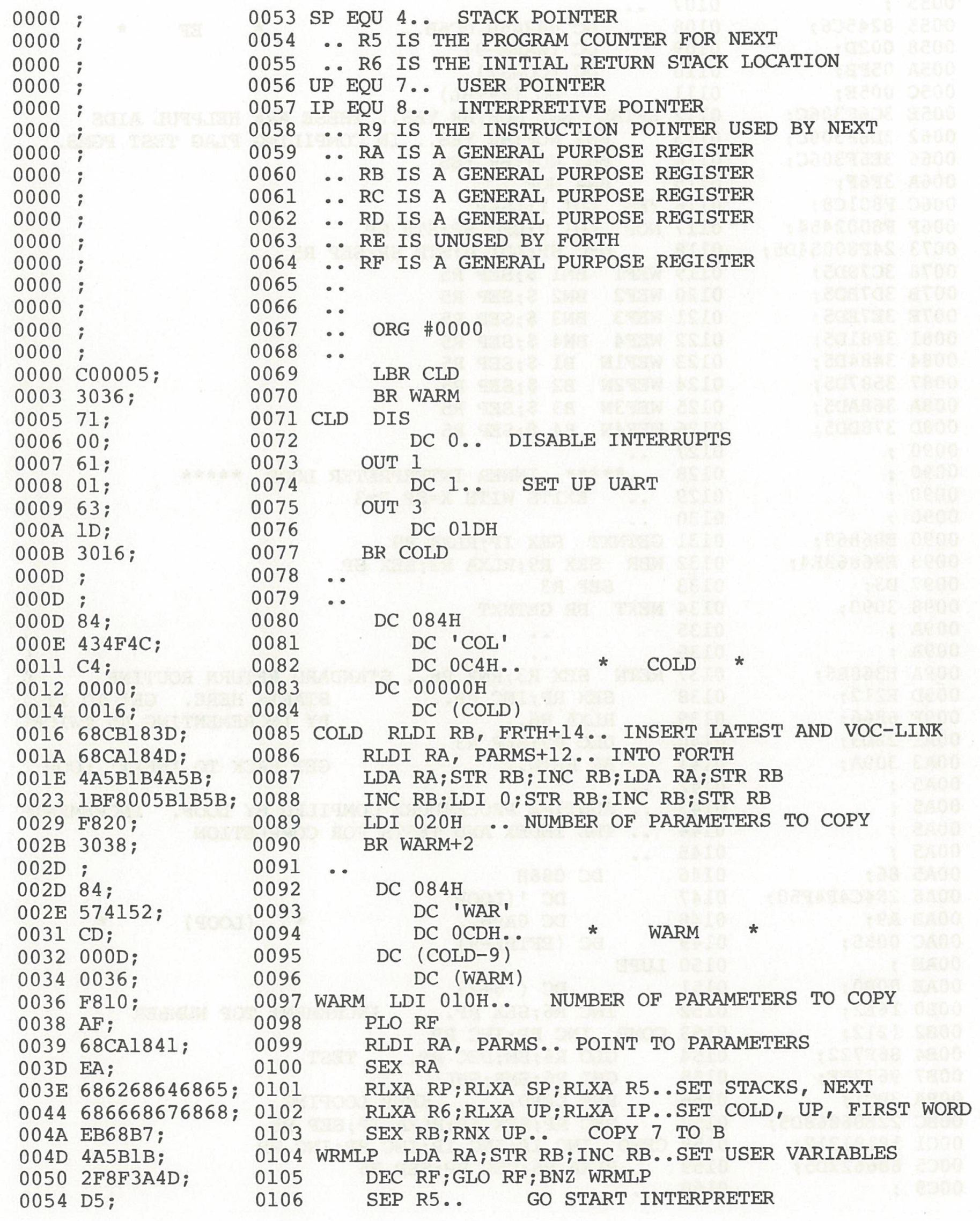


File ID: WFORTH.LST

Disk ID: WOODS HOLE FORTH SOURCE \& LIST VER 1.800

$1130 \quad 84$

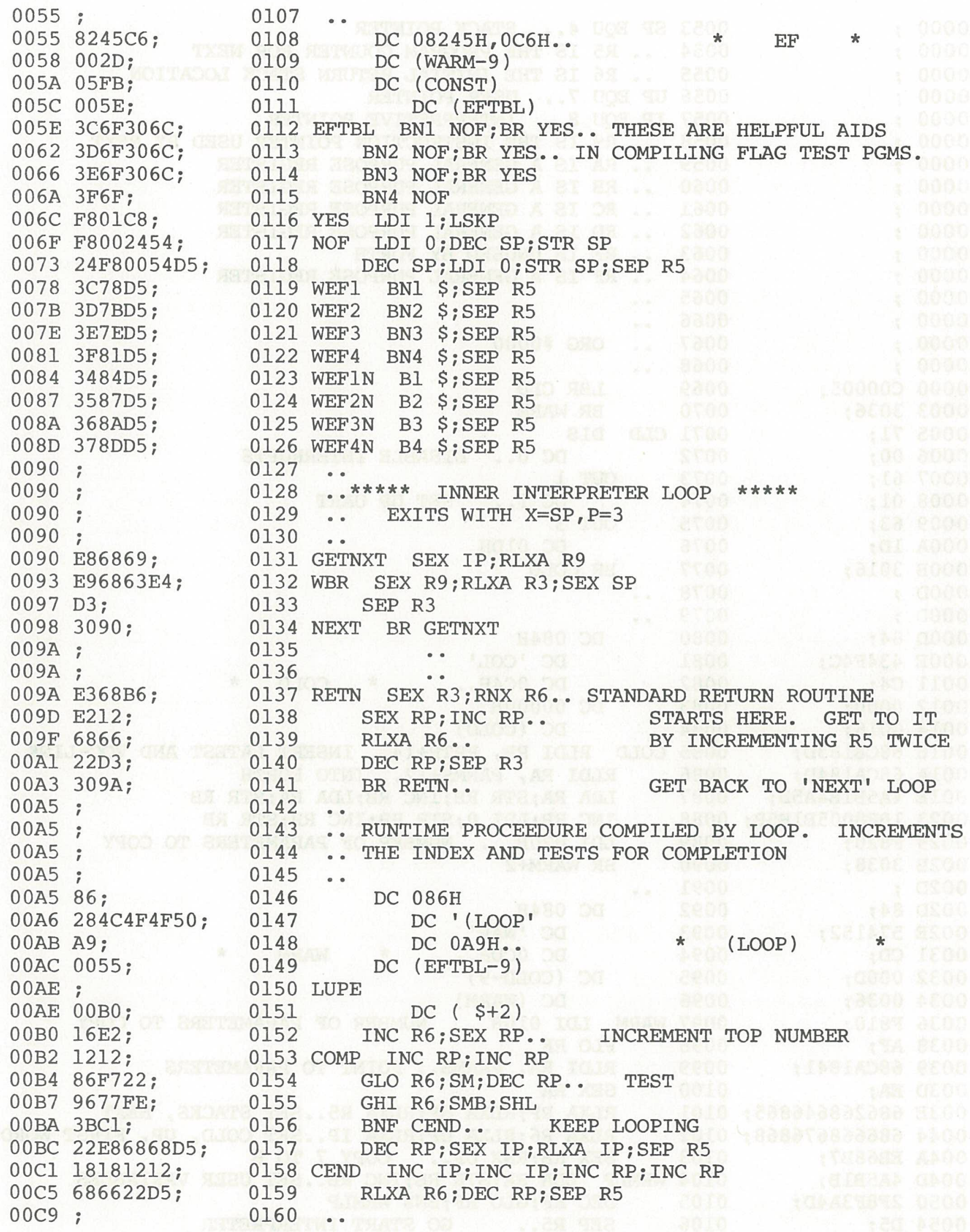


File ID: WFORTH.LST

Disk ID: WOODS HOLE FORTH SOURCE \& LIST VER 1.800

113084

\begin{tabular}{|c|c|c|c|}
\hline $00 C 9$ & ; & 0161 & .. RUNTIME PROCEEDURE COMPILED BY +LOOP. ADDS TOP \\
\hline $00 C 9$ & ; & 0162 & .. TO INDEX \& TESTS FOR COMPLETION \\
\hline $0 \mathrm{Cg}$ & ; & 0163 & . \\
\hline & $87 ;$ & 0164 & DC $087 \mathrm{H}$ \\
\hline & 282B $4 \mathrm{C} 4 \mathrm{~F} 4 \mathrm{~F} 50$; & 0165 & $\mathrm{DC}^{\prime}\left(+\mathrm{LOOP} \mathrm{P}^{\prime}\right.$ \\
\hline & A9; & 0166 & DC $0 A 9 H .$. \\
\hline & $00 A 5$ & 0167 & DC (LUPE-9) \\
\hline & ; & 0168 & PLUPE \\
\hline & 00D5; & 0169 & $\mathrm{DC}(\$+2)$ \\
\hline & $14 ;$ & 0170 & INC SP \\
\hline $0 D 6$ & $86 \mathrm{~F} 4 \mathrm{~A} 624 ;$ & 0171 & GLO R6;ADD;PLO R6;DEC SP \\
\hline DODA & $9674 \mathrm{~B} 64414 \mathrm{E} 2$; & 0172 & GHI R6;ADC;PHI R6;LDA SP;INC SP;SEX RP \\
\hline OEO & FE3BB2 ; & 0173 & SHL;BNF COMP.. BRANCH IF POSITIVE \\
\hline $0 \mathrm{E3}$ & $121286 \mathrm{~F} 522 ;$ & 0174 & INC RP;INC RP;GLO R6;SD;DEC RP \\
\hline $0 \mathrm{E8}$ & 967530B9; & 0175 & GHI R6;SDB;BR COMP+7 \\
\hline $0 \mathrm{EC}$ & i $\quad 2 \quad 1$ & 0176 & s \\
\hline OOEC & ; & 0177 & .. SEARCH DICTIONARY FOR A MATCH, STARTING AT THE \\
\hline OOEC & ; & 0178 & .. ADDRESS ON TOP OF STACK, TRYING FOR A MATCH \\
\hline OOEC & ; & 0179 & .. WITH TEXT AT ADDRESS ONE DOWN ON STACK. RETURNS \\
\hline OOEC & ; & 0180 & .. THE PFA, LENGTH BYTE, AND TRUE FLAG IF A GOOD \\
\hline 00EC & ; & 0181 & .. MATCH WAS FOUND. RETURNS ONLY FALSE FLAG IF \\
\hline OOEC & ; & 0182 & .. THERE WAS NO MATCH. \\
\hline OOEC & ; & 0183 & - \\
\hline OOEC & $86 ;$ & 0184 & DC $086 \mathrm{H}$ \\
\hline OOED & $2846494 \mathrm{E} 44$ & 0185 & DC '(FIND' \\
\hline $00 \mathrm{~F} 2$ & A9; & 0186 & DC $0 A 9 H$ \\
\hline $00 F 3$ & 00C9 ; & 0187 & DC (PLUPE-10) \\
\hline $00 F 5$ & ; & 0188 & FIND \\
\hline $00 \mathrm{~F} 5$ & 00F7 ; & 0189 & DC $(\$+2)$ \\
\hline 00F7 & $686 \mathrm{~A} ;$ & 0190 & RLXA RA \\
\hline 00F9 & 686B24; & 0191 & RLXA RB;DEC SP \\
\hline OOFC & EA0A52; & 0192 & LOOP1 SEX RA;LDN RA;STR RP.. SAVE LENGTH \\
\hline OOFF & $4 \mathrm{BF} 3 \mathrm{FA} 3 \mathrm{~F}$; & 0193 & LDA RB;XOR;ANI 03FH; LBNZ BADLEN.. BRANCH IF BADLEN \\
\hline 0103 & $\mathrm{CA} 0127$ & & \\
\hline 0106 & 1A4BF3FE; & 0194 & NEXCHR INC RA;LDA RB;XOR;SHL.. COMPARE NEXT CHAR. \\
\hline $010 \mathrm{~A}$ & $3 \mathrm{~A} 28$ & 0195 & BNZ BADCHR.. BRANCH IF NO MATCH ON 7 CHARS \\
\hline $010 \mathrm{C}$ & $7 \mathrm{E} 3206$ & 0196 & SHLC;BZ NEXCHR.. LOOP IF NOT LAST CHARACTER \\
\hline $010 \mathrm{~F}$ & 4AFA80 ; & 0197 & LOOP2 LDA RA;ANI 080H.. CHECK FOR END OF STRING \\
\hline 0112 & $320 \mathrm{~F} ;$ & 0198 & BZ LOOP2 \\
\hline 0114 & $\mathrm{E} 48 \mathrm{AFC} 0473$; & 0199 & SEX SP;GLO RA;ADI 4;STXD.. LEAVE PFA \\
\hline 0119 & 9A7C0073; & 0200 & GHI RA;ADCI $0 ; S T X D$ \\
\hline $011 \mathrm{D}$ & $0273 \mathrm{~F} 80073$ & 0201 & LDN RP;STXD;LDI 0;STXD.. LEAVE LENGTH BYTE \\
\hline 0122 & F8FF7354 ; & 0202 & LDI OFFH;STXD;STR SP.. LEAVE TRUE FLAG \\
\hline 0126 & D5; & 0203 & SEP R5 . \\
\hline 0127 & IA; & 0204 & BADLEN INC RA \\
\hline 0128 & 4AFA803228; & 0205 & BADCHR LDA RA;ANI $080 \mathrm{H} ; \mathrm{BZ}$ BADCHR \\
\hline $012 \mathrm{D}$ & ОАЗАЗВ & 0206 & LDN RA;BNZ BOK \\
\hline 0130 & IA0A $2 \mathrm{~A}$; & 0207 & INC RA; LDN RA;DEC RA \\
\hline 0133 & 3АЗВ & 0208 & BNZ BOK \\
\hline 0135 & F800E47354; & 0209 & LDI $0 ;$ SEX SP;STXD;STR SP \\
\hline $013 \mathrm{~A}$ & D5 ; & 0210 & SEP R5 \\
\hline $013 \mathrm{~B}$ & EA686A; & 0211 & BOK SEX RA; RLXA RA \\
\hline $013 \mathrm{E}$ & $24 \mathrm{E} 4$ & 0212 & DEC SP;SEX SP \\
\hline 0140 & C000F9; & 0213 & LBR LOOPI-3 \\
\hline
\end{tabular}

Woods Hole FORTH Version 1.800 Chapter 21

page 147 
File ID: WFORTH.LST

Disk ID: WOODS HOLE FORTH SOURCE \& LIST VER 1.800

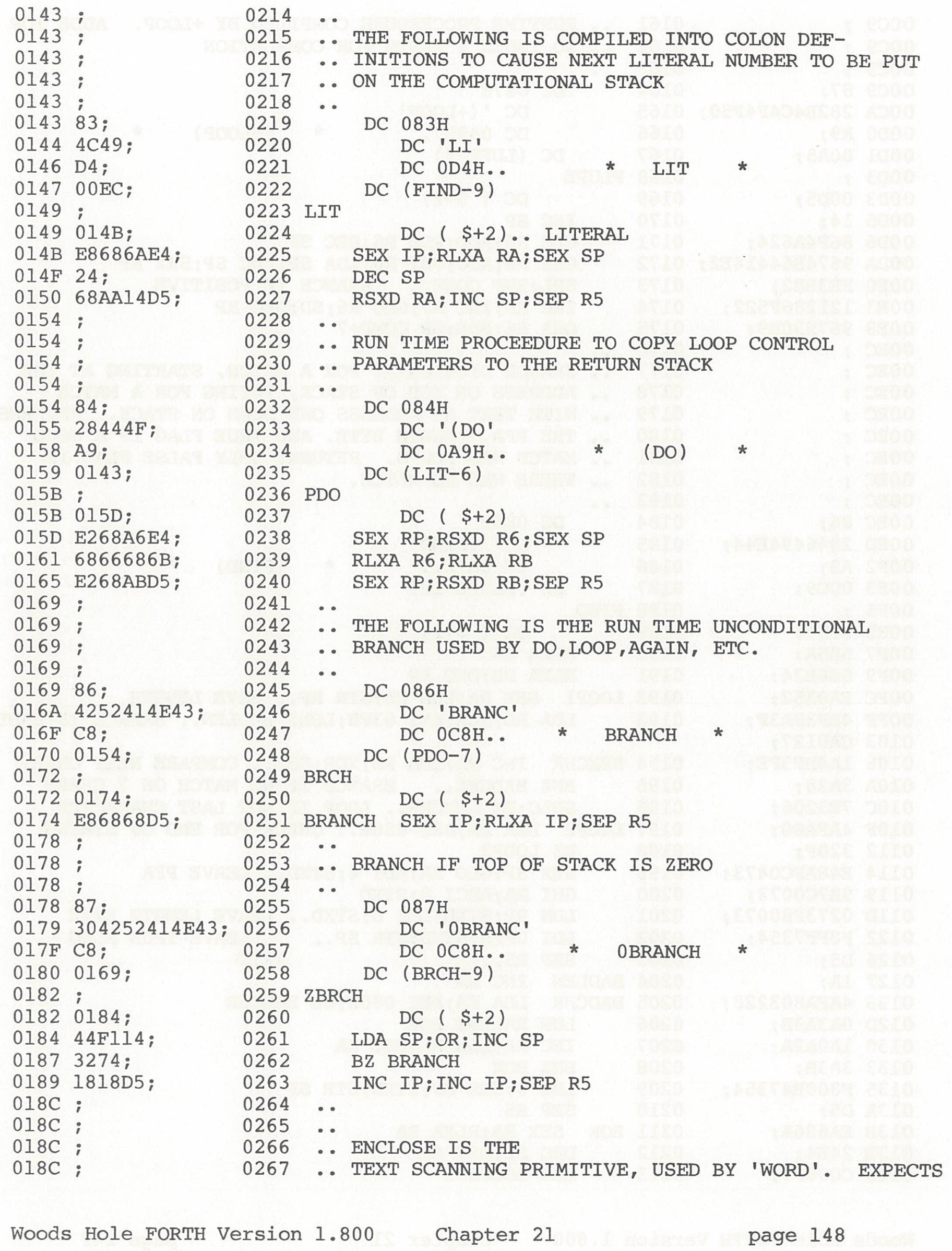


File ID: WFORTH.LST

Disk ID: WOODS HOLE FORTH SOURCE \& LIST VER 1.800

\begin{tabular}{|c|c|c|c|}
\hline $018 \mathrm{C}$ & ; & 0268 & .. ADDRI, C, ON THE STACK. \\
\hline $018 \mathrm{C}$ & ; & 0269 & .. C IS A DELIMITER. IT DELETES C FROM THE \\
\hline $018 \mathrm{C}$ & ; & 0270 & .. STACK, AND ADDS N1 (BYTE OFFSET TO FIRST NON- \\
\hline & ; & 0271 & .. DELIMITER CHARACTER), N2 (OFFSET TO THE FIRST \\
\hline $18 \mathrm{C}$ & ; & 0272 & .. DELIMITER AFTER THE TEXT), AND N3 (OFFSET TO THE \\
\hline $8 \mathrm{C}$ & ; & 0273 & .. FIRST CHARACTER NOT INCLUDED. THIS PROCEDURE \\
\hline & ; & 0274 & .. TREATS A NULL AS AN UNCONDITIONAL DELIMITER. \\
\hline $8 \mathrm{C}$ & ; & 0275 & 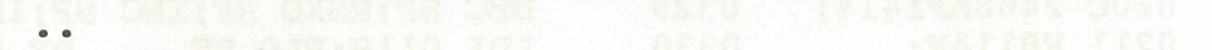 \\
\hline & 87 ; & 0276 & DC $087 \mathrm{H}$ \\
\hline & $454 \mathrm{E} 434 \mathrm{C} 4 \mathrm{~F} 53$ & 0277 & DC 'ENCLOS' \\
\hline & C5; & 0278 & $\mathrm{DC} \quad 0 \mathrm{C} 5 \mathrm{H}$ \\
\hline & 0178 ; & 0279 & DC (ZBRCH-10) \\
\hline 96 & ; & 0280 & ENCL \\
\hline & 0198 ; & 0281 & $\mathrm{DC}(\$+2)$ \\
\hline 98 & $1414 ;$ & 0282 & INC SP;INC SP..POINT TO ADDR \\
\hline & $686 B 242424$ & 0283 & RLXA RB;DEC SP;DEC SP;DEC SP.. RB IS POINTER \\
\hline $19 \mathrm{~F}$ & 0452 ; & 0284 & LDN SP;STR RP.. SAVE DELIMITER \\
\hline lAl & E2F800AA; & 0285 & SEX RP;LDI 0 ;PLO RA. . ZERO CHARACTER COUNT \\
\hline $1 \mathrm{~A} 5$ & $\mathrm{BABC}$; & 0286 & PHI RA;PHI RC \\
\hline $01 \mathrm{~A} 7$ & 0BF7; & 0287 & SAME AS THE DELIMITER? \\
\hline $01 \mathrm{~A} 9$ & ЗАAF ; & 0288 & NO. FOUND FIRST NON-DELIMITER \\
\hline $01 \mathrm{AB}$ & 1B1A30A7; & 0289 & INC RB;INC RA;BR LOPI.. $\quad$ KEEP LOOKING \\
\hline $01 \mathrm{AF}$ & E48A73AC; & 0290 & FRST SEX SP;GLO RA; STXD;PLO RC.. SAVE OFFSET N1 \\
\hline 01B3 & 9А73BC; & 0291 & GHI RA;STXD;PHI RC \\
\hline $01 \mathrm{~B} 6$ & F80073737354; & 0292 & LDI $0 ;$ STXD; STXD;STXD;STR SP \\
\hline $01 B C$ & $14 \mathrm{E} 2 ;$ & 0293 & INC SP;SEX RP.. $\quad$ SP POINTS TO N3 \\
\hline $01 \mathrm{BE}$ & OB32C 8 ; & 0294 & LOP2 LDN RB;BZ NULL.. \\
\hline $01 \mathrm{Cl}$ & F732E3; & 0295 & EXIT WHEN DELIMITER FOUND \\
\hline $01 \mathrm{C} 4$ & 1BІА30BE; & 0296 & INC RB; INC RA;BR LOP2.. LOOP TILL FOUND \\
\hline $01 \mathrm{C} 8$ & $8 \mathrm{AE} 473$; & 0297 & NULL GLO RA;SEX SP;STXD.. SAVE OFFSET TO NULL \\
\hline $01 \mathrm{CB}$ & $9 A 5460$ & 0298 & GHI RA;STR SP;IRX \\
\hline $01 \mathrm{CE}$ & 8CF73AD9; & 0299 & GLO RC;SM;BNZ SKIP.. SAME AS OFFSET NI? \\
\hline 01D2 & 249CF7143AD9; & 0300 & DEC SP;GHI RC;SM;INC SP;BNZ SKIP \\
\hline 01D8 & $1 \mathrm{~A}$; & 0301 & INC RA.. $\quad$ YES. ADD 1 \\
\hline 0109 & $9 A 145$ & 0302 & SKIP GHI RA;INC SP;STR SP;INC SP..POINT TO N2 \\
\hline 01DD & $8 \mathrm{~A} 54$ & 0303 & GLO RA;STR SP.. SAVE IT \\
\hline 01DF & 242424 ; & 0304 & DEC SP;DEC SP;DEC SP.. FIX STACK \\
\hline $01 E 2$ & D5; & 0305 & SEP R5 \\
\hline $01 E 3$ & 1A8A54; & 0306 & DELIM INC RA;GLO RA;STR SP \\
\hline $01 \mathrm{E} 6$ & $249 A 54142 A ;$ & 0307 & DEC SP;GHI RA;STR SP;INC SP;DEC RA \\
\hline OIEB & 30D9; & 0308 & BR SKIP \\
\hline 01ED & ; & 0309 & •. \\
\hline OIED & ; & 0310 & .. \\
\hline OIED & ; & 0311 & .. \\
\hline 01ED & 87 ; & 0312 & DC $087 \mathrm{H}$ \\
\hline OLEE & 455845435554 ; & 0313 & DC 'EXECUT' \\
\hline $01 F 4$ & $\mathrm{C} 5$; & 0314 & DC $0 \mathrm{C} 5 \mathrm{H} .$. \\
\hline $01 F 5$ & $018 \mathrm{C} ;$ & 0315 & DC (ENCL-10) \\
\hline $01 F 7$ & ; & 0316 & EXE \\
\hline $01 F 7$ & $01 F 9$; & 0317 & DC $(\$+2)$ \\
\hline $01 F 9$ & $6869 ;$ & 0318 & RLXA R9 \\
\hline 0IFB & F893A5; & 0319 & LDI A.O(WBR);PLO R5 \\
\hline OIFE & D5; & & SEP R5 \\
\hline & ; & & \\
\hline
\end{tabular}


File ID: WFORTH.LST

Disk ID: WOODS HOLE FORTH SOURCE \& LIST VER 1.800

$1130 \quad 84$

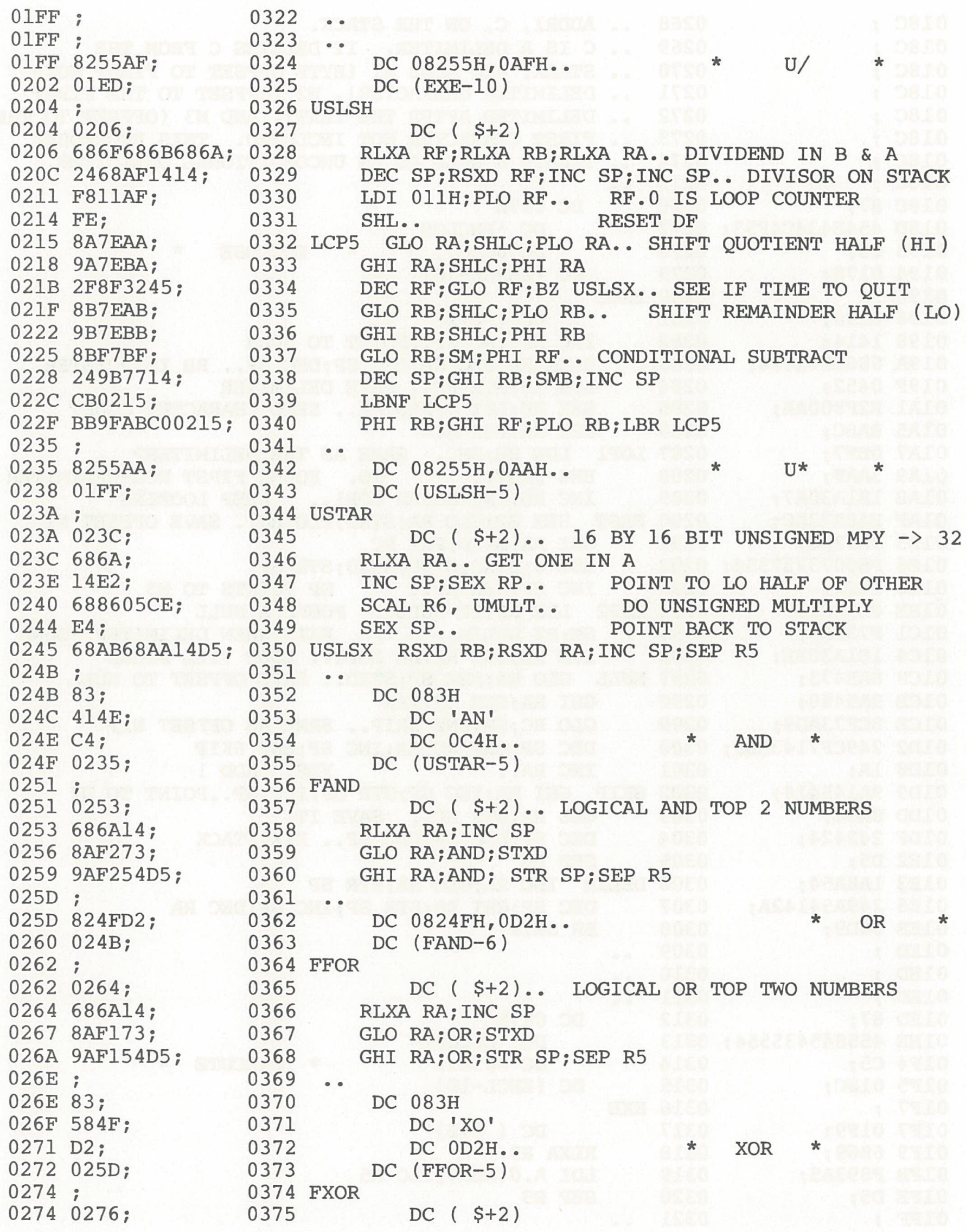


File ID: WFORTH.LST

Disk ID: WOODS HOLE FORTH SOURCE \& LIST VER 1.800

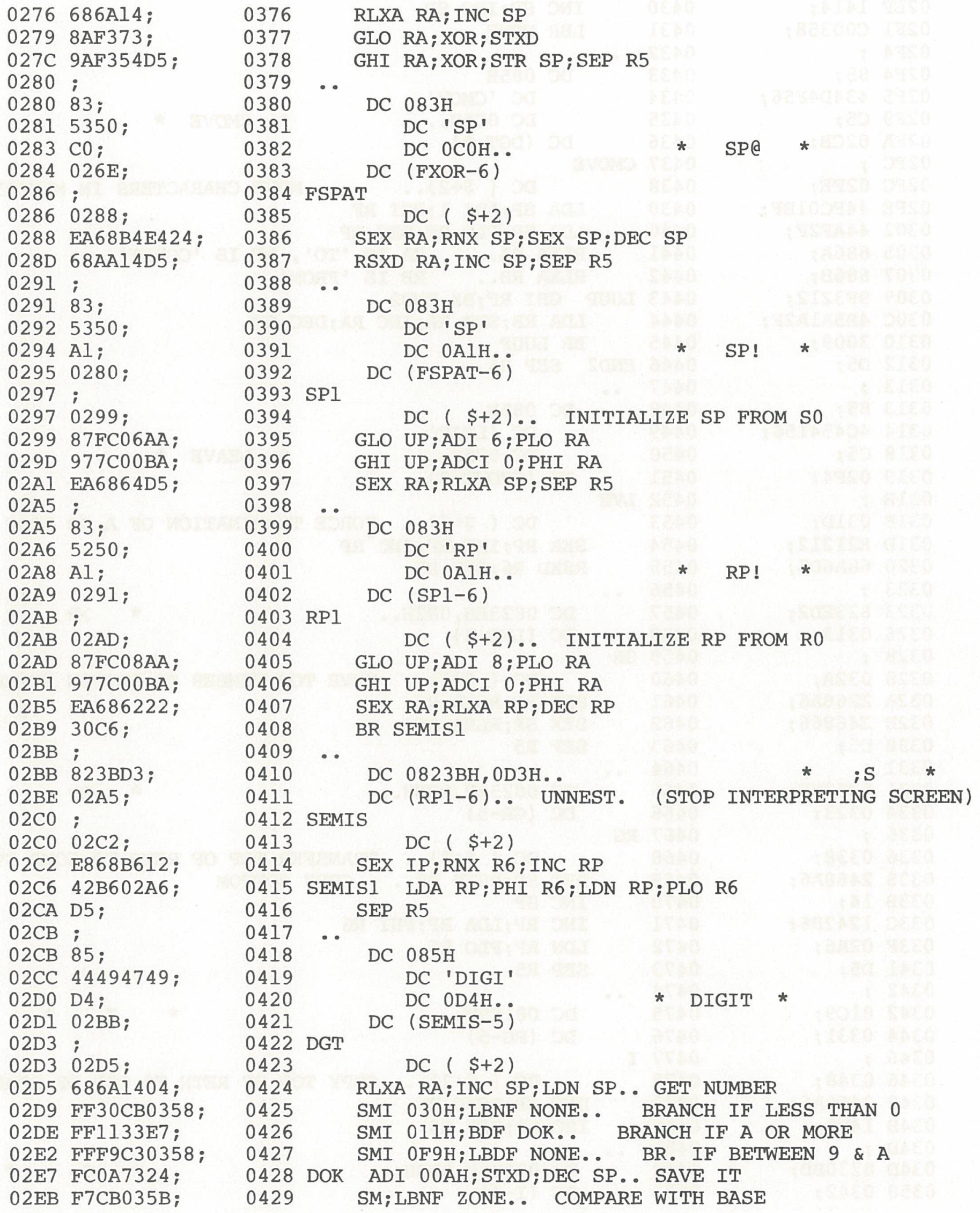


File ID: WFORTH.IST

Disk ID: WOODS HOLE FORTH SOURCE \& LIST VER 1.800

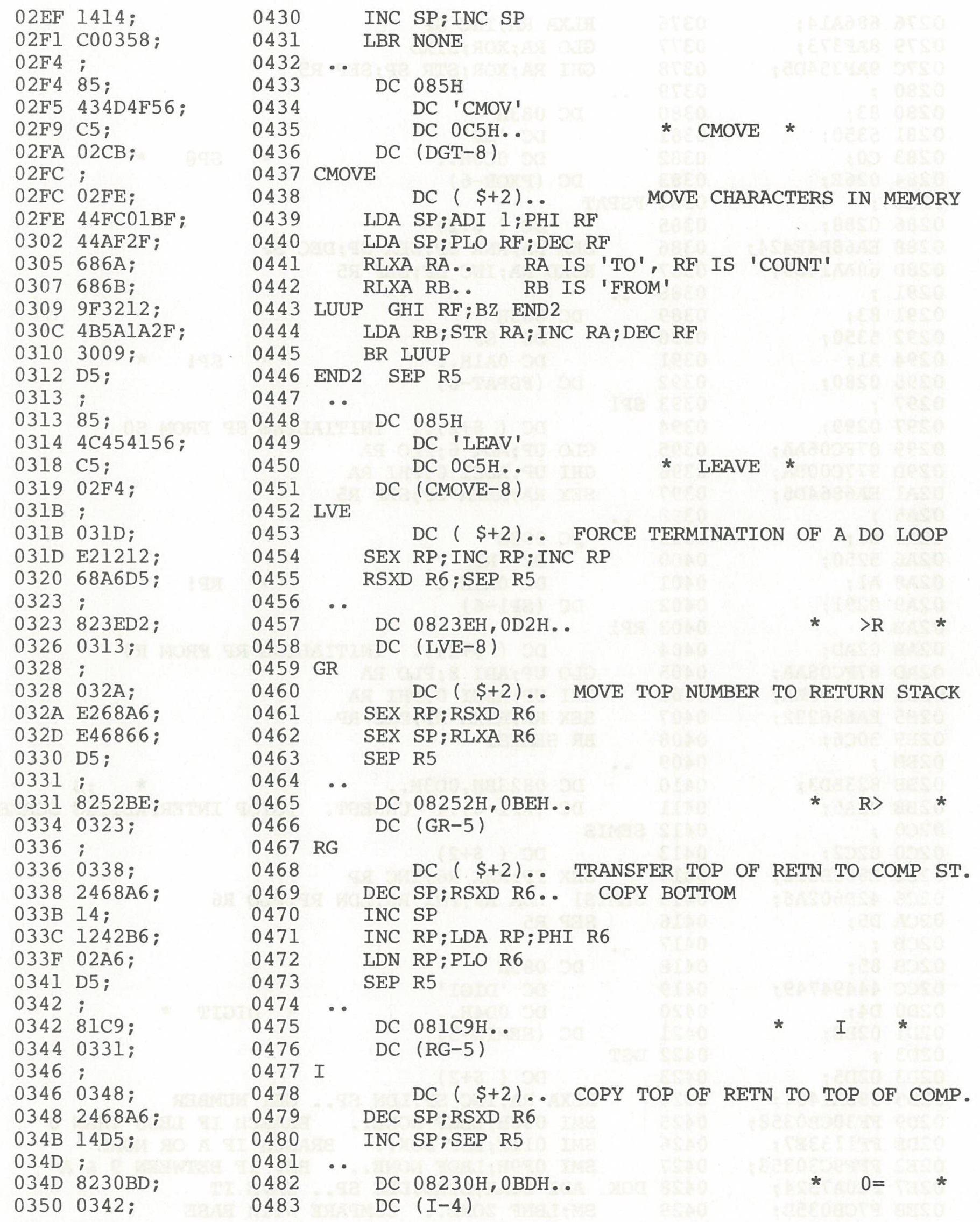


File ID: WFORTH.LST

Disk ID: WOODS HOLE FORTH SOURCE \& LIST VER 1.800

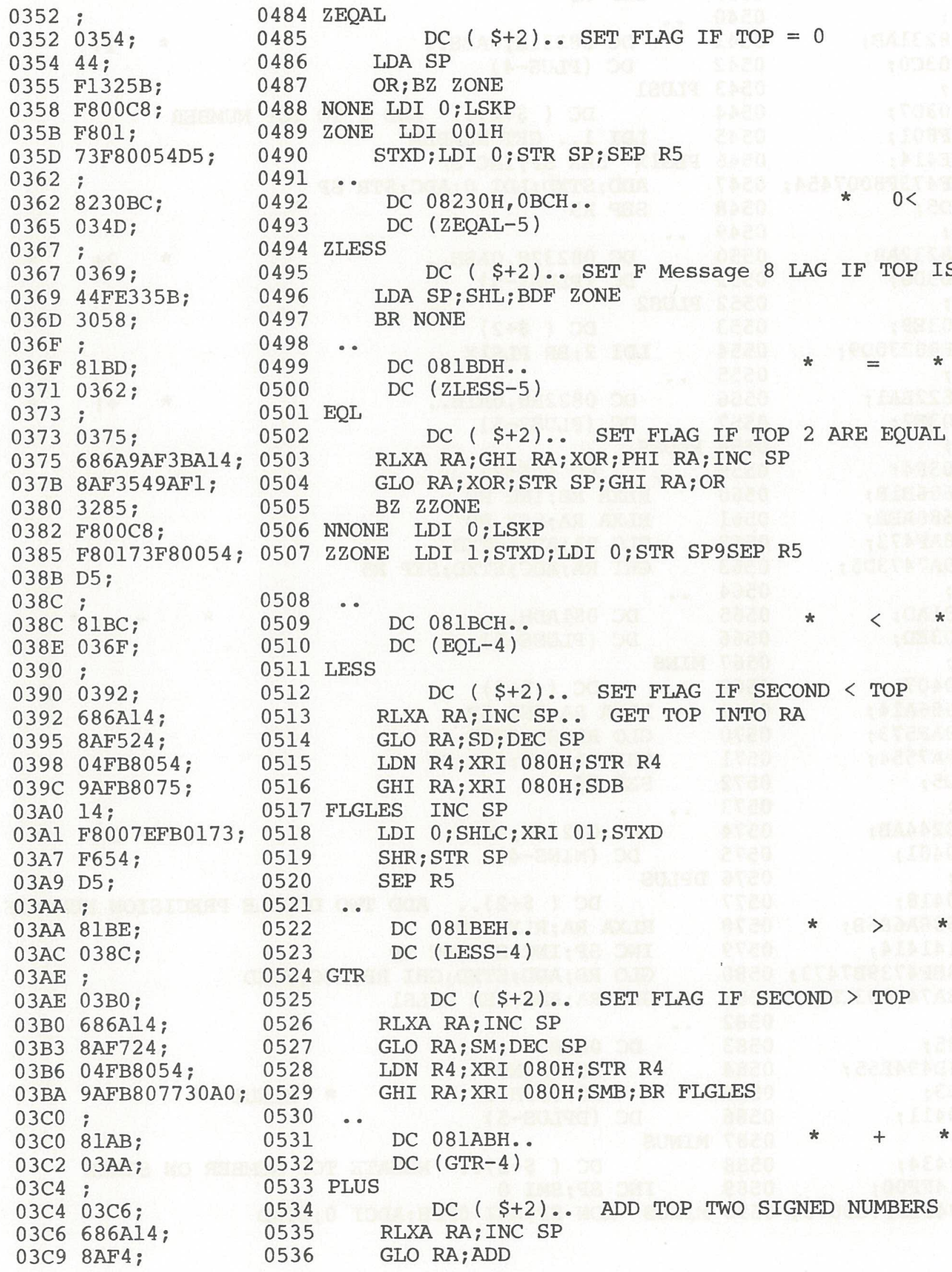


File ID: WFORTH.LST

Disk ID: WOODS HOLE FORTH SOURCE \& LIST VER 1.800

$1130 \quad 84$

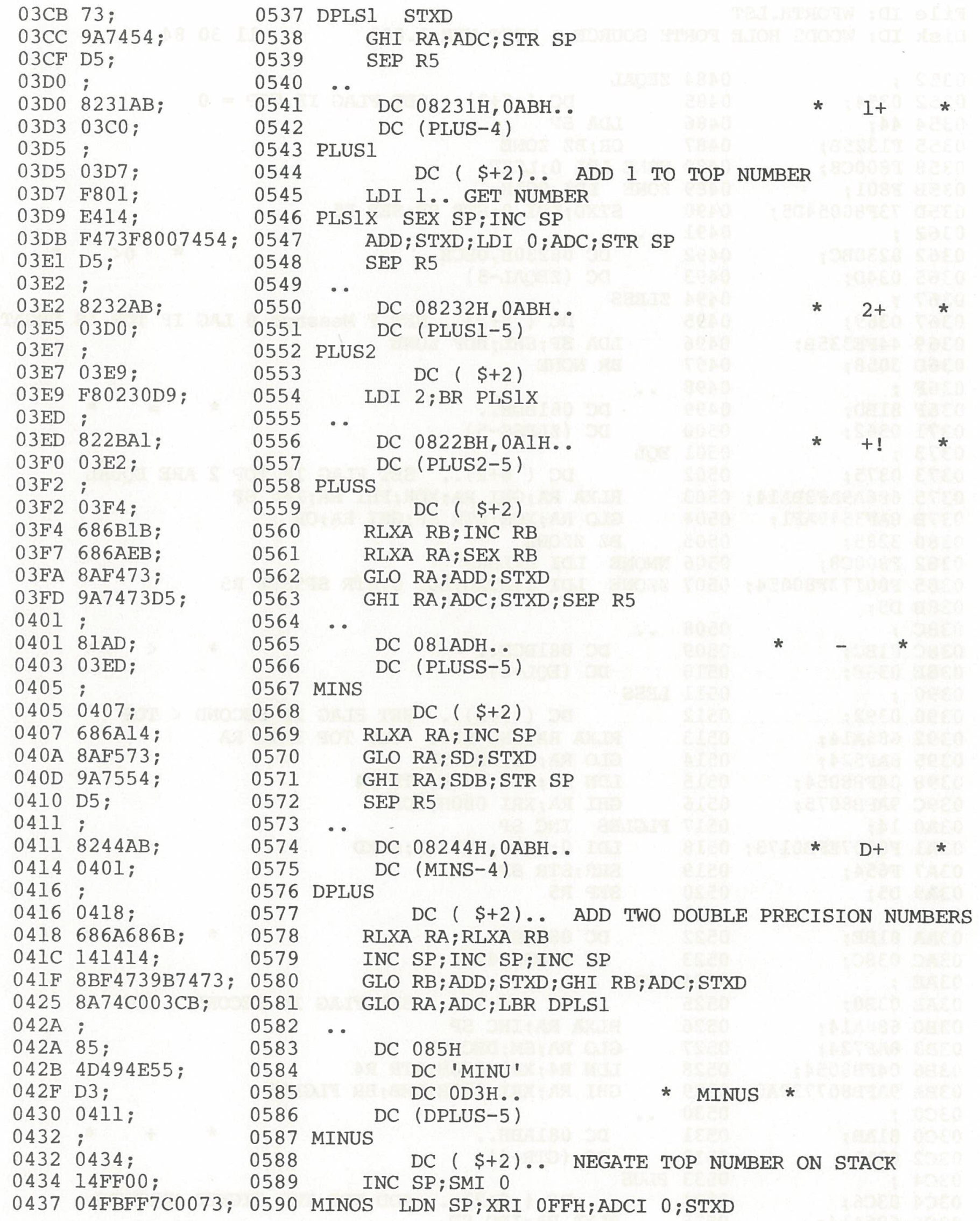


File ID: WFORTH.IST

Disk ID: WOODS HOLE FORTH SOURCE \& LIST VER 1.800

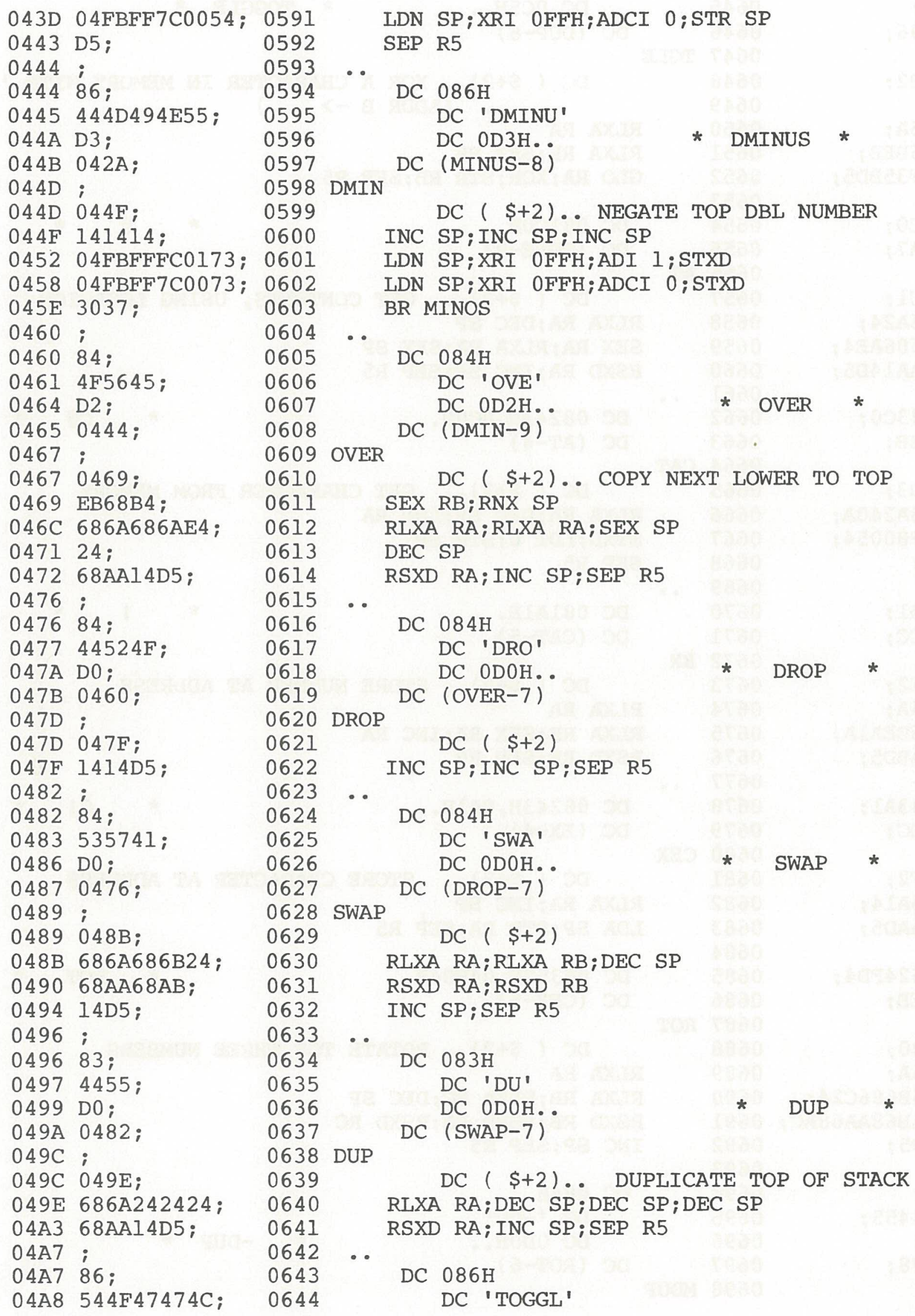


File ID: WFORTH.LST

Disk ID: WOODS HOLE FORTH SOURCE \& LIST VER 1.800 $1130 \quad 84$

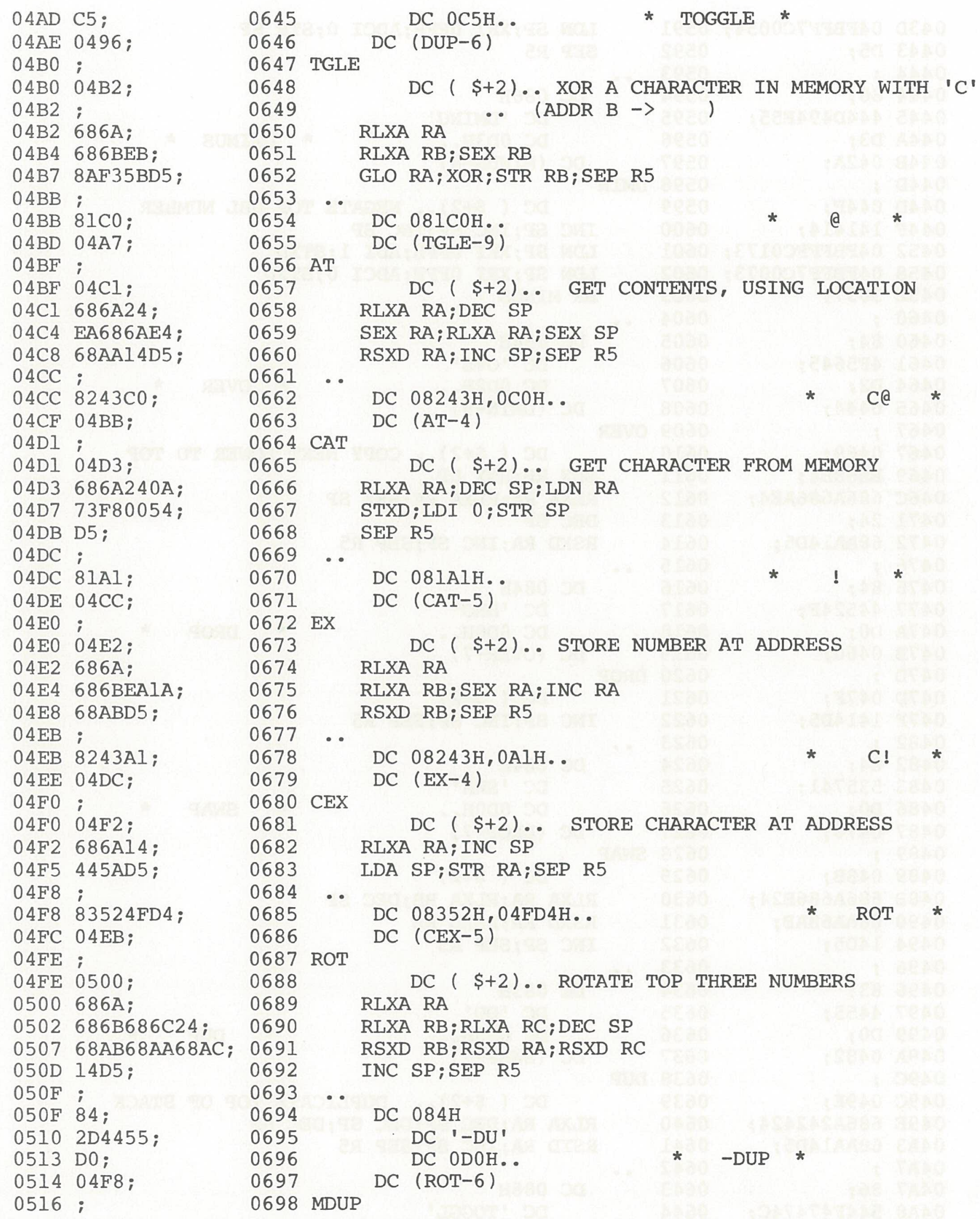


File ID: WFORTH.IST

Disk ID: WOODS HOLE FORTH SOURCE \& LIST VER 1.800

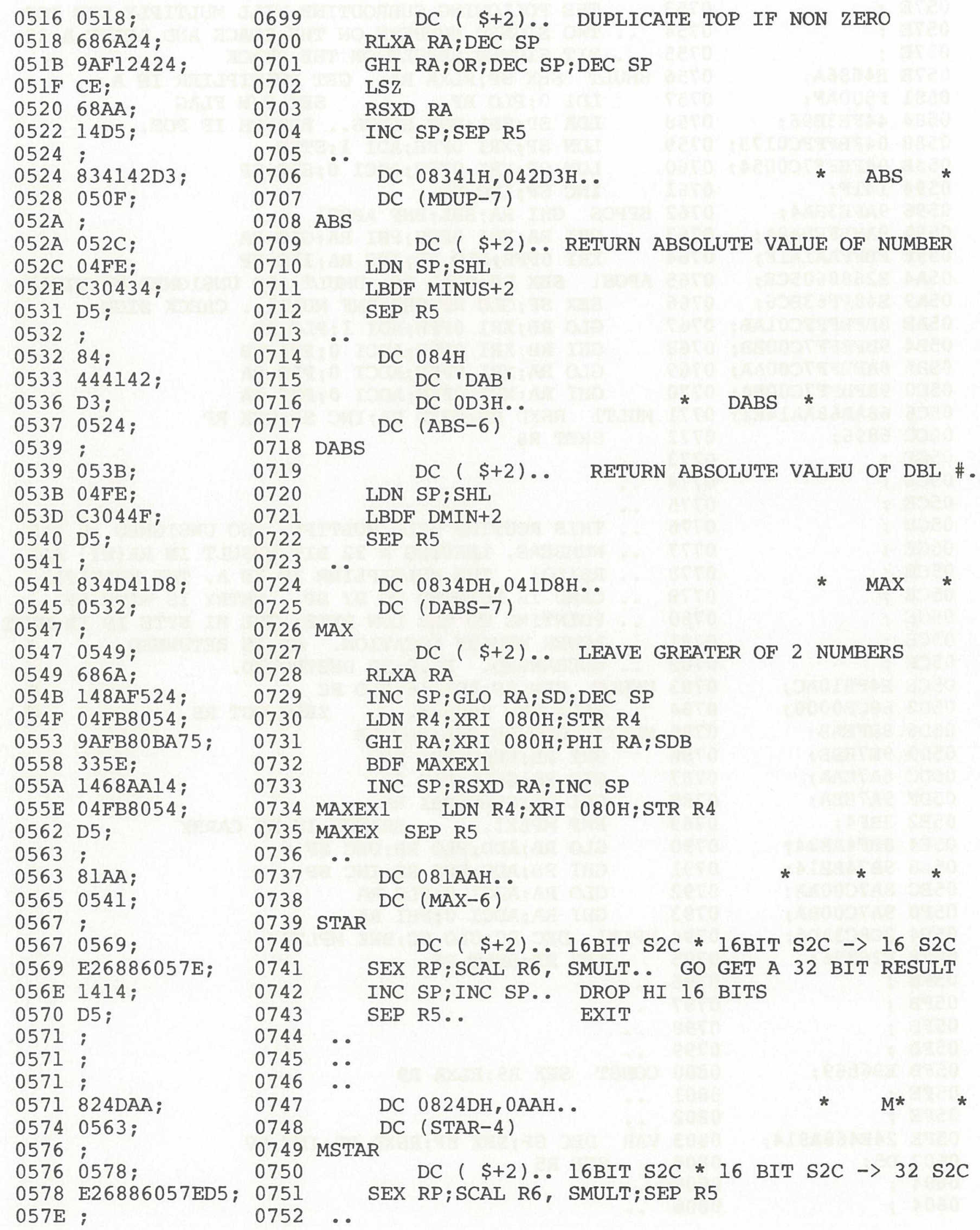


File ID: WFORTH.IST

Disk ID: WOODS HOLE FORTH SOURCE \& LIST VER 1.800

$1130 \quad 84$

\begin{tabular}{|c|c|c|c|}
\hline 057E & ; & 0753 & THE FOLLOWING SUBROUTINE WILI MULTIPLY THE TOP \\
\hline $057 \mathrm{E}$ & ; & 0754 & TWO SIGNED NUMBERS ON THE STACK AND LEAVE A 32 \\
\hline $057 \mathrm{E}$ & ; & 0755 & .. BIT SIGNED RESULT ON THE STACK \\
\hline $57 \mathrm{E}$ & E4686A; & 0756 & SMULT SEX SP;RLXA RA.. GET MULTIPLIER IN A \\
\hline 581 & F800AF ; & 0757 & IDI $0 ;$ PLO RF.. SET P/N FLAG \\
\hline 84 & 44FE3B96; & 0758 & LDA SP;SHL;BNF SPPOS.. BRANCH IF POS. \\
\hline & 04FBFFFC0173; & 0759 & LDN SP;XRI OFFH;ADI $1 ;$ STXD \\
\hline & 04FBFF7C0054; & 0760 & LDN SP;XRI OFFH;ADCI 0 ;STR SP \\
\hline & $141 \mathrm{~F} ;$ & 0761 & INC SP;INC RF \\
\hline & 9AFE3BA4； & 0762 & SPPOS GHI RA; SHL;BNF APOSI \\
\hline & 9AFBFFBA8A; & 0763 & GHI RA; XRI OFFH;PHI RA; GIO RA \\
\hline & FBFFAAIAlF; & 0764 & XRI OFFH;PLO RA;INC RA;INC RF \\
\hline & $\mathrm{E} 2688605 \mathrm{CE}$; & 0765 & APOSl SEX RP;SCAL R6, UMULT..DO UNSIGNED MULTIPLY \\
\hline & $\mathrm{E} 48 \mathrm{FF} 63 \mathrm{BC} 6$; & 0766 & SEX SP;GLO RF;SHR;BNF MULTl.. CHECK SIGN \\
\hline & 8BFBFFFC01AB; & 0767 & GLO RB;XRI OFFH;ADI $1 ;$ PLO RB \\
\hline & 9BFBFF7C00BB; & 0768 & GHI RB;XRI OFFH;ADCI 0 ;PHI RB \\
\hline & 8AFBFF 7C00AA; & 0769 & GLO RA; XRI OFFH;ADCI 0;PLO RA \\
\hline & 9AFBFF7C00BA; & 0770 & GHI RA;XRI OFFH;ADCI 0 ; PHI RA \\
\hline & 68AB68AAI4E2; & 0771 & MULTI RSXD RB;RSXD RA; INC SP;SEX RP \\
\hline & $6896 ;$ & 0772 & SRET R6 \\
\hline & ; & 0773 & - \\
\hline & ; & 0774 & $\cdots$ \\
\hline & ; & 0775 & 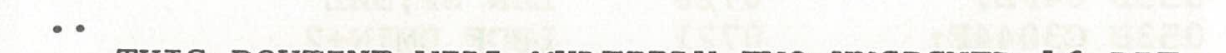 \\
\hline & ; & 0776 & .. THIS ROUTINE WILL MULTIPLY TWO UNSIGNED 16 \\
\hline & ; & 0777 & .. NUMBERS, LEAVING A 32 BIT RESULT IN RA(HI) AND \\
\hline & ; & 0778 & .. RB(LO). THE MULTIPLIER IS IN A, THE MULTIPLI \\
\hline & ; & 0779 & .. CAND IS POINTED TO BY SP. ENTRY IS WITH SP \\
\hline & ; & 0780 & .. POINTING TO THE LOW BYTE. THE HI BYTE IS IN NEX \\
\hline & ; & 0781 & .. LOWER MEMORY LOCATION. SP IS RETURNED \\
\hline & ; & 0782 & .. UNCHANGED. RC.0 IS DESTROYED. \\
\hline & E4F810AC; & 0783 & UMULT SEX SP;LDI $16 ;$ PLO RC \\
\hline & $68 \mathrm{CB} 0000$ & 0784 & RLDI RB，00000H.. ZERO OUT RB \\
\hline & 8BFEAB; & 0785 & MPLX2 GLO RB;SHL;PLO RB \\
\hline & 9B7EBB; & 0786 & GHI RB;SHLC;PHI RB \\
\hline & 8A7EAA; & 0787 & GLO RA; SHLC;PLO RA \\
\hline & 9A7EBA; & 0788 & GHI RA;SHLC;PHI RA \\
\hline & 3BF4; & 0789 & BNF MPLXI.. BRANCH IF NO CARRY \\
\hline & $8 \mathrm{BF} 4 \mathrm{AB} 24$; & 0790 & GLO RB;ADD;PLO RB;DEC SP \\
\hline & 9B74BBI4; & 0791 & GHI RB;ADC; PHI RB; INC SP \\
\hline & 8А7С00AA; & 0792 & GLO RA;ADCI $0 ;$ PLO RA \\
\hline & 9А7СООВА; & 0793 & GHI RA;ADCI $0 ;$ PHI RA \\
\hline & 2C8C3AD6; & 0794 & MPLX1 DEC RC;GLO RC;BNZ MPLX2 \\
\hline & E26896; & 0795 & SEX RP;SRET R6 \\
\hline & ; & 0796 & - \\
\hline & ; & 0797 & $\cdots$ \\
\hline & ; & 0798 & $\cdots$ \\
\hline & i & 0799 & $\ddot{0}$ \\
\hline & E96869; & 0800 & SEX R9;RLXA R9 \\
\hline & ; & 0801 & $\cdots$ \\
\hline & ; & 0802 & 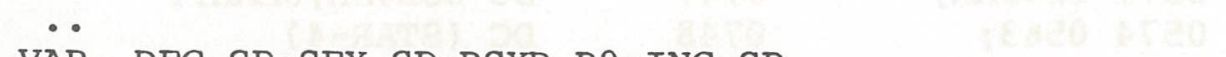 \\
\hline & $24 \mathrm{E} 468 \mathrm{~A} 914$ & 0803 & VAR DEC SP;SEX SP;RSXD R9;INC SP \\
\hline & D5 ; & 0804 & SEP R5 \\
\hline & ; & 0805 & $\cdots$ \\
\hline & i & 0806 & \\
\hline
\end{tabular}

Woods Hole FORTH Version $1.800 \quad$ Chapter $21 \quad$ page 158 
File ID: WFORTH.LST

Disk ID: WOODS HOLE FORTH SOURCE \& LIST VER 1.800

$1130 \quad 84$

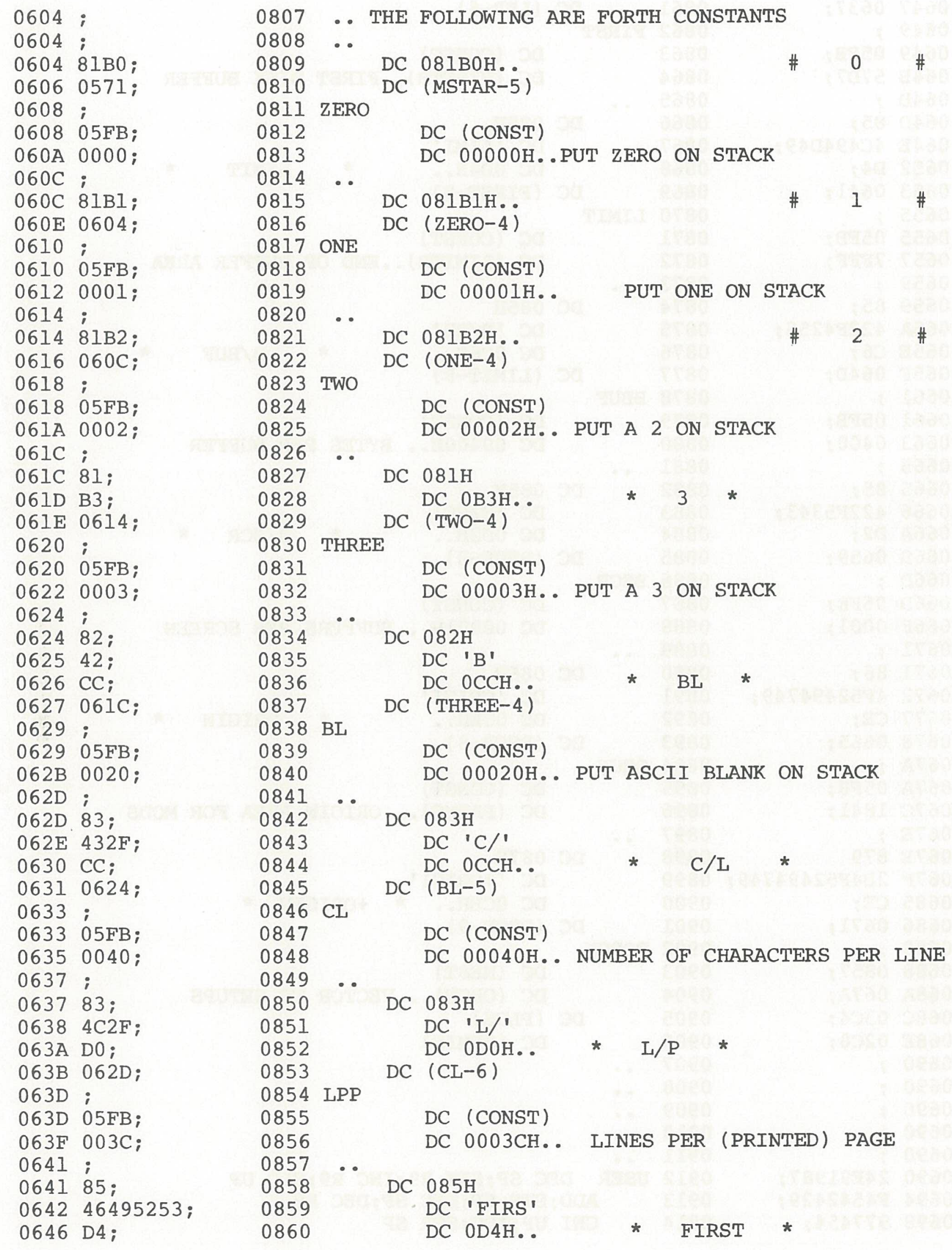


File ID: WFORTH.LST

Disk ID: WOODS HOLE FORTH SOURCE \& LIST VER 1.800

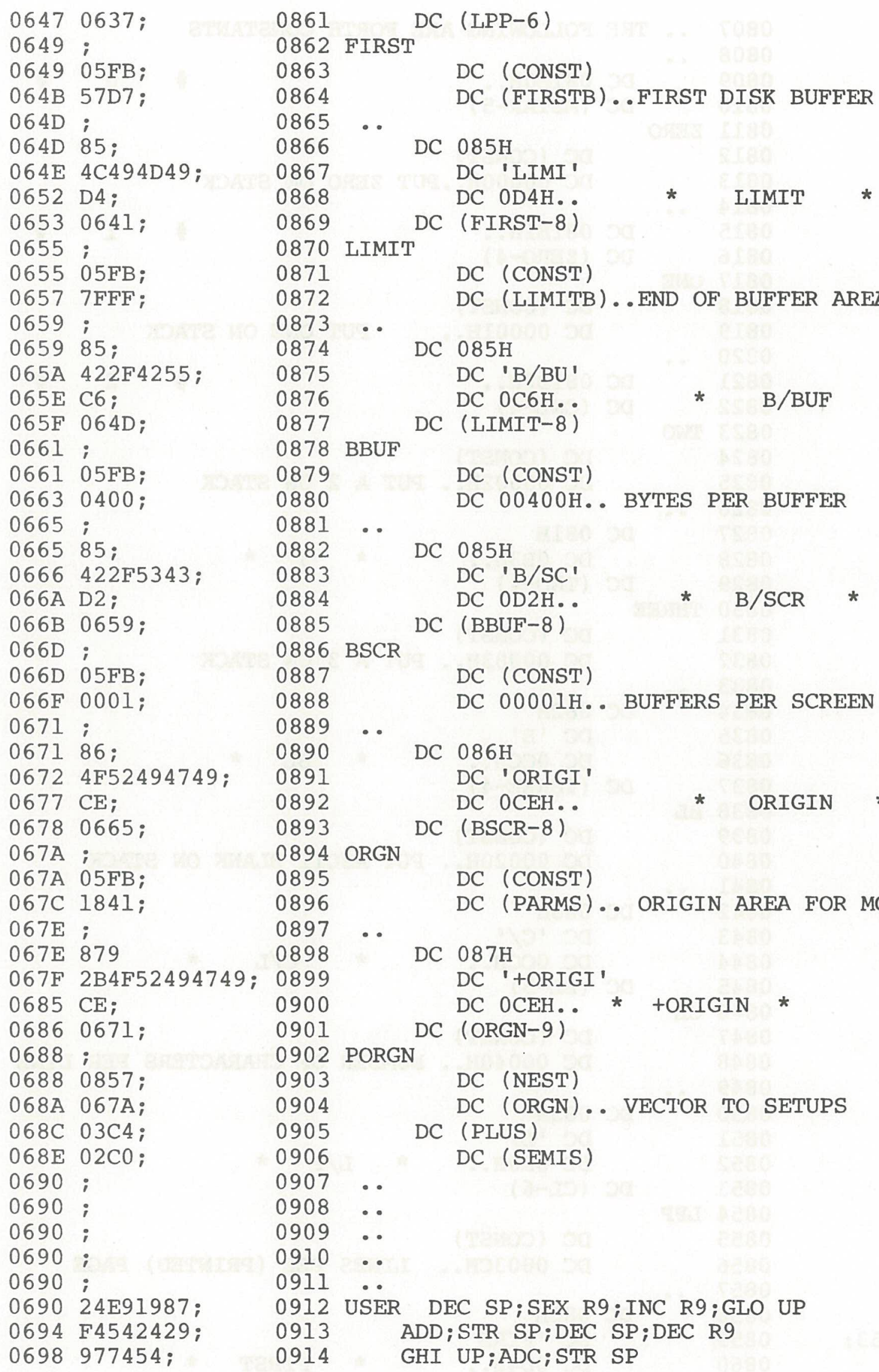


File ID: WFORTH.IST

Disk ID: WOODS HOLE FORTH SOURCE \& LIST VER 1.800

$1130 \quad 84$

\begin{tabular}{|c|c|c|c|c|c|c|c|c|c|c|}
\hline 069B & D5 ; & 0915 & & SEP & R5 & & & & & \\
\hline $069 \mathrm{C}$ & ; & 0916 & .. & & & & & & & \\
\hline $069 \mathrm{C}$ & ; & 0917 & .. & & & & & & & \\
\hline $069 \mathrm{C}$ & ; & 0918 & -. & THE & FOLLOWING ARE USER & VARIABLES & & & & \\
\hline $069 \mathrm{C}$ & ; & 0919 & -. & & & & & & & \\
\hline $069 \mathrm{C}$ & $8253 \mathrm{BO} ;$ & 0920 & & DC & 08253Н, ОВОН.. & & $\$$ & & so & $\$$ \\
\hline $069 \mathrm{~F}$ & $067 \mathrm{E} ;$ & 0921 & & $\mathrm{DC}$ & $($ PORGN-10) & & & & & \\
\hline 06Al & ; & 0922 & so & & & & & & & \\
\hline $06 \mathrm{Al}$ & $0690 ;$ & 0923 & & & DC (USER) & & & & & \\
\hline $06 \mathrm{A3}$ & 0006 ; & 0924 & & & DC $00006 \mathrm{H} .$. STORED $\mathrm{B}$ & ВОTTOM OF & STACK & & & \\
\hline $06 \mathrm{~A} 5$ & ; & 0925 & .. & & & & & & & \\
\hline 06 A5 & $8252 \mathrm{~B} 0$; & 0926 & & DC & $08252 \mathrm{H}, 0 \mathrm{OBOH}$. & & $\$$ & & R0 & $\$$ \\
\hline $06 \mathrm{~A} 8$ & $069 C_{i}$ & 0927 & & $\mathrm{DC}$ & $(\mathrm{SO}-5)$ & & & & & \\
\hline 06AA & ; & 0928 & RO & & & & & & & \\
\hline 06AA & 0690 ; & 0929 & & & DC (USER) & & & & & \\
\hline $06 \mathrm{AC}$ & 0008 & 0930 & & & DC $00008 \mathrm{H} .$. вотTOM & OF RETURN & STACK & & & \\
\hline 06AE & i & 0931 & .. & & & & & & & \\
\hline 06AE & $83 ;$ & 0932 & & DC & $083 \mathrm{H}$ & & & & & \\
\hline $06 \mathrm{AF}$ & $5449 ;$ & 0933 & & & DC 'TI' & & & & & \\
\hline 06Bl & $\mathrm{C} 2$; & 0934 & & & DC $0 \mathrm{C} 2 \mathrm{H}$ & TIB & & $\$$ & & \\
\hline $06 \mathrm{~B} 2$ & 06A5; & 0935 & & $\mathrm{DC}$ & $(\mathrm{RO}-5)$ & & & & & \\
\hline 06B4 & ; & 0936 & TIB & & & & & & & \\
\hline 06B4 & 0690; & 0937 & & & DC (USER) & & & & & \\
\hline 06B6 & 000A; & 0938 & & & DC 0000AH.. TERMINAI & $\mathrm{AL}$ INPUT BU. & JFFER & LOC. & $\therefore$ & \\
\hline 06B8 & i & 0939 & -. & & & & & & & \\
\hline 06B8 & $85 ;$ & 0940 & & DC & $085 \mathrm{H}$ & & & & & \\
\hline 06B9 & 57494454 ; & 0941 & & & DC 'WIDT' & & & & & \\
\hline $06 \mathrm{BD}$ & $\mathrm{C} 8$; & 0942 & & & $\mathrm{DC} \quad 0 \mathrm{C} 8 \mathrm{H}$. & WIDT'H & * & & & \\
\hline $06 \mathrm{BE}$ & 06AE; & 0943 & & $\mathrm{DC}$ & $(\mathrm{TIB}-6)$ & & & & & \\
\hline $06 \mathrm{CO}$ & ; & 0944 & WIDTH & & & & & & & \\
\hline $06 \mathrm{CO}$ & 0690; & 0945 & & & DC (USER) & & & & & \\
\hline $06 \mathrm{C} 2$ & 000C; & 0946 & & & DC $0000 \mathrm{CH} .$. MAX WORL & RD LENGTH & & & & \\
\hline $06 \mathrm{C} 4$ & i & 0947 & - & & & & & & & \\
\hline $06 C 4$ & $87 ;$ & 0948 & & DC & $087 \mathrm{H}$ & & & & & \\
\hline $06 C 5$ & $5741524 \mathrm{E} 494 \mathrm{E} ;$ & 0949 & & & DC 'WARNIN' & & & & & \\
\hline $06 \mathrm{CB}$ & C7 ; & 0950 & & & DC $0 \mathrm{C} 7 \mathrm{H}$ & WARNING & $\$$ & & & \\
\hline $06 \mathrm{CC}$ & $06 \mathrm{~B} 8$; & 0951 & & $\mathrm{DC}$ & (WIDTH-8) & & & & & \\
\hline $06 \mathrm{CE}$ & i & 0952 & WRNG & & & & & & & \\
\hline $06 \mathrm{CE}$ & 0690; & 0953 & & & DC (USER) & & & & & \\
\hline 06D0 & OOOE; & 0954 & & & DC $0000 \mathrm{EH}$ & & & & & \\
\hline 06D2 & i & 0955 & -. & & & & & & & \\
\hline 06D2 & $85 ;$ & 0956 & & DC & $085 \mathrm{H}$ & & & & & \\
\hline $06 \mathrm{D} 3$ & $46454 \mathrm{E} 43$; & 0957 & & & DC 'FENC' & & & & & \\
\hline $06 \mathrm{D} 7$ & $\mathrm{C} 5$ & 0958 & & & $\mathrm{DC} \quad 0 \mathrm{C} 5 \mathrm{H}$. & FENCE & $\$$ & & & \\
\hline 06D8 & $06 \mathrm{C} 4$; & 0959 & & DC & (WRNG-10) & & & & & \\
\hline 06DA & ; & 0960 & FNCE & & & & & & & \\
\hline 06DA & 0690 ; & 0961 & & & DC (USER) & & & & & \\
\hline 06DC & 0010 & 0962 & & & DC $00010 \mathrm{H}_{.}$. DICTION & JARY FENCE & & & & \\
\hline 06DE & i & 0963 & - & & & & & & & \\
\hline 06DE & $8244 \mathrm{DO}$; & 0964 & & DC & $08244 \mathrm{H}, 0 \mathrm{DOH} .$. & & $\$$ & & DP & $\$$ \\
\hline 06El & 06D2; & 0965 & & DC & $($ FNCE-8) & & & & & \\
\hline 06E3 & i & 0966 & DP & & & & & & & \\
\hline $06 \mathrm{E} 3$ & 0690 ; & 0967 & & & DC (USER) & & & & & \\
\hline $06 \mathrm{E} 5$ & 0012 & 0968 & & & DC $00012 \mathrm{H} \ldots \quad$ DICTIOI & DNARY POINT & TER & & & \\
\hline
\end{tabular}


File ID: WFORTH.LST

Disk ID: WOODS HOLE FORTH SOURCE \& LIST VER 1.800

$11 \quad 30 \quad 84$

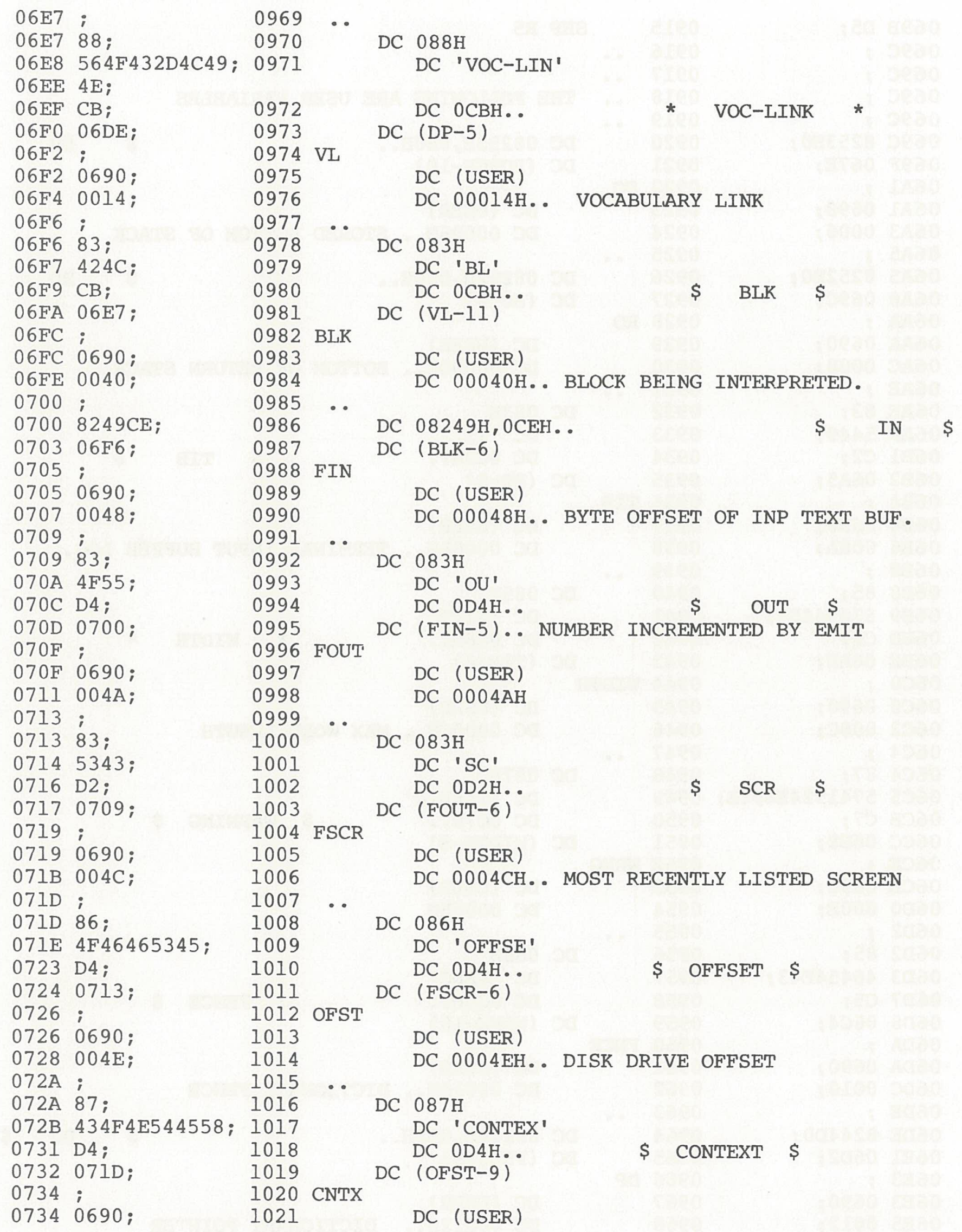


File ID: WFORTH.LST

Disk ID: WOODS HOLE FORTH SOURCE \& LIST VER 1.800

$1130 \quad 84$

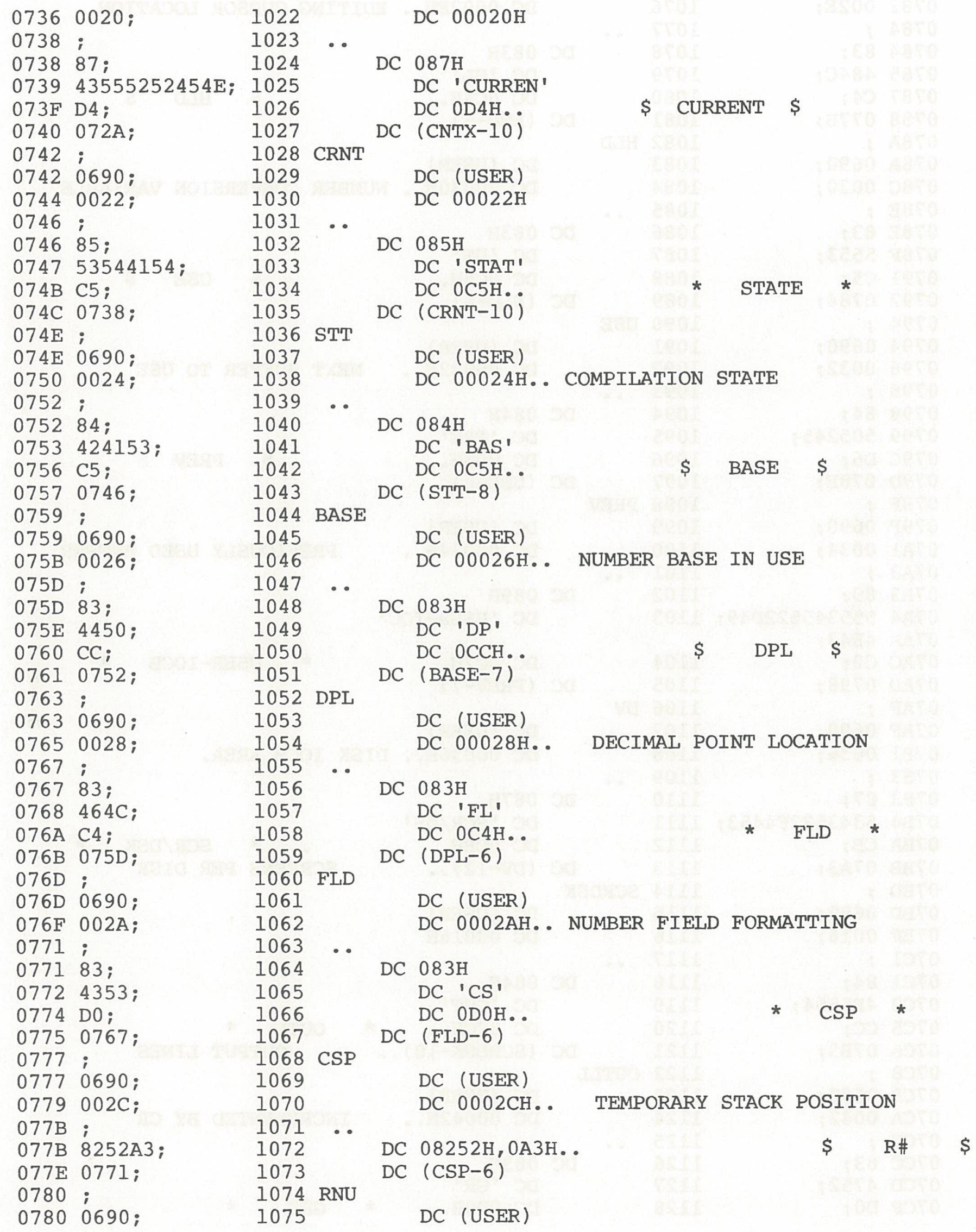


File ID: WFORTH.LST

Disk ID: WOODS HOLE FORTH SOURCE \& LIST VER 1.800

$1130 \quad 84$

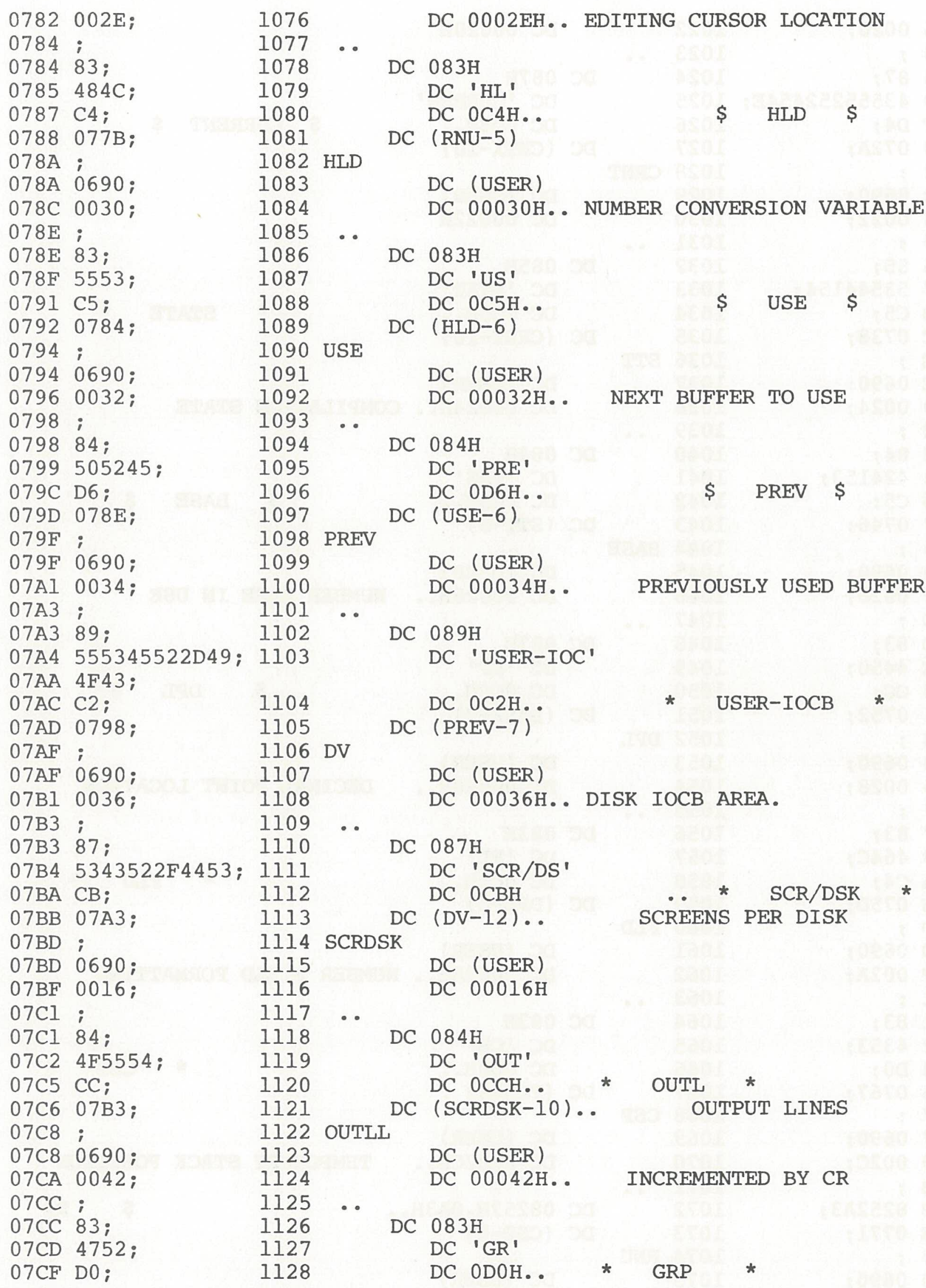


File ID: WFORTH.LST

Disk ID: WOODS HOLE FORTH SOURCE \& LIST VER 1.800

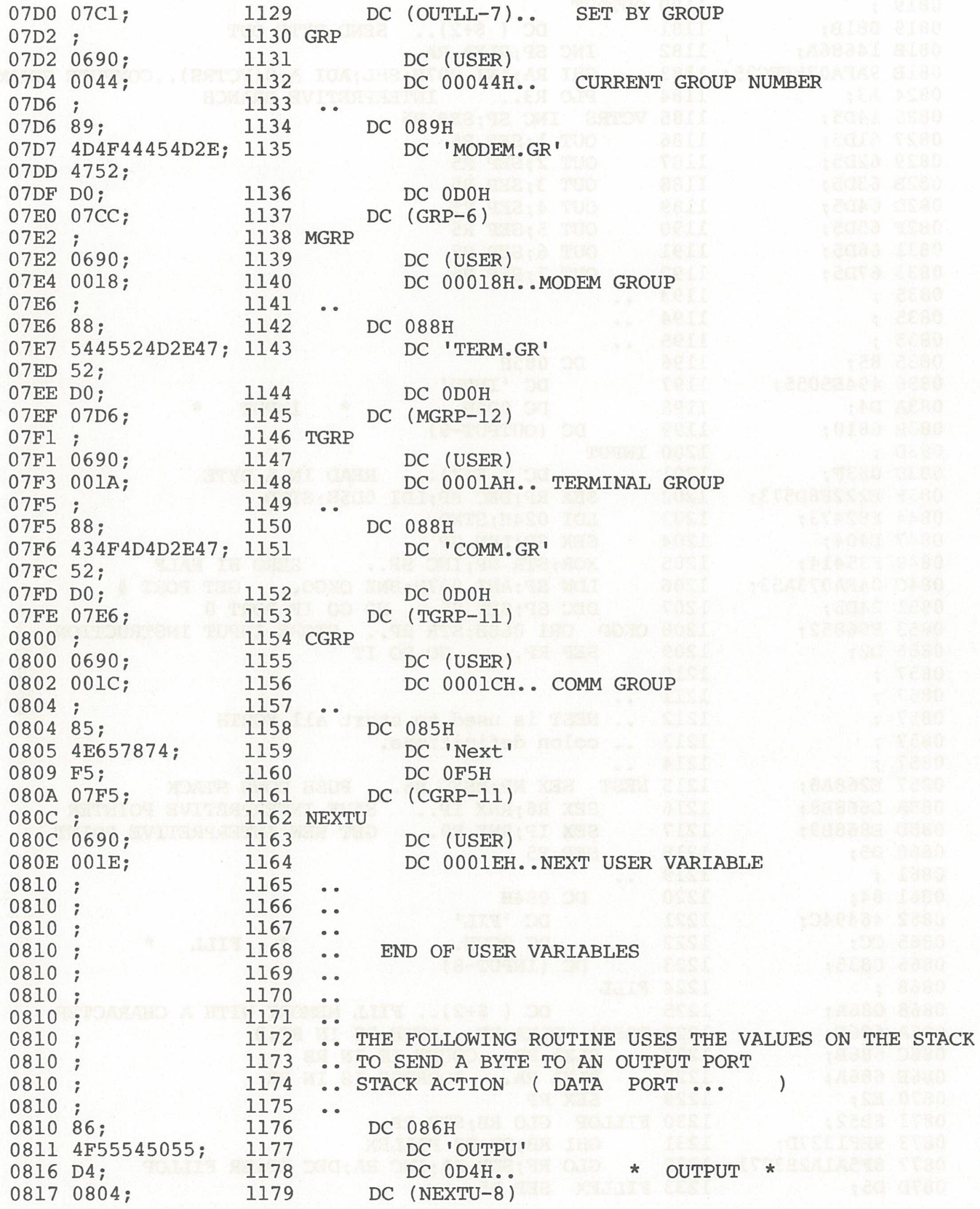


File ID: WFORTH.LST

Disk ID: WOODS HOLE FORTH SOURCE \& IIST VER 1.800

$1130 \quad 84$

\begin{tabular}{|c|c|c|c|}
\hline 0819 & ; & 1180 & OUTPUT \\
\hline 0819 & 081B; & 1181 & SEND BYTE OUT \\
\hline $081 B$ & $14686 \mathrm{~A} ;$ & 1182 & INC SP;RLXA RA \\
\hline $81 \mathrm{E}$ & 9AFA07FEFC25; & 1183 & GHI RA;ANI 007H;SHL;ADI A.0(VCTRS)..COMPUTE \\
\hline 824 & A3; & 1184 & PLO R3.. INTERPRETIVE BRANCH \\
\hline 825 & 14D5； & 1185 & VCTRS INC SP;SEP R5 \\
\hline 827 & 61D5; & 1186 & OUT $1 ;$ SEP R5 \\
\hline 829 & 62D5; & 1187 & OUT 2;SEP R5 \\
\hline $82 \mathrm{~B}$ & 63D5; & 1188 & OUT 3;SEP R5 \\
\hline $82 \mathrm{D}$ & 64D5; & 1189 & OUT 4 ; SEP R5 \\
\hline $82 \mathrm{~F}$ & 65D5; & 1190 & OUT 5; SEP R5 \\
\hline 831 & 66D5; & 1191 & OUT $6 ;$ SEP R5 \\
\hline 833 & 67D5； & 1192 & OUT 7 ; SEP R5 \\
\hline 835 & ; & 1193 & - \\
\hline 835 & ; & 1194 & . \\
\hline 335 & ; & 1195 & .. \\
\hline & $85 ;$ & 1196 & DC $085 \mathrm{H}$ \\
\hline 36 & 494E5055; & 1197 & DC 'INPU' \\
\hline $83 \mathrm{~A}$ & D4 ; & 1198 & DC $0 \mathrm{D} 4 \mathrm{H} \ldots$ \\
\hline $83 \mathrm{~B}$ & 0810; & 1199 & DC (OUTPUT-9) \\
\hline $83 \mathrm{D}$ & i & 1200 & INPUT \\
\hline $83 \mathrm{D}$ & $083 \mathrm{~F} ;$ & 1201 & DC $(\$+2) \ldots \quad$ READ IN A BYTE \\
\hline $83 \mathrm{~F}$ & E222F8D573; & 1202 & SEX RP;DEC RP;LDI OD5H;STXD \\
\hline 844 & F82473; & 1203 & LDI $024 \mathrm{H} ; S T X D$ \\
\hline 847 & E404; & 1204 & SEX SP;LDN SP \\
\hline 849 & F35414; & 1205 & XOR;STR SP;INC SP.. $\quad$ ZERO HI HALF \\
\hline $84 \mathrm{C}$ & 04FA073A53; & 1206 & LDN SP;ANI 007H;BNZ OKGO.. GET PORT \# \\
\hline 851 & 24D5; & 1207 & DEC SP;SEP R5.. NO GO IF PORT 0 \\
\hline 853 & F96852; & 1208 & OKGO ORI 068H;STR RP.. STORE INPUT INSTRUCTION \\
\hline 0856 & D2; & 1209 & SEP RP.. $\quad$ GO DO IT \\
\hline 0857 & ; & 1210 & .. \\
\hline 0857 & i & 1211 & .. \\
\hline 0857 & ; & 1212 & .. NEST is used to start all FORTH \\
\hline 0857 & i & 1213 & .. colon definitions. \\
\hline 0857 & i & 1214 & . \\
\hline 0857 & E268A6; & 1215 & NEST SEX RP;RSXD R6.. PUSH DOWN STACK \\
\hline $085 \AA$ & E668B8; & 1216 & SEX R6;RNX IP.. S SAVE INTERPRETIVE POINTER \\
\hline $085 \mathrm{D}$ & E868B9; & 1217 & SEX IP;RNX R9.. GET NEW INTERPRETIVE POINT \\
\hline 0860 & D5; & 1218 & SEP R5 \\
\hline 0861 & ; & 1219 & .. \\
\hline 0861 & $84 ;$ & 1220 & DC $084 \mathrm{H}$ \\
\hline 0862 & $46494 \mathrm{C}$; & 1221 & DC 'FIL' \\
\hline 0865 & $\mathrm{CC} ;$ & 1222 & $\mathrm{DC} \mathrm{OCCH} .$. \\
\hline 0866 & 0835; & 1223 & DC (INPUT-8) \\
\hline 0868 & i & 1224 & FILL \\
\hline 0868 & 086A; & 1225 & DC $(\$+2) \ldots$ FILL MEMORY WITH A CHARACTER \\
\hline $086 \mathrm{~A}$ & $686 \mathrm{~F} ;$ & 1226 & ERSOl RLXA RF.. BYTE IS IN RF.0 \\
\hline $086 \mathrm{C}$ & 686B; & 1227 & RLXA RB.. COUNT IS IN RB \\
\hline $086 \mathrm{E}$ & 686A; & 1228 & RLXA RA.. ADDRESS IS IN RA \\
\hline 0870 & E2; & 1229 & SEX RP \\
\hline 0871 & 8B52; & 1230 & FILLOP GLO RB;STR RP \\
\hline & 9BF1327D; & 1231 & GHI RB;OR;BZ FILLEX \\
\hline 0877 & 8F5A1A2B3071; & 1232 & GLO RF;STR RA;INC RA;DEC RB;BR FILLOP \\
\hline & D5; & 1233 & FILLEX SEP R5 \\
\hline
\end{tabular}


File ID: WFORTH.LST

Disk ID: WOODS HOLE FORTH SOURCE \& LIST VER 1.800

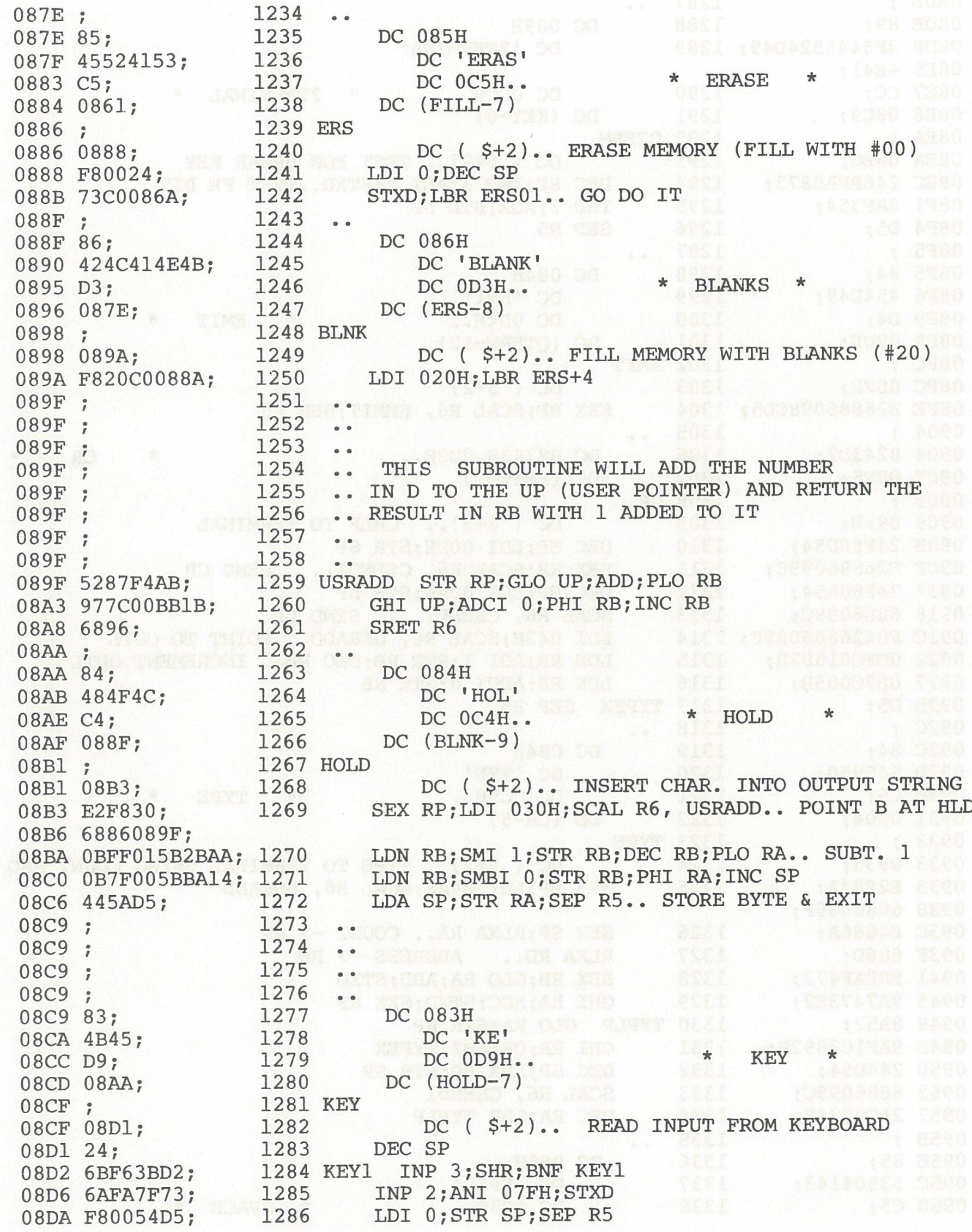


File ID: WFORTH.IST

Disk ID: WOODS HOLE FORTH SOURCE \& LIST VER 1.800

$1130 \quad 84$

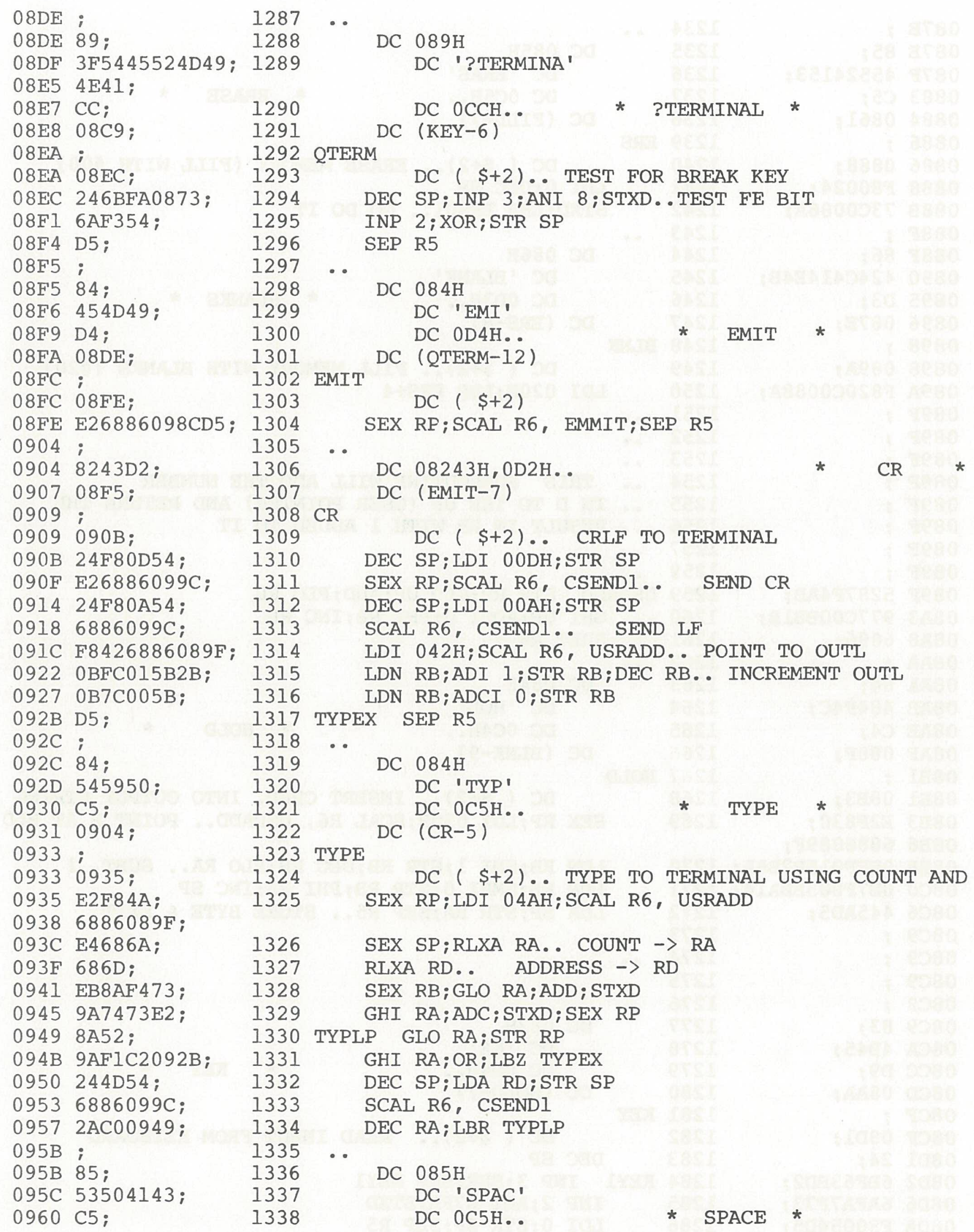

Woods Hole FORTH Version $1.800 \quad$ Chapter $21 \quad$ page 168 
File ID: WFORTH.IST

Disk ID: WOODS HOLE FORTH SOURCE \& LIST VER 1.800

$1130 \quad 84$

\begin{tabular}{|c|c|c|c|}
\hline 0961 & 092C; & 1339 & DC (TYPE-7) \\
\hline 0963 & ; & 1340 & SPC \\
\hline 0963 & $0965 ;$ & 1341 & $\mathrm{DC}(\$+2)$ \\
\hline 965 & 24F82073; & 1342 & DEC SP;LDI 020H;STXD \\
\hline 0969 & $\mathrm{C} 008 \mathrm{FE}$ & 1343 & LBR EMIT+2 \\
\hline $096 \mathrm{C}$ & ; $\quad 2$ & 1344 & - \\
\hline 096C & ; & 1345 & .. THE FOLLOWING ROUTINES ALLOW CALLS INTO THE \\
\hline $96 \mathrm{C}$ & ; & 1346 & .. UTILITY PROGRAM. IT SETS UP R4 AND 5 FOR \\
\hline $96 \mathrm{C}$ & ; & 1347 & ..THE STANDARD CALL AND RETURN ROUTINES \\
\hline 096C & ; & 1348 & 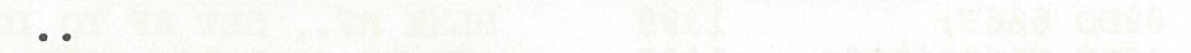 \\
\hline $96 \mathrm{C}$ & $68 \mathrm{A4} ;$ & 1349 & SCRT RSXD R4 .. SAVE R4 \\
\hline $96 \mathrm{E}$ & $68 C 40977$; & 1350 & RLDI R4，CALLR \\
\hline 972 & $15156896 ;$ & 1351 & INC R5;INC R5;SRET R6 \\
\hline 0976 & i $\quad 2 \quad-1$ & 1352 & . \\
\hline 976 & D3; & 1353 & CALLX SEP R3 \\
\hline 0977 & E268A6E668B3; & 1354 & CALLR SEX RP;RSXD R6;SEX R6;RNX R3 \\
\hline 097D & $6863 \mathrm{E} 23076 ;$ & 1355 & RLXA R3;SEX RP;BR CALLX \\
\hline 982 & i $\quad 1 \quad-1$ & 1356 & 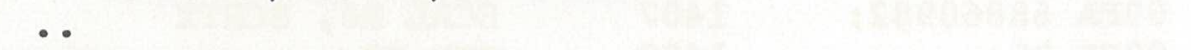 \\
\hline 0982 & $126864 ;$ & 1357 & SCRTX INC RP;RLXA R4 \\
\hline $\begin{array}{l}0985 \\
098 A\end{array}$ & $\begin{array}{l}2268 C 50098 ; \\
6896 ;\end{array}$ & 1358 & DEC RP;RLDI R5, NEXT;SRET R6 \\
\hline $098 \mathrm{C}$ & ; & 1359 & .. \\
\hline $98 \mathrm{C}$ & ; & 1360 & - \\
\hline 098C & ; & 1361 & - \\
\hline $098 \mathrm{C}$ & ; & 1362 & -. THIS SUBROUTINE WILL EMIT A CHARACTER AND \\
\hline 098C & ; & 1363 & .. INCREMENT THE VARIABLE 'OUT' \\
\hline $098 \mathrm{C}$ & ; & 1364 & - \\
\hline $098 \mathrm{C}$ & F84A6886089F; & 1365 & EMMIT LDI 04AH;SCAL R6, USRADD.. POINT TO 'OUT' \\
\hline 0992 & 0BFC015B2B ; & 1366 & LDN RB;ADI l;STR RB;DEC RB.. INCREMENT 'OUT' \\
\hline 0997 & 0B7C005B ; & 1367 & LDN RB;ADCI $0 ; \mathrm{STR} R$ \\
\hline 099B & $14 ;$ & 1368 & INC SP.. $\quad$ POINT TO BYTE TO SEND \\
\hline $099 \mathrm{C}$ & ; & 1369 & - \\
\hline 099C & ; & 1370 & -. THE FOLLOWING SENDS T'HE CHARACTER ON THE STACK \\
\hline 099C & ; & 1371 & .. TO THE TERMINAL. \\
\hline 099C & i $\quad$ i & 1372 & - \\
\hline $099 \mathrm{C}$ & 6BFE3B9C; & 1373 & CSEND1 INP 3;SHL;BNF CSENDI \\
\hline O9A0 & E462E2 ; & 1374 & SEX SP;OUT 2 ;SEX RP \\
\hline $09 A 3$ & $6896 ;$ & 1375 & SRET R6 \\
\hline $09 A 5$ & ; & 1376 & $\cdots$ \\
\hline $09 A 5$ & ; & 1377 & - \\
\hline 09A5 & ; & 1378 & .. \\
\hline 09A5 & $8 \mathrm{~A} ;$ & 1379 & DC $08 \mathrm{AH}$ \\
\hline 09A6 & $424 \mathrm{C} 4 \mathrm{~F} 434 \mathrm{~B} 2 \mathrm{D}$ & 1380 & DC 'BLOCK-REA' \\
\hline O9AC & $524541 ;$ & & \\
\hline 09AF & $\mathrm{C} 4$; & 1381 & * BLOCK-READ * \\
\hline 09B0 & 095B; & 1382 & DC $(S P C-8)$ \\
\hline 09B2 & $i$ & 1383 & BLKRD \\
\hline 09B2 & 09B4; & 1384 & DC $(\$+2) \ldots$ READ A BLOCK INTO BUFFER AREA \\
\hline 09B4 & $686 \mathrm{~F} ;$ & 1385 & RLXA RF.. SET UP RF TO IOCB \\
\hline 09B6 & E26886096C; & 1386 & SEX RP; SCAL R6, SCRT \\
\hline 09BB & 68A868A7 ; & 1387 & RSXD R8;RSXD R7.. SAVE REGISTERS 7 \& 8 \\
\hline 09BF & D487F6D487F9; & 1388 & CALL SEEK;CALL DREAD . . READ 512 BYTES \\
\hline $09 \mathrm{C5}$ & D41778; & 1389 & CALI SETTUP.. SET UP FOR NEXT 512 BYTES \\
\hline $09 \mathrm{C} 8$ & D487F9; & 1390 & READ NEXT 512 BYTES \\
\hline
\end{tabular}


File ID: WFORTH.LST

Disk ID: WOODS HOLE FORTH SOURCE \& LIST VER 1.800

$1130 \quad 84$

\begin{tabular}{|c|c|c|c|}
\hline $09 \mathrm{CB}$ & $30 F 4 ;$ & 1391 & BR BLKEX \\
\hline 09CD & ; & 1392 & .. \\
\hline 09CD & $8 \mathrm{~B} ;$ & 1393 & $\mathrm{DC} \quad 08 \mathrm{BH}$ \\
\hline 09CE & 424C4F434B2D; & 1394 & DC 'BLOCK-WRIT' \\
\hline 09D4 & $57524954 ;$ & & \\
\hline 09D8 & $\mathrm{C} 5 ;$ & 1395 & * BLOCK-WRITE * \\
\hline 09D9 & 09A5; & 1396 & DC (BLKRD-13) \\
\hline 09DB & ; & 1397 & BLKWT \\
\hline 09DB & O9DD; & 1398 & DC $(\$+2) \ldots$ WRITE A BLOCK TO DISK \\
\hline 09DD & $686 \mathrm{~F}$; & 1399 & RLXA RF.. SET RF TO IOCB FROM STACK \\
\hline 09DF & $\mathrm{E} 26886096 \mathrm{C} ;$ & 1400 & SEX RP;SCAL R6, SCRT \\
\hline $09 E 4$ & $68 \mathrm{~A} 868 \mathrm{~A} 7$ & 1401 & RSXD R8;RSXD R7 .. SAVE $7 \& 8$ \\
\hline 09E8 & D487F6D487FC; & 1402 & CALL SEEK;CALI DWRITE \\
\hline 09EE & D41778; & 1403 & CALL SETTUP \\
\hline $09 \mathrm{Fl}$ & $\mathrm{D} 487 \mathrm{FC} ;$ & 1404 & CALL DWRITE \\
\hline $09 F 4$ & $1268676868 ;$ & 1405 & BLKEX INC RP;RLXA R7;RLXA R8 \\
\hline 09F9 & $22 ;$ & 1406 & DEC RP.. FIX RETURN POINTER \\
\hline 09FA & 68860982 ; & 1407 & SCAL R6, SCRTX \\
\hline 09FE & D5 ; & 1408 & SEP R5 \\
\hline $09 \mathrm{FF}$ & i & 1409 & •. \\
\hline 09FF & ; & 1410 & $\cdots$ \\
\hline $09 \mathrm{FF}$ & ; & 1411 & - \\
\hline 09FF & $85 ;$ & 1412 & DC $085 \mathrm{H}$ \\
\hline $0 \mathrm{AOO}$ & 4D2F4D4F ; & 1413 & $\mathrm{DC} \quad \mathrm{M} / \mathrm{MO} \mathrm{I}^{\prime}$ \\
\hline $0 \mathrm{~A} 04$ & $\mathrm{C} 4 ;$ & 1414 & DC $0 \mathrm{C} 4 \mathrm{H}$ \\
\hline $0 A 05$ & 09CD; & 1415 & DC (BLKWT-I4) \\
\hline $0 \mathrm{~A} 07$ & i & 1416 & MSMOD \\
\hline $0 \mathrm{~A} 07$ & 0A09; & 1417 & DC $(\$+2) \ldots($ UDI UN2 -- UR UD3 $)$ \\
\hline $0 A 09$ & $686 \mathrm{~F} ;$ & 1418 & RLXA RF.. DIVISOR TO RF \\
\hline OAOB & $686 \mathrm{~B} 686 \mathrm{~A} ;$ & 1419 & RLXA RB;RLXA RA.. DIVIDEND TO B \& C \\
\hline OAOF & $68 \mathrm{CC} 0000$ & 1420 & RLDI RC, $00000 \mathrm{H} . . \quad$ RC SET UP FOR REMAINDER \\
\hline $0 A 13$ & 248 F739F54 ; & 1421 & DEC SP;GLO RF;STXD;GHI RF;STR SP \\
\hline $0 A 18$ & $14 \mathrm{~F} 821 \mathrm{AF} ;$ & 1422 & INC SP;LDI 33;PLO RF.. SET UP COUNT \\
\hline OAIC & $\mathrm{FE} ;$ & 1423 & $\mathrm{SET} D F=0$ \\
\hline OAID & 8A7EAA; & 1424 & MSMOD3 GLO RA; SHLC;PLO RA.. START 48 BIT SHIFT \\
\hline $0 \mathrm{~A} 20$ & 9A7EBA; & 1425 & GHI RA; SHLC;PHI RA \\
\hline 0 A23 & 8B7EAB; & 1426 & GLO RB; SHLC;PLO RB \\
\hline $0 \mathrm{~A} 26$ & 9B7EBB; & 1427 & GHI RB;SHLC;PHI RB \\
\hline 0 A29 & $2 \mathrm{~F} 8 \mathrm{~F}$; & 1428 & DEC RF;GLO RF.. TEST COUNT BEFORE SHIFTING \\
\hline $0 \mathrm{~A} 2 \mathrm{~B}$ & C20A44; & 1429 & LBZ MSMODX.. $\quad$ RC TO SEE IF DONE. \\
\hline $0 A 2 E$ & 8C7EAC; & 1430 & GLO RC;SHLC;PLO RC \\
\hline $0 A 31$ & 9C7EBC; & 1431 & GHI RC;SHLC;PHI RC.. DONE WITH SHIFT \\
\hline 0A34 & $8 \mathrm{CF} 7 \mathrm{BF}$; & 1432 & GLO RC;SM;PHI RF.. SEE IF DIVISOR WILL GO \\
\hline $0 \AA 37$ & $249 C 7714 ;$ & 1433 & DEC SP;GHI RC;SMB;INC SP \\
\hline ОАЗВ & CBOAID; & 1434 & BRANCH IF IT WONT GO \\
\hline OA3E & $\mathrm{BC} 9 \mathrm{FAC}$; & 1435 & PHI RC;GHI RF;PLO RC.. SUBTRACT IT IF IT WILL \\
\hline $0 A 41$ & COOAID; & 1436 & LBR MSMOD3 \\
\hline OA44 & $68 \mathrm{AC}$ & 1437 & MSMODX RSXD RC.. REMAINDER $\rightarrow$ STACK \\
\hline $0 \mathrm{~A} 46$ & 68AA68AB; & 1438 & RSXD RA;RSXD RB.. QUOTIENT $\rightarrow$ STACK \\
\hline $0 \AA 4 A$ & 14D5; & 1439 & INC SP;SEP R5 \\
\hline $0 A 4 C$ & ; & 1440 & .. \\
\hline $0 A 4 C$ & ; & 1441 & $\cdots$ \\
\hline $0 \mathrm{~A} 4 \mathrm{C}$ & i & 1442 & \\
\hline $0 \mathrm{~A} 4 \mathrm{C}$ & $84 ;$ & 1443 & DC $084 \mathrm{H}$ \\
\hline
\end{tabular}


File ID: WFORTH.LST

Disk ID: WOODS HOLE FORTH SOURCE \& LIST VER 1.800

$\begin{array}{lll}11 & 30 & 84\end{array}$

\begin{tabular}{|c|c|c|c|c|}
\hline $0 A 4 D$ & 484552 ; & 1444 & & DC 'HER' \\
\hline $0 \AA 50$ & $\mathrm{C} 5$ & 1445 & & $\mathrm{DC} \quad 0 \mathrm{C} 5 \mathrm{H}$. \\
\hline $0 \mathrm{~A} 51$ & 09FF; & 1446 & DC & $($ MSMOD-8) \\
\hline $0 A 53$ & ; & 1447 & HERE & \\
\hline $0 A 53$ & $0 \mathrm{A55} ;$ & 1448 & & $\mathrm{DC}(\$+2)$ \\
\hline $0 A 55$ & $\mathrm{E} 2$; & 1449 & SEX & $\mathrm{RP}$ \\
\hline $0 \mathrm{~A} 56$ & F8126886089F ; & 1450 & LDI & 012H;SCAL R6, USRADD. . B=ADDRESS OF DP \\
\hline $0 \mathrm{~A} 5 \mathrm{C}$ & $240 \mathrm{~B} 5424$ & 1451 & $\mathrm{DEC}$ & SP;LDN RB;STR SP;DEC SP.. PUT DP ON STACK \\
\hline $0 A 60$ & 2B0B54D5; & 1452 & $\mathrm{DEC}$ & RB;LDN RB;STR SP;SEP R5 \\
\hline $0 \mathrm{~A} 64$ & ; $\quad 1$ & 1453 & . & \\
\hline $0 \mathrm{~A} 64$ & 85 & 1454 & DC & $085 \mathrm{H}$ \\
\hline $0 \AA 65$ & $414 \mathrm{C} 4 \mathrm{C} 4 \mathrm{~F}$ & 1455 & & DC 'ALLO' \\
\hline $0 A 69$ & D4; & 1456 & & $\mathrm{DC} \quad 0 \mathrm{D} 4 \mathrm{H}$. \\
\hline $0 A 6 \mathrm{~A}$ & $0 A 4 C$ & 1457 & DC & ( HERE-7) \\
\hline $0 \mathrm{~A} 6 \mathrm{C}$ & ; & 1458 & ALLOT & \\
\hline $0 \mathrm{~A} 6 \mathrm{C}$ & OA6E; & 1459 & & ALLOT SPACE AT HERE \\
\hline $0 \mathrm{~A} 6 \mathrm{E}$ & $\mathrm{E} 2$; & 1460 & SEX & $\mathrm{RP}$ \\
\hline $0 A 6 F$ & F8126886089F; & 1461 & LDI & 012H; SCAL R6, USRADD.. $B=A D D R E S S$ OF DP. 0 \\
\hline $0 A 75$ & $\mathrm{E} 4686 \mathrm{~A}$; & 1462 & SEX & SP;RLXA RA.. TOP OF STACK IN A \\
\hline $0 A 78$ & $\mathrm{~EB}$; & 1463 & ALLOTl & ADD IT TO @RB \\
\hline $0 A 79$ & $8 A F 473$ & 1464 & GLO & $\mathrm{RA} ; \mathrm{ADD} ; \mathrm{STXD}$ \\
\hline $0 \mathrm{~A} 7 \mathrm{C}$ & 9A7473D5; & 1465 & GHI & $\mathrm{RA} ; \mathrm{ADC} ; \mathrm{STXD} ; \mathrm{SEP} \quad \mathrm{R} 5$ \\
\hline $0 \mathrm{~A} 80$ & ; & 1466 & . & \\
\hline $0 A 80$ & $81 \mathrm{AC}$ & 1467 & DC & $081 \mathrm{ACH}$ \\
\hline $0 A 82$ & $0 \mathrm{A64}$ & 1468 & DC & (ALLOT-8) \\
\hline $0 A 84$ & ; & 1469 & COMMA & \\
\hline $0 A 84$ & $0 A 86$; & 1470 & & STORE TOP AT HERE AND \\
\hline $0 A 86$ & $\mathrm{E} 2 ;$ & 1471 & SEX & $\mathrm{RP}$ \\
\hline $0 A 87$ & F8116886089F; & 1472 & LDI & 011H;SCAL R6, USRADD.. ALLOT 2 BYTES \\
\hline $0 A 8 D$ & EB686F2B; & 1473 & SEX & RB; RLXA RF;DEC RB.. F=ADDRESS OF HERE \\
\hline $0 A 91$ & $445 \mathrm{~F} 1 \mathrm{~F}$; & 1474 & LDA & SP;STR RF;INC RF.. STORE HI HALF AT HERE \\
\hline $0 \mathrm{~A} 94$ & F802; & 1475 & LDI & 2.. SET UP TO ALLOT 2 \\
\hline $0 \mathrm{~A} 96$ & $\mathrm{AA} 445 \mathrm{~F} ;$ & 1476 & COMMAl & PLO RA;LDA SP;STR RF.. STORE LO HALF \\
\hline $0 A 99$ & F800BAC00A78; & 1477 & LDI & $0 ;$ PHI RA;LBR ALLOTI.. GO ALLOT FOR IT \\
\hline $0 A 9 F$ & ; & 1478 & .. & \\
\hline $0 A 9 F$ & $8243 \mathrm{AC} ;$ & 1479 & DC & $08243 \mathrm{H}, 0 \mathrm{ACH}$. \\
\hline $0 A A 2$ & $0 \mathrm{~A} 80$ & 1480 & DC & $(\mathrm{COMMA}-4)$ \\
\hline OAA4 & ; & 1481 & CCMA & \\
\hline OAA4 & OAA6; & 1482 & & STORE TOP CHAR. AT HERE \\
\hline $0 A A 6$ & E2; & 1483 & SEX & \\
\hline $0 A A 7$ & F8116886089F; & 1484 & LDI & 011H;SCAL R6, USRADD.. AND ALLOT I BYTE \\
\hline$O A A D$ & $\mathrm{~EB} 686 \mathrm{~F} 2 \mathrm{~B}$ & 1485 & SEX & $\mathrm{RB} ; \mathrm{RLXA} \mathrm{RF} ; \mathrm{DEC} \mathrm{RB}$ \\
\hline OABI & $14 \mathrm{~F} 801$ & 1486 & INC & SP;LDI 1 \\
\hline OAB4 & C00A96; & 1487 & LBR & COMMAI... \\
\hline $0 \mathrm{AB} 7$ & ; & 1488 & . & \\
\hline $0 A B 7$ & $8231 \mathrm{AD}$; & 1489 & DC & $08231 \mathrm{H}, 0 \mathrm{ADH} .$. \\
\hline$O A B A$ & OA9F; & 1490 & DC & $(\mathrm{CCMA}-5)$ \\
\hline$O A B C$ & i & 1491 & ONEMIN & \\
\hline $0 A B C$ & $O A B E$; & 1492 & & SUBTRACT 1 FROM TOP \\
\hline $0 A B E$ & F801; & 1493 & LDI & 1 \\
\hline $0 A C 0$ & $14 ;$ & 1494 & ONMNSI & INC SP \\
\hline $\mathrm{OACl}$ & F573F80075; & 1495 & $S D_{i} S$ & STXD;LDI 0 ; SDB \\
\hline $0 A C 6$ & $54 \mathrm{D} 5$ & 1496 & STR & $\mathrm{SP} ; \mathrm{SEP} \quad \mathrm{R} 5$ \\
\hline 0AC8 & ; & 1497 & . & \\
\hline
\end{tabular}


File ID: WFORTH.LST

Disk ID: WOODS HOLE FORTH SOURCE \& LIST VER 1.800

$1130 \quad 84$

\begin{tabular}{|c|c|c|c|c|}
\hline $0 A C 8$ & $8232 \mathrm{AD}$ & 1498 & $\mathrm{DC}$ & $08232 \mathrm{H}, 0 \mathrm{ADH} .$. \\
\hline$O A C B$ & $0 A B 7$ & 1499 & $\mathrm{DC}$ & (ONEMIN-5) \\
\hline$O A C D$ & i & 1500 & TWOMIN & \\
\hline$O A C D$ & $\mathrm{OACF}$; & 1501 & & SUBTRACT 2 FROM TOP \\
\hline OACF & $\mathrm{F} 80230 \mathrm{CO}$; & 1502 & LDI & $2 ; B R$ ONMNSI \\
\hline $0 A D 3$ & ; & 1503 & .. & \\
\hline $0 A D 3$ & $83 ;$ & 1504 & DC & $083 \mathrm{H}$ \\
\hline OAD4 & $4 C 46 ;$ & 1505 & & DC 'LF' \\
\hline OAD 6 & $\mathrm{Cl} ;$ & 1506 & & $\mathrm{DC} \quad \mathrm{OClH}$ \\
\hline $0 A D 7$ & $0 A C 8 ;$ & 1507 & DC & (TWOMIN-5) \\
\hline 0AD9 & ; & 1508 & LFA & \\
\hline OAD9 & $O A D B$ & 1509 & & CONVERT PFA TO LFA \\
\hline$O A D B$ & $\mathrm{~F} 80430 \mathrm{CO}$; & 1510 & LDI & SUBTRACT 4 FROM TOP \\
\hline$O A D F$ & ; & 1511 & .. & \\
\hline OADF & $83 ;$ & 1512 & DC & $083 \mathrm{H}$ \\
\hline OAEO & $4346 ;$ & 1513 & & $\mathrm{DC}{ }^{\prime} \mathrm{CF}{ }^{\prime}$ \\
\hline OAE2 & $\mathrm{Cl} ;$ & 1514 & & $\mathrm{DC} \quad 0 \mathrm{ClH}$ \\
\hline OAE3 & $0 A D 3 ;$ & 1515 & DC & $(L F A-6)$ \\
\hline OAE5 & ; & 1516 & CFA & \\
\hline $0 A E 5$ & OACF ; & 1517 & & CONVERT PFA TO CFA \\
\hline 0AE7 & ; & 1518 & .. & \\
\hline OAE7 & $84214353 \mathrm{D} 0$; & 1519 & DC & $08421 \mathrm{H}, 04353 \mathrm{H}, 0 \mathrm{DOH} \ldots$ \\
\hline OAEC & OADF ; & 1520 & $\mathrm{DC}$ & $(\mathrm{CFA}-6)$ \\
\hline OAEE & ; & 1521 & DCSP & \\
\hline OAEE & $0 \mathrm{AFO}$; & 1522 & & SAVE SP IN CSP \\
\hline OAFO & $\mathrm{E} 2$; & 1523 & SEX & $\mathrm{RP}$ \\
\hline OAFI & F82C6886089F ; & 1524 & LDI & 02CH;SCAL R6, USRADD.. RB=ADDRESS OF CSP. 0 \\
\hline OAF7 & EB68A4D5; & 1525 & SEX & RB; RSXD SP;SEP R5.. SAVE SP THERE \\
\hline OAFB & ; & 1526 & .. & \\
\hline OAFB & $85 ;$ & 1527 & DC & $085 \mathrm{H}$ \\
\hline OAFC & $434 \mathrm{~F} 554 \mathrm{E}$ & 1528 & & DC 'COUN' \\
\hline $\mathrm{OBO} 0$ & D4 ; & 1529 & & DC $0 D 4 H_{.}$. \\
\hline OBOI & 0AE7; & 1530 & DC & $(\mathrm{DCSP}-7)$ \\
\hline OB03 & ; & 1531 & $\mathrm{CNT}$ & \\
\hline $\mathrm{OB} 03$ & OB05; & 1532 & & GET CHARACTER COUNT ON STACK \\
\hline $0 \mathrm{~B} 05$ & $686 \mathrm{~B} 24$ & 1533 & RLXI & A RB;DEC SP \\
\hline OB08 & $4 \mathrm{~B}$ & 1534 & LDA & RB.. GET COUNT \\
\hline OBO9 & $68 \mathrm{AB} ;$ & 1535 & RSXI & D RB.. STORE ADDRESS ON STACK \\
\hline $\mathrm{OBOB}$ & $73 \mathrm{~F} 80054$ & 1536 & STXI & D;IDI 0 ;STR SP.. STORE COUNT ON STACK \\
\hline $\mathrm{OBOF}$ & D5 ; & 1537 & SEP & R5 \\
\hline OBIO & ; & 1538 & .. & \\
\hline OBIO & $89 ;$ & 1539 & DC & 089H \\
\hline $0 \mathrm{~B} 11$ & 2D545241494C; & 1540 & & DC '-TRAILIN' \\
\hline OB17 & $494 \mathrm{E} ;$ & & & \\
\hline OB19 & $\mathrm{C} 7$; & 1541 & & $\mathrm{DC} \quad 0 \mathrm{C} 7 \mathrm{H} \ldots *$ \\
\hline$O B I A$ & OAFB; & 1542 & DC & $(\mathrm{CNT}-8)$ \\
\hline$O B I C$ & $;$ & 1543 & TRLG & \\
\hline $\mathrm{OBIC}$ & OBlE; & 1544 & & DC $(\$+2) \ldots$ ADJUST CHARACTER COUNT TO DELETE \\
\hline OBIE & $686 \mathrm{Bl} 4$; & 1545 & $\mathrm{RLX}$ & A RB;INC SP.. TRAILING BLANKS \\
\hline $0 B 21$ & $8 \mathrm{BF} 4 \mathrm{AA} 24$ & 1546 & GLO & $\mathrm{RB} ; \mathrm{ADD} ; \mathrm{PLO} \mathrm{RA} ; \mathrm{DEC} \mathrm{SP}$ \\
\hline $0 \mathrm{~B} 25$ & $9 B 74 \mathrm{BA} 24 ;$ & 1547 & GHI & RB;ADC;PHI RA;DEC SP.. A=LAST CHAR \\
\hline OB29 & $2 \mathrm{AOAFB} 20$; & 1548 & TRLI DI & EC RA; LDN RA; XRI 020H.. SEE IF BLANK \\
\hline OB2D & 3А 36 ; & 1549 & $\mathrm{BNZ}$ & TRL2.. NO. EXIT \\
\hline $0 B 2 F$ & 2B8B3A29; & 1550 & DEC & RB;GLO RB;BNZ TRLI.. LOOP BACK TILL.. \\
\hline
\end{tabular}


File ID: WFORTH.IST

Disk ID: WOODS HOLE FORTH SOURCE \& LIST VER 1.800 113084

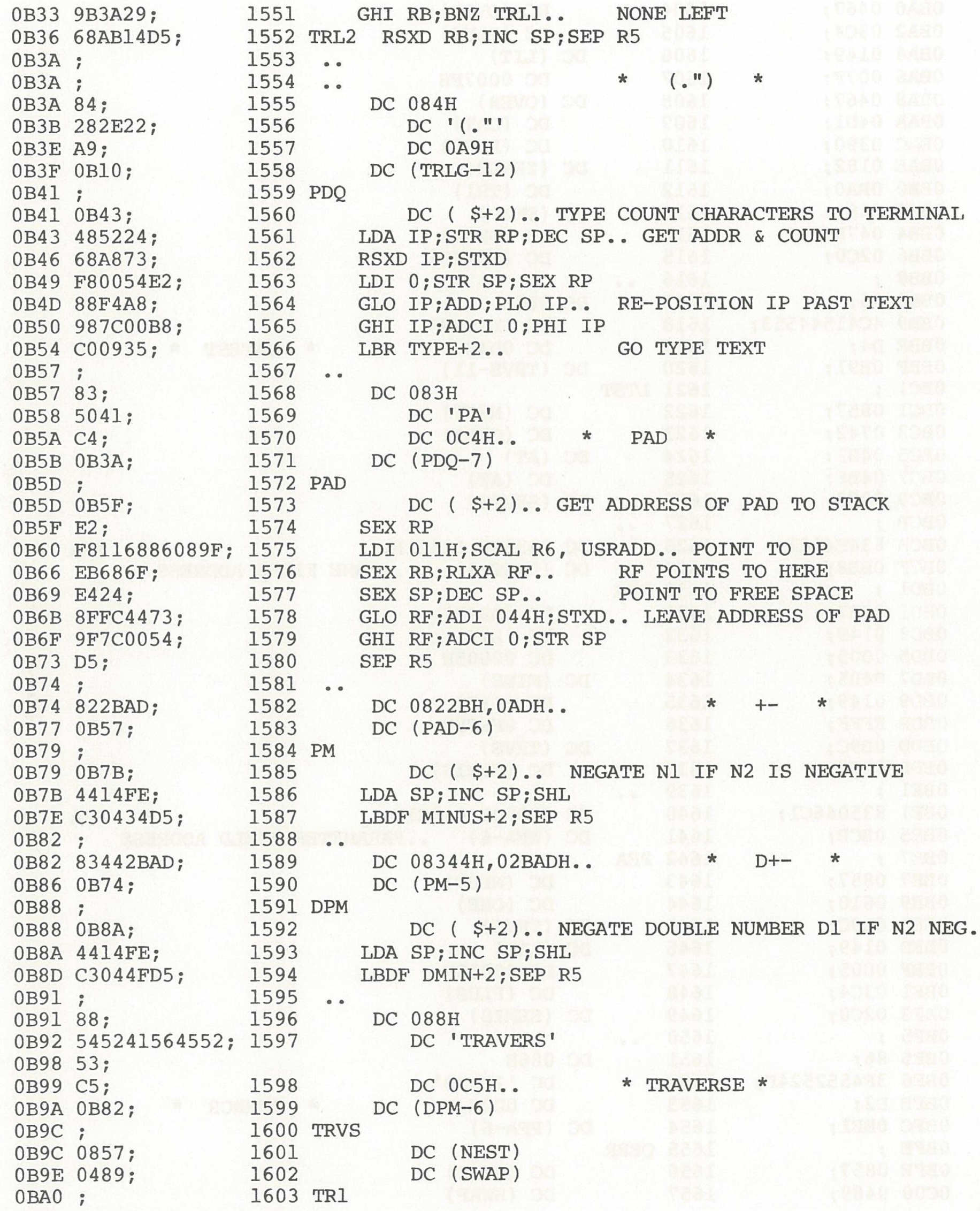


File ID: WFORTH.LST

Disk ID: WOODS HOLE FORTH SOURCE \& LIST VER 1.800 113084

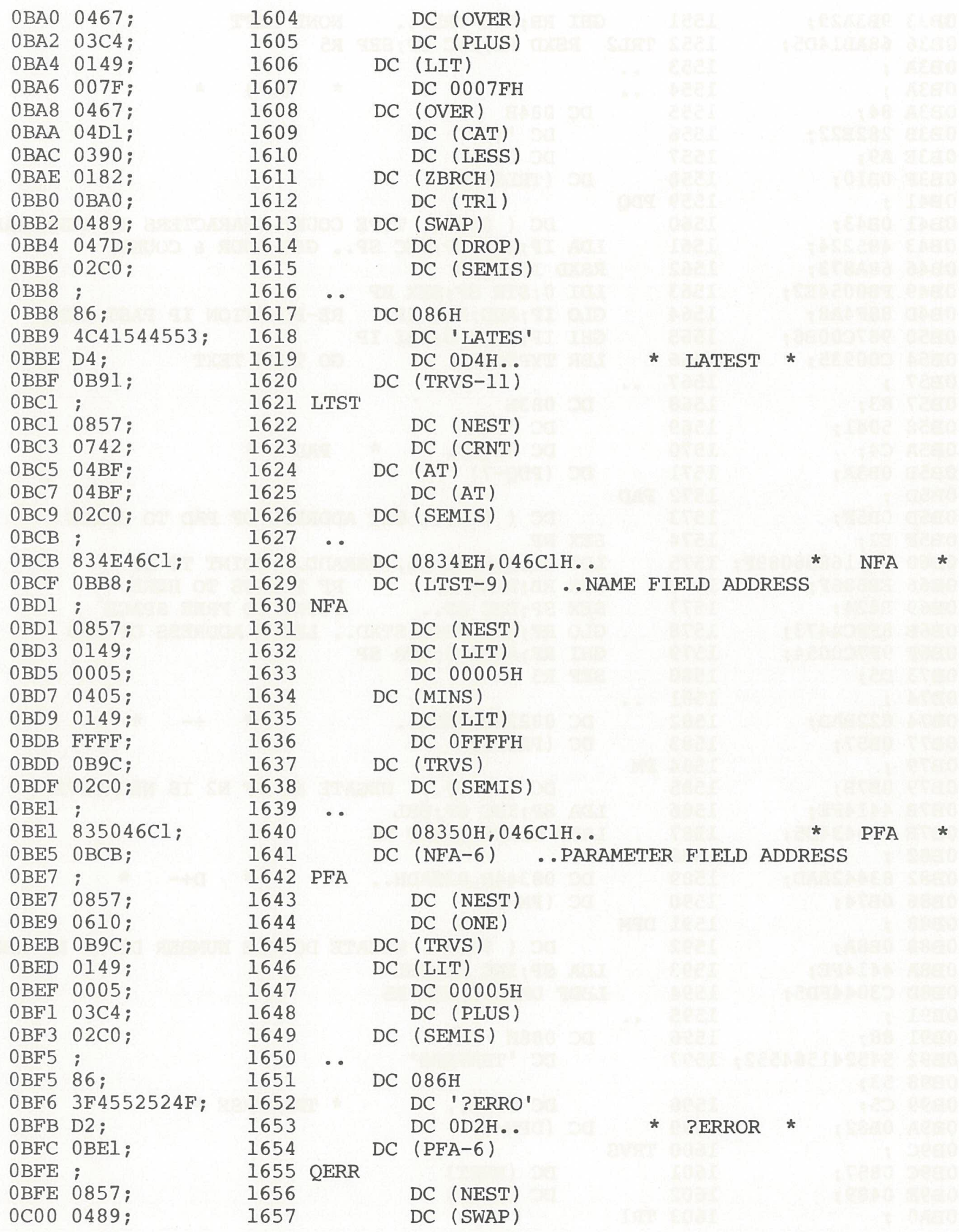


File ID: WFORTH.LST

Disk ID: WOODS HOLE FORTH SOURCE \& LIST VER 1.800

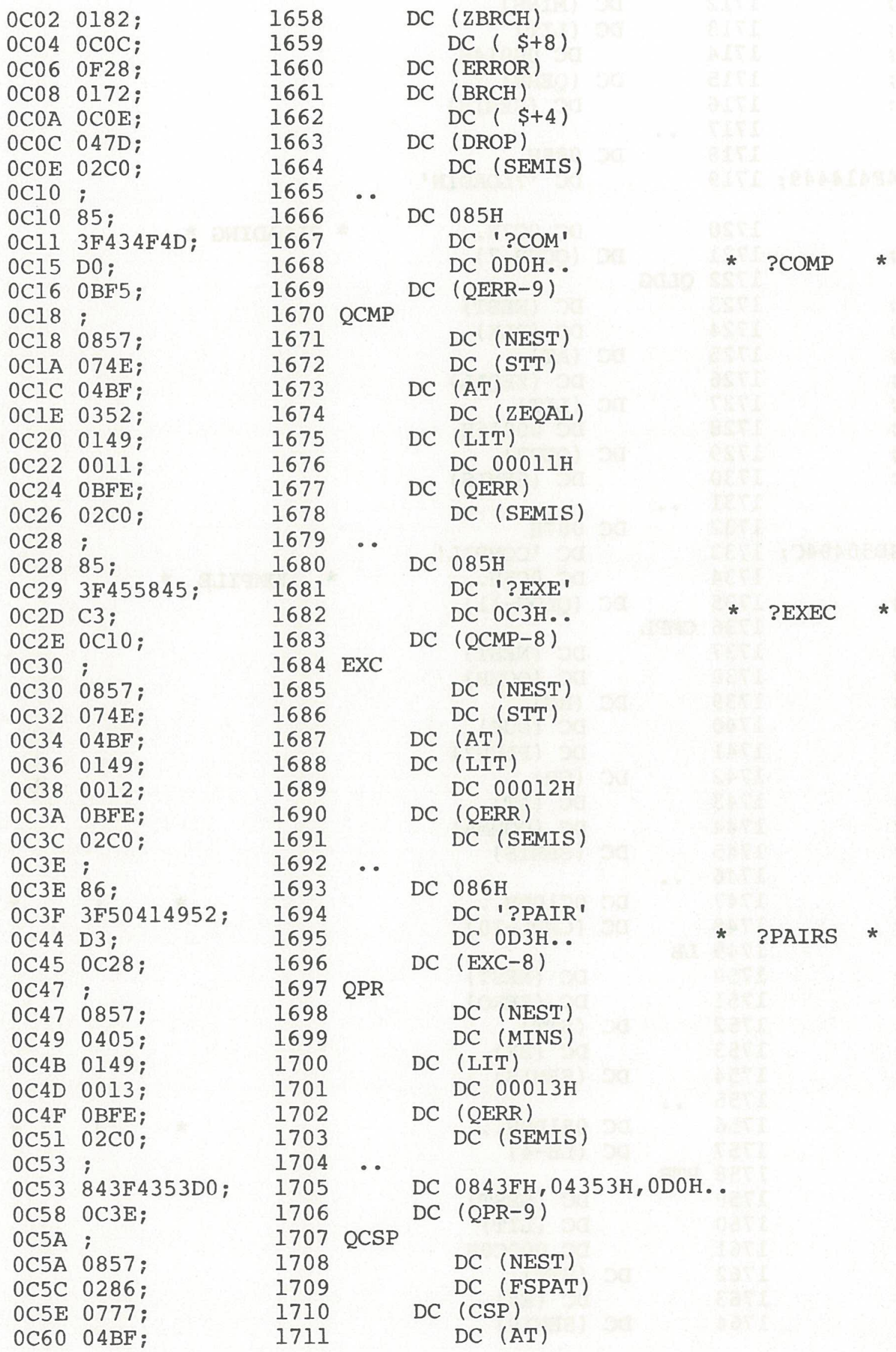


File ID: WFORTH.LST

Disk ID: WOODS HOLE FORTH SOURCE \& LIST VER 1.800

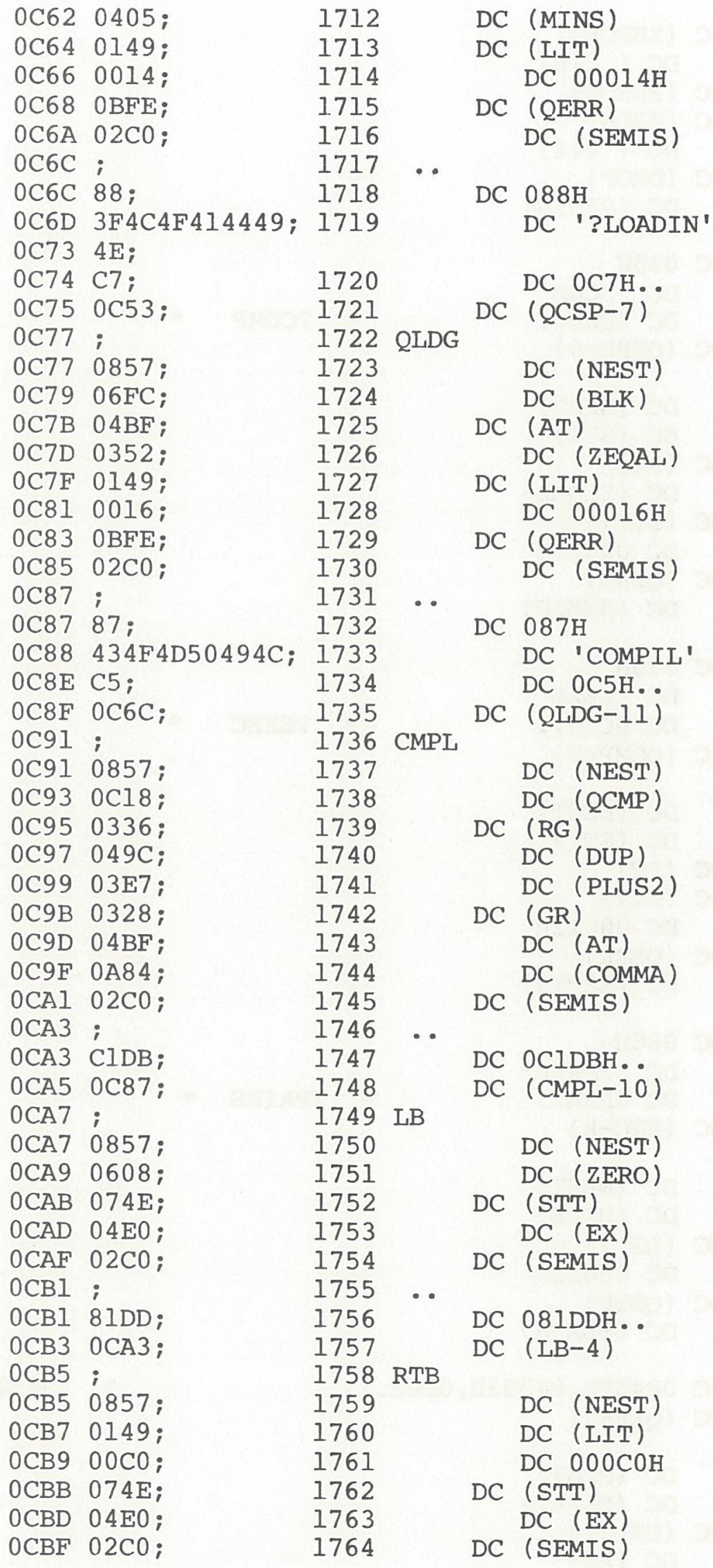

Woods Hole FORTH Version 1.800 Chapter 21 
File ID: WFORTH.LST

Disk ID: WOODS HOLE FORTH SOURCE \& LIST VER 1.800

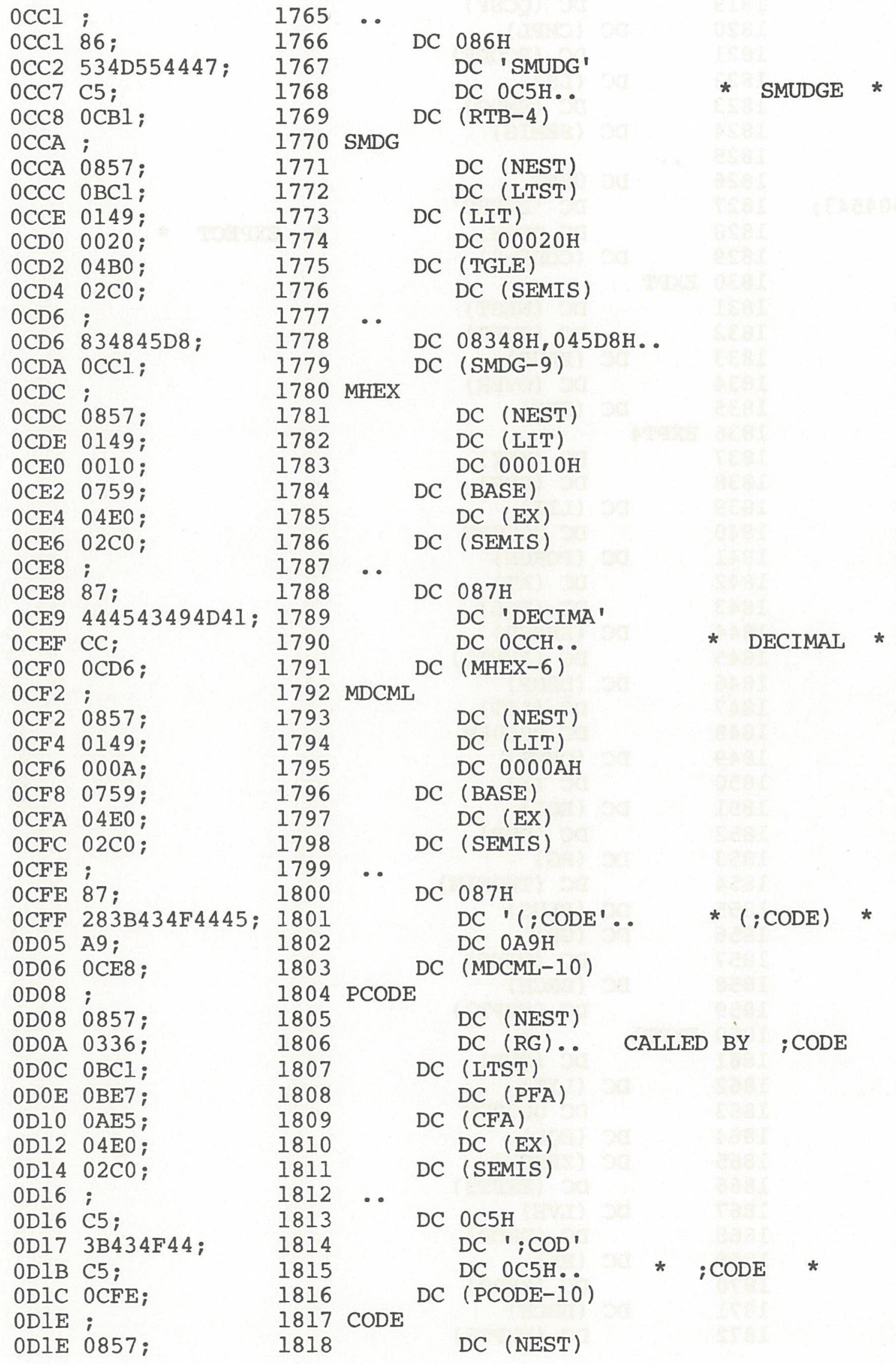


File ID: WFORTH.LST

Disk ID: WOODS HOLE FORTH SOURCE \& IIST VER 1.800

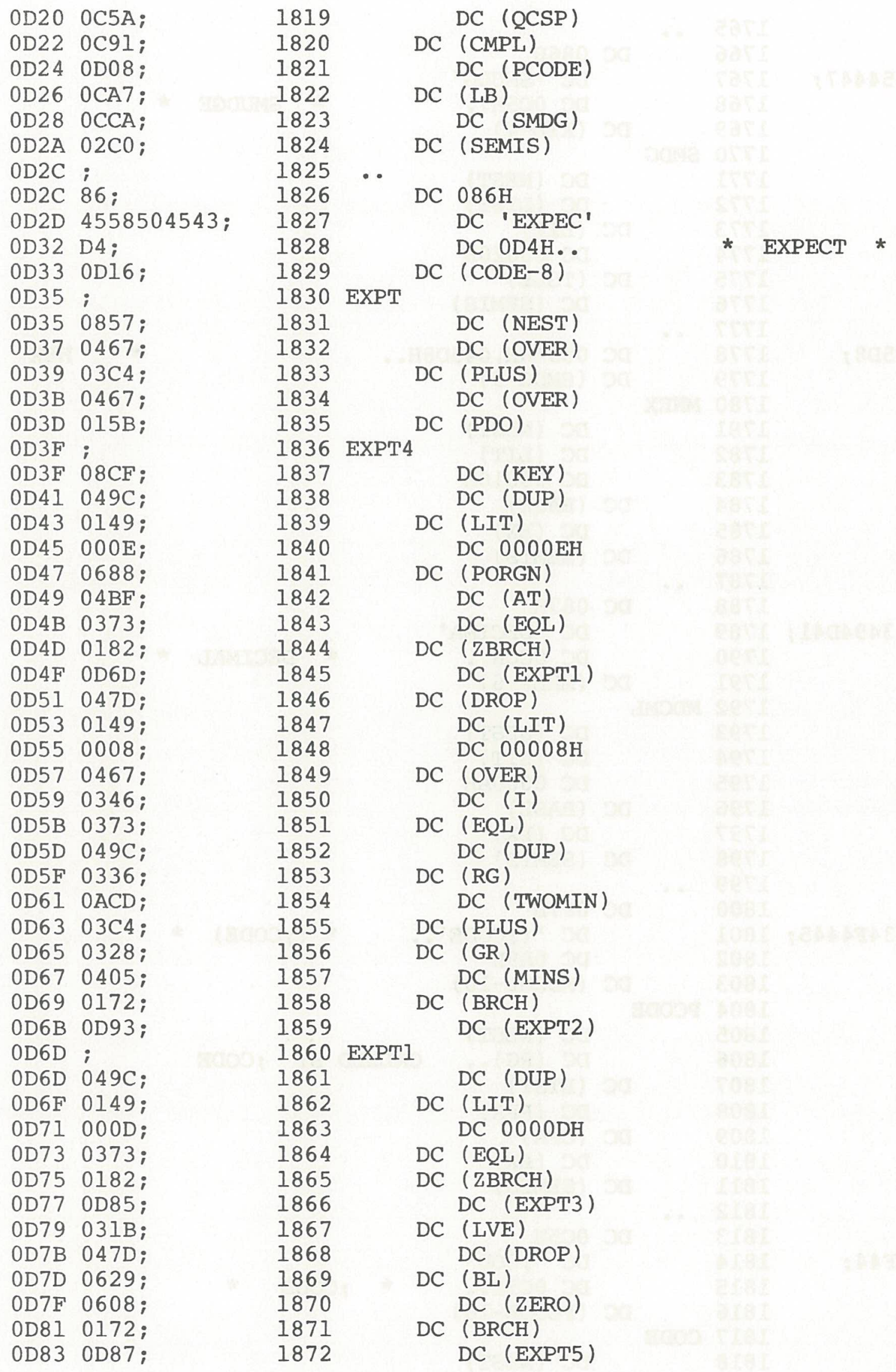


File ID: WFORTH.LST

Disk ID: WOODS HOLE FORTH SOURCE \& LIST VER 1.800

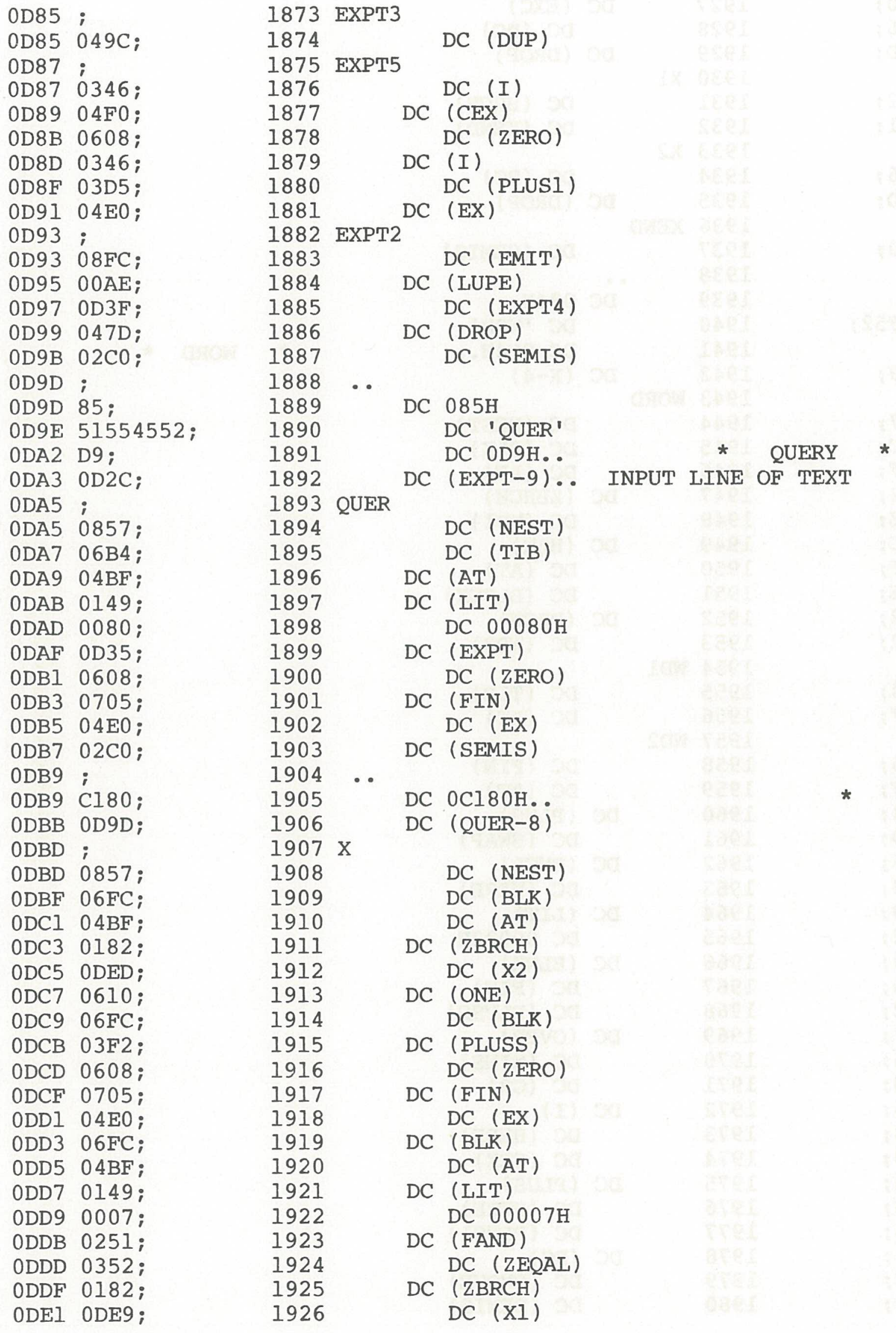


File ID: WFORTH.LST

Disk ID: WOODS HOLE FORTH SOURCE \& LIST VER 1.800

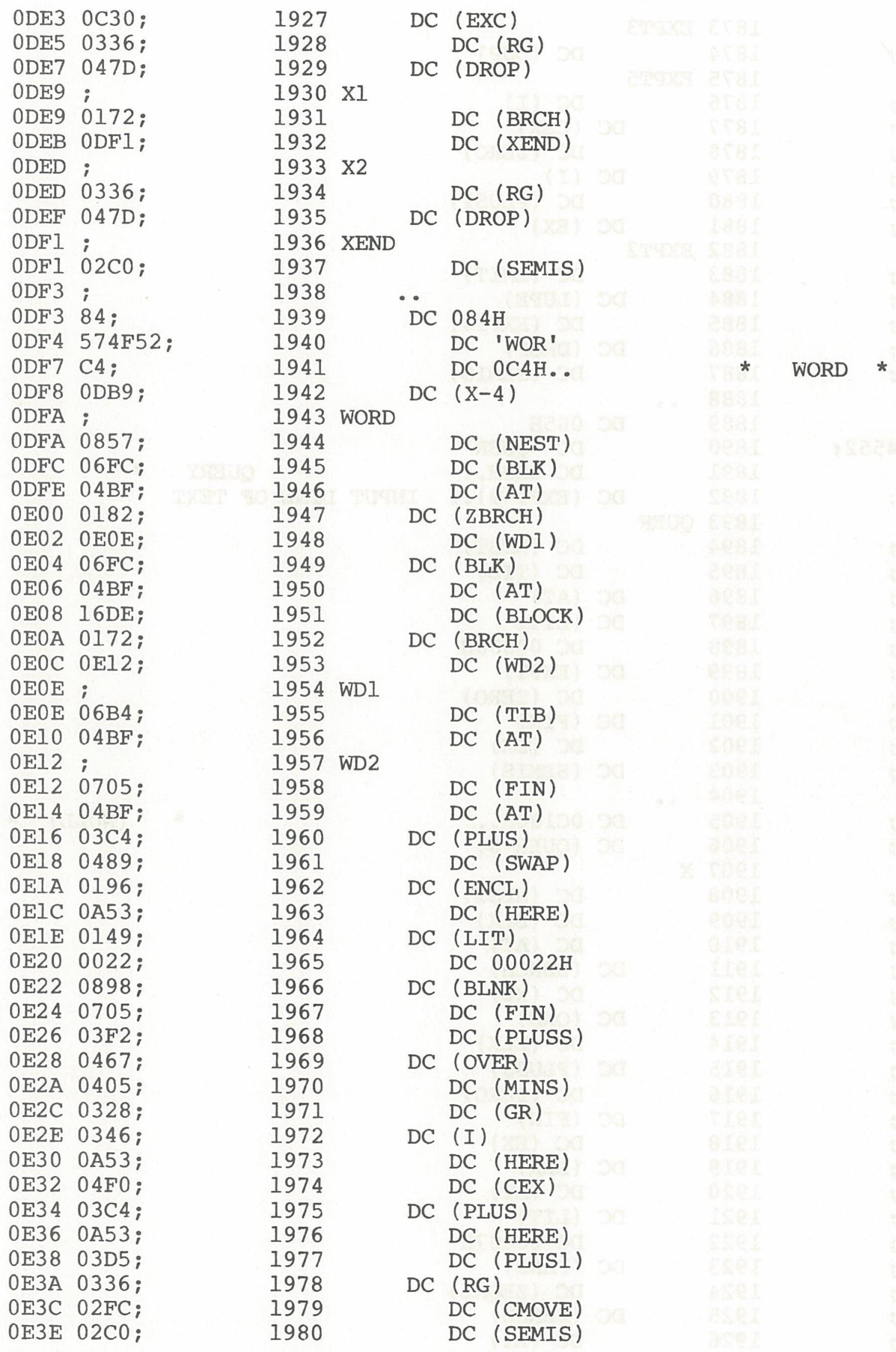


File ID: WFORTH.LST

Disk ID: WOODS HOLE FORTH SOURCE \& LIST VER 1.800

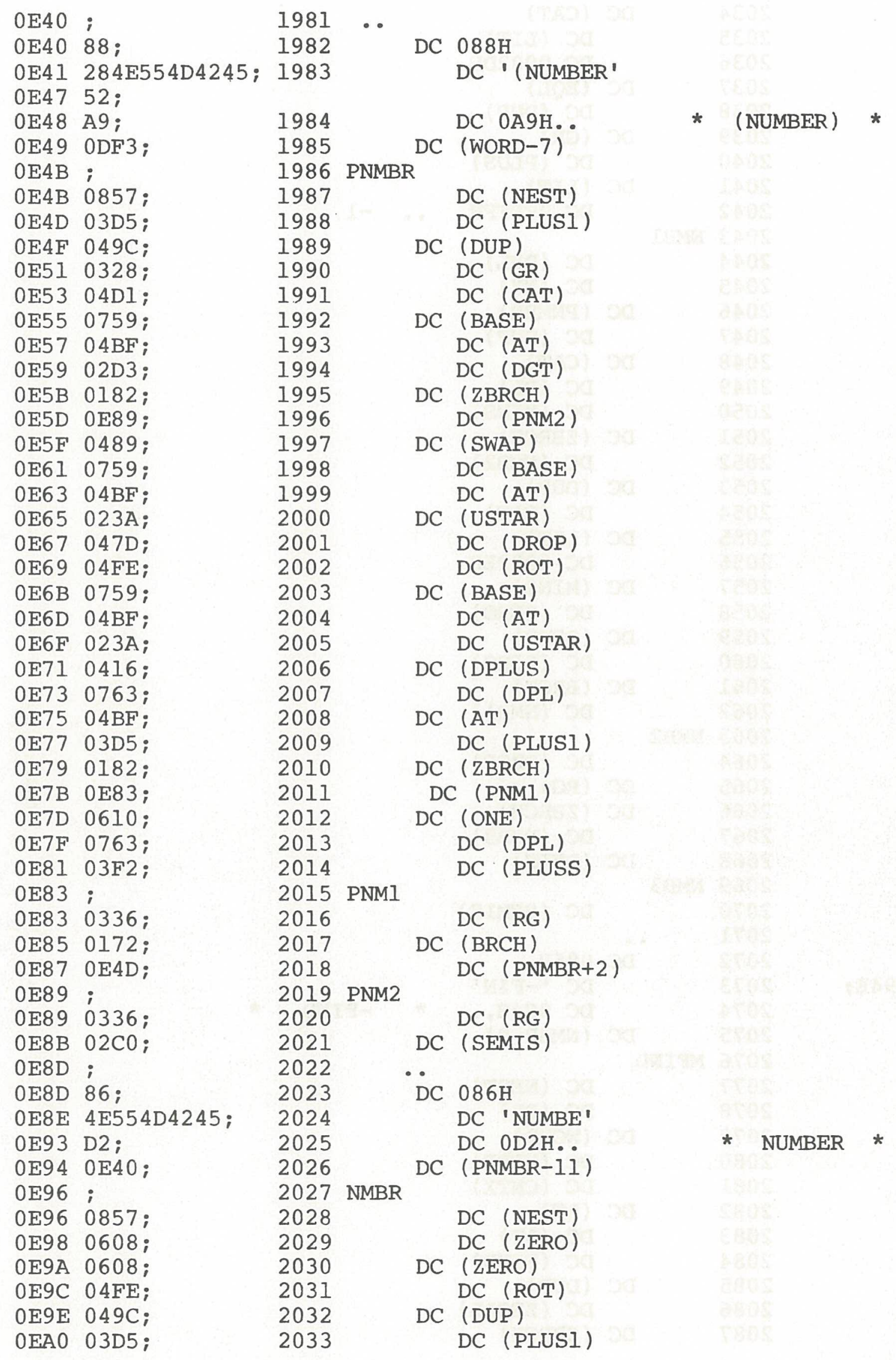


File ID: WFORTH.LST

Disk ID: WOODS HOLE FORTH SOURCE \& LIST VER 1.800

\begin{tabular}{|c|c|c|c|c|c|c|c|}
\hline 0EA2 & 04DI; & 2034 & & DC & (CAT) & & \\
\hline OEA4 & 0149 ; & 2035 & & & DC (LIT) & & \\
\hline OEA6 & 002D; & 2036 & & & $\mathrm{DC} \quad 0002 \mathrm{DH}$ & & \\
\hline 0EA 8 & 0373 ; & 2037 & & DC & (EQL) & & \\
\hline $0 E A A$ & $049 \mathrm{C}$ & 2038 & & & DC (DUP) & & \\
\hline OEAC & 0328 ; & 2039 & & DC & (GR) & & \\
\hline OEAE & $03 \mathrm{C} 4$; & 2040 & & & DC (PLUS) & & \\
\hline OEBO & 0149 ; & 2041 & & DC & (LIT) & & \\
\hline OEB2 & FFFF ; & 2042 & & & DC OFFFFH & .. & -1 \\
\hline OEB4 & ; & 2043 & NMBI & & & & \\
\hline OEB4 & 0763 ; & 2044 & & & DC (DPL) & & \\
\hline 0EB6 & $04 \mathrm{E0}$ & 2045 & & & $\mathrm{DC} \quad(\mathrm{EX})$ & & \\
\hline 0EB8 & OE4B; & 2046 & & DC & (PNMBR) & & \\
\hline OEBA & $049 C_{i}$ & 2047 & & & DC (DUP) & & \\
\hline OEBC & 04DI; & 2048 & & DC & (CAT) & & \\
\hline OEBE & 0629 ; & 2049 & & & $\mathrm{DC} \quad(\mathrm{BL})$ & & \\
\hline OECO & $0405 ;$ & 2050 & & & DC (MINS) & & \\
\hline 0EC2 & 0182 ; & 2051 & & DC & ( $\mathrm{ZBRCH})$ & & \\
\hline OEC4 & OEDA; & 2052 & & & DC (NMB2) & & \\
\hline 0EC6 & $049 \mathrm{C}$ & 2053 & & $\mathrm{DC}$ & (DUP) & & \\
\hline 0EC8 & 04DI; & 2054 & & & DC (CAT) & & \\
\hline OECA & 0149 ; & 2055 & & $\mathrm{DC}$ & (LIT) & & \\
\hline OECC & $002 \mathrm{E}$ & 2056 & & & DC $0002 \mathrm{EH}$ & & \\
\hline OECE & 0405 ; & 2057 & & DC & (MINS) & & \\
\hline OEDO & 0608 ; & 2058 & & & DC (ZERO) & & \\
\hline 0ED2 & OBFE; & 2059 & & DC & (QERR) & & \\
\hline 0ED4 & 0608 ; & 2060 & & & DC (ZERO) & & \\
\hline 0ED6 & 0172 ; & 2061 & & DC & ( $\mathrm{BRCH})$ & & \\
\hline 0ED8 & OEB4; & 2062 & & & DC (NMBI) & & \\
\hline OEDA & ; & 2063 & NMB2 & & & & \\
\hline OEDA & 047D; & 2064 & & & DC (DROP) & & \\
\hline OEDC & 0336 ; & 2065 & & DC & $(\mathrm{RG})$ & & \\
\hline OEDE & 0182 ; & 2066 & & DC & ( $\mathrm{ZBRCH})$ & & \\
\hline OEEO & OEE4; & 2067 & & & DC (NMB3) & & \\
\hline OEE2 & 044D; & 2068 & & $\mathrm{DC}$ & (DMIN) & & \\
\hline OEE4 & ; & 2069 & NMB3 & & & & \\
\hline OEE4 & $02 \mathrm{CO}$; & 2070 & & & DC (SEMIS) & & \\
\hline OEE6 & ; & 2071 & . & - & & & \\
\hline 0EE6 & $85 ;$ & 2072 & & $\mathrm{DC}$ & $085 \mathrm{H}$ & & \\
\hline OEE7 & 2D46494E; & 2073 & & & DC '-FIN' & & \\
\hline OEEB & $\mathrm{C} 4$; & 2074 & & & $\mathrm{DC} \quad 0 \mathrm{C} 4 \mathrm{H}$ & * & -FIND \\
\hline OEEC & OE8D; & 2075 & & $\mathrm{DC}$ & $($ NMBR-9) & & \\
\hline OEEE & ; & 2076 & MFIND & & & & \\
\hline OEEE & 0857 ; & 2077 & & & DC (NEST) & & \\
\hline OEFO & 0629 ; & 2078 & & & $\mathrm{DC} \quad(\mathrm{BL})$ & & \\
\hline OEF2 & ODFA; & 2079 & & DC & (WORD) & & \\
\hline OEF4 & $0 A 53$ & 2080 & & & DC (HERE) & & \\
\hline 0EF6 & 0734 ; & 2081 & & & $\mathrm{DC}$ (CNTX) & & \\
\hline 0EF 8 & $04 \mathrm{BF}$ & 2082 & & DC & $(\mathrm{AT})$ & & \\
\hline OEFA & $04 \mathrm{BF}$ & 2083 & & & $\mathrm{DC} \quad(\mathrm{AT})$ & & \\
\hline OEFC & $00 F 5$ & 2084 & & & DC (FIND) & & \\
\hline OEFE & $049 \mathrm{C}$; & 2085 & & DC & (DUP) & & \\
\hline OFOO & 0352 ; & 2086 & & & DC (ZEQAL) & & \\
\hline 0F02 & 0182 ; & 2087 & & $\mathrm{DC}$ & $(\mathrm{ZBRCH})$ & & \\
\hline
\end{tabular}


File ID: WFORTH.IST

Disk ID: WOODS HOLE FORTH SOURCE \& LIST VER 1.800

$1130 \quad 84$

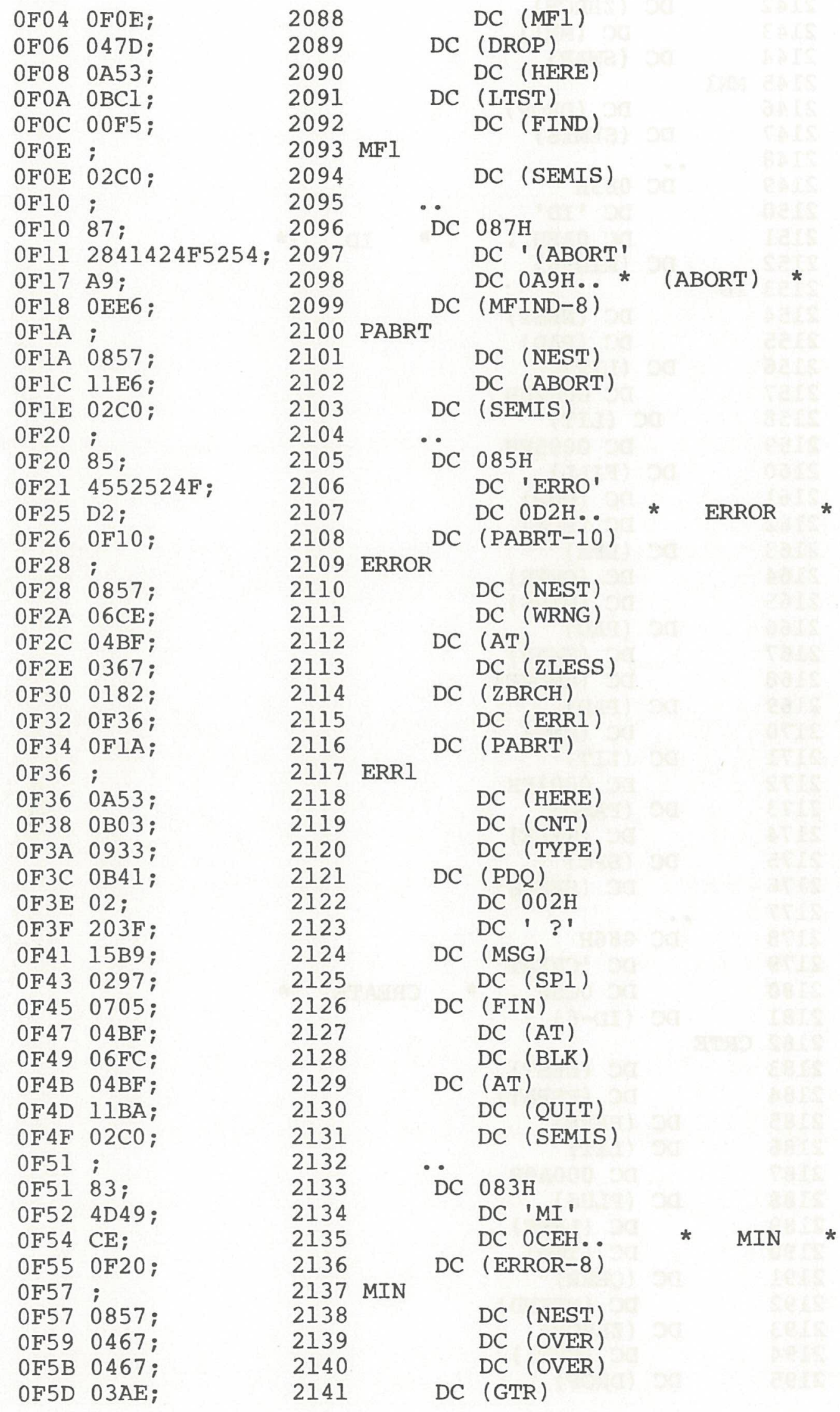


File ID: WFORTH.LST

Disk ID: WOODS HOLE FORTH SOURCE \& IIST VER 1.800

$11 \quad 30 \quad 84$

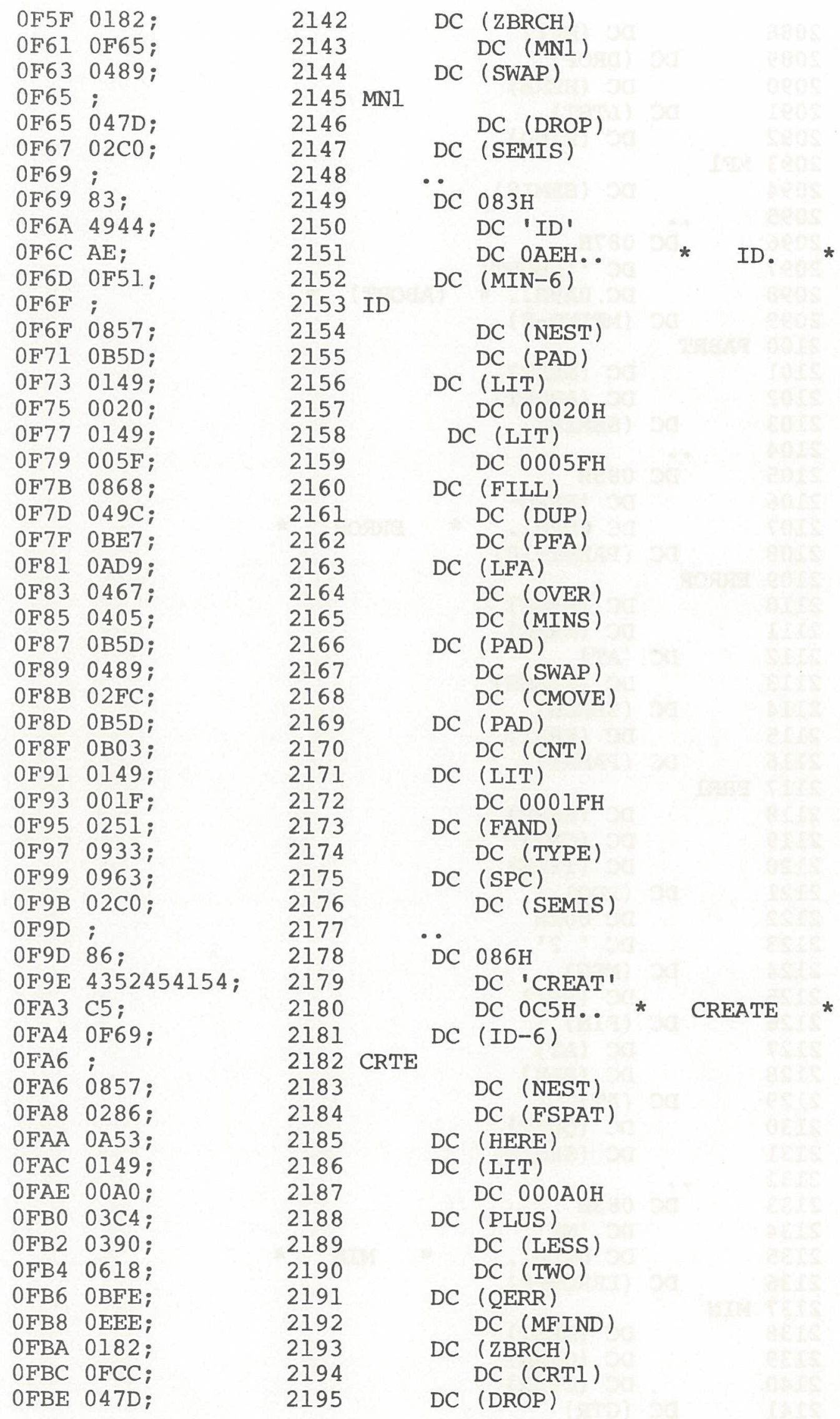


File ID: WFORTH.LST

Disk ID: WOODS HOIE FORTH SOURCE \& LIST VER 1.800

\begin{tabular}{|c|c|c|c|c|c|c|}
\hline OFCO & OBDI； & 2196 & & DC (NFA) & & \\
\hline OFC2 & OF6F; & 2197 & & $D C$ (ID) & & \\
\hline OFC4 & $0149 ;$ & 2198 & DC & $C(\mathrm{LIT})$ & & \\
\hline 0FC6 & $0004 ;$ & 2199 & & DC $00004 \mathrm{H}$ & & \\
\hline 0FC8 & 15B9; & 2200 & DC & (MSG) & & \\
\hline OFCA & $0963 ;$ & 2201 & & DC (SPC) & & \\
\hline OFCC & ; & 2202 & CRTI & & & \\
\hline OFCC & OA53; & 2203 & & DC (HERE) & & \\
\hline OFCE & $049 \mathrm{C} ;$ & 2204 & & DC (DUP) & & \\
\hline OFDO & 04DI; & 2205 & DC & $(\mathrm{CAT})$ & & \\
\hline OFD2 & $06 \mathrm{CO} ;$ & 2206 & & DC (WIDTH) & & \\
\hline OFD4 & $04 \mathrm{BF}$; & 2207 & & $\mathrm{DC} \quad(\mathrm{AT})$ & & \\
\hline OFD6 & 0F57; & 2208 & $D C$ & $C(M I N)$ & & \\
\hline OFD8 & 03D5; & 2209 & & DC (PLUSI) & & \\
\hline OFDA & 0A6C; & 2210 & DC & (ALLOT) & & \\
\hline OFDC & $049 \mathrm{C}$; & 2211 & & DC (DUP) & & \\
\hline OFDE & $0149 ;$ & 2212 & DC & $C \quad(L I T)$ & & \\
\hline OFEO & $00 \mathrm{AO} ;$ & 2213 & & DC OOOAOH & & \\
\hline 0FE2 & $04 \mathrm{BO} ;$ & 2214 & DC & C (TGLE) & & \\
\hline OFE4 & OA53; & 2215 & & DC (HERE) & & \\
\hline 0FE6 & $0610 ;$ & 2216 & DC & C (ONE) & & \\
\hline OFE8 & 0405 ; & 2217 & & DC (MINS) & & \\
\hline OFEA & 0149 ; & 2218 & DC & $(\mathrm{LIT})$ & & \\
\hline OFEC & 0080 ; & 2219 & & DC $00080 \mathrm{H}$ & & \\
\hline OFEE & 04B0; & 2220 & DC & (TGLE) & & \\
\hline OFFO & $\mathrm{OBCl}$; & 2221 & & DC (LTST) & & \\
\hline OFF2 & OA84; & 2222 & & DC (COMMA) & & \\
\hline OFF4 & $0742 ;$ & 2223 & DC & (CRNT) & & \\
\hline OFF6 & 04BF ; & 2224 & & $\mathrm{DC} \quad(\mathrm{AT})$ & & \\
\hline 0FF8 & 04E0; & 2225 & & DC (EX) & & \\
\hline OFFA & OA53; & 2226 & DC & (HERE) & & \\
\hline OFFC & 03E7； & 2227 & & DC (PLUS2) & & \\
\hline OFFE & 0A84; & 2228 & DC & ( & & \\
\hline 1000 & $02 \mathrm{CO}$; & 2229 & & DC (SEMIS) & & \\
\hline 1002 & ; & 2230 & -. & & & \\
\hline 1002 & CIBA; & 2231 & $\mathrm{DC}$ & C OCIBAH.. & & $:$ \\
\hline 1004 & OF9D; & 2232 & $D C$ & C (CRTE-9) & & \\
\hline 1006 & ; & 2233 & COLON & & & \\
\hline 1006 & $0857 ;$ & 2234 & & DC (NEST) & & \\
\hline 1008 & $0 \mathrm{C} 30$; & 2235 & & $\mathrm{DC} \quad(\mathrm{EXC}) \ldots$ & ( IMMEDIATE & EXECUTION) \\
\hline $100 \mathrm{~A}$ & OAEE; & 2236 & $D C$ & $C(\mathrm{DCSP})$ & & \\
\hline $100 \mathrm{C}$ & 0742 ; & 2237 & & DC (CRNT) & & \\
\hline $100 \mathrm{E}$ & 04BF; & 2238 & & $\mathrm{DC} \quad(\mathrm{AT})$ & & \\
\hline 1010 & $0734 ;$ & 2239 & $\mathrm{DC}$ & $C(\mathrm{CNTX})$ & & \\
\hline 1012 & $04 \mathrm{E} 0$; & 2240 & & DC (EX) & & \\
\hline 1014 & OFA6; & 2241 & DC & C (CRTE) & & \\
\hline 1016 & OCB5; & 2242 & & DC (RTB) & & \\
\hline 1018 & $0149 ;$ & 2243 & $D C$ & (IIT) & & \\
\hline $101 \mathrm{~A}$ & FFFE; & 2244 & & DC OFFFEH & -2 & \\
\hline $101 \mathrm{C}$ & 06E3; & 2245 & DC & $C(D P)$ & & \\
\hline $101 \mathrm{E}$ & $03 \mathrm{~F} 2 ;$ & 2246 & & DC (PLUSS) & & \\
\hline 1020 & 0C91; & 2247 & & DC (CMPL) & & \\
\hline 1022 & $0857 ;$ & 2248 & $D C$ & $C($ NEST $)$ & & \\
\hline 1024 & $02 \mathrm{CO}$; & 2249 & & DC (SEMIS) & & \\
\hline
\end{tabular}


File ID: WFORTH.IST

Disk ID: WOODS HOLE FORTH SOURCE \& LIST VER 1.800

$1130 \quad 84$

\begin{tabular}{|c|c|c|c|c|c|c|c|c|c|}
\hline 1026 & i & 2250 & -. & & & & & & \\
\hline 1026 & $85 ;$ & 2251 & $\mathrm{DC}$ & $085 \mathrm{H}$ & & & & & \\
\hline 1027 & $21434 \mathrm{~F} 44$; & 2252 & & $\mathrm{DC} \cdot ! \mathrm{COD}$ ' & & & & & \\
\hline $102 \mathrm{~B}$ & C5 ; & 2253 & & $\mathrm{DC} \quad 0 \mathrm{C} 5 \mathrm{H}$ & & * & !CODE & & * \\
\hline $102 \mathrm{C}$ & 1002 ; & 2254 & DC & $($ COLON-4) & & & & & \\
\hline $102 \mathrm{E}$ & $i$ & 2255 & DCODE & & & & & & \\
\hline $102 \mathrm{E}$ & 0857 ; & 2256 & & DC (NEST) & & & & & \\
\hline 1030 & 0FA6； & 2257 & & DC (CRTE) & & & & & \\
\hline 1032 & $0 C C A_{;}$ & 2258 & DC & (SMDG) & & & & & \\
\hline 1034 & $\mathrm{OBCl}$; & 2259 & & DC (LTST) & & & & & \\
\hline 1036 & OBE7 ; & 2260 & & $\mathrm{DC} \quad(\mathrm{PFA})$ & & & & & \\
\hline 1038 & OAE5; & 2261 & DC & (CFA) & & & & & \\
\hline $103 \mathrm{~A}$ & $04 \mathrm{EO}$; & 2262 & & $\mathrm{DC}(\mathrm{EX})$ & & & & & \\
\hline $103 \mathrm{C}$ & 0A84; & 2263 & & DC (COMMA) & & & & & \\
\hline $103 E$ & $02 \mathrm{CO}$; & 2264 & DC & (SEMIS) & & & & & \\
\hline 1040 & $i$ & 2265 & .. & & & & & & \\
\hline 1040 & 88; & 2266 & DC & $088 \mathrm{H}$ & & & & & \\
\hline 1041 & 434F4E535441; & 2267 & & DC 'CONSTAN' & & & & & \\
\hline 047 & $4 \mathrm{E}$ & & & & & & & & \\
\hline 048 & D4 ; & 2268 & & DC OD4H. & * & CONS & TANT & * & \\
\hline 1049 & $1026 ;$ & 2269 & DC & (DCODE-8) & & & & & \\
\hline $104 \mathrm{~B}$ & $i$ & 2270 & CNST & & & & & & \\
\hline $104 \mathrm{~B}$ & 0857 ; & 2271 & & DC (NEST) & & & & & \\
\hline $104 \mathrm{D}$ & 0149 ; & 2272 & & DC (LIT) & & & & & \\
\hline $104 \mathrm{~F}$ & 05FB; & 2273 & & DC (CONST) & & & & & \\
\hline 1051 & $102 \mathrm{E}$ & 2274 & DC & (DCODE) & & & & & \\
\hline 1053 & $02 \mathrm{CO}$ & 2275 & DC & (SEMIS) & & & & & \\
\hline 1055 & i & 2276 & $\cdots$ & & & & & & \\
\hline 1055 & $88 i$ & 2277 & $\mathrm{DC}$ & $088 \mathrm{H}$ & & & & & \\
\hline 1056 & 564152494142 ; & 2278 & & DC 'VARIABL' & & & & & \\
\hline $105 C$ & $4 C_{i}$ & & & & & & & & \\
\hline 105D & $\mathrm{C} 5$; & 2279 & & DC $0 \mathrm{C} 5 \mathrm{H}_{2}$ & * & VARI & ABLE & * & \\
\hline $105 \mathrm{E}$ & 1040 ; & 2280 & $D C$ & (CNST-11) & & & & & \\
\hline 1060 & $i$ & 2281 & VARB & & & & & & \\
\hline 1060 & 0857 ; & 2282 & & DC (NEST) & & & & & \\
\hline 1062 & 0149 ; & 2283 & & DC （LIT） & & & & & \\
\hline 1064 & 05FE; & 2284 & & DC (VAR) & & & & & \\
\hline 1066 & $102 E_{i}$ & 2285 & DC & (DCODE) & & & & & \\
\hline 1068 & $02 \mathrm{CO}$; & 2286 & & DC (SEMIS) & & & & & \\
\hline $106 \mathrm{~A}$ & i & 2287 & ․ & & & & & & \\
\hline $106 \mathrm{~A}$ & $84 ;$ & 2288 & DC & 084H & & & & & \\
\hline $106 \mathrm{~B}$ & 555345 ; & 2289 & & DC 'USE' & & & & & \\
\hline $106 \mathrm{E}$ & D2; & 2290 & & DC OD2H.. & & * & USER & & * \\
\hline $106 \mathrm{~F}$ & 1055 ; & 2291 & DC & $(V A R B-11)$ & & & & & \\
\hline 1071 & $i$ & 2292 & USR & & & & & & \\
\hline 1071 & 0857 ; & 2293 & & DC (NEST) & & & & & \\
\hline 1073 & 0149 ; & 2294 & & $\mathrm{DC}$ (LIT) & & & & & \\
\hline 1075 & 0690 ; & 2295 & & DC (USER) & & & & & \\
\hline 1077 & $102 \mathrm{E} ;$ & 2296 & DC & (DCODE) & & & & & \\
\hline 1079 & $02 \mathrm{C0}$; & 2297 & & DC (SEMIS) & & & & & \\
\hline 107B & $i$ & 2298 & $\cdots$ & & & & & & \\
\hline 107B & $87 i$ & 2299 & $\mathrm{DC}$ & 087H & & & & & \\
\hline $107 \mathrm{C}$ & $3 C 4255494 C 44 ;$ & 2300 & & DC $\quad<$ BUILD' & & & & & \\
\hline 1082 & D3; & 2301 & & DC OD3H.. & * & $<B U$ & ILDS & * & \\
\hline
\end{tabular}

Woods Hole FORTH Version 1.800 
File ID: WFORTH.LST

Disk ID: WOODS HOLE FORTH SOURCE \& LIST VER 1.800 113084

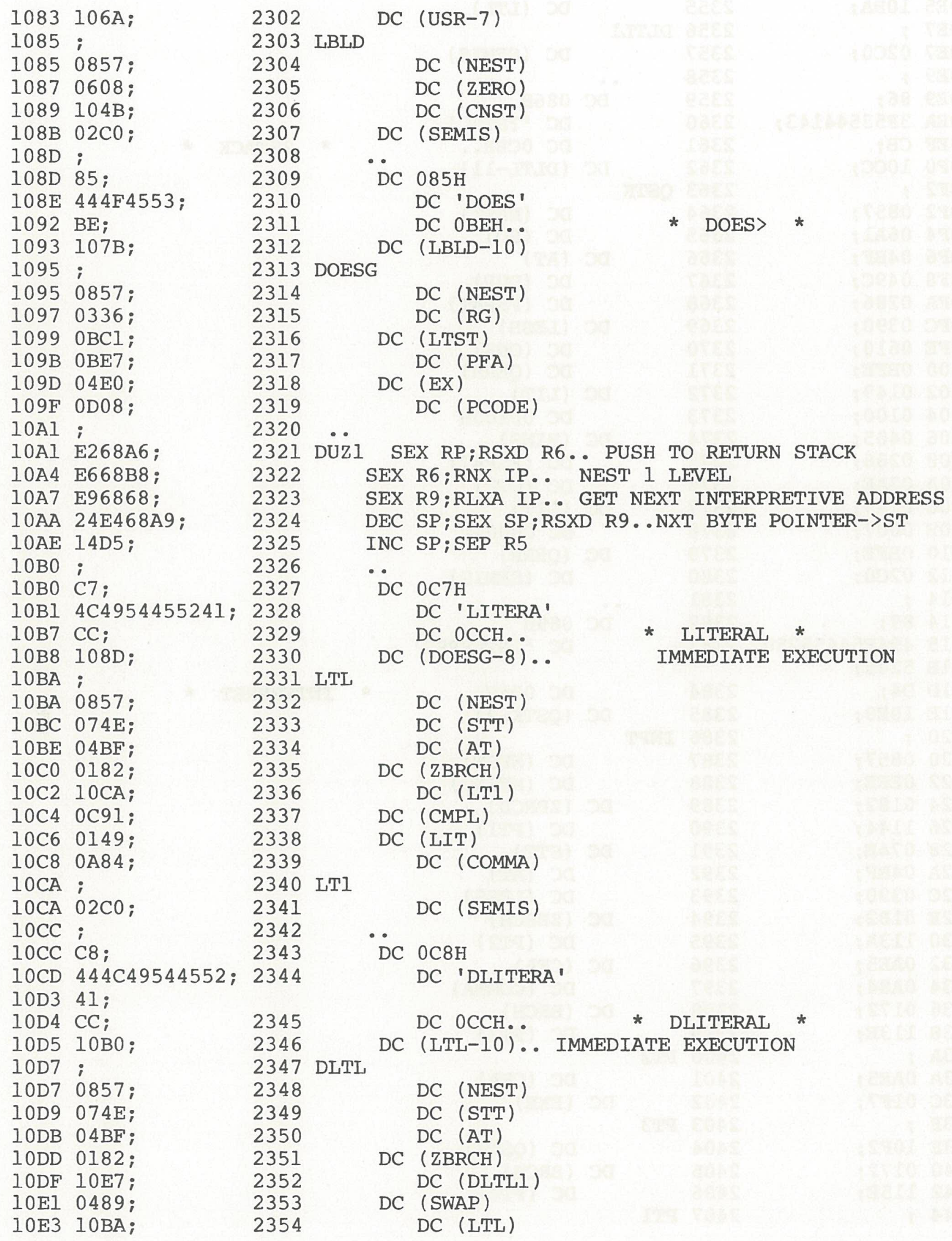


File ID: WFORTH.LST

Disk ID: WOODS HOLE FORTH SOURCE \& LIST VER 1.800

$1130 \quad 84$

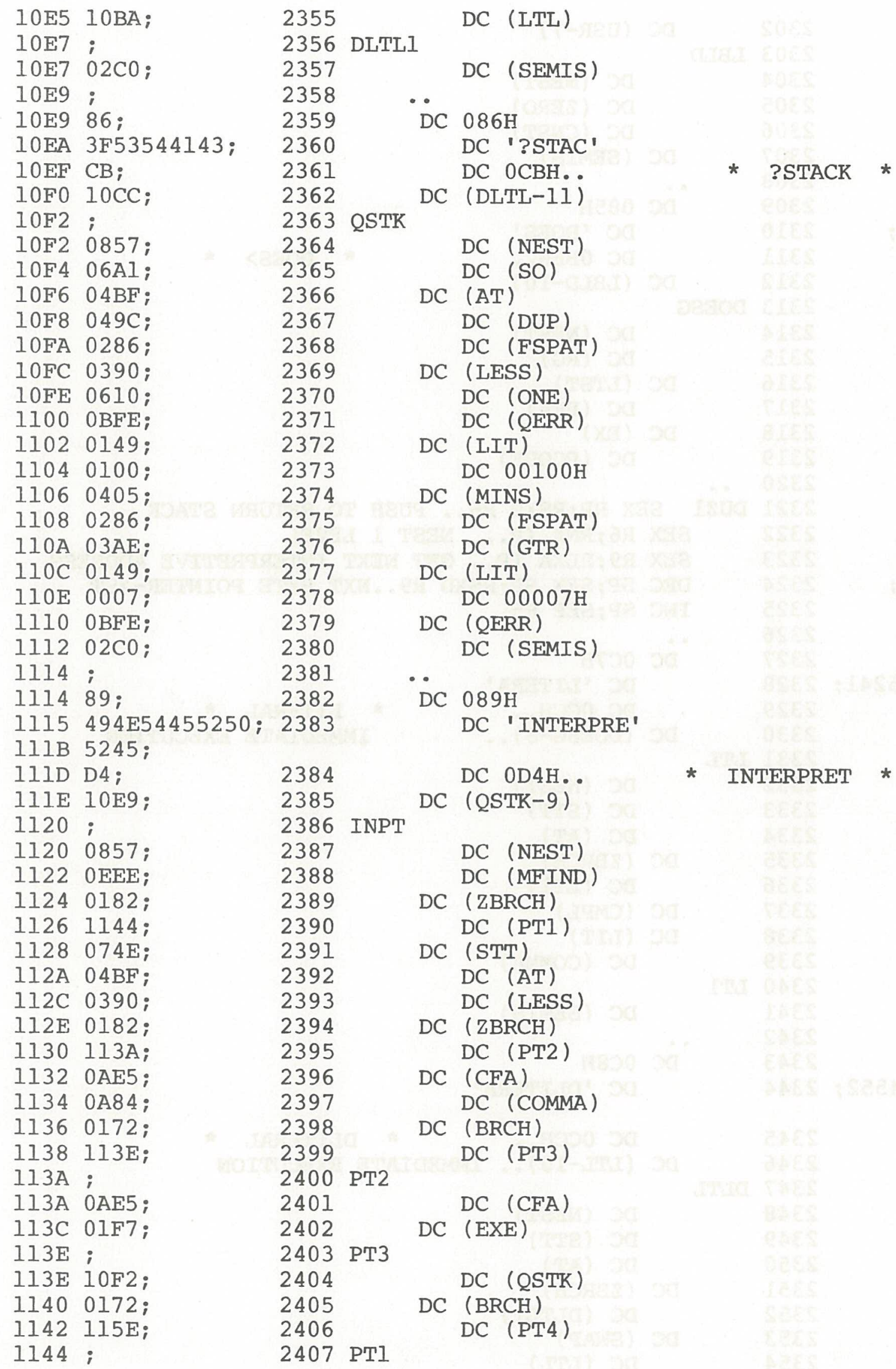

Woods Hole FORTH Version $1.800 \quad$ Chapter 21

page 188 
File ID: WFORTH.LST

Disk ID: WOODS HOLE FORTH SOURCE \& LIST VER 1.800 113084

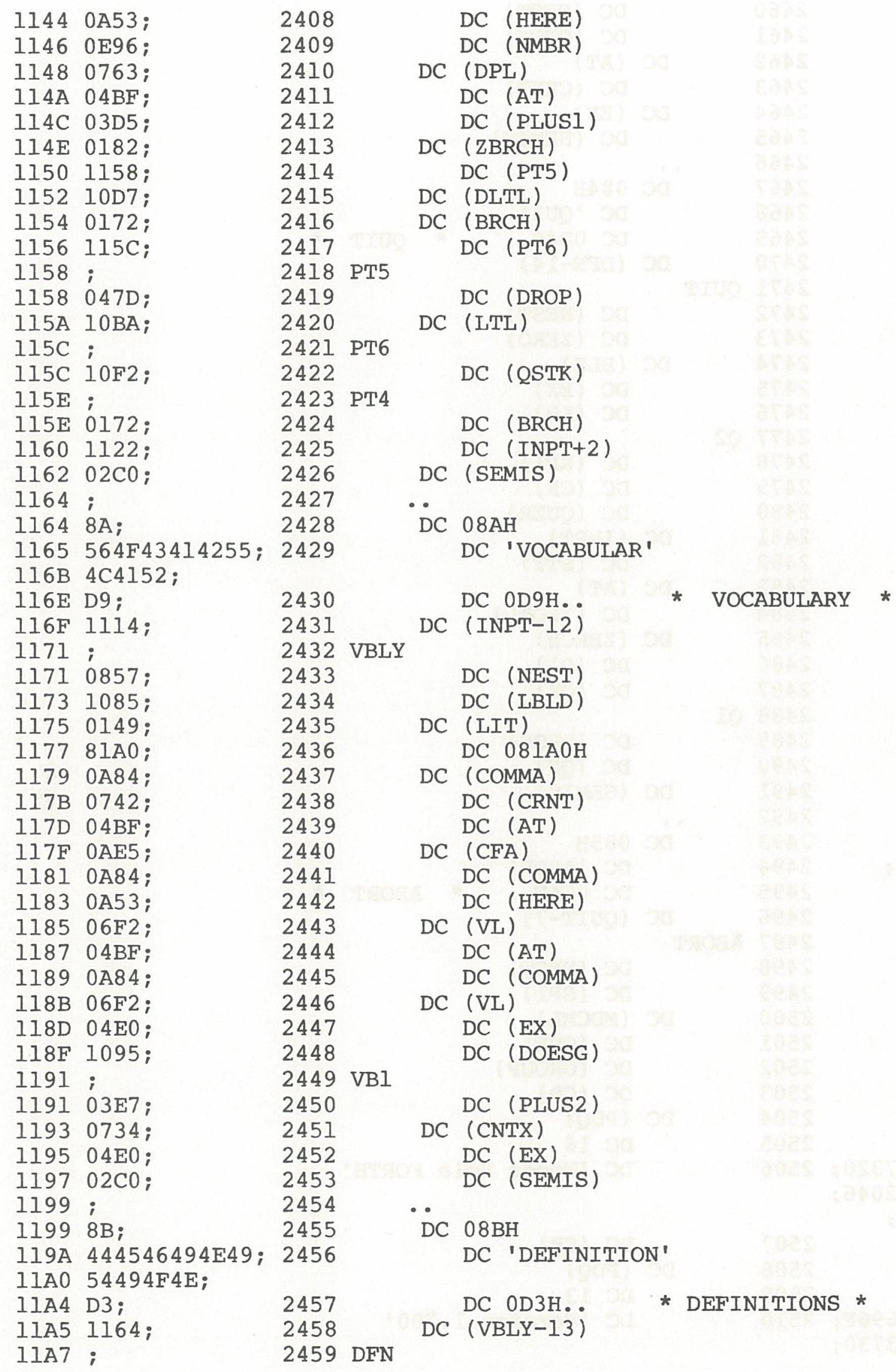


File ID: WFORTH.LST

Disk ID: WOODS HOLE FORTH SOURCE \& LIST VER 1.800

\begin{tabular}{|c|c|c|c|c|c|c|c|c|}
\hline $11 \mathrm{~A} 7$ & 0857; & 2460 & & & DC (NEST) & & & \\
\hline 11 A9 & $0734 ;$ & 2461 & & & DC (CNTX) & & & \\
\hline $11 \mathrm{AB}$ & 04BF； & 2462 & & DC & (AT) & & & \\
\hline $11 \mathrm{AD}$ & 0742; & 2463 & & & DC (CRNT) & & & \\
\hline $11 \mathrm{AF}$ & 04E0； & 2464 & & Xc & (EX) & & & \\
\hline $11 \mathrm{BI}$ & $02 \mathrm{CO}$; & 2465 & & & DC (SEMIS) & & & \\
\hline 11B3 & $i$ & 2466 & - & & & & & \\
\hline $11 \mathrm{~B} 3$ & $84 ;$ & 2467 & & DC & $084 \mathrm{H}$ & & & \\
\hline 11B4 & 515549 ; & 2468 & & & DC 'QUI' & & & \\
\hline $11 \mathrm{~B} 7$ & D4 ; & 2469 & & & DC $0 \mathrm{D} 4 \mathrm{H}$ & * & QUIT & * \\
\hline $11 \mathrm{~B} 8$ & $1199 ;$ & 2470 & & DC & $(\mathrm{DFN}-14)$ & & & \\
\hline $11 \mathrm{BA}$ & ; & 2471 & QUIT & & & & & \\
\hline $11 \mathrm{BA}$ & 0857； & 2472 & & & DC (NEST) & & & \\
\hline $11 \mathrm{BC}$ & 0608 ; & 2473 & & & DC (ZERO) & & & \\
\hline $11 \mathrm{BE}$ & 06FC; & 2474 & & $\mathrm{C}$ & $(\mathrm{BLK})$ & & & \\
\hline $11 \mathrm{C} 0$ & 04E0； & 2475 & & & $D C \quad(E X)$ & & & \\
\hline $11 \mathrm{C} 2$ & 0CA7; & 2476 & & & $D C \quad(L B)$ & & & \\
\hline $11 \mathrm{C} 4$ & ; & 2477 & Q2 & & & & & \\
\hline $11 C 4$ & $02 \mathrm{AB}$; & 2478 & & & DC (RPl) & & & \\
\hline $11 \mathrm{C} 6$ & 0909; & 2479 & & & $\mathrm{DC} \quad(\mathrm{CR})$ & & & \\
\hline $11 \mathrm{C} 8$ & 0DA5； & 2480 & & & DC (QUER) & & & \\
\hline $11 \mathrm{CA}$ & $1120 ;$ & 2481 & & $\mathrm{C}$ & (INPT) & & & \\
\hline $11 \mathrm{CC}$ & 074E; & 2482 & & & $\mathrm{DC} \quad$ (STT) & & & \\
\hline $11 \mathrm{CE}$ & $04 \mathrm{BF}$; & 2483 & & $\mathrm{C}$ & (AT) & & & \\
\hline 11D0 & $0352 ;$ & 2484 & & & DC (ZEQAL) & & & \\
\hline 11D2 & $0182 ;$ & 2485 & & $\mathrm{C}$ & ( $\mathrm{ZBRCH})$ & & & \\
\hline 11D4 & 11D8; & 2486 & & & DC (Q1) & & & \\
\hline $11 D 6$ & 18A9; & 2487 & & & DC (OK) & & & \\
\hline $11 \mathrm{D} 8$ & $i$ & 2488 & Q1 & & & & & \\
\hline $11 \mathrm{D} 8$ & $0172 ;$ & 2489 & & & DC (BRCH) & & & \\
\hline $11 \mathrm{DA}$ & 11C4; & 2490 & & & DC $(Q 2)$ & & & \\
\hline $11 \mathrm{DC}$ & $02 \mathrm{CO} ;$ & 2491 & & $\mathrm{C}$ & (SEMIS) & & & \\
\hline $11 \mathrm{DE}$ & i & 2492 & $\cdots$ & & & & & \\
\hline 11DE & $85 ;$ & 2493 & & $\mathrm{C}$ & $085 \mathrm{H}$ & & & \\
\hline 11DF & $41424 F 52$; & 2494 & & & DC 'ABOR' & & & \\
\hline $11 \mathrm{E} 3$ & D4 ; & 2495 & & & DC OD4H.. & * & ABORT & * \\
\hline $11 \mathrm{E} 4$ & 11B3; & 2496 & & $\mathrm{C}$ & (QUIT-7) & & & \\
\hline $11 \mathrm{E} 6$ & $i$ & 2497 & ABORT & & & & & \\
\hline $11 \mathrm{E} 6$ & 0857 ; & 2498 & & & DC (NEST) & & & \\
\hline $11 \mathrm{E} 8$ & 0297; & 2499 & & & DC (SPI) & & & \\
\hline $11 \mathrm{EA}$ & OCF2; & 2500 & & $\mathrm{C}$ & (MDCML) & & & \\
\hline $11 \mathrm{EC}$ & 0610; & 2501 & & & DC (ONE) & & & \\
\hline $11 \mathrm{EE}$ & 17BC; & 2502 & & & DC (GROUP) & & & \\
\hline $11 \mathrm{~F} 0$ & 0909; & 2503 & & & $\mathrm{DC} \quad(\mathrm{CR})$ & & & \\
\hline $11 \mathrm{~F} 2$ & OB41; & 2504 & & $\mathrm{DC}$ & $(\mathrm{PDQ})$ & & & \\
\hline $11 \mathrm{~F} 4$ & 10; & 2505 & & & DC 16 & & & \\
\hline $11 \mathrm{~F} 5$ & 576F6F647320; & 2506 & & & DC 'Woods & Hole & FORTH' & \\
\hline $11 \mathrm{FB}$ & 486F6C652046; & & & & & & & \\
\hline 1201 & 4F525448； & & & & & & & \\
\hline 1205 & 0909; & 2507 & & & DC (CR) & & & \\
\hline 1207 & OB41; & 2508 & & $\mathrm{C}$ & (PDQ) & & & \\
\hline 1209 & OD; & 2509 & & & DC 13 & & & \\
\hline $120 \mathrm{~A}$ & 56657273696F; & 2510 & & & DC Version & 1.7 & $700^{\circ}$ & \\
\hline & 6E20312E3730; & & & & & & & \\
\hline
\end{tabular}


File ID: WFORTH.LST

Disk ID: WOODS HOLE FORTH SOURCE \& LIST VER 1.800

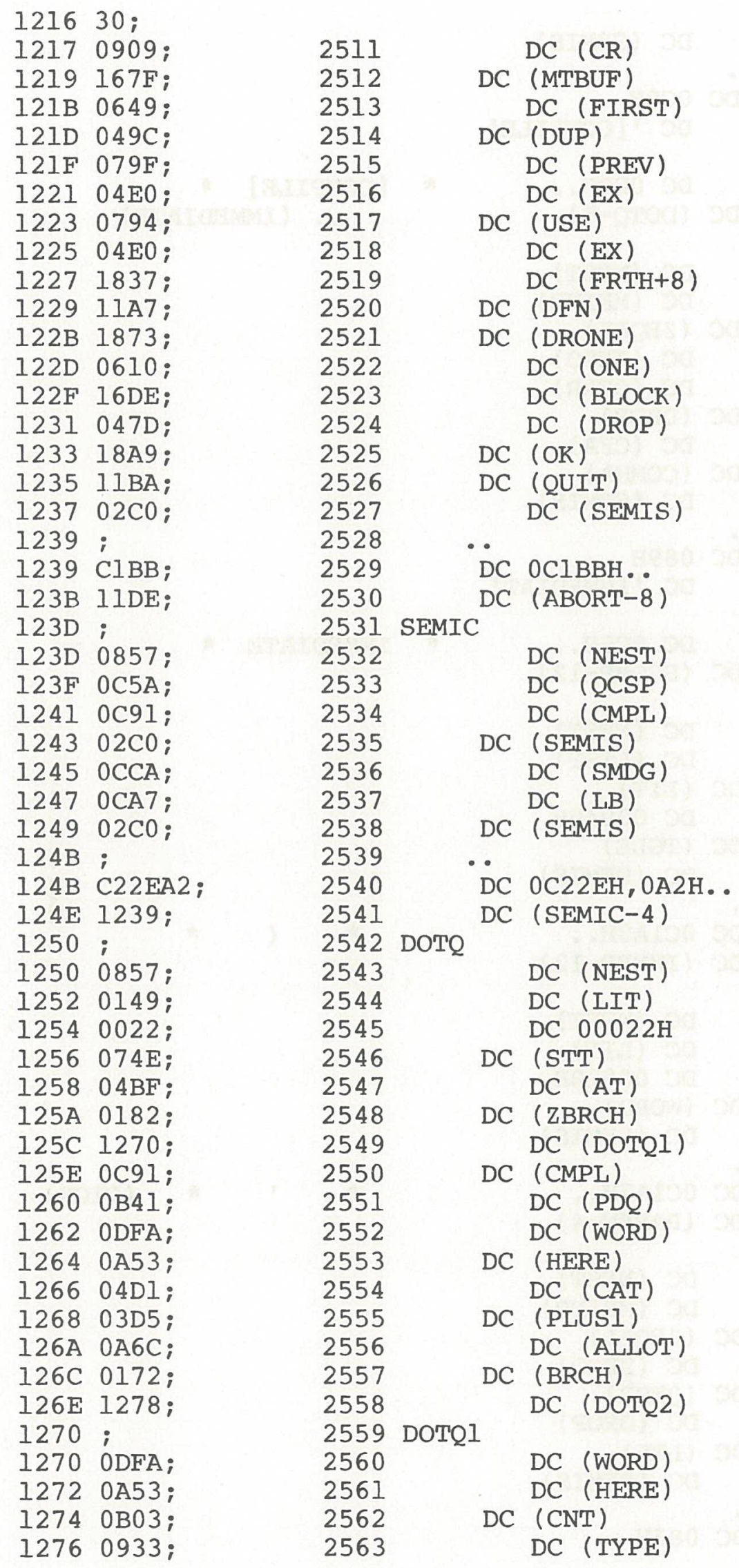

2511

2512

2513

2514

2515

2516

2517

2518

2519

2520

2521

2522

2523

2524

2525

2526

2527

2528

2529

2530

2531 SEMIC

2532

2533

2534

2535

2536

2537

2538

2539

2540

2541

2542 DOTQ

2543

2544

2545

2546

2547

2548

2549

2550

2551

2552

2553

2554

2555

2556

2557

2558

2559 DOTQI

2560

2561

2562

2563

$$
\begin{array}{ll}
\mathrm{DC} \quad(\mathrm{CR}) \\
\mathrm{DC} \quad \text { (MTBUF) } \\
\text { DC (FIRST) }
\end{array}
$$

DC (DUP)

DC (PREV)

DC (EX)

DC (USE)

DC (EX)

DC (FRTH+8)

DC (DFN)

DC (DRONE)

DC (ONE)

DC (BLOCK)

DC (DROP)

DC (OK)

DC (QUIT)

DC (SEMIS)

DC OCIBBH..

DC (ABORT-8)

$$
\begin{array}{ll}
\text { DC } & \text { (NEST) } \\
\text { DC } & \text { (QCSP) }
\end{array}
$$

DC (CMPL)

DC (SEMIS)

DC (SMDG)

DC (LB)

DC (SEMIS)

-

DC $0 \mathrm{C} 22 \mathrm{EH}, 0 \mathrm{~A} 2 \mathrm{H}$.

DC (SEMIC-4)

DOTQ

DC (NEST)

DC (LIT)

DC $00022 \mathrm{H}$

DC (STT)

DC (AT)

DC ( $\mathrm{ZBRCH})$

DC (DOTQI)

DC (CMPL)

DC (PDQ)

DC (WORD)

DC (HERE)

DC (CAT)

DC (PLUSI)

DC (ALLOT)

DC (BRCH)

DC (DOTQ2)

DC (WORD)

DC (HERE)

DC (CNT)

DC (TYPE) 
File ID: WFORTH.LST

Disk ID: WOODS HOLE FORTH SOURCE \& LIST VER 1.800

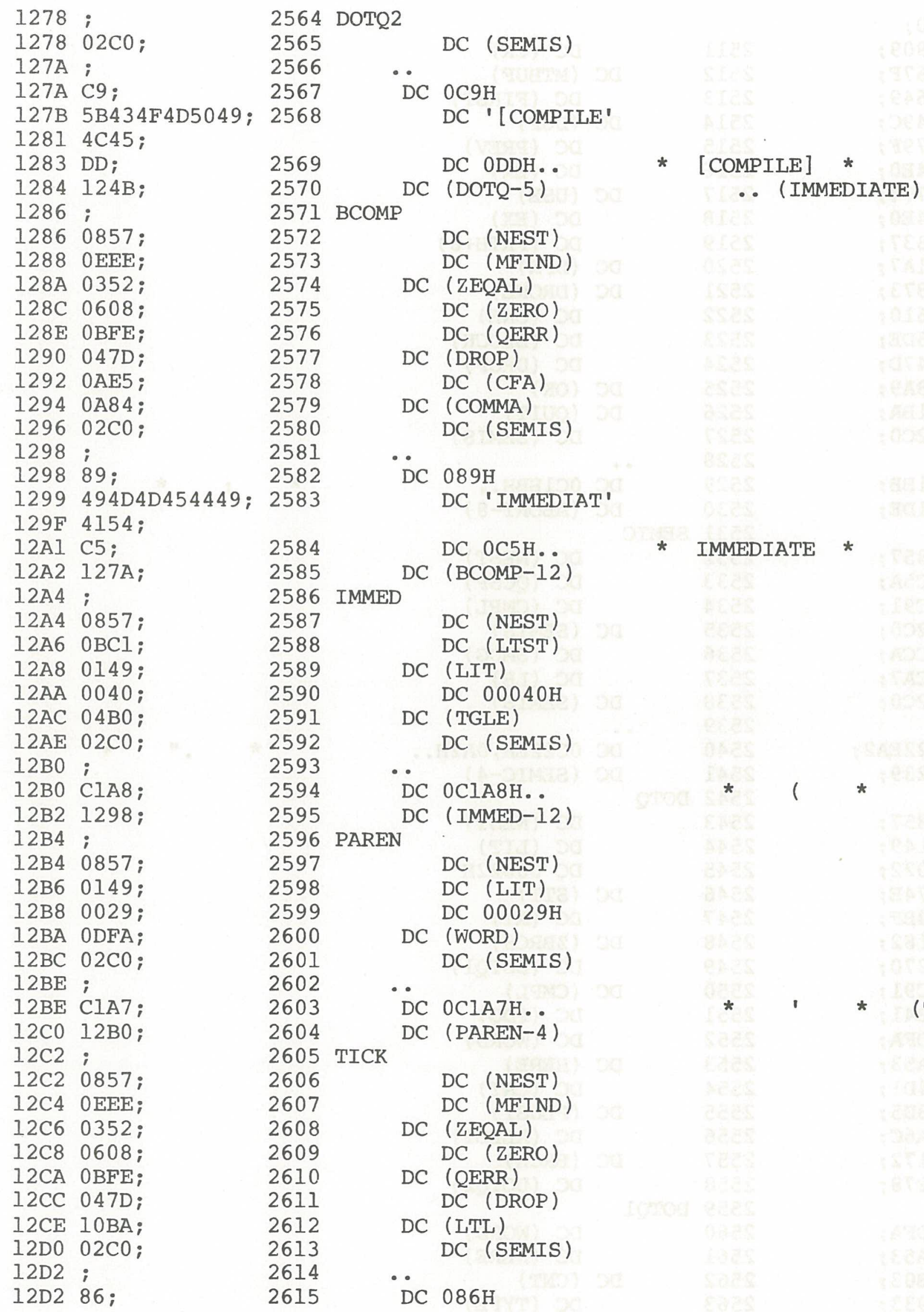

$\begin{array}{ll}1298 \text { 89i } & 2582 \\ 1299494 D 4 D 454449 ; & 2583\end{array}$

2569

2570

2571 BCOMP

2572

2573

2574

2575

2576

2577

2578

2579

2580

2581

48

2584

2585

2586 IMMED

2587

2588

2589

2590

2591

2592

2593

2594

2595

2596

2597

2598

2599

2600

2601

2602

2603

2604

2605 TICK

2606

2607

2608

2609

2610

2611

2612

2613

2614

2615

DC $0 \mathrm{C} 9 \mathrm{H}$

DC ' [ COMPILE'

DC ODDH..

DC (DOTQ-5)

DC (NEST)

DC (MFIND)

DC (ZEQAL)

DC (ZERO)

DC (QERR)

DC (DROP)

DC (CFA)

DC (COMMA)

DC (SEMIS)

$\cdots$

DC $089 \mathrm{H}$

DC 'IMMEDIAT'

PAREN

DC (IMMED-12)

$$
\text { DC (NEST) }
$$

DC (LIT)

DC $00029 \mathrm{H}$

DC (WORD)

DC (SEMIS)

$\cdots$

DC 0 CIA7H..

DC (PAREN-4)

DC $086 \mathrm{H}$ 
File ID: WFORTH.LST

Disk ID: WOODS HOLE FORTH SOURCE \& LIST VER 1.800

$1130 \quad 84$

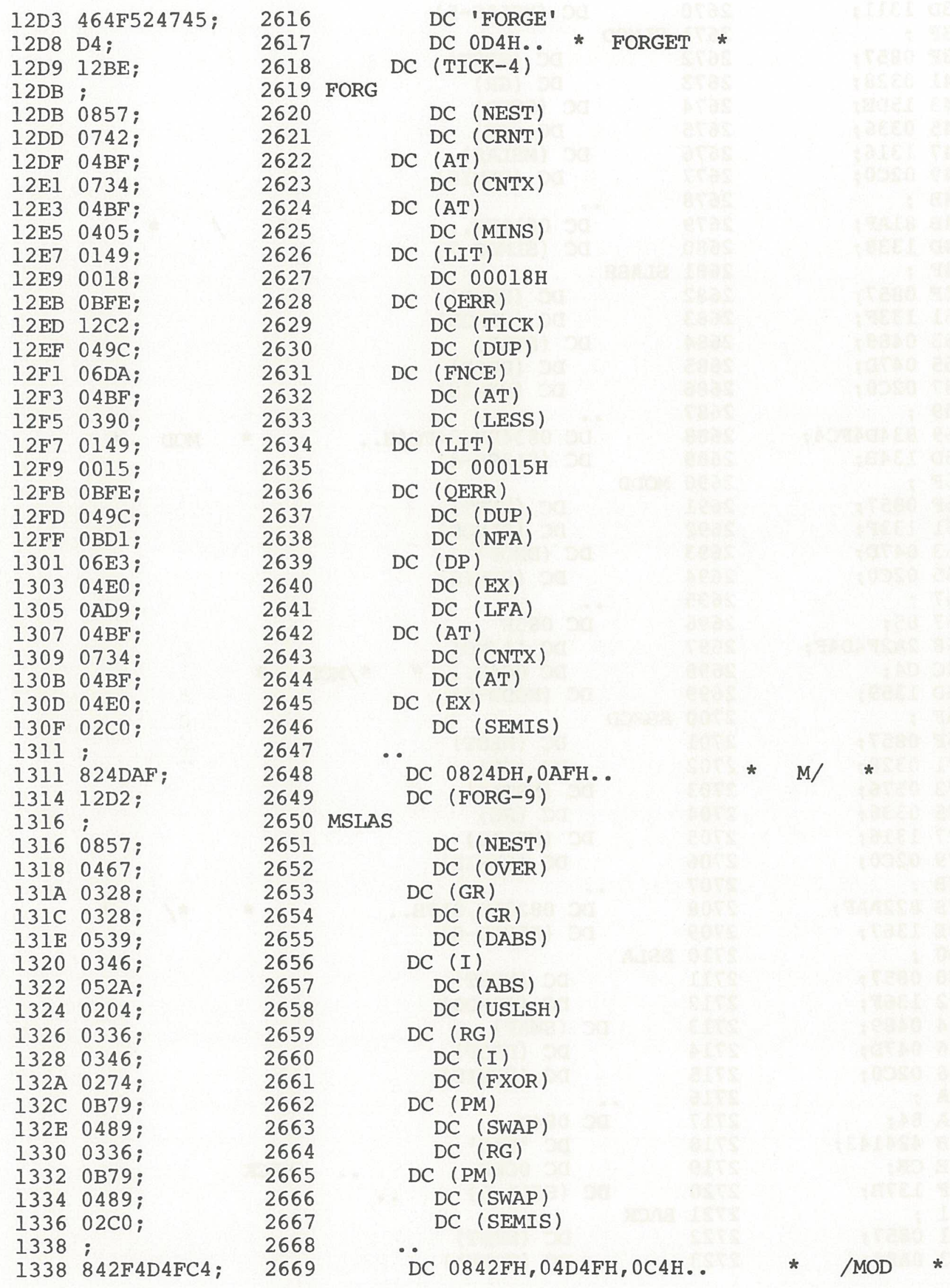


File ID: WFORTH.IST

Disk ID: WOODS HOLE FORTH SOURCE \& LIST VER 1.800

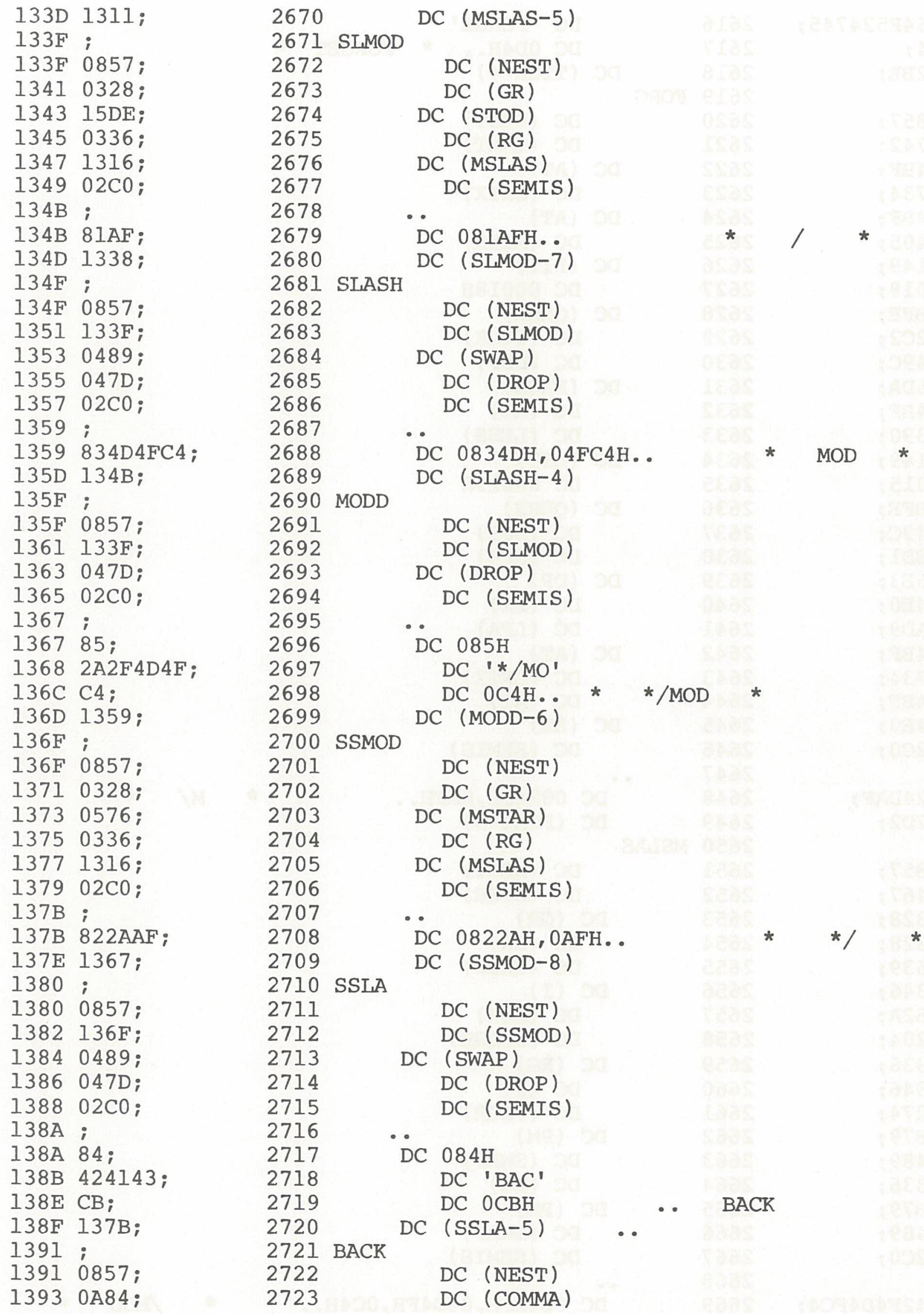


File ID: WFORTH.LST

Disk ID: WOODS HOLE FORTH SOURCE \& LIST VER 1.800

113084

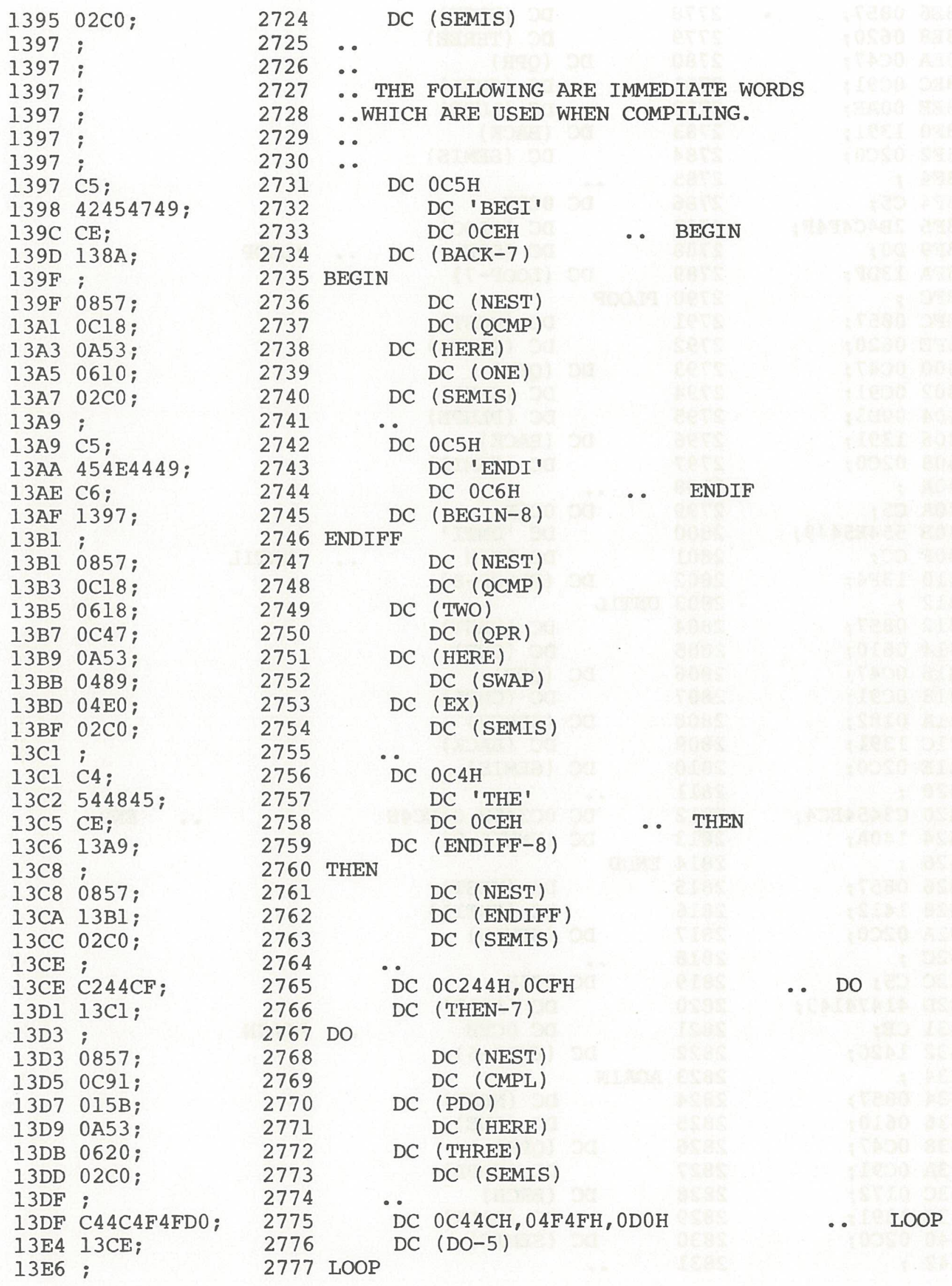


File ID: WFORTH.LST

Disk ID: WOODS HOLE FORTH SOURCE \& LIST VER 1.800

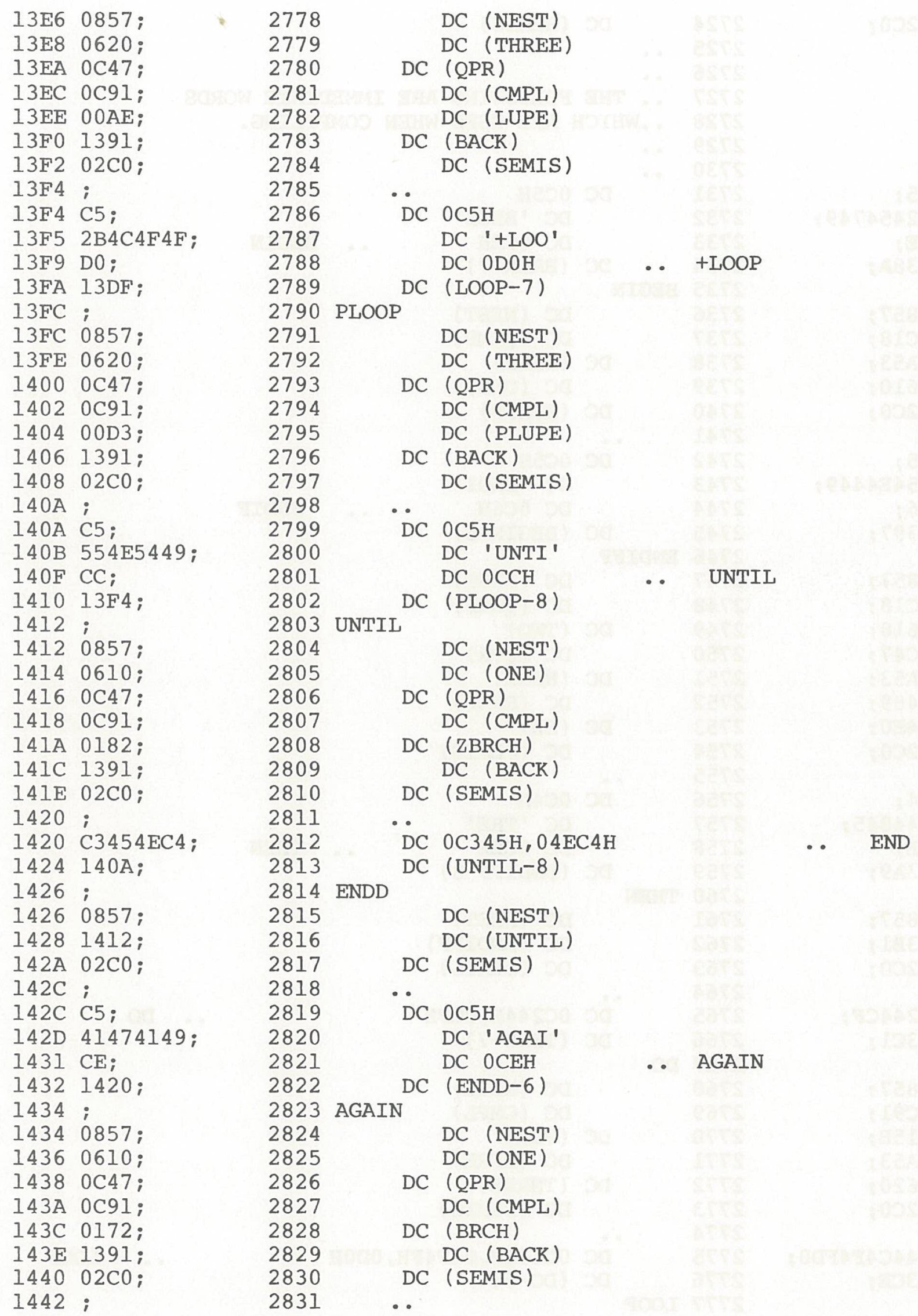


File ID: WFORTH.LST

Disk ID: WOODS HOLE FORTH SOURCE \& LIST VER 1.800

$1130 \quad 84$

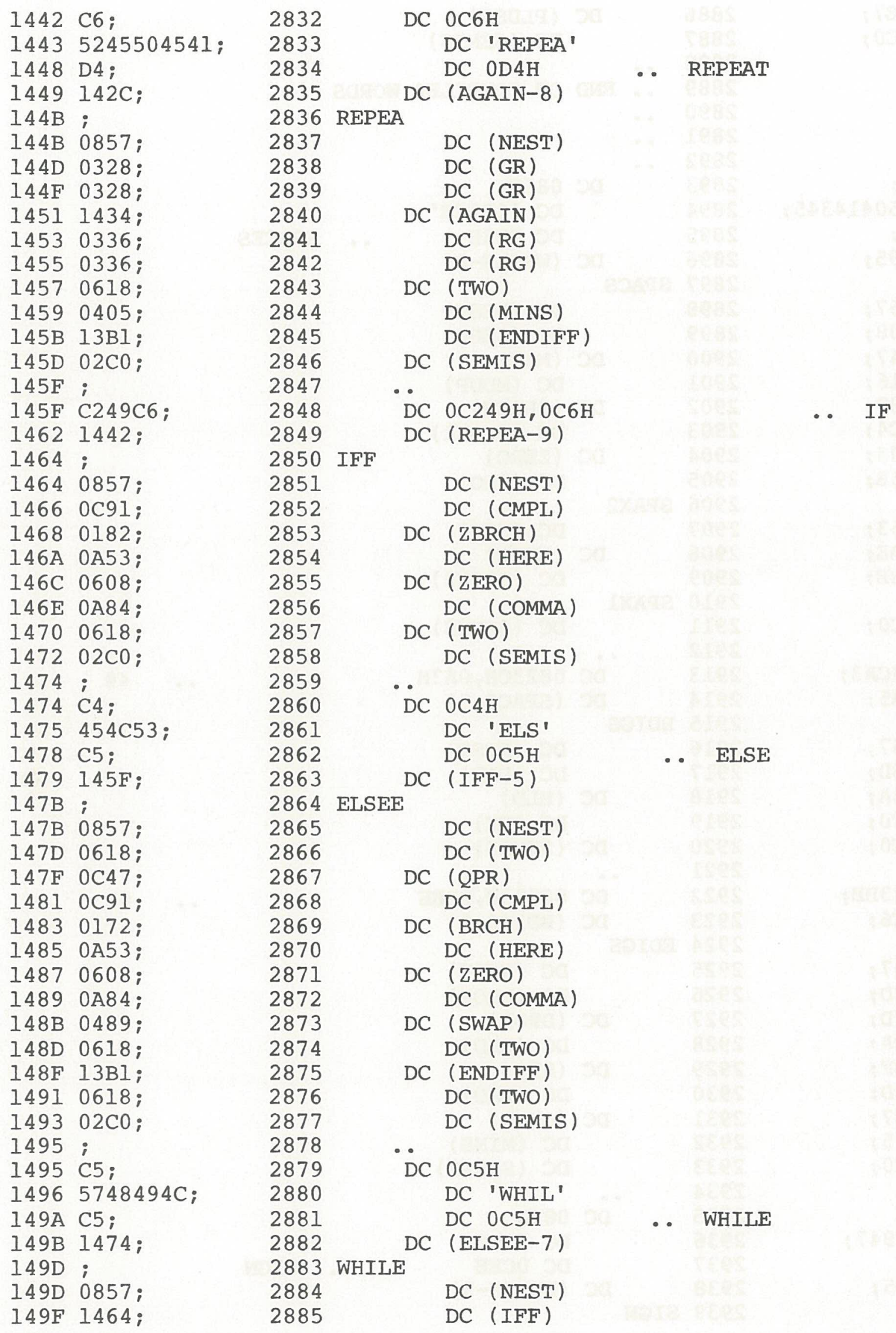


File ID: WFORTH.LST

Disk ID: WOODS HOLE FORTH SOURCE \& LIST VER 1.800

$1130 \quad 84$

\begin{tabular}{|c|c|c|c|c|c|c|c|}
\hline $14 \mathrm{Al}$ & 03E7； & 2886 & $\mathrm{DC}$ & (PLUS2) & & & \\
\hline $14 \mathrm{~A} 3$ & $02 \mathrm{CO} ;$ & 2887 & & DC (SEMIS) & & & \\
\hline $14 \mathrm{~A} 5$ & ; & 2888 & $\cdots$ & & & & \\
\hline $14 \mathrm{~A} 5$ & ; & 2889 & . END & OF COMPILER & WORDS & & \\
\hline $14 \mathrm{~A} 5$ & ; & 2890 & $\ldots$ & & & & \\
\hline $14 \mathrm{~A} 5$ & ; & 2891 & . & & & & \\
\hline $14 \mathrm{~A} 5$ & ; & 2892 & -. & & & & \\
\hline $14 \mathrm{~A} 5$ & $86 ;$ & 2893 & DC & $086 \mathrm{H}$ & & & \\
\hline $14 \mathrm{~A} 6$ & 5350414345 ; & 2894 & & DC 'SPACE' & & & \\
\hline $14 \mathrm{AB}$ & D3; & 2895 & & $\mathrm{DC} \quad 0 \mathrm{D} 3 \mathrm{H}$ & .. & SPACES & \\
\hline $14 \mathrm{AC}$ & $1495 ;$ & 2896 & DC & (WHILE-8) & & & \\
\hline $14 \mathrm{AE}$ & $i$ & 2897 & SPACS & & & & \\
\hline $14 \mathrm{AE}$ & 0857 ; & 2898 & & DC (NEST) & & & \\
\hline $14 \mathrm{BO}$ & 0608 ; & 2899 & & DC (ZERO) & & & \\
\hline $14 \mathrm{~B} 2$ & 0547 ; & 2900 & DC & $(\mathrm{MAX})$ & & & \\
\hline $14 \mathrm{~B} 4$ & 0516; & 2901 & & DC (MDUP) & & & \\
\hline $14 \mathrm{~B} 6$ & 0182 ; & 2902 & DC & ( $\mathrm{ZBRCH})$ & & & \\
\hline $14 \mathrm{~B} 8$ & $14 \mathrm{C} 4$; & 2903 & & DC (SPAXI) & & & \\
\hline $14 \mathrm{BA}$ & 0608 ; & 2904 & DC & (ZERO) & & & \\
\hline $14 \mathrm{BC}$ & 015B; & 2905 & & DC $(\mathrm{PDO})$ & & & \\
\hline $14 \mathrm{BE}$ & ; & 2906 & SPAX2 & & & & \\
\hline $14 \mathrm{BE}$ & 0963 ; & 2907 & & $D C \quad(S P C)$ & & & \\
\hline $14 \mathrm{C} 0$ & OOAE; & 2908 & DC & (LUPE) & & & \\
\hline $14 \mathrm{C} 2$ & $14 \mathrm{BE}$ & 2909 & & DC (SPAX2) & & & \\
\hline $14 \mathrm{C} 4$ & ; & 2910 & SPAXI & & & & \\
\hline $14 \mathrm{C} 4$ & $02 \mathrm{CO}$; & 2911 & & DC (SEMIS) & & & \\
\hline $14 \mathrm{C} 6$ & i & 2912 & .. & & & & \\
\hline $14 \mathrm{C} 6$ & 823СА3; & 2913 & DC & $0823 \mathrm{CH}, 0 \mathrm{~A} 3 \mathrm{H}$ & & & . \\
\hline $14 \mathrm{C} 9$ & $14 \mathrm{~A} 5$ & 2914 & DC & $($ SPACS-9) & & & \\
\hline $14 \mathrm{CB}$ & ; & 2915 & BDIGS & & & & \\
\hline $14 \mathrm{CB}$ & 0857 ; & 2916 & & DC (NEST) & & & \\
\hline $14 \mathrm{CD}$ & OB5D; & 2917 & & $\mathrm{DC} \quad(\mathrm{PAD})$ & & & \\
\hline $14 \mathrm{CF}$ & 078A; & 2918 & DC & (HLD) & & & \\
\hline 14DI & $04 \mathrm{E} 0$; & 2919 & & $\mathrm{DC} \quad(\mathrm{EX})$ & & & \\
\hline $14 \mathrm{D} 3$ & $02 \mathrm{CO}$; & 2920 & DC & (SEMIS) & & & \\
\hline $14 \mathrm{D} 5$ & ; & 2921 & .. & & & & \\
\hline 14D5 & 8223BE; & 2922 & $\mathrm{DC}$ & $08223 \mathrm{H}, 0 \mathrm{BEH}$ & & & -. \\
\hline $14 \mathrm{D} 8$ & $14 \mathrm{C} 6$ & 2923 & DC & $($ BDIGS-5) & & & \\
\hline $14 \mathrm{DA}$ & ; & 2924 & EDIGS & & & & \\
\hline $14 \mathrm{DA}$ & 0857 ; & 2925 & & DC (NEST) & & & \\
\hline $14 \mathrm{DC}$ & 047D; & 2926 & & DC (DROP) & & & \\
\hline $14 \mathrm{DE}$ & 047D; & 2927 & DC & (DROP) & & & \\
\hline $14 \mathrm{E} 0$ & 078A; & 2928 & & DC (HLD) & & & \\
\hline $14 \mathrm{E} 2$ & $04 \mathrm{BF}$; & 2929 & DC & $(\mathrm{AT})$ & & & \\
\hline $14 \mathrm{E} 4$ & OB5D; & 2930 & & DC (PAD) & & & \\
\hline $14 \mathrm{E} 6$ & 0467 ; & 2931 & DC & (OVER) & & & \\
\hline $14 \mathrm{E} 8$ & 0405 & 2932 & & DC (MINS) & & & \\
\hline $14 \mathrm{EA}$ & $02 \mathrm{CO}$ & 2933 & & DC (SEMIS) & & & \\
\hline $14 \mathrm{EC}$ & ; & 2934 & $\cdots$ & & & & \\
\hline $14 \mathrm{EC}$ & $84 ;$ & 2935 & DC & $084 \mathrm{H}$ & & & \\
\hline $14 \mathrm{ED}$ & 534947 ; & 2936 & & DC 'SIG' & & & \\
\hline $14 \mathrm{FO}$ & $\mathrm{CE}_{i}$ & 2937 & & DC OCEH & .. & SIGN & \\
\hline $14 \mathrm{Fl}$ & $14 \mathrm{D} 5$; & 2938 & $\mathrm{DC}$ & (EDIGS-5) & & & \\
\hline $14 \mathrm{~F} 3$ & ; & 2939 & SIGN & & & & \\
\hline
\end{tabular}


File ID: WFORTH.LST

Disk ID: WOODS HOLE FORTH SOURCE \& LIST VER 1.800

\begin{tabular}{|c|c|c|c|c|c|c|c|}
\hline $14 \mathrm{~F} 3$ & $0857 ;$ & 2940 & & DC (NEST) & & & \\
\hline $14 \mathrm{~F} 5$ & 04FE; & 2941 & & DC (ROT) & & & \\
\hline $14 \mathrm{~F} 7$ & 0367 ; & 2942 & DC & (ZLESS) & & & \\
\hline $14 \mathrm{~F} 9$ & 0182; & 2943 & DC & ( $\mathrm{ZBRCH}$ ) & & & \\
\hline $14 \mathrm{FB}$ & 1503; & 2944 & & DC (SIGNI) & & & \\
\hline $14 \mathrm{FD}$ & 0149 ; & 2945 & DC & (IIT) & & & \\
\hline $14 \mathrm{FF}$ & $002 \mathrm{D}$; & 2946 & & $\mathrm{DC} \quad 0002 \mathrm{DH}$ & & & \\
\hline 1501 & 08BI; & 2947 & DC & (HOLD) & & & \\
\hline 1503 & i & 2948 & SIGNI & & & & \\
\hline 1503 & $02 \mathrm{CO}$; & 2949 & & DC (SEMIS) & & & \\
\hline 1505 & $i$ & 2950 & $\cdots$ & & & & \\
\hline 1505 & 81A3; & 2951 & DC & 081АЗН & $\cdots$ & \# & \\
\hline 1507 & $14 \mathrm{EC}$; & 2952 & DC & $(\mathrm{SIGN}-7)$ & & & \\
\hline 1509 & i & 2953 & DIG & & & & \\
\hline 1509 & 0857 ; & 2954 & & DC (NEST) & & & \\
\hline $150 \mathrm{~B}$ & 0759 ; & 2955 & & DC (BASE) & & & \\
\hline 150D & $04 \mathrm{BF}$; & 2956 & & DC (AT) & & & \\
\hline $150 \mathrm{~F}$ & OA07; & 2957 & DC & (MSMOD) & & & \\
\hline 1511 & $04 \mathrm{FE}$; & 2958 & & DC (ROT) & & & \\
\hline 1513 & 0149 ; & 2959 & DC & (LIT) & & & \\
\hline 1515 & 0009 ; & 2960 & & DC $00009 H$ & & & \\
\hline 1517 & 0467 ; & 2961 & DC & (OVER) & & & \\
\hline 1519 & 0390 ; & 2962 & & DC (LESS) & & & \\
\hline $151 \mathrm{~B}$ & $0182 ;$ & 2963 & DC & (ZBRCH) & & & \\
\hline 151D & $1525 ;$ & 2964 & & DC (DIGI) & & & \\
\hline $151 \mathrm{~F}$ & $0149 ;$ & 2965 & DC & (LIT) & & & \\
\hline 1521 & $0007 ;$ & 2966 & & $\mathrm{DC} \quad 00007 \mathrm{H}$ & & & \\
\hline 1523 & $03 C 4 ;$ & 2967 & DC & (PLUS) & & & \\
\hline 1525 & i & 2968 & DIGl & & & & \\
\hline 1525 & $0149 ;$ & 2969 & & DC (LIT) & & & \\
\hline 1527 & 0030i & 2970 & & $\mathrm{DC} 00030 \mathrm{H}$ & & & \\
\hline 1529 & 03C4; & 2971 & DC & (PLUS) & & & \\
\hline $152 \mathrm{~B}$ & 08Bl; & 2972 & & DC (HOLD) & & & \\
\hline $152 \mathrm{D}$ & $02 \mathrm{CO}$; & 2973 & & DC (SEMIS) & & & \\
\hline $152 \mathrm{~F}$ & i & 2974 & $\cdots$ & & & & \\
\hline $152 \mathrm{~F}$ & 8223D3; & 2975 & DC & $08223 \mathrm{H}, 0 \mathrm{D} 3 \mathrm{H}$ & - & - & $\# S$ \\
\hline 1532 & $1505 ;$ & 2976 & DC & $(\mathrm{DIG}-4)$ & & & \\
\hline 1534 & $i$ & 2977 & DIGS & & & & \\
\hline 1534 & 0857 ; & 2978 & & DC (NEST) & & & \\
\hline 1536 & i & 2979 & DIGSI & & & & \\
\hline 1536 & $1509 ;$ & 2980 & & DC (DIG) & & & \\
\hline 1538 & 0467 ; & 2981 & & DC (OVER) & & & \\
\hline $153 \mathrm{~A}$ & 0467 ; & 2982 & $\mathrm{DC}$ & (OVER) & & & \\
\hline $153 C$ & 0262 ; & 2983 & & DC (FFOR) & & & \\
\hline $153 \mathrm{E}$ & 0352 ; & 2984 & & DC (ZEQAL) & & & \\
\hline 1540 & 0182 ; & 2985 & DC & (ZBRCH) & & & \\
\hline 1542 & 1536 ; & 2986 & & DC (DIGSI) & & & \\
\hline 1544 & $02 \mathrm{CO}$; & 2987 & $\mathrm{DC}$ & (SEMIS) & & & \\
\hline 1546 & i & 2988 & 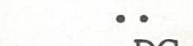 & & & & \\
\hline 1546 & 83442 ED2 ; & 2989 & DC & $08344 \mathrm{H}, 02 \mathrm{ED} 2 \mathrm{H}$ & - & - & $D \cdot R$ \\
\hline $154 \mathrm{~A}$ & $152 \mathrm{~F} ;$ & 2990 & ${ }^{\text {DC }}$ & $(D I G S-5)$ & & & \\
\hline $\begin{array}{l}154 \mathrm{C} \\
154 \mathrm{C}\end{array}$ & i & $\begin{array}{l}2991 \\
2992\end{array}$ & DDOTR & & & & \\
\hline $\begin{array}{l}154 \mathrm{C} \\
154 \mathrm{E}\end{array}$ & $0328 ;$ & 2993 & & $\mathrm{DC}$ (GR) & & & \\
\hline & & & & & & & \\
\hline
\end{tabular}


File ID: WFORTH.LST

Disk ID: WOODS HOLE FORTH SOURCE \& LIST VER 1.800

\begin{tabular}{|c|c|c|c|c|c|c|}
\hline 15500489 ; & 2994 & & $C$ (SWAP) & & & \\
\hline 15520467 ; & 2995 & & DC (OVER) & & & \\
\hline 15540539 ; & 2996 & & DC (DABS) & & & \\
\hline $155614 \mathrm{CB}$; & 2997 & DC & C (BDIGS) & & & \\
\hline $15581534 ;$ & 2998 & & DC (DIGS) & & & \\
\hline 155A 14F3; & 2999 & & DC (SIGN) & & & \\
\hline 155C 14DA; & 3000 & DC & （EDIGS） & & & \\
\hline 155E 0336 ; & 3001 & & DC $\quad(R G)$ & & & \\
\hline 15600467 ; & 3002 & & DC (OVER) & & & \\
\hline 15620405 & 3003 & DC & (MINS) & & & \\
\hline 1564 14AE; & 3004 & & DC (SPACS) & & & \\
\hline 15660933 ; & 3005 & DC & (TYPE) & & & \\
\hline $156802 \mathrm{CO}$ & 3006 & & DC (SEMIS) & & & \\
\hline 156A； & 3007 & $\cdots$ & & & & \\
\hline 156A 822ED2; & 3008 & DC & $0822 \mathrm{EH}, 0 \mathrm{D} 2 \mathrm{H}$ & & - & - $\mathrm{R}$ \\
\hline 156D 1546; & 3009 & DC & (DDOTR-6) & & & \\
\hline $156 \mathrm{~F}$; & 3010 & DOTR & & & & \\
\hline $156 \mathrm{~F} 0857$; & 3011 & & DC (NEST) & & & \\
\hline 15710328 ; & 3012 & & $\mathrm{DC} \quad(\mathrm{GR})$ & & & \\
\hline 15DE; & 3013 & DC & (STOD) & & & \\
\hline 0336 ; & 3014 & & $\mathrm{DC} \quad(\mathrm{RG})$ & & & \\
\hline $154 C_{i}$ & 3015 & DC & (DDOTR) & & & \\
\hline $02 \mathrm{CO} ;$ & 3016 & & DC (SEMIS) & & & \\
\hline $157 \mathrm{~B}$; & 3017 & $\cdots$ & & & & \\
\hline 157B 8244AE; & 3018 & DC & $08244 \mathrm{H}, \mathrm{OAEH}$ & & -. & D. \\
\hline $156 \mathrm{~A} ;$ & 3019 & DC & (DOTR-5) & & & \\
\hline 1580 & 3020 & DDOT & & & & \\
\hline 0857; & 3021 & & DC (NEST) & & & \\
\hline 0608 ; & 3022 & & DC (ZERO) & & & \\
\hline 154C; & 3023 & DC & (DDOTR) & & & \\
\hline 0963; & 3024 & & DC (SPC) & & & \\
\hline $02 \mathrm{CO} ;$ & 3025 & & DC (SEMIS) & & & \\
\hline $158 \mathrm{~A}$ & 3026 & $\cdots$ & & & & \\
\hline 81AE; & 3027 & $\mathrm{DC}$ & O8IAEH & $\cdots$ & - & (DOT) \\
\hline 157B; & 3028 & DC & $($ DDOT-5) & & & \\
\hline $158 \mathrm{E}$ & 3029 & DOT & & & & \\
\hline $158 \mathrm{E} 0857$; & 3030 & & DC (NEST) & & & \\
\hline 15DE; & 3031 & & DC (STOD) & & & \\
\hline $1580 ;$ & 3032 & DC & (DDOT) & & & \\
\hline $02 \mathrm{CO} ;$ & 3033 & & DC (SEMIS) & & & \\
\hline 1596 & 3034 & 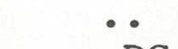 & & & & \\
\hline 81BF; & 3035 & DC & $081 \mathrm{BFH}$ & $\cdots$ & ? & \\
\hline 158A; & 3036 & DC & $(\mathrm{DOT}-4)$ & & & \\
\hline $159 A$ & 3037 & QUES & & & & \\
\hline 0857 ; & 3038 & & DC (NEST) & & & \\
\hline 04BF； & 3039 & & $\mathrm{DC} \quad(\mathrm{AT})$ & & & \\
\hline 159E 158E; & 3040 & DC & (DOT) & & & \\
\hline $15 \mathrm{AO} \quad 02 \mathrm{CO} ;$ & 3041 & & DC (SEMIS) & & & \\
\hline 15A2 i & 3042 & $\cdots$ & & & & \\
\hline $15 \mathrm{~A} 2 \quad 8255 \mathrm{AE}$; & 3043 & $\mathrm{DC}$ & O8255H, OAEH & & $\cdots$ & U. \\
\hline 151596 ; & 3044 & DC & (QUES-4) & & & \\
\hline $7 i$ & 3045 & UDOT & & & & \\
\hline 70857 ; & 3046 & & DC (NEST) & & & \\
\hline 0608 ; & 3047 & & DC (ZERO) & & & \\
\hline
\end{tabular}


File ID: WFORTH.LST

Disk ID: WOODS HOLE FORTH SOURCE \& LIST VER 1.800

113084

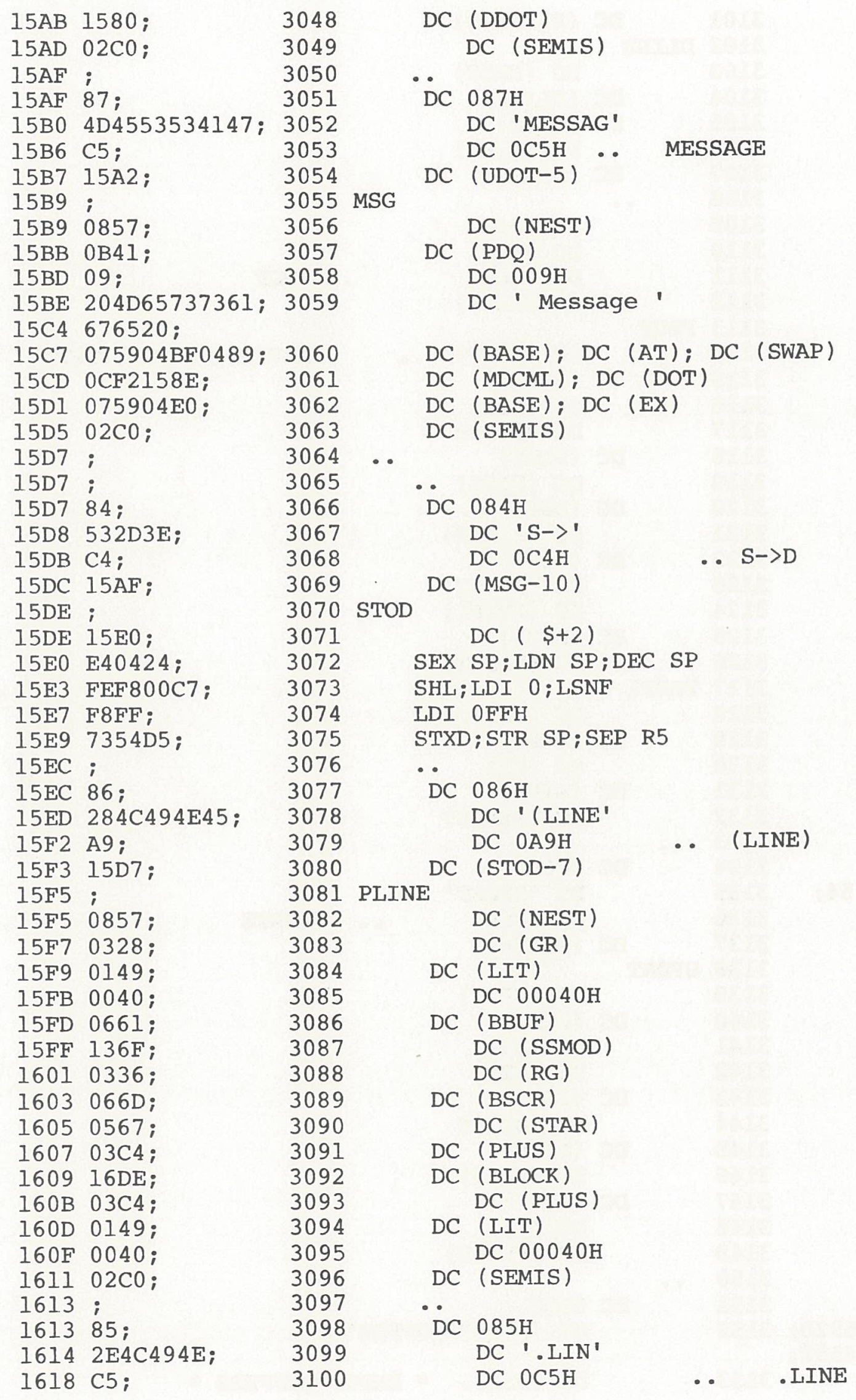

Woods Hole FORTH Version 1.800 Chapter 21

page 201 
File ID: WFORTH.LST

Disk ID: WOODS HOLE FORTH SOURCE \& LIST VER 1.800

$11 \quad 30 \quad 84$

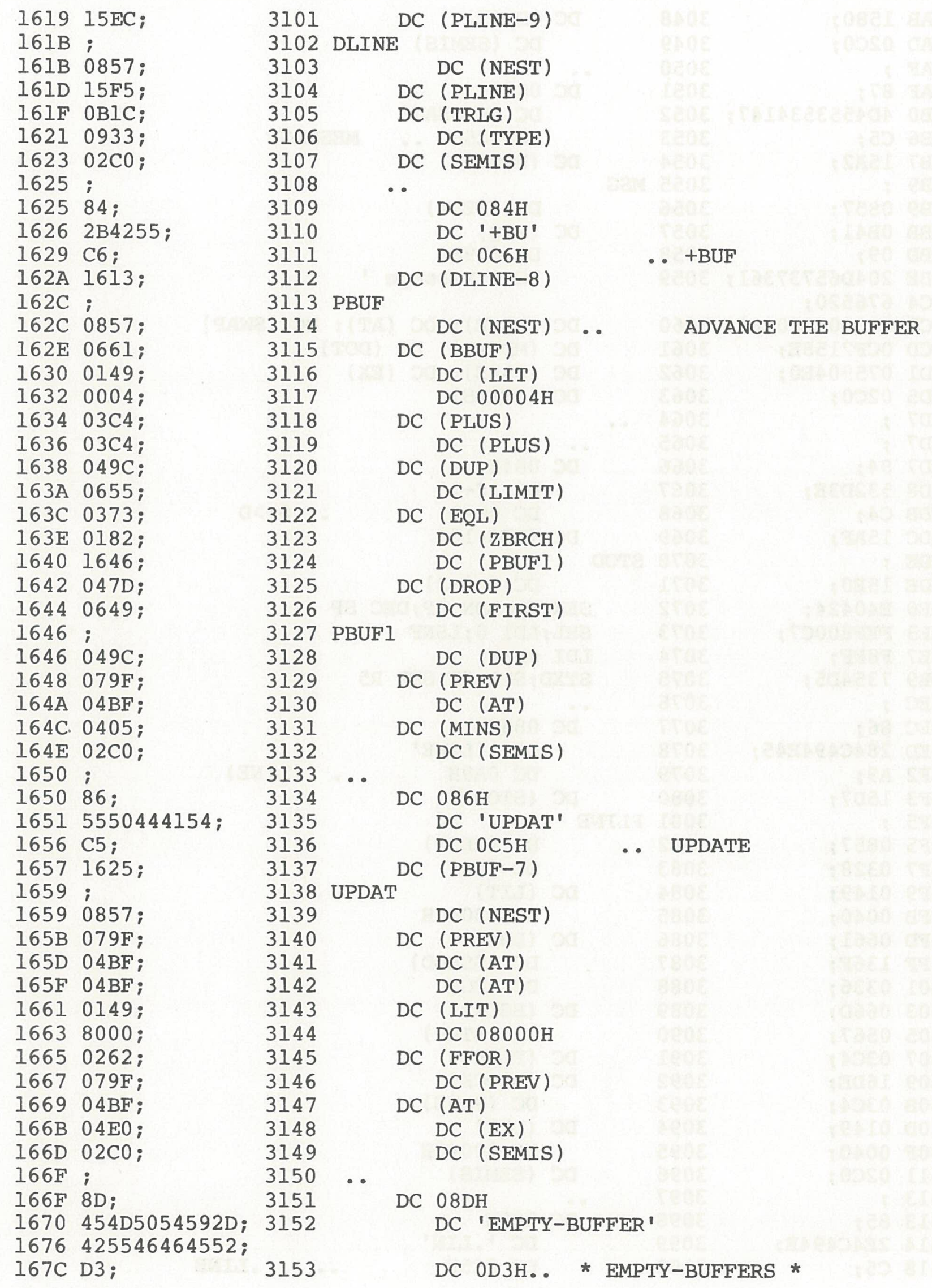


File ID: WFORTH.LST

Disk ID: WOODS HOLE FORTH SOURCE \& LIST VER 1.800

\begin{tabular}{|c|c|c|c|c|c|c|}
\hline $167 \mathrm{D}$ & 1650 ; & 3154 & $\mathrm{DC}$ & (UPDAT-9) & & \\
\hline $167 \mathrm{~F}$ & i & 3155 & MTBUF & & & \\
\hline $167 \mathrm{~F}$ & 0857; & 3156 & & DC (NEST) & & \\
\hline 1681 & $0649 ;$ & 3157 & DC & (FIRST) & & \\
\hline 1683 & 0655 ; & 3158 & & DC (LIMIT) & & \\
\hline 1685 & 0467 ; & 3159 & $\mathrm{DC}$ & (OVER) & & \\
\hline 1687 & 0405 ; & 3160 & DC & (MINS) & & \\
\hline 1689 & 0886 & 3161 & DC & (ERS) & & \\
\hline $168 \mathrm{~B}$ & $02 \mathrm{CO}$ & 3162 & & DC (SEMIS) & & \\
\hline $168 \mathrm{D}$ & ; & 3163 & -. & & & \\
\hline $168 \mathrm{D}$ & $86 ;$ & 3164 & $\mathrm{DC}$ & $086 \mathrm{H}$ & & \\
\hline $168 \mathrm{E}$ & 4255464645 ; & 3165 & & DC 'BUFFE' & & \\
\hline 1693 & D2 ; & 3166 & & $\mathrm{DC} \quad 0 \mathrm{D} 2 \mathrm{H}$ & - & BUFFER \\
\hline 1694 & $166 \mathrm{~F} ;$ & 3167 & DC & (MTBUF-16) & & \\
\hline 1696 & i & 3168 & BUFFE & & & \\
\hline 1696 & 0857 ; & 3169 & & DC (NEST) & & \\
\hline 1698 & 0794 ; & 3170 & DC & (USE) & & \\
\hline $169 \mathrm{~A}$ & $04 \mathrm{BF}$; & 3171 & & $\mathrm{DC}(\mathrm{AT})$ & & \\
\hline $169 \mathrm{C}$ & 049C; & 3172 & $\mathrm{DC}$ & (DUP) & & \\
\hline $169 \mathrm{E}$ & 0328 ; & 3173 & & $\mathrm{DC} \quad(\mathrm{GR})$ & & \\
\hline $16 \mathrm{AO}$ & i & 3174 & BUFFl & & & \\
\hline $16 \mathrm{~A} 0$ & 162C; & 3175 & & DC (PBUF) & & \\
\hline $16 \mathrm{~A} 2$ & 0182 ; & 3176 & DC & ( $\mathrm{ZBRCH})$ & & \\
\hline $16 \mathrm{~A} 4$ & $16 \mathrm{~A} 0$ & 3177 & & DC (BUFFI) & & \\
\hline $16 \mathrm{~A} 6$ & 0794； & 3178 & $\mathrm{DC}$ & (USE) & & \\
\hline $16 \mathrm{~A} 8$ & $04 \mathrm{E} 0$; & 3179 & & $D C \quad(E ; X)$ & & \\
\hline $16 \mathrm{AA}$ & $0346 ;$ & 3180 & $\mathrm{DC}$ & (I) & & \\
\hline $16 \mathrm{AC}$ & $04 \mathrm{BF}$; & 3181 & & $\mathrm{DC} \quad(\mathrm{AT})$ & & \\
\hline $16 \mathrm{AE}$ & 0367 ; & 3182 & DC & (ZLESS) & & \\
\hline 16B0 & 0182; & 3183 & DC & ( $\mathrm{ZBRCH})$ & & \\
\hline $16 \mathrm{~B} 2$ & 16C6; & 3184 & & $\mathrm{DC}$ (BUFF2) & & \\
\hline 16B4 & 0346； & 3185 & DC & (I) & & \\
\hline $16 \mathrm{~B} 6$ & 03E7； & 3186 & & DC (PLUS2) & & \\
\hline 16B8 & 0346 ; & 3187 & DC & (I) & & \\
\hline $16 \mathrm{BA}$ & 04BF ; & 3188 & & $\mathrm{DC} \quad(\mathrm{AT})$ & & \\
\hline $16 \mathrm{BC}$ & 0149 ; & 3189 & DC & (LIT) & & \\
\hline $16 \mathrm{BE}$ & $7 F F F$; & 3190 & & DC $07 F F F H$ & & \\
\hline $16 \mathrm{CO}$ & 0251; & 3191 & DC & (FAND) & & \\
\hline $16 \mathrm{C} 2$ & 0608 ; & 3192 & & DC (ZERO) & & \\
\hline $16 \mathrm{C} 4$ & $173 \mathrm{C} ;$ & 3193 & DC & (RSLW) & & \\
\hline $16 \mathrm{C} 6$ & ; & 3194 & BUFF2 & & & \\
\hline $16 \mathrm{C} 6$ & 0346 ; & 3195 & & DC (I) & & \\
\hline $16 \mathrm{C} 8$ & O4EO & 3196 & $\mathrm{DC}$ & $(\mathrm{EX})$ & & \\
\hline $16 \mathrm{CA}$ & 0346 ; & 3197 & & DC (I) & & \\
\hline $16 \mathrm{CC}$ & 079F; & 3198 & DC & (PREV) & & \\
\hline $16 \mathrm{CE}$ & 04E0; & 3199 & & $\mathrm{DC} \quad(\mathrm{EX})$ & & \\
\hline $16 \mathrm{D} 0$ & 0336 ; & 3200 & DC & (RG) & & \\
\hline $16 \mathrm{D} 2$ & 03E7; & 3201 & & DC (PLUS2) & & \\
\hline 16D4 & $02 \mathrm{CO} ;$ & 3202 & $\mathrm{DC}$ & (SEMIS ) & & \\
\hline 16D6 & $i$ & 3203 & . & & & \\
\hline 16D6 & 85 ; & 3204 & DC & $085 \mathrm{H}$ & & \\
\hline 16D7 & $424 C 4 F 43$ & 3205 & & DC 'BLOC' & & \\
\hline $16 \mathrm{DB}$ & CB ; & 3206 & & DC $0 \mathrm{CBH}$ & -. & BLOCK \\
\hline $16 \mathrm{DC}$ & $168 \mathrm{D}$ & 3207 & DC & (BUFFE-9) & & \\
\hline
\end{tabular}


File ID: WFORTH.IST

Disk ID: WOODS HOLE FORTH SOURCE \& LIST VER 1.800

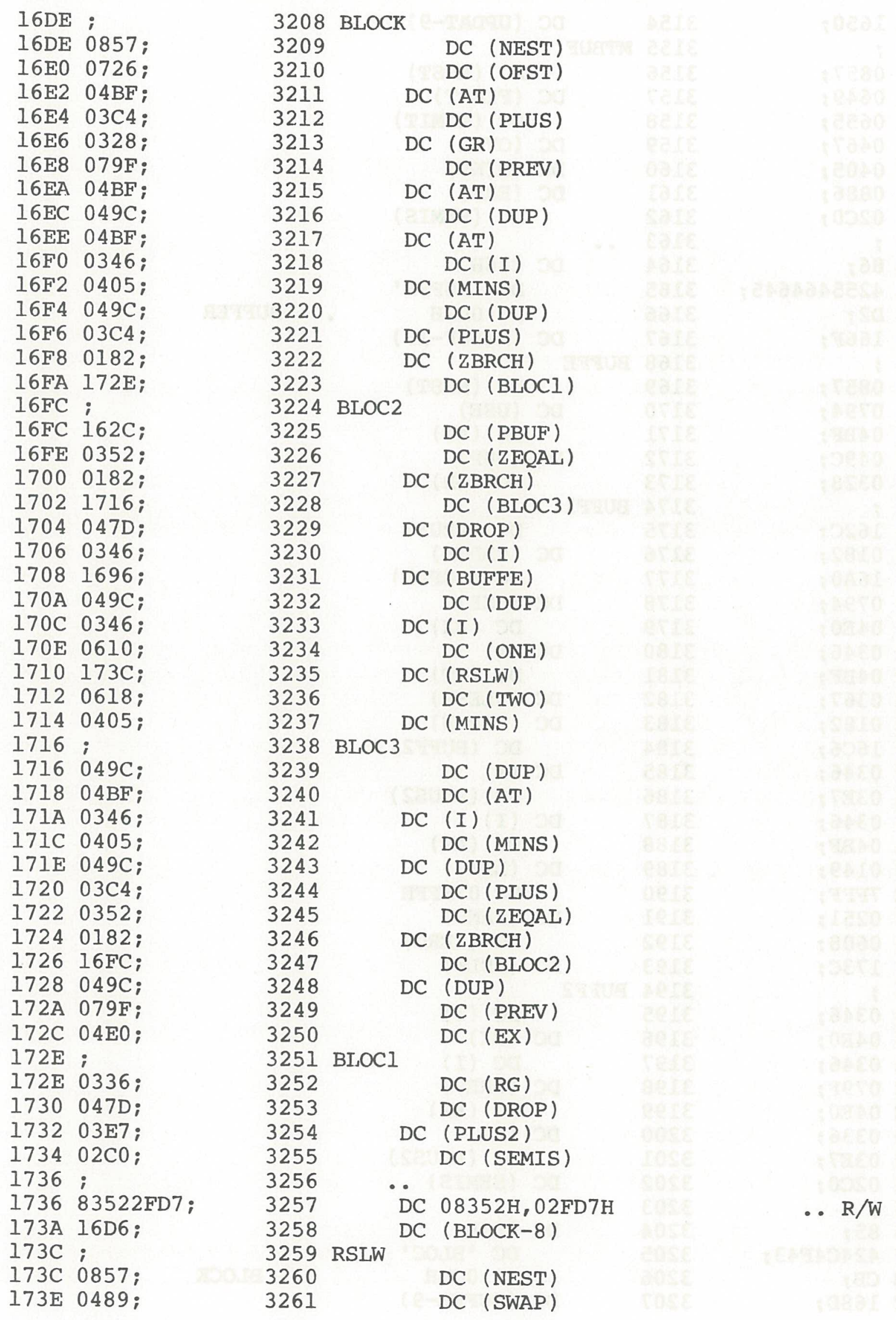


File ID: WFORTH.LST

Disk ID: WOODS HOLE FORTH SOURCE \& LIST VER 1.800 113084

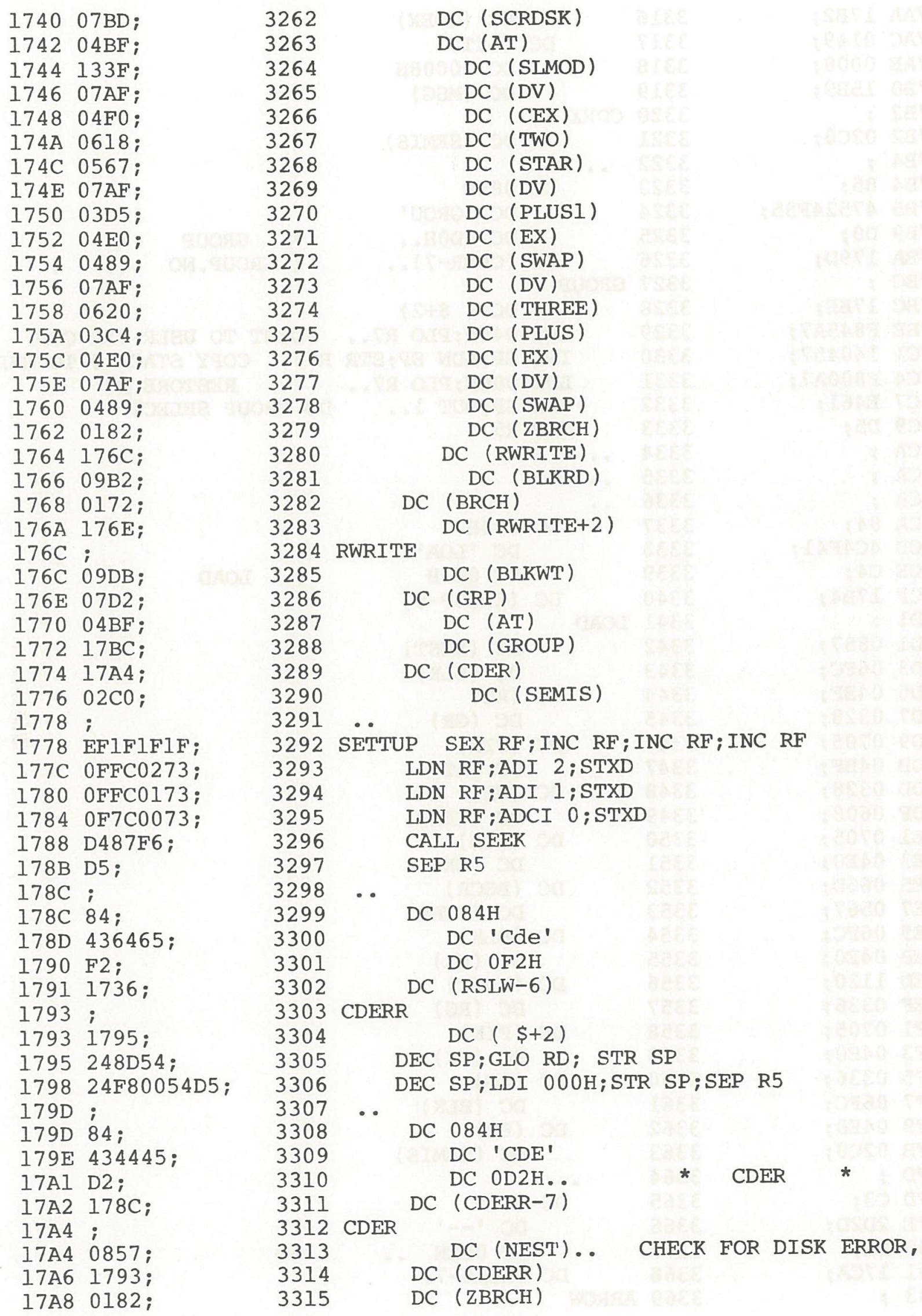

Woods Hole FORTH Version 1.800 Chapter 21 page 205 
File ID: WFORTH.LST

Disk ID: WOODS HOLE FORTH SOURCE \& LIST VER 1.800

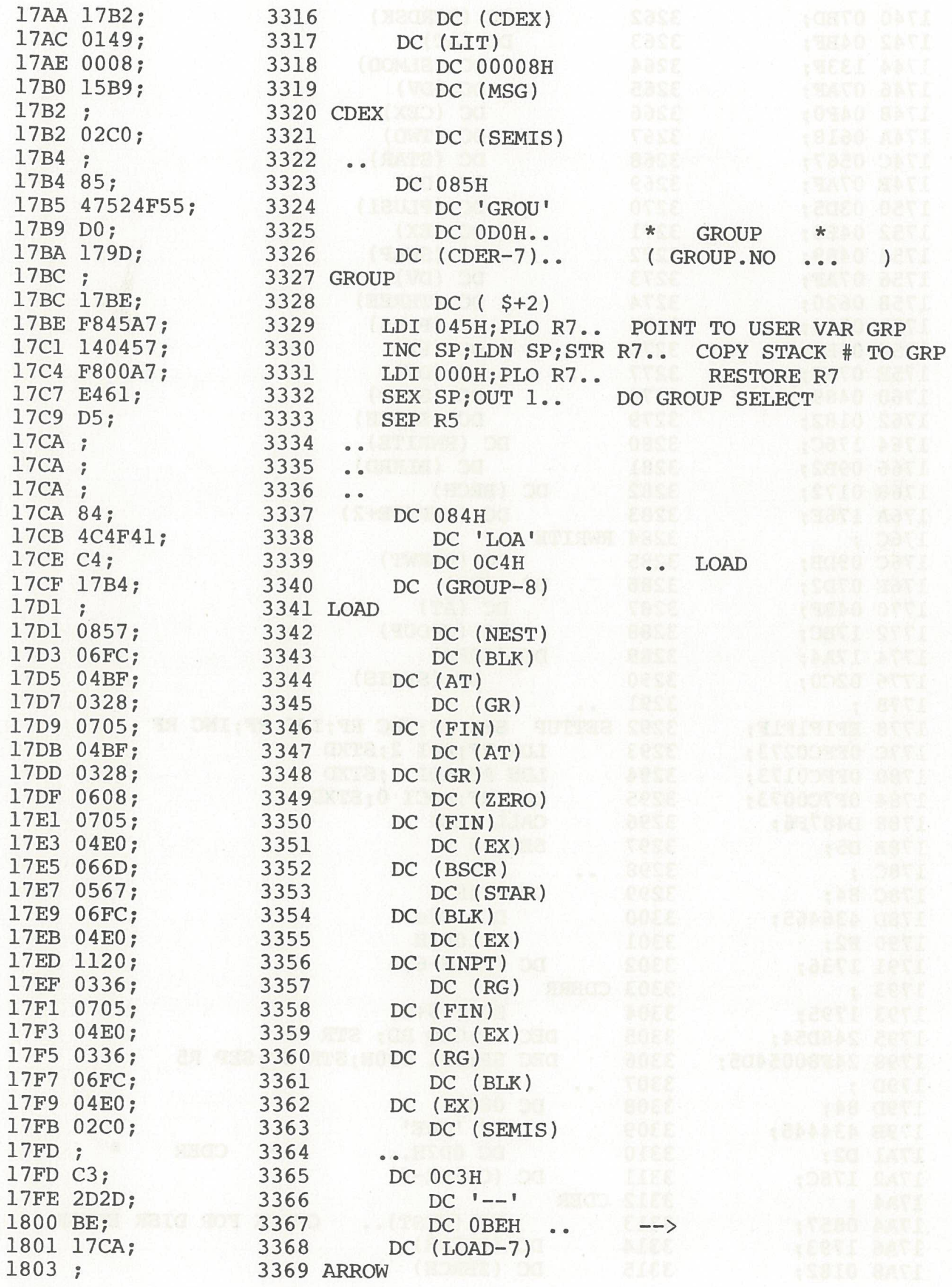


File ID: WFORTH.LST

Disk ID: WOODS HOLE FORTH SOURCE \& LIST VER 1.800

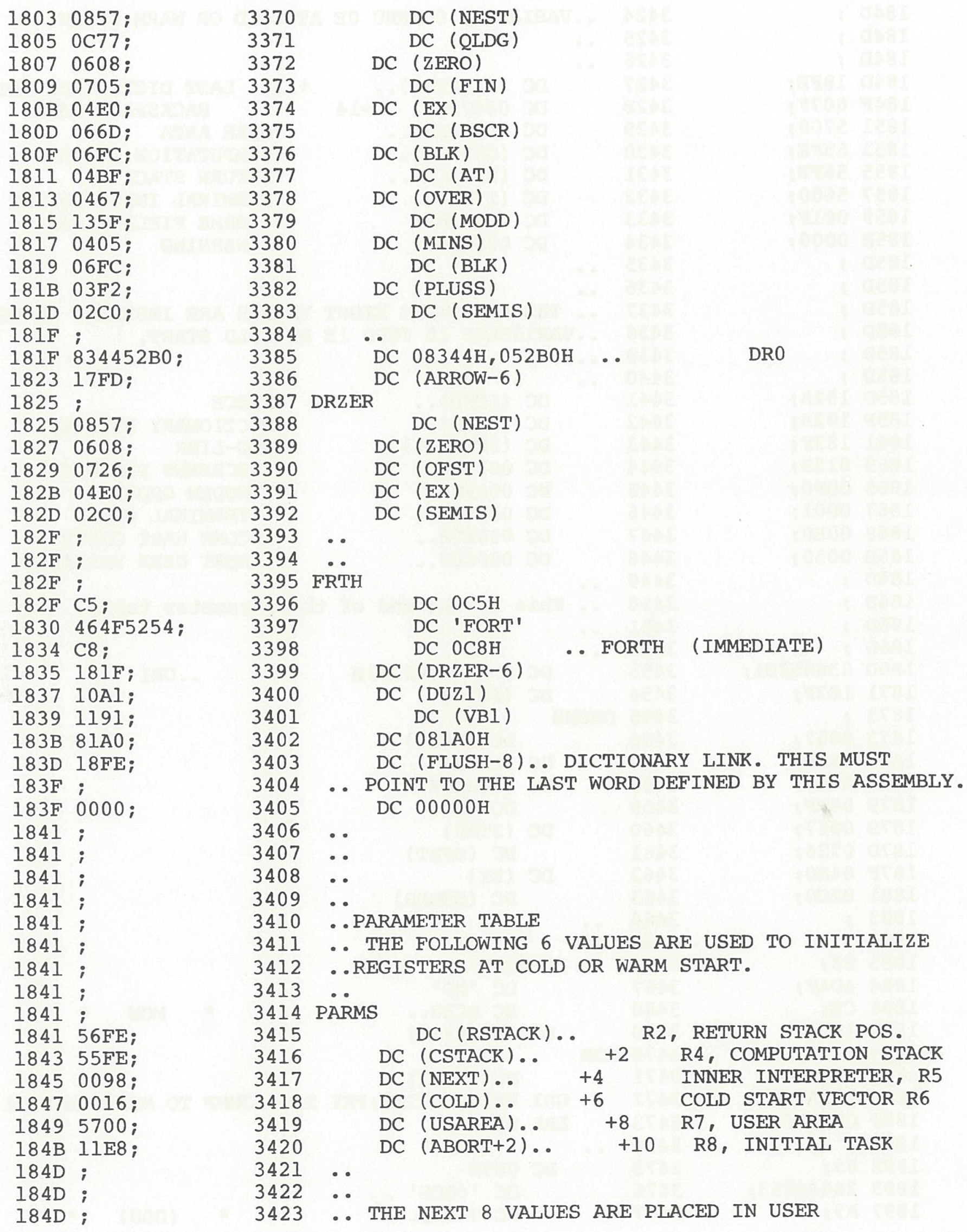

Woods Hole FORTH Version $1.800 \quad$ Chapter $21 \quad$ page 207 
File ID: WFORTH.IST

Disk ID: WOODS HOLE FORTH SOURCE \& LIST VER 1.800

113084

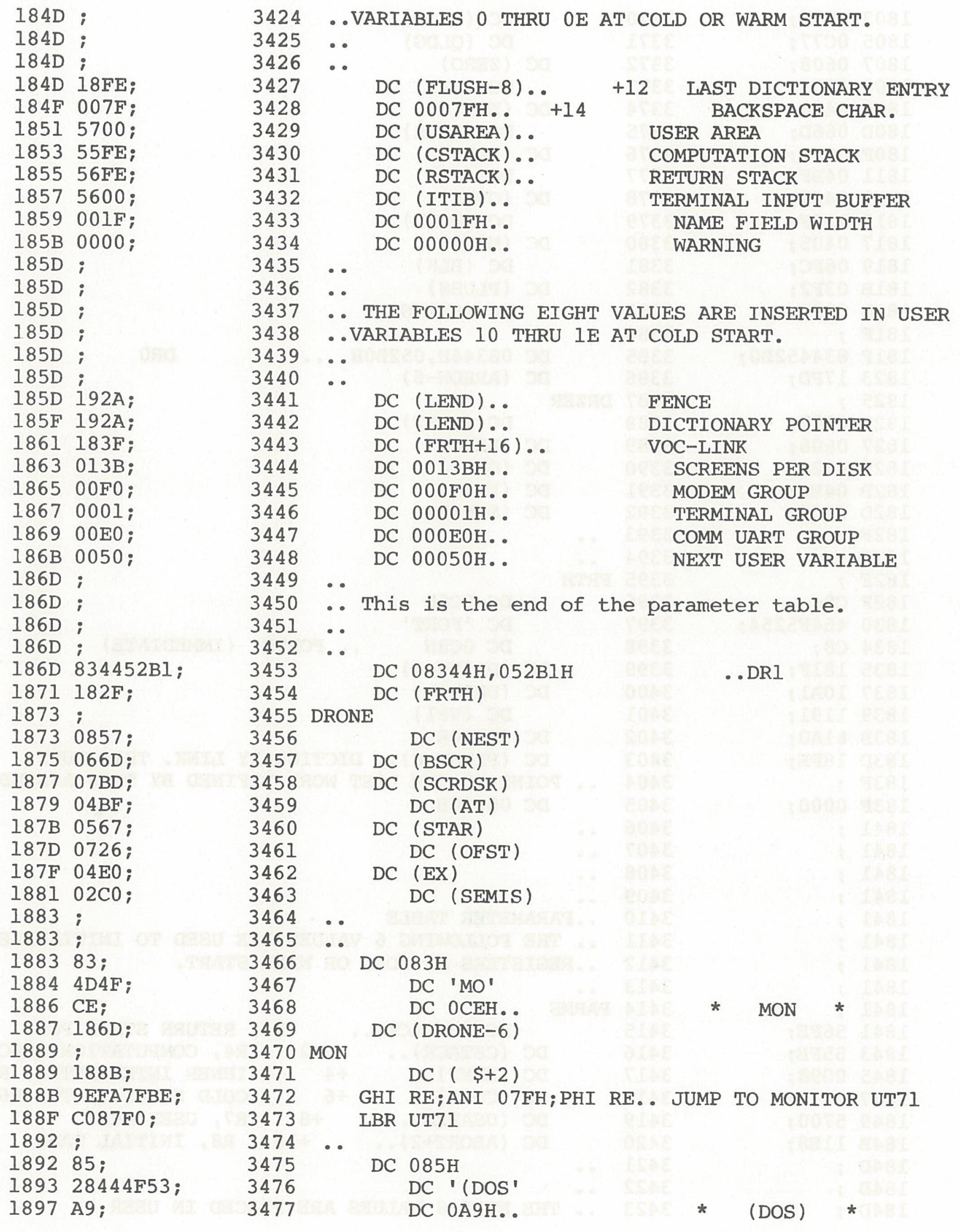


File ID: WFORTH.LST

Disk ID: WOODS HOLE FORTH SOURCE \& LIST VER 1.800 113084

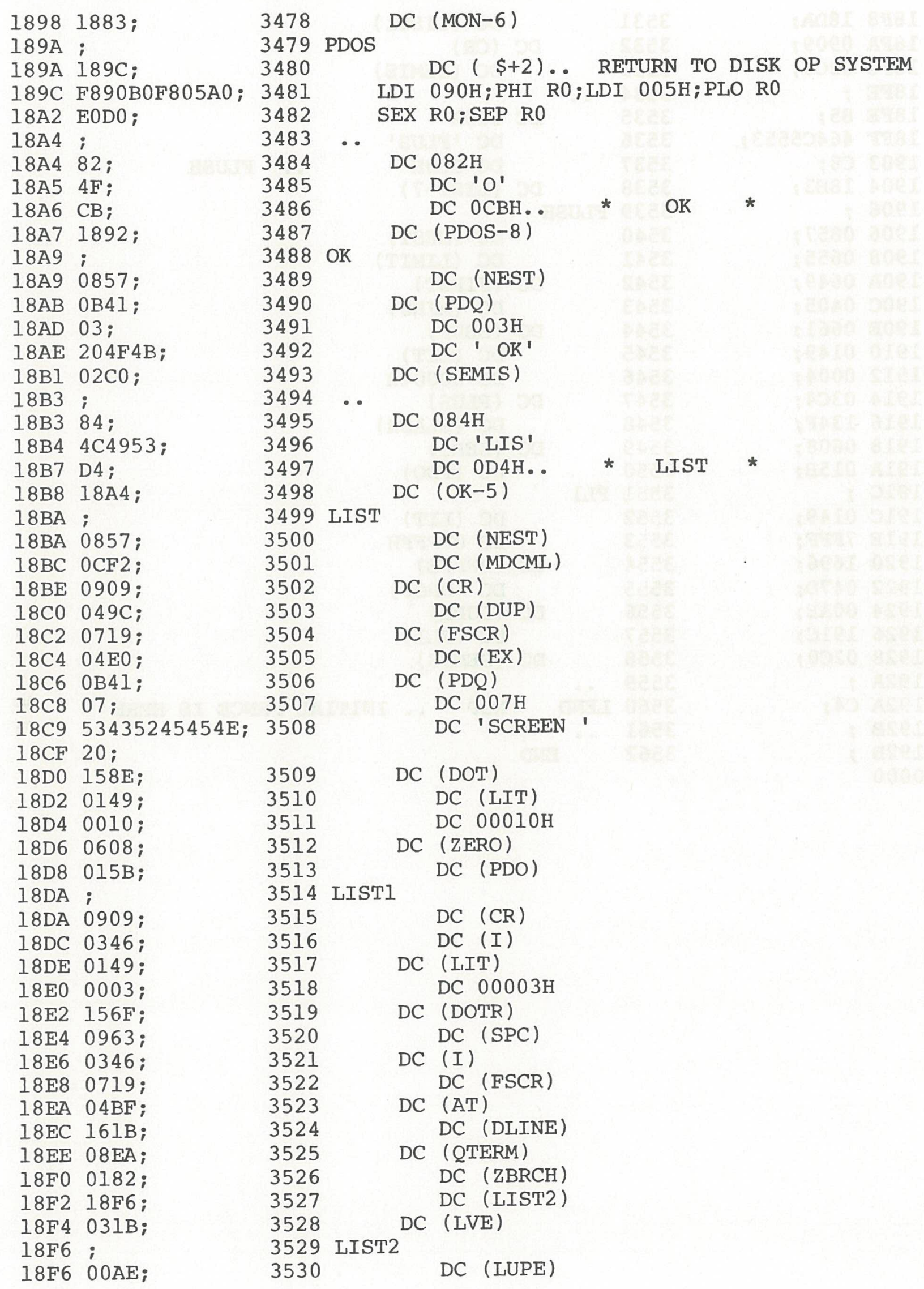

Woods Hole FORTH Version 1.800 Chapter 21 page 209 
File ID: WFORTH.LST

Disk ID: WOODS HOLE FORTH SOURCE \& LIST VER 1.800 113084

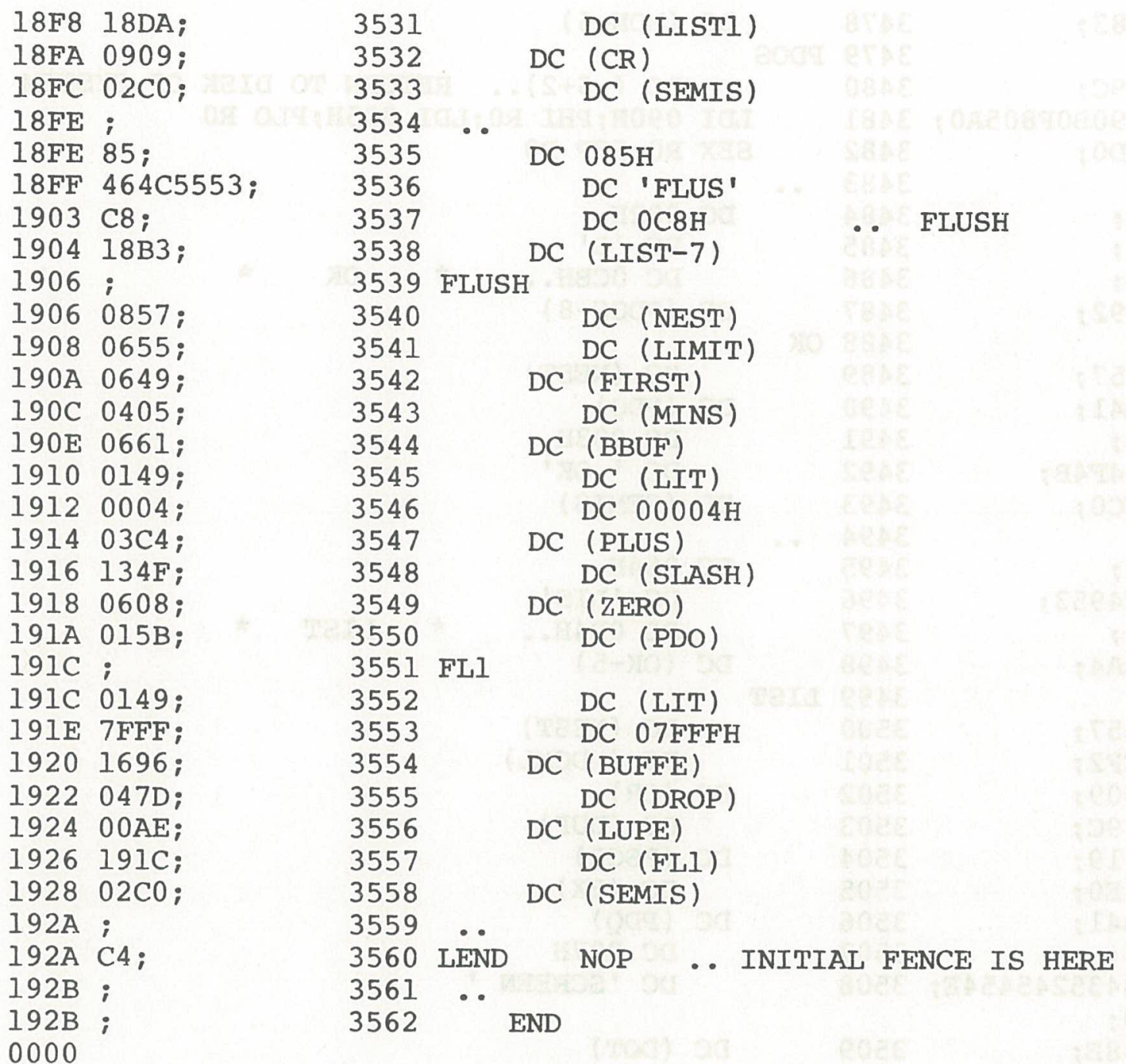


Cross Reference Listing for the Source

The cross reference listing generated by ASM8 is given below. This listing was generated from within FORTH by the word .FILE. The sources for this word are located on screens 232 and 233. 
File ID: XREF .

Disk ID: WOODS HOLE FORTH SOURCE \& LIST VER 1.800

113084

C ROS S RE FERENCE I I S T I N G

\begin{tabular}{|c|c|c|c|c|c|c|c|c|c|c|}
\hline SYMBOL & ADDR & $\mathrm{DEF}$ & REFEF & INCES & & & & & & \\
\hline ABORT' & $11 \mathrm{E} 6$ & 2497 & 2102 & 2530 & 3420 & & & & & \\
\hline ABS & $052 A$ & 0708 & 0717 & 2657 & & & & & & \\
\hline AGAIN & 1434 & 2823 & 2835 & 2840 & & & & & & \\
\hline ALLOT & $0 A 6 C$ & 1458 & 1468 & 2210 & 2556 & & & & & \\
\hline ALIOTI & $0 A 78$ & 1463 & 1477 & & & & & & & \\
\hline APOSI & $05 \mathrm{~A} 4$ & 0765 & 0762 & & & & & & & \\
\hline ARROW & 1803 & 3369 & 3386 & & & & & & & \\
\hline $\mathrm{AT}$ & $04 \mathrm{BF}$ & 0656 & 0663 & 1624 & 1625 & 1673 & 1687 & 1711 & 1725 & 1743 \\
\hline & & & 1842 & 1896 & 1910 & 1920 & 1946 & 1950 & 1956 & 1959 \\
\hline & & & 1993 & 1999 & 2004 & 2008 & 2082 & 2083 & 2112 & 2127 \\
\hline & & & 2129 & 2207 & 2224 & 2238 & 2334 & 2350 & 2366 & 2392 \\
\hline & & & 2411 & 2439 & 2444 & 2462 & 2483 & 2547 & 2622 & 2624 \\
\hline & & & 2632 & 2642 & 2644 & 2929 & 2956 & 3039 & 3060 & 3130 \\
\hline & & & 3141 & 3142 & 3147 & 3171 & 3181 & 3188 & 3211 & 3215 \\
\hline & & & 3217 & 3240 & 3263 & 3287 & 3344 & 3347 & 3377 & 3459 \\
\hline & & & 3523 & & & & & & & \\
\hline BACK & 1391 & 2721 & 2734 & 2783 & 2796 & 2809 & 2829 & & & \\
\hline BADCHR & 0128 & 0205 & 0195 & 0205 & & & & & & \\
\hline BADLEN & 0127 & 0204 & 0193 & & & & & & & \\
\hline BASE & 0759 & 1044 & $\begin{array}{l}1051 \\
3062\end{array}$ & 1784 & 1796 & 1992 & 1998 & 2003 & 2955 & 3060 \\
\hline BBUF & 0661 & 0878 & 0885 & 3086 & 3115 & 3544 & & & & \\
\hline BCOMP & 1286 & 2571 & 2585 & & & & & & & \\
\hline BDIGS & $14 \mathrm{CB}$ & 2915 & 2923 & 2997 & & & & & & \\
\hline BEGIN & $139 \mathrm{~F}$ & 2735 & 2745 & & & & & & & \\
\hline $\mathrm{BL}$ & 0629 & 0838 & 0845 & 1869 & 2049 & 2078 & & & & \\
\hline BLK & $06 \mathrm{FC}$ & 0982 & 0987 & 1724 & 1909 & 1914 & 1919 & 1945 & 1949 & 2128 \\
\hline & & & 2474 & 3343 & 3354 & 3361 & 3376 & 3381 & & \\
\hline BLKEX & $09 \mathrm{~F} 4$ & 1405 & 1391 & & & & & & & \\
\hline BLKRD & 09B2 & 1383 & 1396 & 3281 & & & & & & \\
\hline BLKWT & $09 \mathrm{DB}$ & 1397 & 1415 & 3285 & & & & & & \\
\hline BLNK & 0898 & 1248 & 1266 & 1966 & & & & & & \\
\hline BLOCl & $172 \mathrm{E}$ & 3251 & 3223 & & & & & & & \\
\hline BLOC2 & $16 \mathrm{FC}$ & 3224 & 3247 & & & & & & & \\
\hline BLOC3 & 1716 & 3238 & 3228 & & & & & & & \\
\hline BLOCK & $16 \mathrm{DE}$ & 3208 & 1951 & 2523 & 3092 & 3258 & & & & \\
\hline BOK & $013 B$ & 0211 & 0206 & 0208 & & & & & & \\
\hline BRANCH & 0174 & 0251 & 0262 & & & & & & & \\
\hline $\mathrm{BRCH}$ & 0172 & 0249 & 0258 & 1661 & 1858 & 1871 & 1931 & 1952 & 2017 & 2061 \\
\hline & & & $\begin{array}{l}2398 \\
3282\end{array}$ & 2405 & 2416 & 2424 & 2489 & 2557 & 2828 & 2869 \\
\hline BSCR & $066 \mathrm{D}$ & 0886 & 0893 & 3089 & 3352 & 3375 & 3457 & & & \\
\hline BUFFl & $16 \mathrm{~A} 0$ & 3174 & 3177 & & & & & & & \\
\hline BUFF2 & $16 \mathrm{C} 6$ & 3194 & 3184 & & & & & & & \\
\hline BUFFE & 1696 & 3168 & 3207 & 3231 & 3554 & & & & & \\
\hline CALIR & 0977 & 1354 & 1350 & & & & & & & \\
\hline CALLX & 0976 & 1353 & 1355 & & & & & & & \\
\hline CAT & 04DI & 0664 & 0671 & 1609 & 1991 & 2034 & 2048 & 2054 & 2205 & 2554 \\
\hline CCMA & OAA4 & 1481 & 1490 & & & & & & & \\
\hline
\end{tabular}


File ID: XREF .

Disk ID: WOODS HOLE FORTH SOURCE \& LIST VER 1.800 113084

\begin{tabular}{|c|c|c|c|c|c|c|c|c|c|c|}
\hline CDER & $17 \mathrm{~A} 4$ & 3312 & 3289 & 3326 & & & & & & \\
\hline CDERR & 1793 & 3303 & 3311 & 3314 & & & & & & \\
\hline CDEX & 17B2 & 3320 & 3316 & & & & & & & \\
\hline CEND & $00 \mathrm{Cl}$ & 0158 & 0156 & & & & & & & \\
\hline CEX & $04 \mathrm{FO}$ & 0680 & 0686 & 1877 & 1974 & 3266 & & & & \\
\hline CFA & OAE5 & 1516 & 1520 & 1809 & 2261 & 2396 & 2401 & 2440 & 2578 & \\
\hline CGRP & 0800 & 1154 & 1161 & & & & & & & \\
\hline $\mathrm{CL}$ & 0633 & 0846 & 0853 & & & & & & & \\
\hline CLD & 0005 & 0071 & 0069 & & & & & & & \\
\hline CMOVE & $02 \mathrm{FC}$ & 0437 & 0451 & 1979 & 2168 & & & & & \\
\hline CMPL & $0 C 91$ & 1736 & 1748 & $\begin{array}{l}1820 \\
2807\end{array}$ & $\begin{array}{l}2247 \\
2827\end{array}$ & $\begin{array}{l}2337 \\
2852\end{array}$ & $\begin{array}{l}2534 \\
2868\end{array}$ & 2550 & 2769 & 2781 \\
\hline CNST & $104 \mathrm{~B}$ & 2270 & 2280 & 2306 & & & & & & \\
\hline $\mathrm{CNT}$ & $0 \mathrm{~B} 03$ & 1531 & 1542 & 2119 & 2170 & 2562 & & & & \\
\hline CNTX & 0734 & 1020 & 1027 & 2081 & 2239 & 2451 & 2461 & 2623 & 2643 & \\
\hline CODE & ODIE & 1817 & 1829 & & & & & & & \\
\hline COLD & 0016 & 0085 & 0077 & 0084 & 0095 & 3418 & & & & \\
\hline COI,ON & 1006 & 2233 & 2254 & & & & & & & \\
\hline COMMA & $0 \mathrm{~A} 84$ & 1469 & $\begin{array}{l}1480 \\
2441\end{array}$ & $\begin{array}{l}1744 \\
2445\end{array}$ & $\begin{array}{l}2222 \\
2579\end{array}$ & $\begin{array}{l}2228 \\
2723\end{array}$ & $\begin{array}{l}2263 \\
2856\end{array}$ & $\begin{array}{l}2339 \\
2872\end{array}$ & 2397 & 2437 \\
\hline COMMAI & $0 A 96$ & 1476 & 1487 & & & & & & & \\
\hline COMP & 00B2 & 0153 & 0173 & 0175 & & & & & & \\
\hline CONST & $05 \mathrm{FB}$ & 0800 & $\begin{array}{l}0110 \\
0863\end{array}$ & $\begin{array}{l}0812 \\
0871\end{array}$ & $\begin{array}{l}0818 \\
0879\end{array}$ & $\begin{array}{l}0824 \\
0887\end{array}$ & $\begin{array}{l}0831 \\
0895\end{array}$ & $\begin{array}{l}0839 \\
2273\end{array}$ & 0847 & 0855 \\
\hline $\mathrm{CR}$ & 0909 & 1308 & 1322 & 2479 & 2503 & 2507 & 2511 & 3502 & 3515 & 3532 \\
\hline CRNT & 0742 & 1028 & 1035 & 1623 & 2223 & 2237 & 2438 & 2463 & 2621 & \\
\hline CRTI & OFCC & 2202 & 2194 & & & & & & & \\
\hline CRTE & 0FA6 & 2182 & 2232 & 2241 & 2257 & & & & & \\
\hline CSENDI & 099C & 1373 & 1311 & 1313 & 1333 & 1373 & & & & \\
\hline CSP & 0777 & 1068 & 1073 & 1710 & & & & & & \\
\hline CSTACK & $55 \mathrm{FE}$ & & 0044 & 3416 & 3430 & & & & & \\
\hline DABS & 0539 & 0718 & 0725 & 2655 & 2996 & & & & & \\
\hline DCODE & $102 \mathrm{E}$ & 2255 & 2269 & 2274 & 2285 & 2296 & & & & \\
\hline DCSP & OAEE & 1521 & 1530 & 2236 & & & & & & \\
\hline DDOT & 1580 & 3020 & 3028 & 3032 & 3048 & & & & & \\
\hline DDOTR & $154 \mathrm{C}$ & 2991 & 3009 & 3015 & 3023 & & & & & \\
\hline DELIM & $01 \mathrm{E} 3$ & 0306 & 0295 & & & & & & & \\
\hline $\mathrm{DFN}$ & $11 \mathrm{~A} 7$ & 2459 & 2470 & 2520 & & & & & & \\
\hline DGT & $02 \mathrm{D} 3$ & 0422 & 0436 & 1994 & & & & & & \\
\hline $\mathrm{DIG}$ & 1509 & 2953 & 2976 & 2980 & & & & & & \\
\hline $\mathrm{DIGl}$ & 1525 & 2968 & 2964 & & & & & & & \\
\hline DIGS & 1534 & 2977 & 2990 & 2998 & & & & & & \\
\hline DIGSI & 1536 & 2979 & 2986 & & & & & & & \\
\hline DLINE & $161 \mathrm{~B}$ & 3102 & 3112 & 3524 & & & & & & \\
\hline DLTL & 1007 & 2347 & 2362 & 2415 & & & & & & \\
\hline DLTLI & $10 \mathrm{E} 7$ & 2356 & 2352 & & & & & & & \\
\hline DMIN & $044 \mathrm{D}$ & 0598 & 0608 & 0721 & 1594 & 2068 & & & & \\
\hline DO & 13D3 & 2767 & 2776 & & & & & & & \\
\hline DOESG & 1095 & 2313 & 2330 & 2448 & & & & & & \\
\hline DOK & $02 \mathrm{E} 7$ & 0428 & 0426 & & & & & & & \\
\hline DOT & $158 \mathrm{E}$ & 3029 & 3036 & 3040 & 3061 & 3509 & & & & \\
\hline DOTQ & 1250 & 2542 & 2570 & & & & & & & \\
\hline DOTQ1 & 1270 & 2559 & 2549 & & & & & & & \\
\hline DOTQ22 & 1278 & 2564 & 2558 & & & & & & & \\
\hline
\end{tabular}


File ID: XREF .

Disk ID: WOODS HOLE FORTH SOURCE \& LIST VER 1.800 113084

\begin{tabular}{|c|c|c|c|c|c|c|c|c|c|c|}
\hline DOTR & $156 \mathrm{~F}$ & 3010 & 3019 & 3519 & & & & & & \\
\hline $\mathrm{DP}$ & $06 \mathrm{E} 3$ & 0966 & 0973 & 2245 & 2639 & & & & & \\
\hline DPL & 0763 & 1052 & 1059 & 2007 & 2013 & 2044 & 2410 & & & \\
\hline DPISI & $03 \mathrm{CB}$ & 0537 & 0581 & & & & & & & \\
\hline DPLUS & 0416 & 0576 & 0586 & 2006 & & & & & & \\
\hline DPM & OB88 & 1591 & 1599 & & & & & & & \\
\hline DREAD & $87 \mathrm{~F} 9$ & & 0036 & 1388 & 1390 & & & & & \\
\hline DRONE & 1873 & 3455 & 2521 & 3469 & & & & & & \\
\hline \multirow[t]{4}{*}{ DROP } & $047 D$ & 0620 & 0627 & 1614 & 1663 & 1846 & 1868 & 1886 & 1929 & 1935 \\
\hline & & & 2001 & 2064 & 2089 & 2146 & 2195 & 2419 & 2524 & 2577 \\
\hline & & & 2611 & 2685 & 2693 & 2714 & 2926 & 2927 & 3125 & 3229 \\
\hline & & & 3253 & 3555 & & & & & & \\
\hline DRZER & 1825 & 3387 & 3399 & & & & & & & \\
\hline \multirow[t]{4}{*}{ DUP } & $049 \mathrm{C}$ & 0638 & 0646 & 1740 & 1838 & 1852 & 1861 & 1874 & 1989 & 2032 \\
\hline & & & 2038 & 2047 & 2053 & 2085 & 2161 & 2204 & 2211 & 2367 \\
\hline & & & 2514 & 2630 & 2637 & 3120 & 3128 & 3172 & 3216 & 3220 \\
\hline & & & 3232 & 3239 & 3243 & 3248 & 3503 & & & \\
\hline DUZ1 & $10 \mathrm{Al}$ & 2321 & 3400 & & & & & & & \\
\hline DV & $07 \mathrm{AF}$ & 1106 & 1113 & 3265 & 3269 & 3273 & 3277 & & & \\
\hline DWRITE & $87 \mathrm{FC}$ & & 0037 & 1402 & 1404 & & & & & \\
\hline EDIGS & $14 \mathrm{DA}$ & 2924 & 2938 & 3000 & & & & & & \\
\hline EFTBL & $005 E$ & 0112 & 0111 & 0149 & & & & & & \\
\hline ELSEE & $147 \mathrm{~B}$ & 2864 & 2882 & & & & & & & \\
\hline EMIT & $08 \mathrm{FC}$ & 1302 & 1307 & 1343 & 1883 & & & & & \\
\hline EMMIT & $098 \mathrm{C}$ & 1365 & 1304 & & & & & & & \\
\hline ENCL & 0196 & 0280 & 0315 & 1962 & & & & & & \\
\hline END2 & 0312 & 0446 & 0443 & & & & & & & \\
\hline ENDD & 1426 & 2814 & 2822 & & & & & & & \\
\hline ENDIFF & $13 \mathrm{BI}$ & 2746 & 2759 & 2762 & 2845 & 2875 & & & & \\
\hline EQL & 0373 & 0501 & 0510 & 1843 & 1851 & 1864 & 2037 & 3122 & & \\
\hline ERRI & $0 F 36$ & 2117 & 2115 & & & & & & & \\
\hline ERROR & $0 F 28$ & 2109 & 1660 & 2136 & & & & & & \\
\hline ERS & 0886 & 1239 & 1247 & 1250 & 3161 & & & & & \\
\hline ERS01 & $086 \mathrm{~A}$ & 1226 & 1242 & & & & & & & \\
\hline \multirow[t]{5}{*}{$\mathrm{EX}$} & $04 \mathrm{E} 0$ & 0672 & 0679 & 1753 & 1763 & 1785 & 1797 & 1810 & 1881 & 1902 \\
\hline & & & 1918 & 2045 & 2225 & 2240 & 2262 & 2318 & 2447 & 2452 \\
\hline & & & 2464 & 2475 & 2516 & 2518 & 2640 & 2645 & 2753 & 2919 \\
\hline & & & 3062 & 3148 & 3179 & 3196 & 3199 & 3250 & 3271 & 3276 \\
\hline & & & 3351 & 3355 & 3359 & 3362 & 3374 & 3391 & 3462 & 3505 \\
\hline EXC & $0 \mathrm{C} 30$ & 1684 & 1696 & 1927 & 2235 & & & & & \\
\hline EXE & $01 F 7$ & 0316 & 0325 & 2402 & & & & & & \\
\hline EXPT & 0D35 & 1830 & 1892 & 1899 & & & & & & \\
\hline EXPTI & OD6D & 1860 & 1845 & & & & & & & \\
\hline EXPT2 & 0D93 & 1882 & 1859 & & & & & & & \\
\hline EXPT3 & 0D85 & 1873 & 1866 & & & & & & & \\
\hline EXPT4 & OD3F & 1836 & 1885 & & & & & & & \\
\hline EXPT5 & 0D87 & 1875 & 1872 & & & & & & & \\
\hline FAND & 0251 & 0356 & 0363 & 1923 & 2173 & 3191 & & & & \\
\hline FFOR & 0262 & 0364 & 0373 & 2983 & 3145 & & & & & \\
\hline FILL & 0868 & 1224 & 1238 & 2160 & & & & & & \\
\hline FILLEX & 087D & 1233 & 1231 & & & & & & & \\
\hline FILLOP & 0871 & 1230 & 1232 & & & & & & & \\
\hline \multirow[t]{2}{*}{ FIN } & 0705 & 0988 & 0995 & 1901 & 1917 & 1958 & 1967 & 2126 & 3346 & 3350 \\
\hline & & & 3358 & 3373 & & & & & & \\
\hline
\end{tabular}


File ID: XREF .

Disk ID: WOODS HOLE FORTH SOURCE \& LIST VER 1.800

$1130 \quad 84$

\begin{tabular}{|c|c|c|c|c|c|c|c|c|c|c|}
\hline FIND & $00 F 5$ & 0188 & 0222 & 2084 & 2092 & & & & & \\
\hline FIRST & 0649 & 0862 & 0869 & 2513 & 3126 & 3157 & 3542 & & & \\
\hline FIRSTB & 57D7 & & 0040 & 0864 & & & & & & \\
\hline FLI & $191 \mathrm{C}$ & 3551 & 3557 & & & & & & & \\
\hline FLD & $076 \mathrm{D}$ & 1060 & 1067 & & & & & & & \\
\hline FLGLES & $03 A 0$ & 0517 & 0529 & & & & & & & \\
\hline FLUSH & 1906 & 3539 & 3403 & 3427 & & & & & & \\
\hline FNCE & $06 \mathrm{DA}$ & 0960 & 0965 & 2631 & & & & & & \\
\hline FORG & $12 \mathrm{DB}$ & 2619 & 2649 & & & & & & & \\
\hline FOUT & $070 \mathrm{~F}$ & 0996 & 1003 & & & & & & & \\
\hline FRST & $01 \mathrm{AF}$ & 0290 & 0288 & & & & & & & \\
\hline FRTH & $182 \mathrm{~F}$ & 3395 & 0085 & 2519 & 3443 & 3454 & & & & \\
\hline FSCR & 0719 & 1004 & 1011 & 3504 & 3522 & & & & & \\
\hline FSPAT & 0286 & 0384 & 0392 & 1709 & 2184 & 2368 & 2375 & & & \\
\hline FXOR & 0274 & 0374 & 0383 & 2661 & & & & & & \\
\hline GETNXT & 0090 & 0131 & 0134 & & & & & & & \\
\hline \multirow[t]{3}{*}{ GR } & 0328 & 0459 & 0466 & 1742 & 1856 & 1971 & 1990 & 2039 & 2653 & 2654 \\
\hline & & & 2673 & 2702 & 2838 & 2839 & 2993 & 3012 & 3083 & 3173 \\
\hline & & & 3213 & 3345 & 3348 & & & & & \\
\hline $\begin{array}{l}\text { GROUP } \\
\text { GRP }\end{array}$ & $17 \mathrm{BC}$ & 3327 & 2502 & 3288 & 3340 & & & & & \\
\hline $\begin{array}{l}\text { GRP } \\
\text { GTRR }\end{array}$ & 07D2 & 1130 & 1137 & 3286 & & & & & & \\
\hline $\begin{array}{l}\text { GTR } \\
\text { HERE }\end{array}$ & 03AE & 0524 & 0532. & 2141 & 2376 & & & & & \\
\hline \multirow[t]{2}{*}{ HERE } & $0 A 53$ & 1447 & 1457 & 1963 & 1973 & 1976 & 2080 & 2090 & 2118 & 2185 \\
\hline & & & 2203 & 2215 & 2226 & 2408 & 2442 & 2553 & 2561 & 2738 \\
\hline HLD & & & 2751 & 2771 & 2854 & 2870 & & & & \\
\hline HOLD & $\begin{array}{l}078 \mathrm{~A} \\
08 \mathrm{BI}\end{array}$ & 1082 & 1089 & 2918 & 2928 & & & & & \\
\hline \multirow[t]{3}{*}{ I } & $\begin{array}{l}08 B 1 \\
0346\end{array}$ & $\begin{array}{l}1267 \\
0477\end{array}$ & $\begin{array}{l}1280 \\
0483\end{array}$ & $\begin{array}{l}2947 \\
1850\end{array}$ & $\begin{array}{l}2972 \\
1876\end{array}$ & 1879 & 1972 & & & \\
\hline & 0346 & 0477 & $\begin{array}{l}0483 \\
3185\end{array}$ & $\begin{array}{l}1850 \\
3187\end{array}$ & 3195 & 3197 & 3218 & 3230 & 3233 & $\begin{array}{l}3180 \\
3241\end{array}$ \\
\hline & & & 3516 & 3521 & & & & & & \\
\hline ID & $0 \mathrm{~F} 6 \mathrm{~F}$ & 2153 & 2181 & 2197 & & & & & & \\
\hline IFF & 1464 & 2850 & 2863 & 2885 & & & & & & \\
\hline IMMED & $12 \mathrm{~A} 4$ & 2586 & 2595 & & & & & & & \\
\hline INPT & 1120 & 2386 & 2425 & 2431 & 2481 & 3356 & & & & \\
\hline INPUT & $083 \mathrm{D}$ & 1200 & 1223 & & & & & & & \\
\hline \multirow[t]{3}{*}{ IP } & 0008 & & 0057 & 0102 & 0131 & 0157 & 0157 & 0158 & 0158 & 0225 \\
\hline & & & 0251 & 0251 & 0263 & 0263 & 0414 & 1216 & 1217 & 1561 \\
\hline & & & 1562 & 1564 & 1564 & 1565 & 1565 & 2322 & 2323 & \\
\hline ITIB & 5600 & & 0043 & 3432 & & & & & & \\
\hline KEY & $08 \mathrm{CF}$ & 1281 & 1291 & 1837 & & & & & & \\
\hline KEYI & 08D2 & 1284 & 1284 & & & & & & & \\
\hline LB & $0 \mathrm{CA} 7$ & 1749 & 1757 & 1822 & 2476 & 2537 & & & & \\
\hline LBLD & 1085 & 2303 & 2312 & 2434 & & & & & & \\
\hline LCP5 & 0215 & 0332 & 0339 & 0340 & & & & & & \\
\hline LEND & $192 \mathrm{~A}$ & 3560 & 3441 & 3442 & & & & & & \\
\hline LESS & 0390 & 0511 & 0523 & 1610 & 2189 & 2369 & 2393 & 2633 & 2962 & \\
\hline LFA & OAD9 & 1508 & 1515 & 2163 & 2641 & & & & & \\
\hline IIMIT & 0655 & 0870 & 0877 & 3121 & 3158 & 3541 & & & & \\
\hline LIMITB & $7 \mathrm{FFF}$ & & 0039 & 0872 & & & & & & \\
\hline LIST & $18 \mathrm{BA}$ & 3499 & 3538 & & & & & & & \\
\hline IISTI & $18 \mathrm{DA}$ & 3514 & 3531 & & & & & & & \\
\hline LIST2 & $18 \mathrm{~F} 6$ & 3529 & 3527 & & & & & & & \\
\hline \multirow[t]{2}{*}{ LIT } & 0149 & 0223 & $\begin{array}{l}0235 \\
1713\end{array}$ & $\begin{array}{l}1606 \\
1727\end{array}$ & $\begin{array}{l}1632 \\
1760\end{array}$ & $\begin{array}{l}1635 \\
1773\end{array}$ & $\begin{array}{l}1646 \\
1782\end{array}$ & $\begin{array}{l}1675 \\
1794\end{array}$ & $\begin{array}{l}1688 \\
1839\end{array}$ & $\begin{array}{l}1700 \\
1847\end{array}$ \\
\hline & & & & & & & & & & 1847 \\
\hline
\end{tabular}


File ID: XREF .

Disk ID: WOODS HOLE FORTH SOURCE \& LIST VER 1.800

$1130 \quad 84$

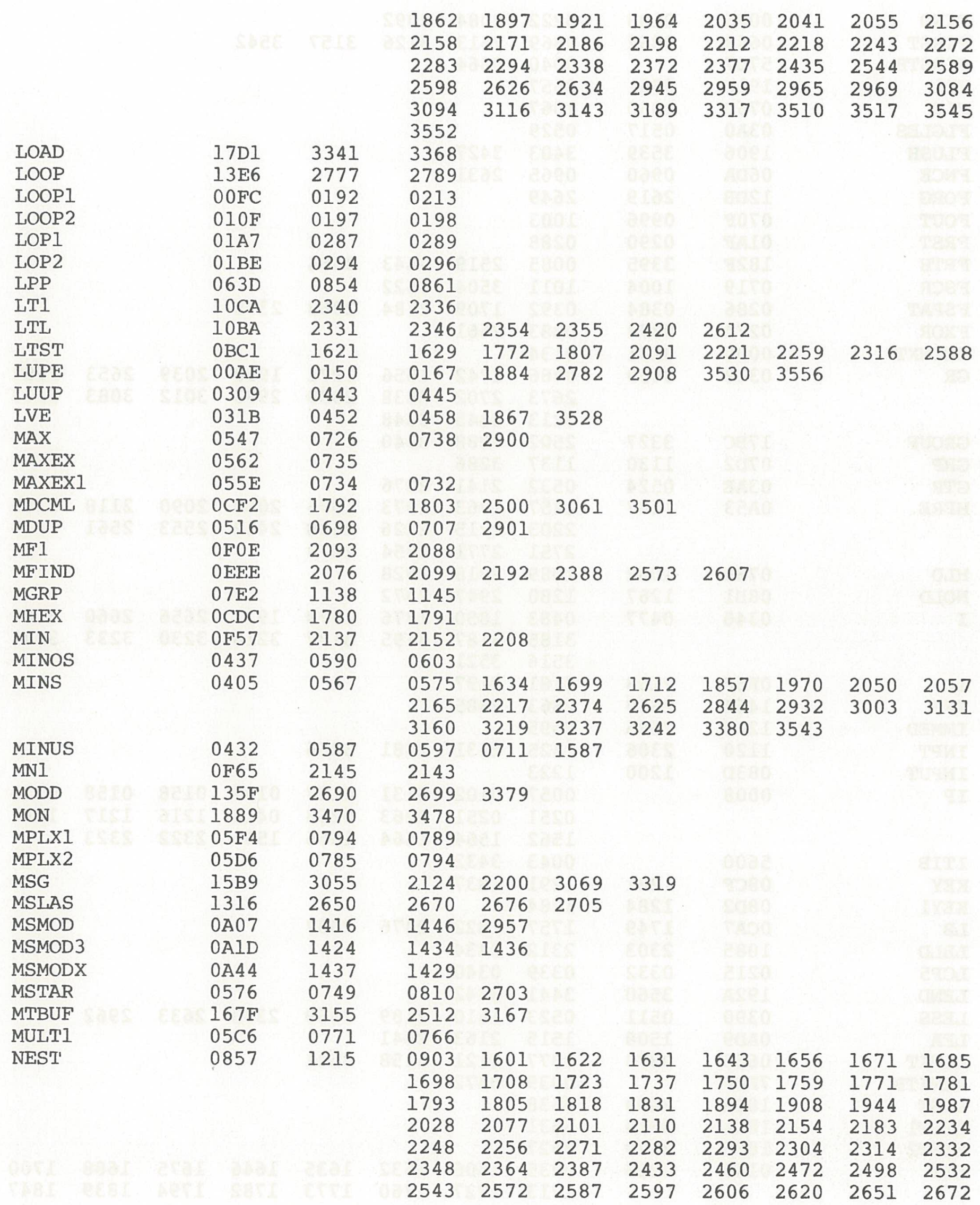


File ID: XREF .

Disk ID: WOODS HOLE FORTH SOURCE \& LIST VER 1.800

$1130 \quad 84$

\begin{tabular}{|c|c|c|c|c|c|c|c|c|c|c|}
\hline & & & $\begin{array}{l}2682 \\
2768 \\
2865 \\
2992 \\
3103 \\
3342\end{array}$ & $\begin{array}{l}2691 \\
2778 \\
2884 \\
3011 \\
3114 \\
3370\end{array}$ & $\begin{array}{l}2701 \\
2791 \\
2898 \\
3021 \\
3139 \\
3388\end{array}$ & $\begin{array}{l}2711 \\
2804 \\
2916 \\
3030 \\
3156 \\
3456\end{array}$ & $\begin{array}{l}2722 \\
2815 \\
2925 \\
3038 \\
3169 \\
3489\end{array}$ & $\begin{array}{l}2736 \\
2824 \\
2940 \\
3046 \\
3209 \\
3500\end{array}$ & $\begin{array}{l}2747 \\
2837 \\
2954 \\
3056 \\
3260 \\
3540\end{array}$ & $\begin{array}{l}2761 \\
2851 \\
2978 \\
3082 \\
3313\end{array}$ \\
\hline NEXCHR & 0106 & 0194 & 0196 & & & & & & & \\
\hline NEXT & 0098 & 0134 & 1358 & 3417 & & & & & & \\
\hline NEXTU & $080 \mathrm{C}$ & 1162 & 1179 & & & & & & & \\
\hline NFA & OBDI & 1630 & 1641 & 2196 & 2638 & & & & & \\
\hline NMBI. & OEB4 & 2043 & 2062 & & & & & & & \\
\hline NMB2 & OEDA & 2063 & 2052 & & & & & & & \\
\hline NMB3 & OEE4 & 2069 & 2067 & & & & & & & \\
\hline NMBR & 0 E96 & 2027 & 2075 & 2409 & & & & & & \\
\hline NNONE & 0382 & 0506 & & & & & & & & \\
\hline NOF & $006 \mathrm{~F}$ & 0117 & 0112 & 0113 & 0114 & 0115 & & & & \\
\hline NONE & 0358 & 0488 & 0425 & 0427 & 0431 & 0497 & & & & \\
\hline NULL & $01 \mathrm{C} 8$ & 0297 & 0294 & & & & & & & \\
\hline OFST & 0726 & 1012 & 1019 & 3210 & 3390 & 3461 & & & & \\
\hline $\mathrm{OK}$ & 18 A9 & 3488 & 2487 & 2525 & 3498 & & & & & \\
\hline OKGO & 0853 & 1208 & 1206 & & & & & & & \\
\hline ONE & 0610 & 0817 & $\begin{array}{l}0822 \\
2739\end{array}$ & $\begin{array}{l}1644 \\
2805\end{array}$ & $\begin{array}{l}1913 \\
2825\end{array}$ & $\begin{array}{l}2012 \\
3234\end{array}$ & 2216 & 2370 & 2501 & 2522 \\
\hline ONEMIN & $O A B C$ & 1491 & 1499 & & & & & & & \\
\hline ONMNSI & OACO & 1494 & 1502 & 1510 & & & & & & \\
\hline ORGN & $067 \mathrm{~A}$ & 0894 & 0901 & 0904 & & & & & & \\
\hline OUTLL & $07 C 8$ & 1122 & 1129 & & & & & & & \\
\hline OUTPUT & 0819 & 1180 & 1199 & & & & & & & \\
\hline OVER & 0467 & 0609 & $\begin{array}{l}0619 \\
2140\end{array}$ & $\begin{array}{l}1604 \\
2164\end{array}$ & $\begin{array}{l}1608 \\
2652\end{array}$ & $\begin{array}{l}1832 \\
2931\end{array}$ & $\begin{array}{l}1834 \\
2961\end{array}$ & $\begin{array}{l}1849 \\
2981\end{array}$ & $\begin{array}{l}1969 \\
2982\end{array}$ & $\begin{array}{l}2139 \\
2995\end{array}$ \\
\hline PABRT & $0 F \perp A$ & 2100 & $\begin{array}{l}3002 \\
2108\end{array}$ & $\begin{array}{l}3159 \\
2116\end{array}$ & 3378 & & & & & \\
\hline PAD & OB5D & 1572 & 1583 & 2155 & 2166 & 2169 & 2917 & 2930 & & \\
\hline PAREN & $12 \mathrm{~B} 4$ & 2596 & 2604 & & & & & & & \\
\hline PARMS & 1841 & 3414 & 0086 & 0099 & 0896 & & & & & \\
\hline PBUF & $162 \mathrm{C}$ & 3113 & 3137 & 3175 & 3225 & & & & & \\
\hline PBUFI & 1646 & 3127 & 3124 & & & & & & & \\
\hline PCODE & 0D0 8 & 1804 & 1816 & 1821 & 2319 & & & & & \\
\hline PDO & $015 B$ & 0236 & 0248 & 1835 & 2770 & 2905 & 3513 & 3550 & & \\
\hline PDOS & $189 A$ & 3479 & 3487 & & & & & & & \\
\hline PDQ & OB41 & 1559 & 1571 & 2121 & 2504 & 2508 & 2551 & 3057 & 3490 & 3506 \\
\hline $\mathrm{PF} \overline{\mathrm{A}}$ & OBE7 & 1642 & 1654 & 1808 & 2162 & 2260 & 2317 & & & \\
\hline PLINE & $15 \mathrm{~F} 5$ & 3081 & 3101 & 3104 & & & & & & \\
\hline PLOOP & $13 \mathrm{FC}$ & 2790 & 2802 & & & & & & & \\
\hline PISIX & 03D9 & 0546 & 0554 & & & & & & & \\
\hline PLUPE & 00D3 & 0168 & 0187 & 2795 & & & & & & \\
\hline PLUS & $03 C 4$ & 0533 & $\begin{array}{l}0542 \\
2040\end{array}$ & $\begin{array}{l}0905 \\
2188\end{array}$ & $\begin{array}{l}1605 \\
2967\end{array}$ & $\begin{array}{l}1648 \\
2971\end{array}$ & $\begin{array}{l}1833 \\
3091\end{array}$ & $\begin{array}{l}1855 \\
3093\end{array}$ & $\begin{array}{l}1960 \\
3118\end{array}$ & $\begin{array}{l}1975 \\
3119\end{array}$ \\
\hline & & & 3212 & 3221 & 3244 & 3275 & 3547 & & & \\
\hline PLUSI & $03 \mathrm{D} 5$ & 0543 & $\begin{array}{l}0551 \\
2555\end{array}$ & $\begin{array}{l}1880 \\
3270\end{array}$ & 1977 & 1988 & 2009 & 2033 & 2209 & 2412 \\
\hline PLUS2 & $03 \mathrm{E} 7$ & 0552 & 0557 & 1741 & 2227 & 2450 & 2886 & 3186 & 3201 & 3254 \\
\hline PLUSS & $03 F 2$ & 0558 & 0566 & 1915 & 1968 & 2014 & 2246 & 3382 & & \\
\hline PM & OB79 & 1584 & 1590 & 2662 & 2665 & & & & & \\
\hline
\end{tabular}


File ID: XREF 。

Disk ID: WOODS HOLE FORTH SOURCE \& LIST VER 1.800

$1130 \quad 84$

\begin{tabular}{|c|c|c|c|c|c|c|c|c|c|c|}
\hline PNMI & 0E83 & 2015 & 2011 & & & & & & & \\
\hline PNM2 & 0E89 & 2019 & 1996 & & & & & & & \\
\hline PNMBR & $0 E 4 B$ & 1986 & 2018 & 2026 & 2046 & & & & & \\
\hline PORGN & 0688 & 0902 & 0921 & 1841 & & & & & & \\
\hline PREV & $079 F$ & 1098 & 1105 & 2515 & 3129 & 3140 & 3146 & 3198 & 3214 & 3249 \\
\hline PT1. & 1144 & 2407 & 2390 & & & & & & & \\
\hline PT2 & $113 \mathrm{~A}$ & 2400 & 2395 & & & & & & & \\
\hline PT3 & $113 \mathrm{E}$ & 2403 & 2399 & & & & & & & \\
\hline PT4 & $115 \mathrm{E}$ & 2423 & 2406 & & & & & & & \\
\hline PT5 & 1158 & 2418 & 2414 & & & & & & & \\
\hline PT6 & $115 \mathrm{C}$ & 2421 & 2417 & & & & & & & \\
\hline Q1 & 11D8 & 2488 & 2486 & & & & & & & \\
\hline Q2 & $11 \mathrm{C} 4$ & 2477 & 2490 & & & & & & & \\
\hline QCMP & $0 \mathrm{Cl} 8$ & 1670 & 1683 & 1738 & 2737 & 2748 & & & & \\
\hline QCSP & $0 \mathrm{C} 5 \mathrm{~A}$ & 1707 & 1721 & 1819 & 2533 & & & & & \\
\hline QERR & OBFE & 1655 & 1669 & 1677 & 1690 & 1702 & 1715 & 1729 & 2059 & 2191 \\
\hline QLDG & $0 \subset 77$ & 1722 & $\begin{array}{l}2371 \\
1735\end{array}$ & $\begin{array}{l}2379 \\
3371\end{array}$ & 2576 & 2610 & 2628 & 2636 & & \\
\hline QPR & $0 C 47$ & 1697 & 1706 & 2750 & 2780 & 2793 & 2806 & 2826 & 2867 & \\
\hline QSTK & $10 F 2$ & 2363 & 2385 & 2404 & 2422 & & & & & \\
\hline QTERM & $08 \mathrm{EA}$ & 1292 & 1301 & 3525 & & & & & & \\
\hline QUER & 0DA5 & 1893 & 1906 & 2480 & & & & & & \\
\hline QUES & $159 A$ & 3037 & 3044 & & & & & & & \\
\hline QUIT & $11 \mathrm{BA}$ & 2471 & 2130 & 2496 & 2526 & & & & & \\
\hline REPEA & $144 \mathrm{~B}$ & 2836 & 2849 & & & & & & & \\
\hline RETN & $009 A$ & 0137 & 0141 & & & & & & & \\
\hline RG & 0336 & 0467 & 0476 & 1739 & 1806 & 1853 & 1928 & 1934 & 1978 & 2016 \\
\hline & & & 2020 & 2065 & 2315 & 2659 & 2664 & 2675 & 2704 & 2841 \\
\hline & & & 2842 & 3001 & 3014 & 3088 & 3200 & 3252 & 3357 & 3360 \\
\hline RNU & 0780 & 1074 & 1081 & & & & & & & \\
\hline RO & $06 \mathrm{AA}$ & 0928 & 0935 & & & & & & & \\
\hline ROT & $04 \mathrm{FE}$ & 0687 & 0697 & 2002 & 2031 & 2941 & 2958 & & & \\
\hline $\mathrm{RP}$ & 0002 & & 0051 & 0101 & 0138 & 0138 & 0140 & 0152 & 0153 & 0153 \\
\hline & & & 0154 & 0157 & 0158 & 0158 & 0159 & 0172 & 0174 & 0174 \\
\hline & & & 0174 & 0192 & 0201 & 0238 & 0240 & 0284 & 0285 & 0293 \\
\hline & & & 0347 & 0407 & 0407 & 0414 & 0415 & 0415 & 0454 & 0454 \\
\hline & & & 0454 & 0461 & 0471 & 0471 & 0472 & 0741 & 0751 & 0765 \\
\hline & & & 0771 & 0795 & 1202 & 1202 & 1208 & 1209 & 1215 & 1229 \\
\hline & & & 1230 & 1259 & 1269 & 1304 & 1311 & 1325 & 1329 & 1330 \\
\hline & & & 1354 & 1355 & 1357 & 1358 & 1374 & 1386 & 1400 & 1405 \\
\hline & & & 1406 & 1449 & 1460 & 1471 & 1483 & 1523 & 1561 & 1563 \\
\hline & & & 1574 & 2321 & & & & & & \\
\hline RPI & $02 \mathrm{AB}$ & 0403 & 0411 & 2478 & & & & & & \\
\hline RSLW & $173 \mathrm{C}$ & 3259 & 3193 & 3235 & 3302 & & & & & \\
\hline RSTACK & $56 \mathrm{FE}$ & & 0042 & 3415 & 3431 & & & & & \\
\hline RTB & 0 CB5 & 1758 & 1769 & 2242 & & & & & & \\
\hline RWRITE & $176 \mathrm{C}$ & 3284 & 3280 & 3283 & & & & & & \\
\hline SCRDSK & $07 \mathrm{BD}$ & 1114 & 1121 & 3262 & 3458 & & & & & \\
\hline SCRT & $096 \mathrm{C}$ & 1349 & 1386 & 1400 & & & & & & \\
\hline SCRTX & 0982 & 1357 & 1407 & & & & & & & \\
\hline SEEK & $87 \mathrm{~F} 6$ & & 0035 & 1388 & 1402 & 3296 & & & & \\
\hline SEMIC & $123 \mathrm{D}$ & 2531 & 2541 & & & & & & & \\
\hline \multirow[t]{2}{*}{ SEMIS } & $02 \mathrm{CO}$ & 0412 & $\begin{array}{l}0421 \\
1691\end{array}$ & $\begin{array}{l}0906 \\
1703\end{array}$ & $\begin{array}{l}1615 \\
1716\end{array}$ & $\begin{array}{l}1626 \\
1730\end{array}$ & $\begin{array}{l}1638 \\
1745\end{array}$ & 1649 & 1664 & 1678 \\
\hline & & & & & $1 / 16$ & $1 / 30$ & & 1754 & 1764 & 1776 \\
\hline
\end{tabular}


File ID: XREF .

Disk ID: WOODS HOLE FORTH SOURCE \& LIST VER 1.800

$1130 \quad 84$

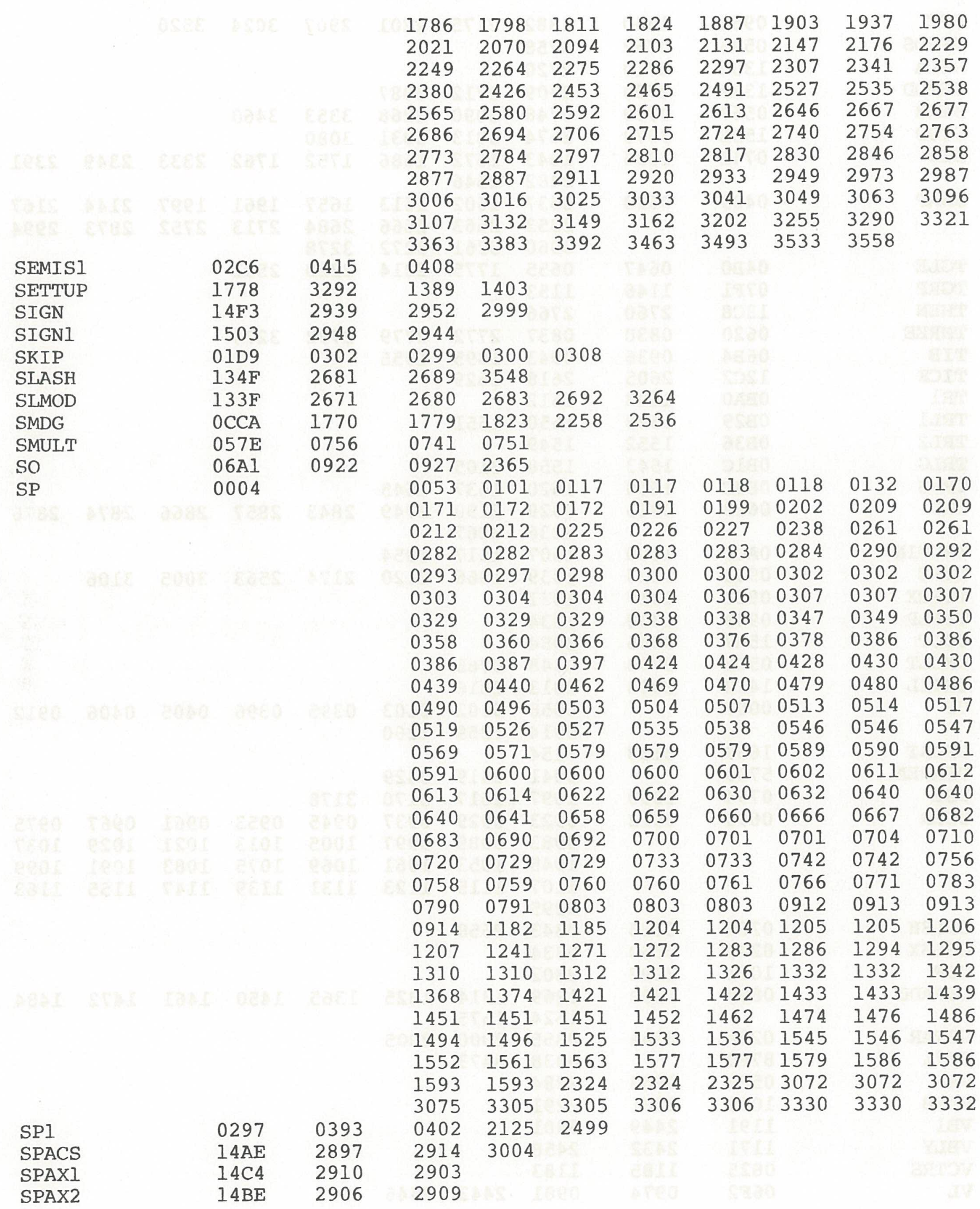


File ID: XREF .

Disk ID: WOODS HOLE FORTH SOURCE \& LIST VER 1.800

$11 \quad 30 \quad 84$

\begin{tabular}{|c|c|c|c|c|c|c|c|c|c|c|}
\hline SPC & 0963 & 1340 & 1382 & 2175 & 2201 & 2907 & 3024 & 3520 & & \\
\hline SPPOS & 0596 & 0762 & 0758 & & & & & & & \\
\hline SSLA & 1380 & 2710 & 2720 & & & & & & & \\
\hline SSMOD & $136 \mathrm{~F}$ & 2700 & 2709 & 2712 & 3087 & & & & & \\
\hline STAR & 0567 & 0739 & 0748 & 3090 & 3268 & 3353 & 3460 & & & \\
\hline STOD & $15 \mathrm{DE}$ & 3070 & 2674 & 3013 & 3031 & 3080 & & & & \\
\hline STT & $074 \mathrm{E}$ & 1036 & $\begin{array}{l}1043 \\
2482\end{array}$ & $\begin{array}{l}1672 \\
2546\end{array}$ & 1686 & 1752 & 1762 & 2333 & 2349 & 2391 \\
\hline SWAP & 0489 & 0628 & $\begin{array}{l}0637 \\
2353 \\
3060\end{array}$ & $\begin{array}{l}1602 \\
2663 \\
3261\end{array}$ & $\begin{array}{l}1613 \\
2666 \\
3272\end{array}$ & $\begin{array}{l}1657 \\
2684 \\
3278\end{array}$ & $\begin{array}{l}1961 \\
2713\end{array}$ & $\begin{array}{l}1997 \\
2752\end{array}$ & $\begin{array}{l}2144 \\
2873\end{array}$ & $\begin{array}{l}2167 \\
2994\end{array}$ \\
\hline TGLE & $04 \mathrm{BO}$ & 0647 & 0655 & 1775 & 2214 & 2220 & 2591 & & & \\
\hline TGRP & $07 \mathrm{Fl}$ & 1146 & 1153 & & & & & & & \\
\hline THEN & $13 \mathrm{C} 8$ & 2760 & 2766 & & & & & & & \\
\hline THREE & 0620 & 0830 & 0837 & 2772 & 2779 & 2792 & 3274 & & & \\
\hline TIB & 06B4 & 0936 & 0943 & 1895 & 1955 & & & & & \\
\hline TICK & $12 \mathrm{C} 2$ & 2605 & 2618 & 2629 & & & & & & \\
\hline TRI & OBAO & 1603 & 1612 & & & & , & & & \\
\hline TRLI & 0B29 & 1548 & 1550 & 1551 & & & & & & \\
\hline TRL2 & $0 B 36$ & 1552 & 1549 & & & & & & & \\
\hline TRLG & OBIC & 1543 & 1558 & 3105 & & & & & & \\
\hline TRVS & OB9C & 1600 & 1.620 & 1637 & 1645 & & & & & \\
\hline TWO & 0618 & 0823 & $\begin{array}{l}0829 \\
3236\end{array}$ & $\begin{array}{l}2190 \\
3267\end{array}$ & 2749 & 2843 & 2857 & 2866 & 2874 & 2876 \\
\hline TWOMIN & OACD & 1500 & 1507 & 1517 & 1854 & & & & & \\
\hline TYPE & 0933 & 1323 & 1339 & 1566 & 2120 & 2174 & 2563 & 3005 & 3106 & \\
\hline TYPEX & 092B & 1317 & 1331 & & & & & & & \\
\hline TYPLP & 0949 & 1330 & 1334 & & & & & & & \\
\hline UDOT & $15 \mathrm{~A} 7$ & 3045 & 3054 & & & & & & & \\
\hline UMUI'T' & $05 \mathrm{CE}$ & 0783 & 0348 & 0765 & & & & & & \\
\hline UNT'IL & 1412 & 2803 & 2813 & 2816 & & & & & & \\
\hline UP & 0007 & & $\begin{array}{l}0056 \\
0914\end{array}$ & $\begin{array}{l}0102 \\
1259\end{array}$ & $\begin{array}{l}0103 \\
1260\end{array}$ & 0395 & 0396 & 0405 & 0406 & 0912 \\
\hline UPDAT & 1659 & 3138 & 3154 & & & & & & & \\
\hline USARFA & 5700 & & 0041 & 3419 & 3429 & & & & & \\
\hline USE & 0794 & 1090 & 1097 & 2517 & 3170 & 3178 & & & & \\
\hline USER & 0690 & 0912 & $\begin{array}{l}0923 \\
0983 \\
1045 \\
1107 \\
2295\end{array}$ & $\begin{array}{l}0929 \\
0989 \\
1053 \\
1115\end{array}$ & $\begin{array}{l}0937 \\
0997 \\
1061 \\
1123\end{array}$ & $\begin{array}{l}0945 \\
1005 \\
1069 \\
1131\end{array}$ & $\begin{array}{l}0953 \\
1013 \\
1075 \\
1139\end{array}$ & $\begin{array}{l}0961 \\
1021 \\
1083 \\
1147\end{array}$ & $\begin{array}{l}0967 \\
1029 \\
1091 \\
1155\end{array}$ & $\begin{array}{l}0975 \\
1037 \\
1099 \\
1163\end{array}$ \\
\hline USLSH & 0204 & 0326 & 0343 & 2658 & & & & & & \\
\hline USLSX & 0245 & 0350 & 0334 & & & & & & & \\
\hline USR & 1071 & 2292 & 2302 & & & & & & & \\
\hline USRADD & $089 \mathrm{~F}$ & 1259 & $\begin{array}{l}1269 \\
1524\end{array}$ & $\begin{array}{l}1314 \\
1575\end{array}$ & 1325 & 1365 & 1450 & 1461 & 1472 & 1484 \\
\hline USTAR & $023 \mathrm{~A}$ & 0344 & 0355 & 2000 & 2005 & & & & & \\
\hline $\begin{array}{l}\text { UT71 } \\
\text { VAR }\end{array}$ & $\begin{array}{l}87 \mathrm{FO} \\
05 \mathrm{FE}\end{array}$ & 0803 & $\begin{array}{l}0038 \\
2284\end{array}$ & 3473 & & & & & & \\
\hline VARB & 1060 & 2281 & 2291 & & & & & & & \\
\hline VBI & 1191 & 2449 & 3401 & & & & & & & \\
\hline VBLY & 1171 & 2432 & 2458 & & & & & & & \\
\hline VCTRS & 0825 & 1185 & 1183 & & & & & & & \\
\hline VI & $06 \mathrm{~F} 2$ & 0974 & 0981 & 2443 & 2446 & & & & & \\
\hline
\end{tabular}


File ID: XREF •

Disk ID: WOODS HOLE FORTH SOURCE \& LIST VER 1.800 113084

\begin{tabular}{|c|c|c|c|c|c|c|c|c|c|c|}
\hline WARM & 0036 & 0097 & 0070 & 0090 & 0096 & 0109 & & & & \\
\hline WBR & 0093 & 0132 & 0319 & & & & & & & \\
\hline WDI & OEOE & 1954 & 1948 & & & & & & & \\
\hline WD2 & 0E12 & 1957 & 1953 & & & & & & & \\
\hline WEFI & 0078 & 0119 & & & & & & & & \\
\hline WEFIN & 0084 & 0123 & & & & & & & & \\
\hline WEF2 & $007 \mathrm{~B}$ & 0120 & & & & & & & & \\
\hline WEF $2 N$ & 0087 & 0124 & & & & & & & & \\
\hline WEF3 & $007 \mathrm{E}$ & 0121 & & & & & & & & \\
\hline WEF3N & $008 \mathrm{~A}$ & 0125 & & & & & & & & \\
\hline WEF 4 & 0081 & 0122 & & & & & & & & \\
\hline WEF 4N & 008D & 0126 & & & & & & & & \\
\hline WHILE & $149 \mathrm{D}$ & 2883 & 2896 & & & & & & & \\
\hline WIDTH & $06 \mathrm{CO}$ & 0944 & 0951 & 2206 & & & & & & \\
\hline WORD & ODFA & 1943 & 1985 & 2079 & 2552 & 2560 & 2600 & & & \\
\hline WRMLP & 004D & 0104 & 0105 & & & & & & & \\
\hline WRNG & $06 \mathrm{CE}$ & 0952 & 0959 & 2111 & & & & & & \\
\hline X & ODBD & 1907 & 1942 & & & & & & & \\
\hline $\mathrm{X} 1$ & ODE9 & 1930 & 1926 & & & & & & & \\
\hline $\mathrm{X} 2$ & ODED & 1933 & 1912 & & & & & & & \\
\hline XEND & ODFl & 1936 & 1932 & & & & & & & \\
\hline YES & $006 \mathrm{C}$ & 0116 & 0112 & 0113 & 0114 & & & & & \\
\hline \multirow[t]{4}{*}{$\mathrm{ZBRCH}$} & 0182 & 0259 & 0279 & 1611 & 1658 & $\begin{array}{l}1844 \\
2066\end{array}$ & $\begin{array}{l}1865 \\
2087\end{array}$ & $\begin{array}{l}1911 \\
2114\end{array}$ & $\begin{array}{l}1925 \\
2142\end{array}$ & $\begin{array}{l}1947 \\
2193\end{array}$ \\
\hline & & & $\begin{array}{l}1995 \\
2335\end{array}$ & $\begin{array}{l}2010 \\
2351\end{array}$ & 2389 & 2394 & 2413 & 2485 & 2548 & 2808 \\
\hline & & & 2853 & 2902 & 2943 & 2963 & 2985 & 3123 & 3176 & 3183 \\
\hline & & & 3222 & 3227 & 3246 & 3279 & 3315 & 3526 & & \\
\hline \multirow[t]{2}{*}{ ZEQAI } & 0352 & 0484 & 0493 & 1674 & 1726 & 1924 & 2086 & 2484 & 2574 & 2608 \\
\hline & & & 2984 & 3226 & 3245 & & & & & \\
\hline \multirow[t]{3}{*}{ ZERO } & 0608 & 0811 & 0816 & 1751 & 1870 & 1878 & 1900 & 1916 & $\begin{array}{l}2029 \\
2855\end{array}$ & $\begin{array}{l}2030 \\
2877\end{array}$ \\
\hline & & & 2058 & 2060 & 2305 & 2473 & $\begin{array}{l}2515 \\
319 ?\end{array}$ & $\begin{array}{l}2609 \\
3349\end{array}$ & 3372 & 3389 \\
\hline & & & 3512 & $\begin{array}{l}2904 \\
3549\end{array}$ & $30<2$ & 2041 & & & & \\
\hline ZLESS & 0367 & 0494 & 0500 & 2113 & 2942 & 3182 & & & & \\
\hline ZONE & $035 B$ & 0489 & 0429 & 0487 & 0496 & & & & & \\
\hline ZZONE & 0385 & 0507 & 0505 & & & & & & & \\
\hline
\end{tabular}


Known Deviations from fig-FORTH

Woods Hole FORTH is modeled after fig-FORTH as outlined in the figFORTH installation manual. There are a few exceptions, however, as listed below. In most cases, these are subtle enhancements and should still allow Fig-FORTH programs to run without problems.

1. ENCLOSE, the text scanning primative used by LOAD, can handle up to 32767 (d) blanks, instead of 255 as in Fig-FORTH. This means that the system will not generate message 7 if more than 255 blank characters are within a screen.

2. CR increments the USER variable OUTL. OUTL is a new USER variable which keeps track of the number of lines which have been printed in a fashion analogous to OUT, the character counter. A new constant L/P (lines per page) has been added. By comparing OUTL with $\mathrm{L} / \mathrm{P}$, the user can decide when to issue form feeds for a printer. The USER variable FLD, unused by fig-FORTH, is used as a page counter. It is incremented by the word PAGE.

3. ABORT has two actions which are different from the fig model. First, ABORT selects drive 1 as the default drive rather than drive zero. This is done so that a library of system programs can be maintained on drive zero, and applications can be completely contained on the disk in drive one. Secondly, ABORT includes the sequence: 1 BLOCK DROP to force a drive head recalibration. This is required after a system reset. For an application that does not have disk hardware, this will cause a system crash. Therefore, the code must be changed to:

$00=$ DROP. This patch is performed by the word NODISK. Screen 83 has the code required to install both of these patches.

4. The word MESSAGE always prints the message number in base 10 (decimal) so there is no confusion as to which message is issued. MESSAGE does not change the current number BASE. When writing 
application programs that do not use disks, it is best to avoid calling ERROR or MESSAGE; rather, when an error is detected, the user should print a text message stored in the dictionary and deal with the problem so execution can continue without interruption.

5. MESSAGE does not access the disk for a text message when WARNING is positive. In the fig-FORTH model, a positive value for WARNING will cause a text message to be printed, rather than a message number.

6. The word INDEX is given special consideration in WFORTH. By convention, all screens reserve line 0 as the index line. The index line is enclosed within parentheses and treated as a comment when loading the screen. Within the index line are the names of all words defined on that screen. The word INDEX uses a word ?INDEX to decide whether to print the index line. An index line will be printed by INDEX only if there are no non-printing characters on line zero and if line 0 contains at least 1 printing character. Therefore, an index over the entire range of screens (DECIMAL 65249 INDFX (CR)) yields a directory to the sources of all words defined on screens, skipping screens without meaningful comment lines.

7. >IN, according to the FORTH 79 standard is included. It is defined as : >IN IN ;. The FORTH 79 word : NOT $0=$; is included also.

8. WORD-79 is included, defined according to FORTH 79. It is defined as : WORD-79 WORD HERE ;

The definition of WORD frequently causes problems when transporting programs from other FORTH systems. Check carefully for the occurrence of WORD in existing FORTH programs.

9. CMOVE> (character move up), a FORTH 83 standard word, is included. This is for memory block moves where the source and destination overlap and the source address is lower than the destination address.

10. EDIT, a full screen editor is included in the Fig-EDITOR vocabulary. FDIT is configured for a Televideo $910+$ terminal and may require 
changes for other terminals.

11. I and $T$ (list and type) which are normally EDITOR words, are also defined in FORTH. When executed in FORTH, they change the context vocabulary to EDITOR and then behave normally as $\mathrm{L}$ and $\mathrm{T}$.

12. There is a non-standard word GROUP, which takes the top number from the stack and uses it to perform the RCA 2 level I/O select. There is also a new USER variable, GRP, which holds the value of the group most recently selected by GROUP. The use of GROUP allows the user to restore the group number to its previous value after executing I/O words.

13. There is a set of communications words which facilitate terminal emulation, modem communication, and uploading and downloading of files and memory blocks.

14. There is a set of disk access words to facilitate reading and modifying operating system files.

15. There is a set of documentation words. The purpose of these words and screens is to make documentation of a particular application version of FORTH easy. Chapters 11 through 22 were generated in this manner.

16. The words PRINTER and NOPRINT allow full printer support. As supplied, PRINTER, and NOPRINT support a parallel printer, using the CDP 185646 card, but executing the word SERPRINTER will redirect all printer output to a uart assigned to the communications group for usewith a serial printer.

17. A single pass Assembler for 1806 mneumonics is included.

18. 32 bit floating point arithmetic is included as a loadable option. It is not rigorously FORTH-like, because the actual program must be loaded separately and called in a slow fashion. The floating point 
words are loaded by the word FLOATING. A file called FFORTH.CM is included which has floating point support already compiled. When floating point is used, it is loaded into the last four screen buffers, reducing the number of buffers available from 10 to 6 . This is essentially transparent to the user, as all memory pointers are reassigned. 
REFERENCES

FORTH ENCYCLOPEDIA, second edition:

Derick and Baker; Mountain View Press, P.O. Box 4656, Mountain View, CA 94040 .

This publication was used as the primary model for Woods Hole fig-FORTH. It is the most important reference work, because of the thorough explanation of each word.

STARTING FORTH:

Leo Brodie

This is an important tutorial, although it describes the FORTH INC. dialect of FORTH, not fig-FORTH. As a result, some differences occur with the editor and with the syntax of individual words. The glossary of Woods Hole FORTH should be used to explain the behavior of WFORTH words, rather than STARTING FORTH.

Fig-FORTH Installation Manual;

William F. Ragsdale; published by the FORTH Interest Group, P.O. Box 1105, San Carlos, CA 94070

Fig-FORTH 1802;

published by the FORTH Interest Group.

These are the publications for the implementation of fig-FORTH. The installation manual contains the original published model, as well as the sources for and a description of the fig-Editor. These publications are indispensable.

Mad-FORTH:

Dick Berger, Micro Amp Designs.

This version of FORTH, based on Fig-FORTH 1802 was the starting point for Woods Hole FORTH.

PC FORTH and Z80 FORTH:

Ray Duncan, Laboratory Microsystems

These two excellent implementations of FORTH, although written in 79 
standard FORTH, were used to insure a high degree of transportability with Woods Hole FORTH. PC FORTH was used on an IBM personal computer. Z80 FORTH was used on an RCA MS2080 development systems. A 2080 development system is composed of:

1 RCA MS2000 development system, with CPU card removed

I RCA MB80 CPU card

1 RCA 18S641 UART card

the TPM operation system (a CP/M clone)

UT80 UTILITY Program

RCA MPM 201 and MPM 201C (supplement)

User Manual for the 1802, 1804, 1805, and 1806 Microprocessors.

These are useful manuals for the 1800 series microprocessors.

$\underline{\text { RCA MPM }} \underline{207}$

Floating point Arithmetic Subroutines.

This document provides the basis for Woods Hole Floating Point.

$\underline{\mathrm{RCA}} \underline{\mathrm{MPM}} 24 \mathrm{2}$

User Manual for the RCA Microdisk Development System.

All operating system programs, including SUBMIT are described here. This is a very good operating system.

\section{FORTH DIMENSIONS}

Published by the FORTH Interest Group. This is the journal

of the FORTH Interest Group. Published approximately quarterly, it provides numerous papers on general FORTH problems, as well as highly specialized applications of FORTH. Many dialects of FORTH are represented here.

Alvin User's Manual and Alvin Data Systems User's Manual

Published by Woods Hole Oceanographic Institution. This publication describes, among other things, the Alvin data system. Much of software for the Alvin data system was written in Woods Hole FORTH. 
Woods Hole FORTH is available from:

Jim Akens

Alvin Group, Woods Hole Oceanographic Institution,

86 Water Street

Woods Hole, Ma 02543

The FORTH ENCYCLOPEDIA and STARTING FORTH are available from:

Mountain View Press

P.O. Box 4656

Mountain View, CA 94040

PC FORTH is available from:

Laboratory Microsystems

4147 Beethoven Street

Los Angeles, CA 90066

The fig-FORTH Installation Manual, fig-FORTH 1802 and FORTH DIMENSIONS are available from:

FORTH INTEREST GROUP

P.O. Box 1105

San Carlos, CA 94070

The MS 2000 and MS 2080 development systems and the RCA documents are available from:

RCA

Route 202

Somerville, NJ 08876 


\section{GLOSSARY}

Each word in the glossary contains 4 entries; the name, computation stack behavior, pronunciation, and a description of the word's behavior. NAME is the actual entry which causes execution. Stack behavior is indicated within < before NAME was executed, the value of BEFORE (a 16 bit value) was on the stack. After NAME is executed, the stack has two values; AFTER.2nd is the lower number, and AFTER.TOP is the top value on the stack. Notice that the indicated stack behavior is only relative to the word being executed. In fact, the stack contains an unknown number of items. The word DEPTH leaves, as the top number on the stack, the number of 16 bit items on the stack. The notation ANY.NAME indicates that a FORTH word is required. There are 8 general types of numbers on the stack.

\section{Examples $\quad$ Meaning}

NUMBER $\mathrm{N}$ represent a single 16 bit signed number;

U represents a 16 bit unsigned number;

DOUBLE D represent a 32 bit signed number;

UD represents an unsigned 32 bit number;

F represents a 32 bit floating point number. The first 8 bits represent a signed exponent (base 10) and the remaining 24 bits represent a signed 24 bit mantissa (base 10).

CHARACTER represents an 8 bit character. Although a 16 bit number is on CHAR the stack, only the least significant 8 bits are used.

ADDRESS represent a 16 bit address, normally interpreted as an ADDR unsigned number.

FLAG represents a flag, a 16 bit number. The value zero represents $\mathrm{f}$ the false condition. Any non-zero value signifies true. 


$$
<\ldots>>
$$

Null

Null is a pseudonym for the ascii character 00 . It is sometimes called $\mathrm{x}$ in fig-FORTH literature. Null causes an exit from INTERPRET. Control is returned to the procedure which called interpret.

$\langle$ NUMBER ADDRESS ... > Store

! stores a 16 bit NUMBER at ADDRESS.

!BLK

$$
\langle\ldots\rangle \text { Store block }
$$

!BLK is a primative used by EDIT.

!CODE

$\langle$ ADDRESS.LIT ADDRESS ... > Store code

!CODE is a Woods Hole FORTH defining word, used in building CONSTANTS, VARIABLES and USER variables.

!CSP

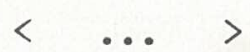

Store Current Stack Pointer

!CSP is a word used by the compiler with CSP and ?CSP for compiler security. It stores the current stack pointer address in the USER variable CSP .

!CUR

$$
\langle\text { CURSOR ... > Store Cursor }
$$

Store cursor location in the USER variable CUR . Check for range 0 to $3 \mathrm{FF}(\mathrm{h})$.

$$
\text { < UNSIGNED.DOUBLE ... DOUBLE. } \underset{\text { SUOTIENT > }}{\text { Sharp }}
$$

Sharp converts 1 digit of the DOUBLE number on the stack to ASCII, using the current number BASE. The output string is constructed, starting with the least significant digit, just before the PAD buffer. Fach time \# is invoked, one digit is converted. See Starting FORTH, by Leo Brodie, for a complete discussion of 〈\# \# \#S and \#〉.

$$
\langle\text { D ... ADDRESS IEENGTH }>
$$

Sharp Greater Than

\#> is used to terminate a pictured numeric output expression. It drops the double number left by \#, and replaces it with the beginning ADDRESS of the converted string and the IENGTH of the string, in a form suitable for TYPE.

$$
\langle\text { UNSIGNED.DOUBLE ... } 00>\text { Sharp S }
$$

Sharp S converts a double unsigned number to an ASCII string located 1 byte before pad. \#S must be used within the 〈\#\#\#〉 structure. The current number BASE is used in the conversion. 
$\langle\ldots \mathrm{PFA}\rangle \quad$ Tick

Tick places the parameter field address of the specified word on the stack. It is used in the form:

- ANY.WORD

(

$(+\mathrm{LOOP})$

$(. ")$

$(; \mathrm{CODE})$

(ABORT)

(DO)

(DOS)

$$
<\ldots>\quad \text { Left Parenthesis }
$$

( is used to mark the start of a comment. ( is a FORTH word; therefore, it must be separated from the comment by one or more spaces. The right parentheses symbol terminates the comment.

Parentheses Plus Loop

$(+L O O P)$ is the run time procedure for +LOOP.

Parentheses Dot Quote

(.") is the run time procedure that is compiled into a definition by ." . It prints the string and moves the FORTH instruction pointer past the string.

$$
<\quad \cdots>
$$

Parentheses SemiColon Code

(;CODE) is the run time procedure for words defined by ;CODE. Refer to 〈BUILDS, DOES>, AND;CODE for a discussion of defining words.

$$
\text { < ALU.STACK.VALUES ... > Parentheses Abort }
$$

(ABORT) is an intermediate word used between ERROR and ABORT. It is normally executed when WARNING is negative, and simply executes ABORT. Replacing the CFA of ABORT in (ABORT) with the CFA of a user's error routine allows the user to customize a task's error trapping. If warning is zero or positive, the message number is printed, and QUIT is executed.

$$
\langle\text { IIMIT INDEX ... > Parentheses Do }
$$

(DO) is the run time procedure compiled by DO. It is not normally called by a user.

$$
<\ldots>\text { Parentheses DOS }
$$

Return to the disk operating system, MicroDos at 9003(h). This is the primative version of the FORTH word DOS. 
(FIND)

(I.INE)

(LOOP)

(NUMBER)

*/

*/MOD

$+$
< STRING.ADDRESS NEA ... PFA LENGTH TRUE.FLAG >

if successful

$\langle$ STRING.ADDRESS NEA ... FALSE.FLAG >

if unsuccessful

Parentheses Find

(FIND) performs a dictionary search for the specified NFA until a match is found (successful) or a link field of zero is encountered (unsuccessful).

$<$ LINE.NUMBER SCREEN.NUMBER ... ADDRESS.BEGIN.LINE LINE.LENGTH >

Parentheses Line

(LINE) is the primative form of .IINE . It finds the line number on the screen number desired by . IINE .

Parentheses Loop

(IOOP) is the execution time procedure for IOOP. It is compiled into a definition by LOOP. This is not normally called by the user.

< DOUBLE.NUMBER STRING.ADDRESS ... DOUBLE.NUMBER CHAR.ADDRESS >

Number

(NUMBER) is a primative used by NUMBER.

$$
\langle\mathrm{N} 1 \mathrm{~N} 2 \ldots \mathrm{N} 3\rangle \quad \text { Times }
$$

* multiplies 2 single ( 16 bit) numbers and leaves their 16 bit signed product. Overflow is possible, and no error message is issued if overflow occurs.

< MULTIPLIER MULTIPLICAND DIVISOR ... QUOTIENT > Times Divide

Times Divide performs the multiplication and division, but uses a 32 bit intermediate result for greater accuracy.

$$
\begin{aligned}
& \text { < MUTTIPLICAND MULTIPLIER DIVISOR ... REMAINDER } \\
& \text { QUOTIENT > }
\end{aligned}
$$

Times Divide Mod

Times Divide Mod performs the indicated operations, but uses an intermediate 32 bit result for greater accuracy.

$$
\langle\mathrm{N} 1 \mathrm{~N} 2 \ldots \mathrm{Nl}+\mathrm{N} 2\rangle \quad \text { Plus }
$$

+ adds two numbers and replaces them with one number, their 16 bit sum. Overflow is possible, and no error message is issued if an overflow occurs. 
+ ! increments the 16 bit number at ADDRESS by the value of INCREMENT.
$\langle\mathrm{NI} N 2 \ldots \mathrm{N} 3\rangle$
Plus Minus
$\mathrm{N} 3$ equals $\mathrm{N} 1$ if $\mathrm{N} 2$ is positive. $\mathrm{N} 3$ equals $-\mathrm{N} 1$ if $\mathrm{N} 2$ is
is negative. In any case $\mathrm{N} 2$ is dropped.
(.CUR

$$
<\text { DELTA } \ldots>>
$$
Plus Print Cursor
+.CUR increases the USER variable CUR by DELTA and moves the cursor. This is a primative used by EDIT.

$$
\begin{gathered}
<\text { BUFFER.ADDRESS ... NEXT.BUFFER.ADDRESS FLAG > } \\
\text { Plus Buffer }
\end{gathered}
$$

Plus buffer advances the buffer pointer to the next block in FORTH's circular array of screen buffers. There are ten buffers in Woods Hole FORTH. The flag is false (zero) if NEXT.BUF is equal to PREV, the USER variable which contains the value of the most recently accessed buffer. +BUF is a primitive used by BLOCK to allocate FORTH's buffers.

+CUR

$$
\text { <EITA ... > Plus Cursor }
$$

Increase the value of CUR by DELTA. This is a primative used by EDIT. Note that the curser itself is not moved.

+ LIN

+ IOOP

HORIGIN
This word is a primative used by EDIT'.

$$
\langle\text { INCREMENT ... > Plus LOOP }
$$

+LOOP is used to terminate a DO loop structure. The INCREMENT is added to the index; if less than the limit, the loop is repeated, if not, the loop is exited and the word following +LOOP is executed.

$$
<\text { OFFSET ... ORIGIN+OFFSET > Plus Origin }
$$

HORIGIN is used to index into the parameter table. OFFSET is added to ORIGIN to compute the actual address. See the ASM8 Source on line 3410, for a complete description of the PARAMETER TABLE.

$$
\langle\mathrm{N} \ldots>>\text { Comma }
$$

Comma stores the number $\mathrm{N}$ into the next two bytes of the dictionary. This is how code words are built using the word CREATE. Note that this is a 16 bit operation.

$$
\langle\mathrm{N} 1 \mathrm{~N} 2 \quad \ldots \quad \mathrm{N} 1-\mathrm{N} 2 \quad\rangle \quad \text { Subtract }
$$

Subtract subtracts the top number (N2) from the second number (NI) and leaves the difference. 


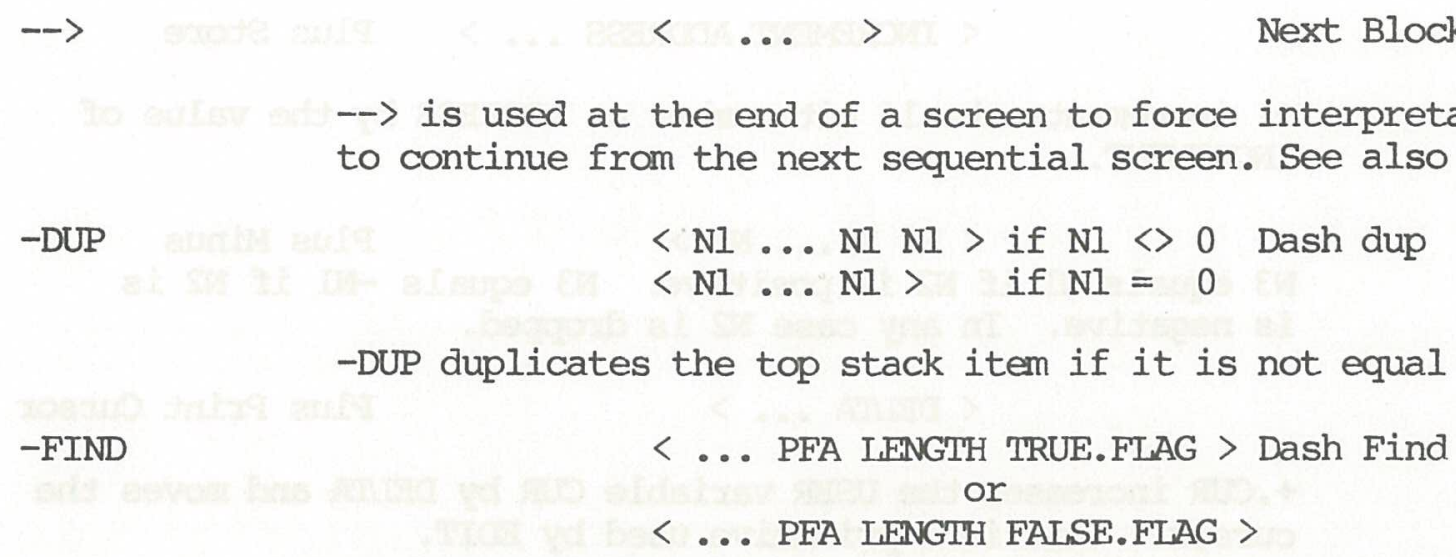

Dash Find reads a word from the input stream and searches the CONTEXT and CURRENT vocabularies for a match. The top number is a true flag (non zero) if a match is found.

-TRAILING will alter the LENGTH of a character string to suppress trailing spaces before calling TYPE.

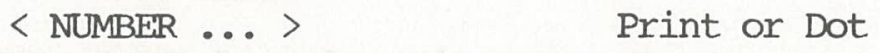

PRINT removes the top item from the stack and prints it as a signed number in the current number BASE.

." is used to send messages to the terminal. It is used in the form: ." This is a message" .

Since dot quote is a FORTH word it must be followed by a space. The terminating delimeter is the quote symbol, and no space is required before the terminator.

$$
\langle\ldots 00\rangle \quad \text { Dot Dot }
$$

This is a bug in fig-FORTH. NUMBER interprets this as the double number 0 . This word is not found in the dictionary.

. $\mathrm{AH}$

$$
\langle\text { NIBBIE } \ldots>>
$$

Print Ascii-hex nibble

- AH interprets the top stack number as a 4 bit nibble and prints it in Ascii-hex notation (0-F). This is a primative used by SEND.

.AHL

$$
\begin{array}{ll}
\langle\text { START.ADDRESS } \ldots .\rangle & \text { Print ASCII } \\
& \text { Hex Line }
\end{array}
$$

This word will print 64 characters starting at START.ADDRESS. This is a primative used by SEND. 
This word prints the character if printable, otherwise, the symbol "." . This is a primative used by DUMP.

-BYTE

$\langle$ NUMBER $\ldots>\rangle$

Print Byte

This is a primative used by SEND. It interprets the NUMBER as an 8 bit byte and prints it in Ascii-hex notation.

.CAT

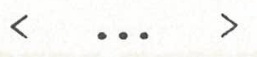

Print Cluster

Allocation Table

This word prints the cluster allocation table for the selected drive. The first bit represents sector 10(d). Since a directory and cluster allocation table (sectors 0-9) are always occupied, from the MicroDos point of view, the first 10 sectors are not represented in the cluster allocation table.

.CUR

$$
<\ldots>\text { Move Cursor }
$$

This is a primative used by EDIT. It moves the curser to the location specified in the USER variable CUR .

-DIRSEC

$<$ SECTOR.BUFFER.ADDRESS $\ldots>>$

Print directory

Print the directory information contained in 1 sector. This is a primative used by DIR . In MDOS, the disk directory occupies the first 9 sectors on the disk.

.FOOT

$$
<\ldots>\text { Print Footer }
$$

This word prints the message: "Woods Hole 1806 FORTH, Version $\mathrm{X} . \mathrm{XXX}$ ", 10 spaces "page" NUMBER, where NUMBER is the value of FLD ; followed by a carriage return. .FOOT resets OUIL, the line counter to zero. .FOOT is normally followed by PAGE .

< LINE. NUMBER SCREEN.NUMBER ... > >

Print Iine

Print line prints 1 line of text from the desired screen. This is the root of the FORTH word INDEX, which prints line 0 over a given range of screens. Trailing blanks are supressed, so typing speed is optimized.

$\cdot R$

$$
\left\langle\text { NUMBER FIEID.WIDTH ...> } \begin{array}{l}
\text { Print Right } \\
\text { Justified }
\end{array}\right.
$$

The NUMBER is printed, padded with leading spaces, for a total number of characters equal to FIEID.WIDTH. The current number BASE is used. 
This word prints all numbers on the stack, bottom number first in the current number BASE. The stack is not altered.

.VHDR

/MOD

0

$0<$

OBRANCH $<\quad \ldots>>$

Print Vocabulary List Header

This is a primative used by VLIST. It prints the header for the vocabulary list.

$$
\begin{array}{r}
\text { DIVIDEND DIVISOR ... QUOTIENT > } \\
\text { Divide }
\end{array}
$$

DIVIDE divides the two signed 16 bit numbers and leaves a signed 16 bit quotient.

$$
<\text { DIVIDEND DIVISOR ... REMAINDER QUOTIENT > }
$$

Divide Mod divides a 16 bit signed number by another and replaces then with a 16 bit signed quotient and 16 bit signed remainder. The remainder takes it sign from the dividend.

$$
\langle\ldots 0\rangle \text { Zero }
$$

0 is a CONSTANT equal to zero. In FORTH, $0,1,2$, and 3 are supplied as constants because execution speed is much faster than that obtained by interpreting with NUMBER.

$$
\text { < NUMBER ... flag > Zero less than }
$$

$0<$ examines the number on the stack and replaces it with a flag. The flag is true (non-zero) if the number is less than zero (negative).

$$
\langle\text { NUMBER ... flag > }
$$

Zero Equal

$0=$ examines NUMBER on the stack and replaces it with a flag. The flag is true (non-zero) if NUMBER is equal to zero.

$$
\text { <flag ... > Zero Branch }
$$

OBRANCH is compiled into definitions by IF, UNIIL and WHIIE to perfom a conditional branch. At run time, OBRANCH examines the flag; if false (zero) a branch is effected to the address compiled by IF, UNTIL or WHILE.

$I$ is a CONSTANT equal to 1.

It adds one to the top number on the computation stack. 
1- subtracts one from the top number on the computation stack.

2 is a CONSTANT equal to 2.

Raise 2 to the Nth power, for $0<\mathrm{N}<256$

$2+$ adds two to the top number on the computation stack.

2- subtracts two from the top number on the computation stack.

This word drops the double number on the computation stack.

This word duplicates the double number on top of the computation stack.

Two Swap

The word performs a double number swap on the computation stack.

$$
\langle\ldots 3\rangle \quad \text { Three }
$$

3 is a CONSTANT equal to 3.

:

$$
<\ldots>\text { Colon }
$$

: is used to start colon definitions. It is used in the form:

: NAME (words to be executed) ;

;

$$
<\ldots\rangle \text { Semi colon }
$$

i is used to terminate a colon definition. It may only be used within a colon definition. 
;CODE $\quad<\ldots>>\quad \begin{aligned} & \text { Parentheses Semi- } \\ & \text { Colon }\end{aligned}$

;CODE is a defining word normally used in conjunction with <BUIIDS . ;CODE is vey similar to DOES> except that ;CODE defines the beginning of a code procedure, while DOES $>$ defines the beginning of a high level procedure. ;CODE starts the machine code procedure which is executed when the defined words are executed. See 〈BUIIDS, ;CODE, and DOES> in the FORTH Encyclopedia.

Semi Colon S

iS is the run time procedure compiled by ; into a colon definition. Its purpose is to return to the calling word by popping the return address from the return stack. It is also used to terminate the interpretation of a screen. Namely, it must be the last FORTH statement on a screen, and anything following it is ignored.

Less than

$<$ comparesthe top two numbers. If $\mathrm{N} 1$ isless than $\mathrm{N} 2$, the top number, the flag is true (non-zero). Notice that $32000-32000<$ works properly.

<\# begins a pictured numberic (binary to ASCII) conversion. Note that the double number to be converted must be positive. Therefore, DABS should be used if the number might be negative.

$$
<\ldots>\quad \text { Builds }
$$

$\angle$ BUIIDS is a defining word. Words created by <BUIIDS are used to create other words. <BUILDS creates the dictionary header for the new definitions. Words between <BUIIDS and DOES> are executed at compile time of the new definitions. The words following DOES> are executed at execution time of the new word. VOCABULARY is a word that uses 〈BUIIDS DOES> to create new vocabularies; however, this is a non-trivial example. Most ASSEMBLER mnemonics are created by <BUIIDS DOES> words and are simple examples of the <BUILDS DOES> construct.

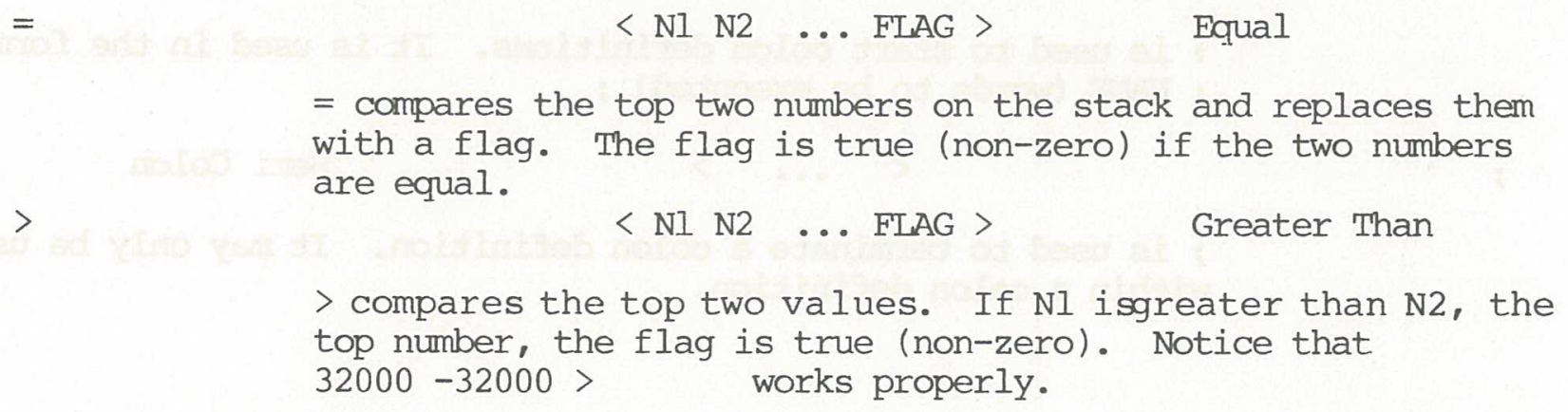


$>\mathrm{R}$

?ASCII

?CARRIER

?COMP

?CSP

?EF1
>IN is a USER variable that points to the byte offset count from the beginning of the currently interpreted block to the end of the word most recently interpreted. This is the FORTH 79 standard version of IN .

$$
\langle\text { NUMBER ... To } R
$$

$>R$ removes a NUMBER from the computation stack and leaves it on the return stack. It is usually used with $R>$ to temporarily store numbers on the return stack. The use of $>R$ and $R>$ must be balanced within a colon definition.

$$
\langle\text { ADDRESS ... > Question Mark }
$$

Question Mark fetches the 16 bit number at ADDRESS and prints it, signed in the current number BASE.

$$
\langle\text { CHARACTER ... FLAG > Question ASCII }
$$

This word returns a true flag if the character is printable $(31 d<$ CHARACTER $<127 d)$.

$$
\langle\ldots \text { FLAG }>\quad \text { Question Carrier }
$$

This word loops for approximately 5 seconds while looking for incoming carrier at the modem. It returns a true flag (non zero) when it detects carrier, or a false flag (0) after 5 seconds. This wora is used by the word ORIGINATE.

Question Compile

?COMP issues MESSAGE 17 (d) and executes QUIT if the system is not in compile mode. The test is to examine the value of the USER variable STATE. STATE is non-zero if compiling.

Question Current Stack Pointer

?CSP checks to see if the current stack pointer position is equal to that stored in the USER variable CSP. If not, message $20(d)$ is issued, followed by QUIT. This is part of the compiler security which is built into the fig-FORTH model.

Question External Flag 1

This word leaves a flag on the stack corresponding to the value of external flag 1 ( $1=$ TRUE $=0$ volts; $0=$ FALSE $=5$ volts $)$. 
This word leaves a flag on the stack corresponding to the value of external flag 2 ( $1=$ TRUE $=0$ volts; $0=$ FALSE $=5$ volts $)$.

?EF3

?EF4

?ERROR

?EXEC

?HEXCHAR

?INDEX

?KEY $\langle\ldots$ FIAG $\rangle$

Question External Flag 3

This word leaves a flag on the stack corresponding to the value of external flag 3 ( $1=$ TRUE $=0$ volts; $0=$ FAISE $=5$ volts $)$.

$$
\langle\ldots \text { FLAG }\rangle
$$

Question External Flag 4

This word leaves a flag on the stack corresponding to the value of external flag $4(1=$ TRUE $=0$ volts; $0=$ FALSE $=5$ volts $)$.

$$
\text { < FLAG MESSAGE.NUMBER ...>> Question Error }
$$

?FRROR issues the specified message and executes QUIT if the flag is true (non-zero).

$$
<\ldots>>
$$

Question Execute

?EXEC issues MFSSAGE $18(d)$ and executes QUIT if the system is not in execute mode. The test is to examine the value of the USER variable STATE. STATE equals 0 if executing.

$$
\left\langle\text { CHARACTER ... FLAG }>\quad \begin{array}{l}
\text { Question Hex } \\
\text { Character }
\end{array}\right.
$$

?HFXCHAR checks to see if the number on the stack is a valid hex character. The flag is true (non zero) if it is a valid character.

$$
\text { < SCREFN.NUMBER ... FLAG > Question Index }
$$

This word returns a true flag (non zero) if the index line (line 0) of SCREEN. NUMBER is considered a valid index. This is so, if there are no non-printing characters and if the index line is not blank. This word works just like ?SCREEN, but only looks at line 0 . Note that this test is not foolproof. A disk which has just been formatted will on certain screens pass the test, although a valid index line does not exist.

$$
\langle\ldots \text { FLAG }>\quad \text { Question Key }
$$

This word checks the status of the selected uart. It returns a true flag if there is a character available, including a break. 
?MONITOR

?PAIRS

?SCREEN

?SPACE

?STACK

?TERMINAL

$? Y / N$
?IOADING checks the USER variable BIK to see if the system is loading from disk. If not, it issues message $22(d)$ and then executes QUIT.

$$
<\ldots>\text { Question Monitor }
$$

This word performs a simple check to see whether it is talking to a standard 1800 utility program. It assumes that communication with the target is via the uart at COMM.GRP. It types "@" to the target, which is an illegal command for the RCA utilities, waits $200 \mathrm{milliseconds} \mathrm{and} \mathrm{looks} \mathrm{at} \mathrm{the} \mathrm{last}$ received character. This should be * for the utility programs. MONABORT is called if this is not true.

$$
\langle\mathrm{Nl} N 2 \ldots\rangle \quad \text { Question Pairs }
$$

?PAIRS campares the top two numbers on the stack. If $\mathrm{Nl}$ does not equal N2, ?PAIRS issues message 19 (D) and executes QUIT. This word is used during compilation to insure that conditional.s are paired properly, such as IF ... THEN.

$$
\text { < SCREFN.NUMBER ... FLAG > Question Screen }
$$

This word returns a true flag (non zero) if SCREFN.NUMBER is considered a valid screen. A screen is considered valid if there are no lower case characters and if the screen is not blank. Note that this test is not foolproof. For instance, a disk which has just been formatted will, on certain screens pass the test, although a valid screen does not exist.

< CHARACT'ER ... FIAG > Question Space

This word returns a true flag if the character is a space (20H).

Question Stack

?STACK checks for stack overflow or underflow. If over or underflow is found, message 7 is issued and QUIT is then executed.

$$
\text { <... FIAG > Question Terminal }
$$

?TERMINAI is used to determine whether the break key has beer depressed. In Woods Hole FORTH, ?TERMINAL only checks for breaks, not any key as some FORTH systems do. A true flag (non-zero) indicates a break condition.

$$
\langle\ldots \text { FILAG }>\quad \text { Question Yes or No }
$$

Wait for a key from the uart at the present group. If character equals $Y$ or $Y$, print " $Y$ ", FLAG $=1$; otherwise print "N", FIAG = 0 
a reads a 16 bit NUMBER from memory at ADDRESS and leaves it on the stack.

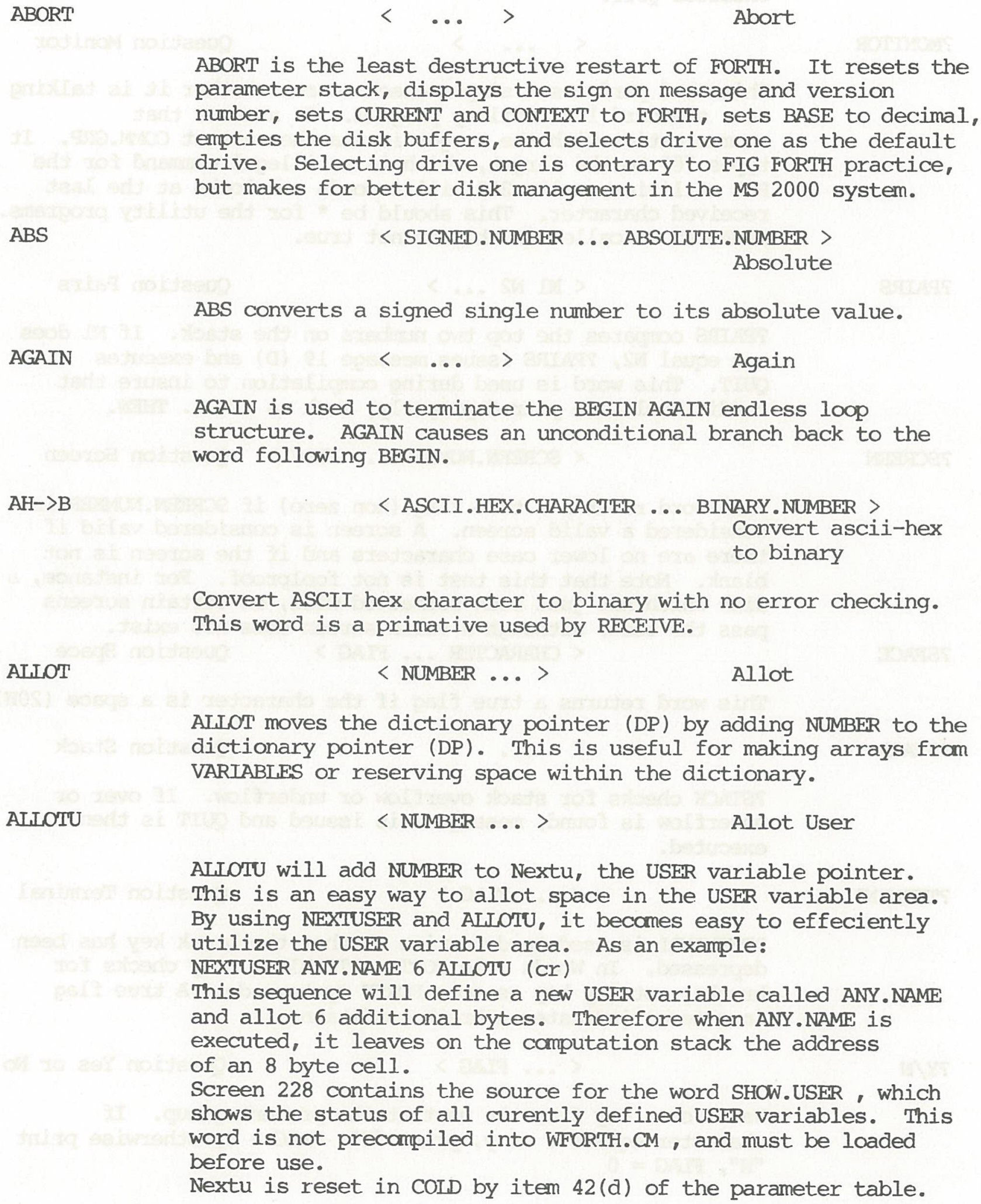

ABORT is the least destructive restart of FORTH. It resets the parameter stack, displays the sign on message and version number, sets CURRENT and CONTEXT to FORTH, sets BASE to decimal, empties the disk buffers, and selects drive one as the default drive. Selecting drive one is contrary to FIG FORTH practice, but makes for better disk management in the MS 2000 system.

ABS

AGAIN

< SIGNED.NUMBER ... ABSOLUTE.NUMBER >

Absolute

ABS converts a signed single number to its absolute value.

AGAIN is used to terminate the BEGIN AGAIN endless loop structure. AGAIN causes an unconditional branch back to the word following BEGIN.

$\mathrm{AH}->\mathrm{B}$

< ASCII.HEX.CHARACTER ... BINARY.NUMBER > Convert ascii-hex to binary

Convert ASCII hex character to binary with no error checking. This word is a primative used by RECEIVE.

ALIOT

$$
\langle\text { NUMBER ... }>\text { Allot }
$$

AILOT moves the dictionary pointer (DP) by adding NUMBER to the dictionary pointer (DP). This is useful for making arrays from VARIABIES or reserving space within the dictionary.

AIIOTU

$$
\langle\text { NUMBER ... } \quad \text { Allot User }
$$

ALIOTU will add NUMBER to Nextu, the USER variable pointer. This is an easy way to allot space in the USER variable area. By using NEXTUSER and ALIOTU, it becomes easy to effeciently utilize the USER variable area. As an example: NEXIUSER ANY.NAME 6 ALIOTU (cr)

This sequence will define a new USER variable called ANY.NAME and allot 6 additional bytes. Therefore when ANY.NAME is executed, it leaves on the computation stack the address of an 8 byte cell.

Screen 228 contains the source for the word SHOw.USER, which shows the status of all curently defined USER variables. This word is not precompiled into WFORTH.CM , and must be loaded before use.

Nextu is reset in COID by item $42(d)$ of the parameter table. 
AND performs a logical AND of the top two 16 bit numbers on the stack and replaces them with the logical result.

ANSWER

$$
<\ldots>\text { Answer }
$$

Initialization

Answer initialization is a primitive used by answer

$\langle\ldots\rangle$ Answer

This word sets the FORTH system for terminal control from the modem group (normally FOh). It waits for a ring on the 18S653VI modem, answers the ring, and changes GRP and TERM.VAR to the modem group. This word allows complete remote access to a FORTH system, in full duplex mode.

ASSEMBIFR

$$
\langle\ldots\rangle \text { Assembler Load }
$$

This word loads the ASSEMBLER and sets the CONTEXT vocabulary to ASSEMBLER. If the ASSEMBIER is already loaded, it merely changes CONIEXT to ASSEMBLER.

ASMBR

$$
<\ldots>\text { Assembly Find }
$$

This word which functions as NO-OPERATION (NO-OP) serves to mark the end of the ASSEMBLER defined FORIH and the start of SCREEN defined FORTH. It merely serves as a convienent word to FORGET when recompiling WFORTH.CM. ASMBR is defined on screen 81. After a new assembler pass, or if you wish to install a different printer and terminal combination, load screen 82 or 243 depending upon desired terminal printer configuration. This will create a new FORTH system by loading screen 80, the Master load screen.

$\mathrm{B} / \mathrm{BUF}$

$$
\langle\ldots \text { VALUE }>
$$

Bytes per Buffer

$\mathrm{B} / \mathrm{BUFF}$ is a CONSTANT whose value equals the number of bytes per BLOCK. In Woods Hole FORTH, B/BUFF equals $1024(\mathrm{~d}) ; 400(\mathrm{~h})$.

$\mathrm{B} / \mathrm{SCR}$

$$
\langle\ldots \text { VAIUE }>\quad \text { Blocks per Screen }
$$

$\mathrm{B} / \mathrm{SCR}$ is a CONSTANT which is equal to the number of blocks per editing screen. B/SCR equals 1 in Woods Hole FORTH. By FORTH convention, a screen is 1024 (d) or 400 (h) characters.

BACK

$$
\text { < STARTING.ADDRESS ... > Back }
$$

BACK is a compiling word used to resolve the backwards branch caused by (LOOP) and (+LOOP) in LOOP structures. 
BASE is a USER variable which contains the current number base used for binary to ASCII (output) and ASCII to binary (input) number conversions.

BEFP

BEGIN

BETL,

$\mathrm{BL}$

BLANKS

BLK

BLOCK

$$
<\ldots>\quad \text { Begin }
$$

Begin is used to mark the beginning of the indefinite loop structures. These are:

BEGIN (any words) UNTII,

BEGIN (any words) END

BEGIN (any words) AGAIN

BEGIN (any words) WHIIE REPEAT

Pseudonym for BEIL. It sends the BELI character (07h) to the terminal.

Beep

$$
<\quad \ldots>
$$

Bell

BEIL sends the ASCII BEIL character $(07 \mathrm{~h})$ to the selected uart.

$$
\langle\ldots 20(\mathrm{~h}\rangle \quad \text { B I }
$$

BL, is a CONSTANT equal to $20(\mathrm{~h})$. This is the ASCII character for blank or space.

$\langle$ BEGINNING.ADDRESS LENGTH $\ldots$.. >

\section{Blanks}

BLANKS fills memory, starting at BEGINNING.ADDRESS for LENGTH bytes, with the ASCII character blank (20h). This is a byte ( 8 bit) operation. IENGTH refers to the number of bytes.

$$
\langle\ldots \text { BLK }\rangle \text { BLK }
$$

BLK is a USER variable which contains the number of the BIOCK currently being interpreted. If BLK contains 0 , the input stream is from the terminal. QUIT sets BLK to zero.

$$
\text { < BLOCK.NUMBER ... DATA.ADDRESS > }
$$

Block

BLOCK is used to transfer data to/from mass storage, normally disk. BLOCK may call BUFFER and R/W. BLOCK can be called by the user; for instance "0 BLOCK DROP" will force a disk head recalibration for the selected drive by forcing the disk drive to seek information on track 0 . This is required immediately after a hardware system reset. 
BLOCK-READ

BLOCK-WRITF:

BRANCH

BRANCH.DSP

BUFFER

BYE

C!

C,

$\mathrm{C} / \mathrm{L}$ $\langle$ IOCB.ADDRESS ... > Block Read

BLOCK-READ is the word which actually performs DISK-reads via utility program UT71. It reads two sectors (1 screen) from the disk.

$\langle$ IOCB.ADDRESS $\ldots>$ Block Write

BLOCK-WRITE is the word which actually performs DISK-writes via utility program UT71. It writes two sectors (1 screen) to disk.

$$
\langle\ldots\rangle \text { Branch }
$$

BRANCH is compiled into definitions by EISE, AGAIN, LOOP, +LOOP and REPEAT. It causes an unconditional branch by adding the compiled offset (stored in the two bytes following the CFA of OBRANCH) to the Instruction Pointer (IP).

$$
\langle\ldots\rangle \quad \text { Branch Display }
$$

Decompile the branch at WORD.PTR. This is a primative used by the decompiler.

$$
\text { < BLOCK.NUMBER ... DATA.ADDRESS > }
$$

Buffer

BUFFER is the word which writes an allocated buffer to disk, if updated, when all buffers are full and one block must leave memory. Buffer is not normally used directly, but rather, is called by the word BLOCK.

$$
\langle\ldots\rangle \quad \text { Bye }
$$

This word calls FLUSH and then returns to the monitor program UT71.

$$
\langle\text { CHARACTER ADDRESS ... > Character Store }
$$

C! stores 1 byte (the low byte of CHARACTER) at ADDRESS.

$$
\langle\text { CHARACTER ... > C Comma }
$$

C, stores the low byte of CHARACTER into the next available dictionary location and increments by 1 the dictionary pointer (DP). Notice that C, only stores the low byte of CHARACTER, not a full 16 bit word.

$$
\langle\ldots \text { VALUE }>\quad \text { Characters Per Line }
$$

$\mathrm{C} / \mathrm{L}$ is a CONSTANT whose value is equal to the number of characters per line on a screen. It is normally 64(d). 
CQ retrieves 1 byte ( 8 bits) from ADDRESS and leaves it as a 16 bit number on the stack. The high 8 bits on the stack are equal to 0 .

CASE

CATLUPDATE

This is the CASE statement by Dr. Charles Eaker. It is described fully in FORTH Dimensions Volume II, page 37. The CASE statements serves as a computed go to in FORTH. It is used in the following form.

: TASK

CASE

NUMBERI OF

NUMBERN OF

(No-Match Words)

ENDCASE ; $\langle$ NUMBER ... >

(Words) ENDOF

(Words) ENDOF

Task expects a number on the stack (NUMBERI through NUMBERN). It then looks for a match with the arguments of the statements. If a match if found, the words between $\mathrm{OF}$ and ENDOF are executed and the word TASK is terminated. If there is no match, the NO-MATCH words are executed. Notice that there are no restrictions on numbers. They can be computed dynamically and FORTH operations may be interleaved between OFENDOF pairs. The stack behavior shown for CASE, OF, ENDOF and FNDCASE is not the actual stack action, but merely the stack action perceived by the programmer. The additional stack numbers are for compiler security. CASE is an IMMEDIATE word.$$
\text { Cluster Allocation }
$$$$
\text { Table Update }
$$

This word marks the cluster allocation table of the diskette in the selected drive, to show sectors 130 through 499 as occupied. This corresponds to screens 65 through 249. This protects screens 65 through 249 from the operating system.

CDER

$$
\langle\ldots\rangle \quad \text { Check Disk Error }
$$

CDER examines register D.O after the call to DWRITE in UT71. If an error is found, message 8 is printed, and execution continues. This is a primative used by $\mathrm{R} / \mathrm{W}$.

CFA

$$
\text { < PARAMETER.FIEID.ADDRESS...CODE.FIETD.ADDRESS > }
$$
CFA

CFA converts a Parameter Field Address (PFA) of a dictionary definition to its Code Field Address (CFA). CFA is defined as : CFA 2- ;
}$$
<\cdots>>
$$ 
This word requires the WHOI uart switcher card. It resets the remote computer connected to CHANNEI.NUMBER. Consult the section on special hardware for the schematic of this card.

CHANNEL-SEI.ECT $\langle$ CHANNEL.NUMBER ... $>$ Channel select

This word switches the uart at CHANNEL. NUMBER of the uart switcher card to the uart at the comm group. The group number in force is not affected.

CHAPTEER

$$
<\text {... ADDRESS > Chapter }
$$

CHAPTFR is a user variable used by the word .FOOT - The user is responsible for initializing and updating CHAPTER . see screens 73 and 74 for examples.

CIEAR

$$
\text { < SCREEN.NUMBER ...> Clear }
$$

This word fills SCREFN. NUMBER with blanks. It does not necessarily write this screen to the disk, however. FLUSH must be called to insure that a blank screen is written to disk.

CIFAR-KEY

$$
<\text {... > Clear Key }
$$

CIFAR-KEY checks the currently selected uart for a character. If there is a character present, it reads it and then drops it.

CIS

$$
<\ldots>\text { Clear Screen }
$$

This word sends the CLEAR-SCREEN character (26d) to the terminal.

CMOVE:

< SOURCE.ADDRESS DEST.ADDRESS IENGTH ... >

Character Move

CMOVE moves LENGTH bytes from SOURCE. ADDRESS to DEST.ADDRESS starting at SOURCE.ADDRESS. If DEST.ADDRESS is greater than SOURCE.ADDRFSS but less than SOURCE.ADDRESS+LFNGTH, use CMOVE> (character move up).

CMOVE>

$$
\text { < SOURCE.ADDRESS DEST'ADDRESS IENGTH } \ldots \text {. > }
$$

Character Move Up

CMOVE> moves IENGTH bytes from SOURCE.ADDRESS to DEST.ADDRESSS, starting at SOURCE.ADDRESS + IENGIH - 1 (the end of the block). CMOVE $>$ is used when the destination string overlaps the source string, and is higher than the source string.

COLD

$$
\langle\text { AII.VALUES } \ldots>>\text { Cold }
$$

Cold initializes USER variable $10(h)$ through $1 E(h)$, FENCE, DP, VOC-LINK SCR/DSK, MODEM.GRP, TERM.GRP, COMM.GRP AND Nextu, and then executes WARM. Entering FORIH at 0000 causes a COID start. 
This word selects the group whose number is contained in the variable COMM.GRP. This is group EO(h) for normal operation.

COMM.CTL

COMM.GRP

COMMINIT

COMPIIE:

CONS

CONST.DSP

CONSTANT

$$
\langle\ldots \text { ADDRESS }>
$$

Communication Control

This VARTABIE hold the value of the control byte for the communications uart. This is normally ID(h) for the CDP1854A uart.

$$
\left\langle\ldots \text { ADDRESS }>\quad \begin{array}{l}
\text { Communication } \\
\text { Group }
\end{array}\right.
$$

This USER variable contains the value of the group number for the communication uart. This is normally E0(h) for the 18S64I uart card or $\mathrm{FO}(\mathrm{h})$ for the 18S653VI modem card.

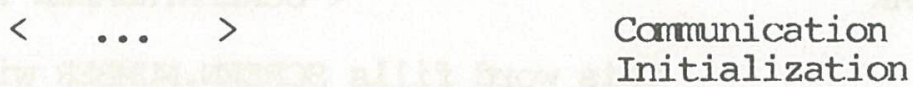

This word initializes the uart at the selected group.

$$
<\ldots>\quad \text { Compile }
$$

COMPILE will compile the Code Field Address (CFA) of the word following COMPIIE in the input stream, into the dictionary.

$$
\langle\ldots\rangle \quad \text { Console }
$$

This word unconditionally selects group 1 for terminal communication. It does not change TERM.VAR.

$$
\langle\ldots\rangle \text { Constant Display }
$$

Display the CONSTANT whose address is contained in the USER variable WORD.PTR. This word is a primative used by the decompiler.

$$
\langle\mathbf{N} \ldots\rangle\rangle \quad \text { Constant }
$$

A CONSTANT is defined in the form:

$\mathrm{N}$ CONSTANT ANY.NAME

where $\mathrm{N}$ is the value of the named CONSTANT.

When a constant is used, the value of the CONSIANT is left on the stack. CONSTANT differs from VARIABIE and USER in that the value is left on the stack when the name is invoked, rather than the address of the value. 
CONTEXT is a USER variable which points to the Name Field Address (NFA) of the most recently defined word of the VOCABUIARY that is to be searched first. ABORT causes CONTEXT to point to FORTH. CONTFXT is changed by executing a VOCABULARY name.

$$
\begin{aligned}
& \text { This word duplicates SOURCE. SCREEN at DESTINATION.SCREEN. Note } \\
& \text { that COPY is a FORTH word, not a FIG Editor word. FLUSH must } \\
& \text { still be called to insure that DESTINATION.SCREEN is written to } \\
& \text { disk. } \\
& \text { COUNT } \\
& \text { <STRING.ADDRESS ... STRING.ADDRESS+1 IENGTH > } \\
& \text { Count }
\end{aligned}
$$

COUNT increments STRING.ADDRESS and leaves the LENGTH of the stringasthetop stack item. COUNT assumes that thebyte pointed to by STRING.ADDRESS contains a count of the number of characters in the following string.

CR

$$
\langle\ldots\rangle \quad \text { Carriage Return }
$$

CR sends carriage return-line feed to the output device. It also increments the USER variable OUTI. This is not FIG-FORTH behavior, but it allows the user to keep track of output lines in a manner analogous to OUT, the USER variable counting output characters. The user is totally responsible for initializing and checking OUIL.

CRFATE

CRS Type carriage return without line feed. This is used when
sending messages to target computers. It is defined as : HEF : CR' OD EMIT ;

$$
<\ldots>>\text { Create }
$$

CREATE is a defining word used to create the header portion of a FORTH definition. It creates the name field, link field, and code field. The word : (COLON) uses CREATE.

$$
\text { < CHANNEL.NUMBER ... > Channel Reset }
$$

This word is the primitive for CHANNEI-RESET, and it requires the WHOI Uart switcher card. See Chapter 2 for the schematic.

$$
\langle\text { CHANNEI.NUMBER ... > Channel select }
$$

This word is the primitive for CHANNEL SETECT, and it requires the WHOI Uart switcher card. See Chapter 2 for the schematic. 
CSP

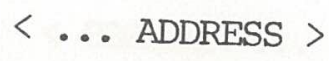

Compiler Stack

Pointer

CSP is a USER variable which is used by the compiler to temporarily store the stack pointer position for compiler
security checking.

$\mathrm{CII}=>$

$\langle$ NUMBER ... >

Control Output

This word sends the control byte on the stack to the uart of the selected group. Normally this means $1 D(h)$ is sent to port 3. CII $\Rightarrow$ is for a CDP1854A uart.

CUR

CURRENT

$$
\langle\ldots \text { ADDRESS }>
$$

Cursor

This USER variable is used by EDIT to store the curser position.

$$
<\text { ADDRESS }>\quad \text { current }
$$

CURRENT is a USER variable which points to the Name Field Address (NFA) of the most recently defined word in the CURRENT vocabulary; the vocabulary to which definitions are currently being appended. When a new word is defined, the

CV.DSP contents of CURRENT become the link of the newly defined word.
Cder

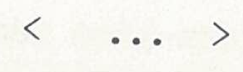
Current value display
Current value display is a primitive used by the decompiler. It types the text message: currently $=$.
Check disk error
Cder is a primitive used by CDER. It checks to see if there
D+

$$
<\mathrm{Dl} \mathrm{D} 2 \ldots \mathrm{D} 1+\mathrm{D} 2>\quad \mathrm{D} \text { Plus }
$$
D+ adds two signed double numbers and replaces them with the double ( 32 bit) signed sum. Overflow is possible, and no

$$
\text { D+- }
$$

$$
<\text { D1 N2 ... D3 > }
$$
Double plus Minus
D3 equals DI if N2 is positive. D3 equals -DI if N2 is This word is a primative used byped from the stack.
D.

$$
\text { < DOUBIE. NUMBER ...> > Double Print }
$$
D. prints the top two stack numbers as a signed double number (32 bit) in the current number BASE. 
D.R

D.RLZ

DABS

$\mathrm{DEC}$.

DECIMAL

DECOMPIIE

DEFINITIONS

DEPTH $\langle$ D FIETD.WIDTH $\ldots>>$
Double Print

Right Justified

The double number $\mathrm{D}$ is printed, padded with leading spaces, for a total number of characters equal to FIFID.WIDTH. If the total is greater than FIFID.WIDTH, all characters are printed.

$\langle$ D FIETD.WIDTH $\ldots>\rangle$

Double Print Right Justified wi.th Leading Zeroes

The double number $\mathrm{D}$ is printed, padded with leading zeroes for a total number of characters equal to FIEID.WIDTH. If the total is greater than FIEID.WIDTH, all characters are printed.

$$
\begin{gathered}
<\text { SIGNFD.DOUBIE } \ldots \text { ABSOLUTE.DOUBIE }> \\
\text { Double Absolute }
\end{gathered}
$$

DABS replaces a signed 32 bit number with its absolute value.

$$
\langle\mathrm{N} \ldots\rangle \quad \text { Decimal print }
$$

Decimal print will print the number $\mathrm{N}$ in decimal, regardless of the current number BASE, without changing BASE.

$$
\langle\ldots\rangle \text { Decimal }
$$

DECIMAI sets the USER variable BASE to $10(\mathrm{D})$. This causes all. Ascii numeric input and output conversions to be performed in base 10 (Decimal).

Usage: DECOMPILE "ANY.WORD". This is Ray Duncan's decompiler, extended to decompile vocabularies, recognize immediate words and generally handle more situations. Chapter 20 has many examples of decompiler utilization.

$$
<\ldots>\text { Definitions }
$$

Definitions is used to specify the VOCABULARY into which new definitions are to be compiled. It simply sets CURRFNT equal to CONTEXT.

$$
\langle\ldots \text { DEPTH }>\quad \text { Depth of Stack }
$$

This word leaves the number of numbers on the stack (before the word was called). This is a primative for.s (print stack). 
This word expects a string to follow it. The delimiter is a space or carriage return. DIAL then examines the string and acts upon it. The valid characters are:

The digits 0 through 9. These are dialed directly. The symbols \# (pound) and *(star). Those are dialed directly. The symbol, (comma). This forces a $250 \mathrm{millisecond} \mathrm{wait.}$ The symbol ; (semicolon). This forces a wait for a dialtone.

DIAL will execute QUIT after 5 seconds without a dialtone. DIAL should be fol lowed by ORIGINATE to enter terminal emulation mode.

DIALINIT

DIALTIONE

DIGIT

DISKID

DISKLIST
DIR

$$
<\ldots>>
$$

Dial Initialization

This word sets the modem for dialing. It is a primative used by DIAL.

$$
<\ldots>\quad \text { Dialtone }
$$

This word loops for approximately 5 seconds while checking for a dialtone at the modem at MODEM.GRP. It executes QUIT if no dialtone is detected after 5 seconds.

$$
\begin{aligned}
& <\text { CHARACTER BASE } \ldots \text { BINARY.DIGIT TRUE.FLAG > or } \\
& <\text { CHARACTER BASE } \ldots \text { FAISE.FIAG }>
\end{aligned}
$$

Digit

DIGIT convers an ASCII character to a binary number if it can. A false flag (zero) indicats that DIGIT could not convert the character using the present BASE.

DISKID

$$
<\ldots>\text { Directory }
$$

Directory prints the name and starting sector number of each file on the disk in the selected drive.

$$
<\ldots>\text { Disk ID }
$$
creation date.

$$
\begin{array}{r}
<\text { STARTING.SCREEN ENDING.SCREFN } \ldots>> \\
\text { Disk List }
\end{array}
$$

This word lists screens STARTING.SCREEN through ENDING.SCREEN. The list can be prematurely terminated with the break key. The listing can be directed to a printer by executing the word PRINTER before executing DISKLIST . 
DLITERAL

$<$ DOUBLE.NUMBER ... > Double Literal

DLITERAL compiles a double number from the stack as a double literal into a colon definition.

DMINUS

< DOUBLE ... NEGATIVE.DOUBLE >

D Minus

DMINUS negates the signed DOUBLE number on the stack by replacing it with the 32 bit 2 's complement number.

DO

$$
<\text { I.imit\# Start\# } \ldots>\text { D D }
$$

DO is used to build a DO LOOp in conjunction with LOOP or +LOOP in the forms:

: EXAMPLE]. Limit\# Start\# "loop body words" LOOP ;

: EXAMPIE2 Iimit\# Start\# DO "loop body words" Increment\# +LOOP ; Within the loop, the value of the index can be brought to the stack with the word I . I,imit\# and Start\# need not be defined in the word that contains the loop. They may be supplied on the stack when the word containing the loop is executed. In the case of both examples, the index is incremented (by 1 for LOOP, or by Increment\# for +LOOP) until the index equals or exceeds Limit\#. At that point the loop is exited and the word following LOOP or HIOOP is executed. DO must be used within a colon definition.

DOES>

DOES.DSP

DOS

$$
<\ldots>\text { Does }
$$

DOES> is a defining word normally used in conjunction with <BUIIDS. DOES> starts the high level words which are executed when the defined words are executed. See <BUILDS in the FORTH Encyclopedia for a thorough explanation.

$$
<\ldots>\text { Does display }
$$

Does display is a primitive used by the decompiler.

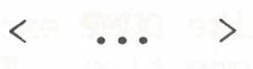

Disk Operating System

This word unconditionally returns to the disk operating system at $9005(\mathrm{~h})$. The disk operating system must have been loaded previously.

DOWNLOAD

$$
\begin{gathered}
<\text { SOURCE.ADDRESS IENGTH TARGET.ADDR ... > } \\
\text { DOwnload }
\end{gathered}
$$

DOWNLOAD moves a block of data from SOURCE.ADDRESS of length LENGTH to a target processor at address TARGET.ADDRESS. The target computer is connected to a uart at the COMM.GRP (communications group). The target computer must be running an RCA utility program. BASE must be HEX to use the word DOWNLOAD. The connection to the target computer is via its terminal input. 
DP is a USER variable which points to the next available dictionary location. This must be changed to point to RAM for an EPROM application in FORTH.

DPL

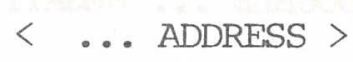

Decimal Point Location

DPL is a USER variable which contains the number of digits found to the right of the last decimal point, when converting ASCII to binary input with the word NUMBER.

DRO

$\mathrm{DR}]$.

$$
<\ldots>\quad \text { Drive Zero }
$$

DR0 stores the value 0 in the USER variable OFFSET, thereby selecting drive zero. In this case, screens 0-314 are located on drive zero, and screens 315-629 are located on drive one.

$<\ldots>\quad$ Drive 1

DRI stores the value 315 (d) into the USER variable OFFSET. This word selects drive 1 for screens $0-314$. Screens 65 through 249 are protected from the operating system by the word CAT.UPDATE. If you plan to use operating system files on any disk, you should restrict yourself to screens 65 through 249 .
DROP
DROP
$<\mathrm{Nl} \ldots>$
Drop
DROP deletes the top item from the stack.
DUMP
$<$ STARTING.ADDRESS LENGTH $\ldots>$
Dump
DUMP-TERM
This word lists memory contents in Hex and ASCII.

D

$$
\text { < START.ADDRESS LENGTH ... > Dump to Terminal }
$$
This word works just like DUMP except that only 16 lines (256 bytes) are printed at one time. The operator is prompted to continue the terminal dump.

\section{DUP}

$$
\langle\mathrm{Nl} \ldots \mathrm{Nl} \mathrm{Nl}\rangle \quad \text { Duplicate }
$$
DUP leaves a copy of the top stack item on the stack.
EDIT$$
\langle\mathrm{N} \ldots\rangle>\quad \text { EDIT }
$$
Edit screen number N. Edit is a full screen editor with full cursor control. GOTOXY, SETEDIT, and NOEDIT must be customized for individual terminals. Also the cursor movement keys may be different. EDIT requires the CASE statement by Charles Eaker and the USER variable CUR . EDIT is terminated by the escape key. EDIT changes the CONIEXX vocabulary to EDITOR. 
EDITT

EDITOR

$\mathrm{EF}$

ELSE

EMIT

EMIT'

EMPTY-BUFFERS

ENCLOSE $\langle\quad \cdots \quad>$

EDIT SCREEN L
Edit the screen at SCN, the screen most recently called by LIST. EDITL changes the CONTEIXT vocabulary to FDITOR.

This word selects the EDITOR vocabulary. The editor is the fig line editor, described in the fig Installation Manual.

$$
\langle\ldots \text { CONSTANT }>\quad \text { External Flag }
$$

$\mathrm{EF}$ is a constant which points to a group of routines for monitoring the external flags. FF is a primative used to create the external flag words, W1 WN2 EF3, etc.

Else is used within an IF ELSE THEN structure at the beginning of the false portion of the test. The use of FISE is optional in the IF ELSE THEN structure.

$$
<\text { CHARACTER ... }>\text { Enit }
$$

FMIT sends CHARACTER to the output device and increments the USER variable OUT. Total output format control is possible using the word OUT. See the source for VLIST as an example.

$$
\langle\text { CHARACTER ... }>\text { Emit Tick. }
$$

FMIT' is a version of EMIT which sends CHARACTER to the uart at COMM.GRP. This is a primitive used by the communication utilities.

$$
<\ldots>\quad \text { Empty Buffers }
$$

Empty buffers clears all buffers to value 00 . The update and terminating words are set to 00. EMPTY-BUFFERS is executed within the word ABORT and therefore by COID and WARM also.

$$
\begin{gathered}
\text { < STRING.ADDRESS DETIMITER ... STRING.ADDRESS } \\
\text { NON. DECIM.OFFSET DECIM.OFFSET TOTAL.OFFSET > } \\
\text { EnclOSE }
\end{gathered}
$$

ENCLOSE performs a parsing function on a character string. ENCLOSE is the primative used by WORD. This version of ENCIOSE does not conform to fig-FORTH in that it allows more than 256 (d) consecutive spaces be contained on a screen before executing is or $\rightarrow$. 
End is a pseudonym for UNTIL, used in the BEGIN UNTIL structure. The flag is examined; if true, the BEGIN-END loop is terminated. If false, there is an unconditional branch to the word following BEGIN. UNTIL is preferred over END.

ENDCASE

$$
\langle\ldots>\quad \text { Find Case }
$$

This word is the final word in the CASE statement construct. ENDCASE is an immediate word. See CASE for a complete discussion of ENDCASE.

ENDIF

$$
<\ldots>\quad \text { End If }
$$

ENDIF is a pseudonym for THEN, used to terminate an IF THEN, or IF ELSE THEN conditional structure. THEN is preferred over ENDIF.

ENDOF

$$
<\ldots>\quad \text { End of }
$$

This word terminates an Of-ENDOF pair in the CASE statement. ENDOF is an immediate word. See CASE for a complete discussion of ENDOF .

EOF

ERASE

$$
\langle\ldots 13 h\rangle
$$

End of File

(Control S)

EOF is a CONSTANT equal to $13(\mathrm{~h})$. This is the end of file character, for files created by the operating system.

$$
\langle\text { BEGINNING.ADDRESS IENGTH ... > }
$$

Frase

ERASE fills memory starting at BEGINNING.ADDRESS for LENGTH bytes with $00(\mathrm{~h})$. This is a byte operation. IENGIH refers to the number of bytes, not words.

ERROR

$$
<\text { MESSAGE.\# ... CONTENT.OF.IN CONTENT.OF.BLK }>
$$

ERROR displays an error message, stops compilation, and starts interpretation from the terminal. The form of the error message is governed by the contents of the USER variable WARNING. If WARNING contains a negative number, (ABORT) is executed. (ABORT) may be modified to call a user error handling routine. If WARNING is zero or positive. Message.\# is printed to the terminal. This is the normal state for WFORTH. The contents of IN and BLK are saved on the stack to help determine the location and cause of the error. 


$$
\langle\ldots 1 \mathrm{~B}(\mathrm{~h})\rangle \quad \text { Escape }
$$

This word is a CONSTANT, which returns the ASCII value of the character escape (lBh).

ESC'

$$
<\ldots>\quad \text { Escape Tick }
$$

This word sends an escape character $1 \mathrm{~B}(\mathrm{~h})$ through the uart at COMM.GRP.

EXECU'TE

EXPECT

F-LFEN

FFNCE

FILEID

FILELOAD

FIIL

$$
<\text { CODE.FIEID.ADDRESS } \ldots>>\text { Execute }
$$

EXECUTE executes the word whose Code Field Address (CFA) is on the stack. This word is called by INTERPRET as part of the indefinite loop QUIT, and is normally only used in this way.

$$
<\text { BUFFER.ADDRESS COUNT } \ldots>>\text { Expect }
$$

EXPECT receives characters from the terminal until COUNT characters are received or a carriage return is encountered. The string is stored at BUFFER.ADDRESS.

$$
\langle\ldots \text { FIIE.IENGTH }>\quad \text { File Length }
$$

File Length is a primative used by SAVE. It returns the length of the file that is presently open or an irrelevant number if no file is open.

$$
\langle\ldots \text { ADDRESS }\rangle \quad \text { Fence }
$$

FENCE is a USER variable which holds the lower limit for FORGET'. FORGET will not forget any word located below FENCE. NEW-FENCE will move the fence to protect all words presently defined, and change the value of FENCE and DP in the boot-up parameter table.

$$
<\ldots>\text { File ID }
$$

This word will print the name of the file most recently opened by OPEN.

\section{FILELOAD}

\section{Usage: FILFLOAD FIIFSPEC (cr)}

File load will load any binary file, into the block of memory specified by the file itself. It loads by complete sectors, however, and will write beyond the actual end of the file if the file doesn't end on a sector boundary.

$$
\text { File load }
$$

$$
\text { < BEGINNING.ADDRESS IENGTH CHARACTER > }
$$

Fill

This word fills memory starting at BEGINNING.ADDRESS for IENGTH bytes with CHARACTER. This is a byte operation. IENGTH refers to the number of bytes. 
FIRST

FLD

FLOATING

FLUSH

FNAME

FORGET

FORTH

GET-SCREEN $\langle\ldots$ First.address $\rangle \quad$ First

FIRST is a CONSTANT whose value is equal to the address of the first available byte in the disk buffer area.

$$
\langle\ldots \text { ADDRESS }\rangle \quad \text { FTD }
$$

FID is a USER variable which was originally intended to control field length in pictured numeric output. It is presently unused in FIG FORTH. In Woods Hole FORTH it is used for page numbering. FLD is incremented by the word PAGE. The user is responsible for initializing FLD.

$$
<\ldots>\text { Floating point }
$$

FLOATING will load the floating point words from screens 143 through 163, into the last 4 screen buffers. Once they have been loaded, FLOATING merely displays a message stating that Floating point is loaded.

$$
\langle\ldots\rangle \quad \text { Flush }
$$

FLUSH writes to disk all buffers that are presently flagged as updated.

File Name

This is a primative used by OPEN.

$$
<\ldots>\quad \text { Forget }
$$

FORGET removes definitions from the dictionary fromthemost recently defined word to the word specified. The CURRENT and CONTEXT vocabularies must be the same or message $24(d)$ is issued, and nothing is forgotten. FORGET searches for the most recent occurrence of the word. If the value contained in FENCE is less than the NFA of the word, then DP (the Dictionary Pointer) is moved to the forgotten word and new words are then compiled there. The usage is: FORGET ANY.NAME ( $\mathrm{Cr})$

$$
<\quad \cdots \quad>
$$

FORTH

Executing FORTH causes CONTEXT to point to the FORTH vocabulary. FORTH is the main VOCABULARY of FORTH systems. All new vocabularies link to FORTH.

$$
\text { < SCREEN.NUMBER ...> > Get Screen }
$$

This word is used to transfer screens between FORTH systems. It assumes that another FORTH system is connected to the uart at COMM.GRP.

To Use: N GET-SCREFN (at the receiving end; then) $M$ SEND-SCREEN (at the sending end)

where $\mathrm{N} 1$ is the screen number being received and $\mathrm{N} 2$ is the screen number being sent 
This is the sequential file access word. Each time it is executed it reads a sector into the buffer and returns the BUFFER.ADDRESS of the next sector in the opened file, or a zero if the end of the file is reached. Notice that a file can be changed by calling GETSEC, changing the file in memory, and then executing: UPDATE FLUSH.

GOTOXY

$$
\langle\mathrm{XY} \ldots\rangle
$$

Move Cursor to $\mathrm{X}, \mathrm{Y}$

This word moves the curser to row $\mathrm{X}$, column $\mathrm{Y} .0,0$ is the top left corner. This word must be changed for different terminals. GOTOXY is used by EDIT, the full screen editor.

$$
\langle\text { NUMBER ... Group }
$$

GROUP does two level group select using the RCA convention by sending NUMBER to port 1 and also storing NUMBER in the USER variable GRP. By combined use of GROUP and GRP, it is possible to perform I/O without disturbing group selections currently in force. $\mathrm{R} / \mathrm{W}$ will restore the group number to its previous value after performing I/O from the disk. This is particularly important if the terminal group is to be something other than group 1. Refer to the source for PARPIR for an example of the usage of GRP and GROUP.

$$
<\ldots \text { ADDRESS }>\quad \text { GRP }
$$

GRP is a user variable which contains the group number most recently selected by the word GROUP. The use of GRP and GROUP allows the programmer to restore a group number after I/O. This is especially useful where I/O might change the group number, as in disk calls to DREAD AND DWRITE in UT71. UT71 sets the group number to 01 after I/O. By executing: GRP a GROUP after non-FORTH I/O the group which was most recently selected by the word GROUP is restored. This allows full terminal operation from a group other than 01 . $R / W$ uses this convention. The word ANSWER relies on this fact to permit full terminal operation from the modem at group $\mathrm{FO}(\mathrm{h})$.

HERE

$$
<\ldots \text { ADDRESS }>\quad \text { Here }
$$

HERE places the address of the next available dictionary location on the stack.

HEX

$$
<\ldots>\text { Hex }
$$

HEX sets the USER variable BASE to 16. This causes all numeric input and output conversions to be performed in base 16(d).

HEX.

$$
<\mathrm{N} \ldots>>\text { Hex print }
$$

Hex print will print the number $\mathrm{N}$ in hex, regardless of the present number BASE, without changing BASE. 
HID is a USER variable which contains the address of the last character of text placed into (below) PAD during binary to ASCII pictured numeric output. <\# initializes HLD, and HOID uses HID.

HOID

HOM

I

ID

ID.

IF

IMMEDIATE

$$
\langle\text { ASCII.CHAR ... H Hold }
$$

HOLD stores an ASCII character into the next available location in a pictured numeric output string. Punctuation may thus be inserted into number strings. Refer to <\#, \#, SIGN, PAD, HID, \#S, and \#> for other operators for pictured numeric output.

$\langle\ldots\rangle \quad$ Home

Move the cursor to the top left corner of the edit screen. This is a primative used by EDIT.

$$
\langle\ldots \text { INDEX }\rangle \quad \text { Loop Index }
$$

I copies the current DO LOOP or DO +LOOP index from the top of the return stack onto the top of the computation stack.

$$
<\ldots>\text { ID }
$$

list the identification of this task. In a multiprocessor environment, it is useful to give each system a different ID, to simplify debugging. In Woods Hole FORTH, the ID is taken from information contained in the word ABORT .

$$
\text { < NAME.FIELD.ADDRESS ... > ID Print }
$$

ID. lists the name field of a word at location NAME.FIFID.ADDRESS.

$$
\langle\mathrm{flag} \ldots\rangle \quad \text { If }
$$

If is used in the IF ELSE THEN structure. The flag is examined; if true (non-zero), the words between IF and EISE are executed. If the flag is false, the words between ELSE and THFN are executed. The use of ELSE is optional. Without it, nothing happens if the flag is false.

$$
<\ldots>\quad \text { Immediate }
$$

IMMEDIATE sets the precedence bit of the most recently created definition. A definition whose precedence bit has been set will be executed immediately by INTERPRET even if the system is in COMPIIE state. It is possible to compile an immediate word by preceding it with [COMPIIE] . The precedence bit is the second most significant bit of the name field's length byte (NFA). 
IN is a USER variable which contains the byte offset from the beginning of the input buffer (whether TIB or disk buffer) to the end of the word most recently interpreted. >IN is the FORTH-79 equivalent for IN.

INDEX

$$
\text { < STARTING.SCREEN ENDING.SCREEN ... > }
$$

Index

This word prints the index line for the screens STARTING.SCREEN through ENDING.SCREEN, if the index line passes the ?INDEX test. In this implementation of FORTH, the names of all words defined on a screen are listed within parentheses on line zero of that screen. If this convention is followed, then the Index serves as a directory to the defining (source) screens.

INPUT

$$
\text { < PORT.NUMBER ... CHARACTER > }
$$

Input

Input will read an I/O port. No group select is performed, the user is responsible for group select.

$$
<\ldots>\text { Interpret }
$$

INTERPRET is referred to as FORTH's outer interpreter. INTERPRET simply interprets the text input stream from the terminal if BLK equals zero, or from disk if BLK is non-zero. INTERPRET first searches the CONTEXT, then CURRENT vocabularies for a match. If one is found, the word is compiled or executed, depending on the state of the system. If no match if found, interpret attempts to convert the word to a number, either placing it on the stack or compiling the number depending on the state of the system. If the word cannot be converted to a number, then message 0 is issued. INIFRPRET Is an endless BEGIN AGAIN loop which is terminated by the word null. INTERPRET is used by 2 words, QUIT and LOAD. 
This is a 9 byte VARIABIE used as an I/O control block for FORTH disk file activity. It is alloted as follows:

IOCB: 2 bytes: Starting sector number.

IOCB 2+: 1 byte: offset into current segment description block for current segment description word.

IOCB 3+: 1 byte: number of contiguous sectors pointed to by the current segment word.

IOCB 4+: 1 byte: number of sectors which have already been read from the current segment description word.

IOCB 5+: 2 bytes: starting sector number for the contiguous block pointed to by the current segment word.

IOCB 7+: 2 bytes: buffer address for the current sector.

For a complete description of the pointer block, refer to RCA document MPM $241 \mathrm{Pl}$, page E-5.

IOCB-SHOW

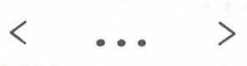

I/O Control block show

This is a teaching word which explains the variable IOCB.

KEY

$$
\langle\ldots \text { CHARACTER }>\quad \text { KeY }
$$

KEY reads one character from the input device. Note that KEY waits for a character. It is therefore possible to hang up when executing KEY, if no character is available at the uart.

L

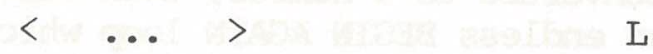

List the screen whose number is stored in the USER variable SCN. Also set the CONTEXT to EDITOR. This is similar to the EDITOR word $I_{\text {, }}$ but because it is a FORTH word, it can be found from any vocabulary.

$\mathrm{L} / \mathrm{P}$

$\langle\ldots$ VALUE $>$

Lines Per Page

$\mathrm{L} / \mathrm{P}$ is a CONSTANT whose value is equal to the number of printed lines per page. This constant can be compared with OUIL when printing, to determine when to issue form feeds, print headers, footers, page numbers, etc. $\mathrm{L} / \mathrm{P}$ is analogous to $\mathrm{C} / \mathrm{L}$ (characters per line) and is normally equal to $60(d)$. 
LATEST places the Name Field Address (NFA) of the most recently defined word in the CURRENT vocabulary on the stack.

LEAVE

$$
\langle\ldots\rangle \quad \text { Leave }
$$

IEAVE is used to prematurely exit a DO-LOOP. It does not immediately force termination of the loop, but rather sets the limit equal to the current index, so that when IOOP or HIOOP is encountered, the loop will be terminated in the normal fashion.

IFA

< PARAMETER.FIETD.ADDRESS...IINK.FIEID.ADDRESS > IFA

IFA converts a Parameter Field Address (PFA) of a dictionary definition to it.s Link Field Address (IFA).

LIMIT

$$
\langle\ldots \text { VALUE }>\quad \text { Iimit }
$$

LIMIT is a CONSTANT whose value is equal to the last available byte in the disk buffer area. The disk buffer area is bounded by FIRST and LIMIT.

LINE

LIST

$$
<\text { IINE.NUMBER ... ADDRESS > I.ine }
$$

LINE leaves the ADDRESS of LINE. NUMBER of the current editing screen. This word is used by the FDIIOR.

$$
\langle\text { SCREEN.NUMBER ... > List }
$$

This word lists SCREEN. NUMBER to the selected UART as 16 rows of 64 columns (1024 characters) of ASCII text. Woods Hole's FORTH system is inflexible with regard to screen size and format.

$$
<\ldots \text { NUMBER }>
$$

LIT

LIT is compiled into a dictionary definition by LITERAL and DLITERAL. It is the run time procedure for these words. IIT places the 16 bit contents of the next dictionary location on the stack.

IITTERAL

$$
\langle\mathrm{N} \ldots\rangle\rangle \quad \text { Literal }
$$

LITERAL compiles a number from the stack as a literal into a colon definition. 
IOAD interprets source text from SCREEN.NUMBER. Only NULU or ;S will terminate loading. Attempting to IOAD screen zero will cause a system crash. IOAD prints the screen number, the value of HERE when the screen is loaded, and the index line (line 0) of SCREEN.NUMBER.

LOADTHRU

$$
\begin{array}{r}
<\text { FIRST.SCREFN LAST.SCREEN ... > > } \\
\text { Load through }
\end{array}
$$

Ioad through will load a consecutive group of screens starting with FIRST.SCREFN, and ending with IAST.SCREEN.

$$
<\ldots>\quad \text { Loop }
$$

Loop is used to terminate a DO LOOP structure. The index is incremented by 1 . If the index equals the limit, the loop is exited and the word following IOOP is executed. If the index is less than the limit, the loop is repeated.

$M^{*}$

$$
\langle\mathrm{N} 1 \mathrm{~N} 2 \ldots \mathrm{D} 2\rangle \quad \mathrm{M} \text { Star }
$$

$M^{*}$ multiplies two single numbers and replaces them with their signed double product.

M Slash divides a 32 bit signed DOUBLE. DIVIDEND by a 16 bit signed DIVISOR and leaves a signed REMAINDER and QUOTIENT. The remainder takes its sign from the dividend.

$\mathrm{M} / \mathrm{MOD}$

$$
\begin{aligned}
& <\text { DOUBLE.DIVIDEND DIVISOR } \ldots \text { REMAINDER DOUBLE. } \\
& \text { QUOTIENT > }
\end{aligned}
$$

M Slash Mod M/MOD divides a DOUBLE.DIVIDEND by a single DIVISOR and returns a single REMAINDER and DOUBLE.QUOTIENT. All operations are unsigned.

MATCH

$$
\begin{aligned}
& <\text { CUR.ADDRESS LENGTH STRING.ADDRESS STRING.COUNT } \\
& \text {... FLAG MOVEMENT' > }
\end{aligned}
$$

Match

MATCH is a very useful sting comparison word. It will search an area starting at CUR.ADDRESS for LENGTH bytes for a match with the string at STRING.ADDRESS of length STRING.COUNT. It returns a true flag (non-zero) if a match if found, and MOVEMENT is the length between CUR.ADDRESS and the start of the found string. MATCH returns a false flag (0) if no match was found, and MOVEMENT is then equal to IENGTH. 
MEMORY-MAP

MESSAGE

MIN

MINUS

MOD

MODEM

MODEM.GRP

MON
MAX examines two signed single numbers and leaves the larger. Notice that $32000-32000$ MAX works correctly.

$$
<\ldots>\quad \text { Memory Map }
$$

This is a teaching word which shows allocation of memory. It. is useful when changing the origin table for ROM applications.

$$
<\text { MFSSAGE.NUMBER ... > Message }
$$

MESSAGE merely prints "Message $\mathrm{N}$ " where $\mathrm{N}$ is MESSAGE. NUMBER. Its action does not depend on the value of WARNING and it does not interrupt execution in any way.

$$
\text { < VALUE VALUE ... MINIMUM.VALUE > }
$$

Minimum

MIN compares the top two stack entries, saves the smaller and discards the larger. Notice that 32000 - 32000 MIN returns -32000 , unlike the FIG model.

$$
\text { < NUMBER ... NEGATIVE.NUMBER > }
$$

Minus

MINUS negates the top stack number by replacing it with its 2 's complement.

$$
\begin{array}{r}
\text { DIVIDEND DIVISOR ... REMAINDER }> \\
\text { Modulo }
\end{array}
$$

MOD performs a 16 bit signed division and leaves a 16 bit signed REMAINDER on the stack. The sign of the remainder is set to equal the sign of the dividend.

$$
<\ldots>\quad \text { Modem }
$$

This word selects the group number contained in the USER variable MODEM.GRP. This is group FO(h) for normal operation.

$$
\langle\ldots \text { ADDRESS }\rangle \quad \text { Modem Group }
$$

This USER variable contains the value of the group number for the modem uart. This is usually FO(h) for the CDP 18S653-VI modem card.

$$
<\ldots>\quad \text { Monitor }
$$

This word is an unconditional branch to UT71 at 87FO(h). The status of SUBMIT in the operating system is not altered. To defeat SUBMIT from UT71, type: $\mathrm{P} 8000$ ( $\mathrm{cr}$ )

to cause a cold restart of the utility program. 
This is a primative called by a ?MONITOR. It selects the terminal group, prints the message "NO MONITOR" and executes QUIT.

MSEC

$\langle\operatorname{TIME} \ldots\rangle$

Milliseconds

This word generates a delay of approximately TIME in milliseconds. The delay is based upon a $2.45 \mathrm{mHz}$ Cpu clock.

Match

Match

This is the primitive form of the word MATCH.

N.

NEW-FENCE

NEWLSN

NEWSDW

NEXTUSER

Nextu $\langle$ NUMBER ... $\quad$ Number Print

This word prints a number twice; once as a 5 column right justified signed decimal number, and once as an unsigned 4 digit hex number enclosed in parentheses.

$$
\langle\ldots\rangle \text { New Fence }
$$

This word protects all current entries in the dictionary. It moves the fence to HERE, and updates tORIGIN (parameter table) items 12, 28, 30 and 32 (decimal).

< NEW.LOGICAL.SECTOR.NUMBER ... BUFFER.ADDRESS >

This is the random access word for files generated by the operating system. It works in a similar fashion to GETSEC, except that the next sector number is taken from the stack. The VARIABLE IOBC is changed. After NEWLSN, GETSEC will. sequentially access the file.

This is a primative used by GETSEC.

$$
<\ldots>\quad \text { Next user }
$$

Example: NEXTUSER TEST.WORD

In the following example, next user automatically builds a USER variable named TEST.WORD in the next available USER variable cell, pointed to by Nextu, for the word TEST.WORD. This is a convenient alternative to the fig-FORTH method of defining USER variables. NEXTUSER TEST.WORD ( $\mathrm{Cr}$ )

$$
<\ldots \text { ADDRESS }>\quad \text { Next User Pointer }
$$

Nextu is a USER variable which contains the address of the next unassigned USER variable. 
NFA converts a Parameter Field Address (PFA) of a dictionary definition to its Name Field Address (NFA).

NODISK

NOEDIT

NOPRINT

NOT

NUMBER

$\mathrm{OF}$

OFFHOOK

OFFSET

OK

$$
\langle\ldots\rangle \text { No Edit }
$$

Disable the terminal for editing. This is a primative used by EDIT. It is written for a Televideo 910+ terminal and may require change or elimination for other terminals.

NODISK patches the word ABORT so that disks are not required in WFORTH.

No Printer

Disables the system printer.

$$
\langle\text { Flag ... Not.flag }>\quad \text { Not }
$$

NOT inverts the Flag on the stack. NOT is a FORTH 79 standard word. It is equivalent to the word $0=$.

$$
<\text { STRING.ADDRESS ... D > Number }
$$

Number uses the current BASE to convert a character string into a signed double precision number. The position of the last decimal point will be left in DPL.

$$
<\text { NUMBER KEY ... > Of }
$$

This is used in the CASE statement construct. If NUMBER equals KEY, then the words between $O F$ and ENDOF are executed and the case is terminated. OF is an immedate word. See CASE for a complete discussion.

$$
<\ldots>\text { Off Hook }
$$

This word sets the 18S653V1 modem to the OFFHOOK state. This is a primitive used by DIAL.

$$
\langle\ldots \text { ADDRESS }>\quad \text { Offset }
$$

OFFSET is a USER variable which contains the block offset to mass storage devices. If OFFSET equals zero, screens 0 through 314 are located on drive 0, and screens 315 through 629 are located on drive 1.

(1)

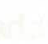

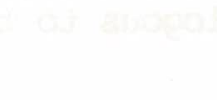

\footnotetext{
This word prints the message " OK".
} 
This word sets the modem to the disconnected or ONHOOK state. Sometimes, due to an untimely exit from ORIGINATE or ANSWER, this word must be used to disconnect the modem from the telephone line.

OPEN

$$
<\text { STARTING.SECTOR.NUMBER } \quad \cdots>>>>\text { Open }
$$

Usage: OPEN FIIESPEC. Open sets up the I/O control block, stored in the VARIABIE IOCB, for sequential reads of an existing file. OPEN selects the proper drive, finds the starting sector number, and calls Open, which is the primative version of OPEN. It sets up the I/O Control Block. Once a file is opened, GETSEC will return the address of the next sector. If GETSEC returns 0000 , it signifies the end of file. Notice that OPEN, and any words which use open, such as SAVE, DOWNLOAD, and SEND will change the default drive number if the specified file is located on another drive.

$\mathrm{OR} \quad\langle\mathrm{N} 1 \mathrm{N2} \ldots \mathrm{N} 3>$ Or

OR performs a logical OR of the top two 16 bit numbers on the stack and replaces them with the logical result.

ORIGIN

$$
<\ldots \text { ORIGIN.ADDRESS }>\quad \text { Origin }
$$

ORIGIN is a CONSTANT which points to the first item in the parameter table. Each item in the parameter table is 2 bytes long, and offsets refer to bytes, not variables. Refer to chapter 7 for details on the parameter table.

ORIGINATE

$$
<\ldots>\quad \text { Originate }
$$

This word sets the 18S653-VI modem at MODEM.GRP to originate mode. 5 seconds after ORIGINATE is called, it checks for incoming carrier and executes QUIT if there is no carrier. It then executes the word RE-ENTER. The escape key terminates ORIGINATE mode.

OUT

$$
<\ldots \text { ADDRESS }>\quad \text { Out }
$$

OUT is a USER variable which is incremented by EMIT. The user is responsible for initializing and referencing OUT. VLIST is an example of a word which uses OUT to perform output formatting.

OUTL

$$
<\ldots \text { ADDRESS }>\quad \text { Output Lines }
$$

OUTL is a USER variable which is incremented by the word CR (Carriage Return). OUTL is intended for line printer page formatting. The behavior of OUTL and CR is analogous to the behavior of OUT and EMIT. 
OUTPUT

$\langle$ CHARACTER PORT.NUMBER ... >

output

OVER

PAD

$\langle\ldots$ ADDRESS $>\quad$ Pad

PAD places the address of the text output buffer on the stack. PAD is always located $68(d)$ bytes past the end of the dictionary. PAD is generally used in two ways. The ASCII type words <\# \# \#> etc., build the output string at PAD -1 , working backwards. The EDITOR words use PAD as a text buffer, with the first character stored at PAD.

PAGE

PARPRINTER

$$
<\ldots>\quad \text { Page }
$$

This word sends a top of form command to the line printer. FLD, the page counter is incremented by 1 .

$$
\langle\ldots\rangle \quad \text { Parallel printer }
$$

Parallel printer selects the parallel printer as the list device. Once this is done, it is merely necessary to execute PRINTER to enable the printer, and NOPRINT to disable the printer. When the printer is enabled, all output is still directed to the terminal also.

PARPTR

PDOTQ.DSP

$$
\langle\text { CHARACTER } \ldots .\rangle
$$

Parallel printer output

This word sends I character to the parallel printer. The character is not sent to the terminal.

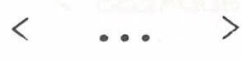

Parentheses Dot Quote Display

This is a primative used by the decompiler to display the the behaviour of words using the FORTH word dot-quote.

< NAME.FIEID.ADDRESS. . .PARAMETER.FIEID.ADDRESS > PFA

PFA converts a Name Field Address (NFA) of a dictionary definition to its Parameter Field Address (PFA).

PREV

$$
\langle\ldots \text { ADDRESS }\rangle \quad \text { Previous }
$$

PREV is a USER variable which contains the address of the most recently (previous) referenced disk block. 
PRINTER enables the system printer. This word, and NOPRINT, have different behaviors for serial or parallel printers. For serial printers attached to the terminal, an escape sequence is sent to the terminal to enable an attached serial printer. With a parallel printer using an $18 \$ 646$ printer card, or a serial printer connected to an 18S641 uart card, a dynamic patch of EMIT is made, to branch to code contained in the word parptr. This code duplicates every character sent through EMIT and sends it to both the terminal and the printer. Placing this code outside of the assembly code makes it easy to change. Placing it before ASMRR allows you to enable a printer during the process of recompiling the system. Because of the patch to EMIT, PRINTER only works in RAM systems. NOPRINT disables the printer by restoring the word FMIT'

parptr

QUERY

QUIT

QUIT.FLAG

QUOTE

$\mathrm{R \#}$

$$
<\ldots>\quad \text { Print Patch }
$$

This word functions as a NOP. It contains the code which duplicates the stack number and sends it to the parallel printer through the CDP 185646 card or to the serial printer through the CDP 18S641 card.

$$
\langle\ldots\rangle \text { Query }
$$

QUERY inputs up to 128 (d) characters from the terminal. Input is terminated by a carriage return. The text is placed in the terminal input buffer (TIB) and IN is set equal to 0 . The carriage return is not stored, but NUIJ (0) and SPACE $(20 \mathrm{~h})$ are appended to the input string. QUERY uses EXPECT.

$$
<\ldots>>\text { Quit }
$$

QUIT stops compilation, resets the return stack pointer, and starts interpretation from the terminal input stream. QUIT is an endless loop which calls the interpreter repeatedly.

$$
<\ldots \text { ADDRESS }>\quad \text { Quit Flag }
$$

This USER variable is used by the decompiler to signify to the CASE statement that decompilation is complete.

Print a ", (22h). This cannot be done directly with the ." (dot quote) format, since the quotation mark is the string terminator.

$$
\langle\ldots \text { ADDRESS }>\quad \text { R Sharp }
$$

R\# is a USER variable which is used by the editors. 
$\mathrm{R} / \mathrm{W}$ performs the actual read or write transfer of data between the disk and FORTH's buffers. It is not normally executed directly by the user.

$$
\langle\ldots \text { ADDRESS }>\quad \text { R zero }
$$

RO is a USER variable which contains the initial value of the return stack location.

$\mathrm{R}>$

RCVBRK

RCVC

RCVCHAR?

RE-ENTER

REPEAT

$$
\langle\ldots \text { NUMBER }\rangle \quad \text { R From }
$$

$\mathrm{R}>$ removes a number from the return stack and leaves it on the computation (parameter) stack. It is usually used with $>\mathrm{R}$ to recover numbers temporarily stored on the return stack. R> must be balanced with $>\mathrm{R}$ within a colon definition.

$$
\langle\ldots\rangle \quad \text { Receive Break }
$$

Receive break drops the character in the COMM.GRP uart and prints the message "Break Received" to the terminal. This is a primative ultimately used by UART. See RCVC •

$$
\langle\text { STATUS ... } \quad \text { Receive Character }
$$

Fxamine the status of the COMM.GRP uart to check for a break. If so, call RCVBRK, otherwise type the character to the terminal. This is a primative used by UART.

$$
\langle\text { STATUS } \ldots\rangle
$$

Receive Character Question

This word checks the status byte of the uart at COMM.GRP. If a character is available, RCVC is called. If a character is not available, execution continues with SENDCHAR? . This word is a primative used by UART.

$$
\langle\ldots\rangle \text { Reenter }
$$

This is the communication word for ORIGINATE. It changes COMM.GRP to the modem group and enters UART mode. Therefore, ESCAPE terminates RE-ENTER. When escape is typed, re-enter asks whether or not you desire to maintain the modem connection. If not, the modem hangs up and the comm group is restored to E0(h). If so, you reenter by typing RE-ENTER (cr).

$$
<\ldots>>\text { Repeat }
$$

Repeat is used at the end of a BEGIN WHIIE REPEAT structure. It causes an unconditional branch back to the word following BEGIN. 
This word resets the Q line of the $1806 \mathrm{CPU}$ to the 0 state (0 volts).

$$
\langle\ldots\rangle \quad \text { Rom It }
$$

ROMIT moves the dictionary pointer to the next 8 page boundary and executes NODISK and NEW-FENCE in preparation for saving a file to be burned in FPROM.

ROT

$$
\langle\mathrm{Nl} N 2 \mathrm{~N} 3 \ldots \mathrm{N} 2 \mathrm{~N} 3 \mathrm{Nl}\rangle \quad \text { Rotate }
$$

ROT rotates the top three items on the stack. The third item comes to the top of the stack.

RP! initializes the return stack pointer to the value contained in the USER variable RO.

RSTBRK

Reset break

This is a primative used by UART.

$S->D$

$$
\langle\mathrm{N} \ldots \mathrm{D}\rangle
$$

Single to Double

S->D converts a single number on the stack to a double number. $S$ to $D$ works simply by duplicating the sign bit of the previous top number in all positions of the new top number.

This is a primative used by EDIT.

$$
<\ldots \text { ADDRESS }>\quad \text { S zero }
$$

S0 is a USER variable which contains the initial address of the bottom of the computation stack.

SAVE

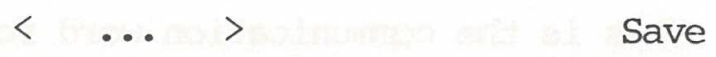

SAVE will save the current FORTH environment into the specified file. The file must already exist, and be long enough to to save from 0 to HERE. If not, SAVE issues Message $26(d)$ and executes QUIT. Remember to call NEW-FENCE first, to protect any newly defined words, and call ROMIT if the file will be burned in EPROM. Usage: SAVE FILESPEC (cr) Example: SAVE WFORTH.CM:I (cr) 
SCR is a USER variable that contains the screen number most recently referenced by LIST. This value can then be referenced by other words, so that the screen number need not be explicitly stated. The words $I$ and EDITL reference SCR.

SCR/DSK

SCREEN-DUMP

SEC

SECTOR

SEND

$$
\langle\ldots \text { ADDRESS }>\quad \text { Screens per Disk }
$$

SCR/DSK is a USER variable which is used by the word DRI to compute OFFSET. SCR/DSK is not a standard Fig-FORTH word.

$$
\langle\text { SCREEN.NUMBER ... > Screen Dump }
$$

This word dumps the contents of the desired screen buffer to the terminal, in Hex and ASCII, 16 lines at one time. The operator is prompted to continue the dump.

$$
\langle\text { TIME ... S Seconds }
$$

This word generates a delay for approximately TIME seconds. The delay is based upon a $2.45 \mathrm{mHz}$ CPU clock.

$$
\begin{array}{r}
\text { SECTOR.NUMBER } \ldots \text { BUFFER.ADDRESS > } \\
\text { Sector }
\end{array}
$$

Sector will retrieve any sector on the selected drive from 0 to 629 and return the buffer address. This is a very useful random access word for disk data.

Send

SEND will search the disk directory for the file named in FILESPEC and transmit the file in ASCII-HEX format through the uart at COMM.GRP. The operation of SEND is to similar XMIT and DOWNLOAD, except that SEND does not assume the presence of a monitor program at the receiving end. Therefore, the receiving system is responsible for capturing the file. The file is transmitted in ASCII-HEX in blocks of 512 (d) bytes ( 1 sector). The blocks are broken up as 16 lines of 64 characters. Each line is followed by CR-LF. After a block is sent, a reply is required from the receiving system. There are 3 valid replies. $Y$ or $y$ signifies that the block was successfully received and that the next block should be sent. Break indicates that the procedure should be terminated ( $A B O R T$ is then executed). Any other character will cause retransmission of the previous block. No end of file mark is sent at any time, a delay should be interpreted as the end of file. Usage: SEND Filespec ( $\mathrm{cr}$ ) Example: SEND ALV00:1 (cr) 
This word is used to transfer screens between FORTH systems. It assumes that another FORTH system is connected to the uart at COMM.GRP.

To Use: NI GET-SCREFN (at the receiving end; then) N2 SEND-SCREEN (at the sending end)

where $\mathrm{N} 1$ is the number of the screen to be received, and N2 is the number of the screen to be sent.

SENDBRK

$$
\langle\ldots\rangle \text { Send Break }
$$

This word sets the uart at COMM.GRP to the break condition. It continues to receive and display characters until the break at the terminal group uart is released. This is a primative used by UART.

SENDC

$$
\langle\text { STATUS ... S Send Character }
$$

This word examines the terminal group uart status word to see if the available key is a break. If so, it sends the break; otherwise it sends the character. SENDC is a primative used by UART.

SENDCHAR?

SERPRINIER

$$
\langle\text { STATUS } \ldots>
$$

Send Character Question

This word checks the status of the terminal group uart. If there is a character available, it is sent via SENDC to the uart at COMM.GRP. If no character is available, execution of the word UART continues with RCVCHAR? - This is a primative used by UART.

$$
<\ldots>\quad \text { Serial printer }
$$

Serial printer selects the device at the COMM.GRP uart as the printer. NOPRINT will disable this device.

SETEDIT

$$
<\ldots>\quad \text { Set Edit }
$$

Set the terminal for editing. SETEDIT is a primative used by EDIT. It is written for a Televideo 910+ terminal, and may require change or elimination for other terminals.

SETQ

$$
<\ldots>\quad \text { Set Q Line }
$$

This word sets the Q line of the $1806 \mathrm{CPU}$ to the true state ( 5 volts). 
SMUDGE

SP!

SP@

SPACE

SPACES

SPREADSCREEN
Sign is used in pictured numeric output <\# \#> to place an ASCII sign symbol in the output stream. If SIGN.FLAG is negative, a minus sign is inserted into the output stream. Refer to <\#, \#, \#S, and \#〉.

$$
\langle\ldots\rangle \quad \text { Smudge }
$$

SMUDGE will toggle the smudge bit ( the third most significant bit) of the length byte of the name field. CREATE and : (which calls CREATE), smudge a word when first named so that it cannot be found until the definition is completed; A word defined using CREATE must be smudged when the word is finished. The FORTH word; does this automatically for colon definitions.

Stack Pointer

Store

SP! initializes the computation stack pointer to the value contained in the USER variable SO.

$$
\begin{array}{ll}
\text {... STACK.POINTER }> & \text { Stack Pointer } \\
\text { Fetch }
\end{array}
$$

SPa returns the present computation stack pointer address to the top of the stack.

$$
<\ldots>\quad \text { Space }
$$

SPACE transmits an ASCII space $(20 \mathrm{~h}$ ) to the output device. The space character is the universal delimeter for FORTH words and numbers.

$$
\langle\text { NUMBER ... } \quad \text { Spaces }
$$

Spaces sends the specified NUMBER of spaces to the output device.

$$
\langle\text { SCREFN.NUMBER } \ldots>\text { Spreadscreen }
$$

This word makes a blank screen within a group of screens. It uses the word ?SCREFN to find the first non-screen above SCREEN. NUMBER. It then moves all intervening screens up 1 screen and clears SCREEN.NUMBER. CAUTION. ?SCREFN assumes that any control character makes a SCREEN a non-screen. Therefore, if SPREADSCREFN finds a screen which has a control character, it will destroy that screen. Notice that neither the FIG editor nor the full screen editor allows the user to place a control character within a screen. 
STATE

STATUS

SWAP

$\mathrm{T}$

TASK

TEAR

TERM

TERM.CTI

TERM.GRP

$$
\langle\ldots \text { ADDRESS }>\quad \text { State }
$$

STATE is a USER variable which shows whether the system is in the compiling $(00 \mathrm{~h})$, or interpreting $(\mathrm{COh})$ state.

$$
\langle\ldots \text { STATUS.WORD }>\quad \text { Status Read }
$$

This word puts the contents of the status register of the selected uart on the stack. The status is for a CDP1854A uart. This is a primative used by UART.

$$
\langle\mathrm{N} 1 \mathrm{~N} 2 \ldots \mathrm{N} 2 \mathrm{Nl}\rangle \quad \text { Swap }
$$

SWAP interchanges the top two 16 bit numbers on the stack.

$$
\langle\text { LINE.NUMBER } \ldots>>\text { T }
$$

List LINE. NUMBER of the screen whose number is stored in the USER variable SCN. Also, set the CONTFEXT vocabulary to EDITOR. This word is similar to the FIG-EDITOR word $T$, but it is a FORTH word and can be found from any vocabulary. Note that this word does not leave LINE. NUMBER on the stack, which is a Fig-EDITOR bug.

$$
\langle\ldots\rangle \quad \text { TASK }
$$

TASK is a Nop word that marks the end of WFORTH. FORGET TASK is a convenient way to reset the dictionary after compiling new words that are no longer required.

$$
<\ldots>\text { Tear }
$$

This word readies the printer for tearing the listing. It issues one form feed and turns the printer off.$$
<\ldots>>\text { Terminal }
$$

This word selects the group number contained in the VARIABIE TERM.GRP This is group 1 for normal operation. This word should be executed after all I/O to restore group numbers.

$$
\langle\ldots \text { ADDRESS }>\quad \text { Terminal Control }
$$

This is a VARIABLE which holds the value of the terminal uart control byte. This is usually ID(h).

$$
<\ldots \text { ADDRESS }>\quad \text { Terminal Group }
$$

This USER variable contains the I/O group number for the terminal UART. This is usually $01(\mathrm{~h})$. 
Usage: DECIMAL 32 TEXT THIS.IS.A.TEST XXXX. TEXT will store the string following TEXT until XXXX at PAD. The string is stored until a character equal to DEIIMETER is found in the string, or until a carriage return is encountered. In this example, the delimeter is the space character.

$$
<\ldots>\text { Then }
$$

THEN is used to terminate an IF THEN or IF ELSE THEN conditional structure. THEN is used by the compiler as a label for conditional branching.

TIB is a USER variable which points to the terminal input buffer (TIB). The buffer is $128(\mathrm{~d})$ bytes. This length is set by a literal in QUERY. TIB is initialized by COLD or WARM from item $+22(d)$ in the parameter table.

TOGGLE

$$
\langle\text { ADDRESS 8.BIT.MASK ... > Toggle }
$$

TOGGLE exclusive or's the byte at ADDRESS with 8.BIT.MASK, and leaves it at ADDRESS.

TONE

$$
\langle\text { CHAR ... Tone }
$$

This word takes a character from the stack and sends it over the modem. This is a primitive word for DIAL. Any invalid characters are ignored. See DIAL for the list of valid characters.

TRAVERSE

$$
\text { < BEGINNING.ADDRESS DIRECTION...ENDING.ADDRESS > }
$$
Traverse

TRAVERSE calculates the address of the opposite end of a name field. If direction equals 1, movement is from low to high, and BEGINNING.ADDRESS must contain the addresses of the length byte of the name field. If DIRECTION equals -1 , movement is from high to low and BEGINNING.ADDRESS must point to the last letter of the name.

TRC

$$
<\ldots>\text { Transmit Character }
$$

This word examines the character at the terminal group uart. If it is an escape character, a flag is set to terminate the UART BEGIN-UNTIL loop, otherwise the character is transmitted to the uart at COMM.GRP. This is a primative for UARI'. 
This word uses a NUMBER from TONE which has been converted from a character. NUMBER is the actual value sent to the modem, not the character being sent. This is a primative for DIAL.

TYPE

$\langle$ STRING.ADDRESS LENGTH $\ldots$.

Type

TYPE outputs a string starting at STRING.ADDRESS to the output device for IENGTH bytes. If IENGTH equals zero, nothing happens.

$\mathrm{U}$ is a pseudonym for UART.

U* multiplies two unsigned single numbers and leaves an unsigned double product.

U.

$$
\langle\mathrm{U} \ldots\rangle
$$

Unsigned Print

The number on top of the stack is printed as a positive number in the present number BASE. This is not the same as printing the absolute value of the number.

U.. $\quad\langle$ NUMBER ... $>$ U Dot Dot

U.. prints an unsigned NUMBER with no trailing space. The current number BASE is used.
$\mathrm{U} \cdot \mathrm{R}$
$\langle\mathrm{UL} \ldots\rangle$
Unsigned Print Right Justified

U.R will print the unsigned number $U$, right justified in $L$ columns followed by one space. The current number BASE is used.

U.RLZ

$$
\langle\text { NUMBER L ... > }
$$

Print unsigned number right justified with leading zeroes

This word prints NUMBER with leading zeroes. I is the number of total columns. If the number needs more than $I$ columns to be printed, then it is printed in full.

$\mathrm{U} /$

U/ divides an unsigned double dividend by an unsigned divisor and leaves an unsigned remainder and unsigned quotient. 
UART

$<\ldots>\quad$ Uart

UART initiates terminal emulation mode between the device at the terminal group (TFRM.GRP) and the device at COMM.GRP. Normally this means a terminal and operator at group 1 (TERM.GRP) communicating with a target computer through the uart at group E0 (h) (COMM.GRP). Note, however, a modem at group $\mathrm{FO}(\mathrm{h})$ is possible for either COMM.GRP or TERM.GRP. U is a pseudonym for UART.

UNTIL

$$
\langle\text { FLAG ... > > }
$$

Until

UNTIL terminates the BEGIN UNTIL loop structure. UNTIL examines the flag, if true, the loop is exited, and the word following UNTII, is executed. If the flag is false, UNTIL causes a branch to the word following BEGIN.

UPDATE

USE

USER

USER-IOCB

$$
<\ldots>\quad \text { Update }
$$

Update sets the update byte of the most recently referenced screen so that the screen will be written to disk when the buffer is re-allocated or the word FLUSH is executed. FLUSH can be executed by the user to force an updated screen to be rewritten to disk.

$$
<\ldots \text { ADDRESS }>\quad \text { Use }
$$

USE is a USER variable which contains the address of the next disk buffer to "use". USE is controlled by +BUF.

$$
\langle\text { OFFSET } \ldots>>\quad \text { User }
$$

USER is used in the form NN USER (Name) to define USER variables. $\mathrm{NN}$ is the offset into the user table and must be less than $\mathrm{D} 4(\mathrm{~h})$ and greater than $50(\mathrm{~h})$. More than 1 name can point to the same USER variable. USER variables can be used in EPROM systems whereas plain variables cannot. This is because USER variables contain an offset into the Ram user table, which does not change as the variable changes. Variables, however, store their value directly into the dictionary, which means that they cannot be changed in a PROM system. Invoking the name of a USER variable leaves the address of the variable on the stack. USER variable 0 thru $4 \mathrm{~F}(\mathrm{~h})$ are considered system variables. USER variables can also be defined sequentially by the sequence: NEXTUSER ANY.NAME (cr)

This method will automatically allocate USER variable space.

$$
\begin{array}{ll}
\langle. . \text { ADDRESS }> & \text { USER I-O Control } \\
& \text { Block }
\end{array}
$$

USER-IOCB is a USER variable that is 10 (d) bytes long. It is used by $\mathrm{R} / \mathrm{W}$ and is the IOCB used by BLOCK-READ and BLOCK-WRITE: to access the disk via the monitor program UT71. 
USERV.DSP

V

$\mathrm{V}$

VAR.DSP

VARIABIE

VCOPY $<\ldots>$

User Variable

Display

Display the USER variable at WORD.PTR. This is a primative for the decompiler.

$$
\text { < TARGET.ADDRESS IFNGTH HOST.ADDRESS ... > }
$$$$
\text { Receive }
$$

This is a block move from a target computer to the host. BASE must be HEX. Communication is through a uart at COMM.GRP (normally EOh) to the RCA utility program of the target. The target computer transmits the data block using the utility D (dump) command.

$$
\begin{gathered}
\text { TARGET.ADDRESS LENGTH HOST.ADRESS } \ldots>> \\
\text { Receive }
\end{gathered}
$$

This is the low level version of $V$. Base need not be Hex. $\checkmark$ performs a simple check to see if it is communicating with a utility program in the target computer via the COMM.GRP uart. It receives IENGTH bytes from TARGET.ADDR and stores them at HOST.ADDR in the host computer.

$$
\langle\ldots\rangle \quad \text { Variable Display }
$$

Display the VARIABLE at WORD.PTR. This is a primative for the decompiler.

$$
\langle\mathrm{N} \ldots\rangle \quad \text { Variable }
$$

VARIABLE is used to define variables in the form: $\mathrm{N}$ VARIABLE ANY.NAME (cr)

where $\mathrm{N}$ is the initial value of the named VARIABLE. Whenever the name is invoked, the address of the VARIABLE is left on the stack. a (fetch) will replace the address with the value. ! (store) will store the second item on the stack at address, thereby updating the VARIABLE. Note that variables cannot be used in Eprom systems because the value of a VARIABLE is stored in the dictionary. Use USER variables for Eprom systems.

$$
\langle\text { PAGE.NUMBER ... > Video Ram COpY }
$$

This word sets the address of the video ram to PAGE. NUMBER and then reads and writes $1024(d)$ bytes. Thus, the video ram will display 4 pages starting at PAGE. NUMBER. This requires the WHOI video ram. See the section on special hardware. 
This word will list the name field address (NFA) and name (ID) for all words in the CONTEXT vocabulary. VLIST starts at the top word in the CONTEXT vocabulary and follows the link field addresses through the CONIEXT vocabulary to the FORTH

VOCABUIARY to the word COLD, the bottom-most word. The break key prematurely terminates VLIST.

VMOVE

VOC-LINK

VOCAB.DSP

VOCAB?

$$
\langle\text { PAGE.NUMBER ... > Video Ram Move }
$$

This word moves the address of the video ram to PAGE. NUMBER. The video ram is not cleared or updated. This word is a primative for VCOPY and VSHADOW. It requires the WHOI video ram. See the section on special hardware.

$$
\langle\ldots \text { ADDRESS }>\quad \text { Vocabulary Link }
$$

VOC-LINK is a USER variable which points to the VOC-LINK field in the vocabulary most recently defined. This is initiallized to point to the VOC-LINK field in the word FORTH by COLD. VOC-LINK is unwittingly used by the word VOCABULARY to extend the dictionary in an Eprom implementation of FORTH. The technique is as follows. Operator keyboard input is underlined.

VOCABULARY FIFTH IMMEDIATE OK HERE 4 = DUP CURRENT ! CONTEXT ! OK : TEST ." HET TEST HETLO OK

VLIST

TEST etc.

The vocabulary FIFTH is created without any problem as part of FORTH, but it cannot be used because FORTH's link is frozen in Eprom. Storing the value of FIFTH's VOC-LINK field in CURRENT and CONTEXT explicitly selects and utilizes the FIFTH vocabulary. QUIT will retain all new definitions but COID or ABORT will forget new definitions.

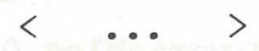

Vocabulary Display

Display the vocabulary at WORD.PTR. This word is a primative for the decompiler.

$$
<\ldots>>
$$

Vocabulary Question

This is a teaching word which displays the name of the CURRENT vocabulary and the name of the CONTEXT vocabulary. 
VOCABUIARY

$$
<\ldots>\quad \text { Vocabulary }
$$

VOCABULARY is used to define new vocabularies in the form: VOCABULARY NAME IMMEDIATE. By using vocabularies, it is possible to have many definitions of the same name, and yet find the different words easily. It is also possible to obscure words which are not particularly valuable to the user, but rather are used to define higher level words. Refer to the FORTH Encyclopedia for complete details.

VSHADOW

Wl

W2

W3

W4

WARM

WARNING

$$
\langle\text { PAGE.NUMBER ... > Video Ram Shadow }
$$

This word first clears the video ram at port 2 and then sets the video ram address to PAGE.NUMBER. VSHADOW will then display data as it is written. This word requires the WHOI video ram. See the section on special hardware.

Wait for External Flag 1

Wl waits for external flag 1 to be true ( 0 volts).

Wait for External Flag 2

W2 waits for external flag 2 to be true ( 0 volts).

Wait for External Flag 3

W3 waits for external flag 3 to be true ( 0 volts).

Wait for External Flag 4

W4 waits for external flag 4 to be true ( 0 volts).

$$
\langle\text { ALL.VALUES ... > Warm }
$$

WARM initializes USER variables 0 through $\mathrm{OE}(\mathrm{h})$ and registers $2,4,5,6,7$, and 8 from the information contained in the parameter table. WARM then executes ABORT. FORTH can be re-entered at location $0003(\mathrm{~h})$ for a WARM start.

$$
\langle\ldots \text { ADDRESS }>\quad \text { Warning }
$$

WARNING is a USER variable which holds a flag. If WARNING is negative, (ABORT) is executed. If warning is 0 or positive, only the message number will be printed. Note that in Woods Hole FORTH, a positive value in warning has no special significance, because error messages are not stored on the screens. 
WHERE

< BLOCK.OFFSET BLOCK.NUMBER ... >

Where

WHFRE is a word which may be called after a screen load error. BLOCK. NUMBER and BLOCK. OFFSET, which are placed on the stack by the word ERROR, point to the word which caused the error, and WHERE uses the values to explain the error.

WHIIE

$$
\langle\text { FIAG ... }>\text { While }
$$

While is used within a BEGIN WHILE REPEAT loop. The flag is examined, and if true the words between WHIIE and REPEAT are executed, returning to BEGIN. If the flag is false, the loop is terminated, and the word following REPEAT is executed.

WIDTH

$$
<\ldots \text { ADDRESS }>
$$

Width

WIDTH is a USER variable which contains the maximum number of characters allowed in the name field of a dictionary definition. WIDTH is 31 (d) in Woods Hole FORTH.

WNI

WN2

This word waits until external flag 1 is not true ( 5 volts).

WN3 WN4

WORD

$<\ldots>>$

Wait not 4

This word waits until external flag 2 is not true (5 volts).

$$
<\ldots>\quad \text { Wait Not } 4
$$

This word waits until external flag 3 is not true (5 volts).

$$
\langle\ldots\rangle \quad \text { Wait Not } 4
$$

This word waits until external flag 4 is not true (5 volts).

\section{WORD}

WORD-79

$$
<\text { DEIIMITER } \ldots>\text { Wora }
$$

WORD parses one word from the input stream. Characters are transferred from the input stream to memory starting at HERE, the next available dictionary location. WORD leaves the character count of the string in the first byte of the string and terminates the string with one or more blanks. Leading delimeters are not transferred. HERE points to the first byte of the string, the byte count. This definition of WORD is the fig-FORTH definition; the FORTH-79 standard version is called WORD-79 in Woods Hole FORTH.

$$
<\text { DEIIMITER ... HERE > Word } 79
$$

WORD-79 is the FORTH-79 standard word for "WORD". It is defined in fig-FORTH as

: WORD-79 WORD HFRE ; 
WORD.DSP

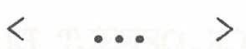

Word Display

Display the NFA, CFA, and ID of the word at WORD.PTR. This is a primative used by the decompiler.

WORD.PTR

$$
\langle\ldots \text { ADDRESS }\rangle \quad \text { Word Pointer }
$$

This USER variable is used by the decompiler to point to the CFA of the word being decompiled.

This word facilitates block moves between computers. It is similar to $\mathrm{V}$ but the direction of the transfer is from host to target. The target must be running an RCA monitor program that supports the I (insert) command. XMIT requires that the base be HEX. It selects the COMM.GRP uart to communicate with the target. 
Xmit

$$
\begin{aligned}
& <\text { HOST.SOURCE.ADDRESS IENGTH } \\
& \text { TARGET.ADDRESS } \ldots>>>\text { Transmit }
\end{aligned}
$$

This is the low level form of XMIT. It checks for a target monitor and uses the Insert command to move the number of bytes specified in IENGTH from HOST.ADDRESS to the target

computer at TARGET.ADDRESS. The target computer is the one connected to the uart at COMM.GRP.

XOR

$$
\langle\mathrm{N} 1 \mathrm{~N} 2 \ldots \mathrm{N} 3\rangle \quad \text { Exclusive Or }
$$

XOR performs an exclusive-or of the two 16 bit numbers on top of the stack and replaces them with the logical. result.

Y?

ZEROES

[COMPILE]

$$
<\ldots>\text { Yes Question }
$$

Wait for a key from the present group's uart. If it does not equal $Y$ or $Y$, execute QUIT, otherwise continue.

$$
\langle\text { NUMBER.OF.ZEROES ... > Zeroes }
$$

This word works just like spaces. It prints number of zeroes. It is a primative for U.RLZ and D.RLZ.

$$
<\ldots>\text { Left Bracket }
$$

[ is used to suspend compilation while in a colon definition. The words which follow [ are executed rather than compiled. Compilation is resumed by executing ].

$$
<\ldots>\text { Bracket Compile }
$$

[COMPIIE] causes the next word in the input stream to be compiled into the dictionary even if it is an immediate word. For instance, [COMPILE] FORTH within a definition will cause FORTH to be the CONIFXX vocabulary when the word is executed, rather than at compile time.

$$
\langle\ldots\rangle \text { Right Bracket }
$$

RIGHT BRACKET places the system into compilation state, usually after compilation was suspended using [ • [COMPIIE] ANY.WORD is the same as [ ANY.WORD ] . 


\section{ERROR MESSAGES}

The word MESSAGE prints all message numbers in decimal.

Message 0 This word is undefined (it cannot be found in the dictionary). The stack now contains 2 numbers. The top number is the screen number which caused the error. The second number contains the offset, in bytes, from the beginning of the screen to the end of the word which caused the error. Message 0 is always followed by QUIT. Message 0 is issued by NUMBER.

Message 1 The stack is empty. This message is issued by ?STACK and followed by QUIT.

Message 2 The dictionary is full. Message 2 is issued by CREATE and is always followed by QUIT.

Message 4 A word with this name already exists. Compilation is not interrupted. This message is issued by CREATE.

Message 7 The stack is full. This message is issued by ?STACK and followed by QUIT.

Message 8 This mesage is issued by R/W. It indicates that there was a disk I/O error. Execution is not interrupted.

Message 17 This message is issued by ?COMP when the system is not in compile STATE. The USER variable STATE is non-zero (COh) if the system is in compile STATE. This is part of the compiler security.

Message 18 This message is issued by ?EXEC when the USER variable STATE is not equal to zero. This indicates that the system is not in EXECUTE STATE.

Message 19 This message is issued by ?PAIRS. If the top two numbers 
on the stack are not equal when ?PAIRS is executed, Message 19 is issued followed by QUIT.

Message 20 This message is issued by ?CSP when ?CSP finds that the current stack pointer is not equal to the user variable CSP. Message 20 is followed by @UIT.

Message 21 This message is issued by FORGET when an attempt is made to forget a word that is protected by the FENCE. QUIT is executed after this message. Change the value of FENCE to fix this error.

Message 22 This message is issued by ?IOADING if the system is not loading from disk when ?IOADING is executed. The test is to see if the USER variable BLK is non-zero. Message 22 is followed by QUIT.

Message 23 This message is issued by LINE in Fig-EDITOR, and does not interrupt execution. It occurs when LINE is asked to find a line number greater than $15(d)$. This message will usually appear when $L$ is executed before any editing has occurred, and therefore the USER variable $\mathrm{R} \#$ is not yet initialized. In this case, the message is unimportant.

Message 24 This message is issued by FORGET and followed by QUIT. It means that the CURRENT and CONTEXT vocabularies are not the same. FORGET will not forget any words unless CURRENT and CONTEXT are the same. 
Message 25 No USER variable space is available. In Woods Hole FORTH, USER variables $50(\mathrm{~h})$ thru $\mathrm{D} 4(\mathrm{~h})$ are available, a total of 66 (d) USER variables. This message is issued by NEXTUSER.

Message 26 This message is issued by SAVE. The file specified is not large enough to store from 0 to HFRE. Message 26 is followed by QUIT.

Message 27 This message is issued by SEND. It means that a break condition was detected by SEND, the transfer is stopped and QUIT is executed.

TEXT MESSAGES

No blank screens There are no blank screens between SCREFN.NUMBER and 249 (d). This message is issued by SPREADSCREEN. No screens are moved, and QUIT is executed.

Break received A break condition exists at the uart in COMM.GRP. This message is issued by UART. Execution is not interrupted.

No dial tone There is no dial tone available to the modem at MODEM.GRP. This message is issued by DIAL. QUIT is executed after this message.

RE-ENTER

This message is issued by RE-ENTER, when the escape key (esc) is pressed at the terminal. Reply with $Y$ if you want to maintain the modem connection, or any other key if you want to hang up. RE-ENTER prompts the user with the message "Type RE-ENTER when ready."

Not found This message is issued by OPEN when the desired file is not found in the disk directory. This message is followed by QUIT. 
No Monitor

This message is issued by $\mathrm{V}$, XMIT, and DOWNLOAD via ?MONITOR, when there is no monitor program (UT71 or UT62) running in the computer connected to the uart at COMM.GRP. It means that FORTH is unable to send or receive information from this computer via the utility program.

Declare HEX

This message is issued by XMIT, $V$, and DOWNLOAD. The base must be hexadecimal when sending or receiving data from remote computers. 
Downloading

is complete

PSN Too Large

End of File

On Screen (num)

(name) caused a

short branch

off page
This message is issued by DOWNLOAD. It means that a block was successfully sent by DOWNLOAD. Execution is not interrupted.

This message is issued by SECTOR when the desired sector is larger than $629(d)$. This message is followed by QUIT.

This message is issued by OPEN and NEWLSN when the end of file mark is read in a file. This message is followed by QUIT.

This message is issued by the ASSFMBIFR. It means that a short branch was detected across a page boundary. Execution is not interrupted, but the assembled code is probably incorrect. 


\section{DOCUMENT LIBRARY}

August 3, 1984

DISTRIBUTION LIST FOR TECHNICAL REPORT EXCHANGE

Institute of Marine Sciences Library

University of Alaska

O'Neill Building

905 Koyukuk Ave. North

Fairbanks, AK

Attn: Stella Sanchez-Wade

Documents Section

Scripps Institution of Oceanography

Library, Mail Code C-075C

La Jolla, CA 92093

Hancock Library of Biology \& Oceanography

Alan Hancock Laboratory

University of Southern California

Los Angeles, CA 90007

Gifts \& Exchanges

Library

Bedford Institute of Oceanography

P.O. Box 1006

Dartmouth, NS, B2Y 4A2, CANADA

Office of the International

Ice Patrol

c/o Coast Guard R\&D Center

Avery Point

Groton, CT 06340

Library

Physical Oceanographic Laboratory

Nova University

8000 N. Ocean Drive

Dania, FL 33304

NOAA/EDIS Miami Library Center

4301 Rickenbacker Causeway

Miami, FL 33149 -

Library

Skidaway Institute of Oceanography

P.O. Box 13687

Savannah, GA 31406

Institute of Geophysics

University of Hawaii

Library Room 252

2525 Correa Road

Honolulu, HI 96822
Library

Chesapeake Bay Institute

4800 Atwell Road

Shady Side, MD 20876

MIT Libraries

Serial Journal Room 14E-210

Cambridge, MA 02139

Director, Ralph M. Parsons Laboratory

Room 48-311

MIT

Cambridge, MA 02139

Marine Resources Information Center

Bldg. E38-320

MIT

Cambridge, MA 02139

Library

Lamont-Doherty Geological Observatory

Colombia University

Palisades, NY 10964

Library

Serials Department

Oregon State University

Corvallis, OR 97331

Pell Marine Science Library

University of Rhode Island

Narragansett Bay Campus

Narragansett, RI 02882

Working Collection

Texas A\&M University

Dept. of Oceanography

College Station, TX 77843

Library

Virginia Institute of Marine Science

Gloucester Point, VA 23062

Fisheries-Oceanography Library

151 Oceanography Teaching Bldg.

University of Washington

Seattle, WA 98195

Library

R.S.M.A.S.

University of Miami

4600 Rickenbacker Causeway

Miami, FL 33149 



\begin{tabular}{|c|c|c|c|}
\hline $\begin{array}{l}\text { REPORT DOCUMENTATION } \\
\text { PAGE } \\
\end{array}$ & $\begin{array}{l}\text { 1. REPORT NO. } \\
\text { WHOI-84-46 }\end{array}$ & 2. & 3. Recipient's Accession No. \\
\hline \multirow{2}{*}{\multicolumn{3}{|c|}{$\begin{array}{l}\text { 4. Title and Subtitle } \\
\text { Woods Hole FORTH }\end{array}$}} & $\begin{array}{l}\text { 5. Report Date } \\
\text { December } 1.984 \\
\end{array}$ \\
\hline & & & 6. \\
\hline \multicolumn{3}{|l|}{$\begin{array}{l}\text { 7. Author(s) } \\
\text { John J. Akens }\end{array}$} & $\begin{array}{l}\text { 8. Performing Orcanization Rept. No. } \\
\text { WHOI-84-46 }\end{array}$ \\
\hline \multirow{2}{*}{\multicolumn{3}{|c|}{$\begin{array}{l}\text { 9. Performing Organization Name and Address } \\
\text { Woods Hole Oceanographic Institution } \\
\text { Woods Hole, Massachusetts } 02543\end{array}$}} & 10. Projoct/Task/Work Unit No. \\
\hline & & & $\begin{array}{l}\text { 11. Contract(C) or Grant(G) No. } \\
\text { (C) } \mathrm{OCE} \mathrm{83-20508} \\
\text { (G) }\end{array}$ \\
\hline \multirow{2}{*}{\multicolumn{3}{|c|}{$\begin{array}{l}\text { 12. Sponsoring Organization Name and Address } \\
\text { National Science Foundation }\end{array}$}} & $\begin{array}{l}\text { 13. Type of Report \& Period Covered } \\
\text { Technical }\end{array}$ \\
\hline & & & 14. \\
\hline
\end{tabular}

This report should be cited as: Woods Hole Oceanog. Inst. Tech. Rept. WHOI-84-46.

16. Abstract (Limit: 200 words)

Woods Hole FORTH, called WFORTH, is a real time language for the RCA 1806 microprocessor. It follows the fig-FORTH standard, and is used by Alvin for data acquisition and display tasks, plus playback and processing tasks at sea and ashore. WFORTH is optimized for simple networking with RS-232 uarts. Programs can be installed in Eprom for use in unattended systems and can be configured to run a specific task when power is applied. The error handling can be customized to permit continued operation when errors are encountered. Extensive utilities and development tools are included such as full screen editor; the fig line editor; a single pass assembler; floating point support; disk file access; communication utilities for uploading or downloading files or blocks of memory; and documentation utilities. The complete sources and a glossary for WFORTH are included in this publication. Versions are supplied which include compiled development, floating point, and assembler utilities.

17. Document Analysis a. Descriptors

1. FORTH

2. real-time

3. Alvin data

b. Identifiers/Open-Ended Terms

c. COSATI Field/Group

18. Availability Statemen:

Approved for publication; distribution unlimited.

\begin{tabular}{|l|l|}
\hline $\begin{array}{l}\text { 19. Security Class (This Report) } \\
\text { UNCLASSIFIFD }\end{array}$ & $\begin{array}{l}\text { 21. No. of Pages } \\
290\end{array}$ \\
\hline 20. Security Class (This Page) & 22. Price \\
\hline
\end{tabular}


